


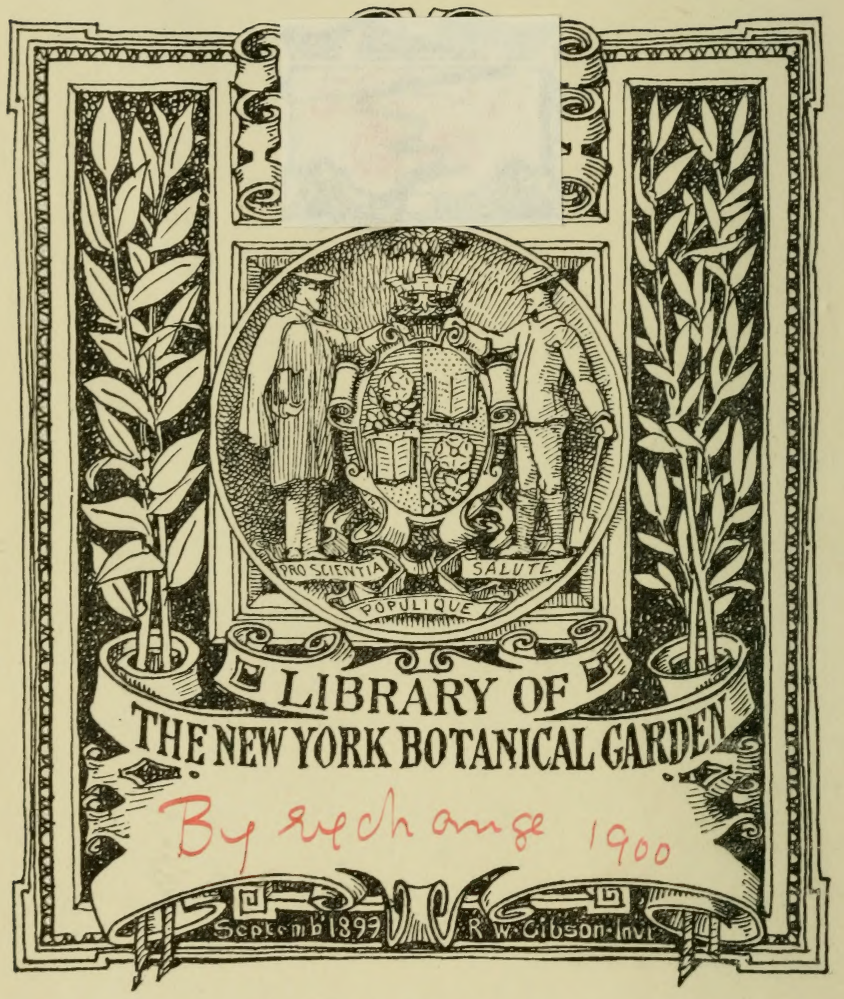








\section{TABULAE RHODOLOGICAE}

EUR OPAE 0-0RIENTALES LOCUPLETISSIMAE 


\section{Ouvrages du même Auteur.}

Révision du genre Crataegus pour les sections des $C$. oxyacantha L. et $C$. oxyacanthoides Thuill. Grand in-8 $8^{\circ}, 1871$.

Catalogus Rosarum Europae ac Orienlis, etc., hucusque cognitarum, novo fundamento instaurandus. In $-4^{\circ}$.

Prodromus monographiae Rosarum europaearum orientaliumque. In- $8^{\circ}$.

Observalions sur les Centaurea decipiens Thuill., tubulosa Chabert et Iransalpina Schl. In-8 ${ }^{\circ}, 1873$.

Collectanea rhodographica, sive Rosarum omnium in Europa confinibusque hucusque cognitarum, enumeratio systematica. In-8 ${ }^{\circ}$.

Revue critique du genre Ulex. In-8 ${ }^{\circ} .1882$.

De guibusdam Senecionis, e grege $S$. erucifolii DC, ac Jacobaeae L. novis speciebus adhuc igitur ignotis. In-8 ${ }^{\circ} .1882$.

Flore Lyonnaise et des départemenls du Sud-Est. Un vol. in-12, 1875.

Decades plantarum novarum praesertim ad floram Europae spectantes :

Fasc. I (1875). In- $8^{\circ}$.

Fasc. II (1876). In- $8^{\circ}$.

Fasc. III (1880). In- $8^{\circ}$.

Fasc. IV (1882). In- $8^{\circ}$.

Fasc. V (1882). In- $8^{\circ}$.

Essai sur une nouvelle classification des Roses de l'Europe, do l'Orient et du bassin méditerranéen, 1876 . In-8.

Ensayo sobre una nueva clasificacion de las Rosas de Europa, Oriente y Region del mediterraneo, por D. Miguel Gandoger, traducido por D. Ramon Martin Cercos, Licenciado en Farmacia. Barcelona in "El Laboratorio, » Calla de Valencia Nr. 332, 1880. In- $8^{\circ}$.

Plantes nouvelles (Roses) pour la Suisse. In-8 ${ }^{\circ}$. (Bulletin des travaux de la Société Murithienne du Valais, fasc. V et VI, 1876; pag. 26-37).

Notice biographique sur Jean-Marie-Michel Bernardin, botaniste lyonnais, 1877. In-8.

Rosae novae Galliam austro-orientalem colentes :

Fascicul. I (1877). In-8 ${ }^{\circ}$.

Fascicul. II (1878). In-8。.

Pugillus plantarum novarum vel minus recte cognitarum, 1882. Un vol. in- $8^{\circ}$.

Menthae novae imprimis Europae, cum tabulis dichotomicis illustratae, 1881. In- $8^{\circ}$. Fascic. I et II.

Tentamen de Verbascis novis, 1882 . In- $8^{\circ}$.

Salices novae (1881). Fasiculus I. In- $8^{\circ}$.

Contributions ad Floram terrae Slavorum meridionalium :

Fasciculus I (species novae). In-8。.

Fasciculus II (genus Rosa). In-8。.

Mémoire sur la propagation des plantes par les graines, considérée au point de vue de l'école analytique. In-8.

Revue du genre Potamogeton. In- $8^{\circ}$.

Revue du genre Jacea (Centaurea). In-8०.

Revue du genre Polygonum. In-8*.

Rubus nouveaux, avec un essai sur la classification du genre. In-8.

Monographie des Saules de l'Europe et du bassin médilerranéen, avec un Appendice sur les Saules des autres parties du globe. 1 vol. in-8 .

Catalogue des plantes récoltées pendant mon séjour en Algérie, de 1877 à 1880. In- $8^{\circ}$. 


\section{ADDENDA ET CORRIGENDA.}

Pag. 29, linea 24, loco croissante, lege décroissante.

- 92, n. 917 , - pedemontana, pone PEDEMONTII.

- 97, n. 1020, - setisepala, - setulisepala.

- 112, n. 1254, - durifolia, - Duriuscula.

- 116, n. 1324, - rostella, - Rostellata.

- 120, n. 1398, - pectinella, - pectinata.

- 122, n. 1434, - orthochlamys, - oRthostepHaNa.

- 292, n. 4266, - berberieofolia, - BERBERIFolia.

- 292, locis lineai, paralble et cineres, lege lineari, parallele et cineres.

- Quae Tu, L. B., in hoc opusculo tanti Generis, minus congrua minusve accurate disposita aut expressa invenias, eorum veniam inde mihi repeto, quod tam diu id prolatum, plurimisque correctionibus ac additamentis interceptum est. Quod si ejus plenitati veritatique profuit, altera ex parte decori haud parvum detraxit. Quamquam ideo majus quod molitus sum, agere mihi non licitum fuit, tamen, si quid hoc minori prodesse potuero, contentus ero.

M. G. 


\title{
TABULAE RHODOLOGICAE
}

\section{EUROPAEO-ORIENTALES LOCUPLETISSIMAE}

\author{
AUCTORE \\ Michat GANDOGER, \\ Plurim. Societ. sodali. \\ < Qui sapit, vidit quanta extricatu et \\ investigatu restant.»

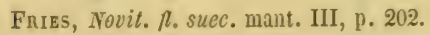

\section{PRÉFACE}

L'un des grands moyens de faciliter la détermination des espèces dans un genre aussi raste que les Roses, c'est assurément celui des tables dichotomiques. - Aucun travail de cette nature n’a encore été, à notre ennnaissance, entrepris sur ce genre; aussi, croyons -nous aller an-derant des désirs des Rhodophiles en publiant les tableaux suivants. Toutefois, nous allons expliquer les changements considérahles apportés dans la classification que nous adoptons, par le commentaire détaillé de notre Essai (1).

(1) Essai sur une nouvelle classification des Roses de l'Europe, de l'Orient et $d u$ bassin méditerranẻen. Paris, 1876, ir $-8 \circ$. 
Le genre Rosa, avons-nous dit, offre à l'étude un intérêt non moins varié qu'attrayant; mais aussi il présente de grandes difficultés. Et en écrivant ceci, nous le savions, nous n'apprenions rien de noureau; tous les botanistes en conviennent et savent en assigner les causes. Depuis quelques années sans doute, ce genre a fait des progrès réels et incontestables; mais tout n'est pas fait encore; le croire serait se flatter d'une étrange illusion, et l'on ne saurait l'affirmer sans faire preuve d'une grande ignorance.

La première question, en effet, qui s'est naturellement posée devant les monographes a été de savoir comment ce genre difficile se laisserait subdiviser. La chose méritait qu'on s'y arrêtât; aussi, il n'est pas de rhodologiste qui n'ait proposé un système particulier de classification. Et, cependant, tout botaniste compétent et sincère l'avouera avec nous : nous n'avons pas encore pour le genre Rosa une classification suffisante et complète; on n'est pas encore parvenu à lui assigner des divisions catégoriquement définies, logiquement et nettement tranchées. Voilà, aujourd'hui, ce qu'on est en droit de constater.

Est-ce à dire que toutes les recherches, que toutes les inventions faites doivent rester inutiles? Rien n'est plus loin de notre pensée. Un système scientifique bien formulé est une grande œurre, et cette œuvre est ordinairement l'ouvrage du temps, le résultat d’efforts combinés, de lumières mises en commun. Tous les systèmes ne peurent pas prévaloir, sans doute, mais tous peurent servir au progrès de la science. Par ses défauts même, un système obtient souvent un résultat heureux en faisant ressortir la vérité et les avantages d'un système opposé.

Les botanistes qui se sont occupés de la classification du genre Rosa n'auront donc pas travaillé en vain s'ils ont 
pu jeter equelque lumière sur la question fondamentale qui nous occupe. Si leur systeme ne préraut pas et s'ils n'ont pas la gloire d'y attacher leur nom, ils n'en n'auront pas moins bien mérité de la science.

Au reste, nous ne prétendons nullement qu'il faille rejeter tous les systemes, car, pour liaire de lit science, il n'est pas nécessaire de faire du nouveau. Parmi tous les systèmes proposés, nous en reconnaissons de légitimes, et mème de très bien imaginés. Notre but primitif a done été uniqguement de choisir le meilleur, d'en corriger les défauts, de le compléter, de le perfectionner.

Sans entrer dans de trop longs détails, qui seraient pour le moins superflus ici, nous nous eontenterons d'esquisser it grands traits et selon l'ordre chronologique les principales classifications du genre Riosa ; nous montrerons ensuite que celle qui nous a paru la meilleure a fait la base du systeme que nous avions à proposer.

Linné (1) qui connaissait seize espèces de lioses, les divisait en : roses à fruits globuleux et roses à fruits orales; division complètement fausse, puisqu'il arrive qu’une mème espèce varie parfois ì fruit arrondi, ovale ou oblong.

C'est à De Candolle 2) que revient la ggloire d'aroir domné le premier une classification bien élémentaire, sans doute, mais qui n'en a pas moins fourni un ji.lon suivi par ses successeurs, en créant sa section des Symstyle.

Desraux (3) s'emparant de cette nourelle sedion, classe les Roses francaises en doux groupes principaux, selon

(1) Systema vegetabilium, Gotting $x, 1794$, in- $8^{\circ}$.

(2) Catalogus plantarum horli botanici Monspeliensis. Monspelii. 1812 , in $-8^{\circ}$.

(3) Journal de Botanique. Paris, 1809-14, 6 vol. in-8。 
qu'elles sont à styles libres ou soudées en colonne. Cette manière d'entendre la classification des Roses a été suivie par la plupart des auteurs français, excepté par Grenier (1) qui fonde son système sur la grandeur relative des stipules, les ovaires sessiles ou stipités, etc., et qui a été ainsi conduit à une classification aussi absurde que bizarre.

Trois ans après, Rau (2), dans un travail justement estimé, divisa les Roses de Wurtzbourg en deux sections basées : $1^{\circ}$ sur la présence ou l'absence des glandes infrafoliaires; 2. sur la forme du fruit. Cette méthode a l'inconvénient grave de séparer des espèces évidemment voisines.

Woods (3) reconnait trois grandes divisions: A. Setigerœe (aculeis saepius rectis). - B. Setis nullis, aculeis rectiusculis. - C. Setis nullis, aculeis uncinatis. - Dans cette dernière division, il admet les Synstylae de De Candolle, car il dit : $1^{\circ}$ Stylis distinctis; $2^{\circ}$ Stylis unitis. - Babington (4) a adopté ce système en y introduisant quelques petites modifications.

Reichenbach (こั) a légèrement changé ces divisions, mais arec cette différence qu'il adopte quelques-uns des groupes proposés par De Candolle dans le Musée helvétique de Seringe (6), dont nous allons parler.

(1) Flore de France. Besançon, 1847-56, 3 vol. in-80.

(2) Enumeratio Rosarum circa Wirceburgum et pagos adjacentes sponte crescentium. Norimbergæ, 1816, in- $8^{\circ}$.

(3) A synopsis of the British species of Rosa - in : Transactions of the Linnean Society, London, in-4 ${ }^{\circ}$ (vol. XII, pp. 159 seq.).

(4) Manual of british botany. London, $1^{\text {re }}$ éd., $1843 ; 2^{\circ}$ éd., 1847, in $-8^{\circ}$.

(5) Flora germanica excursoria. Lipsiæ, 1830-32, 3 vol. in-18.

(6) Musée helvétique d'histoire naturelle (partie botanique). Berne, 1818-23, in $-4^{\circ}$. 
Ce magnifique travail est le fondement de notre classilication actuelle, l'auteur y divise toutes les Roses connues alor's en onze sections : A. Synstylce. B. Rubigince. C. Gallicance. D. Ghinenses. E. Cinnamomece. F. Hebecladce. G. Pimpinellifolice. H. Villosce. I. Centifolice. J. Canince. K. Eglanterice.- Nos grandes sections actuelles, on le voit, se trouvent à peu près toutes indiquées ici. Besser (1), qui connaissait si bien les Roses, a suivi cette classification en y apportant ses changements personnels.

On aurait dû s'attendre à ce que Seringe admit les mêmes sections dans le Prodromus de De Candolle (2) quand il fut appelé à y traiter le genre Rosa; il les réduisit, au contraire, à quatre : Synstylce, Chinenses, Cimnamomece, Canince; en sorte qu'au lieu de former une série naturelle et méthodique, il ne fit du genre Rosa qu un chaos incompréhensible.

Signalons encore les classifications peu connues de Leman (3), qui divise les Roses : $1^{\circ}$ en foliolis simpliciter dentatis; $2^{\circ}$ dentibus foliolorum margine inferiore serratis; $3^{\circ}$ dentibus foliolorum utrinque margine serralis glandulosisve, et de Rafinesque (4), lequel partage les Roses américaines selon qu'elles ont les sépales entiers ou pinnatifides.

En suivant l'ordre chronologique, nous arrivons à

(1) Enumeratio plantarum hucusque in Volhynia, Podolia, gub. Kijoviensi, Bessarabia, etc., collectarum. Vilnæ, 1822, in-8.

(2) Prodromus systematis naturalis rejni vegetabilis. Parisiis, 1824-1870, 16 vol. in- $8^{\circ}$ en 22 tomes.

(3) In Bulletin de la Société philomatique de Paris, année 1818, in $4^{\circ}$.

(4) Annales générales des sciences physiques, publiées par Bory, Drapier et Van Mons, tome V. Bruxelles, 1820, in-8०. 
Thory (1), qui groupe les Roses en cinq sections principales, se subdivisant elles-mêmes en vingt-cinq sous-sections, fondées la plupart sur des caractères de peu d'importance. C'est lui qui a fourni le texte à la magnifique illustration des Roses de Redouté (2).

Lindley (3) adopta à peu près la classification publiée dans Seringe par De Candolle, mais en ajoutant quelques sections nouvelles qui, elles-mêmes, semblent faire double emploi avec celles de De Candolle, puisqu'il admet également onze sections.

Trattinick (4) divisa ses deux cent trente-quatre espèces de Roses en vingt-quatre groupes principaux, dont les noms rappellent ceux de divers botanistes de l'époque, mais dont les diagnoses sont si vagues et si confuses qu'il est impossible d'en faire une analyse sérieuse.

Dumortier groupa d'abord (๖) les Roses en quatre sousgenres; puis, en 1827 (6), il y ajouta quelques sous-divisions tirées de De Candolle, classification qu'il a adopté de nouveau dans son dernier travail sur les Roses ( $\bar{i})$, mais en créant un autre sous-genre, Erhorhodon. Sa classification s'éloigne de toutes les précédentes, en ce qu'il fonde son système sur la forme du nectaire ou du disque, caractère

(1) Prodrome de la Monographie du genre Rosier. Paris, 1820, in-12.

(2) Histoire des Roses. Paris, 3 vol gr. in- $8^{\circ}$.

(3) Rosarum monographia, or a botanical history of Roses. London, 1820 , in $8^{\circ}$.

(4) Rosacearum monographia. Vindobonnæ, 1823-24, 4 vol. in-12.

(5) Notice sur un nouveau genre de plantes: Hulthemia, précédée d'un aperçu sur la classification des Roses. Tournay, 1824, in-8 ${ }^{\circ}$.

(6) Florula belgica. Tornaci Nerviorum, 1827, in-8.

(7) Monographie des Roses de la flore belge. Gand, 1867, in-8॰. 
qui, selon l'auteur, aurait une importance capitale, mais qui, en définitive, devient nul, puisque dans le seul groupe du Rosa canince on trouve tous les intermédiaires, depuis le disque plan jusqu'au disque épais el fortement eonique.

Wallroth (1) publia une classification identique à celle de Rafinesque, classification qui a été aussi suivie par LoiseleurDeslongchamps (2).

Koch (3) partage les Roses d'Allemagne en quatre sections fondées sur la plus ou moins grande dilatation des stipules, sur les rameaux florifères, et sur les ovaires sessiles plus ou moins longuement stipilés. Grenier (loc. cit.) a calqué sa classification sur celle de Koch, laquelle détruit les affinités naturelles des espèces et conduit à un résultat absurde.

Godet (4) revient sur les anciennes données de Desraux, mais avec cette différence qu'il subdivise ses Diastylce ou Roses à styles libres en cinq groupes, basés sur la forme des aiguillons, caractère qui, pour le dire en passant, est loin d'être fixé, même pour les variétés d'une même espèce. Il a reproduit cette classification avec quelques variantes dans son Supplément (こ).

Reuter (6) divise les Roses en deux sections, selon qu'elles

(1) Rosæ plantarum generis historia succincla. Nordhus, 1828 , in-8.

(2) Flora Gallica, éd. 2॰, Lutetix, 1828, 2 vol. in-8॰.

(3) Synopsis floræ germanicx et helvetica, ed. 10, Francofurti, 1837 ; éd. $2 \bullet$, 1843, in-8॰.

(4) Flore du Jura, 1853, in-80.

(5) Supplément à la flore du Jura suisse et français. Neuchátel, 1869 , in- $8^{\circ}$.

(6) Catalogue détaillé des plantes vasculaires qui croissent nalurellement aux environs de Genève. Genève, 20 éd., 1861, in-18. 
ont les sépales caducs ou persistants, caractère excellent en lui-même, mais qui est commun à plusieurs espèces très différentes entre elles, comme les Rosa cinnamomea, alpina, pomifera, etc., par exemple.

M. Déséglise (1) a copié la classification de De Candolle, mais en remaniant les sections et en créant un groupe nouveau, les Alpince. M. l'abbé Cariot (2) et quelques autres ont adopté sa classification. M. Déséglise a reproduit ce classement dans ses trois derniers ouvrages : dans le premier (3) en modifiant certains caractères génériques, et dans le second (4) et le troisième (כ) en remaniant plus ou moins les groupes et les sections principales. Du reste, et ainsi que le fait remarquer M. Crépin (Primitice, fasc. I), cet auteur est loin de comprendre la classification logique du genre, car il range pêle-mêle les unes à côté des autres des espèces très diverses et qui appartiennent à des sections tout à fait différentes. Cet inconvénient parait avoir, en partie, disparu dans son Catalogue, où les erreurs, toutefois, l'ont remplacé en masse.

Grenier (6) abandonnant avec raison la classification proposée par lui dans la Flore de France, partage les Roses

(1) Essai monographique sur cent cinq espèces de Rosiers appartenant à la flore de France. Angers, 1861, in- $8^{\circ}$.

(2) Etude des fleurs, $4^{\circ}$ éd., Lyon, 1865 ; 50 éd., 1872, 3 vol. in-8०.

(3) Observations on the different methods proposed for the classification of the species of the genus Rosa. Huddersfield, 1865, in- $8^{\circ}$.

(4) Enumération cles Rosiers de l'Europe, de l'Asie et de l'Afrique, publiée dans " The Journal of Botany of London. " London, 1874, juin, in-8\%

(5) Catalogue raisonné, etc., in Bull. Soc. Bot. de Belgique, XV, 1876, p. 176 seq. et extr., in-8 ${ }^{\circ}$.

(6) Flore de la chaine jurassique: Paris, 1865, in- $8^{\circ}$. 
jurassiennes en six sections: Dimorphacanthce, Coronatce, Villosce, Ambiguce, Canince et Rubiginosce, basćes sur la forme des aiguillons et sur des caractères importants tirés des organes floraux, mais présentés d'une manière vague et sans points de comparaison, ce qui donne à ses sections une valeur tout à fait inégale.

En 1869, M. Crépin (1) a publié une excellente classification qui, au fond, n'est autre que celle de De Candolle, modifiée par M. Déséglise et améliorée d'une manière heureuse par M. Crépin lui-même. Elle renferme douze sections: Synstylce, Stylosce, Gallicance, Pimpinellifolice, Alpince, Sabince, Montance, Canince, Glandulosce, Rubiginosce, Tomentosce et Villosce. La plupart de ces sections se subdivisent elles-mêmes en plusieurs divisions secondaires qui permettent d'en reconnaitre et d'en étudier facilement les espèces. Malgré l'harmonie parfaite qui semble y régner de prime abord, on doit néanmoins regretter que ces sections n'aient pas toutes la même valeur, et que quelquesunes soient tellement voisines qu'il devient impossible de les séparer sectionnellement. M. l'abbé Cottet, rhodophile suisse distingué, a reproduit cette classification dans son Enumération méthodique des Roses du Valais (2).

M. Baker (3) a adopté les sections de Lindley, à l'exception de celles qui sont étrangères à l'Angleterre; c'est, depuis la monographie de Woods (loc. cit.), le travail le

(1) Primitix monographiæ rosarum. Matériaux pour servir à l'histoire des Roses, fascicule I. Gand, 1869, in- $8^{\circ}$ et fasc. II, Gand, 1872 , in $-8^{\circ}$ et fasc. III, IV, id.

(2) In Bulletin des travaux de la Société Murithienne, fascic. III, 1872-73, in-18.

(3) A Monograph of the british roses (extrait du vol. XI du Linnean Society's Journal Botany), 1870, in-8०. 
plus complet que possèdent sur le genre Rosa les îles Britanniques. Il est fâcheux que l'auteur, partisan à outrance de l'école synthétique, n’ait pas adopté un certain nombre de types regardés universellement comme de véritables espèces.

M. Scheutz (1), de Wexjo en Suède, a publié un prodrome des Roses scandinaves, le meilleur qui ait jamais paru sur les espèces de l'Europe boréale. Il les divise en deux grandes catégories, selon que les tiges sont plus ou moins aiguillonnées et que les sépales sont entiers ou pennatifides. A la première section, il rapporte les Rubiginosee, les Canince et les Villosce; à la seconde, les Cinnamomece et les Pimpinellifolice. Chaque sous-section forme une série assez naturelle, malgré les réductions opérées par l'auteur qui y décrit cependant plusieurs espèces entièrement nouvelles.

M. Boissier (2) divise ses quarante-six Roses orientales en deux grandes séries, fondées sur la disposition des styles qui peuvent être libres ou agglutinés en colonne. Ces séries renferment dix sections, se rapportant pour la plupart à celles de De Candolle, mais dont plusieurs sont tout à fait nouvelles, selon l'auteur (Elymaiticce et Orientales), et renfermant des espèces spéciales à l'Europe australe et à l'Orient. Ces sections semblent assez bien définies; néanmoins, on y voit associées diverses espèces, évidemment de sections différentes, telles que le Rosa pimpinellifolia, par exemple, qui se trouve à côté du Rosa alpina.

(1) Studier ofver de skandinaviska arterna af slagtet Rosa. Wexio, 1872 , in $-4^{\circ}$.

(2) Flora orientalis, sive enumeratio plantarum in Oriente a Græcia et EEgypto ad Indix fines hucusque observatarum. Genevæ, $1867=76$, \& vol, in -8 . 
Récemment, M. le $\mathbf{D}^{\mathrm{r}} \mathrm{H}$. Christ, de Bâle, a donné la première monographie générale des Rosiers de la Suisse (1), ouvrage consciencieux et plein de recherches et d'érudition. Sans donner l'analyse de ce travail, nous ferons remarquer que l'auteur a beaucoup trop généralisé les types primitifs, autour desquels il groupe une foule de variétés secondaires. D'ailleurs, partisan de l'hybridité, - chose très douteuse, pour nous, dans le genre Rosa, - M. Christ n'admet que quatre sections : Cinnamomea, Pimpinellece (sic', Caninea et Gallicanece. Ses Pimpinellece (sic) renferment les Sabinece, de M. Crépin; ses Caninece se subdivisent en quatre sous-sections formées par les Tomenteuses et Villeuses, les Rubigineuses, les Glanduleuses, les Canines proprement dites et les Synstylées, ce qui donne une série fort disparate ; ses Cinnamomeœ et Gallicanœ contiennent seulement les Rosa cinnamomea et Gallica.

Nous-même, dans notre Flore lyonnaise (2), quoique n'ayant pas, à proprement parler, établi de sections tranchées pour le genre Rosa, nous avons néanmoins, autant que possible, tenu compte, dans nos tableaux dichotomiques, de la classitication de De Candolle, telle que l'entend II. Crépin, parce qu'elle seule, si elle ne doit toujours subsister, n'en est et n'en demeurera pas moins le fondement obligé de toutes les classifications futures.

Si l'on nous demande maintenant quel est, à notre avis, le meilleur de tous ces systèmes, nous n'hésiterons pas à

(1) Die Rosen der Schweiz, mit Berücksichtigung der umliegenden Gebiete Mittel-und Süd-Europa's. Basel, Genf, Lyon, 1873, 1 vol. in- $8^{\circ}$.

(2) Flore lyonnaise et des départements du Sud-Est. Paris et Lyon, 1875,1 vol. in-18 jésus. 
répondre : celui de $\mathbf{M}$. Crépin parait le plus facile, le plus simple et le plus logique. Pour le fond, nous l'avons dit, sa classification n'est autre que celle de De Candolle, mais heureusement modifiée et subdivisée en d'autres sections assez bien entendues. Sans doute, nous ne prétendons pas la mettre à l'abri de tout reproche; nous lui en ferons même d'assez graves. Ainsi, elle n'entre pas assez dans les détails, ce qui la rend nécessairement incomplète. Certaines soustribus n'y sont pas non plus logiquement subdivisées : nous n'en voulons pour preure que les Montanes et les Villeuses qui s'y trourent séparées des Alpines et des Tomenteuses. Enfin, on troure dans cette classification assez de coupes anormales, telles que les Stylosées démembrées des Synstylées, quelques sous-divisions de la tribu des Canines, etc., et quelques autres défauts plus ou moins accentués, excusables, d'ailleurs, dans un pareil genre et difficiles à éviter dans l'impossibilité où s'est probablement trouvé l'auteur d'étudier certains tỵpes à l'état virant. Néanmoins, et malgré ces remarques critiques, M. Crépin est, de tous les rhodologistes, celui qui a le mieux défini nos grandes sections européennes du genre Rosa. Nous avons donc choisi sa classification comme étant la meilleure, et nous en avons fait la base du système proposé dans notre Essai (1) il y a quelques années.

Arant d'entrer en matière, avant d'exposer l'innovation que nous arons faite en changeant les anciens noms des Synstylece, Gallicanece, Cinnamomece, etc., nous voulons prévenir une objection qu'on ne manquera pas de nous faire. Il paraitra singulier, en effet, que nous ayions subs-

(1) Gandoger. Essai sur une nouvelle classification des Roses de l'Europe, de l'Orient et du bassin méditerranéen. Paris, 1876, in-80. 
titué à des noms adoptés partout, et cela sans motif apparent, d'autres noms pris, pour ainsi dire, d'une maniere arbitraire. Mieux aurait valu semble-t-il, ou laisser les noms de De Candolle ou revenir à ceux de Trattinick?

D'abord, en agissant ainsi, nous avons cru bien faire en les prenant parmi les rhodologistes les plus renommés de notre époque, et aree lesquels, pour la plupart, nous arons des relations suivies; car, il semble plus naturel de leur dédier une section d'un genre qu'ils ont travaillé pendant toute leur vie et dont ils ont su enrichir l'histoire, que de donner leur nom a des genres de plantes venant de contríes lointaines qu'ils ne connaissent point ou dont l'étude n'est ni facile ni intéressante pour eux. En second lieu, le genre Rosa pouvant ètre scindé en plusieurs sous-genenres, ainsi que nous allons le démontrer ci-après, et les noms des sections actuelles devenant alors insuffisantes, nous avons pensé qu’il était convenable de les changer en en créant de nouveaux.

Cette objection étant écartée, nous le dirons tout d'abord et sans détours : nos Roses européennes et orientales, quoique formant entre elles une série très naturelle, sont cependant susceptibles de constituer des sous-genres nettement tranchés les uns d'avec les autres.

Nous ne nous dissimulons pas combien cette assertion peut paraitre étrange aux yeux de certains botanistes, de ceux surtout qui, ne s'étant jamais donné la peine d’étudier et d'interroger la nature dans la nature elle-mème, se contentent d'un système qu'ils se sont eux-mèmes arbitrairement biti ou qu'ils ont emprunté à un auteur quelconque, sans trop savoir pourquoi. Malgré les réclamations qu’ils pourront faire, malgré leurs fins de non-recevoir, nous n'en maintenons pas moins notre assertion, que nous allons tàcher de prouver par la simple force de la logique et la seule autorité des faits. 
A notre époque, où la botanique semble avoir pris un nouvel essor et s'être dégagée des limites étroites que lui avaient imposées les anciens, on s'occupe activement de l'étude des espèces dites affines, c'est-à-dire des formes séparées entre elles par des caractères peu distincts, souvent même assez difficiles à apprécier, mais se conservant invariablement pendant une suite indéfinie de générations. Or, cette étude a amené les botanistes à reconnaitre que, non seulement les anciens types qu'on appelle linnéens étaient à subdiviser en espèces nouvelles, mais que, pour être logique, il était indispensable aussi de diviser les genres qu'avait établis Linné lui-même. Et ceci est aussi facile à justifier qu’à comprendre.

Supposons que les Rosa Gallica et canina, si différents entre eux, aient été divisés chacun en dix ou quinze autres espèces réputées affines; peut-on croire logiquement qu'une forme démembrée du groupe $R$. Gallica ait la même valeur dans l'ordre systématique des espèces qu'une autre forme tirée du groupe $R$. canina? Evidemment, non : toutes les espèces du groupe $R$. Gallica seront très bien en rapport de similitude entre elles, mais elles n'auront aucune ressemblance avec celles du groupe $R$. canina, parce qu'elles ne seront pas équivalentes. Il faut donc nécessairement conclure de là, que les Rosa Gallica et canina doivent former de petits groupes particuliers ou, pour mieux dire, de véritables petits genres, ayant chacun leurs caractères spéciaux et leurs espèces propres. Et l'on ne saurait démembrer les types linnéens et n'en pas subdiviser les genres sans se mettre en contradiction ouverte. Comment, en effet, pourrait-on appeler naturelle une série qui ne renferme pas exclusivement des êtres similaires? Or, le genre Rosa formera-t-il une série véritablement naturelle s'il se trouve 
composé d'une douzaine de groupes qui comprennent euxmèmes une foule d'êtres, à la vérité voisins, quant à leurs caractères généraux, mais tout à fait dissemblables, respectivement aux groupes auxquels ils appartiennent?

Enfin, pour arriver plus directement au genre Rosa et entrer dans le coeur de la question, peut-on mettre en parallèle le Rosa lutea aux fleurs jaunes, anx sépales persistants, aux styles libres, aux aiguillons dimorphes, avec les Rosa repens ou semperirens, qui ont des caractères tout opposés? Rapprochera-t-on logiquement le R. Jundzilliana, une des plus belles Roses connues, avec le modeste $R$. dumetorum, ou encore songera-t-on à assimiler entre eux les $R$. cinnamomea, pimpinellifolia, mubrifolia, micrantha ou tomentosa? Cela n'est pas possible; et cependant, on veut, dans la longue série des roses européennes, considérer les formes démembrées du $R$. lutea comme équivalentes à celles du $R$. rubrifolia; celles du $R$. cinnamomea à celles du $R$. pimpinellifolia, celles du $R$. alpina à celles du $R$. rubiginosa! Quels rapports, nous le demandons, ces formes ont-elles entre elles? Absolument aucun, si ce n'est un aspect à peu près uniforme, des tiges aiguillonnées, des teuilles imparipennées, des stipules adnées au pétiole, des fleurs ordinairement en corymbe, un calice urcéolé, et le reste. Tandis que, si l’on scrute l'organisation intime des types primaires des Synstylées, Gallicanes, Cinnamomées, etc., on y trouve des différences profondes, des nuances spécifiques tout à fait tranchées, enfin des notes biologiques et morphologiques d'une haute valeur quî, combinées elles-mèmes avec d'autres caractères accessoires, en font autant de petits groupes ou sous-genres qu'il serait illogique et déraisonnable de vouloir assimiler entre eux. 
$\mathrm{Si}$, à tout ce que nous venons de dire, nous ajoutons que dans les genres nombreux en espèces comme le sont les Roses, il est souvent difficile de trouver des noms bien appropriés pour les formes nouvelles, on comprendra mieux encore la nécessité d'entreprendre la coupe yénérique de ce genre. Cette raison n'est pas péremptoire, nous le savons bien; mais il en est une autre d'un ordre différent qui mérite bien qu'on s'y arrête, puisqu'elle est fondée sur l'exemple des plus illustres monographes; nous voulons parler des divisions faites dans des genres à peu près similaires, comme sont les Astragalus de l'Orient, les Erica du cap de Bonne-Espérance, les Saxifraga, les Centaurea et certaines familles : Géraniacées, Ombellifères, Composées, Graminées, etc., où les caractères qui différencient les genres sont souvent loin d'être aussi accentués que ceux de nos sections du genre Rosa. Ici, si nous ne craignions d'être trop long et fastidieux, nous n'hésiterions pas à descendre jusqu'aux détails et à discuter les caractères des innombrables sous-genres proposés par Bunge dans sa belle monographie des Astragalus, par exemple, pour les comparer avec ceux de nos Roses. Nous prouverions ainsi jusqu’à l'évidence que nos nouveaux genres de Roses sont au moins équivalents à ceux établis par Bunge, sinon plus distincts. Pourquoi, alors, ne ferions-nous pas pour les Rosa ce que cet illustre monographe a fait pour les Astragalus ? Pourquoi ne marcherions-nous pas sur la voie de la foule des botanistes qui nous ont précédés ? La science consiste-t-elle à suivre servilement la route tracée par des hommes encore novices, ou bien à agrandir les horizons, à montrer les points de vue nouveaux, à éclaircir enfin les questions qui jusqu'à cette heure sont restées dans l'obscurité ? 
Comme conclusion pratique de tout ceci, il ressort que chaque type linnéen forme une sous-section générique, ou mieux, un genre; autrement il faudrait dire que les genres créés dans les Astragalus, les Saxifraga, les Centaurea, les Erica, etc., ne sont pas distincts. Ou bion admettre ces genres, ou bien réunir les espèces et n’en créer aucune autre; il n’y a pas de milieu, le dilemme est irréfutable.

On objecterait en vain qu’il $\mathrm{y}$ a des espèces qui semblent aussi bien appartenir à une section qu'à une autre; cela ne prouve qu'une chose, c'est qu'on ne les a pas assez étudiées, et il est mainte espèce qui autrefois de douteuse ou d'incertoe sedis, a trouvé une place où elle restera définitivement. Du reste, combien pourrait-on citer de genres en botanique qui n'ont pas leurs espèces mixtes et transitoires? Il suffit de nommer les familles difficiles des Cruciferes, des Ombellifères, des Composées, des Graminées, etc.

De même que dans notre Essai nous avons distribué les Roses européennes et orientales en onze sections, de même aussi nous distribuerons ici les types actuellement connus et nos nombreuses espèces nouvelles en onze sous-genres:

Subgenus : I. - Ripartia (Synstylece DC. el Stylosce Crép.)

- II. - Eurosa (Gallicance DC).

- III. - Scheutzia (Ginnamomece DC. et Orientales Crép.). (1)

- IV. - LAGgeria (Eglanterice DC.).

- V. - Cottetia (Pimpinellifolice DC.).

- VI. - Bakeria (Sabince Crép.).

(1) C'est par une erreur typographique qu'on a imprimé Schentzia dans notre Essai, au lieu de Scheutzia. 
Subgenus : VII. - Ozanonia (Alpinece Déségl. et Montanece Crép.).

- VIII. - Crepinia (Caninece DC.).

- IX. - Chavinia (Gilandulosece Crép.j.

- X. - Chabertia (Rubiginosece DC.).

- XI. - Pugetia (Tomentosece et Villosece DC.).

- XII. - Hultemia Dum.

Un mot maintenant sur l'espèce.

Nous avons exprimé déjà plusieurs fois notre opinion sur ce grave sujet (voy. préf. Decades plant. - Pugillus. Centaurea, etc.) Nous n'y reviendrons ici que pour l'envisager sous un autre de ses côtés.

Remarquons d'abord que, dans l'état actuel de la science, il n'y a logiquement que deux partis à prendre : ou subdiviser les types anciens lorsque les nouvelles espèces offrent assez de caractères distinctifs, ou réunir toutes les espèces aux types desquels elles ont été démembrées. Voilà les deux écoles qui sont en présence: école synthétique d'une part, école analytique de l'autre. Avec la première, on arrive logiquement au darwinisme ou transformisme, système qui révolte autant par ses conséquences que par son absurdité. Avec la seconde, c'est la science scrutée, étudiée, approfondie, livrant ses secrets, étonnant et charmant tout à la fois, par ses résultats, l'observateur judicieux.

Dans le fascicule IV de ses Primitice, M. Crépin abordant la discussion systématique des diverses espèces de Roses, proteste qu'il ne s'occupera pas des nouvelles créations dans le genre.

* Cette discussion, répète-t-il à diverses reprises, serait a stérile, attendu QUE POUR MOI toutes ces espèces ne sont 
"que des variations d'un même type qu'on peut pousser

\& indéfiniment $\mathrm{s}$, etc.

Il est possible que pour Lur ces nourelles espèces ne soient que des variations d'un même type. Mais poun wous, elles sont des êtres parfaitement distincts et parfaitement autonomes.

Sur quoi M. Crépin, partisan à outrance du système du transformisme, fonde-t-il son opinion? Est-ce sur des études sur la nature ?II n’a jamais étudié les Roses à l'état vivant. - Est-ce sur des observations physiologiques? Il n’a connu les Roses qu'à l'état de mort dans les herbiers. Est-ce sur des essais de cultures? Homme de cabinet, il n’a jamais fait aucune expérimentation.

Quand M. Crépin se sera donné la peine de faire comme plusieurs de ses collègues, de nombreux voyages pour étudier les Roses à l'état vivant; quand il les aura scrutées au point de vue physiologique; quand il les aura ensuite passées par le grand criterium de l'expérimentation culturale, alors nous lui reconnaitrons le droit de renir discuter la grave question de la fixité ou de la variabilité de l'espèce qu'il ne connait que d'après les idées qu'il s'est formé dans la solitude de son cabinet.

En attendant, à ses fins de non-recevoir, nous opposons tranquillement les résultats de nos voyages, de nos observations et de notre expérimentation. A ses théories fantaisistes, nous répondons par des faits dûment constatés et mis à la portée de tout le monde. Lorsque, reprenant ces mèmes fiats par les trois moyens ci-dessus indiqqués, il nous aura prouré que nous nous sommes trompé, alors nous nous empresserons de nous ranger à son avis.

II. Crépin a dit encore quelque part que la méthode analytique amène forcément, pour les Roses, à la distinction spécifique du buisson. 
En admettant que ce paradoxe fût vrai sous quelqu'une de ses faces, nous lui demanderons si la méthode synthétique n'amène pas non plus forcément à la réunion de toutes les espèces, de tous les genres et de toutes les familles ?

N’a-t-on pas proposé, il y a un demi-siècle, de ne distinguer que deux Rosa, $R$. sativa pour les espèces cultivées, et $R$. silvestris pour les espèces sauvages (1)? En poussant plus loin les conséquences d'un pareil système, nous ne voyons pas que ces deux Rosa ne puissent être réunis ensemble; que les Rosa ne soient associés aux autres genres de la tribu des Rosées; que cette tribu, par les genres intermédiaires, ne soit identifiée aux Potentillées, et qu’à son tour, la famille entière des Rosacées assimilée aux familles voisines, ne forme plus qu'un seul type qu'on pourra décorer du titre très simple, très facile, très commode de Herba virens ou Planta vulgaris! Absolument comme ceux qui prétendent que tous les êtres ont la même filiation.

Ce résultat est étonnant, n'est-ce pas ? Eh bien, il nous parait, à nous, très naturel, et ne doit pas nous étonner plus que l'excès contraire; car, nous ne sachions pas qu'un phytographe en soit arrivé à distinguer spécifiquement chaque buisson ou chaque brin d'herbe. Il y a là, ce semble, une limite d'urbanité que ne devraient pas franchir les partisans de l'école synthétique dans les reproches qu'ils adressent à ceux de l'école analytique.

Du reste, ainsi que nous l'avons fait remarquer dans la préface de notre Pugillus plantarum novarum, les bota-

(1) Cette méthode est soutenue par M. O. Kuntze, de Leipsik, dans son livre intitulé : Methodik der speciesbeschreibung und Rubus. Leipsik, A Félix, 1879, 1 vol. in-4. Ce qui n'empêche pas l'auteur d'y établir à son tour de nouvelles espèces de Rubus ! 
nistes synthétiques eux, ne dédaignent pas précisément les espèces nouvelles, et il nous serait facile d'en citer parmi eux, à commencer par M. Crépin lui-même, qui, tout en réduisant les espèces des autres en établissent eux-mèmes par douzaine.

Mais, nous dira-t-on, vous êtes arrivé personnellement à la distinction spécifique du buisson en créant de si nombreuses espèces! Oui, nous sommes arrivé à la distinction spécifique du buisson toutes les fois que ce buisson était distinct d'un autre. Mais aussi, dans la plupart des cas, c'était par de nombreux buissons que la même forme se présentait, tout comme c'est ordinairement par centaines et milliers d'individus que les plantes affines herbacées s'ofirent aut botaniste.

Ces objections ne sont done pas sérieuses : les faits, l'expérimentation et la nature, avons-nous dit, sont là pour y répondre. Avec ces trois moyens, chacun peut parfaitement s'éclairer.

Remarquons une chose : avec la méthode analytique telle que nous l'appliquons ici au genre Rosa, nos adversaires y trourent de nouveaux moyens pour inieux apprécier l'espèce. C'est, en effet, par l'étude et l'exposé de toutes les nuances ou variations (selon leur style) d'un mêr.e type primitif, qu'on arrive à connaitre les limites extrêmes de variabilité de ce type. Or, comment connaitre ces limites, si on n'en expose pas, au moyen de l'analyse, les degrés successifs? Par conséquent, les deux écoles y trouvent leur intérèt. Et, comme nous l'avons dit plus haut, si les faits, l'expérimentation (1) et la nature prourent que

(1) L'expérimentation a été faite depuis longtemps, et nous n'avons ici qu’à enregistrer les résultats qu'elle a procurés. 
ces nuances ou variations, ces degrés successifs révélés par l'analyse sont bien en réalité des êtres très distincts et très constants dans leurs caractères constitutifs, notre thèse est prouvée et confirme les principes de la méthode analytique.

Nous avons l'espoir de n'avoir oublié dans ces tables que fort peu de ce que nous venons d'appeler degrés successifs. Sans doute il est possible d'en trouver encore, et nous avons nous-même personnellement négligé certaines autres formes de notre herbier qui auraient pu prendre un nom spécifique. Si nous ne l'avons pas fait, c'est pour éviter la confusion dans ce dédale immense des Roses. Mais nous poursuirrons toujours nos études sur cet intéressant sujet, et aussitôt que des faits nouveaux nous seront acquis, nous nous empresserons de les publier.

Nous ne croyons cependant pas nous tromper en aftirmant que, pour certains types, le dernier mot de l'analyse semble être dit, tant le nombre de formes démembrées est considérable, et tant leur affinité paraît atteindre d'extrêmes limites. Néanmoins, cette affinité n'est pas aussi grande qu'on pourrait le croire; elle est moindre que celle qui existe pour certains groupes : Erophila, Viola, Rubus, Hieracium, Mentha, Polygonum, etc., comme nous nous en sommes très bien convaincus par nos études personnelles.

Cela tient-il à ce que les Roses ont été jusqu'ici moins bien étudiées, ou encore à la grande diversité de leurs types primitifs? Nous ne saurions trop le dire, mais ce qu'il y a de certain, c'est que les Roses, même les plus affines, offrent toujour's un faciès et un ensemble de caractères spécifiques qui frappe. Aussi, leur étude en est-elle relativement plus facile que celle des genres dont nous venons de parler.

Ces considérations faites, disons quelques mots sur les 
matériaux qui ont servi de base à ce travail et sur ce qui en a été publié.

Nos études sur les Roses remontent déjà à plus de 130 ans. Ayant entrevu dès le déljut les résultats féconds que pouvaient nous offrir ces études, nous nous mîmes avec ardeur à réunir les matériaux qui nous manquaient. Une correspondance étendue nous procura bientôt des relations avec les principaux botanistes-collecteurs de l'Europe et d'ailleurs, des échanges nombreux, des achats, des voyages enfin nous ont rendu possesseur d'un matériel immense formant près de 200 paquets.

Quoique nous ayions travaillé divers genres: Lilex, Ciratcegus, Rubus, Sorbus, Hieracium, Centaurea, Verbascum, Mentha, Polygonum, Salix, etc., notre constante préoccupation étaient les Roses, notre genre de prédilection. Nous avons toujours spécialement poussé nos bienveillants et honorables correspondants à nous récolter en masse les diverses formes de ce genre. Les relations qu'il nous a fallu établir, les travaux et les difficultés que nous avons eu à surmonter sont nombreux, mais nous en serons amplement dédommagés si nous avons pu faire avancer d’un pas la science botanique et si notre ouvrage est utile aux Rhodophiles.

Voici les noms de ceux de nos correspondants qui nons ont procuré les collections les plus importantes, ce sont : MIM.

France: Thiébaut, Puget, Motelay, Malbranche Autheman, Debeaux, Magnier, Proal, Ozanon, Garroute, Tuezkiewicz, Héribaud, Gandoger, Chabert, Billot, Lehel, Ripart, Mathonnet (Reliquix Maill.), ete.

Espagne : Zubia, Vayreda, Martin y Cercos, Quet, Laguna, Hegelmaier, Huter, Codorniu, Mayorga, J.-J. Rodriguez. 
Portugal : Welwitsch, Daveau, Henriques.

Italie et Naples : Bertoloni, Gussone, P. Savi, Gibelli, Pasquale, de Notaris, De Cesati, Caldesi, Rostan, Arcangeli, Penzig, etc.

Corse et Sardaigne : Debeaux, Reverchon, Macchiati.

Sicile : Todaro, Gussone, Lo Jacono, Borzi, Nicotra. .

Grèce et Archipel : Orphanidès, De Heldreick, Topali, Spreitzenohfer.

Russie : Becker pour l'Astrakhan, Laupmann pour le sud, Azoff, etc., Golde pour la Volhynie, Meinshausen pour l'Ingrie, Clerc pour l'Oural, et le flora Rossica exsiccata de Bunge et Girgensohn pour la Livonie, la Courlande et la région occidentale.

Pologne : Karo et quelques autres.

Transylvanie : Barth, Csato, Tauscher.

Hongrie : Richter, Tauscher, Vrabelyi, Feichtinger, Borbas, etc.

Croatie: Vukotinovic.

Istrie et Dalmatie : De Visiani, Freyn, Marchesetti, Studniczka, Schalch.

Serbie et Albanie: De Visiani, Pancic.

Autriche : Stein, Wiesbaur, Keck, Oborny.

Tyrol : Kerner, Gremblich, Stein.

Suisse: Christ, Wolf, Gisler, Cottet, Guinet, Lihmann, Sandoz, Thomas, Lerch, Favrat, Delasoie, etc.

Prusse et Allemagne : Haussknecht, Dufft, Kemmler. Hiilsen, Baenilz, Zabel.

Silésie : Straehler, Uechtritz, Ansorge, Hoger, Kuebel.

Bohême : Polak, Studniczka, Patze.

Angleterre : Baker, Briggs, Fraser, Groves, Ley, Webster, Harbord Lewis.

Ecosse : White, Drummond-Hay, Bosivell Syme, Ley. 
Irlande : Stewart.

Belgique : Crépin, De Dieudonné, II. Vanderhatghen.

Hollande : Ankersmit.

Danemarck: Lange, Zahrtmann, Jörgensen, Mortensen.

Scandinavie : Scheutz, Tullberg, Lundquist, Zetterstedt, Dahlgren, Hylten-Cavallius, etc.

Finlande : Brotherus, Enwald, Hollmen, Hult.

Algérie : Debeaux, Choulette, Gandoger.

Canaries, Madère et Açores : Bourgeau, Thiébaut, E. Rodriguez.

Asie occidentale : Aucher-Eloy, Kotschy, Balans?, Blanche, etc. - Sintenis et Rigo pour l'île de Chypre.

Caucase : Hohenacker, Ruprecht, Radde, Brotherus.

Nous passons sous silence d'autres collections d'Amérique ou d'ailleurs qui, ne rentrant pas dans le cadre de nos études, ne sauraient trouver place ici.

Qu'il nous soit permis de remercier publiquement tous nos honorables correspondants pour l'empressement et l'obligeance qu'ils ont mis à nous procurer les matériaux que nous publions aujourd'hui.

Quant à ce que nous avons publié jusqu'ici sur le genre Rosa, il se réduit à peu de chose. II. Cariot, le premier, a édité quelques-unes de nos espèces, entre autres te beitl $R$. incanescens Gdgr in Cariot. Etudes des fl., ̈̈ édit., II. p. 203 (1872 !) R. velutirza Chab., non Clairs., - appelé ensuite $R$. micans, par M. Déséglise, qui parait aroir

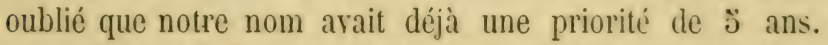
Puis, en 1874, trois de nos nouvelles espèces furent décrites dans le Bulletin de la Société d'échange dauphinoise, fase. I ${ }^{\circ r}$, p. 14-15.

La même année, notre ami, M. Debeaux, nous dédiaitle $R$. Gandogeriana (in Bull. Soc. Bot. de France, XXI, p. 9). 
Dans un mémoire intitulé : Description d'une nouvelle Rose des Pyrénées-Orientales, Paris, 1875, il décrivait à nouveau cette espèce, en y ajoutant une table dichotomique établie par nous pour les Sempervirentes et énumérant 10 espèces inédites, table que M. Crépin a daigné discuter dans le fasc. IV de ses Primitice. Pendant cette même année 187\%, nous décrivions 21 espèces nouvelles dans le fasc. $I^{\text {er }}$ de nos Decades plantarum novarum. En 1876 nous en publiions 3 autres dans le fasc. II, en même temps que paraissait notre Essai sur une nouvelle classification des Roses, où un assez grand nombre de noms nouveaux sont proposés et dont il existe une traduction espagnole (1).Dans notre Flore Lyonnaise, éditée les premiers jours de l'année $1870 \%, 10$ espèces inédites ont encore paru.

En suivant l'ordre chronologique, nons arrrivons à 1876 , époque où la Société Murithienne du Valais, fasc. V et VI, a publié aussi une quinzaine de nos Roses nouvelles. Enfin, en 1877 et 1878, dans nos Rosce novce nous avons

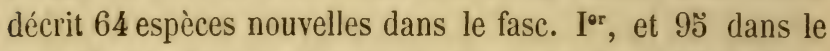
fasc. II. Ajoutons à cette énumératien les fase. III et IV de nos Decades plant. nov., 1880-81, où plusieurs Roses inédites sont indiquées, et enfin les Matériaux pour servir à l'étude monographiquedes Rosiers qui croissent dans les Pyrénées-Orientales, fasc. Irr (1878). In-8, où en collaboration avec M. Debeaux nous avons publié 31 espèces nouvelles. Ce travail n'est que le prélude d'une Monographie des Roses si curieuses des Pyrénées-Orientales, que nous projetons d'exécuter en commun, et dont notre ami, M. 0. Debeaux a, d'après nos instructions, amassé de très nombreux matériaux.

(1) Ensayo sobra una nueva classificacion, etc. Barcelone, 1880. 
M. Déséglise, dans son Catalogue raisonné et dans ses Descriptions de quelques Roses, semble avoir ignoré completement ces divers travaux, qui tous, - à l'exception de ceux postérieurs à 1877 , - sont antérieurs à ces deux ouvrages. Nous ne lui en ferons pas un reproche; mais nous lui demanderons, à lui, qui critique durement Seringe, Godron et d'autres pour quelques omissions insignifiantes dans leurs ouvrages qui passent pour enregistrer tous lez faits connus (sic), s'il a enregistré lui-même tous les faits connus dans son Catalogue qu'il annonce pompeusement comme les connaissant tous? Seringe et les autres étant morts n'ont pu lui répondre ; mais Godron qui vivait alors, s'est assurément chargé de le faire pour eux dans la verte réponse qu'il lui a adressée dans le Bulletin de la Société botanique de Belgique, t. XV, p. 485.

Pour nous, nous nous contenterons, dans l'intérêt de la science, de relever les innombrables erreurs qui pullulent dans ses écrits. Et, dans un très prochain travail, nous publierons les Centuries de ses erreurs, en lui appliquant la méthode employée par M. Baillon à l'égard de M. Decaisne (1).

Tels sont, en résumé, les travaux que nous avons publiés jusqu’à ce jour sur les Roses et qui sont, on le roit, peu de chose en comparaison de ce qui nous reste à faire.

Mais avant que d'entreprendre cette longue série de publications, nous avons jugé utile d'indiquer par des jalons fidèles - les tables dichotomiques, - la marche à suivre dans cette étude. D'autre part, nous ne pouvions tarder plus longtemps à faire connaitre nos découvertes et à débrouiller

(1) Errorum Decaisneanorum graviorum vel minus cognitorum Centurix, $\mathrm{I}-\mathrm{V}$, Paris, $1877-80$, in- $8^{\circ}$. 
la synonymie des nouvelles espèces proposées récemment par quelques auteurs, et dont plusieurs font double emploi.

N'oublions pas non plus d'insister sur l'avantage immense qu'offrent les exsiccatas, surtout pour les plantes criliques. Depuis longtemps déjà, nous en avions nous-même compris toute l'importance. Aussi, dès 1869, avons-nous commencé à récolter en nombre toutes nos nouvelles espèces ou nous les faire envoyer par nos correspondants, pour les distribuer abondamment ensuite avec numéros d'ordre et étiquettes imprimées. En continuant ces récoltes jusqu’à ces derniers temps, nous avons réussi à publier, sous le titre d'Herbarium Rosarum europcearum exsicc., la plus vaste collection qui ait jamais paru sur les Roses, puisque actuellement elle contient 607 numéros.

Un grand nombre de numéros ont été récoltés à 200 exemplaires et plus. Nous avons répandu cetle collection, non seulement dans les grands herbiers publics d'Europe et d'Amérique, mais encore dans une foule l'herbiers particuliers, soit par dons, soit par échanges, soit même, ainsi que nous y avait engagé notre illustre et vénéré correspondant, M. A. De Candolle, par la mise en vente de quelques fascicules, afin d'en augmenter la diffusion.

Si nous ne nous trompons, nous pouvons done affirmer que nos espèces sont très largement répandues par tout le monde scientifique, où chacun peut les examiner de visu et les étudier. Combien il serait à souhaiter que nos maitres dans le genre Rosa - pour ne parler que des Rosa eussent agi ainsi! Nous aurions bien des incertitudes enlerées; la synonymie serait élucidée et l'étude simplifiée.

En terminant, posons une question : Nos espèces nouvelles, indiquées ici, sont-elles réellement publiées par ces simples Tables dichotomiques? Oui. Les raisons en sont 
simples : $1^{\circ}$ Quelques-uns de nos devanciers ont fait ainsi, M. Crépin dans ses Primitice; $2^{\circ}$ il y a ici une véritable diagnose pour chaque espèce. En effet, quoique les éléments de cette diagnose soient épars dans divers endroits, il est facile de la reconstruire en suivant méthodiquement les diverses accolades des Tables.

Du reste, quoique nous regardions ces Tables comme une véritable publication, nous nous proposons d'éditer les diagnoses plus complètes de chacune des espèces indiquées ici. Ce sera dans un avenir très prochain.

Arnas, le 18 mai 1881.

\section{GANDOGER.}

N.-B. - La pubescence caractéristique de chaque espèce a toujours été prise sur les feuilles moyennes de chaque rameau florifère, c'est-à-dire sur la troisième ou quatrième feuille, ou la deuxième ou troisième, selon que le rameau est plus ou moins feuillé. Les folioles inférieures sont toujours velues et les supérieures constamment plus glabres que les moyennes. Ainsi, lorsque les folioles moyennes sont à nervures principales velues, les inférieures sont à poils entreposẹs sur le parenchyme ou entièrement velues en dessous, tandis que les supérieures ont la cóte médiane seule pubescente. Lorsque les moyennes sont à côte saule velue, les inférieures ont les nervures secondaires pubescentes et les supérieures sont entièrement glabres, etc. En un mot, il y a toujours une progression croissante et rẻgulière de la pubescence depuis les folioles inférieures du rameau jusquo dans les supérieures. Ce caractère s'observe dans toutes les Roses à feuillage plus ou moins pubescent, excepté dans les Pugetia (Tomentosac et Villosae). - Pour abréger les diagnoses, nous arons 
donc choisi un terme moyen en prenant les folioles moyennes du rameau floral.

Nous ferons aussi observer qu'il nous a fallu donner une autre signification aux mots ovale, ovoide, obovale-oblong et oblong (pour les folioles, les fruits et les calices). La forme typique est le mot globuleux qui indique, pour le fruit par exemple, un objet exactement sphérique. Quand le fruit ou la foliole dépassait d'un longueur de 1 mill. sa propre largeur, nous l'avons appelé ovale-arrondi; s'il la dẻpassait de 2 mill., ovale; de 3 mill., ovoide; de 4 mill., obovaleoblong; de 5 mill. et au-delà, oblong ou allongé. Cette importante innovation qui, du reste, s'étend à toutes les descriptions de plantes que nous avons déjà publiées, était nécessaire pour arriver à une plus grande précision; autrement, il eût fallu créer des noms nouveaux.

\section{ABBREVIATURAE}

Acul. = Aculei.

Cor. = Corolla.

m. 1. = millimetr. longus.

$\mathrm{Fl}$. = flos vel flores.

Ped. = Pedunculi.

Flor. = floralis.

Pet. = Petioli.

Fol. = Foliola.

Sep. = Sepala.

Fr. = Fructus.

Frut. = Frutex.

Stip. = Stipulae.

Tubus $=$ (calycis vel lacinia $\theta$.

1 = plus minus.

herb. = Herbarium rosarum europaearum exsiccat. 


\section{Subtribus ROSA}

(Tourn. Inst. III p. 636, tab. 408 ; L. gen. p. 254).

Caract. - Calix periantho adhœrens; tubus ore constrictus, annulo glanduloso coactus, urceolatus, globosus, ovatus, ellipsoideus vel oblongus, plus minusve attenuatus quinque laciniis vel sepalis donatus; sepala persistentia aut caduca, integra vel pinnata. Petala 气̆, basi nectario destituta, obcordata, apice plus minus emarginata, alba, rosea, rubra, lutea, passim variegata, margine interdum ciliata. Perfloratio imbricatim contorta. Stamina numero indefinita, antheris ovatis, vel oblongis, bilocularibus, incumbentibus. Carpella plurima, uniovulata, nucamentacea, indehiscentia, libera, inœqualia, glabra aut villosa, inclusa vel lateribus juncta. Styli in columnam cylindricam extus exsertam glabram, villosam vel lanatam plus minus fasciculati disco plano, conico vel acute elevato. Fructus globosus, turbinatus, ovoideus vel oblongus, glaber, glandulosus aculeolatusve, ruber, flavicans interdum niger, præcox aut serotinus, coriaceus vel pulposus. - Frutices vel suffrutices ramosi, veteris orbis et America borealis regionem temperatam præsertim colentes; radix lignosa ; ramis erectis aut procumbentibus, quandoque scandentibus plus minusve aculeatis vel inermibus, rarissime glandulosis vel pubescentibus. Folia imparipinnata (1) foliolis deciduis raro persistentibus,

(1) Rosa simplicifolia Salisb. hort. hallert. p. 359 (Hultemia Dumrt. hult, p. 13, 1824) ab foliorum forma, etc., totorque habitu insignis e genere exclusa est. 
rotundatis, ovatis, oblongis vel lanceolatis, glabris, villosis aut glandulosis, margine serratis, serraturis simplicibus vel compositis. Petioli glabri, villosi, tomentosi vel glandulosi, inermes vel aculeolati. Stipulæ oblongæ petiolo adnatæ integræ vel dentatæ (perraro fimbriatæ) glabræ, pubescentes vel villosæ, apice in auriculas plus minusve divergentes vel acutas productæ. Pedunculi et calicis tubus (vel urceolus) glabri pubescentes, glandulosi aut erinacei. Flores solitarii, geminati, ternati vel corymbosi, terminales, inodori vel suaveolentes, rarissime foetidi.

Subgenus I. - Ripartia Gdgr Essai p. 10 (in sect.) Synstylece DC. monsp. p. 137 et Stylosce Crép. Prim. p. 13.

Styli in columnam glabram aut villosam ultra discum longe producti. Flores plus minus bracteati, albi vel carni raro purpurei, petalis margine haud aut vix ciliatis. Fructus coccineus, præcox, coriaceus, erectus, glaber, villosus vel glandulosus. Laciniæ calycis sæpe sæpius fere omnes integræ et glandulosæ, post anthesim patulæ dein reflexæ, tandem decidux. Pedunculi ordinarie glandulosi rel hispidi. Foliola interdum persistentia, coriacea vel decidua, glabra vel villosa, numquam glandulosa, serrata, serraturis sæpissime simplicibus. Stipulæ angustiores, dentatæ. Aculei sparsi, conformes, adunci vel subrecti, basi plus minus dilatati, rarissime setacei apiceque glanduliferi. Frutices vel suffrutices ramosi, sociales, sæpe procumbentes rel scandantes tunc graciles ac flexuosi, interdum erecti ergo rigidi. Radix haud reptans (an semper ?)

D. Ripart in arte medicandi docto, biturigensi, hoc sub- 
genus dedicamus, qui Rosas Gallia centralis notas modo mirabili fecit.

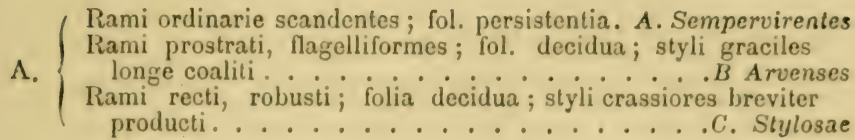

A. Sempervinentes Crép. Prim. p. 12; Gdgr Essai p. 10.

Radix haud reptans; frutices Austrum colentes, elati, ramosi, ramis elongatis, virentibus, flexuosis, scandentibus, plus minusve aculeatis, aculeis conformibus, tenuioribus; foliola lucida, ovata, vel oblonga, persistentia, coriacea ; pedunculi villosi, vel glandulosi ; sepala extus glandulosa, sxpe integra ; corolla parva, alba, vel ex albo-ochroleuca; styli ultra discum ordinarie planum in columnam gracilem tentiorem longe producti; bractea latiuscula; fructus coriaceus, parvus, sepe magnitudinem Pisi amulans.

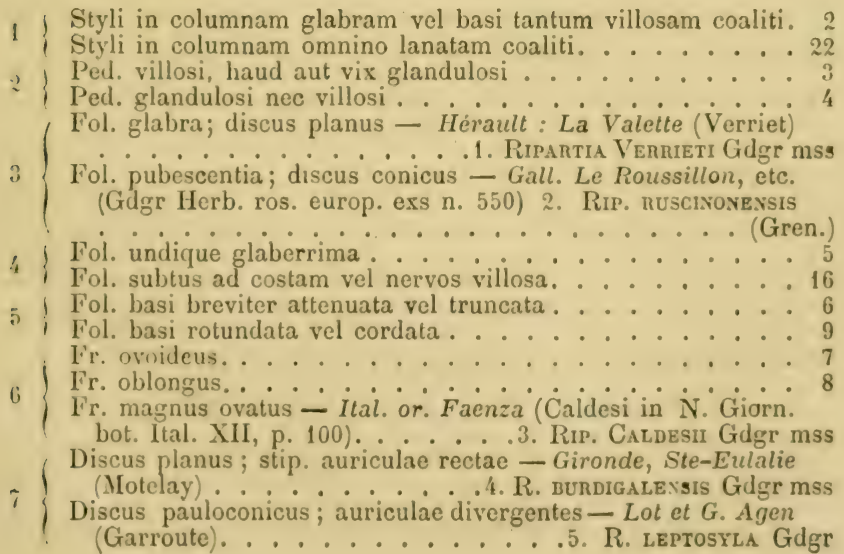


Pet. ad foliola subpilosuli; costa paulo glandulosa - Lot et

8 G. Agen (Debeaux). . . . . 6. R. Garumnae Gdgr mss Pet. glaberrimî ; costa media eglandulosa - Ital. or. Faenza

(Caldesi) . . . . . . . 7. R. LongIPEs Gdgr mss

( Pet. glabri subeglandulosi . . . . . . . 10

9 Pet. dense villoso-glandulosi - Gall. Agen (Garroute). 8 R.

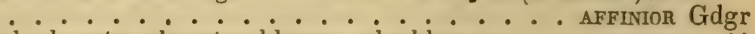

Fol. obovata, obovato-oblonga vel oblonga. ..... 11

10 Fol ovata - Gallia austr. . . . . 9. R. Prostrata (DC)

Fr. ovoideus ................... 12

$11\left\{\begin{array}{l}\text { Fr. oblongus } \\ \text { Fr. ovatus - Gard, Coularou (Diomède Tuezk) } 10 . \text { R. CORIACEA }\end{array}\right.$

Pet. glabri; discus planus - Gironde, Coutras (Motelay).

$12\{$ Pet. villosi; discus conicus - Lot-et-Gar., Brax (Debeaux)

T. . . . 12. R. CORDATIFoliA Gdgr

Tubus eglandulosus; stip. margine I fimbriatae . . . . 14

13 T'ubus glandulosus; stip. haud fimbriatae . . . . . . 15

Fol. obovata apice breviter attenuata; ped. $28-30 \mathrm{~m}$. 1. - Ital.

Bologne (Bertoloni) . . . . 13. R. Bertoloni Gdgr mss

14 Fol oblongo-acuminata; ped. $43-45$ m. I. - Ital. or. Faenza (Caldesi). . . . . . . 14. R. Fimbriata Gdgr mss

Ped. 24-29 m. 1.; fol. amplissime oblonga - Bouches-duRhône, Roquevafour (Autheman).15. R. vireticola Gdgr mss

Ped. 16-17 m. l.; fol parva obovato-oblonga - Lot-et-Garonne, Arasse (Billot exs. No 4020 !). . 16 R. Aduxta Gdgr mss

Fol costa media tantum villosa . . . . . . . . 17

16 Fol. subtus saltem ad nervos villosa . . . . . . 19 Fol. magna, dentes glabrae ; pet. parcissime hirtelli - Lot-et-

17 Garonne, Arasse (Garroute) . 17. R. PHyllomegas Gdgr mss

Fol. parva eorum dentes ciliatae ; pet. villosi. . . . . . 18

Fol. ovato-elliptica acutiuscula; fr. ovatus basi depressus -

Gall. Montpellier. . . . . 18. R. PERvirens (Gren.,

Fol. obovato-obtusuisula, fr. ovatus basi rotundatus - Gard,

Le Vigan (Tuezkiewicz). . . . .19 R pinnatula Gdgr mss

Fol. oblongo-subacuminata; fr. oblongus basilongeattenuatus

- Hisp. Logrono (Zubia). . . . 20 R. Caxtabrica Gdgr mss

Tubus eglandulosus . . . . . . . . 20

Tubus glandulosus. . . . . . . . . . . . 21

Fol. obovato-oblonga acuminata; discus planus Ital. or.

Faenza (Caldesi) . . . . 21. R. HIRTElla Gdgr mss

Fol ovata vix acuta; discus conicus - Ital. or. Faenza (Caldesi) . . . . 22. R. BRAchystylis Gdgr msg.

Fr. ovatus ; discus planus; fol. subtus toto hirta - Var, Le Luc (Hanry) . . . . . . . . 23. R. ALBENS Gdgr Fr. obovato-oblongus; discus planus; costa villosa - Ital. Modène (uibelli). . . . . 24. R. puberula Gdgr mss Fr. obovato-oblongus; discus conicus; nervi villosi - Ital. or. Faenza (Caldesi ..... . 25. R. FAventina Gdgr mss Fol. undique glaberrima ............ 23

Fol. subtus | villosa . . . . . . . . . . . 78

23 Fr. globosus vel subglobosus . . . . . . . . 24 Fr. ovato-rotundatus, ovatus, ovoideus vel oblongus . . . 35 


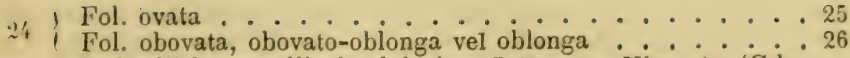
( Ped. alii laeves alii glandulosi - Gall. mer. Nice, etc. (Gdgr

25 herb. ros. n. 480 .......26. R. MERIDIonalis Gidgr Ped. omnes dense glandulosi-Gall. mer.27. R. micnophyLla (DC.)

) Ped. omnes dense glandulosi . . . . . . . . . . 27

26 Ped. I laeves basi vel ad apicem, nec toto glandulosi . . . 32

27 Discus conicus; fol. obovata vel obovato-oblonga. . . . . 28

27 Discus planus; fol. oblonga .......... . 29 Gemma elliptica; ped. 12-16 m. 1. - Catalaunia, Maza

28 (Vayreda)........ 28. R. VArnede Gdgr mss Gemma flor. globosa; ped. 28-31 m.1. - Bouches-du-Rhône, Aubagne (Roux) . . . . . . 29. R. Rouxir Gdgr mss

20 Costa media egIandulosa. . . . . . . . . . . 30

Costa media glandulosa . . . . . . . . . . . 31

Styli staminibus duplo breviores; pet. subinermes - Cata-

30 launia, Dorquès (Vayreda) - . 30. R. Glomigema Gdgr mss Styli stamina requantes; pet. aculeati - Gironde, Bassens (Motelay). . . . . . . . R. Staminosa Gdgr mss

$31\left\{\begin{array}{l}\text { Pet. glabri, eglandulosi ; tubus in 1/3 parte sup. lrvis - } \\ \text { Catalaunia, Sagaro (Vayreda). 32. R. CAtalaunica Gdgr mss }\end{array}\right.$ Pet. sparse pilosi, glandulosi-; tubus toto glandulosus Algeria, Tizi-Ouzou (Gdgr) . . . 33. R KabYLICA Gdgr mss

32 Ped. 29-35 m.l.. . . . . . . . . . . . 33

Ped. 15-22 m. 1.................... . . 34

Tubus toto glandulosus; costa basi aculeolata - Algeria, Kouba (Gdgr Fl. Alger. exs. n. 942).34. R. ALgeriensis

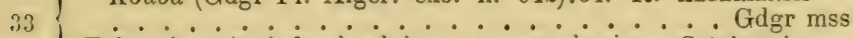
Tubus in $1 / 2$ inf. glandulosus; costa lævis - Catalaunia, Sagaro (Vayreda). . . . . . 35. R. Pluriflona Gdgr mss Pet. inermes ; stip. purpurex - Hisp. Barcelone (Vayreda).

34 . . . . . . . . R. Barcinonensis Gdgr mss Pet. paulo aculeati ; stip. virides - Lot-et-Gar. Agen (Soc. dauph. exs. n. 117.) . . . .37. R. HETERopona Gdgr mss

I Sepala alia pinnata alia integra . . . . . . . . . 36

35 Sepala omnia integra . . . . . . . . . . . 51

$36)$ Fr.ovatus vel ellipticus .............. . . 37

I Fr. obovato-oblongus vel oblongus . . . . . . . . 43

Tubus omnino glandulosus . . . . . . . . . . . . . 38

37 Tubus omnino lævis . . . . . . . . . . 42 Tubus in $1 / 2$ sup. lævis - Catalaunia, Biana (Vayreda). . . . . . . . . 38. R. BREvidexs Gdgr mss

Folia omnia 3-5-foliolata........... . . 39 Folia pleraque 2-foliolata - Bouches-du-Rhone, Martigues (Autheman). ........ R. R. ADENAxtil Gdgr mss

39 Fol. parva obovata . . . . . . . . . . . . . . . . . . . 40 Discus conicus ; pet. glandulosi ; ped. $31-33 \mathrm{~m} . \dot{1}^{\circ}-$ Istria,

40 Pola (Freyn)............ R0. Frexnir Gdgr mss Discus planus; pet. eglandulosi ; ped. 16-18 m. 1.-Bouchesdu-Rhône, Châteauneuf (Autheman).41. R. MYrLadeva Gdgr mss Discus planus; sepala apice angustata - Alger, Hussein-Dey

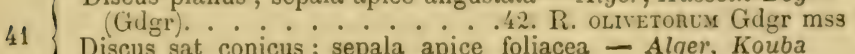
Discus sat conicus; sepala apice foliacea - Alger, Kouba (Gdgr) . . . . . . . .43. R. Mauritavica Gdgr mss 
Folia parvula, condensata apice subrotundata - Istria, Pola

42 (Areschoug). . . . . 44. R. DENsifolis Gdgr mss Fol. amplissima, apice acuminata - Lusit, Italia, Sicilia. ........ 45. R. BRoteri (Tratt.) Tubus vel fr. glandulosus . . . . . . . 44

43 Tubus lævis - Gironde, Sainte-Eulalie d'A mbarès (Motelay)

Fol basi rotundata . . . 46. R. GRANDIS Gdgr mss

44 Fol. basi l attenuata . . . . . . 48

Fr. ovoideus ; pet. aculeati. . . . . . . . . 446

$45 \int$ Fr. oblongus ; pet. inermes. . . . . 47 Fr. obovato-oblongus; pet. inermes-Dalmatie mer. (Visiani!)

Fol. obovata ; sep. apice linearia- $\dot{B}$ ouches-du-Rh., Martigues

46 (Autheman) ............. R. mediterranea Gdgr mss Fol. oblonga; sep. apice foliacea - Gallia, Agen (Puget).

. . . . . . . . . . 49. R. Estuans Gdgr

Ped. 19-22 m. 1.; costa paulo glandulosa - Alger, Birmandreis (Gdgr Fl. Alger. exs. n. 706 ; Gdgr herb. ros. n. 570,

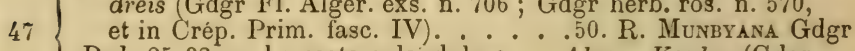
Ped. 25-32 m. 1.; costa eglandulosa - Alger, Kouba (Gdgr Fl. Alger, exs. n. 803) . . . .51. R. Africana Gdgr mss

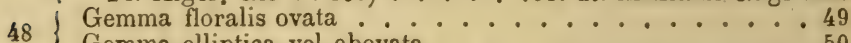

$48\{$ Gemma elliptica vel obovata . . . . . . . . 50 Fol. anguste oblonga; costa lævis - Minorca, Mercadal (Rodriguez). ........52. RodriguezIr Gdgr mss

49 Fol. parva obovata, costa glandulosa - Cantabria, Urberuaya (Zubia) ......... 53. R. BIschyensis Gdgr mss Fol. magna, costa paulo aculeolata - Gironde, Montferrand (Motelay). . . . . . 54. R. setigemala Gdgr mss Ped. sublaeves; folia alia oblonga, alia obovato-oblonga -

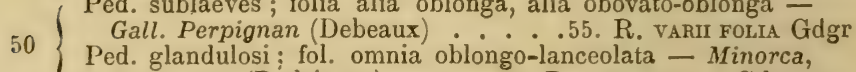
Turmaden (Rodriguez). . . . 56. R. MINORICENsIs Gdgr mss Sepala dorso omnia glandulosa......... 52

51 Sep.dorso alia glandulosa alia necre . . . . . . 74

Fr. ovatus .................... 53

52 Fr. rotundatus vel ovato-rotundatus . . . . 54

Fr. ovato-subovoideus, obovatus vel oblongus . . . . . 59

Ped. 14-16 m. l.; petioli aculeati - Dalmatia, Ragusa (Stud-

$53\left\{\begin{array}{l}\text { niczka) } \\ \text { Ped }\end{array}\right.$ Ped. 29-33 m. l.; pet. inermes - Bouches-du-Rh. Martigues

(Autheman) . . . . . 58. R. Macrostyla Gdgr mss

54 Pet. omnino eglandulosi .......... 55

Pet. plus minus glandulosi . . . . . . . . 56

$55\left\{\begin{array}{l}\text { Fol. minutissime ovato-elliptica obtusa - Istria, Pola (Freyn) } \\ \text { Fol. mediocriter obovata-lanceolata - Europa austr.60. R. }\end{array}\right.$

55 . . . . . . . . Scandens Mill.

Fol. amplissime oblongo-acuminata - Sic. Palerme (Todaro fl. sic. exs. n. 1081). . . . . .61. R. Todaroi Gdgr mss

56 Fol. basi rotundata; fr. parvus globosus ....... 57

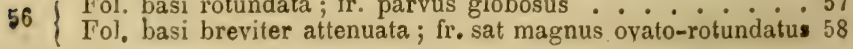


Pet. sparse hirtelli; fol. oblongo-acuminata - Pyrenaci, Perpignan, etc. (Debeaux in Bull. Soc. Bot. Fr. XXI, 1874, p. 9 ; Gdgr herb. ros. exs. n. 554). 62. R. Gaviogeriaxa Pet. glabri ; fol obovato-subacuta - italia, Florence (Caruel) . . . . . . 63. R. Canuelil Gdgr mss Discus subeonicus; costa eglandulosa - Lusit. Lisbonne (Daveau herb. lusit. 1879!) . . 64. R. Lusitavica Gdgr mss Discus planus; costa glandulosa - Lot-et-Gar Moirax (Debeaux; Gdgr Decad. plant. nov. II, p. 38; herb. ros. exs. n. 551 var. oblonga ; $R$. Amansii Gidgr Dec. plant. nov. I, p. 15 non Déségl) . . . . 65. R. Chatmandiasa (idgr Fol. elliptica, ovato-elliptica vel ovato-rotundata . . . . 60 Fol. oblonga vel obovato-lanceolata . . . . . . . 61 Fol. late ovato-elliptica acuminata - Naples, Ischia (Gussone)

Fol. parva rotundata obtusiuscula - Hisp. Barcelone (Bompard). . . . . . . . 67. R. Bompanuil Gidgr

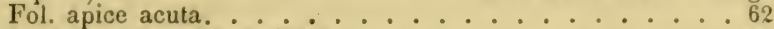
Fol. apice longe acuminata. . . . . . . . . . . . 6́t Aculei haud setacei ; stip. auriculae converyentes . . . . 63 Aculei interdum setacei; auriculae rectae-Vaples (Reimbole), Agen, etc.. . . . . . . 68. R. Ficta Gidgr Pet. glandulosi ; fol. basi rotundata - Istria, Pola (Areschoug) Pet. eglandulosi ; fol. basi attenuata - Naples (Pasquale) . . . . . . 70. R. neapolitava Gdgr mss Pet. eglandulosi. . . . . . . . . . 665

Pet. plus minus glandulosi . . . . . . . . . 67 67

Fol. oh,ovata vel obovato-oblonga. . . . . . . 66 Fol. oblonga ; ped. $32-35$ m. l.; discus valde conicus-Hisp. mer. Ronda (Laguna). . . . . 71. R. ARundava Gdgr mss Fol. oblonga ; ped. 22-25 m. 1.; discus planus - Naples, Castellamare di Stabia (Gussone). .72. R. stabixis Gdgr mss Rami flor. inermes; discus subconicus - Catalaunia, Maza (Vayreda). Ram. flor aculeati; discus planus - Algeria, Bone(Thiebaut)

............. R. BoNensis Gdgr mss Sep. dorso I dense glandulosi ........ 68 Sep. dorso fere eglandulosi - Liguria, Ital. sup.75. R. AтnoStyli staminibus 2-3-plo breviores ; fr. obovato-oblongus apice valde strangulatus. . . . . . . . . . . . 669 Styli stamina / aequantes; fr. ovoideus apice haud vel parum strangulatus. ............ 70 Costa glandulosa; styli staminibus 2 1/2-plo breviore: Alger, Birmandreis (Glgr Fl. Alger, exs, n. 705 bis). Costa erlandulosa; styli s'aminihus 3 1/2-plo breviores Alger, Birmandreis (Gdgr Fl. Alger. exs. n. 705).77. R.

. . . . . . . . rernchocarpa Gdgr mss

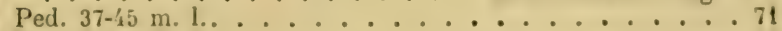

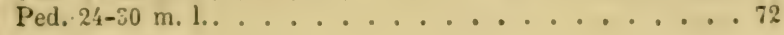


Fol. late oblonga basi rotundata ; costa inferne aculeolata Ital. Terracine (De Not.). . . . .78. R. TERRACINA Gdgr mss

7 Fol. anguste oblonga basi sensim attenuata; costa inferne paulo glandulosa - Ital. mer. Albe (Gibelli).79. R. Romana

. . . . . . . . . . Gdgr mss

Discus planus ; gemma rutundata vel globosa ... . 73

i2 Discus conicus; gemma flor. ovato-elliptica - Dalmatia, Meligne (Studniczka) . . . .80. R. Dalmatica Gdgr mss

Fr. basi attenuatus; styli stamina aequantes - Bouches-

73) du-Rh. Ensuès (Autheman) . . 81. R. Polysticta Gdgr mss or. Gerace (Huter Iter ital. III n.338)82. R. CALABRICA Gdgr mss

71 Fr. ovatus vel ovoideus; bracteae ovato-truncatae . . . . 75

Fr. suboblonщus; bracteae oblongo-lanceolatae - Gall. Avignon (Req.) . . . . . . 83. R. Requienil Gdgr

Fr. ovatus . . . . . . . . . . 76

75 Fr. ovoideus - Europa mer., Africa bor., Canarienses ins.!

(Perez, etc.) . . . . . 84. R. Sempervirens (L.)

Fr. ovatus ; ped. 24-26 in. l.; fol. mediocria. . . . . . 77

76 Fr. rotundatus; ped. $38-50 \mathrm{~m}$. 1.; fol. 46-58 m. 1. - Algeria, Philippeville (Choulette flor. alger. exs. $2^{\circ}$ série n. 132).

85. R. Chouletti Gdgr

Fol. Lasi sensim att.; costa eglandulosa - Corsica, Bonifacio

i7 (Reverchon pl. cors. exs. 1880 n. 325).86. R. consica Gdgr mss

Fol hasi rolundata; costa glandulosa - Algeria, Kouba (Gdgr) . . . . . . 87. R. Durandor Gdgr mss

78 Ped. glandulosi nec villosi . . . . . . . 79

78 Ped. saltem ad basim villosi . . . . . . . 83

$79)$ Pet. minute hirsuti . . . . . . . . . 80

9 Pet glabri vel sparse pilosi 881

Fol. elliptica; fr. rotundatus - Ins. Baleares, Creta.88. R.

Catalaumio Balearica (Desf.) Fol. obovata; fr. ovatus - Catalaunia, Castellfullit (Vayreda)

Fol. obovato-oblonga; fr. ovoideus - Lot-et-Gar., Agen

(Debeaux). . . . . . 90. R. AMIcI Gdgr

(Fol, subtus toto villosa - Syria, Asia occid. (Kotschy n. 77,

81 87, etc.).......... R. PHowicia (Boiss.)

Fol, subtus ad costam tantum villosa . . . . . 82

Tubus laevis ; foliola oblongo-lanceolata; pet. vix glandulosi

82 Baleares, Palma (Sieber). . . 91 bis. R. Palmensis Gdgr

Tubus glandulosus; fol. obovato-oblonga subacuta; pet. dense glandulosi - Istria, Pola (Freyn).92. R. ADENOPHORA Gdgr mss

Tubus villosus ............. 84

Tubus glandulosus; rami flor. sparse glanduliferi - Sicil. Palerne (Todaro fl. sic. exs. n. 1080 ; Guss. syn. sic. I,

83 p. 561 var.) ..... 93. R. panormitana (Tod.) mss Tubus laevis; rami flor. laeves - Gall. Angers (Bor.).94. R.

Fol ovato-acuminala ad costam villosa. . Dupontil (Dés.)

84 Fol. ovato-acuminaia; ad costam villosa

(Sep. corolla breviores; fr. oblongus - A frica bor., Oriens, etc.

$85\left\{\begin{array}{l}\text { Sep. corollam aequantes; fr. ovatus - Algeria, Kouba, Boghar } \\ \text { Soll.) }\end{array}\right.$ 
Fl. numorosissimi ; rami valde aculeati - Tunis, Le Caive,

86 Abyssinia. . . . . . 97. R. Anyssinica (Salt.) Flores pauciores; rami flor. inermes - Corsica (Requien).

.98. R. ERIOPODA Gdgr

B. - Anvenses Crép. Prim., p. 12; Gdgr Essai, p. 11.

Suffrutices fere ubique haud infrequentes, ramosi, flagelliformes ; rami saepe ex virente pruinosi, procumbentes vel reptantes, rarissime erecti, aculeati ; aculei tenues, conformes (necne); foliola rotundata ovata vel oblonga decidua plus minus villosa; pedunculi ordinarie glandulosi violacei; corolla alba magna; sepala ovata haud exserta saepe omnino integra; styli in columnam glabram ultra discum saepissime planum longissime producti, stamina subacquantes vel ea superantes; bracteae angustae ; fructus coriaceus ruber magnitudine pisi.

A. $\left\{\begin{array}{l}\text { Fol. coriacea lucida; rami recti vel rectiusculi. a. Pseudo- } \\ \text { Fol tenuia nec coriacea; rami procumbentes, prostrati vel } \\ \text { reptantes. . . . . . . . Eunverses }\end{array}\right.$

a. - Pseudo-sempervirentes Gdgr Essai, p. 12.

Foliola coriacea rigida lucida; styli glaberrimi ultra discum longissime exserti; fruticuli parsi ramosi, rami recti nec reptantes. - Facies fere subtribus sempervirentium refert, sed foliola equidem submarcescentia tandem decidua nec persistentia. - Grex R. bibracteatae (Bast.).

1 Pet. glabri vel sparse hirtelli. . . . . . . . . . 2

1 Pet. saltem ad basin | villosi . . . . . . . 4

( Tubus inferne nunquam decurrens; nervi subtus fol. virides 3

2 Tubus decurrens; nervi rubentes - Transsilv. Kronstadt. 
. . . 101. R. LETOPHYlla Gdgr mss

Fr. ovoideus, obovato-oblongus vel oblongus. . . . . 5

Fr. magnus glaber rotundatus ......... 8

Fr. ovatus mediocris, glandulosus - Gall. centr., etc. (Billot

n. 1870). . . . . 102. R. Bibracteata (Bast)

; Sep. dorso eglandulosa . . . . . . . . . . 6

Sep. dorso glandulosa . . . . . . . . . . 7

Ped. 44-46 m. 1.; discus planus - Angl. Warwich Chesterton

6) (Fraser). . . . 103. R. Macrocarpa Gdgr mss Ped. 28-32 m. 1.; discus subconicus - Irlande, Leitrim

(Stewart). ....... 104. R. Hibernica Gdgr mss

( Fol. ovato-elliptica, obtusa; fr. ovoideus - Gard, Vigan

(Tuezkiewicz). . . . . . 105. R. obtusata Gdgr mss

Fol. obovato-oblonga acuminata; fr. oblongus - Gard, Aulas (Tuezk.) . . . . . 106. R. villosa Gdgr mss

Ped. in 1/3 inf. laeves; fol. anguste oblongo-acuminata -

Angl. Essex (Groves). . . . 107. R: Glabrifolia Gdgr mss Ped. toto gland.; fol. obovato-subacuta - Rhone, St-Genis

(Chabert). . . . . . . 108. R. prunella Gdgr mss

\section{b. - Euarvenses Gdgr Essai, p. 12.}

Foliola subius saltem villosa, mollia virentia; styli glabri ultra discum longissime et tenuiter producti. - Fruticuli ramosi in Europa media vulgares, rami luridi prostratoreptantes vel radicantes.

1) Styli paulo hirtelli. . . . . . . . . . . 2

1 I Styli glaberrimi. . . . . . . . . . 7

2 Fr. ovatus obovatus vel oblongus . . . . . . . 3

2 Fr. rotundatus vel globosus . . . . . . . . . 4

Rubens; fol. elliptico-acuta - Rhône, Amas (Gidgr herb. ros.

3 n. 1\% ...... 109. R. erythroclada Gdgr mss I Virens; fol. obovata, apice rotundata - Rhône, Arnas (Gdgr)

4 Pet. paulo glandulosi pubescentes. $\ldots \ldots$

Fol. punctala, ovato-rotundata - Gall. austr. (Tratt.). $111 . \mathrm{R}$.

Fol nec punctata, lanceolata-acuta - Rhône, Chaponost (Chaburt). . . . . . 112. R. umbilicata Gdgr mss

(Tubus glandulosus - Rhône, Francheville (Chabert). 113. R.

T. . . . . cYcLOCARPA Gdgr mss Tubus lacvis - Europa centr.. . 114. P. menponnonox (Ehrh) 
Fol: inaeque vil biserrata ........... 8

Fol. omnino uniserrata ............. . . 38

Pet. glabri vel sparse pilosi . . . . . . . . . 9

Pet. villosi . . . . . . 19

Fol. glabra vel ad costam tantum villosa ...... 10

Fol. subtus ad nervos villosa. . . . . . . . . . 18

Fol. glaberrima .................. 11

Fol. ad costam mediam villosa . . . . . . . . . 12 Rami floriferi eglandulosi - Rhône, Arnas (Gdgr herb. ros.,

n. 73) . . . . . 115. R. Neotnia Gdgr mss Rami floriferi glandulosi - Gard, Vigan (Tuezkiewicz).

. . . . 116. R. CEbenvexsis Gdgr mss

Rami floriferi glanduligeri . . . . . . . . . 1

Non . . . . . . . . . . 16

Fr. ovoideus vel oblongus; discus plus minus conicus . . I'

Fr. rotundatus, glaber; discus planus - Belg. Han-sur-Lesse

(Crép.)........ 117. R. glandulifera (Ćrép.)

Fr. globosus toto glandulosus; discus planus - Angl. Warwich

(Fraser). . . . . 118. R. Adexuclada Gdgr mss

Fr. oblongus laevis vel basi glandulosus : fol. obovato-

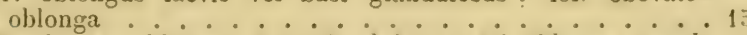

Fr. obovato-oblongus, toto glandulosus; fol. oblonga ; petala ciliata - Angl. Warwich (Fraser). 119. R. eleg.istissima

................... Gdgr mss Sep. anguste pinnata ; discus paulo conicus - Angl. Warwich

(Fraser). . . . . 120. R. Fraseri Gdgr mss Sel. late pinnata; discus conicus - Angl., Gall. occ. 121. R.

. . . . . . Gallicoines (Désẻgl.)

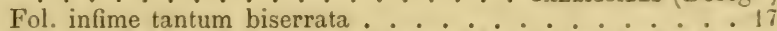

Fol. omnia biserrata - Belg. Namur (Crép.). 122. R. neptass

Fr. globosus to to glandulosus; fol. basi cordata - Gard,

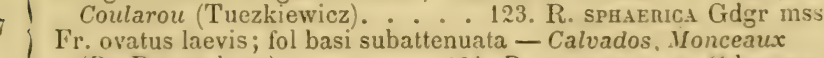

(De Bunnechose). . . . 12'́. R. subbisenrata Gigr ms Fr. et fol. ovati. . . . . . 125. R. ovata (Lej.) Fr. oblongus; fol. orbiculata - Rhone, Arnas, etc. (Gdgr).

126. R. PIsIFonMIs Gdgr miss

Discus planus vel subplanus . . . . . . . . 20

Discus | conicus . . . . . . . . . 30

Fr. ovatus vel ellipticus . . . . . . . . . 21

Fr. ovoideus, obovato-oblongus vel oblongus. . . . . 24

Fol. ovata . . . . . . . . . . . 22

Fol obovata . . . . . . . . . 23

Tubus ellipticus basi glandulosus; pet. valde glandulosi -

Gard, Avèze (Tuezkiewicz). . . 127. R. Durifolia Gdgr mss

Tubus ovatus laevis; pet. vix glandulosi - Rhone. Romanèche (Gdgr). . . . . . . 128. R. belophora Gdgr mss

Fol. ad nervos paulo villosa; pet. vix glandulosi - Rhöne,

Ecully (Chabert). - . 129. R. subintegrisepala Gdgr mss Fol. ad costam vix hirtella; pet. glandulosi - Hibernia, Antrim Crumlin (Stewart). . 130. R. Busaltici Gdgr mass Fr. ovoideus vel obovato-oblongus . . . . . . 25 Fr. elongatus - Hte-Savoie, Annecy (Puget).131. R. tMbel- 
25 Fol. obovata 26

Fol. ovata vel oblonga. . . . . . . . . . 28

Fol. apice / acuta; bracteae obovatae . . . . . 27

26 Fol. apice subrotundata; bracteae oblongo-lineares - Loire, St-Genest-Malifaux (Malarbet). . 132. R. proBata Gdgr mss Pet. omnes villosi; sepala eglandulosa - Isère, Varacieu . . . . . . . . . 133. R. septicola Gdgr mss Pet. omnes villosi; sep. margine subglandulosa - Vosges, Epinal (Berher). . . . 134. R. vogesiaca Gdgr mss Pet. omnes villosi ; sep. dorso glandulosa - Catalaunia, Castellfullit (Vayreda). . . . . 135. R. subcinenea Gdgr mss Pet. nune glabri nunc sparse pilosi ; sep. margine paulo glandulosa - Rhone, Arnas (Gdgr). . .136. R. INAEQuevillosa dulosa - Rhone, Arnas (Gdgr). . . . . . . Gdgr mss

Fol, oblongo-acuminata vel acuta $\ldots 29$

28 Fol. ovato-subattenuata - Haute-Savoie, Cuvaz (Puget) .......... 137. R. Recumbens Gdgr mss Bracteae lineari-lanceolatae; nervi villosi - Rhỏne, Arnas

$29\{$ (Gdgr). . . . . 138. R. BELonoIdes Gdgr mss Bracteae ovato-lanceolatae; costa villosa - Pyren.-Orient., Frexanet (Vayrer'a). . . . 139. R. LATIDENS Gdgr mss

31) Fr. globosus, ovato-rotundatus vel ovatus. . . . . . 31 Fr. ovoideus vel oblongus .............. 35

) Fol. oblonga . . . . . . . . . . . 32

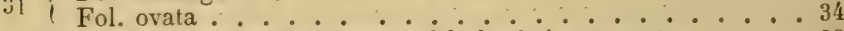

( Fr. rotundatus vel globosus; fol. basi | attenuata . . 33

32 Fr. ovatus; fol. basi subattenuata - Manche; Yvetot (Lebel) . . . . . . . . 140. R. Conifera Gdgr mss (Costa media paulo glandulosa; fol basi subattenuata - Angl.

Cheshire, Bromborough (Harbord L.). 111. R. AxGLICA Gdgr

Costa media eglandulosa; fol inferne valde acuta - Finistère, Cranou (Thiébaut)...... 142. R. DIoxys Gdgr mss

( Ped. giandulosi ; fr. subglobosus - Rhône, Montmęlas (Gdgr 34 herb. no 131). fr. ovato-rotundatus - Rhône, Gleizé (G. herb, n. 53). ......... . . . . . R4. Evolvens Gdgr

.5) Fr. obovato-oblongus vel oblongus - Haute-Savoie, Argonnex (Puget).145. R. SEMI- 36

( Minime $\therefore \therefore \therefore \therefore \therefore$

3 Rami flor glanduliso-setosi - Cher, Givrai (Rip.).146. R.

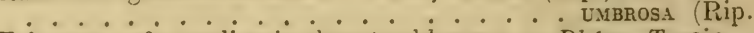

Fol. ovata ; fr. mediocris obovato-oblongus - Rhone, Tassin Billot exs n. 3716) .....147. R. Macrodon Gdgr mss

37 Fol obovata ; fr. mediocris oblongus - Austr. sup. Aistersheim (Keck)........148 R. KECKII Gdgr mss Fol. oblonga; fr. magnus oblongus - Rhône, Arnas (Gdgr) . . 149. R. Gugurbitacea Gdgr mss

38 Pet. laevissimi vel vix subglandulosi. . . . . . 39

38 Pet. omnes | glandulosi. . . . . . . . 50

(Fr. rotundatus vel globosus . . . . . . . . 40

33 Fr. ovatus, bóng vel oblongus . . . . 41 


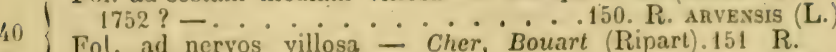
Fol. ad nervos villosa - Cher, Bouart (Ripart).151 R.

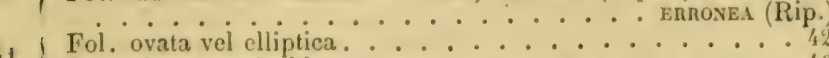

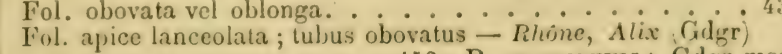

Fol. apice subrotundata ; tubus ovatus - Hisp. bor. Sierra de Cameros (Zubia) . . . . 153. R. Asturica Gdgr mss Fol, apice acuta ; tubus ovatus - Rhône, Charbonnieres (Chabert). . . . . . 154. R. sepicola Gdgr mss Fol. ohovata. . . . . . . . 44

Fol. oblonga .................. 45

Discus planus; costa eglandulosa - Vosges, Epinal (Berher)

Discus a conicus; costa glandulosa. R. SEMINuda Gdgr mss (Verlot). . . . . 156. R. gratianopolitana Gdgr mss Pet. sparse glandulosi ; discus planus - Rhone, Liergues $45\left\{\begin{array}{l}\text { (Gdgr). } \\ \text { Pet eglandulosi ; discus subconicus - 157. R. APTA Gdgr mss }\end{array}\right.$ (Kemm.) . . . . . 158. R. Kemmleri Gdgr mss

46 Fol. ovata vel ovato-rotundata . . . . . . 47

Fol. obovata vel oblonga. . . . . . . . . 48 Fol, late ovato-rotundata - Rhóne, Beaunant (Gdgr).159 R. 47 Fol. parva elliptico-ovata - Rhóne, Alix (Gdgr).160. R.

Pet villosi, fol oblonga

48 P'et. glabri; fol. obovata - Isère, Charancieu (G. herb. ros. n. 360$) \ldots . .161$. R. PHALACROPODA Gdgr Petala eciliata ; fol. oblonga basi rotundata - Pyren. centr.,

491 Gèdre (Bordère). . . . . . 162. R. NudATA Gdgr mss Petala paulo ciliata ; fol. obovata basi subattenuata - Gironde. Bassens (Motelay). . . . . 163. R. IMpressa Gdgr mss

50 Fol. ad costam (rarius paulo ad nervos) villosa. . . . . . 51

$50 \quad$ Fol. ad nervos vel subtus toto villosa. . . . . . . . . 67

5) Fol. ovato elliptica vel obovata . . . . . . . . 52

4 Fol, obovato-oblonga vel oblonga. . . . . . . . 62

52 Fr. ovatus vel ovoideus . . . . . . . . . . 53

52 Fr, obovato-oblongus vel oblongus. . . . . . . 58

53 I Fr. ovatus ................ 54

3 Fr. ovoideus . . . . 55 Rami floriferi aculeati ; fol. ovata rel rotundata. Europa med. et mer. . . . . . . . . 164. R. RePEss (Scop.) Rami flor. aculeati; fol. olovalo-attenuata - Gard, Vigan (Tuezk.). . . . . . 165. R. marcescens Gdgr mss Ram. flor. inermes ; fol. obovata - Isére, Grande-Chartreuse (Gdgr). . . . . 166. R. Alpestris Gdgr miss

Fol. basi rotundata. ........... 56

55 Fol. basi sensim attenuata - Rhone, Arnas (Gdgr heb ros. n. 358 . . . 167. R. silvelicola Gdgr Discus planus; fr. basi contractus. . . . . . . 57

56 Discus subconicus; fr. hasi rotundatus - Pyren, centr., Gèdre (Bordere). . . . 168. R. LEPTODos Gdgr mss 


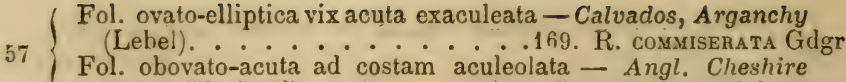
Oxton (Harbord). . . . . 170. R. Spininaeva Gdgr mss

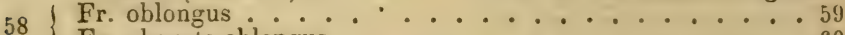

Fr. obovato-oblongus.

(Fol. basi cordata; petala ciliata - Isère, Le Sappey (Verlot) $59\{$ Fol. basi subattenuata; petala eciliata - Rhône, Liergues

(Gdgr)

$60\left\{\begin{array}{l}\text { Pet. alii glabri alii villosi; fol obovata } \\ \text { Pet. omnes villosi ; fol. ovata }- \text { Haute-Savoie, Pringy (Puget) }\end{array}\right.$

. 173. R. Confertiramea Gdgr ms

Rami floriferi inermes; discus planus - Isère, Bourgoin

$61\{$ (Gdgr) . . . . 174. R. GLABREscens Gdgr

Rami flor. aculeati; discus sat conicus - Pyren. centr.

Gèdre (Bordère) . . . . . . 175. R. JACENs Gdgr mss

62 Fr. oblongus ........................... 63

62 Fr. obovatus vel oblongus. . . . . . . 64 Aculei inclinati; fol. oblonga - Ain, Sathonay (Chabert)

63 A 176 . R. IDIONOCARPA Gdgr mss Aculei valde uncinati ; fol. obovato-oblonga - Isère, Charancieu(Gdgr herb. ros. n. 123).177. R. ODONTOpHora Gdgr mss

( Fol. tenua ; pet. eglanlulosi . . . . . . 65

Fol. tenua, basi acuta ; pet. sat glandulosi - Pyren. centr.,

64 Chichaubé (Bordère). . . . 178 R. oxyphylla Gdgr mss

Fol. coriacea basi rotundata ; pet. valde glandulosi - Angl. mer. Devonshire (Briggs, nec Bripp). .179. R. BRIGGSII Gdgr

Tubus laevis; pet. villosi eglandulosi . . . . . . 66 65 Tubus basi glandulosus; pet. sparse villosn-glandulosi $-\dot{-}$

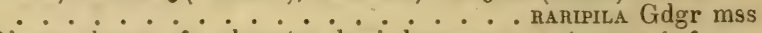

Discus planus ; fr. obovatus basi depressus - Austrıa inf., Kalk $\backsim$ burg (Wiesbaur). . . 181. R. Austriaca Gdgr mss $66\left\{\begin{array}{c}\text { Discus sat conicus; fr. ovoideus basi attenuatus - Westphalia, } \\ \text { Annen (Scheman). : . . 182. R. Guestphalica Gdgr. mss }\end{array}\right.$

Discus valde conicus; fr. obovato-oblongus, basi decurrens - Bavaria, Schloss Zeil (Kugler).183. R. BAvarica

Fol. subtus toto villosa. $\therefore \therefore \cdots$ Gdgr mss

67 Fol. subtus ad nervos villosa $: \therefore 68$

(ji $\int$ Sepala dorso eglandulosa. . . . . 69

(i8 Sepala dorso glandulosa - Ital. or. Faenza (Caldesi).184. R.

$\mathrm{Fr}$ ovoideus vel obovatus ..... DISCoLon Gdgr mss

$69\{$ Fr. ovato-rotundatus vel ovato-ellipticus. . . . 73

69 Fr. oblongus - Ital. orient. Faenza (Caldesi). $185^{\circ} \dot{\mathrm{R}}$ LEIOSE-

$70 \mid$ Discus i conicus $\because \because \cdots$ PALA Gdgr mss

$70\{$ Discus planus .

Ped. in $1 / 2$ inf. laeves; folia lata basi rotundata - Naples,

71 monte di Mezzo Campania (Gussone).186. R. Gussoni

Ped. toto glandulosi; fol parva basi subattenuata - Istria, Canfanaro (Freyn) . . . . 187. R. AdRiatica Gdgr mss 
(Petala subciliata; fol. basi rotundata - Rhône, Arnas (Gdgr)

72 Minime ; fol basi sensim attenuata - Croatia, Agrans Gdgr mss tinovic).189. R. veLuTins Guko-

3 Discus planus; ped. in $1 / 2$ vel $1 / 3$ inf. laeves . . . . . 71

73 Diseus subconicus; ped. toto glandulosi - Carniolia, Cilli (Studniczka) ......190 R. canxıolica Gdgr mss

Tubus laevis; fol. basi longe attenuata - Croatia, Sestine

(Vukotinovic) . . . . 191. R. croatica Gdgr mss

Tubus in $1 / 4$ inf glandulosus; fol. basi vix attenuata Tyrol, m. Baldo (Kern.); Croatia Agram (Vukot.).192. R. .................. BaldENSIS (Kern.)

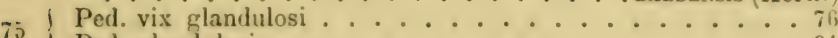

5 I Ped. glandulosi............... . . 84

76 Fol. ovata vel ovato-elliptica ............ 77

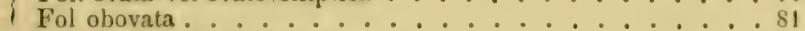

77 Fol. basi paulo attenuata. . . . . . . . . . 78

Fol. basi rotundata vel cordata . . . . . . . . 79

Fr. subovatus - Rhône, Arnas (Gdgr). 193. R. strata B. 78 Fr. saepius ovoideus - Rhóne, Arnas (Gdgr). 194. R. DUME.................. TICOLA Gdgr mss

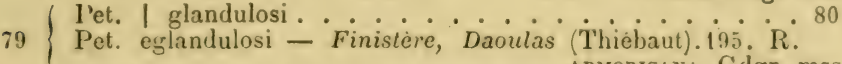

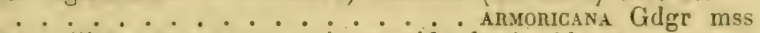

(Pet. eciliata ; fr. magnus, apice turgidus basi valde attenuatus

- Rhöne, Arnas (Gdgr herb. ros. n. 311).196. R. TAMNoides

80 . . . . . . . . . . Gdgr

Petala ciliata; fr. mediocris haud turgidus, basi vix attenuatus

- Rhóne, Arnas (Gdgr herb. ros. n. 320).197. R. strata Gdg̈r

81 Intense purpureo-vinosa ; petala basi paulo ciliata . . . 8?

I Minime. . . . . . . . . 83

Discus subconicus; aculei minute adunci-Finistere. Daoulas

8: I (Thiébaut) . . . 198. R. oenopiryla Gdgr mss

(Discus planus; aculei recti - Rhone, Arnas (Gdgr herb. ros.

n. 350). ......... 199. R. Dumetigena Gdgr mss

Fr. ovatus; costa paulo aciculata - Helvet. Neuchatel (Mor-

83 thier). . 200. R. viridula Gdgr mss Fr. ovoideus ; minime - Ain, Les Echeyx (Chabert).201. R.

. . . . . . . . . . LUGEXS Gdgr mss

St Rami recti, robusti ............. 85

Rami prostrati, tenues. . . . . . 86

Glauca ; costa villosa - Mayenne; Rhin $(R$. glauca Dierb.

85 f. Heidelb. II, p. 140). . 202. R. Dienbacuin Gdgr Virescens ; costa glabra - Borussia, Coblentz (Wirtgen exs.

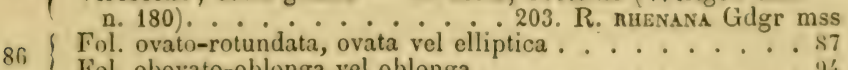

86 Fol. obovato-oblonga vel oblonga . . . . . . . .

87 Fol basi breviter attenuata. . . . . . . . 88

87 Fol. basi rotundata .58

( Purpurea ; petala paulo ciliata - Hte-Savoie, Pringy (Pugret)

ss $\quad \ldots \ldots . \cdots 205$. R. oenocallos Gdgr mss Virens; minime - Irispania bor., Sierra de Cameros (Zubia) 206. R. Zublae Gdgr mss 


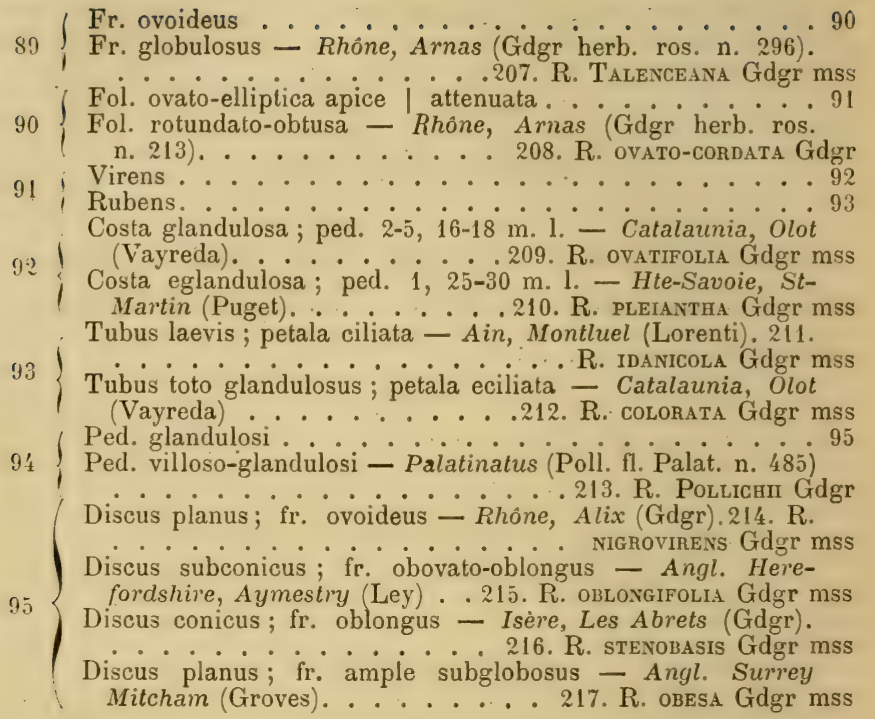

\section{C. - Stylosae Gdgr Essai p. 12.}

Radix haud reptans; frutices hinc inde sat rari elati ramosi erecti, rami rigidi robusti aculeati; aculei conformes adunci Dasi late dilatati ; folia imparipinnata 3 -ร̆-foliolata, foliola ovata vel oblonga decidua, supra nitida plus minus villosa; pedunculi glandulosi ; sepala oblongo-lanceolata mox decidua, extus plus minus glandulosa, 2 integra, 3 late inciso-pinnata ultra gemmam longe producta ; corolla majuscula saepe rosea, late bracteata; styli in columnam ordinarie glabram parumque exsertam ultra discum longe conicum coaliti staminibusque breviores; fructus coccineus crectus sat magnus, coriaceus. 
A f Foliola simpliciter vel inaeque serrata. . . . . a. Eustylosae Foliola omnino biserrata. . . . . . b. Tomentelloidae

\section{a. - Eustylosae Gdgr Essai p. 13.}

Foliola simpliciter vel inaeque (nec omnino) serrata; pedunculi plerumque glandulosi - Grex verae $R$. stylosae (Desv.).

( Fol. omnino simpliciter serrata . . . . . . . 2

Fol. inaeque serrata . . . . . . . . . . 52

( Fol. subtus toto villosa . . . . . . . . . . .

2 Fol. glabra vel subtus ad costam nervosve villosa .... 28

3 f Pet. eglandulosi. . . . . . . . . . 4

Pet. evidenter glandulosi. . . . . . 16

4 Pet inermes . . . . . . . . . . 5

| Pet. | aculeati ............... . . 11

5 Aculei caulinarii maximi dilatato-adunci . . . . . . 6

I Aculei caulinarii parvi. . . . . . . . . . 7

Fol. supra glabra ; tubus ovatus - Europa centr. (Gdgr herb. ros. n. 453). . . . . 218. R. fastigiata (Bast.) Fol. supra paulo villosa ; tubus obovalo-oblongus - Hte-Sav.

Pringy (Puget). . . . . 219. R. BRevistipulata Gdgr mss

7 Sep. late copioseque pinnata . . . . . . . 8

Sep. anguste parceque pinnata . . . . . . . . 10

( Ped. dense glandulosi . . . . . . . . 9

8 Ped. sublaeves (2-5-gland.) - Hte-Savoie, Annecy (Puget).

. . . . . . . 220. R. FLACCIDA Gdgr mss

Fr. rotundatus; fol. reflexa obovato-subcordata - Gironde,

9 Montferrand (Motelay). . 221. R. REFLExifolia Gdgr mss

9 Fr. obovatus; fol. recta oblonga l,asi breviter attenuata Isère, Paladru (Gdgr herb. ros, n. 269).222. R. INsignita

. : . . . . . . . . . Gdgr mss

Rami flor. inermes ; fr. basi longe attenuatus - Isère, Anthon

11) (Boullu). . . . . . 223. R. MYriolepis Gdgr mss

Rami flor. aculeati ; fr. basi haud aut vix attenuatus - IIle-

Savoie. Argonnex (Puget); Manche, Carteret (Lebel).225.

(Ped. hispidi $\therefore \ldots \ldots$ R. LaEtevinens Gdgr mss

11 Ped. laeves - Lot-et-Garonnne, Nanau (Garroute).226. R.

1 Virens $\ldots \ldots \ldots$ barbatula Gdgr mss

12 Purpurea $\therefore 13$

13. Fr. basi rotundatus . . . . . . 14

13 Fr. basi attenuatus - Rhone, Vaux-en-Velin (Chabert), 227. 
Fol. saturate virentia ; tubus ovatus - Hte-Savoie, Pringy

14 (Puget) ......... 228. R. IntybacEa Gdgr mss

Fol. pallide virentia ; tubus oblongus - Hte-Savoie Pringy

(Puget) . . . . . 229. R. pachyacantha Gdgr mss

Fol. ovato-elliptica; sepala late copioseque pinnata - Hte-

15 Savoie, Pringy (Puget). . . 230 R. thamnocharis Gidgr mss

Fol. obovato-oblonga ; sep. anguste parceque pinnata - Lot-

et-Gar. Agen (Garroute). . . 231. R. Rubrisepala Gidgr mss

16 Fr. obovato-oblongus vel oblongus ......... 17

16 Fr ovatus . . . . . . 2 23

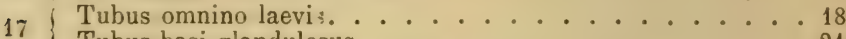

Tubus basi glandulosus . . . . . . . . . 21

18 Ped. sublaeves; corolla pallide rosea . . . . . . . 19

Ped. glandulosi ; corolla alba vel intense rosea . . . . 20

(Fol. ovata ; pet. villosi - Cher (R. puberula Rip. non Desv.)

19 Fol. obovato-oblonga pet. sparse pilosi - Rhóne, Charbonnières - Valognes (Lebel). . .233. R. veducta Gdgr mss Corolla magna alba; ped. glandulosi - Gallia, etc. (Gdgr

$20\{$ herb. ros. n. 457 ; Billot n. 1483). . 23!. R. STYLosA (Desv.)

Corolla parva amoene rosea; ped. villoso-glandulosi - Ain, Montluel (Lorenti) . . . . . 235. R. CALOPODA Gdgr mss

(Sep. intus rubra; fol amoene virentia . . . . . . . 22

21 Sep. intus albida ; fol. pallide virentia - Hte-Savoie, Pringy (Puget) . . . . . . 236. R. Lamprotes Gdgr mss Sep. dorso paulo glandulosa - Hte-Savoie, Pringy (Puget) $22\{$ Sep. dorso eglandulosa Finistère, Daoulas (Thiébaut). 238 .

. . . . . . . . R. oceanica Gdgr mss

23 Pet. sparse pilosi ............. 24

23 Pet omnino villosi $\ldots 27$

21 Fr. rotundatus . . . . . . . . . . 25

24 ! Fr ovatus .................... 26 Costa glandulosa; petala eciliata - Rhône, Arnas (Gdgr

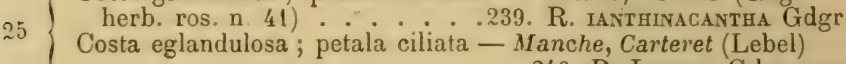

. . . . . . 240. R. LeBELII Gdgr mss

Fol. amplissime obovato-oblonga; petala paulo ciliata - Lyon

26 (Chab.) . . . . 241. R. Hispidobasis Gdgr mss Fol. anguste oblongo-lanceolata; petala eciliata - Rhône, Ecully, etc. (Gdgr herh. ros. n. 64). .242. R. Microdon Gdgr Fol. oblonga; pet. laxe pilosi - Isère, Villette d'Anthon (Chabert). . . . . . 243. R. collinita Gdgr mss Fol. ovata ; pet. dense pilosi - Lyon (Gdgr herb. ros n. 415)

(Fol ad nervos villosa. . . . . . . 29

28 Fol. ad costam villosa . . . . . . . . 44

Fol. glaberrima . . . . . . . . . . . 47

29 Pet. glabri vel sparse pilosi . . . . . . . . 30

Pet. saltem inferiores villosi . . . . . . . . . 34

Ped, sublaeves ; tubus eglandulosus . . . . . . . . . 31

30 Ped hispidi ; tubus basi glandulosus . 33

Ped. glaberrimi - Angl. Hants Holmsley (Groves).245. R. 
Fr. ovalus vel subrotundatus; pet. aculeati

Fr. anguste oblongus ; pet. subinermes - Gironde, Montferrand (Motelay) . . . . 246. R. semicalva Gdgr mss

Discus subconicus; fr. rotundatus - Ital. Apenn. Modene (Gibelli) ......... 247. R. APENNINA Gdgr mgs

Discus conicus; fr. ovatus - Rhone, Charbonnieres (Chabert)

. . . . . . . . . 248. R. Finmata Gidgr mss

Fr. ovatus ; discus conicus - Rhone, Ecully (Chabert). 249.

33 Fr oblongus; discus subplanus - Gironde, Montferrand

(Motelay) . . . . . 250. R. Laxistigma Gdgr mss

34 Fr. obovateus oblongus vel oblongus : $: \therefore 35$

Corolla pallide rosea. . . . . . . . . . 36

35 Cor. rosea ; ful. adulta flavescentia - Eur. centr. (Gdgr herb. ros.n. 408). . . . . 251. R. LeucochnoA (Desv.)

(Fol. ovata vel obovata . . . . . . . . . 37

36 Fol. anguste oblonga - Rhône, Gleizé, (Gdgr herb. ros. n. 137) . . . . . 252. R. LENTISGIFOLIA Gdgr msS Sep. anguste pinnata; fol. parva obovata - Isère, Chavanoz (Gariod). . . . . . 253. R. Gariodiana Gigr mes Sep. late pinnata; fol. ample ovata - Eur. centr. (Gdgrhrb. n. 467 ; Billot n. 1663) . .... 254. R. systyla (Bast.)

Fol. ovata vel obovata ................ 39

Fol. obovato-oblonga vel oblonga . . . 40

Cor. alba vel rosella; fol. virentia. - Rhöne, La Tour (Chabert). . . . . . . 255. R. subovata Gdgr mss Cor. saturate rosea ; fol glauca - Lot-et-Gar. Arasse (Garroute) . . . . . 256. R. Conspersa (Pug. et Rip.)

40 i Tubus eglandulosus .............. 4

40 , T'ubus basi hispidus .................. 45

(Fr. obovato-oblongus ; ped. $15-20 \mathrm{~m}$. i. . . . . 42

41 Fr. oblongus ; ped. 25-28 m. 1.-Helvetia : Vevey (R. stylosa Grem. excfl. p. 174) . . . . . 257. R. GremLir Gdgr mss

Fol. basi sensim attenuata - Lot-et-Garonne, Arasse (Gar-

42 route) . . . . . . 258. R. Neglecta Gdgr mss Fol. basi sensim rotundala - Rhone, Dracé (Fray).259. R.

. . . . . . . . . PERYotata Gdgr mss

Pet. sup. subglabri ; fr. obovato-oblongus - Rhone, Salva-

43 gny, etc. (Chab.). . . 260. R. vacillans Gdgr mss Pet. omnes villosi ; fr. oblongus - Gironde, Montferrand (Motelay) . . . . . . 261. R. Rubripes Gdgr mss

4) Ped. slandulosi ; rami flor inermes $\ldots . . .256$

) Ped. omnes glaberrimi - Bouches-du-RhGne, Martigues (Autheman). . . . . . 262. R. singulanis Gdgr mss Fol. amplissime oblongo-acuminata - Gall. Agen (Garroute) 45 Foi. parva obovato-acuta - Gironde, Blanquefort (Motelay) . . . . . . 264. R. Glaucocanpa Gdgr mss

Styli conliti ; flores albi - Rhome, Charbonnieres (Gdgr herb. ros. n. $\{00$; Hibernia (Lindl., R. systyla f. lanceolata Trat.). . . . . . 26j. R. Trateivickir Gdgr Styli vix cnaliti; flores amoene rosei - Gall. Agen (Garroute in Soc. dauph. exs. 11. 374 et bis) .266. R. Garnoutei (Png.) 
Ped. glandulosi .............. . 48

47 Ped. laeves vel sublaeves ............ 49 Petala ciliata; tubus oblongus - Manche, Yvetot (Lebel).

$48\{$. . . 267. R. cuspidifers Gdgr mss Petala eciliata ; tubus ovatus - Cher : Servanterie, etc.268. f.......... Rusticana (Dés.) Styli glabri...................... 50

49 Styli basi lanati - Helvet. Neuchatel, La Clutelle (Lerch). . . . . . . . 269. R. RIGEScENs Gdgr mss Pet. aculeati pauloque glandulosi . . . . . . . 51

50 Pet. inermes eglandulosi - Cher, Marmagne, etc. $270 . \mathrm{R}$.

. . . . . . . . . rmitrs (Dés.) Fr. oblongus ; ped. 6-9 m. 1. - Madère, Funchal (Thiébaut)

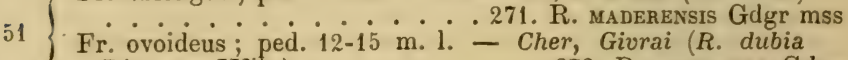
Rip. non Wib.) . . . . . 272. R. DECLINATA Gdgr Styli glabri. . . . . . . . . . . 53

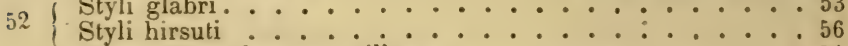
Fol. subtus ad nervos villosa. . . . . . . . . 54

53 Fol. subtus toto vel ad costam tantum villosa . . . . . 55

Fol. glaucescentia et glaberrima - Cher, Marçay (Dés.).273.

.......... . . R. Fissidens Gdgr mss Fr. rotundatus; costa eglandulosa - Hte-Savoie, Pringy

54 (Puget) . . . . . . 274. R. GlobUlifERA (Rip.) Fr. obovato-oblongus ; costa paulo glandulosa - Rhône, Alix (Gdgr herb. ros. n. 23 et 491). . .275. R. Carnatula Gdgr Fol. subtus omnino villosa - Manche, Yvetot (Lebel).276.

55 Fol. subtus ad costam villosa, nervi Rubhirtelli - Gard, Vigan (Tuezk.). . . . 277. R. DEcalvata Gdgr mss Fol. subtus ad costam solam villosa - Hte-Savoie, etc. (Puget). . . . . . . . 278. R. congesta (Rip.) Fol. undique glaberrima - Lot-et-Garonne, Agen (R. pusilla Rip. non Raf.) . . . . . . . 279. R. Exigua Gdgr Fol. subtus ad nervos villosa - Istria, Fasana (Freyn). 280 .

............ R. AnIsodonta Gdgr mss Fol. subtus toto hirsuta - Gall. Toulouse, Bouconne (Timbal). 281. R. Clotildea (Timb.)

\section{b. - Tomentelloidae Gdgr Essai p. 13.}

Foliola omnino biserrata; pedunculi saepius glabri. Facies quarumdam Crepiniarum (Canineae Auct.), bene vero ob stylos evidenter coalitos huc adnumerandae. Subtribus omnino nova, a meipso jam anno 1873 , indicata.

1 Fol. undique glaberrima . . . . . . . . . 2

Fol. subtus | pubescentia............ 6 
Styli glabri . . . . . . . . . . .

Styli hirsuti. . . . . . . . . . . . .

Pet., ped. petalaque paulo hirsuti - Manche, Yvetot (Lebel).

p. 282. R. Mcass Grilgr mss Pet., ped. et petala glabra - Insula Madère (Kuliler).283.

....... K. Klmleni (Crép.)

Fr. oblongus - Vaucluse, Caromb (Autheman). 283 bis. R.

4

Fr. ovatus - Pyrén.-Orient, Vernet-les-Bains (Roffavier).

. . . . . 284. Mi. heterogiva Gidgr mss

(Fol. oblongo-lanceolata, basi paulo attenuata - Pyrén. cent.,

5 Fol. obovata, basi cordata - Rhöne, Gleize (Gdyr herb. ros

n. 63); Gall or. (R. seposita Dés. 1880).286. Li. PATEL is

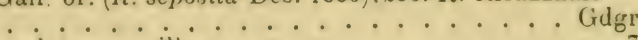

Fol. subtus ad nervos villosa.......... . . 7

6 Fol. subtus ad costam villosa - Deux-Sevres, La Mothe.287.

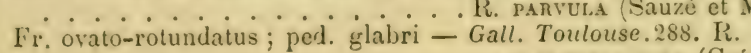

f . . . . . tomentelloidea (Crép.)

Fr. olsovatus; ped. paulo hirsuti - Manche, Yvetot (Lebel).

. . . . . . 289. R. popophylla Gdgr mss

r. ovoideus; ped. glaberrimi - Gall. bor. Provins (Bou-

teiller) . . . . . 290. R. ANCEPS (Bouteiller)

Subgenus II. - Rosa (Eurosa) Gdgr Essai p. 13; Gallicaneae DC. in Ser. Mus. helv. (1818) I, p. 2; Crép. Prim. p. 13.

Styli glabri villosi aut lanati plerumque liberi. Flores anguste bracteati, rubri rosei rarissime albi ndorati, ad unguem nonnunquam ciliati. Fructus coriaceus serotinus atrorubens saepe glandulosus. Sepala plus minus glandulosa, post anthesim reflexa mox decidua, 2 integra, 3 pinnata. Pedunculi semper glandulosi vel aculeolati. Folia imparifinnata, foliola coriacea marcescentia rel sero decidua, glabra villosa rel ad costam mediam saltem glandulosa, serrata; serraturae simplices aut compositae. Stipulae angustae, earum auriculae breves conformes. dculei sparsi dimorphi nune adunco-dilatati nune setaceo-glanduliferi. - 
Suffrutices humiles erecti solitarii, Europam mediam et australem, Orientem cum Africa boreali hinc inde colentes nec frequentes, rami setiferi graciles luridi; radix longissime reptans.

Putavimus quod dignum esset genus Rosa ab opere nostro non omnino evanesceret; illud igitur tanquam titulum dedimus Gallicanaeis in quibus Rosae tam pulchrae quam inventu difficiles includuntur.

A Styli liberi ............... B

A Styli in columnam exsertam coaliti. ...... Spuriae

B Styli glabri ........... 2. Transitoriae

B Styli pubescentes . . . . . . . Geminatae

Styli dense lanati. ........... 4. Gallicanae

\section{1. - Spuriae Gdgr Essai p. 14.}

Styli evidenter in columnam glabram vel villosam ultra discum plus minus producti, staminibus breviores vel ea aequantes; corolla magna alba vel pallide rosea; fructus parvus saepius abortivus; rami flexuosi arcuati. - Subtribus haec intermedium optimum praebet inter Ripartias (Synstyleae) et Eurosas (Gallicanae).

1 Styli glaberrimi .............. 2

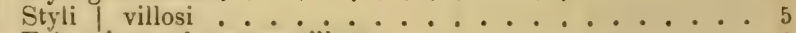

Fol. subtus ad costam villosa. . . . . . . . 3

2 Fol. subtus toto villosa ........... 4 Fol. ad nervos villosa - Bade; Helv. Fribourg.291. Rosa

Fol. biserrata, basi attenuata - Rhône, Tassin (Chabert). $3\{$ Fol. inaequeserrata, basi rotundata - Rhône, Charbonnières (chab.). . . . . . 293. R. curtistyla Gdgr mis

(Albida; tubus laevis - Rhône, Charbonnières (Chabert). 4 Virens; tubus glandulosus - Helv. Fribourg (R. varia var. Spen.). . ......... 295. R. SpenNerI Gdgr 
- i Fol. ad costam villosa ............ 6

Fol. ad nervos vel subtus toto villosa . . . . . . 10

6) Discus | conicus. ............. 7

Discus planus . . . . . . . . . . 8

Fol. ovata mediocria - Eur. centr. (Gdgr herb. ros. n. 416).

7 . . . . . 296. R. Anvira (Bchwenk.)

Fol. amplissime oblonga - Rhöne, Charbonnières (Billot $\mathrm{n}$. $3,578) \ldots . .297$. R. Desidenata Gdgr mss

Flos magnus; fol. obovata vel elliptica . . . . . . 9 9

8 Flos parrus; fol. ovato-rotundata - Germ. Altenbourg 298.

. . . R. Warziana (Waitz.)

Fr. owatus; fol. clliptica - Rhine, Dardilly Gilgr herb. ros.

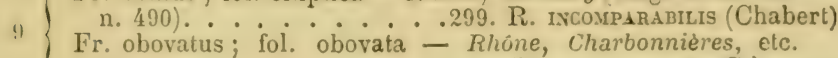

Fr. obovatus; fol. obovata - Rhône, Charbonnieres, etc.

(Chabert). . . . . . 300. R. Cartilagivosa Gdgr mss

10 Fol. subtus ad nervos villosa. . . . . . . . . . 11

(Styli in columnam rectam producti . . . . . . 12

11 Styli in columnam tortuoso-replicatam producti $R$ hône, Charhonnieres (Chab.).....301. R. streptogrya Gdgr mss

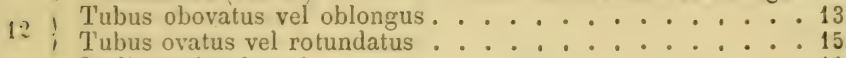

Styli omnino lanati ............ 14

13 Styli basi tantum subhirsuti - Rhone, Tassm (Chaljert).302.

$14\left\{\begin{array}{l}\text { Fol. elliptico-subobovata - Vallesia, Joux brulle (Thomas). } \\ \text { Fol. amplissime oblonga - Rhône, Tassin (Gdgr herb. ros. }\end{array}\right.$ n. 482) . . . . . . 304. R. surnномвоеA Gdgr mss

Discus subplanus; tubus subglobosus - Tolhynia, ete.305.

$15\left\{\begin{array}{l}\text { Discus valde conicus; tubus ovatus - Volhynia, etc. } 306 \text {. R. } \\ \text { R.) }\end{array}\right.$

16 Fr. rotundatus vel globosus $\because \ldots$ Wolfgavgrana (Bess.)

6 Fr. ovatus vel obovatus . $: \ldots 18$

Pet. lanati; tubus hispidus - Helv. Fribourg.307. R. LADEN-

17 Pet. pilosuli; iubus glaber - Rhöne, Charionnieres (Chabert).

Fol . 308: R. nEMOTA Gdgr ms

Fol. elliptica vel obovata. . . . . . . . . 19

is Fol. rotundata saepius obtusissima . . . . . . 20

Fol. ample oblongo-lanceolata - Rhône, Charbonnières (G. herb. n. 394). . . . 309. R. ABortwi Gdyr mss

Styli toto villosi ; tubus glaber - Europa centr. (Gdgr herb. 19 ros. n. 456 , etc.). Styli inferne villosi ; tubus basi glandul. - Rhone, Grézieu

(Chabert)............. R. INDECORA Gdgr mss

( Pet. villosi ; tubus hispidus . . . . . . . . 21

20 Pet. sparse pilosi ; tubus laevis - Rhone, Charbonnieres

(Chabert) . . . . . 312. R. odovitotricha Gdgr mss Fol. apice dilatata spathulata - Rhine, Dardilly (Chab.). 21 Fol. aequalia orbiculata - Rhone, Charbonnieres (Chab.). .314. R. nugsulata Gdgr mss 


\section{2. - Transitoriae Gdgr Essai, p. 14.}

Styli omnino liberi, glabri raro pilis quibusdam obsiti ; discus interdum longe conicus; corolla magna speciosa rosea aut variegata; fructus nunc fertilis nunc abortivus, coriaceus ; rami erecti rigidi.

1 Discus planus . . . . . . . . . . 2

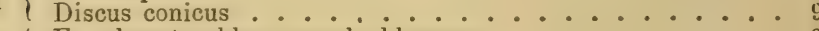

2 Fr. obovato-oblongus vel oblongus ......... 3

$2\{$ Fr. ovatus vel rotundatus. . . . . . . . . 8

3 Fol. ovata vel elliptica.............. 4

Fol. oblonga . . . . . . . . . . 6

(Fol. subtus toto vel ad nervos villosa . . . . . . 5

Fol. undique glaberrima - Ital. or. Faenza (Caldesi).315. R.

Fol. subtus ad costam villosa - Rhône, Tassin (Chabert)

. . . . . 316. R. actinacantha Gdgr mss

Fol. ad nervos villosa; tubus laevis - Rhône, Charbonnieres Rhône, Francheville (Chab.). .318. R. Conyulformis Gdgr mss

(Gemma obovata; fol. subtus toto villosa. . . . . . 7

6 Gemma oblonga ; costa villosa - Rhóne, Dardilly (Chabert).

. 319. R. PSEUDO-ACUTIFORMIS Gdgr msS

( Sep. dorso glandulosa; tubus basi glandulosus - Rhône, $7\left\{\begin{array}{l}\text { Tassin (Chab.) didosa ; tubus laevis - Rhone, Ste-Consorce } \\ \text { Sep. margine glandulos }\end{array}\right.$ (Chab.) . . . . . 321. R. Acutiformis Gdgr mss ( Tubus laevis; fol. elliptica - Rhône, Francheville (Chabert).

8 THa. . . 322. R. opacifolia (Chab.) ubus glandulosus ; fol. orbiculata - Rhône, Charbonnières (Chab.). . . . . 323. R. GLYGYPHYLla Gdgr mss

9 Bracteae nullae vel caducae .......... 10

I Bracteae persistentes. . . . . . . . . . 11

$10 \int$ Fol. ovato-olstusa - Rhône, Charbonnières (Gdgr herb. ros.

10 n. 388)..........324. R. EbRACTEAta Gdgr mss Fol. lanceolata - Cher, Marmagne (Rip.).325. R. NEMoRum (Rip.)

11 Tubus saltem basi hispidus. .......... 12

11 , Tubus omnino laevis. . . . . . . . . . 21

12 Fr. ovatus vel ovoideus. . . . . . . . . 13

12 Fr. obovato-oblongus vel oblongus ........... 14 
Fr. ovoideus; fol. ad costam villosa - Rhône, Charbonnières (Chab.). . . . . . . 326. R. LATEOVATA Gdgr mss

Fr. ovoideus; fol. subtus toto villosa - Rhône, Ste-Consorce

(Boullu) . . . . . . 327. R. LUGDUNENSIS Gdgr mss

Fr. ovatus; fol. subtus toto villosa - Rhóne, Soucieu (Cha-

bert) . . . . . . . 328. R. pRopinqua Gdgr mss

Fol. elliptica .............. 15

f Fol obovata . . . . . . . . . 18

Fol. subtus toto villosa. . . . . . . . . 16

15 Fol. subtus ad costam villosa - Rhone, Dardilly (Chabert).

(....... 329. R. DebILIOR Gdgr mss

Sepala dorso eglandulosa $\ldots \ldots 17$

16 Sep. dorso glandulosa - Rhone, Tassin (Boullu). 330. R.

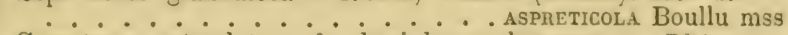

Serraturae rotundatae; fr. basi longe decurrens - Rhône,

17 Tassin (Billot exs. n. 4021) . .331. R. Leiosepala Gdgr mss Serralurae acutae; fr. basi attenuatu : - Rhone, Pont d'Alai (Chab.)....... 332. R. DExTIFERA Gdgr mss

Fol. omnia simpliciter serrata . . . . . . . 19

18 Fol. onnia biserrata. 20 Fol. inaequeserrata - Italia, Modène (Gibelli). 333. R.

( Sep. eglandulosa; nervi subglabri - Rhóne, Francheville

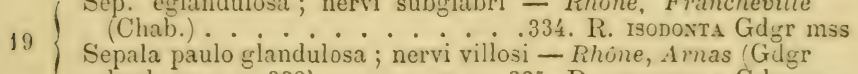
herb. ros. n 338) .....335. R. chlonella Gdgr mss

20 Fol. utrinque subrotunda - Rhone, Tassin (Chabert).336. R. Fol. basi cordata apice retusa - Gard, Massargues (Tuezkiewicz) . . . . . 337. R. CEBENyexsis Gdgr mss

Fol. subtus glabra. . . . . . . . . . . 23

21 Fol. ad nervos villosa; styli glaberrimi 22 Fol. subtus toto villosa; styli paulo hirtelli - Rhöne, SteConsorce . . . . . . 338. R. marabils (Dés.) Corolla pallida; tubus ovatus - Rhône, Chátoux, etc. (G.

2.) I herb. n. 274) . . . . 339. R. Nemocmanus Gdgr mss Corolla rosea ; tubus rotundatus - Rhone, Dardilly (G. herb. ros. n. $4 \$ 3) . . . . . .310$. R. triflora Gdgr miss

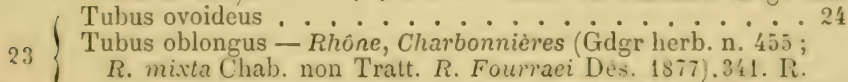
. . . . . . DENDROIDEA Gdgr (1875)

$24\left\{\begin{array}{l}\text { Fol. glaberrima, apice lanceolata - Rhóne, Francheville (G. } \\ \text { herb. n. 61). insuta, ipice acuta - Ital. bor. m. Ballo } \\ \text { Fol. ad costam hirsuta }\end{array}\right.$ (Sprengel) $R$, ambigens et $R$, arvensi-gallica Gremli 343 . (Sprengel) $R$. ambigens et $R$. arvensi-gallica Gremli 343.

\section{3. - Geminatae Gdgr Essai, p. 15.}

\section{Styli liberi semper villosi (nec lanati); corolla ampla}




\section{speciose rosea alba aut variegata interdum velutino-purpu-}

rea.

Fol. undique glaberrima ............. . 2

Fol. subtus ad costam villosa ............ 3

1 Fol. subtus ad nervos villosa................ 11

Fol. subtus omnino villosa. . . . . . . . . . 24

(Discus planus; fr. oblongus - Ital. or. Faenza (Caldesi).

valde conicus; fr. ovoideus - Ital. or. liaenza (Caldesi) . . . . . . . 345. R. AlbIDA Gdgr mss

( 1 Discus conicus .............. 4

I Discus planus . . . . . . . . . . . . . 7

4 Tubus globosus vel ovatus . ........... . . . .

Tubus ovoideus vel obovato-oblongus . . . . . . 6

Styli subglabri ; tubus basi glandulosus - Rhône, Ecully

(Chabert). . . . . . 346. R. ADPRESSA Gdgr mss

Styli pubescentes; tubus in $1 / 2$ inf. glandul. - Croatia, Agram (Vukotinovic). . . . . 347. R. croatica Gdgr mss

Styli dense villosi ; tubus toto glandulosus - Gall. Angers, etc. . . . . . . 348. R. Boraeana (Bèraud)

Pet. aculeati ; fol. subtus glaucescentia - Gall. centr. (R. laevis

6 Boullu) . . . . . 349. R. incarnata (Mill.) Pet. inermes; fol. subtus viridia - Rhône, Charbonniéres (Chab.). . . . . . 350. R. commixta Gdgr mss

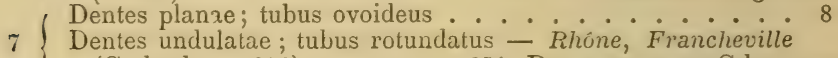
(G. herb. n. 396). . . . 351. R. FLectidenta Gdgr mss

8 I Corolla rubro-velutina; styli villosissimi . . . . . 9

, Corolla rosea; styli parce villosi . . . . . 10

Fol. oblongo-lanceolata; fr. ovoideus - Gall. med.352. R. 9 Fol. obovata ; fr. oblongus - Naples, Ischia (GIRssone). 353 .

Fr. oblongus utrinque longe attenuatus - Ital. apenn. Parme

10 (Gibelli)....... 354. R. ApENnna Gdgr mss Fr. ovoideus vix attenuatus - Rhône, Tassin (Chabert).355.

Fr. globosus ovatus vel ovoideus . R. Pubigera Gdgr mss

. . 12

Fr. oblonuus ..................... 20

Fol. simpliciter serrata . . . . . . . . 13

12 Fol. omnino biserrata ................ 14

Fol. inaeque serrata - Rhône, Francheville (Chabert).356.

. . . . . . . . R. EXIMIA Gdgr mss

$13\left\{\begin{array}{l}\text { Pet. sparse pilosi ; fr. rotundatus - Rhône, Charbonnieres } \\ \text { (Chab.) } \\ \text { Pet. villosi; fr. ovatus - Rhône, Francheville (Chabert).358. }\end{array}\right.$ Pet. villosi; fr. ovatus - Rhône, Francheville (Chabert). 358.

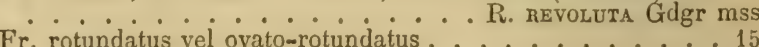

14 Fr. rotundatus vel ovato-rotundatus . . . . . . . 15

Fr. ovoideus . . . . . . . . . 18

15 Pet. aculeati ; fr. laevis vel basi tantum glandulosus . . . 16

Pet. inermes; fr. toto glandulosus ......... 17 
('orolla pallida; fol. rotundala - Europa med. abiger herh. n. 401, 489; Billol n. .378) . . . . 359. R. gemisata (Ran) Cor. amone msea; fol. late rhombon - Iihone, St-lient; (Chabert) . . . . . 360. R. aspenvata Gdgr mss Dentes acutac; fol. oblongo-lanceolata - Rhone, Charbon-

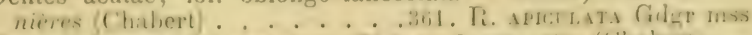

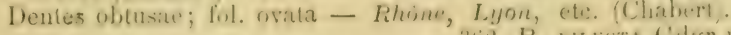

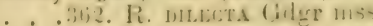

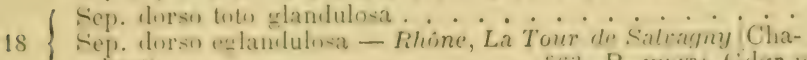

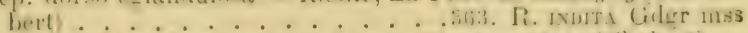
Styli hirkilli; tulus laevis - Rhome, rhatumieres (chaliert)

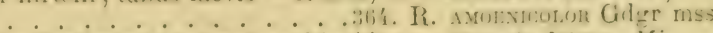
Styli villosissimi; tuhus hasi hispichus - Puy-de-lJome, Mirabelle (Des. herb. ros. n. 51) . . 365. R. amptoximata (Des.)

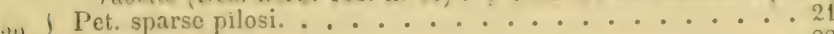

Pet. toto villosi.................... 22

Fol. ovato-acutiuscula - Rhône, Charbonnières (Cliabert)

:1 . . . . 366. R. intentexta Gdgr mss Fol. amplissime oblongo-lanceolata - Rhône, Francheville, (Chabert) ........ . 367. R. ELATA Gdgr mss Fol. obovato-oblonga vel oblonga... Fol. ovata ; tubus toto glandulosus - Rhône, Lyon, etc. 368.

Fol obovata; tubus basi tantum glandulosus - Rhône, La Tour (Chab.). . . 369. R. Gyvocrates Gdgr miss Fr. ovalus toto glandulosus - Rhóne, Charbonniẻres (Gdgr

.. I herb. ros.n. 407). . . . . .370. R. numess (Chah). Ir. obovato-ol,longus, in $1 / 2$ inf. clandulosus - Iihine, Tas$\sin$ (Chabert). . . . .71. R. nigiditsctla Gidgr miss

I Fr. globosus vel rotundatus. . . . . . . . 25

24 Fr. ovatus ovoideus vel oblongus. . . . . . . 27

Tubus laevis vel basi tantum hispidus. . . . . . 26

25 Tubus toto hispidus - Bade (R. agrestis Gm. fl. bad.-als. II, p. 406). . - . . 372. R. BADExsIs Gdgr mss Sep. brevia; tubus basi hispidus - Europa centr. (Gdgr

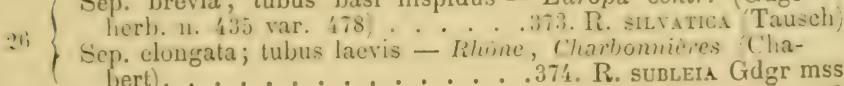
bert). . . . . . . . 374. R. subleia Gdgr mss

Discus planus. . . . . . . . . . . 28

Discus conicus. . . . . . . . . . . . . 31

Tubus toto glandulosus. . . . . . . . 29

Tubus basi tantum glandulosus. ...................... 30 Tubus toto lacvis - Angl. Yorkshire, Engleton.375. R. PUL-

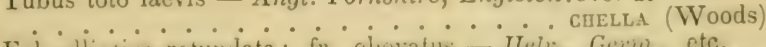

Fol. ellipuro-rotumdata; fr. obovatus - Ireix., Germ., ete.

Fol. obovata; fr. oblongus; petala ciliata - Contia, Agram

(Vukotinovic). . . . . . 377. R. sLavica Gdgr mss

Fol. obovato-oblonga; fr. oblongus; pet. eciliata - Pedemont.

Vallées Vaudoises (Rostan). . . 378. R. Rosta.si Gdgr mss

30 Fr. oblongus; fol. ovata - Germ., Rhöne (Gdgr herb. ros. n. 466)............... R79. Wuldexowil (Tratt.) 
Fr. et folia obovata - Rhône, Dardilly (Chabert; Gdgr berb.

30 n. 209) . . . 380 R. LAureola Gdgr mss Fr. et folia oblonga - Pedemontium, Vallées Vaudoises (Ros-

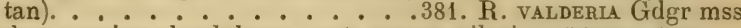
Tubus omnino glandulosus; pet. sparse pilosi - Rhône, Tas$\sin$ (Chabert). . . . . 382. R. BICHROophylla Gdgr mss

Tnbus toto glandulosus; pet. villosi - Rhône, St Genis (Chabert). . . . . . . 383. R. ATroviridis Gdgr mss

Tubus laevis vel basi tantum glandulosus. . . . . . 32

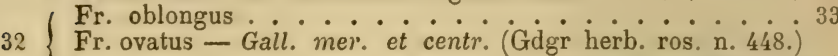

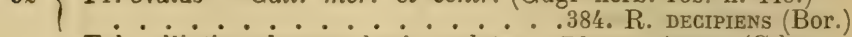
Fol. elliptico-obovata basi cordata - Rhône, Arnas (Gdgr

33 herb. ros. n. 66). . . . . 385. R. ARNASSENSIS Gdgr Fol. ample oblonga, basi sensim attenuata - Rhône, Fancheville (Chab.). . . . 386. R. transmutata Gdgr mss

\section{4. - Gallicanae Gdgr Essai p. 15.}

Styli liberi albo-lanati; corolla speciosissima atropurpureo-relutina vel maculis albis punctata interdum pallida ; sepala post anthesim saepe usque ad colorationem fructus persistentia, tandem decidua magis pinnata quam in 3 sectınibus praecedentibus; radix longissime reptans. - Suffructices humiliores magis rigidi et setiferi floribus solitariis saepe instructi.

( Fol. subtus ad costam mediam villosa. . . . . . . . 2

Fol. subtus ad nervos villosa. . . . . . . . . . 8

Fol. subtus toto villosa. . . . . . . . . . 37

2 Petioli sparse pilosi . . . . . . . . . . . 3

Petioli minute villosi. . . . . . . . . . . . 6

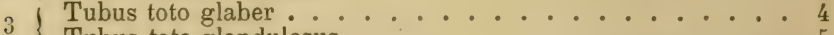

Tubus toto glandulosus . $\ldots$

Fr. oblongus; fol. ovata - Bohemia, Prague.387. R. Fulgens

4 Fr. ovoideus ; fol obovato-oblonga - Rhône, Charbonnières

(Chabert). . . . . 388. R. subglabrescens Gdgr mss

(Fr. ovoideus - Rhône, Francheville (Chabert).389. R. AEQUA

Fr. subglobosus - Rhône, Charbonnières (Chabert).390. R. NOTATA Gdgr mss 
Fr. rotundatus - Cher, Bourges, ete. 391. R. ntritis (I)és.).

Fr. ovatus; ful. elliptica - Rossia mer., Caucasus, I'ersia luror.

Fr, ovoideus; fol oblonga - Rhóne, etc.393. R. Allanthifo-

Fr. oblongus ful subolilonga - Italia, Florence (Areanceli)

. . . . 39't. R. Flonextrisa Gilgr mas

7 Fr. Globosus vel rotundatus. . . . . . . . .

7 Fr. ovatus olovalus vel oblongus. . . . . . . . l'

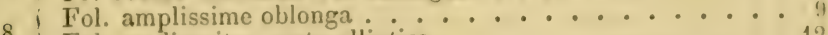

8 , Fol. mediocriter ovato-elliptica .......... 12

9 Fol apico rotundata................ 10

I Fol apice acuta ................... 11

Sep. dorso glandulosa; discus planus - Rhone, Dardilly

10 Minime; discus sat conicus - Rhone, Charbonnieres (Ozanon)

Folovata discus subplanus- Rhe cine, Francherille (Pu m*s Fol. oblonga; discus conicus - Gall. Lyon; Rh. Amas (Gdgr)

iscus subconicus; auriculae stipul. R. INTEgriuscula Gdgr mss

$12\left\{\begin{array}{l}\text { Discus subconicus; } \\ \text { Discus planus ; auriculae divaricatae }- \text { Rhóne, Charbonnieres }\end{array}\right.$ (Chab.). . . . . 399. R. HYPomelayocanpa Gdgr mss Pet. villosi; tubus hispidus - Helv. Fribourg.400. R. VARI

$13\left\{\begin{array}{l}\text { Pet. sparse pilosi ; tubus laevis - Gall. Lyon (Gdgr herb. } \\ \text { ros. n. 469). }\end{array}\right.$ ros. n. 469). . . . . . .401. R. RHodAsi (Chab.)

4 Pet plerique sparse pilosi . . . . . . . 15

Pet. minute villosi. . . . . . . . . 20

15 Fr. obovato-oblongus vel oblongus . . . . . 16

15 Fr. ovatus vel ovoideus. . . . . . . . 17

Discus conicus; fr. oblongus - Rhône, Charbonnières (Cha-

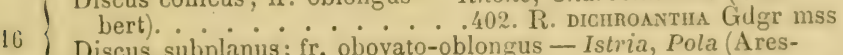

Discus subplanus; fr. obovato-oblongus - Istria, Pola (Areschoug). . . . . . 403. R. platysepala Gdgr mss

17 Fol. sat parva elliptica. . . . . . . . 18

17 Fol amplissime obovata vel oblonga. Charbonnières (Gdgr 19

18 herb. n. 468) . . . 404. R. velutivaeflona (Ozanon) Dentes compositae; fr. obovatus - Rhône, Tassin, etc. 405.

........... R. Densinamea Gdgr mss

Petala eciliata; discus planus - Rhône, Dardilly (Chabert).

Petala ciliata; discus conicus - Rhône, Charbonnieres Chr mss bert). . . . . . 407. R. Diospynos Gdgr mss

20 Tubus laevissimus. . . . . . . . . 21 Tubus saltem inferne glandulosus . . . . . . . 23

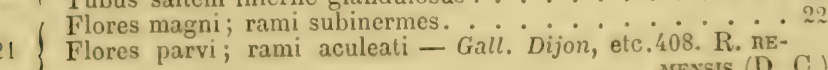

21 Flores parvi, rami aculeat - Gassis (D. C.) Dentes compositac; tubus obovatus - Gall. Orléans (G. herb.

$? 2$ n. 470 ; Dés. n. 41). . . - .409. R. Anenivaga (Jullien) herb. ros. n. 477). . . : 410 . R. subINERM! (Chab.) 
23 Fol. apice rotundata vel obtusa. . . . . . . 24

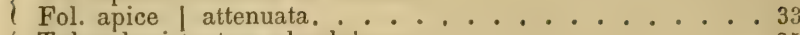

24 Tubus basi tantum glandulosus . . . . . . . 25

24 Tubus omnino glandulosus . . . . . . . 26 Coroll $\iota$ amœne rosea; fol. biserrata - Europa med--centr.

25 (Willd. !) . . . . . 411. R. PARVIFOLIA (Ehrh) Corolla pallide rosea ; fol. simpliciter serrata - Rhone, Charbonnières (Billot n. 3718). .412. R. PSEUDO-RURAIIS Gdgr mss

26 Fol. oblonga vel suboblonga. . . . . . . . . 27

Fol. ovata vel obovata. 28

Discus conicus; fol basi sensim attenuata - Rhône, Char-

27 bonnières (Chab.) ....413. R. Polyadena Gdgr mss Discus planus; fol. basi cordata - Phône, Charbonnières (Chabert) ...... . . . R14. RHomboidalis Gdgr mss

$28\left\{\begin{array}{l}\text { Fol. obovata } \\ \text { Fol ovata vel elliptica } \ldots \ldots\end{array}\right.$

( Petala eciliata; fol. apice plerumque obtusa . . . . . . 30

29 Petala ciliata; fol. apice subacuta - Rhône, Charbonnières (Chabert)..... 415. R. Excogrtata Gdgr mss Discus planus; aculei adunci nulli - Rhone, Charbonnières

$30\{$ (Chab.) ........... R16. Macrosepala Gdgr mss Discus conicus; aculei adunci sat copiosi - Ital. or. Faenza (Caldesi)........417. R. stenodendron Gdgr mss

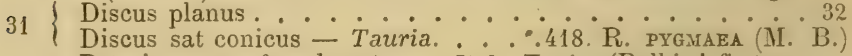
Pet. inermes; fr. obovatus - Ital. Turin (Balbis! fl. taur. $32\left\{\begin{array}{l}\text { p. } 81 \text { ). } \\ \text { Pet. subaculeati; fr. obovato-oblongus }- \text { Rhône, Charbon- }\end{array}\right.$ nières (Chab.). . . 420. R. attenuatocalyx Grlgr mss

$33\left\{\begin{array}{l}\text { Discus conicus; fr. ovoideus. } \text { Discus subplanus; fr. obovato-oblongus - Rhône, Charbon- } \\ \text { - }\end{array}\right.$ nières (Chab.). . . . . 421. R. Concessa Gdgr mss

34 Gemma ovata vel ovoidea. . . . . . . . . . 35

Gemma oblonga. . . . . . . . . . . 36

Petala paulo ciliata; fol. superne simpliciter serrata - Croa-

$35\{$ tia, Agram (Vukot.). : : .422. R. Vukotinorici Gdgr mss Petala eciliata; fol. omnia biserrata - Rhône, Tassin (Chabert). . . . . . 423. R. BETUlina Gdgr mss

( Ped. 20-25 m. 1.; discus planus - Camiolia Cilli (Studniczka) $36\left\{\begin{array}{l}\text { Ped. } 1_{t}-16 \text { m. i.; discus conicus - Ital. or. Faenza (Caldesi) } \\ \text { Pass }\end{array}\right.$

Fr. ovatus ovoideus vel oblongus

$37\left\{\begin{array}{l}\text { Fr. ovatus ovoideus vel oblongus } \ldots . \cdots 38 \\ \text { Fr. globosus vel rotundatus }\end{array}\right.$

38 Tubus laevis vel glandulosus in 1/3 inf. . . . . . . 39

Tubus toto glandulosus . . . . . . . . . 4 41

Fol. ovato-elliptica ............. . . 40

Fol. ample oblonga; tubus laevis - Navarre, Caparroso

39 (Ruiz). . . . 426. R. Fusifonmis Gdgr mss

Fol. obovato-oblonga; tubus basi glandulosus - Rh. Francheville (Chab.). . . .427. R. cuspididenta Gdgr mss

$40\left\{\begin{array}{l}\text { Pet. inermes; petala coiliata - Hisp. Pamplune (Lacoizqueta) } \\ \text { Pet. aculeati ; petala ciliata - Rhône, Tassin (Cariot).429. }\end{array}\right.$ 
41 Tubus ovatus vel ovoideus' ...... 42

41 Tubus obovato-oblongus vel oblongus . . . . . . . 54

4: Flores albo-punctati . . . . . . . . . 43

Flores rosei. ...................44

Caulis pet. aculeati ; discus pi bescens - Iihone, Charbon-

4. nières (Gdgr herb. ros. n. 434; Soc. dauph. n. 771. - R.

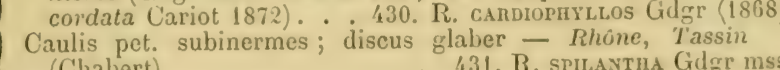
(Chabert) . . . . . 431. R. spilavtha Gdgr mss

Fol. ovata vel elliptica. . . . . . . . . 45

4 t Fol obovato-oblonga vel oblonga. . . . . . 48

45 Tubus glaber vel in $1 / 3$ parte inf. glandulosus $\ldots . . .447$

Tubus laevis; petala pubescentia - Valais (Hoppe exs. n. 78 ).

4 (j) . . . . . 432. R. Holosenicea (Ser.) Tubus inferne glandulosus; petala glabra - Bohemia. 433.

Fr. ovoideus ; fol. late ovala - Gallopiorincia, Le lne

(Champ.).....434. R. Cirampagaeuxu Gdgr mss

Fr. ovatus vel rotundatus; fol. mediocriter ovato-elliptica Europa med.; Caucasus; Libanus (Glgr herb. ros. n. 438 cum var. n. 411, 436, 437, '414 et 4'15; Rchb n. 2250).425.

Noті. - Species secjuentes hibridae dictac ad alias sectionepertinent, nempe $\quad R$. gallieo $\times$ biserrata, gallico $\times$ dumetorum, gallico $\times$ dumetoro $\times$ obtusifo!ia, gallico $\times$ lutetiana, gallico $\times$ mollissima et gallien $\times$ tomentosa (in Christ Rosen der. Schw. indicatae).

Tubus omnino glandulosus ........... 49

\&s $\{$ Tubus basi tantum glandulosus - Rhône, Denicé (Ġgr) 43 Fol. obovata vel obovato-oblonga $\cdot 43 \dot{R}^{\circ}$ Condifolia (IIost) Fol. rotundata - Austria; Hung. . .437. R. Condifolia (Ilost)

io ( Pet. inermes - Rhône, Brouilly (Gdgr) 438. R. Pustuliferi

Fol basi cordata . . . . . 5 ?

51 Fol. basi sensim attenuata - Rhone, Charbonnières (Chabert).

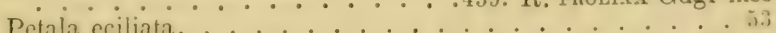

52) Petala ciliata - Hungaria, Presburg (Schneller). 440. R.

1................. Trichopetala Gdgr mss Discus conicus ; nervi glandulosi - Ital. or. Faenza (Caldesi)

53 . . . . 441. R. Adniatica Gdgr mss Discus planus ; nervi eglandulosi - Rhone, Dardilly (Chabert).. . . . . . 442, R. echisavtia Gdgr mss Minime. . . . . . . . . . 55

i4 Flores valde corymboso-viscosi - Oriens? (non vidi). 443 .

\} . . R. Damascena (Mill.) Fol. ovato-elliptica vel oblonga ......... . . J6

5.) Fol obovata . . . . . . . . . 58

Fol. amplissime oblonga; discus $\mid$ conicus . . . . . . 57

j6) Fol. ovato-elliptica; discus planus - Rhône, Charbonnières , (Chabert).......444. R. LATICAlix Gdgr mss 
( Nervi glandulosi ; discus conicus - Rhône, Dardilly (Cha$57\left\{\begin{array}{l}\text { bert) } \\ \text { Minime ; discus paulo conicus - Silesia, Breslau (Ansorge). }\end{array}\right.$

58 Petala ciliata $\ldots . . .446$. R. silesiaca Gdgr mss

Petala eciliata. . . . 60

$59\left\{\begin{array}{l}\text { Gemma obovata; fr. depressus - Rhône, Charbonnières } \\ \text { (Chabert). } ; \text { fr. longe attenuatus - Bohemia, Prague }\end{array}\right.$ Gemma oblonga ; fr. longe attenuatus - Bohemia, Prague

(Polak). . . ....448. R. BонEMica Gdgr mss

Fr. ovoideus ; fol. basi sensim attenuata - Rhône, Franche-

$60\left\{\begin{array}{l}\text { ville (Chab.) } \\ \text { Fr. ohlongus; fol. basi cordata - Hung. Csepel (Feichtinger) }\end{array}\right.$

Tubus inferne tantum glandulosus ........... CRIBROSA Gdgr

$61\left\{\begin{array}{l}\text { Tubus toto glandulosus } \ldots \ldots \\ 0\end{array}\right.$

Tubus villosus haud aut vix glandulosus - Europa med? . . . . . . 451. R. BELGICA (Mill.)

62 Sepala dorso glandulosa .

Minime - Rhône, St-Genis (Chabert).452. R. Festiva Gdgr mss

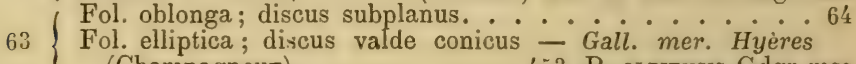

(Champagneux). . . 453. R. olBIENSIS Gdgr mss

Tubus aculeatus, rotundato-campanulatus - Alger, Birman-

$64\left\{\begin{array}{l}\text { dreis (Gdgr Flora Alger. exs. n. 704).454. R. MacrosphaERa } \\ \text { Tubus glandulosus, rotundatus - Rhóne, Charbonnieres }\end{array}\right.$

(Chabert) . . . 455. R. ERYTHROPHORA Gdgr mss

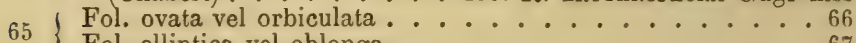

Fol. elliptica vel oblonga. . . . . . . . 67

Costa glandulosa; pet. subaculeati - Rhône, Francheville

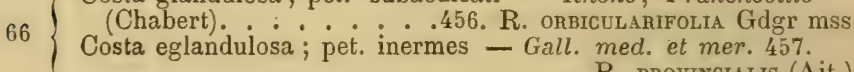

. . . . . . . . R. Provincialis (Ait.)

Fol. omnia ovato-elliptica - Eur. med. Gdgr herb. n. 403

$67\left\{\begin{array}{l}\text { et } 418 \text { var.) } \\ \text { Fol. nunc elliptica nunc oblonga - Rhone, Charbonnières }\end{array}\right.$

(Chabert). .... . 459. R. Dimorphophylla Gdgr mss

Subgenus III. - Scheutzia Gdgr Essai, p. 16. -

Cinnamomeae DC. in Ser. Mus. helv. (1818) I, p. 2.

Styli liberi sessiles villoso-lanati; discus rarissime conicus; flores albi vel rosei amplissime bracteati; fructus praecox coriaceus vel subpulposus, erectus vel arcuatus purpureus, glaber vel glandulosus; sepala eglandulosa necne integer- 
rima, post anthesim erecta conniventia et persistentia; pedunculi glabri villosi vel glandulosi ; folia imparipinnata ; foliola molliter plerumque pubescentia eglandulosa et simpliciter serrata, decidua; stipulae superiores valde dilatatae, glaucescentes denticuiatae; aculei stipulares recti adunci interdumque subulati; radix reptans. - Frutices vel sufirutices plerumque regionem borealem totius Veteris Orbis incolentes, ubi parum frequentes, parvi subflexuosi, ramis gracilibus conspicue bruneo-rubentibus gaudentes.

Genus hocce dedicatum est am. J.-N. Scheutz, suecico, de scientia rhodologica optime merito.

( Ped. glaberrimi . . . . . . . . . . 2

1 Ped. villosi vel glandulosi . . . . . . . . . 43

2 Fol. undique pubescentia. . . . . . . . . 3

2 Fol. glabra vel subtus tantum villosa . . . . . . 26

3 Fr. globosus vel turbinatus. ........... 4

3 Fr. ovatus ovoideus vel oblongus .......... 19

4 Pet. aculeati ............... 5

Pet. omnino inermes. . . . . . . . . 8

5 Fol. elliptica vel oblonga . . . . . . . . 6

Fol. ovato-obtusa - Scandinavia. 460. Scheutzia cinerea (Sw.) Styli lanati; flores corymbosi.

6 Styli subglabri; flores geminati - Tauria, Astrakhan.461.

. . . . . . . . Scheutzia taurica (M B.) Fol. basi rotundata; sep. apice dilatata - Europa bor., med.

7 Asia bor. . . . . . . 462. S. Cinvamomea (L.) Fol. basi truncata; sep. apice linearia - Rossia Wolgae inf. Sarepta (Becker) . . . 463. S. Desentonum Gdgr mss

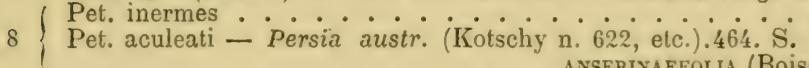

Tubus turbinatus $\ldots \ldots$ ANserlvaefola (Boiss.)

9 Tubus globosus Fol. ovata; pet. aculeati - Europa bor.465. S. Foecuxdissina

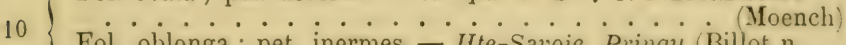
Fol. oblonga ; pet. inermes - Ite-Savoie, Pringy (Billot n. 3845). … 466. S. Mollifolia Gdgr mss Sep. omnino eglandulosa. . . . . . . . . 12

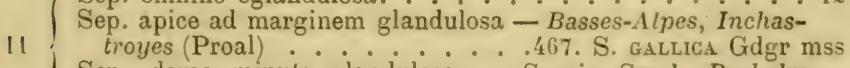
Sep. dorso minute glandulosa - Suecia Smol. Backaby (Scheutz) . . . . . 468. S. AdENosepala Gdgr mss

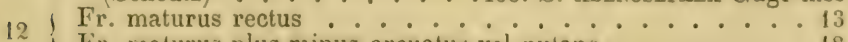

2 Fr. maturus plus minus arcuatus vel nutans . . . . 18 
Rami flor. inermes; discus planus .......... 14 Rami flor. aculeati ; discus subconicus - Silesia (Weichler).

S . . . . . 469. S. PEROBLONGA Gdgr mss Stip. virides; fr. rectus .......... 15

Stip. sat purpureae; fr. rectus - Suecia, Ostrog. Wardsberg

(Dahlgren). . . . . . 470. S. G0THICA Gdgr mss Stip. purpureae; fr. subflexuosus - Suecia, Scania (Tullberg).

. . . . . . 471. S. TOMENTELla Gdgr mss

15 Ped. 3 ; rami flor. aculeati - Suecia, Gotland Barlingbo 1

(Lindman) ....... . 472. S. TrIfLORA Gdgr mss $16\left\{\begin{array}{l}\text { Sep. apice integra } \\ \text { Sep. apice dentata - Suecia, Dalecarl. Silpberg (Hagerstrom) }\end{array}\right.$

. . . . . . . 473. S. DALECARLICA Gdgr msS

Dentes convergentes ; fr. rotundatus - Harz, Steigerthal

17 (Vocke) . . . . . . . S74. Hercynica Gdgr mss

Dentes apertae; fr. globosus - Rossia mer. Tscherkask (Laupmann). . . . 475. S. BORYsTHENICA Gdgr msS

Fol. utrinque rotundata; stip. virentes - Suecia, Hall. Kristinehed (Lundquist). . . . 476. S. CREMOcepHaLA Gdgr mss

Fol. apice rotundata basi sensim attenuata; stip. rubrae Suecia, Smol. Wrigstad (Johansson).477. S. suecica Gdgr mss

Fol. apice obtusa basi valde cuneata; stip. virentes - Suecia Ostrog. Wardsberg (Dahlgren). .478. S. CunEIFolia Gdgr mss

Fol. apice acutiuscula basi truncata; stip. virentes - Valais, Zermatt (Jaccard) . . . . . . 479. S. ALPINA Gdgr mss

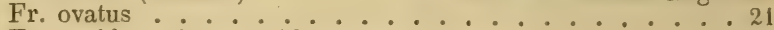

Fr, ovoideus obovato-oblongus vel oblongus . . . . 20

Fr. obovato-oblongus; fol. basi sensim rotundata - Rossia

Wolga inf. Sarepta (Becker) .480. S. Astrachanica Gdgr mss

Fr. ovoideus; fol. basi cuneata - Rossia, Astrakhan, Sarepta

(Becker). . . . . . . 481. S. WolgensIs Gdgr mss

Fr. oblongus; fol. simpliciter serrata - Ross. Astrakhan, Sarepta (Becker). . . . . 482. S. Beckeri Gdgr mss

Fr. oblongus; fol. biserrata - Rossia Astrakhan, Sarepta

(Becker). . . . . . 483. S. AdENodontos Gdgr mss

Fol. oblonga . . . . . . . . . 22

Fol. ovato-elliptica vel obtusa . . . . . . . 25

Sep. paulo glandulosa, integerrima . . . . . . . . 23

22 Sep. eglandulosa, integerrima . . . . . . 24

Sep. dorso eglandulosa, paulo pinnata - Gall. Metz.484. S.

. . . . . . . obcuneata Gdgr mss

Discus planus; sep. dorso glandulosa - Bavaria, Bartolzheim

2.3 (Dumoulin). . . . . 485. S. NERvosa Gdgr msS Discus subconicus; sep. margine glandulosa - Finlandia, Abo (Hollmén). . . - 486. S. Fennica Gdgr mss

$24\left\{\begin{array}{l}\text { Sep. glabra; stip villosa; fol. basi rotundata - Puy-de- } \\ \text { Dôme, Pont de Naud (Lacroze).487. S. Arvernensis Gdgr mss }\end{array}\right.$ Sep. villosa; stip. glabra; fol. basi attenuata - Sibiria, Irkutzk (Augustinowicz). . . .488. S. IRcutiana Gdgr mss Fol. ovato-obtusa; sep. extus viridia - Germ. bor.489. S. 25 Fol. ovato-elliptica; sep. extus rubra - Vallesia Helv. StNicolas (Vuitel) . . . . 490. S. HYPOLEUCA Gdgr mss 
. Fol. subtus toto villosa.

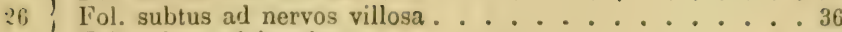

Fol. subtus glaberrima. ........... . . 39

27 Tubus ( $(\mathbf{r}$.) globosus vel turbinatus . . . . . . . . 28

27, Tubus (fr.) ovatus ovoideus vel oblongus . . . . . . 33

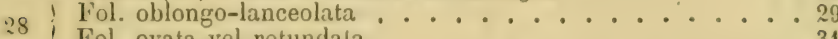

8 i Fol. ovata vel rotundala. . . . . . . . . . 31

Fol. simpliciter serrata. . . . . . . . . 30

29 Fol. inaeque vel hiserrata - Rossia mer.491. S. Gonkikns

Pet. aculeati ; tubus globosus - Dania; Scand. 492 . S.

30 Pet. inermes; tubus globoso-depressu . . MaJalis (Herm.) .......... 493. S. GeMella (Willd.)

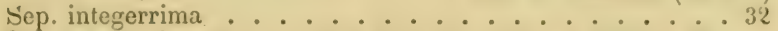

31 Sep. paulo pinnata - Germ. bor.-occ.; Dania; Suecia.494.

.. S. Fluviatilis (Retz)

Fr. turbinatus - Suecia (Fries herb. norm. VIII, n. 46 A.).

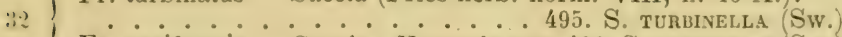

Fr. pyriformis - Suecia; Norvegia mer.496. S. PYRIFonMIS (Sw.)

33 Caulis inermis vel subinermis . . . . . . . 34

Caulis aculeatus . . . . . . . . . 35

Fol. late ovato-oblonga ; pet. inermes - Dania (Fr. herb. ViI).

it . . . . . . 497. S. mutica (Fl. dan.) Fol. parva ovata; pet. aculeati - Germ. alp. Salzbourg (Hoppe exs.) . . . . . . . 498. S. HoppII Gdgr mss Aculeı dilatati ; cor. saturate rosea - Baleares, Iviça (Gdgr herb. ros. n. 56) . . . . 499. S. IVICENsIS Gdgr mss . . . . . . . . S. Moravensis Gdgr mss Aculei sparsi haud setacei ............ . . 37

36 Aculei copiosi aciculati - Sibiria orient. et subspont.501. S. T. . . . . . . *amrschatica (Vent.) Tubus haud attenuatus; sep. integra ........ 38

$: 3$ Tubus attenuatus; sep. pinnata - Subspont. (ex America bor.)

. . . . . . , 502. S. Fastuosa (Tratt.)

Fr. globosus; fol. oblongo-lanccolata - Subspont. (ex Amer.

is bor.) . . . . . . . 503. S. Solandr (Tratt.) Fr. ovoideus; fol. elliptica - Rossia (Steudel); Oural med. (Clerc). . . . . . . . 504. S. Fischenuana (Bess.) Fr. globosus vel ovatus; cor alba vel saturate rosea . . 40

39 Fr. obovato-oblongus; cor. pallide rosea - Ross. bor. Slatoust, Oural ..... 505. S. GLabrifolia (C. A. Mey.) Cor, saturate rosea; fol. subtus eglandulosa . . . . . 41

40 Cor. alba; fol. paulo glandulosa-Corsica, Sicilia et Graecia mont. . . . . . . . . 506. S. sicula (Tratt.) Stip. dentatae; pet. inermes - Germ. occ. (Wirtg. exs. n.

41 464); Gall. or. . . . . 507. S. FrAxLvifolia (Borkh.) \{ Stip. fimbriatac; pet. aculeati - Subspont. (Insulae Brit.?).

. . . . 508. S. Woods (Lindl.)

Fol. undique villosa $\ldots \ldots . . . .44$

43 Fol. subtus tantum villosa . . . . . . 54

Fol. glabra .................... 54 
Tubus ovoideus ..................... 45

44 Tubus oblongus . . . . . 47

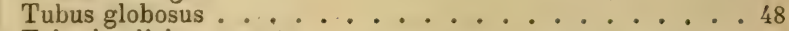

Fol. simpliciler serrata . . . . . . . . . 46

Fol. inaeque serrata; sep. eglandulosa-Ross. Astrakhan,

45 Sarepta (Becker) ..... 509. S. Ledebourdana Gdgr mss

Fol. biserrata ; sep. extus glandulosa - Ross. Azoff, Tscherkask (Laupmann). . . . . 510. S. BISERrata Gdgr mss

Ped. villosi ; tubus laevis - S.-Petersbourg (Meinshausen fl. Ingr. exs. n. 217). . . . . 511. S. INGricA Gdgr mss

Ped. minutissime glandulosi ; tubus laevis - Sibiria, Ussuri

Kengka (Maack). . . . . .512. S. sibIRICA Gdgr mss

Ped. hispidi ; tubus basi aculeolatus - Ross. Astrakhan, Sarepta (Becker). . . . . 513, S. SAREptana Gdgr mss Fol. biserrata ; tubus hispidus - Graecia, etc.514. S. OrPHA-

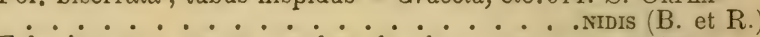

Fol. inaeque serrata; tubus laevis - Ross. Astrakhan, Sarepta (Becker) . . . . . 515. S. PEndula Gdgr mss Fol. simpliciter serrata ; tubus laevis - Persia bor.516. S.

Pitis (B. el Bushe)

Ped, villosi nec glandulosi glandulosi . . . . . 59

Rami floriferi glabri. . . . . . . . . . 50

49 Rami flor. velutino-sericei - Asia, Bagdad, Babylonia. 517.

Fol. orbiculato-obtusissima. $\therefore$. S. Assyraca Gdgr mss

50 Fol. ovata vel oblonga. . . . . . . . 52

(Dentes compositae; sep. integra - Sicilia; Graecia ; Libanus.

Dentes simplices; sep. pinnata - Naples, $m$. Vésuve (Pasquale). . . . . . . 519. S. vesuviana Gdgr Cor. alba ; fol. biserrata ovata - Persia, Affghania.520. S.

Cor. alba; fol. biserrata obovata - Affghania mont. (Stocks)

C......520 a. Stocksiana Gdgr mss

Cor. rubra; fol. simpliciter serrata - Batavia, Apeldoorn (Ankersmit). . . . . . . 521. S. Batavica Gdgr mss Tubus hispidus; fol. subrotundata - Asia occid. (Kotschy n. 343,539 , etc.) $\ldots . . .522$. S. ORIENTALIS (Dup.)

Tubus laevis; fol. obovato-oblonga - Rossia, Dorpat (Girg.

fl. ross. exs. n. 252) . . . 523. S. GirgensoHnI Gdgr mss

Fol. subtus ad nervos villosa . . . . . . . . 55

54 Fol. subtus toto villosa. . . . . . . 57

Fol. glaberrima - Subspont. (ex America bor.). 524 . S. Lucida

......................... Ehrh)

Tubus globosus; pet. inermes $\ldots \ldots 56$

55 Tubus turbinatus; pet. aculeati - Germ. bor.525. S. Franco-

. . . . . . . . . Furtana (Borkh.) Ped. omnino glandulosi - Germ. bor. Rostock; Russia occ.

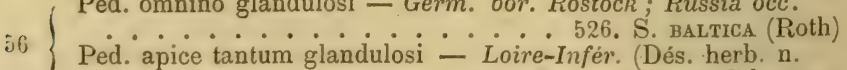

46); Batavia occid.. . . . 527. S. occidentalis Gdgr mss

Fol. subtus eglandulosa; aculei parvi . . . . . . . 58

57 Fol. subtus glandulosa; aculei robusti - Tauria; Caucasus. 
Ped, et sep. glandulosa - Subspont. (ex America bor.). 529 .

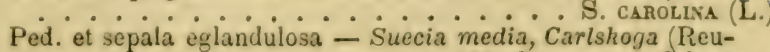
terman) ....... 530. S. Elegans Gdgr mss

Subgenus IV. - Laggeria Gdgr Essai, p. 18; Eglanteriae DC. in Ser. Mus. helv. (1818) I, p. 2.

Styli liberi villosi vel lanati ultra discum breviter capitati; flores ebracteati, lutei vel sulphurei interdum intus flavopurpurei, cimiciodori vel foetidi; fructus sat praecox, coriareus coccineus, erectus; sepala plus minus glandulosa, post anthesim reflexa tandem decidua, integra vel pinnata; pedunculi glabri villosi vel hispidi; folia 3-ò-foliolata, subtus ad nervos glandulosa parce rillosa, argute biserrato-glandulosa ; stipulae conformes, sat angustae, margine glandulosae ; aculei sparsi setacei vel rectiusculi. - Frutices praesertim orientales, aut in Europa temperata rarissime vel vix sponte crescentes, erecti graciles sociales, ramorum cortex saepius bruneus. - Radix plerumque ignota ; in L. eglanteria (L.) et speciebus affinibus subrepens.

Hocce dicavimus Beat. Dr Lagger in Botanica et Rodologia clarissimo.

Ped. laeves . . . . . . . . . . . . 2

Ped. villosi vel glandulosi $\ldots \ldots$

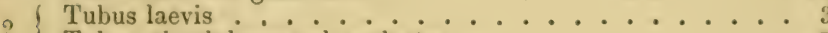

2 Tubus glandulosus vel aculeatus $\ldots 7$

3 Flores omnino lutei .............. 4

3 Flores intus rubri extus flavi . . . . . . . . . 6

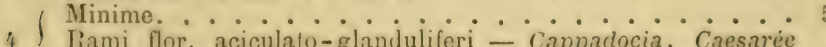

4 Iami flor. aciculato-glanduliferi - Cappadocia, Caesarée (Balansa exs. an. 1856) . 531. Laggeria caesarea (B. et B.)

Tubus globosus; fol. ovato-rotundata - Oriens; subspont. 
Fol. ovato-rotundata - Colitur (ex Oriente).534. L. PUNICEA

6 Fol. obovata - Saxonia (Krieger). 535. L. Dichroopertala

Fol. elliptico-lanceolata - Rhóne, Bully subsp. (Gdgr) Gdgr mss

Tubus apice tantum glandulosus ... L. IGNESGENS Gdgr mss

Fol, ad nervos villosa ; pet. aculeati Fol. ad costam villosa; pet. inermes - Valais, Branson (Gdgr herb. n. 365) …538. L. CHRYsocepHaLA Gdgr mss Flores omnino sulfurei . . . . . . . . . 10 Flores omnino luteo-aurei - Rossia mer. Novo-Tscherkask

9 (Laupmann).. . . . . . . . 539. L. cHersoneNsIS Gdgr mss Flores extus lutei intus rubri - Colit. ex Asia occid. 540. L. BI...................... . . Jacq.

$101 \ldots \ldots$. . Oriens (auct.; Gdgr herb. ros. n. 27 var.

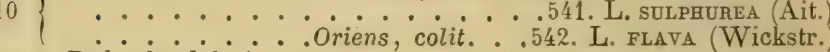

11 Ped. glandulosi . Oriens, colit. 542. L. FLAVA (Wickstr.

11 Ped glandulosi ............. . . .

12 Flores lutei ................... 16

Fl. rubri $\ldots \ldots 15$

13 Fol. glabra biserrata; tubus laevis . . . . . . 14

Fol. rillosa simpliciter serrata; tubus glandulosus - Persia

bor. (Bushe exs. n. 437). . . . . . .543. L. Bungeana (B.)

(Caulis elatus; aculei haud setacei - Europa mer. (rr) Oriens.

14 Caulis nanus ; aculei setacei- Persia (544. L. EGLANTERIA (L.) p. 671). . . 545. L. Yellotiviava II,

Fol. orato-elliptica ad nervos glaudulosa - Germ.; Rossia

15 Fol ovato-cordata eglandulo - 546. L. CAMPANULATA (Ehrh) (. Ovato-cordata eglandulosa - Austria inf. Laxembourg

(Halacsy), etc. ....... 547. L. TURBinata (Ait.) Fol. subtus ad nervos villosa . . . . . . . . . . 17 Fol. ad costam villosa - Rhône, Bully (Gdgr, subspont.).

.......... 548. L. ARGUTIDENTA Gdgr mss Fol. undique villosa; rami hispidi - Phrygia, Ouchak (Bal. exs. n. 1171). . . . . . . 549. L. Rapini (Boiss.) Fr. obovatus ; fol. subtus ad nervos glandulosa - Loiret, Malsherbes (Wuitel); Valais . .550. L. WuITELII Gdgr mss Fr. ovatus, costa sola glandulosa - Helvetia occid. (Dés.) Fr. globosus ; costa sola glandulosa - Italia, Modène (Gibelli)

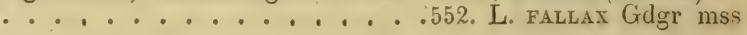


Subgenus V. - Cottetia Gidgr Essai p. 18; Pimpinellifoliecue DC in Ser. Mus. helv. (1818) I, p. 2.

Styli liberi plerumque lanati, ultra discum brevissime capitati; flores latiuscule breviterque bracteati, albi interdum rosei, petala ample cordata eciliata; fructus præcox coriaceus, lucide niger vel atrosanguineus, laevis vel aciculatus , rarius nutans; sepala integra, glandulosa necne, post anthesim erecto-persistentia ; pedunculi laeves aut hispidi; folia imparipinnata, foliola parva glabra vel subhirsuta, subglauca, subtus interdum glandulosa, simpliciter vel biserrata; stipulae dimorphae basi angustiores, ad apicem ample dilatatae; aculei setacei recti subteretes saepissime copiosissimi; radix haud reptans. - Suffrutices humiles erecti sociales, dense foliosi, in Europa media et australi (tunc alpinae vel montanae regionis) solo calcareo passim crescentes, ramis bruneo vel rubro suffultis rigidis ad normam aculeatissimis gaudentes.

Dicavi in honorem D. Cottet presbyteris Friburgensis qui, conatos adhibuit suos ut Rosae Helvetiae occidentalis clarius noscerentur.

( Fol. simpliciter vel inaeque serrata. . . 1. Pimpinellifoliag A Fol. omnino biserrata, subtus interdum glandulosa. 2. Ruвi-

\section{Pimpinellifoliae Gdgr Essai p. 19.}

Foliola simpliciter vel inaeque serrata, subtus ad costam mediam interdum glandulosa necne. 
2 Fol. undique glaberrima ............. 3

$2\{$ Fol. subtus | villosa . . . . . . . . . 44 a

( Pet. glaberrimi ............... 4

3 Pet. hirtelli, glandulosi vel sparse pilosi. . . . 22

4 Pet. omnino inermes. . . . . . . . . . 5

4 Pet. aculeati. . . . . . . . . 16

5 Ped. hispidi. . . . . . . . . . . . . 6

5 I'ed. glabri .................. . . 9

( Ped. dense glandulosi . . . . . . . . . . 7

6 Ped. 2-4-aculeolati - Sabaudia, Annecy (Puget). 553. Cot.............. tetia apposita Gdgr mss

(Sep. eglandulosa. . . . . . . . . . 8

7 Sep. basi paulo glandulosa-Wurtemb. Donnstetten (Kemmier) ......... . 554. C. maculans Gdgr mss

Fol ovato-rotundata; fr. globosus - Europa; Caucas. (Billot n. 1182). ...... . . 555. C. PIMPINELlifolia (L.) Fol. obovata; fr. rotundatus - Manche, Carteret (Lebel).

8 Fol. oblonga; fr globoso-depressus - Bohemia, Prague (Polak).........557. C. BoHemica Gdgr mss Fol. obovato-oblonga; fr. globosus - Hung. Csepel (Tauscher)

. 558. C. Danubialis Gdgr mss Fol. obovata vel suboblonga ............ 9 9

Fol. ovata vel elliptica ............ 11

Fr. ovoideus; aculei rarissimi. . . . . . . . . 10

Fr. ovato-ellipticus; aculei rarissimi - Catalaunia, Monseny

(Vayreda). . . . . . 559. C. catalaunica Gdgr mss

Fr. subglobosus; aculei copiosi - Scotia; Suecia.560. C. Bo-

. . . . . . . . . . . REAlis (Tratt.)

Ped. 19-21 m. 1.; stip. virertes - Sabaudia Mt Salève (Sandoz)

Ped. $12-14$ m. l.; stip. purpureae - Basses-Alpes, Bouzolières

(Proal) . . . . . . 562. C. Laevissima Gdgr mss

11 Styli pubescentes. . . . . . . . . . 12

11 Styli lanati.................... 13

(Sep. pinnata glandulosa; tubus ovatus - Carnia. 562 bis. $C$.

12 Sep. integra eglandulosa; tubus globosus - Delph., Sabaudia

(Dès. herb. n. 8) . . . . . 563. C. Spreta (Dés.)

13 Rami aculeati; fol basi attenuata. . . . . . 14

13 Rami subinermes; fol. basi rotundata. . . . . . . 15

Fr. ovato-subrotundatus; ped. haud carnosi - Palatinat

14 Dürkheim (C.H Sch. bip.). . . .564. C. Caroli Gdgr mss

Fr. magnus sphaericus; ped. carnosi-Gall. Rhône (Gdgr

herb. ros. n. 26). . . . .565. C. PAGHY PODA Gdgr mss

Ped. apice inflati; costa eglandulosa - Helv. Bex (Thomas).

15 Ped. haud turgidi; costa paulo glandulosa - Gall. Rouen

(Letendre) ......567. C. Rothomagensis Gdgr mss

16 Ped. hispidi. . . . . . . . . . . 17

6 Ped laeves $: \ldots 21$

(Sep. eglandulosa; tubus laevis vel basi tantum glandulosus. 18

17 Sep. et tubus paulo glandulosi - Carnia; Frioul. 568. C. 
18 Fol. parva rolundata vel ovato-obtusa ........ 19

Fol. anguste obovato-oblonga . . . . . . . . . 20

Tubus laevis; fol. basi sensim attenuata - Austr. inf. Kalksburg (Wiesbaur). ...... 569, C. vinescevs Gdgr mss Tubus basi aculeatus; fol. inferne rotundata - Istria $m$. Maggiore (Freyn) . . . . . .570. C. UTzKa Gdgr mss Fr. globosus ; ped. hispidi - Europa; Asia occid. et bor.!

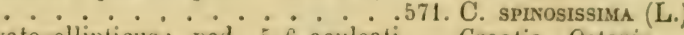
. Orato-ellipticus; ped. 5-6-aculeati - Croatia, Ostarje (Vukotinovic) . . . . . 572. C. croatica Gdgr mss Fr. globosus haud attenuatus ; fol ovata - Gall. Monts Domes (Aunier). . . . . . .573. C. ABscondra Gdgr mss Fr. conicus ovato-ellipticus basi rotundus; fol. olovato-oblonga - Montenegro, Vucia (Pantocsek). 574. C. PANтocsekrI F. . . . . . . . . . Gdgr mss Fr. obovato-oblongus, utrinque atten.; fol. nbovata - Pyren. Or. Mt Louis (Debeaux) . . . .575. C. DIchrocarPA Gdgr Pet. sparse hirtelli vel paulo glandulosi sat puberuli.. . . . 23 Pet. villosi - Gall. Rhóne; mer. .576. C. Ventenatiana (Thory) Ped. glandulosi vel hispidi. . . . . . . . . 24 Rami valde aculeati

Rami inermes vel subinermes. . . . . . . . . 32 Sep. omnino eglandulosa. . . . . . . . . . 26 Sep. dorso glandulosa vel aculeata . . . . . . . . 30 Fr. globosus. . . . . . . . . . . . . 27 Fr. ovatus - Basses-Alpes, Bouzolières (Proal). 577. C. ACANFr. ovoideus - Angl. Surrey Heat (Groves). 578. C. AngLICA

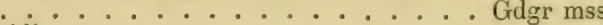
Ped toto hispidi. . . . . 2 Ped. basi vel in $1 / 3$ parte inf. paulo aculeati. . . . . . 29 (Costa eglandulosa; pet. inermes - Vosges, Epinal (Berher) Costa paulo glandulosa ; pet. aculeati - Manche, Carteret (de Bonnechose). . . . . . . 580. C. maritima Gdgr mss Ped. 23-25 m. 1.; fol. obtusa - Scotia, Perth Dunkeld (Drummond) . . . . . . . . . C. Studurtina Gdgr mss Ped. 8-10 m. l.; fol. apice sensim acuta - Sabaudia, Thonon (Puget). . . . . . . . 582. C. Pugeti Gdgr mss Sep. dorso (cum tubo) aculeata . . . . . . . . 31 Sep. dorso paulo glandulosa; tubus laevis - Inng. Csepel. (Tauscher). . . . . . 583. C. TAuscheri Gdgr mss Aculei dimorphi ; fr. ovoideus basi aculeatus - Istria, $m$. Magyiore (Freyn) . . . 584. C. Macroacantua Gdgr mss Aculei conformes; fr. globosus toto aculeatus - Delphinatus . . . . . . . . 585. C. NeYratana Gdgr mss Fr. subglobosus basi hispidus - Sabaudia.586. C. CoNTING Exs

Fr. ovoideus laevis - Italia, Modène (Gibelli).587. C. Amoena 
Rami flor. inermes; cor. alba - Europa centr. 588. C. Miris-

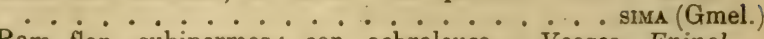

(Berher). . . . . . 589. C. Latebrans Gdgr mss Ram. flor. subinermes ; cor. rosea - Gall. centr. (Boreau). . . . . . . . 590. C. mitissimoides Gdgr mss

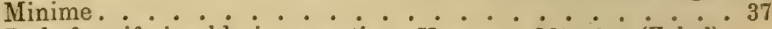
Ped. fructiferi valde incrassati - Hanovre, Münden (Zabel).

Fol elliptica $\cdots \cdots 38$

Fol. apice rotundata - Ain, St-Rambert (Gdgr herb. ros. $n$.

68) . . . . . . 592. C. MELANopoda Gdgr msS Fol. apice lanceolata - Isère, Villard de Lans (Aunier). 593.

. . . . . . . . . . C. Lansaeensis Gdgr mss Costa paulo glandulosa; fol. 7 mill. lata - Sabaudia, Annecy

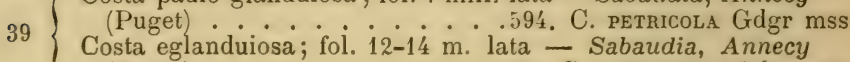
(Puget) . . . . . . 595. C. interjecta Gdgr mss

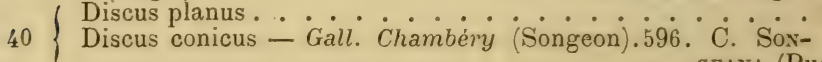

Fr. globosus vel rotundatus. $\therefore$. . GEANa (Pug.)

41 Fr ovatus . . . 43

(Fol. obovata basi rotundata - Helvet. Berne (Seringe). 597 .

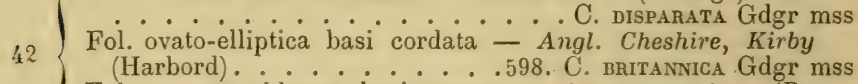
Fol. anguste oblonga basi cuneata - Calvados, Livry (De Bonnechose). . . . . 599. C. oblongIfolia Gdgr mss

Fol. ovata vel elliptica. . . . . . . . . . 44

Fol, obovato-suboblonga . . . . . . 45

( Rami super. inermes; ped. 11-13 m. 1. - Sabaudia, Annecy

44 (Puget). . . . . . 600. C. Aciculosa Gdgr mss Rami super. dense aculeati; ped. 7-9 m. 1. - Hisp. Burgos, Soncillo (Estebanez) . . . . .601. C. Legronensis Gdgr mss Ped. 11-13 m. l.; rami flor. paulo aculeati - Sabaudia,

45 Annecy (Puget) . . . . 602. C. Insueta Gdgr mss Ped. 25-28 m. l.; rami flor. inermes - Helv. Neuchatel; Chaumont (Sire). . . . . 603. C. Juratensis Gdgr mss

44 a $\left\{\begin{array}{l}\text { Fol. subtus ad costam villosa. } \\ \text { Fol. subtus toto villosa. }\end{array}\right.$ Fol. subtus ad nervos villosa - Sicilia austr. Caltanisetta (Reimbole). . . . . 604. C. Acanthodesma Gdgr mss

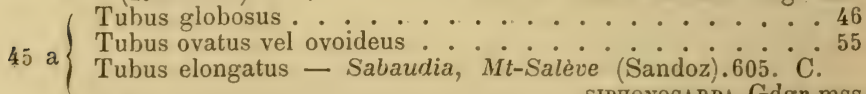
Tubus elongatus - Sabaudia, Mt-Salève (Sandoz).605. C.

46 Fol. rolundata ovato-elliptica vel obtusa . . . . . 47

46 Fol obovato-oblonga oblonga vel lanceolata $\ldots \ldots 1$

$47\left\{\begin{array}{l}\text { Styli lanati - Delphin La Grave (Dés. herb. n. } 9 \text { ?).606 } \\ \text { Styli glabri - } 48\end{array}\right.$ 
Flores albi vel pallide rosei nec punctati. . . . . . 49

48 Flores albi rosen-variegati - Delph. Villard d'Arène (Dés. herb. n. 5) . ..... .607. C. mariaebungensis Gdgr Ped. glabri; fol, ovato-elliptica. . . . . . 50 Ped. villoso-glandulosi ; fol. rotundato-obtusa - Tauria! Caucasus!..........08. C. oxyacantha (II B.) Sepala dorso paulo glandulosa - Italia, Tarente (Reimbole).

50 . . . . 609. C. Retmbolei Gdgr mss Minime - Marne, Chálons (Debeaux).610. C. Immxta Gdgr mss Styli liberi; pet. minute aculeati . . . . . . . 52

51 Styli liberi; pet, inermes . . . . . . . . . 53 Styli subcoaliti ; pet. aculeati - Rossia mer.! Caucasus !611.

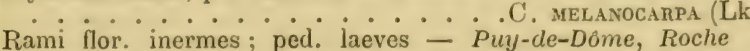

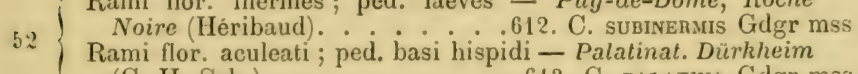
(C. H. Sch.) . . . . 613. C. Palativa Gdgr mss Ped. et tubus laeves . . . . . . . . . . . . 5't Ped. villosi ; tubus laevis - Montenegro, Vucia (l'antocsek).

. . . . . 614. C. Montenegrina Gdgr mss Ped. et tubus (inferne) longe aculeati - Pedem. Vallees Vaudoises (Rostan) . . . .615. C. valdensium Gdgr mss

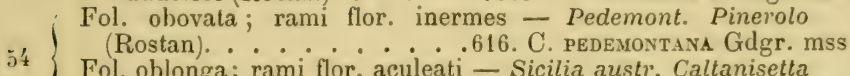
Fol. oblonga; rami flor, aculeati - Sicilia austr. Caltanisetta (Reimbole). . . . . 617. C. Caltanisetrensis Gdgr mss

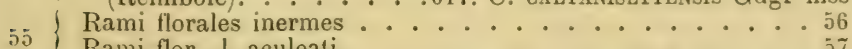

55 Rami flor. | aculeati. . . . . . . . . 57 Fol. ovata; pet. inermes - Delph. La Grave (Dés. herb. 11. 45)

56 Fol. obovata basi truncata; pet. inermes - Basses-Alpes Condamine (Proal). . . . 619. C. DELPHINENsis Gdgr mss Fol obovata basi cordata; pet. inf. aculeati - Basses-Alpes Condamine (Proal) . . . 620. C. Alpestris Gdgr mss Ped. saltem glandulosi - - Hautes-Alpes, Boscodon (Mathon- 58
Ped. et tubus laevissimi net). . . . . . . . 621. C. Nubigena Gdgr mss Ped. $8-10 \mathrm{~m}$. $\mathrm{l}$. laeves in $1 / 2 \mathrm{inf}$; fr. ovatus laevis - BassesAlpes, Condamine (Proal). : . 622. C. Isoacantua Gdgr mss Ped. $11-14 \mathrm{~m}$. 1. glandulosus in $1 / 2$ inf.; fr. ovatus laevis Aragonia austr. Sierra de Mosqueruela (Martin). 623. C.

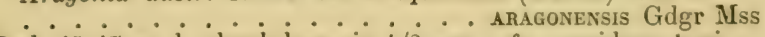
Ped. $15-17 \mathrm{~m}$. 1 . glandulosus in $1 / 2$ sup.; fr. ovoideus basi hispidus - Aragonia austr. Sierra de Mosqueruela (Martin). 59 Ped. glandulosi $\ldots .624$. C. Hirtella Gdgr mss

59 Ped. laeves - Helvet. Wintherthur! 625. C. velutiva Clairv.

60 Fol. rotundato-obtusa - Hungaria.626. C. ADENopHona (Willd.)

60 Fol. ovato-elliptica-Hung. Leibnitz. 627. C. Leibnitzensis Gdgr

61 Fol. glaberrima ............... 62

61 Fol. ad costam villosa . . . . . . . . 72

$62)$ Ped. glandulosi ................ 63

Ped. laeves ................... 66 
Styli omnino lanati .............. 64

Styli glabri - Rossia mer.-occid.; Mare Casp.! 628. C. BEs-

Minime................ SERI (Tratt.

64 Sep. dorso paulo glandulosa - Sabaudia, Annecy (Puget). . . . . . . . 629. C. oENocarpa ùdgr mss Fr. rotundatus; ped. $10-13 \mathrm{~m}$. i. nunc laeves nunc paulo glandulosi - Finistère, Crozon (Thiébaut). 630. C. ARFNosa

Fr. rotundatus ; ped. $9-10 \mathrm{~m}$ l. omnes sparse aculeati - Scotia, Aberdeen (Ley). . 631. C. septentrionalis Gdgr mss Fr. ovoideus ; ped. 19-22 m. 1. omnes valde hispidi - Angl.

Warwich Chesterton (Fraser). 632. C. PYCNACANTHA Gdgr mss

Fr. globosus rotundatus vel ovatus ......... . 67

Fr. ovoideus vel obovato-oblongus. . . . . . . . 70

Fr. globosus. . . . . . . . . . . . 68

Fr. ovatus. . . . . . . . . . . 69

Fr. turbinatus - Jurass. idanensis (Gdgr herb. ros. n. 25).

. . . . . . . 633. C. acanthocaulon Gdgr mss

Fol. obovato-oblonga ; pet. inermes - Htes-Alpes, La Grave

(Reliq. Maill. n. 1085 ex parte). 634. C. Mailleana Gdgr mss Fol. rotundata; pet. aculeati - Scotia; Apenn. 635. C. scoticA

Rami flor. valde aculeati; ped. 13-16 m. 1. - Angl. Cheshire, Kirby (Harbord) . . . .636. C. platyacantha Gdgr mss Rami flor. inermes; ped. 9-10 m. 1. - Gall. Mont Dore (Hèribaud). . . . . . 637. C. Montis Aunei Gdgr mss

Fr. ovoideus; fol obovata. . . . . . . . 71

70 Fr. obovato-oblongus; fol. elliptica - Sabaudia, Annecy (Puget). . . . . . . 638. C. suboblonga Gdgr mss Pet. aculeati; dentes plerumque compositae - Sabaudia, Annecy (Puget). . . . . 639. C. Glabrinaeva Gdgr mss

71 Pet. inermes; dentes plerumque simplices - Htes-Alpes, La Grave (Reliq. Maill. n. 1085 parte). 640. C. Mathonneti

Pet. eglandulosi .............. . . 73

Pet. glandulosi . . . . . . . . . . . 77

Fr. rotundatus ovatus vel ovato-ellipticus . . . . . . 74 Fr. globoso-cucurbitaceus - Prusse, Brandebourg Lycken

73 (Heiland)....... 641. C. Platycalyx Gdgr mss Fr. ovoideus - Delphin. La Gde Chartreuse (Gdgr). 642. C. ........ FISSIFolia Gdgr mss

74 Ped. laeves .............. 75

Ped. glandulosi vel hispidi . . . 76

Fr. nutans, valde globoso-depressus - Htes-Alpes, Lautaret

75 (Aunier). . . . . . 643. C. Autaretica Gdgr mss Fr. rectus, ovato-ellipticus apice valde attenuatus - Hispan. Aragonia austr. Sierra de Mosqueruela (Martin). 644. C. HIS-

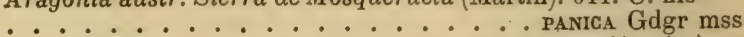
Ped. pubescentes; fr. ovatus - Htes-Alpes, Mt Viso (Aunier)

76 Ped. minute glandulosi; fr. ovato 645. C. Montigena Gdyr mss austr. Sierra de Mosqueruela (Martin). 646. C. Martini .................... Gdgr mss 
$77 \quad$ Ped. dense glandulosi . . . . . . . . . 78

Ped. laeves vel vix aculeolati . . . . . . . . . 80

Tubus basi glandulosus ............ . . 79

Tubus laevis; pet. inermes - Yanche, Granville (Lenormand).

Tubus laevis; pet. paulo aculeati - ${ }^{\circ 47}$ Scolia, Forfar Gilen South Eok (Ley). . . . . . . . 648. C. LeYI Gdgr mss

Ped. 9-13 m. i.; sep. dorso eglandulosa - Scotia, Aberdeen

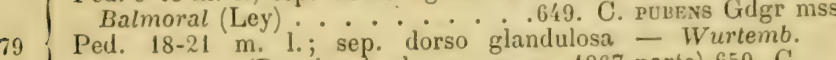
Donnstetten (Baenitz herb, europ. Wurtemb.

........ Kemmeni Gdgr mss

80 Ped laevissimi . . . . . . . . . 81

80 Ped. I glandulosi. . . . . . . . . . 83

Fr. ovoideus; fol. ovato-elliptica. . . . . . . . 82

81 Fr. globoso-depressus; fol. obovata - Hibernia, Larne (Stewart). . . . . . . 651. C. Hibennica Gdgr mss Costa glandulosa; cor alba - Aragonia austr. Sierra de

82 Mosqueruela (Martin). . . . 652. C. Glaucescens Gdgr mss Mosqueruela (Mllide rosea - Belg. Namur (Crép.). 653. C.

Minime; cor. pallide rosea - . . spinosissimo $\times$ cononata (Crép.)

Vinime. . . . . 8t

83 Sep. dorso glandulosa - Sabaudia, It Saleve (Thomas).

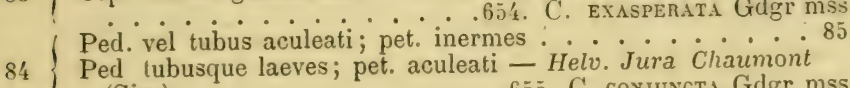

Ped tubusque laeves; pet. aculeati - Helv. Jura Chaumont (Sire) . . . . 655. C. consuncta Gdgr mss Ped. basi paulo aculeati; tubus laevis - Aragonia austr. 85 Sierra de Mosqueruela(R. Martin). 656. C. Assodva Gdgr mss . Trogenes (Ozan.)

\section{2. - Rubiginoidae Gdgr Essai, p. 20.}

Foliola crebre biserrata, subtus nonnunquam glandulosa. - Fruticuli humiliores condensati plerumque aculeatissimi, Europae australis partes calidiores habitanteș.

Fol. subtus glaberrima. ............ 2

Fol. subtus saltem ad costam villosa . . . . . . . 13

Pet. pubescentes. . . . . . . . . . . 3

2 Pet. glabri vel sparse pilosi glandulosi $\ldots \ldots \ldots \ldots$

3 Fol. subtus omnino vel ad nervos glandulosa. . . . . 5 
4 Ped. laeves; fr. subglobosus - Styria ; Carniolia.658. C. GLAPed. paulo glandulosi fr ovoldeus - Croatia Sestinata (Vest) tinovic) . . . . . . . 659. C. Dinarica Gdyr mss

5 Minime................... 6 Fol. supra glandulosa - Armenia, etc. 660 . C. Tuschetica (Boiss.) Minime.............. 7

6 Sep. dorso glandulosa - Gall. Montrellier (Roffavier) 661 . - C. HORREFACTA Gdgr mss ( Ped. apice carnosi ; fr. ample pomiformis - Cher, Givrai, etc. Minime ; fr, globosus - - 662. C. MACrOPODA (Rip. herb. n. 7). . . . . 663. C. Ripartir (Dés.) Aculei tantum setacei Aculei glanduliferi - Turkestan, Samarkande (Bge!).664.

Rami . . . . . . . Maracandica (Bge)

9 Rami inermes - Istria, $m$. iraggiore $(\dot{R}$. affinis Stern. non Rau). . . . . . . 665. C. Sternbergil Gdgr

10 Sep. dorso vel margine glandulosa . . . . . . 11 Sep. omnino eglandulosa. . . . . . . . . 12 Styli glabri ; fr. ovoideus - Pyren. centr. Bué (Bordère). 666.

• • . . • . C. glandulosissima Gidgr mss Styli lanati ; fr. ovoideus; fol. late obovato-oblonga-Bouchesdu-Rhône, St-Cyr (H. Roux) . . .667. C LaCINiosı Gdgr mss Styli lanati ; fr. globosus; fol. minutissime elliptica - Delph. Gap. (Bompard). . . . . 668. C. LEPIDOPHORA Gdgr mss Fr. laevis, ovoideus apice longe attenuatus - Gall. mer.

12 Cette (Tuezkiewicz). . . 669. C. ReBiglvoidea Gdgr mss Fr. aculeatus, globosus - Gall. mer. Narbonne (Bompard).

. . . . . . 670. C. APrICA Gdgr mss

13 Fol. subtus ad nervos glandulosa . . . . . . . 14 Fol. eglandulosa vel tantum ad costam glandulosa . . . 19

14 Fol. 6-10 m. lata, 8-13 m. longa . . . . . . . 15

14 Fol. $4 \mathrm{~m}$. lata, 5-6 m. longa - Gall. irontpellier (Bompard). . . . . 671. C. GRAMMICOLEPIS Gdgr mss

15 Sepala dorso glandulosa ............ 16 Minime. . . . . . . . . . . . 18

$16\left\{\begin{array}{l}\text { Fr. laevis. } \\ \text { Fr. dense aculeatus - Gall. mer.; Caucasus (non vidi).672. }\end{array}\right.$

Fol. supra glabra subtus ad costam villosa-A. Mragonia, Sierra

17 de Mosqueruela (Martin). . . 673. C. MYriadena Gdgr mss Fol. fere undique pubescentia - Helv. Neuchatel. 674. .......... C. DICHROA (Lerch) Pet. aculeati ; fr. hispidus - Hisp. austr. Sierra de Alfacar (Willk. exs. n. 1159). . . . 675. C. Granatensis (Willk.) Pet. et fr. inermes - Hisp. Tarragone, Monsent (Pujol).676.

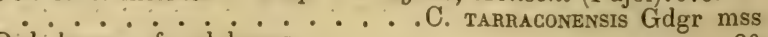

Ped. glandulosi ; fr. ovatus. . . . . . . . . 21 ( Sep. eglandulosa ; fr. amplissime globoso-depressus - Hisp. austr. Sierra Nevada, S. Geronimo (Hegelmaier). 677. Sep. margine glandulosa ; fr. mediocris rotundatus - Gall. Toulon (Bompard) .....678. C. TELONENSIs Gdgr mss 
Fr. echinatus; aculei saepe glanduliferi - Hung.; Istria;

Dalm. . . . . . . 679. C. reversa (W. K.)

Fr. laevis ; aculei setacei - Aragonia austr. Sierra de Mos-

queruela (Martin). . . . . 680. C. Loscosians Gdgr mss

Subgenus VI. - Bakeria Gdgr Essai, p. 20. -

Sabineae Crép. Prim., p. 13.

Styli liberi, lanati vel pubescentes ultra discum planum brevissime capitati; flores praecoces longuiscule angusteque bracteati, saepius amoene rosei, petala parva convexa interdum ciliata; fructus coccineus, glaber glandulosus vel erinaceus, erectus nutansve, praccox, saepe pulposus; sepala extus plerumque glandulosa, integra vel pinnata, post anthesim erecto-persistentia; pedunculi ordinarie hispidi ; tolia imparipinnata, foliola parva glabra vel tomentosa subtus quandoque glandulosa; stipulae conformes angustae, auriculis acutis; aculei dimorphi sparsi, nunc setace 0 -glanduliferi nunc falcato-dilatati; radix reptans (an semper?). - Frutices parvi humiles erecti sociales in insulis Britannicis et Sabaudia praesertim hucusque inventi, ramosissimi, ramis tenuibus foliolatis condensatis plus minus aculeato-glanduliferis corticeque rubro vel purpureo constantes.

Pulcherrimum genus hoc nomen induit D. Baker, qui, operibus de Rosis britannicis patriam suam ditavit.

1 Fol. undique villosa $\ldots \ldots . . . .2$

Fol. supra glabra subtus plus minus villosa ....... 20

Fol. undique glaberrima............. . 26

2 Fol. subtus eglandulosa ............... 3

2 Fol. subtus | glandulosa ............. 7

3 Fol. simpliciter serrata ............ 4

Fol. biserrata. . . . . . . . . . . 5 
Sep. integra ; fr. ovatus - Angl,; Hibern.; Scotia.681.

Sep. pinnata; fr. sphaericus - Armenia; Caucasus.682.

r. rotundatus; ped. $15-20{ }^{\circ} \cdot$ : BAKERIA ARMENA (Boiss.

Fr. ovato-ellipticus; ped. 14-16 m. 1. - Sabaudia; Mt-

Salève (Sandoz) . . . . . 683. B. tomentosa Gdgr mss

Fr. oblongus; ped. 3-5 m. 1. - Aragonia austr. Sierra de Mosqueruela (Martin) . . . .684. B. HJspanica Gdgr mss Aculei breves; fr. sphaericus - Angl.; Hibernia. 685.

Aculei longissimi; fr. rotundatus - Sabaudia, Mt-Salève (Guinet). . . . . . . 686. B. PILosa Gdgr mss

7 Fol. subtus ad costam glandulosa . . . . . . . 8

7 Fol. subtus ad nervos vel toto glandulosa . . . . . . 15

f Foliola biserrata . . . . . . . . . . 9 Fol. inaeque serrata - Angl. Northumberland (Baker ros. exs. n. 3). ....... 687. B. Robertsoni (Baker) Minime. ............ 10

9 Rami aculeato-glanduliferi .......... 11 Fol. ovato-elliptica ; sep. brevia - Sabaudia, Mt-Salève $(R$.

10 cinerea Rap. non Sw.) . . . . 688. B. Tephrosa Gdgr Fol. oblonga; sep. longissima - Angl. Cheshire, Hoylake (Harbord). . . . . 689. B. Longisepala Gdgr mss

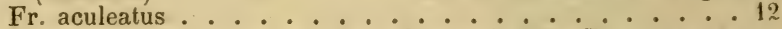

11 Fr. laevis - Hibernia, Belfast (Stewart). 690. B. LEIoCARPA Fr, ovoideus vel obovato-oblongus . . . . 13 Fr. ovatus ; fol. obovato-oblonga - Hibernia, Slemish (Ste-

12 wart) ....691. B. RHIPIDOPHLAEA Gdgr mss Fr. globosus; fol. elliptica - Insulae Brit.692. B. Gracrus

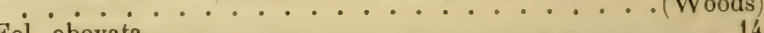

13 Fol. ovata - Angl. Yorkshire, Brafferton (Webster). 693 .

........... Dubia Gdgr mss Ram. flor. subinermes; sep. apice angusta - Angl. Derby

14 Deepe Dale (Ley). . . . 694. B. AngLICA Gdgr mss Ram. flor. aculeati ; sep. apice foliacea - Angl. Worcester (Fraser).... 695. B. Normalis Gdgr mss

15 Cor. purpurea; aculei glanduliferi . . . . 16

15 Cor. alba vel pallida; aculei haud glanduliferi. . . . . 17

Styli hirsuti; fr. globosus - Insulae Brit. (Baker exs. ros.

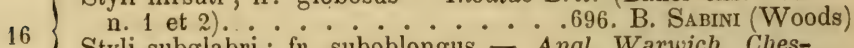
Styli subglabri ; fr. suboblongus - Angl. Warwich, Chesterton (Fraser) . . . . 697. B. Echinantha Gdgr mss Ped. tubusque glandulosi; styli lanati. . . . . . . 18

17 Ped. tubusque glandulosi ; styli subglabri . . . . . . 19 Ped. tubusque laeves; styli lanati - Turkestan, Samarkande (Lehm.). . . . . . . 698. B. Lehmanniana (Bge)

Fr. basi attenuatus; fol. obovato-acuta - Sabaudia, MtSalève (Reut., etc.) …699. B. Reuteriana Gdgr mss

$18\{$ Fr. basi rotundatus; fol. oblongo-obtusa - Angl. Cheshire, Moreton (Harbord) . . . . . 700. B. BRITANnICA Gdgr ms8 
Fr. apice vix attenuatus - Belg. (Reliq. Maill. n. 438). 701.

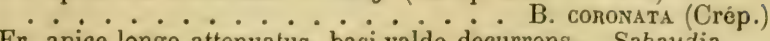
Mt-Salève (Sandoz) . . . . .702. B. crassibasis Gdgr mss

Fr. apice longissime attenuatus, basi rotundatus - Belg. Han-sur-Lesse (Billot exs. n. 4023). 703. B. Conovata

Ped dense glandulosi

Ped laeves

21 Aculei glanduliferi. . . . . . . . . . . 22

I Minime. . . . . . . . . . 23

( Tubus et sep. omnino glandulosi - Scotia; Hibernia.704. $22\{$ Tubus basi hispidus; sep. laevia - Helv.; Gall. or., etc. 705.

Fr. rotundatus hispidus - . . B. ADENOPHLAEA Gdgr mss Gall. centr. (Gdgr herb, ros. n. 347 . . . . . . 706. B. pomponia (DC.)

. 707. B. Malyi (Kern.)

Fr. obovato-oblongus laevis - Sabaudia, Chambéry (Perrier). . . . . . 708. B. MEgalochlaMys Gdgr

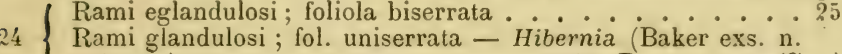
4 var.)......... 709. B. HIBERNICA (Sm.)

( Tubus ovatus; fol. elliptico-ovata - Helvetia.710. B. HELve-

25 Tubus globosus; fol rotundato-obtusa - Gall TICA (Hall. f.) (Schultz exs, n. 1445) . . . .711. B. BITuRgensis (Bor.)

26 Ped. glabri.................... 27

\{ Ped. glandulosi . . . . . . . . 28

( Sepala glandulosa - Dania (Fl. dan. III, tab. 388 !).712. B.

27 Minime - Angl. Cheshire, Thurtasto

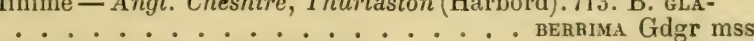

$28 \mid$ Costa media subtus glandulosa . . . . . . . . 29

Minime . . . . . . . . . 30

(Cor. intense rosea ; aculei rari - Europa? 714. B. suavis (Willd.)

29 Cor. pallida; aculei copiosi - Sabaudia, Mt-Salève. 715.

Fr. globosus; corolla saturate rosea - Insulae Brit. 716 . B.

$30\left\{\begin{array}{l}\text { Fr. ovatus vel ellipticus; cor. pallida-Istria, Mt-Maggiore, etc. } \\ \text { B. Buter }\end{array}\right.$

717. B. GENTILIS (Sternb.)

Subgenus VII. - Ozanonia Gdgr Essai, p. 21. Alpineae et Montaneae Auct. recent.

Styli liberi saepissime lanati ultra discum planum vel 
conicum plus minus breviter capitati flores late vel angnste. bracteati, intense purpurei rarissime albi, petala haud ciliata; fructus serotinus pulposus sanguineus saepe nutans, glaber et hispidus; sepala glandulosa necne; pinnata vel integra, post anthesim erecta tandem conniventia, in veris Alpinis et Montanis semper persistentia in quibusdam (raro quidem) formis aliis sero decidua; pedunculi recti vel arcuati, glabri vel glandulosi; foliola glauca vel viridia, glabra vel subhirtella rariusque subtus toto glandulosa, argute simpliciter necne serrata; stipulae conformes dilatatae glaucescentes; aculei sparsi tunc adunci, vel stipulares ergo recti, plus minus copiosi vel nulli, - Suffrutices solitarii graciles tunc ramis inermibus cortice purpureo radiceque reptante constantes, vel frutices robustiores sociales ergo ramıs aculeatis saepe glaucis radiceque haud aut vix reptante sistentes, nihilominus omnes floritione serotina in alpinis vel montanis totius fere Europae et Asiae occidentalis non rari. Dedicatum D. Ch. Ozanon, gallico, de rhodologia jam longo tempore, bene merito.

\footnotetext{
(Rami rubri haud aut vix glaucescentes; fol. virentia vix nerA vosa; sepala saepius integerrima; aculei nulli vel setacei.

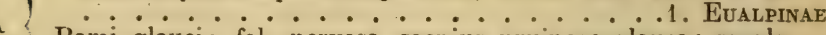
Rami glauci; fol. nervosa saepius pruinoso-glauca; sepala plus minus pinnata; aculei saepissime falcato-dilatati.

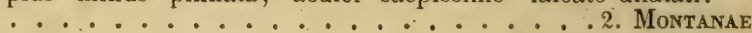

\section{1. - Eualpinae Gdgr Essai, p. 22.}

Suffrutices graciles solitarii rubentes; rami flexuosi inermes vel aculeis stipularibus subulatis praediti (basi caulium hornotinorum excepta) purpurei; radix reptans; foliola elongata tenuia virentia, nervi subtus haud aut vix promi- 
nuli; sepala integra lanceolata, erecto-persistentia ; corolla mediocris purpurea; flores anguste parumque bracteati solitarii vel 2-3-ni, pedunculati; styli ultra discum plerumque planum sessili lanati; fructus pulposus nutans fere axillaris saepius elongatus et utrinque attenuatus. - Plantae regionem alpinam omnino colentes.

1) Fol. undique glaberrima . . . . . . . . . . . 2

1 Fol subtus saltem ad costam villosa... . . . . . . 79

Fr. oblongus vel elongatus . . . . . . . . . . . 3

2 Fr. globosus rotundatus ovatus vel ovoideus . . . . . . 54

3 Tubus laevis. . . . . . . . . . . . 4

1 Tubus glandulosus. . . . . . . . . . . . 40

, Pet. glabri ............... . . 5

4 Pet. villosi vel sparse pilosi. . . . . . . . . . . 30

5 Fol biserrata ............. . . 6

5 Fol inaeque vel simpliciter serrata. . . . . . . . . 25

6 Ped. I dense glandulosi. . . . . . . . . . . 7

6 Ped. laeves, villosi vel 1-3-glandulosi ......... 21

7 Sep. extus omnino eglandulosa . . . . . . . . . 8

7 Sep. dorso vel margine glandulosa. . . . . . . . . . 12

( Fol. late obovato-oblonga vel oblonga. . . . . . . 9

Fol. anguste elongata - Delph. La Grande Chartreuse (Gdgr)

718. Ozanonia carthusianorum Gdgr mss

Fr. oblongus; ped. usque ad 2/3 vel 1/4 sup. glandulosi . . 10

9 Fr. late oblongus; ped. omnino et dense glandulosi. . . . 11 Fr. longissimus cylindricus; ped. in $1 / 4$ inf. laeves $-A l p$.

Germ. bor.-occid. (Zabel).719. Ozax. crlivdrocarpa Gdgr mss Ramr paulo aculeati ; ped. $12-15 \mathrm{~m}$. 1. usque ad $2 / 3$ sup. glandulosi - Pyrenaei Or. Costabona (Vayreda).720. OzA-

........... NONIA VAYREdAE Gdgr mss Rami inermes; ped. 22-25 m. 1. in $1 / 4$ inf. glandulosi Cantal, Le Plomb (Héribaud) . .721. O. PAllidipes Gdgr mss Sep. omnino eglandulosa; fr. rectus - Croatia, Plitvica Borbas). . . . . . . 722. O. Borbasi Gdgr mss Sep. margine glandulosa; fr. nutans - Pyrenaei Orient. Canigou (Gautier).. • . . 723. O. MEgatiannos Gdgr mss Fol. obovato-oblonga vel oblonga. . . . . . . . 13

2 Fol. ovalo-elliptica - Europa med. et austr.724. O. Alpiva (L.)

13 ) Sep. apice eglandulosa . . . . . . . . . . . 14

3 , Minime....................... 20

14 Sep. dorso omnino glandulosa. . . . . . . . 15 Sep. apice tantum vel margine glandulosa . . . . . . 17 Fr. apice strangulatus; fol. basi subattenuato-truncata . . 16

15 Minime; fol. basi subrotundata - Helv: Jura, Chaumont

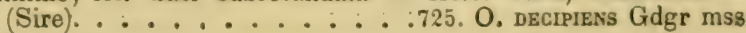


(Fr. arcuatus ; ped. 25-28 m. 1. in $1 / 2$ parte sup. laeves Scotia Perth, Richemont Hill (Boswell S.).726. O. scotica

Fr. subinclinatus ; ped. $15-20 \mathrm{~m}$. 1 . toto glandulosus - Isère, Gde Chartreuse (Gdgr) . . . . 727. O. GRAcILIs Gdgr mss

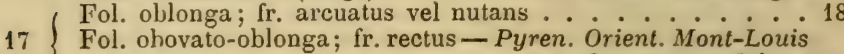
(Debeaux). . . . . . 728. O. DETONSA Gdgr mss

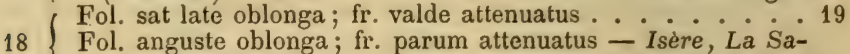
lette (Puget). . . 729. O. Mariana Gdgr mss

Ped. parce glandulosi ; fol. oblongo-acuta - Isère, Gde Char-

19 treuse (Gdgr). . . . . . . S30. SipHo Gdgr mss Ped. dense glandulosi ; fol. obtuse obovato-oblonga - Istria, Mt Maggioie (Freyn). . . . .731. O. Utzka Gdgr mss

Sep. apice foliacea; fol. longiora $=38-40 \mathrm{~m}$. 1. - Hte-Sa-

$20\left\{\begin{array}{l}\text { voie, Habère-Poche (Puget) } .732 \text {. O. AMYgdalordes Gdgr mss } \\ \text { Minime; fol. longiora }=20-30 \mathrm{~m} \text {. l. Hte-Savoie, Habere- }\end{array}\right.$ Poche (Puget). . . . . 733. O. CONGREgata Gdgr mss

Ped. laevissimi ................... 22

Ped. 1-3-glandulosi; fr. basi subrotundatus - Pyren. Or.

21 Vernet-les-Bains (Nou). . . .734. O. BIADENA Gdgr mss Ped. villosi ; fr. basi valde attenuatus - Hte-Savoie, HabèrePoche (Puget). . . . . . 735. O. PU BIPEs Gdgr mss

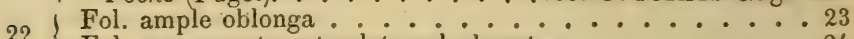

Fol. parva ovato-rotundata vel obovata . . . . . 24 Sep. eglandulosa; fol. lanceolata - Htes-Alpes, La Grave

23 (Bompard) . . . . 736. O. GRAVENSIs Gdgr mss Sep. apice glandulosa; fol. rotundata - Alp. Europae bor. (Tullberg! . . . . . 737. O. Platyphylla Gdgr mss Fol. minutissime suborbiculata; sep. margine glandulosa -

24 Gall. Mt-Viso (Bompard). . . . 738. O. vesula Gdgr mss Fol. latuiscule obovata ; sep. eglandulosa-Delph. Gde Chartreuse (Gdgr). . . . . . 739. O. FRIGIDA Gdgr mss

25 Rami aculeati.............. 26

25 Rami inermes. . . . . . . . 28 28

(Fol. biserrata; aculei basi dilatati. . . . . . . 27 Fol. inaeque serrata; aculei setacei - Suecia bor.; Ross. bor. (Fr. herb. norm. XIII n. 59) . . . 740. O. CARELICA (Fr.) Ped. et sepala eglandulosi - Ital. Apenn. Pistoje Boscolungo

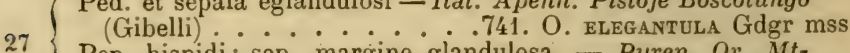
Pep. hispidi ; sep. margine glandulosa. - Pyren. Or. MtLouis (Debeaux). . . . . 742. O. ACANthopHora Gdgr mss

$28\left\{\begin{array}{l}\text { Fol. oblongo-lanceolata; cor purpurea. } \\ \text { Fol. ovata; cor. pallide rosea }- \text { Europ. cenlr. mont. } 743 \text {. } 29\end{array}\right.$

Fr. basi rotundadus - Scotia (non vidi).744. O. ANDre (Mill.) $29\left\{\begin{array}{l}\text { Fr. basi longe decurrens - Pyrenaei orient. Mt-Louis (De- } \\ \text { bratt.) }\end{array}\right.$ beaux) ......... 745. O. aucuparioldes Gdgr

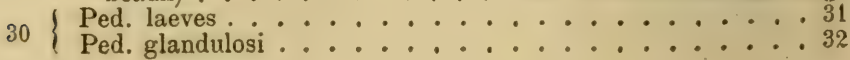


Sep. paulo pinnata; fr. obovato-oblongus - Italia Mt-Cenis Gautier). .......747. O obtusata Gdgr mss Sep. I glandulosa. . . . . . . . . . 33

Sep. eglandulosa. . . . . . . . . . . . 37

33 Fol. obovata vel obovato-oblonga .......... 34

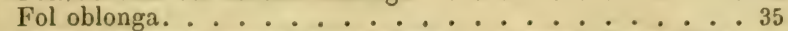
(Rami inermes; costa eglandulosa - Austr.; Hung. 748. 34 Rami aculeati; costa glandulosa - Hte-Savoie, Brenthonne (Puget) . . . . . 749. O. acanthophlaEa Gdgr mss

( Sep. apice dilatata. . . . . . . . . 36

35 Sep. apice linearia - Italia, Mt-Cenis (Gdgr).750. O. ontrex....................... Gatgr ms Fr. superne valde strangulatus; sep. apice foliacea - Hte-

36 Savoie, Habère-Poche (Puget).751. O. Amoenevinfns Gdgr mss Fr. attenuatus; sep. apice subangustata - Savoie, Mouxy (Puget). . . . . . . 752. O. Misera Gdgr mss

37 Fr. in $1 / 3$ parte sup. valde turgidus. . . . . . 38 Ninime. . . . . . . . 39 Rubens ; pet. inermes - Italia, Mt-Cenis (Aunier).753. O. Virens; pet. aculeati - Delph. Gde-Chartreuse (Gdgr).754. O.

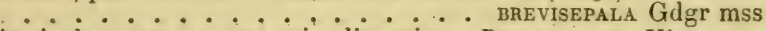
Auriculae rectae; sep. apice linearia - Pyren. centr. Vigne-

$39 \int$ male (Bordere) . . . . 755. O. stenoriphaea Gdgr mss Auriculae divaricatae ; sep. apice dilatata - Savoie, MtCormet (Puget) ....... 756. O. FAusta Gdgr mss

( Ped. tubusque glandulosi. . . . . . . . . 41

40 Ped. glaber; tubus glandulosus - Jura, Val-de-Joux (Thomas) . . . . . . 757. O. Juratensis Gdgr mss

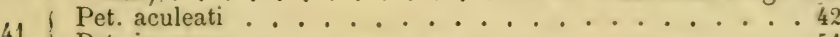

41 Pet inermes $\ldots$

Fol, rotundata vel ovato-elliptica ........ 43

42 Fol obovato-oblonga .................. 44

Fol. oblonga .................. . . . 46 Fol. ovato-elliptica; tubus glanduloso-aculeatus - Pyren.

43 centr. Gavarnie (Bordère). 758. O. stictophylla Gdgr mss Fol. subrotundata; tubus tantum glandulosus - Pyren. centr. Gavarnie (Bordère). . . . .759. O. politula Gdgr mss Scp. et foliola subtus glandulosa . . . . . . . 45

44 Sep. eglandulosa; fol. subtus eglandulosa - Delph Lautaret (Aunier) . . . . . . 760. O. Gracillima Gdgr mss Rami flor. dense aculeati ; fr. rectus - Dalmatia, Mt-Vellebit

45 (Visiani) ........ 761. O. Dalmatica Gdgr mss Rami flor. inermes; fr. nulans - Pyren. centr. Pic du Midi (Debeaux). . . . . . . 762. O. RuBiginosa Gdgr mss Fol. inaeque serrata. . . . . . . . . 4 47 Fol. biserrata. . . . . . . . . . . 48 Sep. basi laeves; tubus sparse hispidus - Delph. Gde-Chartreuse (Gdgr). . . . . . 763. O. PERMIXTA Gdgr mss Sep. tubusque dense hispidi - Vallesia, Louèche (Lorenti). 764. O. LAGENIFORMis Gdgr mss 


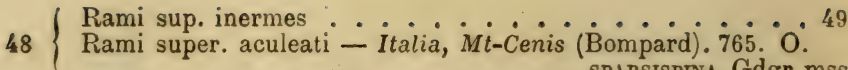
Stip subtus gand - . SPARsispra Gdgr mss

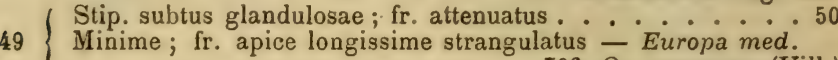
Minime; fr. apice longissime strangulatus - Europa med. Sep. apice glandulosa ; dentes mediocri - Helv. St-Gall

$50\{$ (Schlasser)....... 767. O. Longrpes Gdgr mss Minime; dentes profundae - Delph. Gde-Chartreuse (Gdgr).

51 Rami sup inermes. $\therefore . . . . .53$

\{ Rami | aculeati. . . . . . . . . . 52 Sep. et fructus (amplissimus) hispidissimi - Pyren. centr.

52 La Maladetta (Bordère) . . 769. O. sarcocarpa Gdgr mss Sep. in $1 / 2$ inf. laeves; fr. angustatus parce glandulosus Hte-Savoie, Habère-Lullin (Puget). 770. O. Angustituba

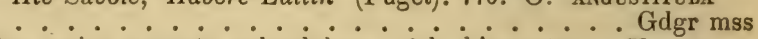
Sep. apice angusta eglandulosa; fol. biserrata - Vosges, Gerardmer (Millot) . . . . 771. O. Incumbens Gdgr mss

53 Sep. apice foliacea glandulosa; fol. biserrata - Delph. Lautaret (Aunier). ....772. O. HORRIPILA Gdgr mss Sep. apice foliacea eglandulosa; fol. inaeque serrata - Silesia, Landeshut (Patze). . . 773. O. Neilreichiana Gdgr mss

54 Rami flor toto inermes .......... . 55

54 Rami flor | aculeati ................. 70

55 Ped. laeves................ 56

Ped. glandulosi . . . . . . . . . . 57 Dentes rigidae; pet. minute aculeati - Pedem.; Lomb.774.

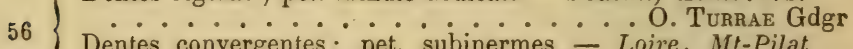
Dentes convergentes; pet. subinermes - Loire, Mt-Pilat (Malarbet) ...... 775. O. ToRTIDENTA Gdgr mss

57 Tubus laevis ............ . . 58

Tubus hispidus ................ 68

58 Sep. integerrima $\ldots . .259$ Sep. pinnata - Dalmatia; Transylv.776. O. Portenschlagiana

. . . . . . . . . (Tratt.)

Fol. rotundata ovata vel elliptica $\therefore \therefore 62$

59 Fol. obovato-oblonga ........... . . 60 60

Fol. oblonga ............... 63

Minime ; fol utrinque subrotundata . . 61

60 Sep. glandulosa; fol. utrinque attenuata - Hte-Savoie, HabèreLullin (Puget) . . . . . 777. O. volenus Gdgr mss Fr. ovato-rotundatus; ped. $15-20$ m. 1. - Valais, Zermatt

$61\{$ (Wolf).......... . . S78. SPAERICA Gdgr mss Fr. ovoideus ; ped. 25-28 m. 1. - Ital. Bonon. Mte-Corno (Bert.). . . . . 779. O. Bertolonu Gdgr mss (Fol. ovato-elliptica; fr. ovatus vel rotundatus - Alp. Pyren.

62 Fol. elliptico-obtusa; fr. obovato-oblongus - Pyren. centr. Gavarnie (Bordère). . . 781. O. crassifolia Gdgr mss Fol. ovato-rotundata ; fr. obovatus - Pyren. centr. Gavarnie (Bordère)........782. O. subRotunda Gdgr mss 
Fr. ovoideus apice vix attenuatus . . . . . . 54

Fr. ovoideus apice valde strangulatus . . . . . . . 65

Fr. obwato-oblonsus apice strangulatus - Hie-Savore.

Habère-Lullin (Pu-et). . . 783. O. gymмostephana Gigr mss

Fol. late ohlongo-jhtusa - Italia Abruzz. Aquila (Ġussone). 34 Fol. anguste oblongo-acuta - Hte-Savore, Habère-Lrillin

65 Fr. patulo-arcuatus vel flexuosus; perl $13-16 \mathrm{~m} .1$. . . . . fff Fr. rectus; perl. 17-19 m. 1. toto glandulosi-Pedem. Vallees Vaudoises (Rostan). . . . .786. O. Nervulosa Gdgr mss Sep. apice integra . . . . . . . . . . . 67

66 Sep. apice late foliaceo-dentata - Pyren. orient. Bosch Carbone (Vayreda) . . . 787. O. odoNtostephaxa Gdgr mss Fol. parva obovato-oblonga; dentes convergentes - Cata-

67 launia, Nuria (Tremols). . .788. O. catalaunica Gdgr mss Fol. late oblonga; dentes patulae - Croatia, Agram (Vukotinovic) : . . . . . 789. O. croAtica Gdgr mss Fr. sphaericus ovoideus basi hisididu:- Doubs, Villers-le-Lac (Flora 69 Sequaniae exs. n. 439 parte). . .790. O. ADscira Gdgr mss Fr. obovato-oblongus toto hispidus - Hte-Savoie, HabereLullin (Puget). . . . . 791. O. SETicalyx Gdgr mss Tubus toto hispidus; sep. eglandulosa - Silesia (Krock.); Bohem. (Pohil) . . . . . .792. O. HISPIOA (Krock.) Tubus et sep. basi laevia - Pyren. orient. La Breste (Vayreda). . . . . 793. O. GLoBosa Gdgr mss

70 Tubus larvis Tubus glandulosus. . . . . . . . . . . . . . . 71

1 Fr. ovoideus $\ldots 72$

Fr. globosus ovatus vel ovato-ellipticus $\ldots 73$ Fol. parva ovata; sep. laevia - Italia, Pise Tre potenze 72 Fol. late obovato-oblonga; sep. glandulosa - Pyren. centr. Esquierry (Debx). . . . 795. O. Debeauxi Gdgr mss

73 Sep. basi saltem eglandulosa. . . . . . . 74 Sep. extus toto glandulosa - Austria.796. $\mathrm{O}^{\circ}$ Wrefexi (Tratt.) Aculei verticillati; fr. subglobosus - Europa, Sibir. (O. filispina Delax 1878, . . . . . .797. O. stbcrassa Gilgr

Aculei sparsi ; fr. oratn-ellipticus basi subrotund. - Pyren. or. Bosch Carbone (Vayreda) .798. O. Prcxacastha Gdgr mss Acule stipulares; fr. oratus basi attenuatus - Loire, Pierresur-Haule (Gdgr). . . .799. O. Glaucopurpurea Gdgr mss Aculei stipulares; fr. subrotundatus basi rotund. - Loire, Pierre-sur-Haute (Gdgr) . . . 800. O. BRETrúba Gdgr mss Sep. pinnata . . . . . . . . . . . 76 Sp integerrima . . . . . . . . . . . . . . i7 Aculei crassi dilatati - Caucasus. - .801. Q. optrstres (Boiss.)

76 Aculei subulati - Asia occid., Larislan; Caucasus. 802.

Discus planus ; cor purpurea . . O. DJLMLExsts (Boiss.)

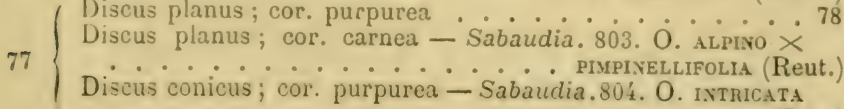


Ped. et sepala dense glandulosa - Dalmatia, alp. Dinarici

$78\left\{\right.$ (Visiani) ..... ${ }^{805}$ O. DINARICA (Vis.! in litt.) Ped. sublaeves; sep. apice margine glandulosa $-D a^{\prime} m$. alp.

Dinarici .......806. O. Adriatica Gdgr mss

79 Fol. subtus ad costam villosa. . . . . . . 80

79 Fol. subtus toto vel undique villosa . . . . . . 134

80 Tubus laevis .................... 81

Tubus tuasi saltem vel apice glandulosus. .......117

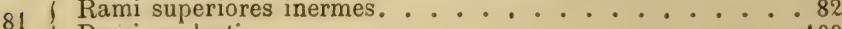

1 Rami aculeati. . . . . . . . . . 109

82 ) Pet. inermes ................. 83

82 Pet. aculeati . . . . . . . . . . . 97

$83)$ Ped. laeves . . . . . . . . . . 84

( Ped. glandulosi . . . . . . . . . . . . 89

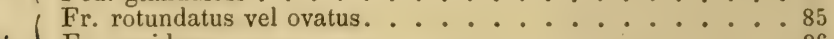

84 Fr. ovoideus ................ 86

Fr. obovato-oblongus vel oblongus . . . . . . . 87

Fol. simpliciter serrata - Delph. Gde-Chartreuse (Gdgr).807.

O. ABIAEFOLIA Gdgr mss

Fol. biserrata - Hie-Savoie, Fessy (Puget).808. O CREMoce-

Fol obtuse elliptico-ovata - Europae mont. (Ser. exs. n. 26)

86 . . . . . . . . . . P09. PEndina (Ait.)

Fol. oblongo-acuta - Hte-Savoie, Habère-Poche (Puget).810.

$\ldots \ldots \ldots$ O. Latidens Gdgr mss

(Fol. oblonga; tubus laevissimus . . . . . . . 88

87 Fol. obovala; tubus basi glandulosus - Helvet. Bex (Thomas)

..... 811. O. Bactiacensis Gdgr mss Sep. omnia glandulnsa; fr. obovato-oblongus - Pyren. occid. Tortes (Bordere) . . . 812. O. AnISOPODA Gdgr mss Sep. 2 glandulosa, 3 laevia; fr. oblongus - Jura, Creux de l'Enfer (Fray) . . . . 813. O. ANgustifolia Gdgr mss

89 Sep. omnino eglandulosa. . . . . . . . . 90

89 Sep. I glanduiosa. . . . . . . . . . . 94

90 ( Fr. ovoideus ..................... 91

( Fr. obovato-oblongus vel oblongus ........ 92

Fol. obovato-oblonga utrinque attenuata - Hte-Savoie, Fessy

91 (Puget)............ 814. O. Nutans Gdgr mss Fol. oblonga utrinqne rotundata - Gall. Le Bugey (Gdgr).

. . . . . 815. O. Beugesiaca Gdgr mss

Rubens; fr. oblongus basi valde attenuatus semi-arcuatus. 93

Rubens; fr. anguste ob!ongus utrinque abrupte contractus arcuatus - Hte-Savoie, Habère-Lullin (Puget). 816. O.

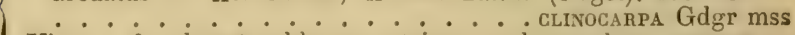
Virens; fr obovato-oblongus utrinque subrotundatus erectus - Hie-Savoie, Habere-Poche (Puget).817. O. Collecta Gdgr mss Fol. lanceolata ; sep. apice angusta - Gall., Grenoble, stAnge (Verlot) . . . . . . 18 . O. DEMISSA Gdgr mss Fol. apice rolundata; sep. foliacea - Basses-Alpes, Tournoux (Proal). . . . . 819. O. TnichopodA Gdgr mss

Fol. magna oblongo-lanceolata ........ 95

Fol. mediocria obtuse obovato-oblonga - Ital. Pistoje L'Abe-

tone (Gibelli) ...... . 820. O. ApENNINA Gdgr mss Fol. parva obovato-elliptica - Jura, Mt Faucille (Fray).821. 
Ped. omnes glandulosi. . . . . . . . . 96

Ped. alii laeves alii glandulosi - IIte-Savoie, Habère-Lullin (Pug:t)............ O. IDIonopoda Gdgr mss Ped. 14-17 m. i.; fr. nutans - Vosges, Le IIohneck (Berher).

Ped bed, Cantal, Le Plomb (F. Heribaud). . . . . . 824. O. MEgACARPA Gdgr mss

Sep. laevia . . . . . . . . . . . 98

Sep dorso saltem in $1 / 2$ sup glandulosa. $\therefore 105$

Fr. obovato-oblongus. . . . . . . . . . . 99

Fr. oblongus vel elongatus . . . $\therefore 100$

Pet tralri - IIte-Savoie, Habère-Lullin (Puget).825. $\dot{0}^{\circ}$.

99 . . . . . . Attenuatifolia Gdgr mss Pet. sparse pilosi - Gall. Gde-Cluartreuse; Mt Pilat Gilgr)

. . 826. O. gracilifolia Gdgr mss

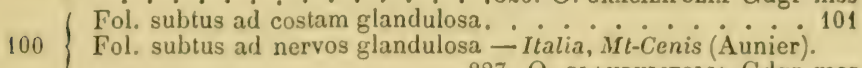

. . . . . 827. O. glandulifolia Gdgr mss

( Fol. obovata - Vaucluse, Mt-Ventoux (Autheman).828. O.

101 Fol. oblinga.

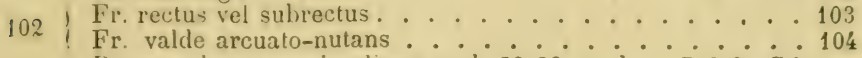

Dentes pleraeque simplices; ped. $23-26$ m. l. - Delph. Gde-

103 Chartreuse (Gdgr). . . . 829. O. Lerantha Gdgr mss Dentes compositae; ped. $15-18 \mathrm{~m} .1$. Delph. Gde-l'hartieuse

(Gdgr). . . . . 830. O. A Bieticola Gidgr mss

Fol. lanceolata - Htes-Alpes, La Grave (Aunier).831. O.

104 . . . . . . ECGREMOCARPA Gdgr ms

Fol. apice rotundata - Delph. La Gde-Chartreuse (Gdgr).832.

. . . . . . . . . O. Ampuona Gdgr mss

105 Fol. subtus ad nervos glandulosa. . . . . 106

Fol. subtus ad costam glandulosa vel laevia. . . . . 107

Fr. ovoideus - Pyren. centr. Gavarnie (Bordère).833. O.

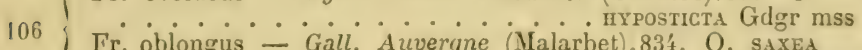

Fr. oblongus - Gall. Auvergne (Malarbet).831. O. SAXEA

. . . . . . . . . . G Gdyr mss

Fr. basi attenuatus; fol. attenuata . . . . . . 108

Fr. basi rotundatus; fol. valde lanceolata - Sabaudia, Mt-

Cormet (Puget). . . . 835, O. onelsosa (Rip.) mss

Ped. rectus; fol. apice vix attenuata - Vallesia, Louéche

108 (Lorenti) . . . . 836. O. Applanata Gdgr mss

Ped. arcuatus; fol. longe attenuata - Pyren. centr. Gavarnie

(Bordère). . . . . 837. O. PHoENiceoclada Gdgr mss

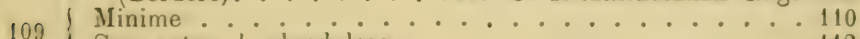

Sep extus | glandulosa . . . . . . . . . 112

Ped. glantulosi ; fr. oblongus . . . . . . . . 111

110 Ped. hirsuti: fr. ovatus. Hte-Savoie, Habère-Lullin (I'ugél)

838. O. Leioserala Gdgr mss

Ped. recli, alii glabri alii glandulosi; fr. utrinque subrotundatus - Ite-sacoie, Brenthonne (Puget). 839. O. Alpes-

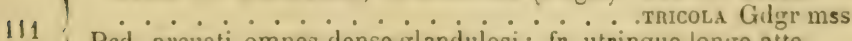

Ped. arcuati omnes dense glandulosi ; fr. utrinque longe attenuatus - Loire, Pierre-sur-Haute (Gdgr). 840. O. Fontina- 
Ped. arcuati in $1 / 4$ inf. laeves ; fr. longissimus - Alp. Germ bor.-occid. (Hort. Goetting. et Münden).841. O. CYlindro• . . . . . . . . . CARPA Gdgr mss

$112\left\{\begin{array}{l}\text { Fol. obovato-oblonga vel oblongo-lanceolata. . . . . . . } 113 \\ \text { Fol. ovata vel elliptica | obtusa. . } 115\end{array}\right.$

Ped. 2-3-ni ; fr. ovoideus . . . . . . . . 114

113 Ped. 1 ; fr. obovato-oblongus - Jura, Mt-Dôle (Reliquiae Maill. n. 1083)........... 842. O. Jugorum Gdgr mss

Fol. obovato-oblonga; aculei verticillati copiosissimi - Ital. Pistoje, L'Abetone apenn. (Gibelli).843.O. PISTORIEnsis Gdgr mss Fol. oblongo-lanceolata; aculei rari stipulares - Loire, Pierre-sur-Haute (Malarbet). . . 844. O. MInA Gdgr mss. Fol. ovata vel rotundata; ped glandulosi. . . . . . 116

115 Fol. elliptico-obovata; ped. sublaeves - Aragonia MontTorla (Bordère). . . . 845. O. Sarcostephana Gidgr mss Fr. oblongus; costa glandulosa - Hautes-Alpes, La Grave

116 (Dés. herb. n. 59) ..... 846. O. alpestris (Dès.) Fr. ovato-ellipticus; nervi glandulosi - Hisp. Navarre, Ostiz, (Lacoizqueta). . . . 847. O. NAvarrensis Gdgr mss

117 Rami flor, aculeati ............. 118 Rami flor inermes . . . . . . . . 120

Fol. biserrata; sep. glandulosa. . . . . 119

118 Fol. simpliciter serrata; sep. laeria - Suecia inquil. (Fr. herb. norm. VIII n, 46); Sibiria. . . 848. O. LAXA (Retz) Sep. laevia; fr. oblongus arcuatus - Pyren. centr. Gavarnie! (Bordère) . . . . . . 849.0. pyrenaica (Gou.)

119 Sep. laevia; fr. ovatus rectus - Dalmatia, Mt-Vellebit (Visiani) ...... . . . . . Visianil Grdgr mss Sep. apice glandulosa ; fr. ovoideus inclinatus - Loire, Pierresur-Haute (Gilgr) . . . . 851. O. Auriculata Gdgr mss Sep. saltem in $1 / 3$ inf. eglindulosa. . . . . . . 121

120 Sep. toto glandulosa .............. 124 sep. toto glandulosa et insuper basi aculeolata . . . . 131

121 Fr. oblongus. . . . . . . . . 122 Fr. ovatus . . . . . . . . . . . . 123 Fr. et fol. longissima - Hte-Savoie, Habère-Lullin (Puget)

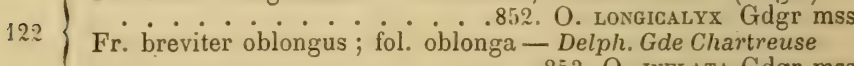
. . . . . . . 853 O. Inflata Gdgr mss Fol. ovato-elliptica; fr. dense hispidus - Delph. Connexe (Vill. dauph. III, p. 554). . . .854. O. Villarsil (Tratt.)

Fol. oblonga ; fr. basi tantum hispidus - Basses-Alpes, Tournoux (l'roal). . . . . 855. O. colorata Gdgr mss Fol. obovato-obtusuiscula; fr. sublaevis - Loire, Pierre-surHaute, $(\mathrm{Gdgr})$. . . . 856. O. Coronularia Gdgr mss

(Pet. sparse hirsuti ............ 125 Pet villosi. . . . . . . . . 128 Pet. glabri ............. 127 Sep. apice foliaceo-dentata; pet. inermes . . . . 126

$125\left\{\begin{array}{l}\text { Sep. apice dilatalo-dentata; pet. aculeolati - Helv. Vaud, } m \text {. } \\ \text { Lavarraz (Thomas) . . . 857. O. vaudensis Gdgr mss }\end{array}\right.$ Sep. apice linearia integra; pet. inermes - Hte-Savoie, Habere Poche (Puget). . .858. O. Dictamnifolia Gdgr mss 
Ped. 20-23 m. 1.; fr. apice tantum glandulosus - Vaucluse.

1261 Mt-Ventoux (Autheman) . . 859. O. cyt.nenica Gdgr mss Ped. 15-18 m. 1.; fr. toto hispidissimus - Pedem. Vallées Vaudoises (Rostan) . . . 8rio. O. Tnicroc un Gi Gremss Sep. apice glandulosa et angustata - Ilaute-Savoie, Ilabère-

127 Lullin (Puget). . . . . 861. O. Glabnion Gdyr mss Sep. foliacea a $1 / 3$ parte inf. usque ad apicem glandulosa IIte-Savoie, /labère-Lullin ('uget).862. O. subalpina Gigr mss

128 Fol. subtus ad nervos interdum villosa: . . . . . 129

Fol. subtus ad costam villosa . . . . . . . 130

Fol. obovato-elliptica utrinque rotundata - Italia, Mt-Cenis

129 (Aunter) . . . . . . 863. O. onstinata Gdgr mss Fol. oblonga, basi paulo attenuata - Ite-Savoie, HabèreLullin ('Puget). . . . 864. O. siphuncula Gdgr mss Fr. ovoideus toto glandulosus, basi subrotundatus - Jura, Mt-Cenis (Fray). . . . .865. O. camptopoda Gdgr mss Fr. oblongus toto hispidissimus, basi attenuatus - Pyren. or. Ml-Louis (Debx). . . . 866. O. echinulata Gdgr mss Fr. obiongus inferne laevis et longissime attenuatus - Sabaudia, Habère-Lullin (Puget). . .867. O. Interposita Gidgr mss Fol. ovato-obtusa vel obovata ......... 132

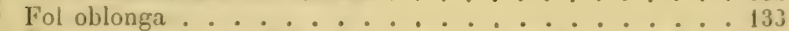
Tubus basi glandulosus; fol. ovata - Sabaudia, Mt-Salève

13: (Rap.) (Rap.) Tubus toto glandulosus; fol. obovata - Helv. Bex, m. Bovonnaz (Thomas). . . . . . 869. O. EfFugtens Gdgr mss

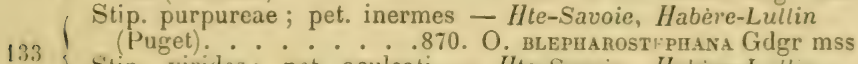
Stip. virides; pet. aculeati - IIte-Savoie, Habère-Lullun (Puget) . . . . . . 871. O. setisepala Gdgr mss Fol. subtus ad nervos villosa. . . . . . . . 135

13: Fol. supra glabra subtus toto villosa . . . . . . 141

Fol. undique villosa . . . . . . . . . . . . 148

Aculei nulli vel subulati. . . . . . . . . 136

13. Aculei plerumque falcato-dilatati - Caucasus orient.872 ............... oxyodon (Boiss.) Sepala extus glandulosa. . . . . . . . . . 137

136 Minime; fol. parva oblonga vel suboblonga; fr. rectus. . 140 Minime; fol. late obovata; fr. subnutans - Ilelv. Jura, Chaumont (Sire). . . . . .873. O. cuneata Gdgr mss

Fol. biserrala ........... 138 Fol. inaeque serrata; fr. ovoideus subglandulosus - Jura, $\dot{L a}_{a}$ Dóle (F. Orbanis). . . . 874. O. Onbanislaxa Gdgr mss

Fol. uniserrata; fr ; ovatus laevis - Rossiabor. (R. acicularis b. stipularis Rupr.) . . . . 875. G. LISsINessis Gdgr mss Tubus laevissimus . . . . . . . . . . . . 139 'Tubus basi hispidus; ped. 18-20 m. 1. - Helv. Winterthur (Hohenacker). . . . . . 876. O. BnEvifnoss Gidgr 'Tubus in 1/2 inf. hispidus; ped. 10-11 m. 1. - Austr. sup. m. Traunfall (Keck). . . . 877. O. austriaca Gdgr mss Fol. inferiora ad nervos glandulosa - Pyrenaei centr. Gavarnie (Bordère). . . . . . 878. O. Bondent Gdgr mss Minime - Montenegro, m. Durmitor (Pantocsek).879. O. Mus- 
$140\left\{\begin{array}{l}\text { lers (Fl Sequan. exs. n. } 439 \text { parle).880. O. Mrrtula Gidgr mss } \\ \text { Caules inferne aculeati ; sep. linearia - Basses-Alpes, }\end{array}\right.$ Tournoux (Proal) .....881. O. BREvifolia Gdgr mss

Fr. obovatus vel oblongus. . . . . . . . . 142

Fr. ovatus - Rossia, S. Petersbourg (Meinshausen fl. Ingrica

141 exs. n. 216). . . . 882. O. Petersburgensis Gdgr mss Fr. ovato-rotundatus - Bohemia (Sieber Herb. austr. n. 447; ej. Dendr. exs. n. 56). . . . . 883. O. PresliI Gdgr

142 Fr. laevis ....................... 143

Fr. glandulosus . . . . . . . . . . 147

Sep. omnino eglandulosa . . . . . . . . . . 144

143 Sep. in $2 / 3$ sup. glandulosa,. .146

Sep. extus toto glandulosa - Lapponia, Pitea (Ándersson, etc.). . . . . . 884. O. LAPPONICA Gdgr msS

$144\left\{\begin{array}{l}\text { Rami flor inermes } \\ \text { Rami flor. densissime aculeati - Finlandia bor. Kuopio }\end{array}\right.$ (Boldt). . . . . . . 885. O. FenNica Gdgr mss Ped. 16-19 m. 1.; fr. oblongus - Basses-Alpes, Uvernet (Proal) . . . . . 886. O. PILosula Gdgr mss

145 Ped. $35-40$ m. 1.; fr. ovoideus - Basses-Alpes, Tournoux (Proal) . . . . 887. O. Tenurpubens Gdgr mss Ped. $25-30$ m. l.; fr. oblongus - Hung. Heves, m. Bagolyko (Vrabely). . . . . . 888. O. Vrabelyi Gdgr mss Rami flor. inermes - Hung. Matra (Vrabely).889. O. Hirsuta $146\left\{\begin{array}{l}\text { Rami flor. valde aculeati - Finlandia bor. Kuopio (Enwald) } \\ \text { Ras }\end{array}\right.$

. . . . . . 890. O. Enwaldil Gdgr mss Stip. subtus valde glandulosae; fol obovato-oblonga - Hung. Parad, etc ..... 891. O. Kitaibelin Gdgr mss

147 Stip. dorso glandulosae; fol ample oblonga - Croatia, Samobor (Vuk.). . . . 892. O. Vukotinovici Gdgr mss Stip. eglandulosae; fol anguste oblonga - Silesia, Langwaltersdorf (Christ) ..... 893. O. PuBEscens Gdgr mss Fol. apice exaristata . . . . . . . . 149

148 Fol. apice aristata - Pyren centr. Barèges (Lap.) 894.

- . . . . . . . aristata (Lap.)

149 Fr. ovatus vel ovato-ellipticus . . . . . . 150

Fr. ohovato-oblongus vel oblongus. . . . . 152

Fol. uniserrata; aculei subulati. . . . 151

150 Fol. biserrata; aculei robusti - Pyren. centr. Barèges (R. parviflora (Lap.) … . . 895. O. LAPEynousil Gdgr

$151\left\{\begin{array}{l}\text { Fol. ovato-aruta - Mt-Oural; Sibiria (Eschscholtz pl. sib. } \\ \text { exs.!). }\end{array}\right.$ Fol. obovato-obtusa - Sibiria, Irkutzk (Augustinowicz).897.

. . . . . . . O. Ircutiana Gdgr mss Ped. tantum glandulosi vel parce pubentes Piberia, Ussuri (R. tomentosa Rgl. fl.
Ped. tomentosi - Sibe

Ussur.). . . . . 898. O. Desertonum Gdgr

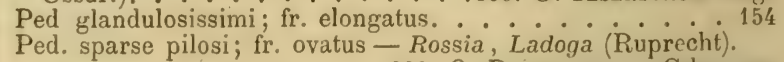

153 Ped. sparse pilosi; fr. ovatus - Rossia, Ladoga (Ruprecht). Fol. biserrata; rami inermes ......... 155

$154\left\{\begin{array}{c}\text { Fol. uniserrata; rami aculeati - Scand. bor.; Rossia bor } 900 \\ \ldots \ldots\end{array}\right.$ 


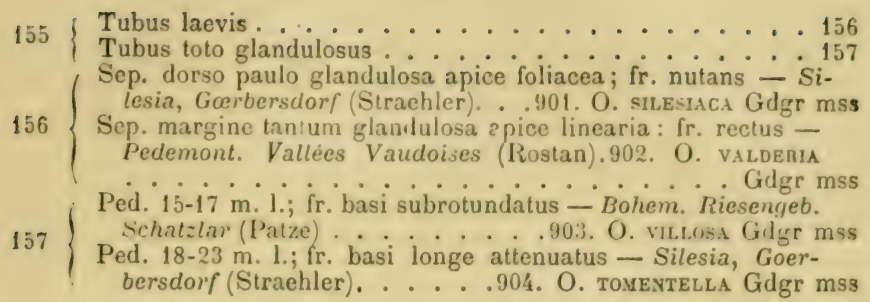

\section{2. - Montanae Gdgr Essai, p. 23.}

Frutices plus minus robusti sociales glaucescentes, rami rigidi glauci aculeati; aculei conformes majusculi falcatodilatati sparsi; radix haud reptans; foliola crassa opaca glaucescentia coriacea, subtus prominule nervosa; sepala plus minus pinnata, post anthesim erecto-conniventia, rarissime serius subdecidua; corolla majuscula alba vel purpurea; flores late bracteati, corymbosi breviterque pedunculati; styli plerumque lanati ultra discum planum vel conicum capitati liberi ; fructus ordinarie magnus subcoriaceus erectus terminalis. - Tribus haec intermedium praebet inter Ozanonias (Alpinae) et Crepinias (Canineae) sed plerumque istis magis affinis, ita ut, nihil nisi earum gregem peculiarem atque valde curiosam sit.

1 Fol. inaeque serrata ............. 58

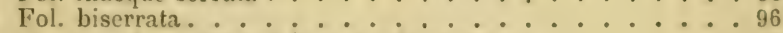

2 Costa media subtus glabra et eglandulosa . . . . . . . . 3

2 Costa sulitus glandulosa aciculata vel villosa. . . . . . 46

3 Pet. glaberrimi .. . . . . . . . . . . . 4

3 Pet. sparse pilosi vel villoso-glandulosi. . . . . . . 17

4 Fr. globosus ................ . 5

4 Fr. ovoideus vel oblongus ............ 10

5 Sep. omnino eglandulosa. . . . . . . . . . . 6

5 Sep. I glandulosa. . . . . . . . . . . . . 7 
Fol, obovato-oblonga; sep. apice linearia - Istria, Mi-Maggiore (Freyn). . . . . . 905. O. 1striACA Gdgr mss Fol. elliptico-obovata; sep. dilatata - Sabaudia, Hauteluce (Billot exs. n. 3581). . . . .906. O. conjugata Gdgr mss Sep. margine glandulosa; fol. basi attenuala. . . . . 8

Sep. dorso glandulosa; fol. basi sensim attenuata. . . . . . 9

Sep. apice tantum glandulosa; fol. basi rotund. - Suecia, Scania, m. Kullaberg (Tullberg). 907. O. RuberRima Gidgr mss Sep. dorso glandulosa; fol. rotundata - Alp. Pyren. (Billot n. 1183). . . . . . . 908. O. RuBRifolia (Vill.) Rami flor. inermes; ped. 15-18 m. 1. - Isère, Villard de Lans (Soc. dauph. exs. n. 116). . . .909. O. FARINosa Gdgr mss Rami aculeati ; ped. 8-10 m. I. - Delph. La Grave (Reliq. Maill. n. 1087 parte) . . 910. O. Mathonneti Gdgr mss Glauco-purpurea ; fr. ovato-rotundatus - Gall. Puy-de-Dômẹ (Lamotte) . . . . . . 911. O. LamotTi Gdgr mss Glauca; fr. globosus - Pedemont. Vallées Vaudoises (Rostan).

. . . . . 912. O. Cerasifera Gdgr mss Saturate glauco-purpurea; fr. ovatus - Croatia, Ogulin (Vukotinovic). . . . 913. O. Schlosseriana Gdgr mss Ped. omnes laeves. . . . . . . . . . . . 11 Ped. omnes glandulosi............. 16 Ped, alii laeves alii glandulosi - Tyrol, Pusterthal (Schonach) . . . . . . 914. O. TYRolensis Gdgr msS Fr. ovoideus vel obovato-oblongus . 12 Fr. oblongo-conicus - Gall. Puy-de-Dôme (Malarbet). 915. . . . . . . . . . O. conocalyx Gdgr mss Sep. dorso glandulosa .............. 13

12 Sep. omnino eglandulosa ............... 14 Sep. margine glandulosa - Doubs, Villers-le-Lac (Fl. Sequaniae exs. n. 440). . . . . 916. O. vinosa Gdgr mss Rami flor. aculeati; dentes courergentes - Pedem. Vallées Vaudoises (Rostan). ... . 917. O. pedemontana Gdgr mss Rami flor. inermes ; dentes apertae - Helv. Jura, Couvet) (Baenitz herb. eur. a. 1879) . . 918. O. Helvetica Gdgr mss Fol. oblonga; sep. apice filiformia. . . . . . . . . 15 Fol. ovato-elliptica; sep. apice angusta - Hung. Hradeh (Ilse).

14 Fol. minute obovato-obtusa; sep. angusta - Angl. Surrey Barnes (Groves). . . . . . .920. O. GrovesiI (Baker) Fol. late obovata; sep. foliacea - Hte-Savoie, Bellevaux (Puget). . . . . . 921. O. sciacephala Gdyr mss Ped. 6-8 m. l.; quaedam dentes inaequales - Saxonia, Haardorf (Haussknecht) . . . .922. O. Pallidifolia Gdgr mss Ped. 10-12 m. l.; dentes omnino simplices - Galloprov. Aiguines (Albert). . . . . 923. O. Provincialis Gdgr mss Sep. integerrima; tubus et ped. laevia - Alp. Sabaudiae 16 Bell. in Mem. acad. Turin 1790, p. 229 tab. 6).924. O. Sep. pinnata ; ped tubusque glandulosa - Jura, Cütes de StCergues (Gaud. fl. helv. IIl, p. 347) . .925. O. Decrosil Gdgr Fr. globosus. . . . . . . . . 18

Fr. rotundatus vel ovatus. $\ldots 22$ Fr. ovoideus obovato-oblongus rel oblongus. . . . . . 27 
Ped. laeves .......... . 19

Ped. glandulosi . . . . 20

Sep. ylandulosa; fol. elliptico-ovata - Angl. (Baker exs.

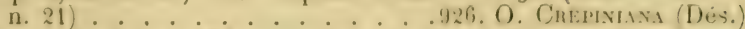
Sep. oglandulosa; fol. orlsiculata - Gall. Bomeges (Sichultz herb. norm. n. 43). . . . . .927. O. Schultzi (Rip.)

Sep. dorso glandulosa; fol. obovato-oblonga - Hte-Savoie, Bellevaux (Puget). . . . . . 928. O. cxcua Gdgr mss

Sep. eglandulosa vel 2 extus sparse muricata. . . . . . . 21

Sep. omnia dense glandulosa - Sabaudia, Annecy (Puget).

Sep. apice tantum glandulosa - Suecia, Scania ('Tullberg).

.....930. O. pulchenrima Gdgr mss

Fr. globosus; fol. apice rotundata - Ilte-Savoie, Reyvroz

(Puget) . . . . . . 931. O. actinosirpala Gidgr mss

I'r. ovato-rotundatus; fol. attenuata - Ilte-Savoie, Reyvroz

(Puget). . . . . . . . .932. O. propris Gdgr mss

. Fol. utrinque aut saltem basi attenuata . . . . . . 23

Fol. utrinque rotundata - Pyren. centr. Gédre (Bordère).

......... . 933. O. macrostepuara Gdgr mss

Ped. paulo glandulosi . . . . . . . . . . . 24

Ped. laeves. . . . . . . . . . . 25

Tubus laevis; sep. margine glandulosa - Tyrol Trins

(Kerner). . . . . . . . 934. O. Kenveri Gdgr mss

Tubus paulo glandul.; sep. dorso glandul. - Ital. Apenn. Modène (Gibelli). . . . . . . . 935. O. Gibelli Gdgr mss

Sep. pinnata .....

Sepala integerrima - Ain, Songieu (Bichet).936. O. Bricueті . . . . . . . . . . . . . Gdgr mss Sep. eglandulosa; fol. elliptico-lanceolata - Belg. Namur Gravet). . . . . . . 937. O. Gravetil (Crép. mss)

Sep. margine glandulosa; fol. oblongo-retusa - Gard, Camprieux (Anthouard) . . . . 939. O. cebennensis Gdgr mss Sep. dorso glandulosa; fol. oblongo-acuta - Delphl. La Grave (Reliq. Maill. n. 1087 parte). .1'0. O. nermminessis Gderr mss Sep. extus glandulosa . . . . . . . . . . 28

27 Sep. eglandulosa; pet. et ped. gland - Suecia Scania, Nobbelof (Lundquist) . . . . .941. O. ADENopodA Gdgr mss Sep. laevia vel margine tantum glandulosa. . . . . . . 29 Ped. alii laeves alii glandulosi — Loire, MI-Pilat (Malarbet) Ped. omnes glandulosi - Gall. Puy-de-Dóme (F. Héribaud) .................... O. elaners Gidgr mss Pet inermes. . . . . . . . . . . . . . . 30 Pet. aculeati. .................. 41

30 Fructus oblongus . . . . . . . . . . . . . 31

Fr. obovatus. . . . . . . . . . . . . . . 35

31 lol olovata. . . . . . . . . . . . . . 32 Fol oblonga............................ 34

( Sep. apice angustata, fr. amplissimus. . . . . . . . 33

39 Sep. dilatato; fr. mediocris-l'edem. Mt-Cenis, Lans-leBourg (Morthier). . . . 944. O. PEdemontaxa Gdgr mss 
Pet. aculeati ; sep. valde pinnata - Delph. La Grave (Reliq. Maill. n. 1082 parte) ‥945. O. HeterocarPa Gdgr mss Pet. inermes; sep. parce pinnata - Gall. Orient.946. O. GRA-

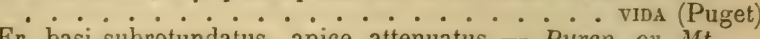
Fr. basi subrotundatus, apice attenuatus - Pyren. or. Mt-

34 Louis $(\mathrm{Debx}) \ldots . . .947$. O. Hastulata Gugr mss Fr. basi longissime attenuatus, apice turgidus - Scania, Pinelierna (Lundquist). ... 948. O. cLarata Gdgr mss

Fol. elliptico-obovata vel obovata. . . . . . . . 36

Fol. oblonga ................ . . 37

Fol. obovato-oblonga . . . . . . . . . 38 Styli glabri vel glabrescentes - Ardèche, Le Mezenc (Malar$36\left\{\begin{array}{l}\text { bet) } \\ \text { Styli sublanati - Helv. Fribourg, La Cernioz (Cottet).950. O. }\end{array}\right.$

*........ Bombax Gdgr mss Albo-glauca; ped. 4-6 m. 1. - Pedemont. Vallées Vaudoises

37 Virens ; ped. $9-10$ m. l. - Pyren. orient. Mt-Louis (Debeaux)...........952. O. Pelleti (Debx)

Fol. basi rotundata ................... 39

Fol basi attenuata . . . . . . . . . 440 Styli conico-capitati ; auriculae divaricatae - Suecia, Scania

39 Elleholm (Scheutz).....953. O. conostyla Gdgr mss Minime; auriculae convergentes - Helv. Fribourg, La Cernioz (Cottet). . . 954. O. вомвах C. Cotteti Gdgr mss Ramı flor. inermes; fr. basi rotundatus - Suecia Smol. Calmar (Lundquist). . . . . 955. O. LEIOCLADA Gdgr mss Rami flor. aculeati; fr. basi decurrens - Suecia Smol. Wexio (Scheutz). . . . 956. O. Atrovirens Gdgr mss Rami parce aculeati; fr. basi depressus - Suecia Westrog. Kinnekulle (Lundquist) . . .957. O. AcipHyLloines Gdgr mss Fol. obovata vel obovato-elliptica . . . . . . . . 42 Fol. oblonga ............... . . 45

4 Ped. omnes glabri. . . . . . . . . . . 43 Ped. alii villosi alii glandulosi - Helv. Vaud, St-Cergues (Favrat) . . . . . . 958. O. DimorPHA Gdgr mss Fr. ovoideus; styli sublanati . . . . . . . . 44

Fr. anguste oblongus; styli paulo villosi - Delph. Gde-

43 Chartreuse (Gdgr) . . . . 959. O. Fagetorum Gdgr mss Fr. amplissime oblongus; styli lanati - Basses-Alpes, Bouzolières (Proal) . . . . 960. O. ventricosa Gdgr mss Discus planus; fol. anguste oblongo-acuta - Ital. Pistoje Boscolungo (Gibelli). . . . .961. O. ITALiCA Gdgr mss

44 Discus planus; fol. ample obovato-acuta - Basses-Alpes, Bouzclières (Proal). . . .962. O. Macrosepala Gdgr mss Discus subconicus; fol. obovato-elliptica - Helv. Fribourg, La Cernioz (Cottet) .963. O. Bombax B. Aculeolata Gdgr mss Rami flor. subinermes; fr. oblongus basi depressus - Suecia Vestrog. Kinnekulle (Lundquist) .964. O. Bonealis Gdgr mss

45 Rami flor. valde aculeati ; fr. ovoideus, centralis valde decurrens - Hte-Savoie, Habère-Poche (Puget). 965. O. SPINI- 
Fol. subtus ad costam glandulosa . . . . . . . 47

Fol. subtus ad nervos villosa . . . . . . . . . 54

Fol. subtus ad costam villosa. . . . . . . . . 55

Fol. subtus omnino villosa - IItes-Alpes, Villard d'Arène

(Chabert). . . . . 966. O. crataegina Gidgr mss

Ped. omnes laeves. . . . . . . . . . . . 48

Ped. omnes paulo glandulosi . . . . . . . . . 51

I'ed alii laeves alii 1-3-glandulosi - Suecia Vestrog. Mariestad (Lundquist). . . . .967. O. scandica Gdgr mss Sep. glandulosa............... 49

Sep. laevia valde foliacea ......... . 50

Fr. ovatus basi rotundatus; fol. oblongo-lanceolata - Loire, Pilat, (Malarbet). ....968. O. Gymnopoda Gidgr mss

Fr. ovatus basi attenuatus; fol. oblongo-acuta - Valais, Saas (Woll). . . . . 969. O. punpurans Gdgr mss

Fr. ovoideus, basi rotundatus; fol. ovato-acuta - Valais, Martigny (Wolf). . . 970. O. Latibasis Gdgr mss Fr. oblongus; rami flor. valde aculeati - Suecia Eland,

50 Tveta (Scheutz) . . . . .971. O. Calycisa Grlgr mss Fr. ovoideus; rami inermes - Ital. Apenn. Modène, Boscolungo (Gibelli). . . . . . 972. O. Mutinensis Gdgr mss

$51\left\{\begin{array}{l}\text { Tubus oratus } \\ \text { Tubus obovato-oblongus } \ldots\end{array}\right.$ Tubus obovato-oblongus ; fol. ovato-subrotundata - Helv.

5 ( Vaud, Jerogne (Thomas). . . .973. O. BINATa Gdgr mss Sep. laevia ; fol. oblonga - Carniolia, Mt-Nanos (Borbas).

. . . . . 974. O. carniolica Gdgr mss Discus conicus ; pet. sparse pilosi - Sabaudia, Mt-Salève Discus planus; pet. basi minute villosi - Helv. Fribourg, Montbovon (Cottet) . . . . 976. O. DRYophila Gdgr mss Ped. laeves; rami flor. inermes - Hte-Savoie, MIt-Sion

54 (Puget) . . . . 977. O. sericonateva Gdgr mss Ped. sulglandulosi ; rami aculeati - Hte-Savoie, Mt-Sion (Puget). . . . . 978. O. rubroxotata Gdgr mss

5 5. Ped. glandulosi . . . . . . . . 56

Ped. laeves . . . . . . . . . . . . 57 Fr. subglobosus ; fol ovata - Austria - 979. O. LIvids (Host)

36 Fr. oblongus; fol. anguste oblongo-lanceolata - Helv. m. Lavarraz Bex (Thomas) . . . . 980 O. Ludexs Gdgr mss Flores 60-80-corymbosi - Helvetia (non vidi).981. O. LURIDA

Flores 1-4-ni ; fr. globosus ; sep. glandulosa - Helv. Fribourg - . 982. O. ontwosepala (Schimp. et Spen.) Flores 1-4-ni ; fr. oblongus; sep. laevia - Delph. Gde-Chartreuse (Gdgr). . . . . 983. O. condexsata Gdgr mss Pet. glaberrimi . . . . . . . . . . . . 5?

I Pet. I villosi vel glandulosi ............. . . . . . . .

59 Fr. obovoideus vel suboblongus. . . . . . . 60

Fr. rotundatus sub subglobosus. Serchate Geneveys, ete.
Ped. fr. et sep. hispida - Helv. Neuchat Minime - Lyon; Sabaudia, etc . . 985. O. Falcata (Pug.) 
Ped. tubus et sep. laevia. . . . . . . . . . 62

61 Ped. tubus et sep.glandulosa - Hte-Savoie, Bellevaux (Puget).

.........986. O. trinsiens Gdgr mss Fol. ovato-obtusa basi rotundata - Europa centr.987. O.

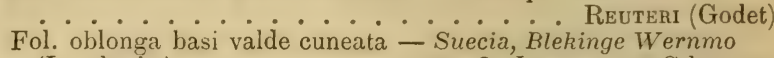

988. O. Lundeuistil Gdgr mss

63 Fr. globosus vel subglobosus. . . . . . . 64 64

63 Fr. ovatus ovoideus vel oblongus . . . . . . . 69

64 Sep. laevia ................ 65

64 Sep. I glandulosa. . . . . . . . . . . 68

65 / Fol oblonga ................ 66

Fol. obovato-oblonga - $\dot{S}^{-} \dot{\text { Vestrog. Sattra (Lundquist) }} 67$

66 . . 9 . O. Fallacina Gdgr mss

Rubens ; pet. eglandulosi - Hte-Savoie Brenthonne (Puget).

. . . . 990. O. pseudo-Gerasus Gdgr mss

Fol. basi rotundata; pet. aculeati - Hte-Savoie, Habère-Poche

(Puget) . . . . . . 991. O. вомBycifera Gdgr mss

Fol. basi subcuneata; pet. subinermes - Hte-Savoie, Bellevaux (Puget). . . . . . 992. O. REFracta Gdgr mss

Ped. parce glandulosi ; pet. eglandulosi - Hte-Savoie, Habère-

Poche (Puget). . . . . . 993. O. setipes Gdgr mss

Ped. laeves; pet. sparse glandulosi - Hte-Savoie, HaberePoche (Puget) . . . . . 994. O. Adversa Gdgr mss

( Sep. ad margines glandulosa. . . . . . . . . . 70

69 Sep dorso glandulosa . . . . . . . . . 72

Sep. omnino eglandulosa. . . . . . . . . . . 85

Fr. oblongus .................... 71

Fr. obovato-oblongus; ped. 12-14 m. 1. - Sabaudia, MtSalève (Guinet). . . . . .995. O. Rarispina Gdgr mss

Fr. ovatus; ped. 7-9 m. 1. - Suecia, Vestrog. Kinnekulle (Zetterstedt). . . . . 996. O. Macrocarpa Gdgr mss

Ped. 12-14 m. 1.; fol. ovato-elliptica - Helv. La Dôle, m. Bauloz (Favrat). . . . 997. O. Marginata Gdgr mss Ped. 2-5 m. l.; fol. oblonga - Savoie, Mt-Sion (Puget).998.

........... O. alpivaga Gdgr mss

Fr. obovato-oblongus .......... 73

Fr. ovatus . . . . . . . . . . . . 74

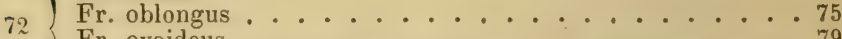

Fr. ovoideus . . . . . . . . 79

Fr. amplissime globosus - Angl. Warwich, Solihull (Fraser)

. . . . . . . 999. O. MacrosphaERA Gdgr mss

Ped. laeves; discus planus - Hte-Savoie, Habère-Lullin

73 (Puget) . . . . 1000. O. stictochlamys Gdgr mss Ped. subaculeati; discus sat conicus - Istria, Mt-Maggiore (Freyn) . . . . . . . 1001. O. UBERTA Gdgr mss Styli subglabri ; fol. late ovato-acuta - Helv. Vaud, Leysins

74 (Favrat) : . . . . 1002. O. ovata Gdgr mss Styli hirsuti ; fol obovata apice rotundata - Gall. Mts Dores, Cantal, etc. . . . . 1003. O. ARvernensis Gdgr mss Fol. obovata vel obovato-oblonga; pet. | hirsuti. . . . . 76

75 Fol. ovato-elliptica ; pet. glandulosi. . . . . . 78 Fol. obovato-oblonga ; pet. egiandulosi sparse pilosi - Dania, Helleboek (Lundquist). . . . 1004. O. DANiCA Gdgr mss 
Fol. oblongo-acuta; pet. inermes glandulosi - Suecia, Wexio (Scheutz). . . . . 1005. O. Scneurzir Gdgr mss Ped. laeves . . . . . . . . . . . . . 77 Ped. hispidi; rami flor. aculeati; aculei falcati - BassesAlpes, Bouzolieres (Proal). . 1006. O. Chaixiana Gidgr mss Ped. hispidi ; rami inermes; aculei subulati - Silesia, Landeshut (Hoger; O. salaevensis f. Uechtritziana Christ in Jahr. Schl. Cult. 1876, p. 135).1007. O. Uechтнiтzi Gdgr mss Costa eglandulosa ; pet. subinermes - Scotia Perth, Seggieden (1)rummond) . . . . . 1008. (). Balformana Gidgr mas Costa glandulosa; pet. aculeati - Suecia, Eland, Tveta (Scheutz). . . . . . 1009. O. ERrostyla Gdgr mss Rami flor. inermes; ped. 8-10 m. 1. - Basses-Alpes, Bouzolières (Proal) . . . . . 1010. O. Ficoldes Gidgr mss Rami flor. aculeati ; ped. 12-15 m. 1. - Cantal, Le Lioran (F. Héribaud). . . . . 10l1. O. cantalica Gugr mss Perl. | glandulosi. . . . . . . . . . . 80 Ped laeves . . . . . . . . . . . 82 Rami flor. parce aculeati ; fol. obovata . . . . . 81 Rami flor. inermes; fol. elliptica utrinque rotundata - Creta, Sfakia (Sieber) .1012. O. sfakiensis Gdgr mss Rami aculeatissimi; fol. oblonga utrinque attenuata - Suecia, Scania Kironovall (Tullberto). . 1013. O. Abevophona Gidgr mss Tubus basi laevis - Suecia, Vestroyot'?. Sattra Luntquist). Tubus basi selosus - Basses-Alpes, Bouzolières (Prial). . . . . . . . 1015. O. calcarea Gdgr mss Fol. obovata vel elliptica. . . . . 83

82 Fol. anguste lanceolata - Gall. Auvergne (Malarbet). 1016. . . . . . . . . O subsessilis Gdgr mss

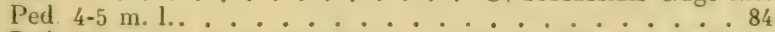
Ped. 7-9 m. 1.; costa glandulosa - Suecia Vestrog. Sattra (Lundquist). . . . . 1017. O. polychada Gdyr mss Ped. 9-11 m. l.; costa eglandulosa - Sabaudia, eic. (Dès. herb. n. 16). . . . 1018. O. Caballicensis (Pug.) Fr. amplissimus; sep. apice paulo glandulosa - Basses-

84 Alpes, Bouzolièes (Proal) 1019. O. macrostigka Gidgr mss Fr. mediocris; sep. toto glandulosa - Wurtemb. Donnstelten (Kemmler). . . . . 1020. O. setisepala Gdgr mss Fol. ovato-elliptica. . . . . . . . . . 86 Fol. obovata vel oblonga . . . . . . . . . 87 Fr. ovato-rotundatus - Suecia med. Storgarden (Zetterstedt). . . . . . 1021. O. platystigma Gdgr mas Fr. obovato-oblongus; fol. basi sensim rotundata - Brunswick, Nussberg (Braun). . . . 1022. O. Braunir Gdgr mss Fr. ovoideus apice vix attenuatus; fol. basi cordata - Suecia Vestrog. Kinnekulle (Zetterstedt, etc.).1023. O. PExiolulata . . . . . . . . . . . . Gdgr mss Fr. ovoideus apice valde attenuatus; fol. basi rotundata Isère, Le Sappey (Boullu). .1024. O. nuxwenocalyx Gidgr mss Fol. obovata vel obovato-oblonga . . . . . . . 88 Fol. oblonga vel lanceolata. . . . . . . . . . . 93 Fol. elliptica - Loire, Mt-Pilat (Gdgr).1025. O. striexs Gdgr mss 
Aculei | dilatato-adunci . . . . . . . . . 89

Aculei recti subulati - Sabaudia, Salève, Brezon (Billot exs. n. 3583). . . . . 1026. O. salaevensis (Rap.) Aculei tenuiores parum falcati ; ped. laeves . . . . . 990 Aculei magni; ped. interdum glandulosi - Loire, Mt-Pilat (Malarbet). . 1027. O. SAlaev. f. aculeis recurvis Gdyr msS Fr, ovoideus ................... 91 Fr. oblongus . . . . . . . . . . . 92 Fr. ovatus - Suecia Vestrog. m. Kinnekulle (Zetterstedt). . . . . . . 1028. O. Zetterstedtir Gdyr mss Fol. obovato-oblonga basi sensim rotundata - Scotia, Fife (Boswell Syme). . . . . . 1029. O. Symir Gdgr mss Fol. obovata, basi sensim attenuata - Hte-Savoie, HabèrePoche (Puget) . . . . 1030. O. semidecidua Gdgr mss Discus conicus ; ped. 11-13 m.1.-Vallesia, Martigny (Wolf) . . . . . . . 1031. O. cuspidens Gdgr mss Discus planus ; ped. $4-5$ m. 1. - Suecia Smol., Dref (Hylten Cav.). . . . . . . 1032. O. PILOSA Gdgr mss Fol. oblongo-lanceolata; fr. basi rotundatus vel subdepressus 94 Fol. oblongo-lanceolata; fr. basi valde contractus . . . 995 Fol. apice rotundata; fr. basi attenuato-decurrens - HteSavoie, Habère-Lullin (Puget) .1033. O. Incrassans Gdgr mss Fr. oblongus basi subdepressus - Pyren. orient., Mt-Louis (Debeaux). . . . . 1034. O. NERvifolia Gdgr mss Fr. ovoideus basi depressus - Istria, Mt-Maggiore (Freyn) . . . . . . 1035. O. Haceuetil Gdgr msis Fr. ovoideus basi rotundatus - Rhone, Francheville (Boullu) . . . . . 1036. O: Salaevensis f. Boulluil Gdgr mss Dentes plerumque compositae ; ped. $21 / 2-3 \mathrm{~m}$. 1. - Suecia, Wexio (scheutz). . . . 1037. O. smolandica Gdyr mss Dentes plerumque compositae; ped. 8-10 m. 1. - Aragonia aust. Sierra de Mosqueruela (Martin).1038. O. ARAgonensis . . . . . . . . . . . Gidgr mss Dentes inf. tantum compositae; ped. 5-6 m. 1. - Suecia, Kalmar (Scheulz, etc.). . . . 1039. O. Baltica Gdgr mss Fol. subtus ad costam glabra vel glandulosa . . . . . 97 Fol. subtus ad costam villosa . . . . . . . . . 162 Fr. globosus vel rotundatus . . . . . . . . . 98 Fr. ovatus ovoideus vel oblongus . . . . . . . . 107 Ped. I glandulosi . . . . . . . . . . 999 Ped laeves . . . . . . . . . . 102 Pet. paulo pubescentes . . . . . . . . 100 Pet. glabri - Carinthia, Malnitz, Taurer.1040. O. GLAuces-

Ped. sparse glandulosi ; sep. dorso glandulosa - c cens (Wulf) Ped. et sep. dense glandulosi - Anı,l. Cheshire, Thingwall (Harbord). . . . . 1041. O. Micromegas Gdgr mss Ped. dense glandulosi ; sep. sublaevia - Pyren. centr., Héas 10. Crepini Mlieg. non Dés.) .1042. O. Miegevillei Gdgr mss Fol. ovato-elliptica; pet. aculeati - Angl. Keld Swaldale (Baker). . . . 1043. O. pseudo-subcristata Gidgr mss Fol. obovato-oblonga; pet. inermes - Angl. Warwich, Solihull (Fraser). . . . 1044. O. Conglobata Gdgr mss Sepala extus glandulosa .............. . . . . . . . . . Sep. laevia ........................... 
103 Aculei basi dilatati - Angl. Yorkshire, etc.1045. O. SubCrisMinime - Sabaudia. etc. (Dés herb. n. 64 ) 1046. O. Impo-

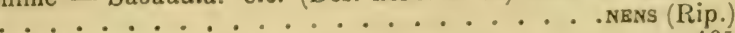

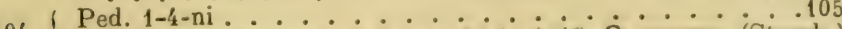
Ped. 15-20 corymbosi - Istria, Idria.1047. O. vestira (Sternb.)

Fol obovata

105 Fol. orbiculata - Ite-Savoie, St-Gervais (Puget) 1048.

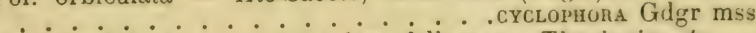
Pet. glandulosi; sep. apice late foliacea - Thuringia, etc. $106\left\{\begin{array}{l}\text { (Zabel). } \\ \text { Pet. eglandulosi ; sep. apice angustata- Isere, Villard de } \\ \text {. } 1050 \text {. O. Crassispiva Gdgr mss }\end{array}\right.$ Lans (Boullu). . . . 1050. O. crassispina Gdgr mss

107 Ped. glabri vel villosi . . . . . . . . . 108

$107\{$ Ped. glandulosi . . . . . . . . . . . 119

$108\left\{\begin{array}{l}\text { Fr. ovatus. ovoideus obovato-oblongus vel oblongus } \ldots 109 \\ \text { Fr. } \ldots 11\end{array}\right.$

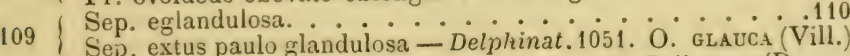
Fr. ovoideus; ped. 12-20 m. 1. - Hte-Savoie, Bellevaux (Puget). . . . . . . 1052. O. PERsonata Gdgr mss

110 Fr. ovatus; ped. 7-8 m. 1. - Suecia, Carlscrona (Scheutz) Fr. ovato-ellipticus; ped. $2-4 \mathrm{~m}$. 1. - Thuringia, Weimar (Haussknecht) . . . . . 1054. O. sessilis Gdgr mss

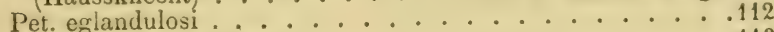

11 Pet. sparse glandulosi . . . . . . . . . . . . . . . . . . . .

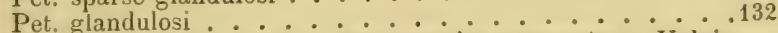
Fr. ovoideus; fol. oblonga basi sensim attenuata - Valais,

112 Bovernier (Wolf). - * - 1055. O. Montosicola Gdgr mss Smol. Moheda (Hyltén). . . . 1056. O. E.cLINENs Gdgr mss

113 Rami flor inermes. . . . . . . . . . 114 Rami flor aculeati. . . . . . . . . 116

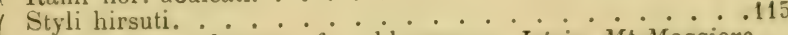
Styli paulo pubentes; fr. oblongus - Istria, Mt-Maggiore (Freyn) ....... 1057. O. FreryI Gdgr mss Styli glabri ; r. oblongus - Var, Ste-Baume (Roux). 1058.

. Sanctae Balmae Gdgr ms Styli glabri ; fr. ovoideus - Angl. Hereford Wormbridge Leg). . . . . 1059. O. Leiostrla Gdgr mss Fr. obovoideus; fol. ovata - Valais (Lagger, etc). 1060.

. . . . Delasoli (Lagg. et Pug.) Fr. elongatus; fol. oblonga - Sabaudia, Mt-Salève (Guinet). 1061. O. Grandifolia Gdgr mss Discus planus; fr. oblongus . . . . . . . 117 Discus planus fr. ovoideus. . . . . . . . . . 118

116 Discus valde conicus; fr. oblongus - Aragonia austr. Sierra de Mosqueruela (Martin). . . 1062. O. Hispanica Gdgr mss Ped. $15-18$ m. 1.; rami flor. aculeati - Cantal, Le Lioran $117\left\{\begin{array}{l}\text { (F. Héribaud). Póni flor. inermes - Austria, Znaim (Oborny) } \\ \text { Ped. 6-8 m. l.; rams }\end{array}\right.$

.1064. O. MORAvica Gdgr mss 
Ped. 3-4 m. 1.; fol. obovato-oblonga - Thuringia, Rudolstadt

$118\left\{\begin{array}{l}\text { (Dufft) } \\ \text { Ped. } 8-9 \text { m. l.; } \text { fol. ovata - 1065. O. Thuecia Vestrog. Kinnekulle }\end{array}\right.$ (Zetterstedt). . . . . 1066. O. cuspidata Gdyr mss

Discus planus. . . . . . . . . . . . 120

119 Discus valde conicus - Italia, Mit-Cenis (Fray). $1067^{\circ}$ O. ALPI. . . . . . . . . . GENA Gulgr mss

120 Sep. inferne ad margines glandulosa. . . . . . . 121

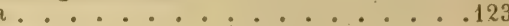

Sep. omnino eglandulosa. . . . . . . . . . 125

$121\left\{\begin{array}{l}\text { Fr. ovoideus. } \\ \text { Fr. oblongus - Suecia Scania, Wollsjo (Tullberg) } 1068 .\end{array}\right.$ ........... . . . . Tullbergil Grdgr mss Sep. apice linearia; fol. basi subattenuata - Wurtemb.

122 Donnstetten (Kemmler). . . 1069. O. opacifolia Gdgr mss Sep. valde foliacea; fol. basi rotundata - Suecia Vestrog.

Kinnekulle (Zutteret.) . . . 1070. O. BISERRATA Gdgr mss

Fol. ovata vel ovato-rotundata . . . . . . . 124

123 Fol. obovata - Hte-Savoie, Draillant (Puget). 1071. O. verri-

I'ed. glabri; fr. basi attenuatus - Savoie, Chambery (Billot

124 n. 3581 bis). ..... 1072. O. Parisil Gdgr mss

Ped. villosi ; fr. basi late rotundatus - Htes-Alpes, Chaudun

(Burle). . . . . 1073. O. Caturigensis Gdgr mss

125 Aculei dimorphi subulati necne . . . . . . . 126

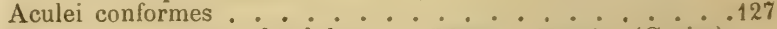

Fr. ovatus; costa eglandulosa - Rhône, Tassin (Cariot).

. . . . . 1074. O. stephanocarpa B. Carioti Gdgr mss

Fr. ovatus; costa glandulosa - Helv. Fribourg, Montbovon

(Cottet). . . . 1075. O. stepgan. C. Friburgensis Gdgr mss

Fr. ovoideus - Cher, Terrieux, etc.1076. O. STEPHANocARPA

. . . . . . . . . (D. et Rip.)

127

oblongus

Fr. ovoideus vel obovato-oblongus. . . . . . . . 130

128 Fol. late obovata vel oblonga; pet. aculeati. . . . . . 129

Fol. parva ovato-elliptica; pet. inermes - Sabaudia, it-

Salève (Bouvier). . . . . 1077 O. Parvifolia Gdgr mss

Fol. obovata; sep. apice foliacea-Suecia, Vestrog. Kinnekulle

(Lundquist) . . . . . . 1078. O. GотнICA Gdgr mss

Fol. oblonga; sep. linearia-Suecia, Scania, Kivik (Tullherg).

. . . . . . 1079. O. PlatycarPa Gdgr mss

Fol. obovata. . . . . . . . . . . . . .

Fol. ovata basi rotundata; ped. 2-4 m. 1. - Pyren. occ.

Tortes (Bordère). . . 1080. O. CALYPTOPODA Gdgr mss

Fol. oblonga basi attenuata; ped. 6-7 m.1. - Suecia Smol.

Backaby (Scheutz). . . 1081. O. oblongrfolia Gdyr mss

Ped. 3-5 m. 1.; fol. basi attenuata - Saxonia, Weimar

131 (Haussknecht). . . 1081 bis. O. Haussknechit Gdgr mss Ped. 7-9 m. 1.; lol. basi rotundata-Prusse, Dantzig (Baenitz herb, eur. n. 2237) . . . 1082. O. FAlCATULA Gdgr mss

132. Tubus toto hispidus .............................

Tubus laevis vel basi tantum glandulosus . . . . . 148

$133 \int$ Fr. ovoideus vel obovato oblongus. $\ldots . .134$

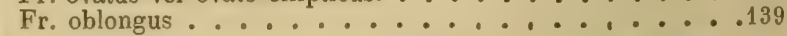

(Suite au prochain Bulletin). 
fol. obovala vel oblonga. . . . . . .

134 Fol. ovata vel elliption-olitusa; pet. aculeati . . . . . .137

Fol. ovato-acuta; pel. inermes - Isère, forençon (Boullu;.

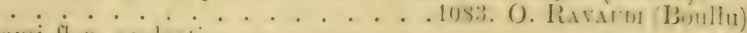

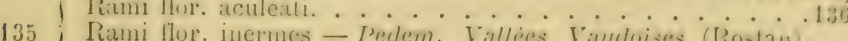
Vindorises (liostan.

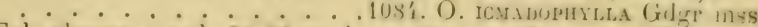
Fol. olovita; peel. 23-28 in 1. - Valais, Bovernier (Delasoie, elc.) . . . . 1085. O. Luvieplitwcutata (Delannie)

136 Fol. obovato-ullonga virentia; perl. 11-1:2 m. 1. - Talais,

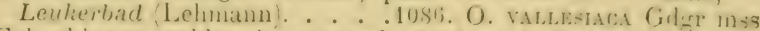

Fol. oblonga valile glauca; ped. S-11) m. 1. Tedem. Vallees Tamdonses (Rostant. . . . . lus7. (). Rustaxi Gilgr mss

Rimmi flor. inermes; 11 amplissinus - Basse-1lpes, Bonzo-

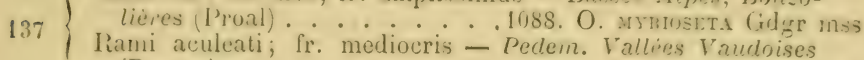
(Rostan) . . . . 1089. O. Allionir Gdgr mis Pet. glabri ; fol. obtuse rotundata - Delph., Pedem., Helv.

138 Pet. glabri; fol. obtuse elliptica - Basses-Alpes, Digne (Roux). . 1091. O. obesa Gdgr mss Ped. minte villosi; fol. acule elliptica - Itte-Saroie, Bellevaux (Pluget), . . . . . 1092. O. oxyonovera Giler mss Sep. I pinnata, valde hispidissimo-glandulosa ... . . 140

139 Sep. saepius integra, parce hispida - Delph. Lautaret (Aunier) . . . . 1093. O. acavthocarpa Gdgr mss

$140\{$ Fol. apice obtusa vel retusa. . . . . . . . 141 Fol. apice | acuta. . . . . . . 147 Discus planus; pet. glabri; fr. basi evidenter vel longe attenuatus . . . . . . . . . . 11 .

141 Discus planus; pet. sparse pilosi ; fr. basi vix attenuatus . 143 Discus planus; pet. inferne villosi ; fr. basi | attenuatus: . 145 Discus conicus, pet. unifariam paulo villosi - Valais, Approz (Woli). . . . . . . 109'. O. strnetusi Gder mss I'ed. 4-i m. l.; fol. ovata - Sabaudia, Mt-Salieve (Puret).

142 1 Ped 1 Ped. 12-1'4 m. 1.; fol obovata - Sabaudia, IIt-salcre (Guinet).

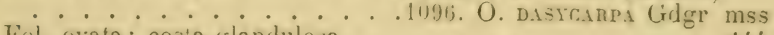
Fol. orata; costa slandulosa. . . . . . . . . . 144

143 Fol. oratu-rotundata; costa acieulata - Delph. Briançon (Aunier) . . . . 1097. G. Brigavtiaca Gdgr mss

$141\left\{\begin{array}{c}\text { Sep. apice integra; ped. 2-ni-13asses-A/pes, La Conda- } \\ \text { mine (Proal). } \\ \text { Sep. apice dentata; ped. } 1 \text { - Basses-Alpes, La Condamine }\end{array}\right.$ Sep. apice dentata; ped. 1 - Basses-Alpes, La Condamine (Proal). . . 1099. O. netusa Gdyr miss

14.5 Rami flor aculeati; fr. I magnus . Rami inermes ; fr. amplissimus - Basses-Alpes, Bouzolières (Proal) ..... 1100. O. Admirabilis Gdgr mss Fr. basi attenuatus; ped. 11-13 m. 1. - Basses-Alpes, La

146 Condamine (Proal). - . 1101. O. MEgapmYla Gdgr mss Fr. basi subrotundatus; ped. 6-8 m. 1.-Basses-Alpes, Bouzolières (Proal). . . . 1102. O. oppositisplya Gdgr mss 
Rami flor. inermes - Valais, Mayens de Sion (Wolf).1103.

147

Ramorum florif, aculei conformes recti - Valais, Bovernier

(Wolf) ............ . Wolfir Gdgr mss

Ramorum flor. aculei alii glanduliferi, alii dilatati - Valais

(Christ ros. p. 162). . . 1105. O. Hispidissima Gdgr mss

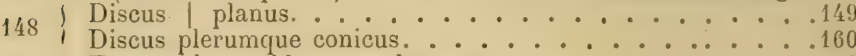

(Fr. ovoideus, oblongus vel ovatus. . . . . . . . 150

Fr. rotundatus - Sabaudia (Dés. herb. n. 15 et bis).1106

Tubus basi vel in $1 / 3$ inf. hispidus - . O. Discreta Gdgr mss

150 Tubus laevissimus. . . . . . . . . . . 156

151 Fol. oblonga; ram. sup. omnino inermes. . . . . . . 152

151 Fol. ovata vel obovata; rami aculeati ................

Ped. 10-12 m. l.; fol. oblongo-lanceolata - Thuringia, Heili-

$152\{$ genstadt (Hampe!). . . . 1107. O. Hampeana (Gris.)

Ped. 7-8 m.1.; fol. elliptico-obtusa - Basses-Alpes, Bouzolières (Proal) . . . . . 1108. O. FusifonMis Gdgr mss

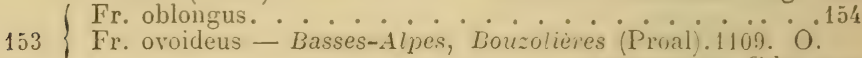

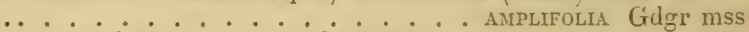

Tubus basi hispidus; costa glandulosa. . . . . . . 155

154 Tubus in $1 / 2$ inf. hispidus; costa aciculata - Hies-Alpes, Mt-Aurouse (Aunier). . . 1110. O. Aurosica Gdgr mss

Fol. basi sensim attenuata; ped. 12-14 m. 1. - Sabaudia,

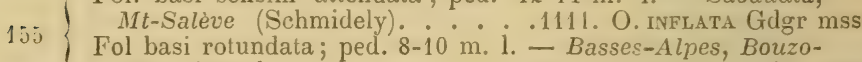
lières (Proal). . . . . 1112. O. editicola Gdgr mss

Fol. subtus ad costam eglandulosa. . . . . . . . . 157

156 Fol. ad costam glandulosa, supra glaberrima. . . . . 159

Fol. supra adsperse pilosa, ad costam glandulosa - Valais, Mayens de Sion (Wolf). . .1113. O. suprapilosa Gdgr mss

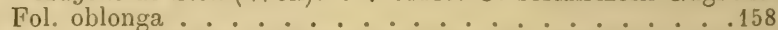

Fol. obovato-acuta - Cantal, Le Lioran (F. Héribaud). 1114.

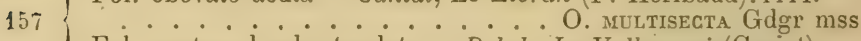

Fol. ovata vel subrotundata - Delph. Le Valbonnais(Cariot). . . . . . 1115. O. valbonnaisiensis Gdgr mss

$158\left\{\begin{array}{l}\text { Rami flor. aculeati; fol. basi rotundata - Angl. Cheshire, } \\ \text { Raby (Harbord) }\end{array}\right.$ Rami inermes; fol. basi cuneata - Hte-Savoie, Bellevaux (Puget). . . . . 1117. O. DidymonirксHA Gdgr mss Fr. ovato-rotundatus; rami flor. inermes-Alp. Delph..1118.

159 . . . . . . . . . montana (Chaix) Fr. oblongus; rami flor. aculeati - Sabaudia, Mt-Salève (Guinet). . . . 1119. O. Testudinaria Gdgr mss Discus conicus; fol. late ovato-acuta ; fr. rectus . . . . .161

Discus conicus: fol. parra elliptica; fr. nutans - Gall.

$160\left\{\begin{array}{c}\text { Chambéry (Billot exs. 3584). 1120. O. Perrieri (Songeon) } \\ \text { Discus planus; fol. late obovato-oblonga - Pyren. or. Mit- }\end{array}\right.$ Louis (Debeaux) : . . 1121. O. Patenti-Ramea (Debx)

Rami flor. aculeati ; fr. ovoideus - Sabaudia, Mt-Saleve

161 (Rap.). . . . . . 1122. O. Chavini (Rap.) Rami inermes; fr. oblongus - Basses-Alpes, La Condamine (Proal) ........ 1123. O. PoDopHylla Gdgr mss 
162 Fr. rotundatus. . . . . . . . 163

Fr. obovatus vel oblongus . . . . . . . . 164

Ped. et tubus glabra - Tyrol, Mulhau, etc.1124. O. IncLIvata

163 Ped. tubusque hispida - Lombardia, Mt-Generoso (Thomas).

.............. O. Assurgens Gdgr mas

164 ) Rami flor inermes. . . . . . . . . . 165

161 Tami flor aculeati. 165 Tubus et sepala laevia. . . . . . . . . . . . . . 1668

Tubus et sep. glandulosa. . . . . . . . . 167

Pet. aculeati; sep. apice dentata - Gall. Mt-Dore (Aunier).

166 . . . . . . 1126. O. MYnIodon Gdgr mss

Pet. inermes; sep. apice integra - Silesia, Waldenburg

(Straehler). . . . . 1127. O. sunetica Gidgr miss

Discus subconicus; tubus dense aculeatus - Helv. Vand,

167 Chesiéres (Rap.!). . . . 1128. O. Lerescrim (Rap.)

Discus planus; tubus sparse glandulosus - Gall. Mt-Dore (Aunier). . . . . . . 1129. O. vulcaxonus Gidgr mss

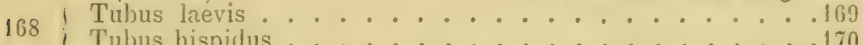

Tubus hispidus ................... . . .

Pet. aculeati; sep. paulo pinnata et glandulosa - Sabaudia, 169 It-Saleve (Billot n. 3583) Pet. inermes; sep integra et laevia - Creta, Sfakia (Sieber).

170 (Puget). . . ovato-elliptica - Sabaudia, Mt-Salève

Tubus obovato-oblongus; fol, oblongo-lanceolata (Pug. mss. Vaud, Morcles (Thomas). . . 1133. O. gratiosa Gdgr mss

Subgenus VIII. - Grepinia Gdgr Essai p. 2̊̃ (in sect.);

Ganineae D. C. in Ser. Mus. helv. I (1818) p. 2.

Styli liberi, glabri rillosi ant lanati, ultra discum planum vel conicum capitati vel subfasciculati. Flores late longeque lracteati, albi rosei vel purpurei, petalis amplis cordatis nonnunquam ad marginem ciliatis donati. Fructus praecox, corymbosus, pulposus, coccineus, erectus, glaber raro glandulusus. Sepala extus glabra interdum glandulosa, 2 integra 3 semper plus minus pinnata, post anthesim deflexa, mox (rarissime serius) decidua. Pedunculi erecti, glabri villosi aut glandulosi. Foliorum imparipinnatorum foliola glabra villosa eglandulosa (costa media excepta in 
quibusdam), virentia vel glauca, serrata, eorum serraturis simplicibus vel composito-glandulosis. Stipulae conformes latiusculae. Aculei sparsi, majusculi, adunci, basique late dilatati (saltem in caulibus hernotinis, Radix (in aliqnibus exceptis) haud reptans. - Frutices elati, a regionibus borealibus totius Veteris Orbis necnon Americae ad plagas Africae septentrionalis usque ad Indiae fines (in australioribus montani vel alpestres et quidem infrequentes) ubique ac frequentissime crescentes, erecti, sociales, ramosi, ramis virentibus glaucis interdumque purpureis rigidis aculeatis praediti.

Generis hujus immensi nomen assumet illum Cl. F. Crépin belgici qui, opera plurima atque pretiosa rhodologica in lucem protulit.

\footnotetext{
(Fol. undique glaberrima; ped. glabri vel villosi eglandulosi.

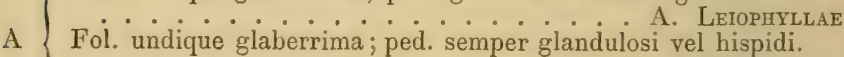
semper glandulosi vel hispidi

Fol. subtus saltem plus minus villosa. . C. Trichophyllae
}

\section{A. - Leiophyllae Gdgr Essai p. 26. Canineae nudae Auct.}

Foliola undique glaberrima, 1-2-3-serrata, serraturis glandulosis necne; costa media non raro glandulosa. Sepala deflexa mox decidua, dor'so semper eglandulosa, pinnata, eorum pinnulis integris vel dentato-glandulosis. Styli liberi, ultra discum planum vel conicum producti. Corolla ampla alba vel rosea; fructus pulposus. Pedunculi glabri villosi eglandulosi. - Aculei, saltem caulium vetustorum, majores validi conformes, e basi dilatata falcato-compressi. Radix halld reptans. 
Foliola simpliciter serrata. . . . . . . a. Isodontae

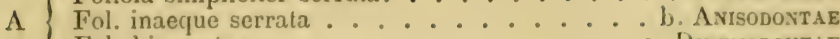
Fol. biserrala .................. Didysodontae

\section{a. - Isodontae Gdgr Essai p. 26. Lutetianae Crép. Prim. p. 17.}

Foliolis omnino uniserratis (simpliciter), serraturis saepius argutissime filcatis; foliis nonnunquam glaucescentibus pallideve virentibus, eorum nervis parum prominulis, ad costam mediam ordinarie eglandulosis. Turionum aculeis validioribus.

Styli glaberrimi vel vix parcissime hirsuti. . . . . . . 2

Styli paulo hirsuti. . . . . . . . . . . . . 17

Styli villoso-hirsuti vel lanati ............ . 58

Fr. globosus rotundatus vel ovatus. . . . . . . . . 3

2 Fr. ovoideus vel obovato-oblongus. . . . . . . . . 4

Fr. oblongus. . . . . . . . . . . . . 12

Fr. globosus; ped. 7-8 m. 1. - Aisne, S. Quentin (Magnier)

. 1134. Crepinia Magnieri Gdjr mss

Fr. rotundatus; ped. 16-18 m. 1. - Isère, Paladru (Gdgr)

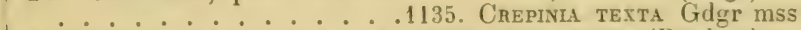

Fr. ovatus; ped. $5-8$ m. 1. - Pyrenaei centr. Gèdre (Bordère)

Fol. basi attenuata. basi rotundata vel paulo truncata. . . . . . . . . 5

Ped. juniores laeves; pet. aculeati. . . . . . . . . 6

Ped. juniores laeves; pet. inermes. - Gard, A ulas (Diomède)

Ped. hirtelli ; pet. subinermes. - Pyrenaei, Gedre (Bordère)

(i) Fol. ovata apice rotundata. - Cher; Angl. bor' $1139 .{ }^{\circ} \mathrm{C}$.

l. . . . . . . . . . . Flexibilis (Dés.)

Rami flor. inermes; discus valde conicus. - Serbia, Belgrade - Ramiflor aculeati ; discus parum conicus - Pyrenaei, Gedre

(Bordère) .........1141. C. Ixsulsa Gdgr mss

Fol. obovata vel obovato-oblonga ........ . . 9

s I Fol. ovato-clliptica; fr. basi subrotundatus. . . . . . 11 Fol. oblonga; fr. basi depressus, - Isère, Charancieu (Gdgr)

.1142 . C. cremoclada Gigr mss 
Discus planus ; costa eglandulosa . . . . . . . 10 Discus paulo conicus; costa eglandulosa - Helv. Neuchatel (Lerch) . . . . . . . . 1143. C. Lerchi Gdgr mss Discus conicus; costa basi paulo aculeolata - Angl., Cornouailles (Briggs) . . . . 1144. C. Convubiensis Gdgr mss Ped. 7-9 m. 1.; sep. apice dentata - Puy-de-Dôme, S. Mau-

10 rice (Dubosclard). . . . 1145. C. Rigidiramea Gdgr mss Ped. 12-14 m. l.; sep. apice integra - Hisp. Logrono, Sierra de Cameros (Zubia) . . . 1146. C. CANTABRica Gdgr mss Costa aculeata; rami saepe inermes - Pyren. Perpignan

11 (Debeaux) . . . 1147. C. Mcrostrla Gdgr mss Costa laevis; rami aculeati - Angl., Gall. etc.1148. C. MU-

. . . . . CRonulata (Dés.)

Fol. oblonga. ......................

12 Fol. obovata vel obovato-oblonga . . 14 Fol. parva ovata - Basses-Alpes, La Condamine (Proal) . . . . . . 1149. C. Alpicola Gdgr mss Rami florales valde aculeati - Dalmatia, Raguse (Studniczka)

13 Rami flor. inermes; ped. 9 -1 1 m. 1. - C. Ratia, Edimburg (Drummond) . . . . . 1151. C. edimburgensis Gdgr mss Rami flor. inermes; ped. 19-22 m. 1. - Calvados, Monceaux (de Bonnechose). . . . . 1152. C. oligopoda Gdgr mss

14 Fr. apice valde strangulato-attenuatus. . . . . . 15

Fr. apice plus minus attenuatus. . . . . . . . 16

Fr. basi attenuatus; costa glandulosa; ped. 7-9 m. 1. -

15 Istria, M. Maggiore (Freyn).1153. C. DiDyмorhyncha Gdgr mss Fr. basi decurrens; costa egland; ped. 13-15 m. 1. - Vallesia, Sierre (Favrat). . . . 1154. C. Collaris Gdgr mss Rami flor. inermes; fol. basi-cordata - Pyrenaei Orient,

$16 \int$ Fenouillet (Debx). - . 1155. C. Pounretiana Gdgr mss Rami flor. aculeati; fol. basi sensim attenuata - Rhône, Pommiers (Gdgr). . . . . 1156. C. DonnIENs Gdgr mss

17 Fr. rotundatus ovatus ovoideus vel obovato-oblongus. . . . 18

17 Fr. oblongus vel elongatus ............ 39

18 Ped. villosi................ 19

\{ Ped. glabri . . . . . . . . . . . . 2 23

$19\left\{\begin{array}{l}\text { Fol. obovato-oblonga. } \\ \text { Fol. ovata vel elliptica } \\ \text { Fol. oblonga }- \text { Suecia, Scania : Pinelierna (Tullberg) } 1157 .\end{array}\right.$ Fol. oblonga - Suecia, Scania : Pinelierna (Tullberg).1157.

. . . . . . . C. Macrosphaera Gdgr mss

Pet. inermes eglandulosi; fr. basi valde depressus - Ain,

Néron (Gdgr herb. ros. n. 228).1158. C. oroinituba Gdgr mss Pet. aculeati sparse glandulosi; fr. basi subrotundatus Rhône, Arnas (Gdgr herb. ros. n. 105).1159. C. CRassion . . . . . . . . . . . . Gdgr mss Fr. late ovoideus; aculei adunci; rami robusti glauci. . . 22 Fr. parvus ovoideus; aculei rectinsculi; rami gracillimi virentes Sabaudia (Puget in Billot exs. n. 3579 ex parte)

. . . . . 1160. C. tenuigaulis Gdgr mss Fr. globosus - Hung. Sisatovoc /C. glabrata Kanitz in Fora XXXII, 1863 non Vest). . . . 1161. C. GLabella Gdgr 
Discus parum conicus; pet. inermes; fr maximus - Helv. occid. Montbovon (Cottet; Puget in Bull. Soc. Murith. III, 1874, p. 41). . . . . . 1162. C. Gavdogeri (Puget)

Discus conicus; pet. aculeati ; fr. parvus - IIisp. bor., Sierra de Cameros (Zubia). . .1163. C. caveniva Gdgr mss

Pet. inermes,

24

Pet. aculeati............. . . . 30

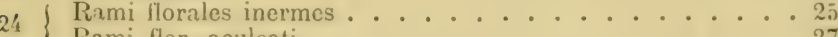

Rami flor aculeati. . . . . . . . . . . 27

(Fol. oblongo-lanceolata. . . . . . . . . 26

Fol. ovato-rotundata ; pet. villosuli - Germ.; Gall. (Gdgr herb. ros. n. 547) . . . . 1164. C. Ramosissima (Rau) Fol. ovala; 1ret. glahri - Rhone, Amas (Gidgl).1165.) C.

. . . . . . . . . . . . PICACEA Gdgr msS

Discus planus; fr. ovatus - Gironde, Marcamp (Motelay)

26 ............... Cal66. Gdgr mss

Discus conicus; fr. ovoideus - Saône-et-Loire, Autun (Déséglise) . . . . . 1167. C. Intacta Gidgr mss

27 Fol. acute elliptica, basi rotundata . . . . . . 28

\{ Fol. oblonga basi attenuata. . . . . . . . . . . 29

Discus conicus; pet. inermes eglandulosi - Rhône, Montmelas $(G$. herb. ros. n. 549). .1168. C. TERETispiva Gidgr mss

Discus subplanus; pet. paulo aculeati et glandulosi - Rhóne, St-Cyr-cle-Châtoux (Gdgr herb. ros. n. 538) .1169. C. ConY-

. . . . . . . . . . . licola Gdgr mss

Fr. basi attenuato-decurrens; pet. glabri - Vallesia, S. Mau-

29 rice (Favrat). . . . 1170. C. Favrati Gdgr mss

Fr. basi depressus; pet. sparse pilosi - Austria inf., Kalksburg (Wiesbaur). . . . 1171. C. PARvispiNi Gdgr mss

Rami flor inermes. . . . . . . . . . . . 31

Rami flor aculeati. . . . . . . . 32

Fol. basi truncata; discus subplanus - Puy-de-Dôme,

31 Pionsat (Dubosclard). . 1172. C. Laeviranea Gidgr mss

Fol. basi longissime attenuata; discus conicus - Sabaudia (Puget). . . . . . 1173. C. oxrpirlla (Rép.)

3.) Fol, basi breviter attenuata. . . . . . . . . 33

32 Fol. basi rotundata . . . . . . . . . 35

Fol. basi attenuata. . . . . . . . . . 36

Pet. glabri ; costa media haud aut vix glandulosa. . . . 3'

33 Pet. Dasi villosi; costa glandulosa - Rhüne, Brignais; Ain, La Pape (Chabert). . . . . . 117'. C. Modica Gdgr mss

Discus conicus; costa eylandulosa - Maute-Savoie, IIabere-

$34 \mid$ Lullin (Puget) . . . . . 1175. C. Rivalis Gdgr mss Discus subconicus; costa glandulosa - Anglia, IIereford: Eaton (Ley) . . . . . . . 1176. C. LeYI Gdgr mss

Fr. ovato-rotundatus; pet. subhirtelli - Europa media (Gdgr

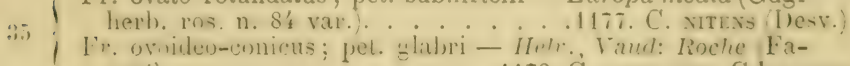
vrat) . . . . . 1178. C. covocanpa Gdgr mss

36 I Anome purpurea ; fol. liasi valie aruta. . . . . . . .77 Virens; fol. basi tantum attenuata. . . . 38 ['ol. virentia; petala amoene rosea basi ciliata - Rhóne, Amas (G. herb. ros. n. 569). .1179. C. oexophlata Gdgr mss Fol. purpurea; petala albido-rosea basi glabra - I:"

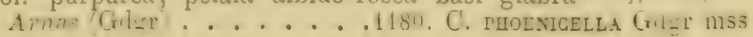


Fol. obovato-elliptica; fr. ovato-conicus - Scandinavia ; Gall., Rhône (Gdgr herb. ros. n. 559 var.).1181. C. SENTicosa Fol ob - Achar.

- Pyrenaei Orient., Oletteles-Bains (Debeaux)... . 1182. C. Camptodes Gdgr mss

39 Pet. glabri vel basi sparse et vix hirsuti. . . . . . . 40 Pet. saltem a basi usque ad 2 um jugum foliol. minute villosi. 49

40 Discus planus vel subplanus . . . . . . . . . . 41 Discus valde conicus. . . . . . . . 45

(Fol. obovata vel oblonga basi plus minus attenuata. . . . 42

41 Fol. ovata inferne late cordata - Rhône, Arnas (Gdgr). 1183.

Rami floriferi inermes . . . C. ApPENdiculosa Gdgr msS

42 Rami flor aculeati $\ldots 43$

Fol. late oblonga, serraturae apertae; ped. 16-18 m. 1. Suecia, Vestrog. m. Kinnekulle (Zetterstedt).1184. C. AM-

Fol. mediocriter obovata, eorum dentes convergentes; ped. 7-9 m. l.-Rhône, Pommiers (Gdgr herb. ros. n. 18). 1185.

. . . . . . . . C. valdefoliosa Gdgr msS

Ped. 8-10 m. l.; cor. intense rosea; fr. bası decurrens Phóne, Montmelas (Gdgr herb. ros. n. 331).1186. C. PHOE-

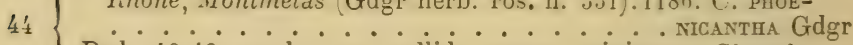

Ped. 16-19 m. l.; cor. pallide rosea; minime - Gironde, Coutras (Motelay) . . . . 1187. C. PETiolulata Gdgr mss

Fol. basi rotundata. ................... 46

45 Fol. basi acute attenuata. . . . . . . 47

Fol. basi abrupte truncata - Hung. Budapesth (L. Richter).

. . . . . . 1188. C. Kitaibeliana Gdgr mss

Fol. acuta obovato-oblonga; pet. glandulosi aculeati - Isère,

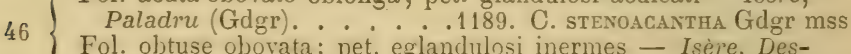

Fol. obtuse obovata; pet. eglandulnsi inermes - Isère, Dessine (Gdgr herb. ros. n. 129).1190. C. obattenuata Gdgr mss

Rami florales inermes; fr. utrinque aequaliter depressus vel attenuatus. . . . . . . . . 48

Rami flor. aculeati; fr. apice valde attenuatus basi subde-

47 pressus; foliola obovata - Hisp. Aragonia: Boucharo (G. herb. ros. n. 36) . . . 1191. C. vivinalis Gdgr mss

Rami flor. aculeati; fr. apice subattenuatus basi vero longe decurrens; fol. late elliptica - Rhône, Marcy (Boullu). . . . . . 1192. C. Pseudo-Fallax Gdgr mss

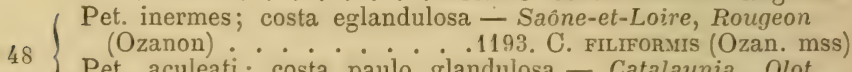
Pet. aculeati; costa paulo glandulosa - Catalaunia, Olot (Vayreda). . . . . . 1194. C. Catalaunica Gidgr mss

49 Ped. glabri . . . . . . . . . . 50

49 Ped. pubescentes $\ldots 57$

$50\{$ Discus planus vel subplanus ........... 51

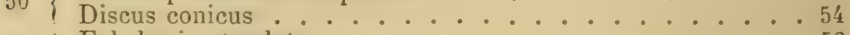

51 Fol basi rotundata. . . . . . . . . 52

Fol. basi attenuata, dentes convergentes - Gallia bor. 1195 .
Virens; fol. tenera,

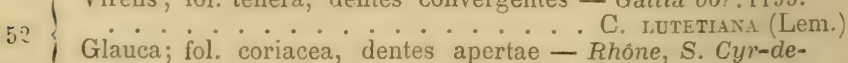
Chatoux (Gdgr. herb. ros. n. 170). 1196. C. GRAvitica Gdgr 
Glanco-virons; rami flor. aculeati ; fol. elliptico-obovata Sabaudia, Pringy (Puget). . 1197. C. Funcellania Grdgr mss Purpurea ; rami flor. inermes; fol. obovato-oblonga - Austr. inf., Modling (Wiesbaur) . . .1198. C. nubnfolia Gdgr mss

Fol basi rotundata ..............55

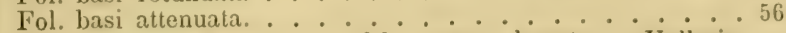

Fr. inferne vix depressus; fol. parva obovata - Vallesia, Ypresse (Wolf). ......1199. C. Fallacina Gdgr mss

Fr. basi valde attenuatus; fol. late ovato-elliptica - Ital. or., Faenza (Caldesi) ..... 1200. C. Caldesir Gdgr mss Ped. 9-11 m. l.; fol. elliptica - IIte-Savoie, Pringy (Puget).

Ped. 1 i-i2 m. i.; fol. oblongo-lanceolata - Dalmatia, La Trinita (Studniczka). . . 1202. C. Visianiana Gulgr mss Ped. 15-17 m. 1.; fol. oblonga - Isère, Recoin (Gdgr). 1203.

Fol oblongo-lanceolata basi subrotundata C. mutispisi Grlyr mss 1204. C. Finitima Gdgr mss ol. obovato-oblonga lanceolata basi attenuata - Italia or. Faenza (Caldesi) . . . 1205. C. DEcipiess Gdgr mss

Fol. late obovato-subacuta basi attenuata - Rhône, Arnas (Gdgr herb. n. 328). . . 1206. C. crassiuscula Gdgr mss

Fr. globosus vel ovato-subrotundatus ....... . 59

58 Fr, ovoideus vel obovato-oblongus. . . . . . . 76

Fr. oblongus . . . . . . 134

Fr apice haud attenuatus ........660

59 Fr. apice attenuatus vel valde strangulatus. . . . . 72

60 Rami florales et stipulae haud aut vix purpurea . . . . 61

Rami florales et stipulae purpureo-glaucescentia . . . . 68

Discus planus.

61 Discus conicus. . . . . . . . 65

62 Rami floriferi inermes . . . . . . . . 63

Rami floriferi aculeati . . 64

Fol. basi rotundata; pet. basi villosi ; fr. ample rotundatus - Helv. Uri (Lagger) . . . 1207. C. exarmata (Crép. mss)

63 Fol. basi acuta ; pet. glabri ; fr. parvus globoso-depressus Anglia, Cornouailles: Tregunnus (Briggs).1208. C. cyclo. . . . . . . CARPA Gdgr mss

Ped. 16-20 m. l.; fol. obovata, costa glandulosa-Hte-Savoie, Habere-Lullin (Puget). . .1208 bis. C. Acutiuscula Gdgr mss

Ped. 11-14 m. 1.; fol. oblonga, costa glandulosa - Doubs, Rosemont (Paillot flora sequaniensis exsicc. n. 52) 1209

. . . . . . C. Sequaniensis Gdgr mss Fol hasi rotundata. . . . . . . . . . . 96

65 Fol. basi attenuata - Gallia, Jura, etc. 1209 bis. C. spHAERICA ............. (Gren.) Rami floriferi aculeati .......... . . 67

66 Rami floriferi inermes - Isère, Paladru (Gdgr).1210. C. pasR.......... curcola Gdgr mss Pet. adsperse pilosi ; fr. maximus basi attenuatus - Rhóne, A'nas (G. lierb. ros. n. 272). . . 1211. C. Robusta Gdgr Pet. adsperse pilosi ; fr. maximus basi subrotundatus - B.-duRhône, Martigues (Autheman).1212. C. Axplicscula Gdgr mss Pet. glabri; fr. mediocris basi subrotundatus - Helv., Jura. 
Fol. ovata vel elliptica . . . . . . . 69 Fol. late oblongo-acuminata - Silesia, Kreunerlehue (Frachen). . . . . 1214. C. HETEROCARPA Gdgr mss Ped omnino laeves; fol. basi rotundata. . . . . . . 70

69 Ped. subglandulosi; fol. basi attenuata - Sabaudia.1215. . . . . . . . . C. condensata (Puget) Discus planus; fr. globosus . . . . . . . . . 71

70 Discus conicus; fr. ovatus - Helv. Vaud: Morcles (Favrat).

.............. C. FISSIFoliA Gdgr mss Sep apice 1-2 dentata; fol. superne attenuata - Hte-Savoie,

71 Brenthonne (Puget). . . . 1217. (. Boxbycina Gilgr mss Sep. apice integra; fol. superne rotundata - Hte-Savoie, Habère-Lullin (Puget) . . . . 1218. C. TURGIDA Gdgr mss

72 Fol. elliptica vel obovata............. . 73

Fol, oblonga .................... . . . .

Fr. ample subglobosus apice strangulatus; fol. basi sensim attenuata - Hte-Savoie, Habère-Poche (Puget). 1219. Fr. parvus anguste ovatus apice attenuatus; fol. inferne acuta - Helv., Bâle (Cottet). .1220. C. Aggerrvaga Gdgr mss

74 Rami flor. et petioli inermes; fr. rotundatus vel ovato-conicus. 73 Rami flor. et petioli aculeati ; fr. ovatus - Hte-Savoie, Habère-Poche (Puget) . . . . . 1221. C. PODANTHA Gdgr mss

Ped. 16-18 m. 1.; fr. rotundatus - Suecia, Scania: Alnarp

$75 \mid$ (Tullberg). . . . 1222. C. Gracilis Gdgr mss Ped. 12-13 m. 1.; fr. ovato-conicus - Aisne, St-Quentin (Magnier). . . . . . . 1223. C. ANTIQUA Gdgr mss

( Pet. glabri vel basi pilis raris parcissime obsiti. . . . . 77

76 Pet. I saltem usque ad $1 / 2$ infer. partem minute vel laxe pilosi. . . . . . . . . . . . . . . . . . 25

. Rami florales inermes vel 1-2 aculeati. . . . . . . 78

i7 / Rami florales | aculeati. . . . . . . . . 94

78 I Petioli inermes . . . . . . . . . 79

78 , Petioli aculeati ................ 88

$79)$ Discus planus. . . . . . . . . . . 80

79 Discus | conicus ............. 84

$80)$ Fol. basi truncata . . . . . . . . . . 81

Fol. basi rotundata. . . . . . . . . . 82

Flores amoene rosei; fr. basi rotundatus - Rhône, Montmelas (Gdgr herb. ros. n. 178). .1224. C. JUNIPEROBUM Gdgr

Flores pallide rosei ; fr. basi longe attenuatus; ped. 7-9 m. 1

- Suecia, Vestrog.: Mt-Kinnekulle (Zetterstedt; Lundquist).

*. . . . 1225. C. MESOPODA udgr mss

Flores pallide rosei; fr. basi depressus; ped. 3-5 m. l. Suecia: Mt-Kinnekulle (Zett.). .1226. C. CONFERTIOR Gdgr mss

$8:\left\{\begin{array}{l}\text { Fol. apice paulo attenuata; cor. alba. Fol. apice rotundata; cor. rosea-Hte-Savoie, Pringy (Puget). } \\ \text { Fol }\end{array}\right.$

Pet. inermes; ped. 10-12 m. l. subhirsuti - Finlandia, Abo

8. 1 (Hollmén). . . . . 12:8. C. Fexwat Gdgr mss ; Pet. subinermes; ped. 3-5 m. l. glabri - Rhône, Montmelas

(G. herb. ros. n. 194) . . . 1229. C. Bellojocensis Gdgr

8 i Fol. inferne attenuata . . . . . . . . 85

Fol. inferne cordata . . . . . . . . . . . 86 
Ped. 12-14 m. 1.; fol. obovata - Pyrenaei, Gèdre (Bordère).

Ped. 16-17 m. i, fol, oblonga - Hisp., Logrono: Sierra de Cameros (Zubia) . . . 1231. C. Asturica Gdgr mss

Fol. ovato-elliptica vel elliptica . . . . . . . . 87

86 Fol. obovata - Isère, Charancieu (Gdgr herb. ros. n. 146).

Cor. subalba; fol. atrovirentia acuminata - Pyrenaei Gèdre

(G. herb. ros. n. 313) ... 1233. C. praestans Gdgr mss Cor. rosea; fol. pallide virentia apice subacuta - Rhóne,
Arnas (Gdgr). . . . . 1234. C. oBlEcta Gdgr mss

88 Pet. sparse glandulosi . . . . . . . . 89

Petioli eglandulosi.
Fol. olutusa; fr. hasi rotundatus - Rhone, Pommiers iG. $89\left\{\begin{array}{l}\text { lierb. 11. 267; Soc. dauph. 1874). 123j. C. Cer.tophori Gidgr } \\ \text { Fol. acuta; fr. basi attenuatus - Rhône, Montmelas (Gdgr }\end{array}\right.$ herb. n. 224) . . . . . 1236. C. Luconum Gdgr

90 Fol oblonga. . . . . . . . . . . 91

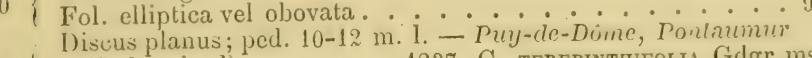

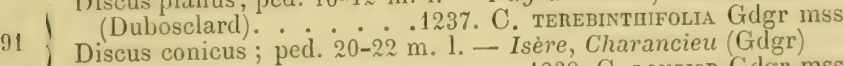

( Discus paulo conicus - a . 9 93

12 Discus planus - Hte-Savoie, Pringy (Puget).1239. C. TENESep. pinnulae integrae; fol. basi attenuata - Rhône, Pom-

93 miers (G. herb. ros. n. 80). . 1240. C. petrivaga Gdgr mss Sep. pinnulae dentatae; fol. basi subtruncata - Isère, Charancieu (Gdgr). . . . . 1241. C. vallicola Gdgr mss Fol. ovata, ovato-elliptica vel elliptica....... . 95

94 Fol. obovato-oblonga vel oblonga . . . . . . 105 Fol. obovata - Bouches-du-Rhône, Martigues (Autheman)

Fol. basi rotundata vel cordatia . . . . . . . . . . 969 96

| Fol. basi cuneata vel | attenuata. . . . . . . . 103

Petioli omnino inermes. . . . . . . . . . 97

96 Petioli | aculeati .............. 99

$\left\{\begin{array}{l}\text { Ped. 3-9 m. l. ; fr. ovoideus fr obovato-oblongus - Isère, Recoin (Gdgr) } \\ \text { Ped. 13-18 m. 1.; fr. os }\end{array}\right.$

97 Ped.13-18 m. 1.; fr. obovato-oblongus-12!3. C. IDErI.t. Gdgr mss Cor. basi ciliata; fol plana virentia - Rhône, Pommiers

98 (Gdor). . . . 1214. C. Btxigexi Grigr mss Cor. eciliata ; fol plicata glauca - Rhône, Montmelas (Gdgr herb. ros. n. 368). . . . 1245. C. plicAta Gdgr mss

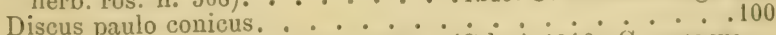

99 Discus planus - Isere, Montferrat (Gंdgr). 1246. C. vianum

Discus planus — fsere, Mdontferrat (Gidgr). . Gs

100 i Fol. apice obtusa . . . . . . . . . . . . 101

Fol. apice acuta. . . . . 102

Costa et pet. glanduloso-aculeolata - Rhone, Francheville

101 Costa et petioli eglandulosa - 1247. C. GLandulixatera Gugr mss ros. n. 71 var.)......1248. C. Rubricolor Gidgr mss 


\section{$-112-$}

(Ped. 17-21 m. 1.; aculei parvi - Sabaudia; Helv. Fribourg (Cottet). . . . . . 1249. C. ACIPHYlloides (Crép. mss) Ped. 6-10 m. 1.; aculei validi - Rhône, Montmelas (Gdgr herb. ros. n. 327). . . . . 1250. C. DURA Gdgr Discus conicus; ped. 9-16 m. l.; petala glabra. . . . . 104

103 Discus planus; ped. 5-7 m. 1.; petala extus hirta - Rhône, Arnas (G. herb. ros. n. 8) . . . 1251. C. pogovorasis Gdgr Fol. utrinque valde acuta amoene virentia - Isère, Les

104 Abrets (Gdgr). . . . . 1252. C. BIAcuta Gdgr mss Fol basi sensim attenuata atrovirentia - Isère, Charancieu (G. herb. ros. n. 201) . . . 1253. C. PRovirens Gdgr mss

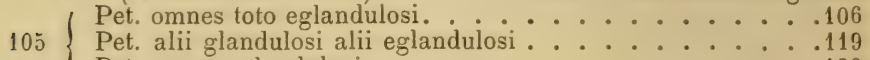
Pet. omnes glandulosi ............. 123

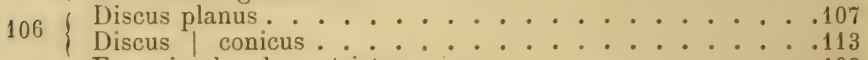

107 Fr. apice haud constrictus - . - . - . 108

107 Fr. apice anguste constrictus - Rhône, Francheville (Chabert)......... 1254. C. DuRifolia Gdgr mss

108 / Minime. . . . . . . . . . . 109

Fr. apice in $2 / 3$ super turgidus. . . . . . . . . . 112

Ped. glaberrimi; fol. plana. . . . . . . . 110

109 Ped. saepius paulo hirsuti; fol. plicata - Gallia, Lorraine P. . . 1255. C. Ramulosa (Godr.) Pet. inermes; ped. 4-6 m. l. . . 111

110 Pet. aculeati; ped. $9-11$ m. 1. -Gard, Le Vigan (Dioméde)

. . . . . . . 1256. C. DAsyphylla Gdgr mss

$111 \int \begin{aligned} & \text { Sep. apice integra; pet. basi sparse pilosi - Suecia, Scania : } \\ & \text { Pinelierna (Tulherg). . - . 1257. C. PAchyprona Gdgr mss } \\ & \text { Sep a pice dentata; pet. glabri - Rossia, Riga (Bienert, in }\end{aligned}$

111 Sep. apice dentata; pet. glabri - Rossia, Riga (Bienert, in flora ross. bor.-occid. exsicc. n. 253).1258. C. Bienerti .................... Gdgr mss Petala basi ciliata - Rhône, Arnas (Gdgr herb. ros. n. 364)

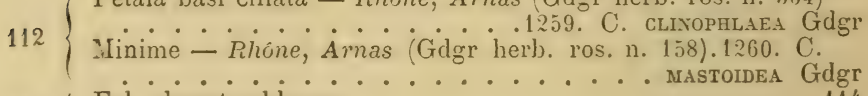

113 Fol. obovato-oblonga. $\therefore \ldots \ldots \ldots$ Fol oblonga. . . . . . . . . . . . 117

114 Petioli aculeati. . . . . . 115 Pet. inermes - Rhóne, Montmelas (Gdgr herb. ros. n. 524)

Ped. glabri; fol basi | attenuata. . . . . . . . . 116

115 Ped. subhirsuti; fol. basi subtruncata - Rhone, Arnas (Gdgr) Ped. $12-14^{\circ}$ m. l. $^{\circ}$ fol. hasi attenuata - Suecia, Scania : Andrarum (Tullberg). . . . 1263. C. ScAvica Gdgr mss

116 Ped. 4-5 m. 1.; fol. basi subattenuata - Rossia, Astrakhan: Sarepta (Becker). . . . . 1264. C. Wolgexsis Gdgr mss Ped. 5-6 m. 1.; fol. basi lanceolata - Calvados, Ellon (Bonnechose). . . . . 1265. C. ERronea Gdgr mss

$117\left\{\begin{array}{l}\text { Pet. inf. vel super. inermes. } \\ \text { Pet. omnes aculeati - Silesia, Lausitz (Uechtritz).1266. C. }\end{array}\right.$ 
Ped. 1-7 m. 1.; fol. flavo-maculata-Rhone, Arnas (Gidgr)

118 Ped. $8-10$ m. l.; fol. immaculata - Isère, Paladru Gidgr her mss ros. n. 50) ....... 1268. C. umbrigent Gerb.

119 Discus planus. . . . . . . . . . . . . .

Discus paulo conicus. . . . . . . . . . . 121

Fr. parvus oroideus; ped. 10-14 m. 1. - Pyrenaei, Gèdre

120 (Bordère) . . . . 1269. C. strivocaulis Gdgr mss

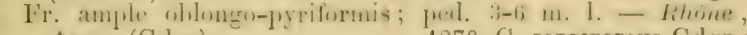
Amas (Gdgr). . . . 1270. C. sonbifonmis Gdgr mss Ped. glabri; fol. supra virentia anguste oblongo-acuta. . . 122 Ped. glabri; fol. virentia parva obovato-lanceolata - BassesAlpes, Condamine (Prozl). . 1271. C. Lavivesiana Gdgr mss

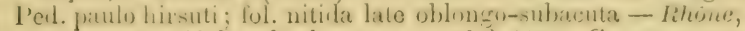
Montmelas (Gdgr herb. ros. n. 367).1272. C. subsitens

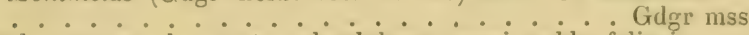

Ped. 8-10 m. l.; costa eglandulosa; rami valde foliosi -

1:2) Rhone, Arnas (Gdgr herb. ros. n. 341).1273. C. CAMpylo-

Laxe foliosa ; ped. $13-16$ m. $1 . ;$ costa paulo glandulosaIsève, Paladru (Gdgr). . . 1274. C. Consolationis Gdgr mss

Pétala basi ciliata; fol, inferne acuta . . . . . . 124

123 Petala eciliata; fol. basi rotundata - Ain, Les Echeyx (Chabert). . . . . . 1275. C. crmigena Gdgr mss

12 1 $\left\{\begin{array}{l}\text { Ped. corymbulosi } 13-15 \mathrm{~m} .1 .- \text { Puy-de-Dôme, Savi (Dubos- } \\ \text { clard) }\end{array}\right.$ Ped. solitarii 3-5 m. 1. - Rhône, Arnas (Gdgr herb. ros. n. 305 . . . . 1277. C. cunvidens Gdgr mss

125 ) Discus conicus . . . . . . . . . 126

Discus planus, . . . . . . . . . . . 129

1261 Fol. obovata. . . . . . . . . . . 127

Fol. oblonga . . . . . . . . . . . . 128

Ped. 9-15 m. l.; fol. acutiuscula - Hte-Savoie, Bellevaux

$1: 27$ (Puget) . . . 1278. C. Densiuscula Gdgr mss Ped. 4-6 m. 1.; fol. apice obtusa - Dalmatia (Schalch).1279

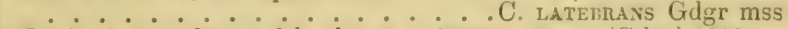

Fr. basi rotundatus ; fol. glauca - Rhône, Arnas (Gdgr) 1280

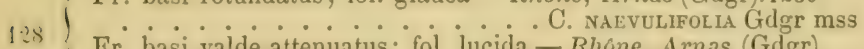
Fr. basi valde attenuatus; fol. lucida - Rhone, Arnas (Gdgr)

Fol . 1281. C. MEgalopHYLLA Gdgr mss

Fol. ovata vel elliptica basi rotundata. . . . . . . 130

129 Fol. obovata basi attenuata vel truncata ......... 132 Fol. oblonga basi valde acuta - Isère, Charancieu (Gdgr herb. ros. n. 120) ..... 1282. C. Patatil Gdgr mss Pet. sparse vel basi tantum pilosuli . . . . . . . 131

130 Pet. toto densiuscule villosi - Hte-Savoic, Bellevaux (Puget)

. . . . . . 1283. C. Tricuophora Gdgr mss Sep. apice foliacea; fr. superne subdepressus - Htc-Savoie,

$131 !$ Bellevaux (Puget). . . . . 1284. C. patula Gdgr mss Sep. apice angustata; fr. valde attenuatus - Itte-Savoie, Pringy (Puget) . . . . . 1285. C. rrimaria Gdgr mss

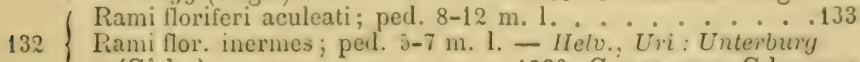
(Gisler) . . . . . . 1286. C. PERMIxTA Gdgr mss 
Ped. 8-9 m. 1. ; fr. basi depressus - Loire, Sauvain (Gdgr) . . . . 1287. C. Foresiaca Gdgr mss Ped. 10-12 m. i.; fr. basi longe decurrens - Suecia, Sm. : Wexio (Scheutz). . . . . 1288. C. smolandica Gdgr mss

13' Fol. ovato-elliptica vel obovato-oblonga basi rotundata . . .135 Fol. obovato-oblonga vel oblonga basi | attenuata ... 141

135 | Discus planus . . . . . . . . . . . 130 Discus | conicus . . . . . . . . . . . . . . . Fr. globosus - Cher, Savoie, Helv. . 1289. C. avaloga (Dés.) Fr. oblongus; rami flor. inermes - Cher; Mt Salève.1290

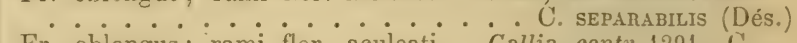
Fr. oblongus; rami flor. aculeati - Gallia centr.1291. C.

. . . . . Touravgiviana (Dés. et R.) Pet. omnes inermes. . . . . . . . . . . 138

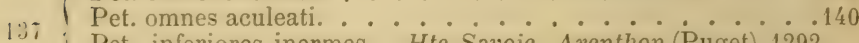
Pet. inferiores inermes - Hte-Savoie, Arenthon (Puget) 1292

Fol . . . . . C. Conda Gdgr mss Fol. obovato-oblonga; ped $14-16 \mathrm{~m} .1 . \ldots 139$ Fol. obovato-oblonga; ped. 9-11 m. 1. - Pyren. Orient., S. Antoine de G. (Debx). . . 1293. C. StEPHarodon Gdgr mss Fol. subrotundata; ped. 12-18 m. 1. - Hte-Savoie, Charly (Puget) . . . . . 1294. C. Laevidscula Gdgr mss Purpurea ; petala ciliata; ped. glalri - Ty,ol, Trente Gelmi)

139 ; Glauca; pet. eciliata; ped. sparse pilosi - Westphalia, Braunschweig(G. Braun). . . . 1296. C. Braunir Gdgr mss Pet. glalıri; ped. 8-10 m. 1. - Europa, Oriens (Grlgr heri).

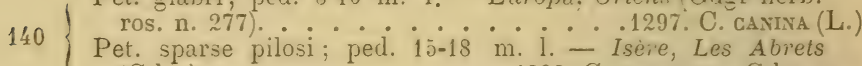
(Gdgr). . . . . . . 1298. C. Avpmiloga Gdgr mss

141 Fol. dentes torto-convergentes ad limbum contiguae. . . . .142 Fol. dentes rectae vel patulae. . . . . . . 143 Fol. ollongo-lanceolata utrinque valde attenuata; ped. glabri

142 - Hte-Savoie, Pringy (Puget). .1299. C. Fallax (Puget mss) Fol. elliptica utrinque breviter attenuata; ped. hirsuti Rhóne, Arnas (Gdgr herb. ros. n. 69 ; Billot exs. n. 4024)

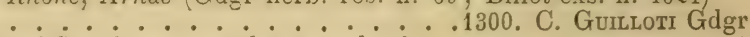

143 | Rami flor. inermes, vel $1-3$ aculeati. . . . . . . . . . . . 144

144 I Pet. omnino inermes. . . . . . . . . . . 145

Pet. aculeati. . . . . . . . . . . 146 Fol. oblonga basi attenuata; discus planus - Rhône, Mont-

1:5 melas (G. herb. ros. n. 354) ..1301. C. PINETORUM Gdgr mss Fol. elliptica basi subrotundata; discus subconicus - Aragonia, Torla (G. herb. ros. n. 31). . 1302. C. Melastoma Gdgr mss

Fol. oblongo-lanceolata; pet. toto villosi - Gallia, Perpignan (Debeaux) . . . . 1303. C. CRAssidens Gdgr mss Fol. sat ample obovato-oblonga; pet. basi unifariam villosiGard, Aulas (Diomède) . . . 1304. C. Fissidens Gdgr mss

146 Fol. parva obtuse ovato-elliptica; pet. adsperse pilosi Suecia, Mt-Kinnekulle (Zett.) • 1305. C. Dininuta Gdgr mss Fol. obovato-acutiuscula; pet. adsperse pilosi - Rhone, Montmelas (G. herb. ros. n. 135). .1306. C. LATEdentata

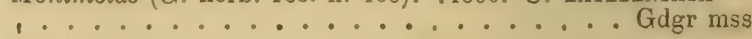


Pet. saepius omnes eglandulosi ............ 148

Pet. I copiose glandulosi . . . . . . . 155

Diseus planus. . . . . . . . . . . 149

Discus | conicus. . . . . . . . . . . 150

Fol. oblongo-lanceolata; costa paulo aculeolata; pet. aculeolati ..................... 149 bis

Fol. oblongo-acuta; costa eglandulosa; pet. subinermes Rhoue, Amat (filer herb ros. 11. 174. . .1307. ('. Isasis

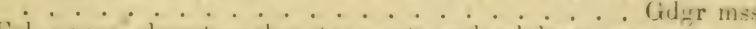

Fol. parva olusato-subacuta; i:osta erlandulosa; pet. nunc: aculeati nune inermes - Iihone, Charbonnieves (1'. Chaloert).

•. . . . . . . . . 1308. disidenabilis Gilgr mss

Pet. Loto rillosi ; fr. basi decurrens - Italia or., Faenza

(Caldesi) . . . . . . 1309. C. FAventiva Gdgr mss

149 Pet. basi tantum villosi ; fr. basi depressus - Suecia, Ves-

bis trogothia: Mt-Kinnekulle (Lundquist). 1310. C. SIPHoxordes . . . . . . . . . . . Gdgr mss

Eul. ollonga ................... . . .

1,50 Fol. obovato-oblonga. . . . . . . . 153

Fol. obovata vel elliptica. . . . . . . 154

$151\left\{\begin{array}{l}\text { Fr. elongatus oblongus - Calvados, Monceaux de Bonne- } \\ \text { Fr. }\end{array}\right.$ chose). . . . . . . 1311. C. Dusosa Gdgr mss Ped. glabri; fol. glauca - Hte-Savoie, Pringy (Puget). 1312.

15: $\ldots \ldots$. . . . DEDolata Gdgr mass Ped. sparse pilosi; fol. lucida - Rhône, Tour-de-Salvagny (Chabert)....... . 1313. C. tubifonsis Gdgr mss

$153\left\{\begin{array}{l}\text { Sep. apice dilatata; perl. 12-19 m. 1. - Sabaurlia, Thoron } \\ \text { (Dés. herb. ros. n. 12). …1314. C. CAprifica Gdgr mss }\end{array}\right.$ Sep. apice filiformia; ped. 7-10 m. 1. - Rhône, Arnas (Gdgr)

. . . . 1315. C. ANXIA Gdgr mss Fol. apice breviter acuta; pet. aculeati; fr. superne subdepressus - Rhône, Gleizé (Gdgr).1316. C. seprvaga Gdgr mss Fol. apice subrotundata; pet. inermes; fr. apice longe attenuato-conicus - Rhône, Montmelas (Gdgr. herb. ros. n. 355) • . . . . . 1317. C. SpILopmylla Gdgr mss Fol. apice acuta ; pet. infer. inermes; fr. apice valde attenuatus - Rhöe, Gleizé (Gdgr herb. ros. eur. n. 92).1318. C. RE-

15, Pet. villosi vel subsparse glandulosi. . . Liglosa Gdgr mss Pet. laevissimi . . . . 158

Fol. amplissime oblongo-lanceolata; ped. villosi $6-10$ in. 1.

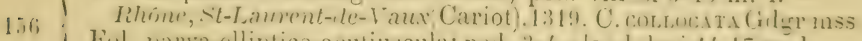

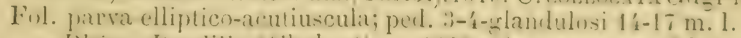

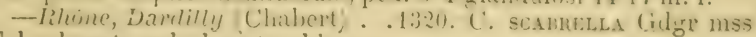
Fol. obovala vel obovato-oblonga ....................

158 Fol. oblongo-acuminata - Aragonia, Boucharo (Bördère).

Pet . . . 1321. C. ARAGONENSIS Gdgr mss

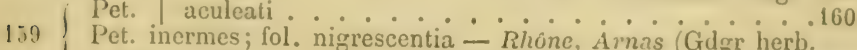
ros. n. 108). 1322, C. yetavocutonos Gerb.

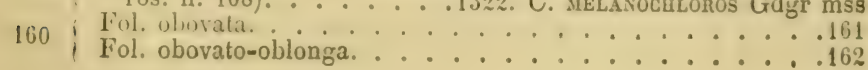


Ped. 4-12 m. 1.; fr. basi paulo attenuatus - Rhône, Montsuit (Chabert). . . . . 1323. C. CAloRHYNCHA Gdgr mss Ped. 15-20 m. l.; fr. basi longe attenuatus - Rhône, Montmelas (G. herb. ros. n. 260). . . 1324. C. Rostella Gdgr Fol. glauca; petala basi eciliata - Gallia (C. glaucescens $162($ Desv. non alior.) . . . 1325. C. Desvauxir Gdgr Fol. nitida ; petala parce ciliata - Rhône, Montmelas (Gdgr herb. ros. n. 210) . . . . 1326. C. CALOdonta Gdgr

\section{b. - Anisodontae Gdgr Essai p. 26. Transitoriae Crep. Prim.p. 17.}

Foliolis aliis simpliciter aliis biserratis plus minusque argutis: serraturae simplices foliolorum inferiorum partem occupant, bene vero compositae sunt superius. Folia virentia rel glaucescentia, ad costam mediam glandulosa necne, nervis subtus plus minusve prominulis; aculeis caulium hornotinorum saepe minoribus quam in speciebus sectionis $a$.

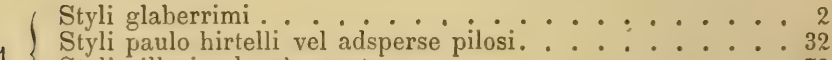

Styli villosi vel pubescentes. . . . . . . . . 72

Styli densissime villoso-lanati. . . . . . . . . . 133

2 $\{$ Petioli eglandulosi. . . . . . . . . . . 3

2 Petioli | glandulosi. . . . . . . . 9

. Fol. ovato-elliptica . . . . . . . . 4

\{ Fol. obovata obovato-oblonga vel oblonga . . . . . . 6

(Fr. ovoideus vel oblongus; rami flor. inermes . . . . . 5

3 Fr. subglobosus ; rami flor. aculeati - Helv., Bâle (Cottet).

. . . 1327. C. BRACHYCALYX Gdgr mss Discus subplanus; fr. oblongus - Pedem, Riva Valsesia

. (Carestia in soc. helv. 1877) . 1328. C. Carestiae Gdgr mss

Discus conicus; fr. ovoideus - Suecia, Mt Kinnekulle (Zettersiedt). . . . . . . 1329. C. subinenMis Gdgr mss Fr. oblongus; sep. eglandulosa vel 1-3 dentata. . . . . 7

Fr. obovato-oblongus; sep. dentato-glandulosa - Hte-Savoie,

(i) Pringy (Puget) . . . . 1330. C. ololeia (Rip. mss) Fr. ovoideus; sep. eglandulosa - Cher, Les Aix (Rip).1331.

- Rami flor. aculeati; fol basi rotundata . . . . . . 8 8 Rami flor. inermes; fol. basi attenuata - Vosges, Epinal (Berher). . . . . 1331 bis. C. TENuIon Gdgr mss 
Ped. villosi 12-14 m. 1.; pet. aculeati - Italia, Modene
(Gibelli). 1332. C. Gracilituba Gdgr mss Ped. glabri 9-10 m. l.; pet. inermes - Austr. inf., Kalhsburg

(Wiesbaur) . . . . 1333. C. Jacquiniana Gdgr mss

9 Fr. ovatus vel olovovatus. . . . . . . . . 10

Fr. ohilongus. . . . . . . . . . . . 19

Fol, ovata vel ovato-elliptica . . . . . . . . 11

101 Fol. obovato-oblonga vel oblonga. . . . . . . 14

Fol. et fr. obovato-oblonga - Tyrol, Trente (Gelmi).1534.

, Discus planus $\because \cdots \cdots \ldots$

Discus | conicus . . . . . . . . 13

Fr. ovatus; fol. parvá bảsi attenuata - Pyrenaei or., Camélas

12 (Debeaux) . . . 1335. C. Calcarea Gdgr

Fr. ovoideus; fol. ampla basi rotundata - Pyrenaei, Gèdre

G. herb. ros. n. 247) . . . 1336. C. Acmodoxta Gdgr mss

Fol. basi rotundata; fr. inferne depressus; ped. 5-8 m. l. -

Borussia occ., Nahe. . . . 1337. C. ExILIs (Crép. et Wirtg.)

Fol. et fr. basi attenuata ; ped. 11-14 m. l. - Rhône, Pommiers (G. herb. ros. n. 186). .1338. C. anbutifolia Gidgr mss

Fol. basi attenuata; fr. inferne rotundatus; ped. 15-20 m. 1 . - Hte-Savoie, Pringy (Puget).1339. C. ovatifolia Gdgr mss

Discus conicus. . . . . . . . . . . 15

Discus planus. . . . . . . . . . 16

Fol. basi cordata amplissima; pet. infer. inermes - Isère, Charancieu (Gdgr herb. ros. n. 142).1340. U. ULMifonMIS

Fol. minora basi truncata; pet. omnes aculeati - Gironde, Pauillac (Motelay) . . . 1341. C. Fruteticola Gdgr mss

Fol. merlincria hasi attenuala; pet. omnes parce aculeati l'yienaei, (iedre (Bordere). . 134?. C. Rimondixa Gidgr mes

Fr. ovoideus ; fol. obovato-oblonga; cor. eciliata. . . . . 17

16 Ir. oswideus; fol. oblonga; cor. valde ciliata. . . . . 18

I'r. parrus ovatus ; fol. oblonga - Angl., Cheshire (J. M.

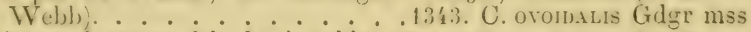
Discus elevatus; fol. basi valde attenuatir - IIelv., Taud: Tartegnins (Favrat). . . . 1344. C. uxcivula Gdgr mss Discus planus; fol. basi sensim attenuata - Hisp. bor. Logrono (Zubia). . . . 1345. C. Echeandiaxa Gdgr mss Sep. dorso glandulosa; fol. basi attenuata - Angl., Cor-

18 nouailles (Briggs). . . 1346. C. obloxgIFolia Gdgr mss Sep. dorso eglandulosa; fol. basi rotundata - Hisp. bor., Logrono (Zubia) . . . . 1347. C. PERMutata Gdgr mss

19 Fol. oblonga elliptica vel obovato-oblonga . . . . . . 24

20 Rami lloriferi inermes .............. 21

20 Rami flor. I aculeati . . . . . . . . . . 23

( Glaucescens; ped. 10-12 m. 1.; pet. inermes vel subinermes. 22 Atrovirens; ped. 7-8 m. 1.; pet. aculeati - Suecia mer., Ystad ('Tullberg, elc.). . . 1348. C. Atnovinens Gdgr mss Discus subconicus ; costa eglandulosa-Thuringia, Goldnhau

22 (Haussknecht). . . 1349. C. PAllescexs Gdgr mss Discus valde conicus; costa paulo glandulosa - Gallia, Clermont (Lamotte). . . . . 1350. C. Lamotti Gdgr mss 
Fr. basi longe attenuatus ; ped. 13-16 m. l.; pet. parce aculeati Rhône, Arnas (Gdgr herb. ros. n. 288).1351. C. POETiCa Gdgr Isère, Charancieu (Gdgr herb. ros. n. 6).1352. C. Exsertis-

Rami floriferi inermes . . . . . . . . 25

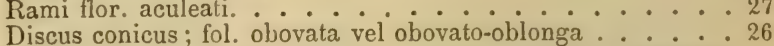

25 Discus subconicus; fol. elliptica - Rhône, Dardilly (Chabert).

. . . . . . 1353. C. Adornata Gdgr mss

Fol. basi subrotundata; pet. omnes parce aculeati - Rhóne, Montmelas (G. herb. n. 253) . .1354. C. Congruens Gdgr mss

Fol. basi subattenuata; pet. sup. inermes - Italia, Modène (Gibelli). . . . . . . 1355. C. Italica Gdgr mss

Fol. basi truncato-acuta; pet. omnes inermes - Bouches-duRhône, Berre (Autheman). . . . 1356. C. Pruva Gdgr mss

Discus planus. . . . . . . . . . . . . . . 28

Discus / conicus . . . . . . . . . . . . 29

Ped. 10-15 m. 1.; fol. obovato-elliptica basi subrotundata Rhône, Arnas (Gdgr). . . . 1357. C. Exoptata Gdgr mss

Ped. 7-9 m. 1.; fol. ovata basi breviter attenuata - Helv., Bâle (Cottet) . . . . . 1358, C. SEPTICA Gdgr mss

Fol. basi cordata vel rotundata. . . . . . . . . 30

Fol basi | attenuata ................... 31

Fr. basi longissime attenuatus; fol. obovato-oblonga - HteSavoie, Pringy (Puget). . . 1359. C. Elisantha Gdgr mss

Fr. basi attenuatus ; fol. obovala - Bouches-du-Rhỏne, Martigues (Autheman) . . . 1360. C. MAssILIEnsis Gdgr mss

Fr. basi depressus; fol. obovata - Basses-Alpes, Bouzolieres (Proal) ........ 1361. C. Polycephala Gdgr mss Fr. basi paulo depressus; fol: inferne paulo attenuata Gallia, Perpignan (Debeaux). 1362. C. Companyonis Gdgr mss

31 Fr. basi longe decurrens; fol, basi longe cuneata - Aragonia austr., Sierra de Mosqueruela (Martin).1363. C. Pardoana . . . . . . . . . Gdgr mss

32 i Costa media enlandulosa, interdum basi 1-2-aculeolata. . . . 33 Costa media glandulosa, interdum | aculeolata. . . . . 49

33 Fol. basi late rotundata vel cordata......... 34

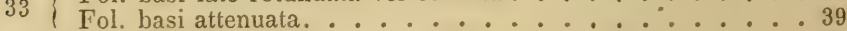

Fr. oblongus .................... 35

34 Fr. obovatus vel obovato-oblongus. . . . . 37 Fr. globosus - Angl., Ambleside (Woods).1364. C. NUDA

Pet . . . . . . (Woods)

$35 \int$ Pet. aculeati, fol . . . . . . 36 Pet. inermes; fol. anguste oblongo-lanceolata - Rhône, Pommiers (Gdgr) . . . . 1365. C. Apiceacuta Gdgr mss

$36\left\{\begin{array}{l}\text { Rami flor. aculeati; fol. lata-Sabaudia; Helv.1366. C. Fallens } \\ \text { D. (Dégégl.) }\end{array}\right.$ Rami flor. inermes; fol. parva - Catalaunia, Olot (Vayreda) . . 1367. C. Vayredae Gdgr mss Rami flor. I aculeati. . . . . . . 3 38 Rami flor. inermes - Pyrenaei, Lourdes (Bordère).1368. C. . CORDigera Gdgr mss 
Fol. ovato-elliptica ; discus conicus - Rhône, Arnas (Gidgr herb. ros. n. 243). . . . . 1369. C. Thevenetil Gidgr mss

Fol. ovato-elliptica; discus planus - Rhöne, Craponne (Cariot). . . . . 1370. C. privata Gidgr mss

Fol. oblongo-lanceolata ; discus paulo conicus - Isère, Les Abrets (Gdgr) . . . . . 1371. C. scapigera Gidgr mss

Fr. globosus vel subglobosus . . . . . . . . . 40

Fr. ovatus. . . . . . . . . . . . . 41

39 Fr. ovoideus vil obovato-oblongus. . . . . . . 42

Fr. oblongus - Tyrol, Trente (Gelmi).1372. C. SEMminta

Discus planus ; fol. ovato-elliptica - Gallia centr. 1373. C.

Discus conicus; fol. obovata - Hte Savoie, IIabere-Lullin (Puget). . . . . 1371. C. pubens Gdgr mas

Fol. ovato-elliptica; pet. paulo aculeati - Pyrenaei centr., Vallee d'Ossau (Bord.; Gdgr herb. ros. n. 97).1375. C.

Fol. nlusata ; pet. fere inermes - Rhone, Lyon Grlat . 1376.

i... Conylordes Gdgr ms

4. 1 Rami floriferi inermes.............. 43

1 Rami flor aculeati. . . . . . . . . . 46

4.: Discus planus. . . . . . . . . . . 丢

Discus paulo conicus. . . . . . . . . . 45

Fol. obovato-oblonga; ped. 13-1'́ m. 1. - Angl., Cheshire

44 (J.M. WVebb). . . . . 1377. C. Microcarpa Gdgr mss Fol. obovata; ped. 9-11 m. 1. - Thuringia, Goldnhau (Haussknecht) . . . . 1378. C. THurisgidCa Gdgr mss Pet. eglandulosi; ped. 13-14 m. 1. - Helv., Vaud: Tar-

45 tegnins (Favrat). . . . 1379. C. Microvegas Gdgr miss ( Pet. glandulosi ; ped. 5-7 m. 1. - Loire, Mt-Pilat (Gdgr)

1380. C. praemissa Gdgr mss

46 Fol obovata. ..... 47

Fol. obovato-oblonga vel oblonga . . . . . . 48

Discus planus; ped. 5-7 m. 1. - Manche, Yrceto! (Lebel) 47 Discus conicus; ped. $13-15$ m. 1. 1381 - C. Instr. inf., Kalksburg

(Wiesbaur). . . . . . 1352. C. umbilicata Gidgr mss

Ped. 5-7 m. 1.; pet eglandulosi - Angl. bor., Thirsk (Baker)

Ped. $9-14$ m. 1.; pet. sparse glandulosi - Ain, La Pape (P

Chabert). . . . . . 1384. C. Gracilion Gidgr mss

Rami flor. inermes, rarius $1-2$ aculeati. . . . . . 50

50 Pet. saepius omnes inermes. . . . . . . . . 51

Pet. aculeati. . . . . . . . . . 54

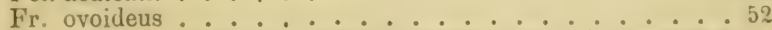

Fr. ovatus - Pyrenaei, Gèdre (Bordère).1385. C. Avgustion

Fr. valde elongatus - Rhône, La Tour-de-Salvagny (Chabert)

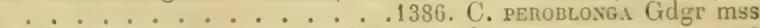

Fol. elliptica vel obovata ; ped. 6-12 m. . . ..... 53

52 Fol. ovata; ped. $12-16 \mathrm{~m}$.1. - Pyrenaei centr., Ossau Gdgr herb, ros. n. 127). . . . 1387. C. REDoless Gdgr mss 
$53\left\{\begin{array}{c}\text { Arnas (Gdgr) } \\ \text { Discus conicus; fol. obovata sensim attenuata - Isère, Cha- }\end{array}\right.$ rancieu (G. herb. ros. n. 181) . 1389. C. FAstibILIS Gdgr mss

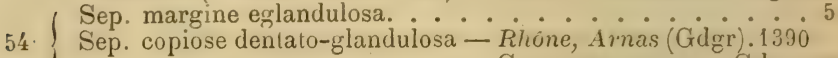

. . . . . . . C. glandulisepala Gdgr mss

Fol oblonga . . . . . . . . . . 56

Fol. ovato-cordata; costa valde aculeata - Pyrenaei, Ossau

55 Fol. elliptico-attenuata; costa 1-3-aculeata - Rhône, Arnas

Fol. elliptico-attenuata; costa 1-3-aculeata - Rhône, Arnas
(G. berb. ros. n. 176). . 1392. C. Appropinquata Gdgr mss

Fol. obovata; costa laevis - Puy-de-Dôme, Montbaubier (Dubosclard) . . . . . 1393. C. subconferta Gdgr mss

Discus planus; rami flor. subinermes - Rhóne, Denicé (Gdgr

56 herb. n. 100) .......1394. C. Saltivaga Gdgr mss Discus valde conicus; rami flor. inermes - Bouches-duRhône, Roquefavour (Autheman). .1395. C. Incurva Gdgr mss

57 Sepala copiose marginato-glandulosa. . . . . . . 58

57 Sep. margine eglandulosa vel 3 -4-dentata. . . . . . . 59

Cor. ciliata ; Ir. olorato-oblongus; discus sulsconicus -

Rhône, Arnas (Gdgr). . . 1396. C. Astreata Gigr mss

58 Cor. eciliata; fr. ovoideus; discus ennions - Phinne, Arnas (Gdgr)......... . 1397. C. Lenta Gdgr mss

Cor. eciliata ; fr. oblongus; discus valile conicus - IIisp. bor., Logrono (Zubia). . . 1398. С. Pectinella Gdgr mss

(Fol. basi cordata vel rotundata . . . . . . . . 60

59 Fol. basi sensim attenuata vel truncata . . . . . . 65

Fol. basi valde acuta. . . . . . . . . . 70

Fol. obovata vel obovato-oblonga . . . . . . . . 61

60 Fol. ovata. . . . . . 63

Fol. elliptica - Pyrenaei cent., Benqué (Bordère).1399. C.

•.......... Falcatula Gdgr mss

Fr. oblongus; fol dentes plerumque biserratae. . . . . 62

61 Fr. obovatus; fol. dentes plerumque simplices - Rhône, Arnas (G. herb. ros. n. 160) .1400. C. strictidenta Gdgr mss

$62\left\{\begin{array}{l}\text { Pet. eglandulosi; discus subconicus - Hung., Albe : Erd } \\ \text { (Tauscher). }\end{array}\right.$ Pet. valde glandulosi ; discus subplanus - Rhône, Arnas (Gdgr herb. ros. n. 126) . . . . . 1402. C. scrupea Gdgr

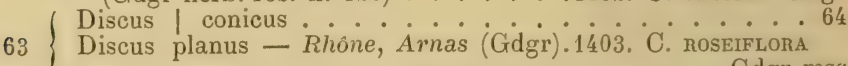

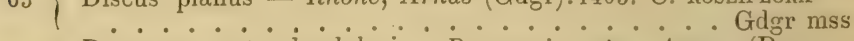
Purpurea; pet. glandulosi - Pyrenaei centr., Arran (Bor-

64 dère). . . . 1404. C. vinosa Gdgr mss Virens ; pet. eglandulosi - Pyrenaei, Gèdre (Gdgr herb. ros. n. 353). . . . 1405. C. Curvispina Gdgr msS

65 Discus planus vel subplanus . . . . . . 66

Discus conicus . . . . . . . 667

Ped. glabri 10-14 m. l. ; fr. basi valde attenuatus - Rhône, Arnas (Gdgr). . . . . . 1406. C. Lupulina Gdgr mss

Ped. glabri 7-8 m. 1.; fr. basi depressus - Ain, Néron (P. Chabert) . . . . 1407. C. argutiserrata Gdgr mss Ped. villosi $12-14 \mathrm{~m}$. 1.; fr. basi rotundalus - Bavaria, Schloss Zeil (Kugler). . . . 1408. C. BAvarica Gdgr mss 
Fr. oblongus basi rotundatus vel depressus. . . . . . . 68

Fr, ovoideus basi | attenuatus . . . . . . . . 669 Dentes convergentes; sep. apice integra - Basses-Alpes, Condamine (Proal). . . . 1409. C. DELPHINEXsis Gdgr mss Dentes apertae; sep. apice dentata - Rhône, Charbonnières (P. Chabert). . . . . 1410. P. BREvcollis Gdgr mss

Fol. oblonga; pet. aculeati - Pyrenaei Orient., St Antoine de Galamus (Debx) . .1411. ('. Timbaliana Gdgr et Debeaux Fol. obovato-oblonga; pet. inermes - Austr. inf., Kalksburg (Wiesbaur). . . . . . 1412. C. Foliolosa Gdgr mss Fol. et fr. obovata vel obovato-oblonga . . . . . . . 71

70 Fol. et fr. apice constrictus) oblonga - Puy-de-Dome, Savy (Dubosclard) . . . . . . 1413. C. PRESSA Gdgr msS Discus subconicus; sep. pinnulae 4-6-dentatae - Rhône, Arnas (Gdgr) . . . . . 1414. C. odontoxys Gdgr mss Discus valde conicus; sep. pinnulae integerrimae - B.-duRhône, Pas-des-Lancier's (Autheman).1415. C. AMoene-

virens Gdgr ms

72 Sep. I copiose glanduloso-marginata . . . . . . 73

Sep. margine eglandulosa. . . . . . . . . . 86

Fr. ovatus ovoideus vel obovato-oblongus. . . . . . . 74

73 Fr. oblongus ............... . 78 Fr. globosus - Pyrenaei, Gèdre (Bordère).1416. C. venset. . . . . . . . . Gdgr mss

74 ( Fol. ovata vel ovato-elliptica; discus conicus. . . . . . 75 Ful. nbovato-oblonga vel oblonga; discus planus vel subplanus ................. 76

$75\left\{\begin{array}{l}\text { Ped. 3-7 m. li ;ol. glauca basi subattenuata - Rhône, Mont- } \\ \text { melas (Gdgr) } \\ \text { Med }\end{array}\right.$ Ped. 8-10 m. l.; fol. virentia basi rotundata - Gallia, Perpignan (Debeaux) . . . 1418. C. TRIaxgularis Gdgr mss

76 Fol. oblonga basi attenuata. . . . . . . . 77 Fol. obovato-oblonga basi rotundata - Bomussia, Dantzig (Baenitz). . . . . 1419. C. pomeranica Gdgr mss Ped. 3-5 m. l.; fol. lanceolata - Thuringia, Goldnhau

7 (Haussknecht). ..... 1420. C. TENuIfoliA Gdgr mss Ped. 11-13 m. 1.; fol. acuminata - Oise, Noyon (Nagnier) . . . . . . . 1421. C. MigropapPa Gdgr mss

( Fol. ovata vel ovato-elliptica . . . . . . . 79

is Fol. obovata vel obovato-oblonga ......... . . 80 Fol. oblonga ..................... 85 Cor. pallide rosea : discus planus - Thime, Amas (Grlgr) Cor amoene rosea; discus conicus - Rhône, Arnas (Gdgr herb. ros. n. 306) . . . 1423. C. AdENochlanyda Gdgr Discus evidentius conicus. . . . . . . . . 81

I)iseus paulo conicus; rani flor. et petioli inermes - Suecia,

80 Scania : Pinelierna (Tullberg).1424. C. cLadophora Gdgr mss Discus planus ; rami flor. et pet. aculeati - Rhône, Arnas (G. herb. ros. n. 278) . . . 1425. C. Dentosa Gdgr mss 81 Tami flnrales inermes . . . . . . . . . 82 Rami flor. aculeati. . . . . . . . . 83 
Pet. et costa media glandulosa - Helv., Neuchátel (Morthier) .......... 1426. Collincold Gdgr mss Pet. et costa eglandulosa - Austr. inf., Kalksburg (Wiesbaur) . . . . . . . 1427. C. InCOMPTA Gdgr mss Costa media tantum glandulosa . . . . . . . . 84 Costa glanduloso-aculeolata - Silesia, Landeshut (Uechtritz) . . . . . . 1428. C. AciculIfolia Gdgr mss Fol. obovata basi subattenuata; ped. 11-15 m. 1. - Loire, Mt-Pilat (Gdgr) . . . . 1429. C. Diphthenitis Gdgr mss Fol. obovato-oblonga basi subrotundata; ped. 9-10 m. 1. Rhône, Montmelas (Gdgr herb. ros. n. 133).1430. C.

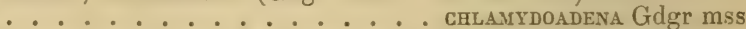
Pet. aculeati ; rami flor. inermes - Thuringia, Oesterfeld (Haussknecht). . . . . 1431. C. Involucrata Gdgr mss Pet. inermes; rami paulo aculeati - Silesia, Brachoogel (Straehler). . . . . . 1432. C. silesiaca Gdgr mss

86 Tota amoene purpureo-violacea .......... 87 Tota virescens vel haud aut vix purpurea.

87 Fr. ovatus vel rotundatus. . . . . . . . . 88 \{ Fr. ovoideus vel obovato-oblongus. . . . . . . . 89 Discus subconicus; fol. ovata vel rotundata - Rhône, Alix $88\left\{\begin{array}{l}\text { (Gdgr herb. ros. n. 297) . 1433. C. PYCNopHylla Gdgr mss } \\ \text { Discus sat conicus; fol. oblonga - Rhône, Ville-sur-Jarnioux }\end{array}\right.$ (Gdgr). . . . . 1434. C. onthochlanys Gdgr mss Fol. ovata vel ovato-elliptica . . . . . . . . 990

89 Fol oblonga. . . . . . . . . 91 Fol. obovato-oblonga - Rhône, Pommiers (Grdgr).1435. C. . . . . . . . . cruentata Gdgr mss Pet. glandulosi ; fol. acuta basi subrotundata - Sabaudia, etc.

90 (Billot n. 3579) ......... 1436. C. spuris (Puget) Pet. eglandulosi; fol. lanceolata basi attenuata - Gallia, Annecy (Puget) . . . . 1437. C. ozwophora Gdgr mss Discus planus; ped. 6-7 m. 1. - Loîe, Mt-Pilat (Gdgr).1438

Discus conicus; ped. $9-11$ m. 1. - Thuringia, Oesterfeld (Haussknecht) . . . . 1439. C. subhirta Gdgr mss Pet. glabri vel parce hirtelli. . . . . . . . . 993

2 Pet. minute villosi vel usque ad 2 a folia infer. sparse hirsuti.127

93 Fr. ovatus ovoideus vel obovato-oblongus . . . . . 94

, Fr. oblongus ..........................

9 ( Rami floriferi inermes . . . . . . . . . . 95

Rami flor. I aculeati ............ 98

95 Discus conicus. . . . . . . . . . 96 Discus planus vel subplanus . . . . . . . . . 97 Pet. inermes; fol. obovata basi rotundata - Rhóne, Arnas

96 (Gdgr herb. ros. n. 156). . . . 1440. C. LAevis Gdgr mss Pet. aculeati ; fol. obovato-oblonga basi attenuata - Suecia, Scania : Alnarp (Tullberg). . 1441. C. Divaricata Gidgr mss Fol. oblonga; ped. 6-8 m. 1. - Austr. inf., Kalksburn (Wiesbaur) .............. . . Mit2. Gidgr mss Fol. obovato-oblonga; ped. 12-14 m.1. - Rhône, Montmelas (Gdgr herb. ros. n. 51) . . . 1443. C. mammosa Gdgr mss Fol. obovata; ped. 6-8 m. 1. - Suecia, Scania : Alnarp (Tullberg). . . . . 1444. C. MYriophyLla Gdgr mss 


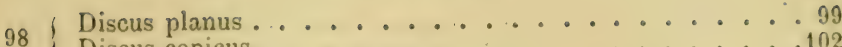

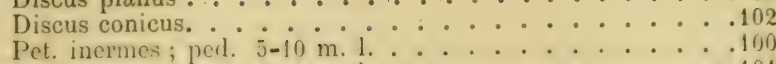

99 Pet. aculeati; perl. $12-15 \mathrm{~m}$. 1. . . . . . . . 101

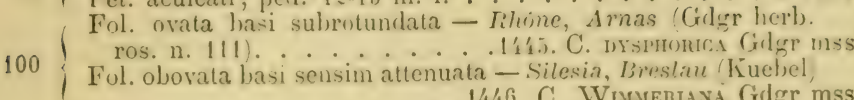
Fol. obovata basi sensim attenuata - Silesia, Breslan (Kuebel

Fol. ovato-elliptica hasi cuncata - Phhore, Pommiers Golgr mss

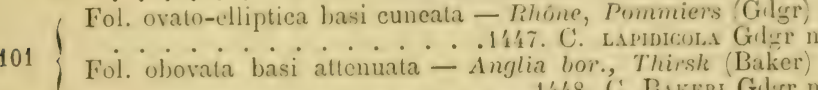

Fol. obovata basi attonuata - Anglia bor., Thirsk (Baker)

Fol. obovato-oblonga basi attenuata 144. C. Baken Grder mss

102 Fol. obovata basi rotundata . . . . . . 104 Fol. ovata basi rotundata - Rhône, Alix (Gdgr).1449. C.

Petala eciliata; ped. 7-10 m. 1. - Rhöne, Arnas (Gdgr herb. $103\left\{\begin{array}{l}\text { ros. n. 152) } \\ \text { Petala ciliata ; ped. 12-14 m. i. } 1450 \text {. Rhône, Montmelas (Gdgr). }\end{array}\right.$

Peil. $18-20 \mathrm{~m} . \mathrm{i}$; fol. basi late cordata - Bouches-du-Tih., Roquefavour (Autheman). 1452. C'. Castagieasa Gdgr mss

104 Ped. 11-13 m. 1.; fol. basi rotundata - Hisp. bor., Logrono (Zubia) . . . . . 1453. C. Zublae Gdgr mss

Rami floriferi inermes ..................

i Rami flor aculeati ................ . . . . . .

106 Fol. basi rotundata vel cordata. . . . . . 107

Fol basi attenuata. . . . . . . . . 109

Fol. obovata; discus planus vel subconicus . . . . . 108

Fol. ovata; discus conicus - Rossia austr., Odessa.1454. U. 107 Fol. elliptica; discus paulo conicus - Thuringia, Rudolstadt

Fol oblongo-cordata; discus conicus - Suecia, Scania : Orup

(Tullberg). . . . 1456. C. Tullbergir Gdgr mss

Pet. inermes; ped. 7-8 m. 1. - Italia mer., Albe (Gibelli) $108\left\{\begin{array}{l}\text { Pet. aculeati ; ped. } 10-15 \mathrm{~m} \text {. 1. - Cher, St Ursin (Ripart) } \\ \text { - } 1457 \text {. C. Rovara Gdgr mss }\end{array}\right.$

Discus | conicus; ped. glabri. ............ . .

$109\{$ Discus planus; ped. villosi - Rhône, Amas (Gdgr). 1459. C.

(Ped. $8-15 \mathrm{~m} .1$. fr. basi attenurtus. . . . . . . 111

Pud. $18-23 \mathrm{~m}$. 1,; ir. hasi longe dentrens - Rhine, Charbonnières (Cariot) .....1460. C. Acicarpa Gdgr mss Pet. | aculeati; discus paulo conicus. . . . . . .112

111 Pet. omnes inermes; discus valde conicus - Serbia, Belgrade (Pancic). . . . . . 1461. C. Pancicil Gdgr mss Pet. sup. inermes; ped. 12-15 m. 1. - Isère, Charancieu

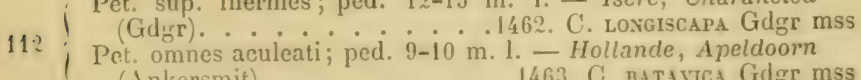

(Ankersmit) . . . . . 1463. C. Batavica Gdgr mss

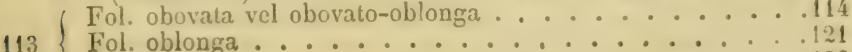

Fol ovata. ................. 126 
114 Discus planus. ................... . . . .

Discus conicus. . . . . . . . . . . . . . .

Cor. ciliata; fol. basi subrotundata - Manche, Valognes

115 (Lebel). . . . . 1464. C. pogonopetala Gdgr mss Cor. eciliata; fol. attenuata - Thuringia, Wallendorf (Haussknecht). . . . . . . 1465. C. oBLONGA Gdgr mss

116 ( Fol. obovata. . . . . . . . . . . . . . . . . . . . .

116 Fol obovato-oblonga. . . . . . 120 $117\left\{\begin{array}{l}\text { Pet. aculeati; fol. basi I rotundata. } \\ \text { Pet. inermes; fol. basi attenuata }- \text { Silesia, Mts-Géants }\end{array}\right.$ (Uechtritz).......... 1466. C. MODEsTA Gdgr mss

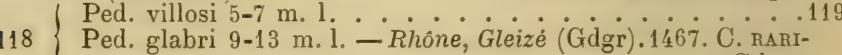
Ped. glabri 9-13 m. 1. - Rhône, Gleizé (Gdgr).1467. C. RARI-

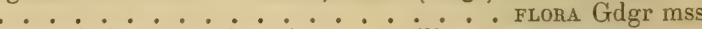

Fol. minutissima obtusiuscula; cor. ciliata - Hisp., bor., Logrono (Zubia) . . . . . 1468. C. MicRoideA Gdgr mss

119 Fol. abrupte acuminata; cor. eciliata - Aragonia austr. Sierra de Mosqueruela (Martin).1469. C. Mosqueruelensis

. . . . Gdgr mss

Ped. 8-11 m. 1.; fol. basi breviter attenuata-Gallia, Besançon (Paillot, Fl. Sequaniae exs., n. 50).1470. C. Criacantha

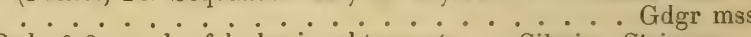

Ped. 6-8 m. l.; fol. basi subtruncata - Silesia, Striegau (Uechtritz) . . . . . 1471. C. virens Gdgr msS

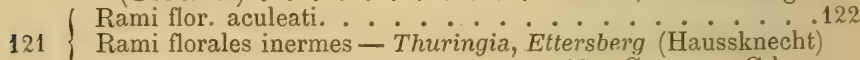
........... 1472. C. AGUTA Gdgr mss

Fol, virentia inferne attenuata. . . . . . . . . 123

122 Fol. coerulescentia basi rotundata - Rhône, Arnas (Gdgr herb. ros. n. 220). . . . 1473. C. coerulescens Gdgr mss

Pet. omnes aculeati; ped. $14-18 \mathrm{~m}$. 1. . . . . . . 124

Pet. subinermes; ped. 9-11 m. 1. - Vosges, Epinal (Berher).

Pet. omnes inermes; ped. 10-13 m. 1. - Rhône, la Tour-deSalvagny (Chabert).... 1475. C. Illustrata Gdgr mss

$124\left\{\begin{array}{l}\text { Pet. paulo glandulosi ; fr. basi longissime attenuato-decurrens. } 125 \\ \text { Pet. eglandulosi; fr. basi subdepressus - Suecia, Eland: }\end{array}\right.$ Albrenna (Scheutz). . . 1476. C. oELAvdica Gdgr mss Sep. apice angustata; discus conicus - Suecia, Scania:

125 Alnarp (Tullberg). . . . 1477. C. MELANomea Gdgr mss Sep. apice foliacea ; discus paulo conicus - Vosges, Epinal Berher) . . . . . . 1478. C. Nigrescens Gdgr mss Sep. dentata; fr. utrinque sensim attenuatus - Rhône, Arnas Gdgr). . . . . . . 1479. C. Rubriflora Gdgr mss

126 Sep. integra; fr. basi subrotundatus apice valde strangulatus - Basses-Alpes, Bouzolières (Proal).1480. C. Minuscula .............. Gdgr mss

Fr. oblongus . . . . . . . . . . . . 128 Fr. et fol. obovato-oblonga - Rhône, Arnas Gdgr herb. ros. n. 139). . . . . 1481. C. SPISsa Gdgr Fr. ovoideus; fol. ovata - Rhône; Cher.1482. C. Horridula (Dés.)

127 Fr. ovatus; fol. minutissime elliptica - Carnia, Laybach (C. canína myrtilloides Tratt. ros. II, p. 20).1488. C. MYRFr. globosus; fol. oblonga - Germ. occid., Wurtzburg (Rau). ............. C. Aciphylla (Rau) 


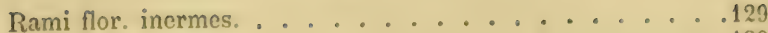

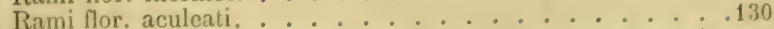

Fol. oblonga basi attenuata; ped. glabri - Belg., Rochefort (Crépin). . . . 1185. C. Clavata (Crép. mss) Fol. obovata basi attenuata; ped. villosi - Rhone, Ecully

129 (Chabert). . . . . 1486. C. Enocta Gidgr mss Fol. obovata basi rotundata ; ped. glabri - Pyrenaei, Gedre (Bordère) . . . . 1487. C. phoenicocanpa Gidgr mss Fol. basi cordata. . . . . . . . . . . . . . . .

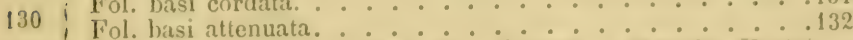
Fr. inferne paulo depressus; fol. oblonga - Manche, Yvetot $131\left\{\begin{array}{l}\text { (Lebel) } \\ \text { Fr. basi valde attenuatus; fol. ovata - Rhone, Arnas (Gdgr } \\ \text { herb, ros. n. 229). }\end{array}\right.$ herb, ros. n. 229). . . 1489. C. icmanopiryla Gdgr mss Ped. glabri 9-12 m. i.; fol. basi acuta - Rhóne, Amas (Gdgr) 132 Ped. villosi $8-9 \mathrm{~m}$. $1 . ;$ fol. basi attenuata - Tyrol, llall Gremblich)........ 1491. C. puberula Gdgr mss

i Fr. ovato-cllipticus ovatus vel rotundatus. . . . . . 134

133 Fr. ovoideus obovato-oblongus vel oblongus . . . . 157 Ped. glabri vel adsperse hirtelli, vel basi tantum unifariam

134 subhirsuti. . . . . . . . . . 135 Pet. undique saepius ad apicem usque laxe villosi. . . . 154

Fol. basi rotundata vel cordata ........... . . 136

135 Fol basi / attenuata vel acuta. . . . . . . . . . . . . .

136 I Rami florales aculeati . . . . . . . . . 137

136 I Rami flor omnino inermes. . . . . . . . . 133

Fr. ovatus: ped. 6-8 m. . . . . . . . . 138

137 Fr. rotundatus; ped. $13-15 \mathrm{~m} .1$ - Vallesia, Tourbillon (Favrat). . . 1492. C. Subglobosa Gdgr mss Pet. inermes ; costa eglandulosa - Anglia, York (Webster).

138 Pet. aculeati; costa paulo glandulosa-Rossia, St-Pétersbourg (Meinshausen Herb. flor. Ingr. n. 218).1494. C. PETEnsBUrGENsis Gdgr mss Pet inermes; ped glabri. . . . . . . . 140

139 Pet. aculeati; ped. villosi. . . . . . . 112 Fol. mediocriter ohovato-oblonga; fr. inferne depressus. . .141

140 Fol. amplissime obovata; fr. basi rotundatus - Rhöne, Chervinges $(\mathrm{Gdgr}) . . . .1495$. C. Figrroria Gdgr mss Pet omnes inermes; ped. 6-8 m. 1. - Scotia, IIt-Clova (DrumDiscus conicus ; pet. glandulosi - Rhöne, Arnas (Gdgr herb. ros, n. 107). 1498. C. grossulanordes Gdgr mss

1'? Discus planus; pet. eglandulosi-Pyrenaei, Gèdre (Bordere). 1499. C. eunicturos Gdgr mss

143 Fol ovata obovata vel obovato-oblonga . . . . . 144 Ful oluma . . . . 1.0

144 , Rami florileri inermes . . . . . . . . . 145 Rami flor, aculeati. . . . . . . 146 
( Fr. rotundatus; fol. ovata - Sabaudia, etc. (Billot exs. n. 3580 ; Dés. herb. n. 61) . . . 1500. C. montivaga (Dés.) Fr. globosus; fol. obovata - Hte-Savoie, Habère-Poche (Puget). . . . . 1501. C. Henicycha Gdgr mss

146 I Discus planus . . . . . . . . . . . . . . . . . . . Discus | conicus. . . . . . . . 149 Ped. 8-15 m.1.; fol. obovato-oblonga lanceolata . . . .148

147 Ped. 5-7 m. l.; fol. acutiuscule obovata-Thuringia, Oesterfeld (Haussknecht). ..... 1502. C. CERAsiformis Gdgr mss Ped. 13-15 m. 1.; pet. inermes - Anglia, York (Webster). $148\left\{\begin{array}{l}\text { Ped. } 7-11 \text { m. l.; pet. aculeati - Suecia, Eland: Farjestaden }\end{array}\right.$ (Scheutz). . . . . . 1504. C. globosa Gdgr mss $149\left\{\begin{array}{c}\text { Fr. basi rotundatus; fol. minutissime obovata }- \text { Rhône, Beau- } \\ \text { nant (Cariot) }\end{array}\right.$ Fr. basi abrupte decurrens; fol ampliuscule obovato-oblonga - Helv., Lausanne (Favrat).1506. C. stexonhyncha Gdgr mss

150 i Rami flor. aculeati; discus paulo conicus. . . . . . 151

150 Rami flor. inermes; discus planus. . . . . . 152

$151\left\{\begin{array}{l}\text { Ped. 1?-15 m. I.; fr. basi rotundatus - Rhône, Dardilly } \\ \text { (Chabert). } \text { Ped. 8-9 m. i.; fr. depressus - Angl. Yorkshire: Boltby }\end{array}\right.$ Ped. 8-9 m. 1.; tr. depressus - Angl., Yorkshire: Boltby (Baker). . . . . . 1508. C. BRitannica Gdgr mss Pet. glandulosi ped. $16-20 \mathrm{~m} . \mathrm{l} . \ldots . . . . .153$

152 Pet. eglandulosi; ped. 8-9 m. l. - Austr. inf., Kalksburg (Wiesbaur) . . . . . 1509. C. AMOENA Gdgr mss

$153\left\{\begin{array}{l}\text { Sep. foliaceo-laciniata; fol. basi vix attenuata Hte-Savoie, } \\ \text { Habère-Lullin (Puget). } 0 \text {. } 1510 \text {. C. CLINELLA Gdgr mss } \\ \text { Sep. sat angustata; fol, basi longe attenuata - Gironde, Le }\end{array}\right.$ Sep. sat angustata; fol. basi longe attenuata - Gironde, Le Verdon (Motelay). . . . 1511. C. GREgaria Gdgr mss Pet. eglandulosi aculeati $\ldots . . . .155$

154 Pet. glandulosi inermes vel subinermes . . . . . . 156

154 i Pet. eglandulosi inermes - Croatia, Sestine (Vukotinovic). . . . . . . . 1512. C. croatica Gdgr mss Discus planus; sep. apice foliacea - Hte-Savoie, Habère-

$155\left\{\begin{array}{l}\text { Poche (Puget). . . . . Dicus conicus; sep. apice filiformia - Aragonia austr., } S^{a} \text { de } \\ \text { Discus }\end{array}\right.$ Mosqueruela (Martin) . . . 1514. C. Loscosiana Gdgr mss

156 Costa paulo glandulosa; sep. integra eglandulosa-Hte-Savoie, Costa eglandulosa; sep. dentato-glandulosa - Hte-Savoie, Arenthon (Puget) . . . 1516. C. coxsobrina Gdgr mss

157 , Pet. eglandulosi vel ad basin 1-3 glandulosi . . . . . 158 151 Pet. omnino I copiose glandulosi a plosi, vel inferne tantum unifariam 188

1.5 ( Pet glabri vel adsperse pilosi, vel inferne tantum unifariam

Pet. saltem usque ad $2^{\text {a }}$ folia undique villosi. . . . . 183

159 ( Fr. ovoideus vel obovato-oblongus. . . . . . . 160

159 Fr oblongus . . . . . . . . . 174

Fol. ovata vel ovato-elliptica . . . . . . . . . 161

160 Fol. obovata vel olsovato-oblonga . . . 163

Fol. oblonga . . . . . . . . . . . 168

161 Sep. dorso eglandulosa. . - 162 Sep. dorso minute glandulosa - Scotia, Aberdeen : Braemar (Drummond). . . . . 1517, C. Donniana Gdgr mss 


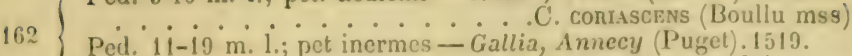
Ped. 11-19 m. 1.; pet inermes - Gallia, Annecy (Puget). 1519.

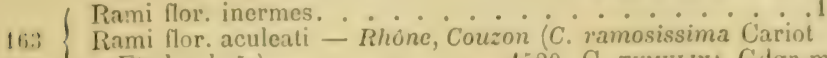
Etud. ed. $5^{\circ}$. . . . . 1520. C. tumulisa Gidgr mss

Fol basi attenuata. . . . . . . . . . 165 Fol. basi rotundata - Hite-Savoie, Argonnex (Puget). 1521.

lii.) ( Pet inernes. . . . . . . . . . . 166

Pet. aculeati. . . . . . . . 167

Fr. basi subdepressus; fol. obovato-oblonga - Suecia, MI

166 Finnehulle (Lundquist) . . . 1522. C. Gotmica Gdgr mss Fr. valde altenuatus; fol. obovata - Tyrol, Hall (Gremblich) 1523. C. TrRolevsis Gidgr mss Ped. 12-14 m. 1.; fr. apice depressus - Austr. inf., Kalks-

167 burg (Wiesbaur). . . 1524. C. intnicata Gidgr mss Ped. 7-8 m. 1.; fr. apice longe strangulatus - Thurinjia, Oesterfeld (Haussknecht). . 1525. C. Gracilinamea Gdgr mss

168 | Discus planus. . . . . . . . . . . 169 Discus conicus. $\ldots . .2172$

| Rami florales aculeati. . . . . . . . . . . 170

169 Rami flor inermes. . . . . . . 171 Ped. 8-10 m. 1.; fol. basi valde attenuata - Silesia, Neisse

170 (Uechtritz). . . . 1526. C. AcMopHyLla Gdgr mss Ped. 6-7 m. 1.; fol, basi sensim attenuata - Suecia, Wexio (Scheutz) . . . . 1527. C. vexrovessis Gdgr mss Fr. apice valde attenuatus; ped. 10-12 m. 1. - Suecia,

171 Alnarp (Tullberg). . 1528. C. LAxcifolia Gidgr mss Fr. apice paulo strangulatus; ped. 9-10 m. 1. - Bohemia, Riesengebirge (Patze). . . . 1529. C. sudetica Gdgr mss Fol, basi altenuata. . . . . . . . . 173

172 Fol. basi rotundata - Isère, Charancieu (Gdgr).1530. C.

. . . . . . CoMrnessispixa Gdgr mss Rami flor. aculeati ; discus vix conicus - Rhône, Pommiers (Gdgr). . . . . 1531. C. confertifolia Gidgr mss Rumi flor. subinermes; discus conicus - Austr. inf., Kalks-

173 Rumirg (IViesbaur). . 1532. C. PunpurasceNs Gdgr mss Rami flor. inermes; discus conicus - Vallesia, Ifontorge

(Favrat) ....... 1533. C. platyspiaera Gidgr mss Fol. ovata obovata vel obovato-oblonga. . . . . . 175

lit Fol oblonga. . . . . . . . . . . . . . .

17. Rami floriferi inermes . . . . . . . . . . 176

17 Rami flor aculeati. . . . . . . . . 177 Ped. paulo villosi $13-20 \mathrm{~m}$. $\mathrm{l}$; discus vix conicus - Rhone, Arnas (Gdgr). . . . 1534. C. Flexibilis Gdgr (1872)

176 Ped. villosi 7-8 m. 1.; discus planus - Saúne-el-Loire, Rougeon (Ozanon) . . . . 1535. C. Ozarosir Gdgr mss Ped. glalori 8-10 m. 1.; discus valde conicus - Silesia, Breslau (Uechitritz) . . . 1536. C. vratisLaviexsis Gdgr mss

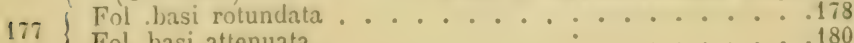
Fol. basi attenuata. . . . . . . . . 180 
Fol. obovato-oblonga; discus | conicus . . . . . . 179

178 Fol. ovata; discus planus - Podolia aust. (Andrz.). $1537 .{ }^{\circ} \mathrm{C}$.

(ev.) Ped. 8-15 m. l.; fr. amplissimus - Rhône, Alix (Gdgr herb.

179 ros. n. 11) . . . . 1538. C. Gastroidea Gdgr mss Ped. 7-8 m. 1.; fr. mediocris - Basses-Alpes, Bouzolières (Proal) . . . . . . 1539. C. Albistyla Gdgr mss Ped. 7-9 m. l.; fol. obovata - Rhône, Montmelas (Gdgr)

180 Ped. $5-7$ m. l.; fol. ovata - Htes-Alpes, Les Lauzieres (Reliq. Maill. n. 170 parte) .1541. C. Mathonseti Gdgr mss

Ped. 13-16 m. 1.; fol. obovato-oblonga - Thuringia, Ettersberg (Haussknecht) ... 1542. C. conymbulosa Gdgr mss

181 Rami florales aculeati. - * . 182

181 Rami flor. inermes - Silesia, Bresiau (Uechtritz). 1543. C.

Discus planus; fol. basi rotundata - Italia, Selva Pisana (P. Savi). . . . . . . 1544. C. SAvil Gdgr mss

Discus subplanus; fol. basi attenuata - Oise, Noyon (Magnier). . . . . 1545. C. BELLA Gdgr msS

Discus conicus; fol. basi attenuata - Hte-Savoie, Pringy (Puget). . . . . . 1546. C. Longrtuba Gdgr mss Discus conicus; fol. basi sensim attenuata - Istria, Pola (Freyn). . . . . . . 1547. C. Istriaca Gdgr mss

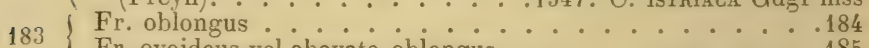

Fr, ovoideus vel obovato-oblongus. $\ldots 185$

Discus planus; fol. atrovirentia oblonga - Suecia, Scania : Esperod (Tullberg). . . . 1548. C. NERvifolia Gdgr mss

18: Discus conicus; fol. viridia late elliptico-obovata - Bolzemia, Prague (Polak). . . . . . 1549. C. BoHesica Gdgr mss

Discus valde conicus; fol. glauca obovato-oblonga - Helv., Corcelles (Morthier) . . . 1550. C. Grandiflora Gdgr mss

Fol. basi rotundata. . . . . . . 186

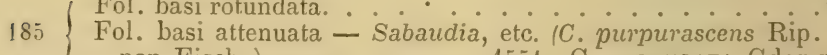
non Fisch.) . . . . 1551. C. Purpurata Gdgr mss

Ped. 9-16 m. l; fol. ovato-elliptica vel obovata. . . . . .187

186 Ped. 4-7 m. 1.; fol. ovato-oblusa - Helv., Montbovon (Cottet). . . 1552.C. purpurata B helvetica Gdgr mss Costa eglandulosa; ped. 13-16 m. 1. - IIte-Saroie, Pringy

137 (Puget). . . . . . . . Palonet Gdgr mss Costa glandulosa; ped. 9-12 m. 1. - Rhône, Arnas (Gdgr herb. ros. n. 286). . . 1554. C. Illibata Gdgr mss

1s. Fol. basi attenuata truncata vel acuta. . . . . . 189

Is Fol. basi rotundata vel vix subattenuata. . . . . 203

189 Sep. eglandulosa vel tantum 1-3-dentato-glandulosa . . . 190

189 Sep. I copiose marginato-glandulosa. . . . . . 199

$190\left\{\begin{array}{l}\text { Fol. oblonga ovata obovata vel obovato-oblonga. } \\ \text { Fol. }\end{array}\right.$

191 Rami florales aculeati d discus planus. . . . . . 192

191 Rami flor. inermes vel subinermes; discus conicus . . . 194

11.) $\{$ Fr. basi attenuatus vel decurrens; ped. $11-17 \mathrm{~m} .1 .2 .193$ feld (Haussknecht) . . . . 1555. C. Pnostrata Gdgr mss 
Ped. 11-13 m. 1.; dentes plerumque compositae - Hisp.

$193\left\{\begin{array}{l}\text { bor., Sa de Cameros (Zubia) . 1556. C. Sierrae Gdgr mss } \\ \text { Ped. } 14-17 \mathrm{~m} \text {. 1.; dentes aeque simplices et compositae- }\end{array}\right.$ Hisp., Logrono (Zubia). . . 1557. C. Laxcaefolia Gdgr mss Cor. ciliata; ped. 9-11 m. 1. - Gironde, Montferrand

194 (Motelay). . . 1558. C. Motelayi Gidgr Essai Cor. eciliata ; ped. 13-17 m.1. - Hte-Savoie, Brenthonne (Puget). . . . . . 1559. C. mentacea (Pug. mss) Rami florales aculeati...................

195 Rami flor. inermes - Rhöne, Arnas (Gdgr).1560. C. DrcraFol. obovata vel obovato-oblonga; aculei omnes adunci. . 197 $196\left\{\begin{array}{c}\text { Fol. ovata; aculei nunc recti nunc adunci - Gallia, Annecy } \\ \text { (Pugel). . . . . . . Multispina (Pug. mss) }\end{array}\right.$ Discus | conicus ; perl slabri . . . . . . . . . 198

$197\{$ Discus planus; peu. villosi - Rikine, irontmelas (idgr herb.

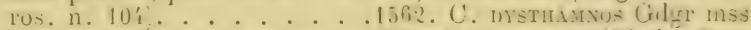
Ful. ohovata; discus conicus - Rhoune, (\%nponost (Chabert) 198 Fol. obovato-oblonga; discus parum conicus - Rhone, Francheville (Chabert). . . . 1564. C. FInMIor Gdgr mss

199 Fol. obovato-oblonga vel oblonga . . . . . . . 200 Fol. clliptica vel obovata. . . . . . . . . . .201 Ped. 9-11 m. l.; fr. ovoideus - Puy-de-Dôme, Montbaubier

200 (Dubosclard)......1565. C. Dubosclandil Gdgr mss Ped. 12-15 m.1.; fr. oblongus - Loire, 1It-Pilat (Gdgr) . . . 1566. C. Genistaecola Gdgr mss Rami flor. et petioli aculeata . . . . . . .202 Rami flor, et pet. inermia - Rhône, Arnas (Gdgr).1567.C.

I Rami flor. inermes; pet. paulo aculeati - Pyrenaei, Gèdre (Bordèe). . . . . . 1568. C. Prrenaica Gdgr mss Costa eglandulosa; fol. elliptico-subattenuata - Rhône,

202 Arnas (Gdgr herb. ros. n. 47) .1569. C. xulticeps Gdgr mss Costa glandulosa; fol. ohorata utrincue acuta - Rlowme, Ville-sur-Jamioux (Gdgr) . . 1570. C. pulrpoda Gelgr mss

203 i Rami flor inermes vel 1-2-aculeati. . . . . . . .204 Rami flor aculeati. . . . . . . . . . 208

20 i Fr ovoideus. . . . . . . . . . . 205 Fr. oblongus . . . . . . . . . . . . . . 2065 Discus paulo conicus; $\mathrm{fr}$. apice valde attenuatus - Hte-

205 Savoie, Habère-Lullin (Puget).1571. C. BRETICALYx Gdgr mss

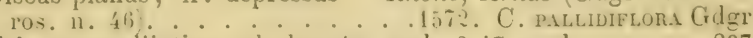
Fol. ovato-elliptica vel obovata; ped.6-17 m.1. ... .207

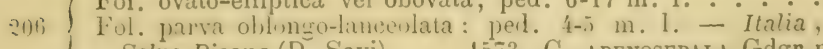
Selva Pisana (P. Savi) . .1573. C. adexosepala Gdgr mss Costa paulo glandulosa; pinnulae subintegrae - Isère, Charancieu (Gdgr herb, ros, n. 118).1574. C. JoNGeCononata

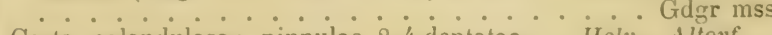
Costa eglandulosa; pinnulae 2-4-dentatac - Helv., Altorf (Gisler). . . . . 1575. C. Geracastea Gdgr

208 Fol. ovata elliplica vel obovato-elliptica $\ldots . .2092$ 
Discus conicus. . . . . . . . . . . 210

Discus planus. . . . . . . . . . . 211

Cor. rosea; fr. parvus ovatus - Rhône, Francheville (P.

210 Chabert). . . . 1576. C. oвcondata Gdgr mss Cor. alba ; fr. ample oblongus - Cher, etc.1577. C. ADSCITA

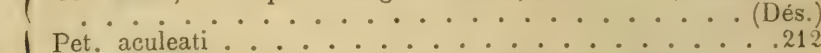
Pet. inermes - Borussia, Dantzig (Baenitz Herb. europ.

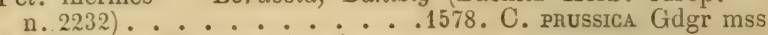

Fol. obovato-elliptica apice $\mid$ acuta. . . . . . . 213

$212\{$ Fol. late ovata apice rotundata - Rhône, Francheville (P. Chabert). . . . . . 1579. C. sublustris Gdgr mss Ped. $3-10 \mathrm{~m}$. 1.; fr. basi rotundatus - Belg., Rochefort

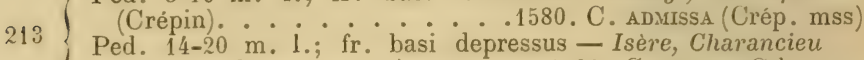
(Gdgr herb. ros. n. 35). . . . 1581. C. PATENs Gdgr mss

214 Fr. ovoideus. . . . . . . . . . . . . . . . . . . . . .

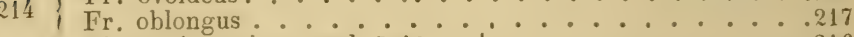

$215\{$ Discus | conicus; ped, 5-10 m. 1. . . . . . . 216 Discus planus; ped. 11-15 m.1. - Rhône, Gleizé (Gdgr herb. ros. n. 162) …1582. C. Hebeghlamys Gdgr mss

$216\left\{\begin{array}{l}\text { Pinnulae 2-4-dentatae : stip. rubentes - Rhône, La Tour (P. } \\ \text { Chabert) }\end{array}\right.$ Pinnulae integrae; stip. virentes - Rhône, Charbonnières (P. Chabert). . . . . 1584. C. LYTerobsciura Gdgr mss

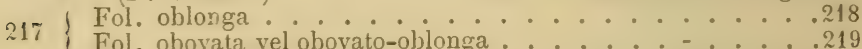
Fol. obovata vel obovato-oblonga . . . . . . . . 219 Sep. eglandulosa; ped. 11-15 m. 1. - Rhône, Francheville

218 (Chabert) . . . 1585. C. Allanthoides Gdgr mss Sep. dentato-glandulosa; ped. 9-10 m. 1. - Sabaudia, Thonon (Dés. herb. ros. n. 48).1586. C. Elongata Gdgr mss

$219\left\{\begin{array}{l}\text { Ped. } 9-12 \mathrm{~m} .1 \text {. } \\ \text { Ped. } 5-7 \mathrm{~m} .1 \text {; }\end{array}\right.$ Jitomir (Golde). . . . 1587. C. volnyxica Gdgr mss

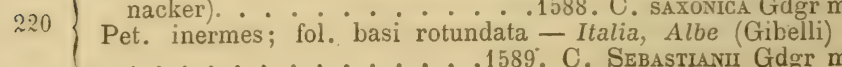
. . . . . . 1589. C. Sebastianil Gdgr mss

\section{c. - Didymodontae Gdgr Essai p. 27 ; Biserratae Crép. Prim. p. 17.}

Foliolis omnibus crebre et argute biserratis; foliis lucide virentilus, ad costam mediam saepe glandulosis, subtus glaucescentibus eorum nervis magis convergentibus prominulisque. Aculeis caulium vetustorum ordinarie minoribus 
quan in 2 sectionibus praccedentibus; corolla magis rosea, et ramis etiam videntur tenuioribus.

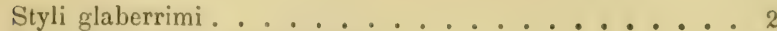

Styli basi tantum paulo hirtelli . . . . . . 48

Styli | copiose villosi ............. . . . 96

Styli densissime lanati. . . . . . 152

$2 \quad$ Sepala copiose marginato-glandulosa . . . . . . . . 3

Sep. eglandulosa vel 2-6-marginato-glandulosa. . . . 10

Fr. oblongus ............. 4

Fr, ovoideus; pinnulae angustatae - Rhône, Pommiers

(Gdgr herb, ros. n. 106) . 1590. C. streptoclada Gdgr mss

Fr. ovatus; pinnulae foliaceae - Lyon (Gdgr herb. ros. n.

386, 417, 441 et 442) ....1591. C. Cariotr (Chabert)

Fr. ovalus; pinnulac angustatae - Belg., Tournai.1592.

. . . . . . C. glabernima (Dumrt.)

Fr. globosus vel rotundatus - Rhône, Gleizé (Gdgr herb. ros. n. 303). . . . . 1593. C. subeffusa Gdgr mss

lol. ovala vel ovato-elliptica . . . . . . 5

Fol. obovato-oblonga; ped. glabri - Rhône, Francheville

(Chabert) . . . . . 1594. C. tuisulosa Gdgr mss

4 Fol. oblonga; ped. villosi - Saône-et-Loire, Rougeon (Billot exs. n. 4025). . . . . 1595. C. Billotiana Gdgr mss Fol. obovata ; ram. flor. inermes - Rhône, Caluire (Chabert).

5 Rami flor inermes. . . . . . . 6

Pet. paulo aculeati; fol. ovata - Cher, Auron (Ripart). $1597^{\circ}$

6) . . . . . . . . le . . . . .

Pet. subinermes; fol. obovata - Rhône, Amas (Gdgr herb. ros. n. 298) . . . . . 1598. C. DEvotA Gdgr mss

7 1 Discus planus vel subconicus. . . . . . . . . 8

1 Discus valde conicus. . . . . . . . . 9

Pet. aculeati; fol. utrinque attenuata - Tyrol Innsbruck, etc.

8 Pet. inermes; fol. basi rotundata - Rhône, Ecully (Chabert).

........ 1600. C. strangulata Gdgr mss

Fol. obovata basi acuta; ped. pubescentes - Rhône, Alix (Golgr herh). ros. n. 379; C. aciphylla B. gracilescens Gdgr in Cariot Etudes ed. 5o II, p. 186).1601. C. scbgracilis

Fol ob (E. Quet). 1602. C. LegroNensis Gdgr ms

Fol. ovato-obtusa basi rotundata - Naples, Castellamare (Pasquale). . . . . 1603. C. stabiana Gdgr mss

10 Fr. rotundatus ovatus ovoideus vel obovato-oblongus .... 11

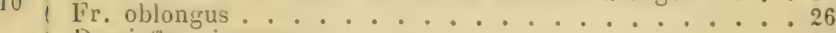

11 Rami flor inermes . . . . . . . . 12

, liami flor aculeati............21

12 I ol. ohorata rel orata. . . . . . . . . 13

Fol. obovato-oblonga vel oblonga . . . . . . 17

1: $\mid$ Fol. basi | attenuata ..................... 14

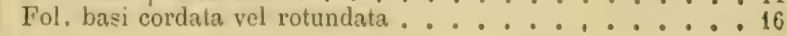


I Pet. omnes aculeati; ped glabri . . . . . 15 Pet. subinermes; ped. villosi - Ain, Le Plantay (Gdgr). ......... 1604. C. DoMBENsIs Gdgr mss Discus valde conicus; ped. $14-16 \mathrm{~m}$. 1. - Loire Mt-Filat

15 (Gdgr)........... C. Millebfoliata Gdgr mss Discus vix conicus; ped. 6-9 m. 1. - Rhône, Gleizé (Gidgr herb. ros. n. 326) . . 1606. C. THERMopHILA Gdgr mss Ped. 5-7 m. 1.; pet. sat villosi - Rhône, Villefranche (Gdgr $16\left\{\begin{array}{l}\text { herb. ros. n. 541). - glabri - Lusit., Coimbre (Henriques } \\ \text { Ped. 11-12 m. 1.; pet. glar }\end{array}\right.$

fl. lus. exs. sine num.). 1608. C. Conturricensis Gdgr mss

1 - $\{$ Pet. omnes | aculeati ............... 18

17 Pet. inf. inermes ................. 19

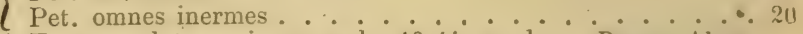

Fr. rotundato-conicus; ped. 13-14 m. 1.-Basses-Alpes, Bouzolières (Proal). . 1609. C. Pseudo-Cerasus Gdyr mss

18 Fr. ovato-rotundatus; ped. 9-11 m. 1.-Rhône, Arnas (Gdgr herb. ros.n. 261)......1610. C. POLYoDoN Gdgr Fr. obovato-oblongus; ped. 12-14 m. 1. - Gironde, Montferrand (Motelay). . . 1611. C. Torrentum Gdgr mss Discus planus; ir. globosus - Phone, Chenelelle Gurr).

19 … 1612. C. SPHAERophora Gdgr mss Discus conicus; fr. obvato-oblongus - Pyrenaei, Gèdre (Bordère). . . . . . . 1613. C. Lepidiflora Gdgr mss Discus cınicus; fol. basi attenuata - Rhône, Theizé (Gdgr).

20 . . . . . 1614. C. PYramidula Gdgr mss Discus paulo conicus; fol. basi rotundata - Phóne, Francheville (Chabert). . . 1615. C. pRuxordes Gdgr mss Fol. ovata elliptica vel obovata. . . . . . . 22

21 Fol. oblonga - Gall., Perpignan, etc. (Gdgr herb. ros.

n. 55.;: C. serrulata Dés. Cat.) . . 1616. C. Niras Gidgr

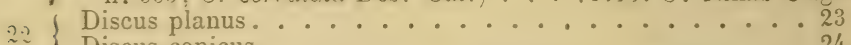

Discus conicus. . . . . . . . . . 24 Fol. parva ovato-elliptica; pet. glandulosi - Rhône, Fran-

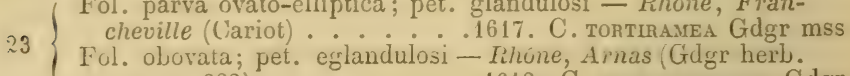
ros. n. 332). . . . 1618. C. RHIPIDOdENDRon Gidgr

\{ Pet. omnes | aculeati . . . . . . 25

24 Pet. sup. inermes - Rhöne, Arnas (Gdgr herb. ros. n. 254).

. . . 1619. C. CHLAMrdocalyx Gdgr mss 2.) $\left\{\begin{array}{l}\text { Fol. late ovato-cordata - Rhône, Couzon (Boullu).1620. } \\ \text { Fol. late obovata basi rotundata - P. subcordata Gdgr mss }\end{array}\right.$ herb. ros. n. 191). . . . 1621. C. Bortrayara Gdgr mss

26 I Pet. glabri vel parcissime pilosi ......... 27

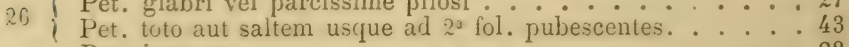

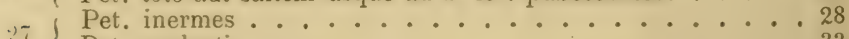

27 Pet. aculeati ..................... 33

24 ( Pami flor aculeati . . . . . . . . . 29

Prami flor inermes . . . . . . . 30

Ped. 3-6 m. 1.; fol. basi vix attenuata - Puy-de-Dôme, St

29 Maurice (Gdgr herb. ros. n. 234).1622. C. PERonata

Ped. 8-10 m. i.; fol attenuata-Puy-de-Dôme, St Maurice (Gdgr herb. ros. n. 380) . 1623. C. SubseGundA Gdgr mss 
30 , Discus conicus. . . . . . . . . . 31

Discus planus vel subconicus. . . . . . . . . 32

Lor. rosea; serraturae patulae - IIte-Savoie, etc.1624. 31 Cor. alba; serraturac rectae - Hie-Savoie, Pringy (Puget).

. . . . . Microcalyx Gdgr mss

Ped. 12-13 m. 1.; cor. amoene rosea - Isère, Bourgoin

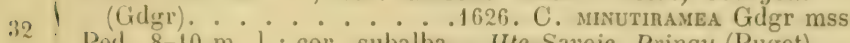
1 Ped. 8-10 m. 1.; cor. subalba - IIte-Savoie, Pringy (Puget)

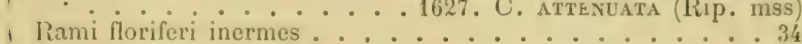

I Rami flor aculeati . . . . . . . . . . 38

I lol. basi rotundata; discus conicus. . . . . . . 35

3 Fol. basi rotundata; discus planus . . . . . . . 36

Fol. basi attenuata. . . . . . . . . . . 37

Ped. 8-9 m. 1.; fr. basi attenuatus - Austr. sup., Aisters-

haim (Keck). . . . . 1628. C. cyclordeA Gdgr mss

Ped. 11-1/4 m. 1.; fr. basi depressus - Istria, Mt Maggiore

35 (Freyn).......... 1629. C. Utzka Gdgr mss Ped. 5-6 m. 1.; fr. basi depressus - Oise, Noyon (Magnier).

. . . . . 1630. C. EDOCENDA Gidgr mss

$\mathrm{Fr}$. basi attenuatus apice longissime constrictus - IIisp.,

36 ! Lofrono (Zubia). . . . . 1631. C. LuCnoniensis Gdgr mss

Fr. basi subrotundatus apice attenuatus - Finistère, Crozon

(Thiébault) . . . . . 1632. C. Armoricava Gdgr mss

Fol. ovata; fr. apice longissime attenuatus - Cher, Bourges, etc., etc. . . . . . 1633. C. rmynchocarpa (Rip.)

Fol. elliptica; fr. apice depressus - Puy-de-Döme, St Maurice $(G$. herb. ros. n. 24). .1634. C. eusynpuylla Gdgr mss

l'ol. ample obovala; fr. longissime attenuatus - Calcados,

Monceaux (Bomnechose) - . . 1635. C. Laglificla Gdgr mss

Fol. obovato-oblonga; fr. apice attenuatus - Thuringia, Ilaardorf (Haussknecht). . . 1636. C. LEIosepala Gdgr mss

as I Fol basi rotundata . . . . . . . . 39

Fol. basi attenuata. . . . . . . . . 40

Pet. rlandulosissimi; discus value conicus - IIisp. bor..

.3! I Simra de Cameros (Zubia) \& . 1637. U. Querraxil Gidgr mss P'et. vix mandulosi ; discus subplanus - Cóle-dor, Meursault (Ozanon) . . . . 1638. C. Flexical Lis (Crép. mss) Fol obovata. . . . . . . . . . . il Fol. ovata basi acula - Gallia, Annecy (Pugel). i639.

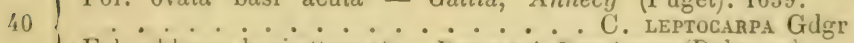
| Fol. oblongra basi attenuata - I'yrenaei, I'erpignan (Debeaux). . . . . . . . 1640. C. stexodiscus Gdgr mss

Ped.5-8 m. 1.; fol. basi attenuata . . . . . . . 42

41 Ped. 11-12 m. l.; fol. basi truncata - Gard, Le Vigan (Diomède). . . . . . 1641. C. cebennensis Gdgr mss Sep. apice dentata; ped. 4-8 m. 1. - Manche, Yvetüt (Lehel). Mosqueruela (Martin) . . . 1643. C. Martini Gdgr mss

43 Rami lloriferi inermes . . . . . . . . . 44 Rami llor, aculeati .................... 46 
44 Discus conicus; pet. aculeati........... 45 Discus planus; pet. inermes - Vosges, Epinal (Berher). ...... 1644. C. confusa Gdgr mss Fol. oblonga - Rhône, Arnas (Gdgr herb. ros. n. 273). • . . 1645. C. Acridentata Gdgr mas Gard, Pommiers (Diomède). . 1646. C. Telocarpa Gdgr mss Fol. ovato-elliptica; fr. apice tantum attenuatus - Sabaudia.

Fol. obovato-oblonga; ped. glabrip. C. macroacantha (Rip.)

$46\left\{\begin{array}{l}\text { Fol. ovato-elliptica; ped. villosi - Helv. Montbovon } \\ \text { Fol }\end{array}\right.$

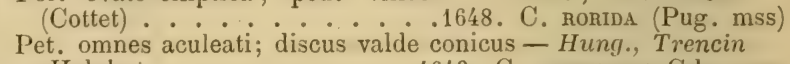

47 (Holuby). . . 1649. C. HUNGarica Gdgr mss Pet. inf. inermes; discus sat conicus - Hte-Savoie, Pringy (Puget) . . . . 1650. C. laburnifolia Gdgr mss Sep. margine valde dentato-glandulosa. . . . . . 49

48 Sep. margine eglandulosa vel 2-5-dentato-glaudulosa . . . 58 Sep. ad pinnulas integra sed margine glandulosa - Tyrol, Trente (Gelmi) ...... 1651. C. TRIDENTINA Gdgr mss Fr. ovoideus. . . . . . . . . . . 50

49 Fr. oblongus. . . . . 52 Fr. ovato-rotundatus - Anglia, Cheshire : Newtown (Webb). . . . . . . 1652. C. microacantha Gdgr mss Rami florales aculeati $\therefore \ldots \ldots$ Rami flor. inermes; discus paulo conicus - Drôme, Chabeuil

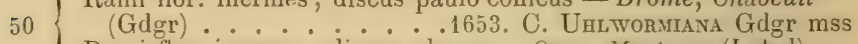
Rami flor. inermes; discus planus - Orne, Mortagne (Lebel).

( Fol. obovata apice subrotundata; discus valde conicus. - Ca-

51 talaunia, Sagaro (Vayreda) : 1655. C. CRinisepala Gdgr mss Fol. obovato-oblonga acuta; discus paulo conicus - Rhône, Arnas (Gdgr). : . . . 1656. C. Holophaea Gdgr mss

52 Pami flor. aculeati. . . . . . . . . . 5.?

Rami flor inermes. $\ldots \ldots \ldots . \therefore 54$

53 Fol. ovata. - Rhône, Limonest (Chabert) 1657. C. DIscostruA Ped. $7-9$ m. i.; fol. basi rotundata - Austr. inf., Kalksburg

54 (Wiesbaur) Ped. 11-15 m. 1.; fol. basi cordata - Hte-Savoie, St-Martin (Puget) . . . . . 1659. C. LAxifolit Gdgr mss

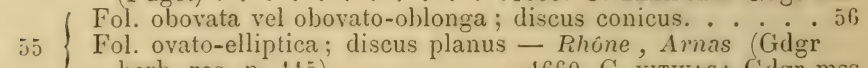
herb. ros. n. 115). . . 1660. C. vitivaga Gdgr mss

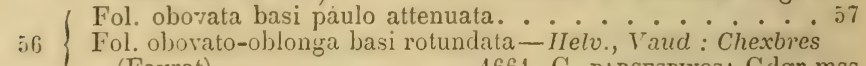
(Favrat). . . . . 1661. C. PARCEspinosa Gdgr mss

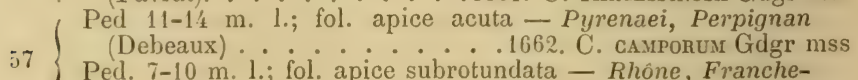
Ped. 7-10 m. l.; fol. apice subrotundata - Rhône, Francheville (Chabert) . . . . . 1663. C. PECTINELLA Gdgr mss

js Rani floriferi inermes . . . . . . . . . 5? Rami flor, aculeati. . . . . . . . . . . 71 
Fr. ovoideus vel obovato-oblongus. . . . . . 6 60

Fr. oblongus. . . . . . . . . . I'r. ovatus - Rhone, Gleizé (Gdgr herb. ros. n. 67).1664.

Discus conicus. . . . . . C. pampinosa Gdgr mss

60 Discus planus - Isère, Páda 61

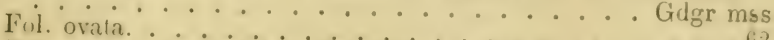

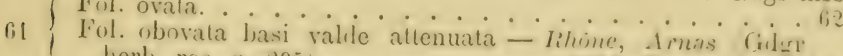
herb. ros. n 295 . The

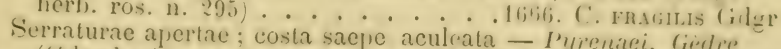

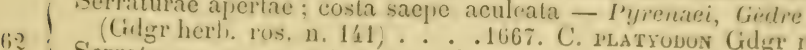

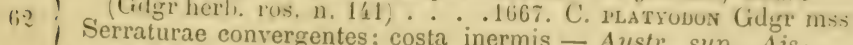
tershaim (Keck).

. 1668. C. Keckir Gdgr mss

63 Fol ovala rel ol . . . . . 64

lít Pet. incrmes. . . . . . . . 67

l'el aculeati. . . . . . . . . . . . 155

fi.) (Chabert, - fol. purpurea hasi acuta - A in, La Pape

Discus conicus; fol virentia basi subrotundata - Thuring ms

Fol. basi subrotundata; ped. 10-18 m. 1. - Sabaudia, Thonon

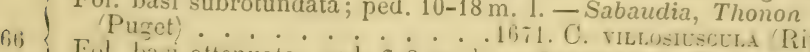

Ful, has attenuata jed. i-s m. 1. - Pomerania, stayliown.) (IIülsen) . . . . 1672. C. Acicularis Gider ms

fiт \{ Pet. inermes . . . . . . . . . . . . . . . . . . . . . .

Pet aculeati. $\cdot 0.0 .0 .090$

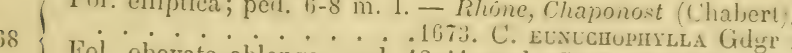
$\left\{\begin{array}{l}\text { Fol. obovato-oblonga; ped. } 12-14 \mathrm{~m} \text {. 1. Gard, Molière (Dio- } \\ \text { mède). . }\end{array}\right.$

Discus conicus; fol obovata vel 1674. C. Delileana Gdgr mss

$69 \int$ Discus planusolonga. • . 70

Discus planus; fol. ovato-cordata - Gallia, Annecy (Puget).

Ped. 6-8 m. l.; costa glandulosissima - Sicilia, Mirto (Tod.
fl. sic. exs. n. 1082)

70 Ped. 11-19 m. 1.; costa vix glandulosa - 1676. C. Sicula (Tod. mss) (Puget). . . . . 1677. C. Acte-Savoie, Pringy

- Fol. basi rotundata vel cordata 1077 . C. Actixoclada Gdgr mss

71 Fol. basi | attenuata. . . . . . . . 72

72 Fol ovata vel ovato-elliptica . . . . . 81

2 Fol. obovata obovato-oblonga vel oblong. . . . . 73

( Discus I conicus . . . . . . . . 76

73) Discus planus - Rhône, Arnas (Ġdgr).i678. C. Scotophrlat

Fr, ovoideus. . . . . . . . Gdgr mss

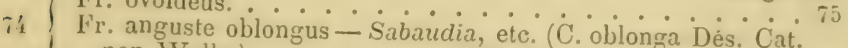

non Wallr.)

7. Cor. eciliata; fol. obtusa - Rhone, Arnas (Gdgr). 1680.

i) Cor. valde ciliata; fol. acuta - Rhóne Prnamdta Gdgr mss ros, n. 363).

Ped. villosi . . . . 1681. C. BLeplanopetala Gdgr mss

Ped. glabri. . . . . . . . . . . . 
Fol. oblonga, discus paulo conicus - Rhône, Arnas (Gdgr).

Fol. obovato-oblonga; discus valde conicus - Hisp., Logrono

(Zubia) . . . . . 1683. C. conophora Gdgr mss

Pet. inf. inermes; fol. obovata apice subrotundata. . . . . 79

8 Pet. omnes inermes. . . . . . . . . . 80

Pet. inf. inermes; fol. obovato-oblonga apice attenuata Rhöne, Arnas (Gdgr) . . . . 1684. C. exsul Gdgr mss Stip. virentes; fr. inflatus basi depressus - Oise, Noyon

79 (Magnier) . . . . . 1685. C. lustrata Gdgr mss Stip. purpureæ; fr. basi attenuatus - Rhône, Chaponost

(Chabert) . . . . . 1686. C. Ruinarum Gdgr mss

Sep. apice laciniata; ped. 9-12 m. 1. - Aragonia austr., Sa

de Mosqueruela (Martin). . 1687. C. Loeflixgiaxa Gdgr ms

Sep. apice integra; ped. 13-15 m. 1. - Tyrol, Zirl pr. Hall Gremblich). . . . . 1688. C. Haussmaniana Gdgr mss

(Fol. obovata............... 82

81 Fol. ovata - Cher, Grange St-Jean (Ripart).1689. C. virginea

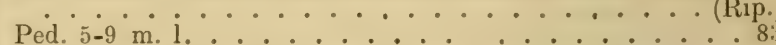

Ped. 14-16 m. l.; sep. foliacea-Silesia, Storchberg (Straehler).

8. Ped. 12-13 m. l.; sep angustata-Rhône, Chaponost (Chabert).

Pet glabri . . . . . 1691. C. LEPTOcLada Gdgr mss

1 (Puget)

83 Pet. villosi ; sep. dilatata - Gard, Le Vigan (Diomède). 1693.

. . . . . . . . . C. Didymocarpa Gdgr mss

8 ( l'et. inermes vel sup. tantum aculeati . . . . . . 85

Pet. omnes aculeati . . . . . . . . . . 87

Fol. obovato-oblonga. . . . . . . . . 86

Fol. obovata; discus conicus - Pyrenaei, Perpignan (Debeaux). . . . . . 1694. C. FAlcinella Gdgr mss

Fol. elliptica; discus conicus - Rhône, Ville-sur-Jarnioux

(Gdgr). . . . . . . 1695. C. SYNPHYLLOCLADA Gdgr msS

Fol. elliptica; discus planus - Rhône, Arnas (Gdgr herb. ros. n. 334). . . . . 1696. C. Serapionis Gdgr mss Cor. pallida ciliata; costa glandulosa - Puy-de-Dôme, St-

Maurice (Dubosclard). . . . . 1697. C. scita Gdgr mss

Cor. rosea eciliata; costa eglandulosa - Rhône, Les Charpennes (Gdgr). . . . . 1698. C. Rosalis Gdgr mss

Discus planus. . . . . . . . . . . 88

Discus conicus. . . . . . . . . . 990

Fr. oblongus; fol. obovato-oblonga vel oblonga. . . . . 89

88 Fr. oroideus; fol. obovata - Isère, Charancieu (Gdgr). 1699.

. . . . . . C. EdITA Gidgr mss (1872)

Ped. 12-13 m. l.; fr. apice longe strangulatus - Aisne, St$89\left\{\begin{array}{l}\text { Quentin (Magnier) } \text { Ped. 7-10 m l.; fr. apice attenuatus - Hisp., Logrono (Zubia) } \\ \text { Ped }\end{array}\right.$

I Fr. ovoideus. $\ldots \ldots$

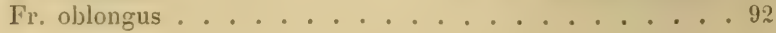


Discus conicus; ped. 12-15 m. 1. - Rhône, Pommiers (Gdgr)

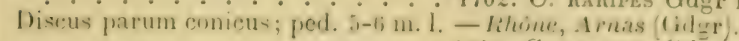

1703. C, Rigidion Gider mss

Fol. oluvala.

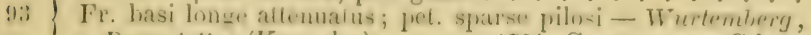
Donnstetten (Kemmler) . . . 1704. C. apiculata Gdgr mss Costa glandulosa ; sep. apice integra - IIisp., Gerona (Vay-

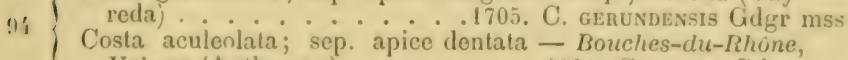
Velaux (Autheman)..... 1706. C. VAFRA Gdgr mss Fr. apice subattemuatus; pet. glandulosi-Finistere, Darmias

$9 . \quad$ (Thiébaut)........ 1707. C. nabrass Gdgr mss Fr. longe altenuatus; pet. eqlandulosi - Bouches-du-lihime,

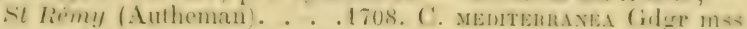
Sep. valde dentato-glandulosa. . . . . . . . . 97

Sep. margine haud aut parce dentato-glandulosa. . . . .111

97 Fr. ovoideus . . . . . . . . . . 98 I Fr oblongus . . . . . . . 104

Is $/$ Rami floriferi inermes . . . . . . . . . . . 99

IS Rami flor aculeati. . . . . . 102

$99 \quad$ Discus planus. . . . . . . . . 100

Discus | conicus . . . . . . . . 101 Fol. elliptica basi vix atlenuata; pet. inf. inermes - Rhone, $100\left\{\begin{array}{c}\text { Amas (Gdgr) } \\ \text { Fol. obovata basi attenuata; pet. omnes aculeati - Hanovre, }\end{array}\right.$ Franeforl a.M. (Kesselmayer). 1710. C. Howoteraxa Gilyr mss Ped. 8-10 m. l.; fol. basi acuta - Gallia, Autun (Déségl.).

101 Ped. 5-6 m. i.; fol. basi late rotundata-Anglanss Gidgr ms: mere (Baker). 1712. C IYsul Gor-

Fol. oblonga basi attenuata. ... 103

Fol. obovata basi vix attenuata - Rhone, Lyon (Gdgr).1713.

102 . . . . . . . . . V. Vaga Gdgr mss

Fol. ovata basi cordata - Dalmatia (Schalch). 17it. C. vinI-

. . . . . . . . . . diglauca Gdgr ms s Discus planus ; pet. vix glandulosi - Suecia, Halland.1715.

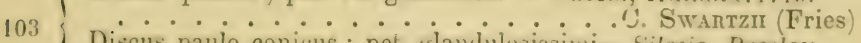
Discus paulo conicus ; pet. Glandulosigsini-Silesia, Breslau (Uechtritz). . . . . . 1716. C. scamnata Gdgr mss

104 I Rami floriferi inermes . . . . . . . . . 10 Rami flor aculeati. . . . . . . . . 106 Fol. oblonga basi attenuata; discus conicus - Suecia, Eland: Isgarda (Scheutz). . . . . 1717. C. ADExorHor Gdgr mss Fol. obovato-oblonga basi attenuata ; discus planus - Anglia, Surrey barnes (Groves) . . . 1718. C. Cuntisiani Gdigr mss

Fol. obovata basi rotundata; discus conicus - Bavaria, Schloss Zeil (Kugler). . , . . . 1719. C. suevica Gdgr mss

106 I Fol. basi rotundata. . . . . . . . . . 107

Fol basi attenuata. . . . . . . 108 
(Ped. 17-19 m. 1.; discus planus - Hisp., Logrono (Zubia) 107 Ped. $10-12$ m. l.; discus conicus-Oise, Noyon (Magnier).1721.

OENOPHYLLA Gdgr mSS

108 Fol. oblonga; discus paulo conicus . . . 110 Fol. oblonga; discus planus - Tyrol, Telfs (Gremblich). 1722.

...... C. MYriosticta Gdgr mss

Pet. villosi; fr. oblongo-oviformis - Germania, Wurtzburg

109 (Rau). . . . . . . . 1723. C. AfFinis (Rau)

Pet. sparse pilosi; fr. oblongo-attenuatus - Hte-Savoie, Pringy (Puget). . . . . 1724. C. Leptosticta Gdgr mss Sep. foliacea; pet. inermes - Suecia, Halland (Fries). 1725.

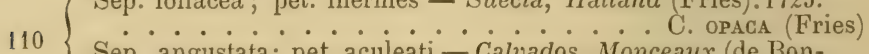

Sep. angustata; pet. aculeati - Calvados, Monceaux (de Bonnechose). . . . . . 1726. C. CRINITA Gdgr mss

(Fr. globosus vel ovato-rotundatus. ........112

111 Fr. ovatus ovoideus vel obovato-sublongus. . . . 116

Fr. oblongus. . . . . . . . . . . . . . 138

11. Rami flor. aculeati; pet. glabri :. . . . . . . . . . .

Rami flor. inermes; pet. villosi - Saône-et-Loire, Rougeon (Ozanon) . . . . . 1727. C. Rougeonensis (Crêp. mss)

Pet. omnes aculeati .................. 114

113 Pet. sup. inermes - Hte-Savoie, St-Germain (Puget).1728.

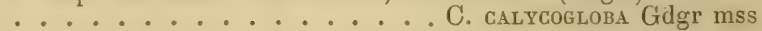

$114\{$ Discus I conicus . . . . . . . . . . 115 Discus planus - Belg., Spa, etc.1729. C. Malmundariensis (Lej.) Fol. ovata basi subattenuata - Cher, Soie, etc.1730. C. BRA$115\left\{\begin{array}{l}\text { Fol. elliptica basi attenuata - Phrygia, Ouchak (Balansa). } \\ \text { - Pars }\end{array}\right.$

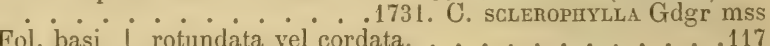

116 Fol. basi I rotundata vel cordata. . . . . . . . . . .

117 Rami floriferi inermes . . . . . . . . . . . . . . .

117 Rami flor aculeati. . . . . . . . . . 120

1 (Fol. obovata vel oblonga. . . . . . . . . 119

118 Fol. ovata - Rhône, Arnas (Gdgr herb. ros. n. 275) 1732. Ped. $2-5$ m. i.; petala valde ciliata - Puy-de-Dôme, Pionsat

119 (Dubosclard). . . 1733. C. Calyptocalyx Gdgr mss Ped. 15-20 m. 1.; cor. eciliata - Hte-Savoie, Argonnex (Puget). . . . 1734. C. Callicanthos Gdgr mss

$120\left\{\begin{array}{l}\text { Fol. ovata elliptica vel obovata } \text { Fol. oblonga - Rhône, Arnas (Gdgr).1735. C. xanthochlora } \\ \text { C. } 121\end{array}\right.$

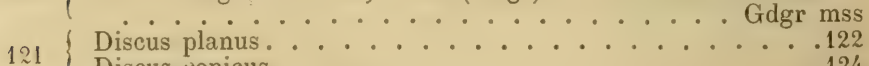

121 Discus conicus. . . . . . . . . . . . . . . . . . .

$122\left\{\begin{array}{l}\text { Fol. obovata; ped. } 3-6 \mathrm{~m} . \mathrm{l} \\ \text { Fol }\end{array}\right.$ ol. ovata; ped. $10-12 \mathrm{~m} .1 .-$ Italia, Apennini Pistoje :
Boscolungo (Gibelli) . . . . 1736. C. APENNina Gdgr mss Aculei geminati ; fol. breviter acuta - Austr. inf., Kalks-

$123\{$ burg (Wiesbaur). . . . 1737. C. Dolata Gdgr mss Aculei sparsi; fol. lanceolata - Rhône, St-Lager (Gdgr). ......... 1738. C. Sarmentoides (Pug. mss) 
124 Discus conicus ; pet. omnes aculeati. . . . . . . 125

Discus parum conicus; pet. inf. inermes - Puy-de-Dôme, Riom (Gdgr herb, ros. n. 40). . . 1739. C. FALSA Gdgr mss

125 ( Fol. obnvata. . . . . . . . . . . . . 26 Fol. ovata. . . . . . . . . 127 Fr. ample ovato-ellipticus conicus basi rotundatus - Gard,

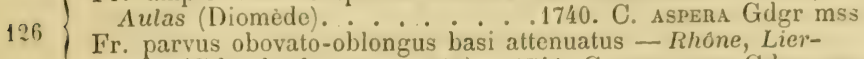
Fr. parvus obovato-oblongus basi attenuatus - Rhône, Liergues (Gdgr herb. ros. n. 219). .1741. C. Dysclados Gdgr mss

127 Fol. basi late cordata. . . . . . . . . . 128

Fol. basi subrotundaca . . . . . . . . . . 129 Ped. 5-7 m. l.; fol. terminale basi aculeolatum - Rhône, $1: 8\left\{\begin{array}{c}\text { Gleizé }(\mathrm{Gdgr}) \text {. } \text { minime - Pyrenaei orient., Amélie-lès- } \\ \text { Ped. 8-10 m. 1. }\end{array}\right.$ Bains (Debeaux) . . . . . 1743. C. INDIFFERENs Gdgr Stip. latae; cor. pallida - Pyrenaei, Perpignan (Debeaux)

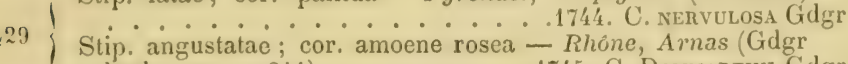
herb. ros. n. 211). .....1745. C. Dommantini Gdgr

i Rami flor normaliter inermes. . . . . . . . . . 131

130 Rami flor aculeati. . . . . . . . 136

131 ( Ped. villosi . . . . . . . . . . 132

, Ped. glabri ... . . . . . . . . . 133

Fol. obovato-oblonga; pet. aculeati - Rhône, Pommiers

132 (Gdgr). . . . . 1746. C. subrectispina Gdgr mss

Fol. oblonga ; pet. inermes - Scotia, Perth Seggieden (Drummond). . . . 1747. C. CALEDonionum Gdgr mss

133 \& Discus planus. . . . . . . . . . . 131

Discus conicus.

Pet. inermes; fol. elliptica basi truncata - Pyrenaei, Gèdre Pet. aculeati ; fol. obovata basi attenuata - Suecia; Podolia (Bess. fl. gal. I, p. 325) . . . . . 1749. C. venosa (Sw.) Fol. obovato-oblonga subatlenuata; pet. inermes - Rhöne, Amas (Gdgr herb. ros. 11. 138). .1750. C. o1: TLsinanea Gdgr

135 Fol. oblongo-lanceolata; pet. paulo aculeati - Rhóne, Arnas (Gdgr) . . . . . . 1751. C. unibrosa Gdgr mss Fol. obovato-oblonga vel oblonga . . . . . . . . . 137 Fol. obovata - Ain, La Pape (Chabert).1752. C. Dicranordes ....................... Gdgr mss Fol. ovata basi attenuata; ped. glabri - Germ., Wurtzbourg (Rau). . . . . . 1753. C. seuarrosa (Rau) Fol. ovata basi rotundata; ped. villosi - Rhône, Francheville (Chabert) ....... 1754. C. Aculeata Gdgr mss Pet. inermes; ped. 6-8 m. 1. - Rhône, Francheville (Cha-

137 bert). . . . . 1755. C. RHodella Gdgr mss Pet. aculeati; ped. 11-13 m. 1. - Rhône, Gleizé (Gdgr herb. ros. n. 188). . . . . 1756. C. Asclepiadea Gdgr

138 Pet. glabri vel sparse pilosi. . . . . . . . . 139

138 Pet basi saltem hirsuti. . . . . . . . . . . . .

139 Rami floriferi inermes... . . . . . . . 140

Fol basi cordata; discus conicus $\cdots 140$ bis

140 Fol. basi attenuata; discus parum conicus - Rhóne, Arnas (Gdgr herb. ros. n. 190). . . . 1757. C. eucharis Gdgr mss 


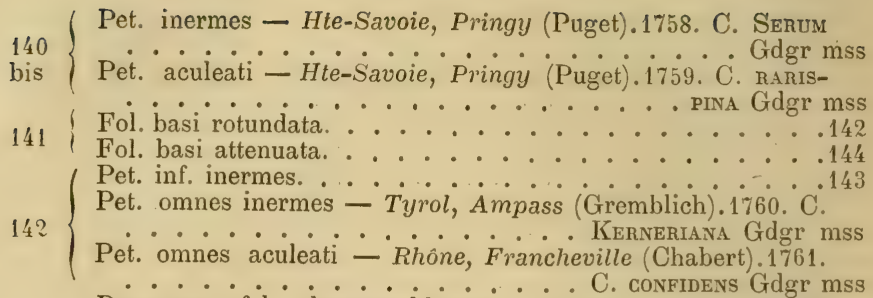

( Purpurea, fol. obovato-oblonga - Tyrol, Trente (Gelmi) 143 Virens; ; fol. obovata - Rhône, La Tour-de-Salvagny (Chabert)
Ped. 7-10 m. l.; fol. obovato-oblonga acuta - Helv., Zurich

144 (Lehmann) ........ 1764. C. Turicensis Gdgr mss Ped. 16-20 m. l.; fol. oblongo-lanceolata - Pyrenaei, Gèdre (Bordère): . . . . . . . 1765. C. RubENs Gdgr mss

145 ( Rami flor inermes. . . . . . . . . . .

Rami flor aculeati. . . . . . . . . . 149

Fol. basi | attenuata. . . . . . . . . . . 147

Fol. obovata basi cordata; discus subconicus - Hte-Savoie, Pringy (Puget). . . . . 1766. C. Anisophlata Gdgr mss

146 Fol. obovato-oblonga basi rotundata; discus subconicus Pyrenaei, Gèdre (Bordère). . . 1767. C. PAPILlOsa Gdgr mss

Fol. ovato-elliptica cordata; discus valde conicus - HteSavoie. Pringy (Puget) . . . 1768. C. Macrodiscus Gdgr mss Cor. eciliata ; discus | conicus. . . . . . . 148

147 Cor. valde ciliata; discus planus - Helv., Zurich (Lehmann)

(Pet. inermes; ped. $4-7^{\circ}$ m. 1. $^{-}$Hte-Savoie, St-Martin (Puget)

148 . . . . 1770. C. Caulifolia Gdgr mss Pet. aculeati; ped. 10-12 m. 1. - Hisp. centr., Escorial (Tremols). . . . . . 1771. C. CARPETANA Gdgr mss $149\left\{\begin{array}{l}\text { Fol. obovata obovato-oblonga vel oblonga; inferne attenuata.150 } \\ \text { Fol. rotundata inferne cordata - Helv., Vaud : Morcles }\end{array}\right.$ (Favrat) .......1772. C. CYCLOPHYLLA Gdgr msS

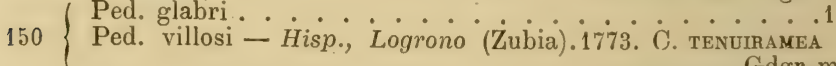

Fol obovata discus . Gdgr mss

Fol, obovata ; discus conicus - Austr. inf., Kalksburg (Wiesbaur)........1774. C. Wiesbauril Gdgr mss Fol. obovata; discus vix conicus - Isère, Charancieu (Gdgr)

151 . . . . 1775. C. EFFusa Gdgr mss Fol. obovato-oblonga; discus conicus - Manche, Yvetôt (Lebel). . . . . . 1776. C. onthodon Gdgr mss Fol. oblonga; discus subplanus - Gallia, Narbonne (Gautier)

$152\left\{\begin{array}{l}\text { Sep. omnino eglandulosa } \\ \text { Sep. paulo dentato-glandulosa. }\end{array}\right.$

Sep. valde pectinato-glandulosa. . . . . . . . 252 
Fr. ovatus vel rotundato-globosus . . . . . . . 154

Fr. ovoideus. . . . . . . . . . . 166

Fr, obovato-oblongus. . . . . . . . . . 175

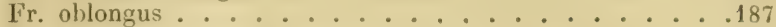

154 ( Pet. glabri vel sparse pilosi. . . . . . . . . . 155

Pet. saltem basi hirsuti. . . . . . . . . . . .159

1.5 Rami flor inermes. . . . . . . . . . 156

Rami flor aculeati. . . . . . . . . 158

Fol. obovata vel obovato-oblonga. . . . . . . . . 157

1.56 Fol. ovato-elliptica; styli subcoaliti-Cher, Mehun, etc.1778.

. . . . . . . . SPHAERoIdA (Rip.)

Fr. ample subglobosus; fol. basi sensim attenuata - IIteFr. parvus ovato-rotundatus; fol. cuneata - Côte-d'Or, Puligny (Ozanon). . . . 1780. C. tomentelloides (Ozan. mss) Fol. obovata basi rotundata; ped. $9-11 \mathrm{~m} .1 .-$ Paris.1781.

. . . . . . . . . . . C. stipularis (Mèrat) Fol. obovato-oblonga basi subattenuata; ped. $14-21 \mathrm{~m} .1 .-$ Belg., Rochefort (Crépin). . .1782. C. Lejeunir (Urép. mss)

Fol. minute ovata basi rotundata; ped. 2-5 m. 1. - Cher, Chapelle-St-Ursin (Rip.). . 1783. C. Armatissima (D. et Rip.)

159 Rami flor inermes. . . . . . . . . 160

Rami flor aculeati. ................ . . . .

Discus planus; ped. 17-19 m. 1. - Gironde, Le Verdon (Mo-

$160\{$ telay). . . . . 1784. C. Gregaria Gdgr mss Discus conicus; ped. 6-13 m. 1. - Pyrenaei occid., Col de Tortes (Bordère) . . . . 1785. C. BREvispina Gdgr mss

161 Fol. obovato-oblonga ................. 162

Fol. ovata . . . . . . . . . . . . 163

Discus subconicus; pet. omnes aculeati-Rhône, Francheville

$162\left\{\begin{array}{l}\text { (Billot exs. n. 3719). . . 1786. C. Avellana Gdgr mss } \\ \text { Discus planus; pet. sup. inermes. - Gard, Le Vigan (Billot }\end{array}\right.$ exs. n. 3721 parte). . . 1787. C. confertiflora Gdgr mss

163 ( Discus planus. . . . . . . . . . 164

Discus conicus .................... 165

Fol. basi rotundata - Helv., Montbovon (Cottet) $1788^{\circ}$.

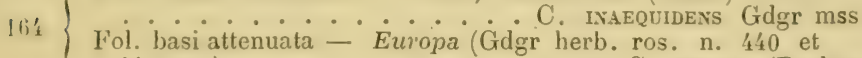
447 var.). . . . . 1789. C. Dumalis (Bechts Pet. omnes aculeati basi hirsuti - Gallia centr.1790. C.

165 Pet. inf. inermes toto dense villosi - IIte-Savoie, IIabèrePoche (Puget) . . . 1791. C. valdepubens Gdgr mss

160 Fol. basi rotundata vel cordata. . . . . . 167

166 Fol. attenuata vel cuneata. . . . . . . . . . . . .

167 Rami flor omnino inermes. . . . . . . . 168

Rami flor. plus minus aculeati . . . . . . . . . 169

Fol. ovato-olliptica; pet. sparse pilosi-Austr. inf., Iodling

(Wiesbaur) . . . . . 1792. C. confenta Gdgr mss

168 Fol. obovata; pet. sparse pilosi-Pyrenaei, Gèdre (Bordere)

. . . . . . 1793. C. Manchantiana Gdgr mss

Fol. late ol,ongo-lanceolata ; pet. hasi villosi - Lragonia bor. Torla (Bordère). . . . . 1794. C. singularis Gdgr mss 
Discus conicus; serraturae apertae - Helv., Montbovon

169 (Cottet)......... 1795. C. RAMigera Gdgr mss Discus planus; serraturae convergentes - Gallia, Besançon (Billot n. 3720) . . . . 1796. C. BREgIllensis Gdgr mss

170 | Rami flor inermes. . . . . . . . . . 171

Rami flor aculeati.

171 Fol obovato-oblonga. . . . . . . . . . 172

171 Fol. ovato-elliptica - Cher, etc. 1797. C. Rublirifora (Rip.)

Ped. 4-6 m. 1.; discus conicus - Hte-Savoie, St-Martin (Pu-

172 get) ........... C. MYriotricha Gdgr mss Ped. 10-18 m. 1. ; discus subconicus - Hte-Savoie, HabereLullin (Puget). . . . . 1799. C. villigera Gdgr mss

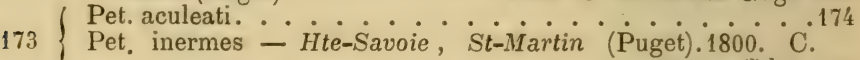

Fol. obovato-acuta; pet. laxe pilosi- Helv. . TINcta Gdgr mss tet).

17' Fol. obovato-oblonga subacula; pet. subglabri - Isère, Le Sappey (Boullu) . . . . 1802. C. SARCophylla Gdgr mśs

Fol. oblongo-acuminata; pet. villosi - Bohemia, Kuchelbad (Opiz) . . . . . . 1803. C. lanceolata (Opiz)

Fol. parva (5-8 m. lata, 12-21 m. longa) ovato-cordata apice attenuata. ...................... 176

175 Fol. late $(20-25 \mathrm{~m}$. lata, 22-28 m. longa) ovata vel rotundata, basi cordata media vero ad apicem obtusa. . . . 178

Fol. sate late $(12-16 \mathrm{~m}$. lata, 18-30 m. longa) obovata vel oblonga. . . . . . . . . . 180

( Discus valde conicus ; rami recti. . . . . . . 177 Discus subplanus; rami arcuati - Rhóne, Gleizé (Gdgr) 1804 .

$176\{$. . . . . . . Humcampta Gdgr mss Pinnulae sat angustatae; ped. 8-11 m. l. - Hte-Savoie,

177 Pringy (Puget).....1805. C. curtifolia Gdgr mss Pinnulae dilatatae; ped. $4-6 \mathrm{~m} .1$. - Hte-Savoie, Pringy (Puget). . . . . . 1806. C. Plicatilis Gdgr mss

178 (Discus plus minus conicus. . . . . 179

178 Discus planus - Bohemia, Kuchelbad (Opiz).1807. C. Glau-

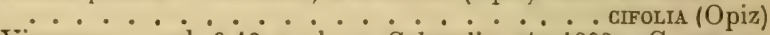

Virescens; ped. $9-13$ m. 1. - Sabaudia, etc.1808. C. ME-

179 Glauco-purpurea; ped. $4-8$ m. i. - Hte-Savoie, St-Martin (Puget). . . . . 1809. C. GLauconubra Gdgr mss . . . . . . Insulae Madère.1810. C. Mandonir (Dés.)

180 | Rami flor inermes. . . . . . . . . . . 181

Rami flor aculeati. . . . . . . 182

Fol. oblonga basi subrotundata - Hte-Savoie, St-Martin (Puget). . . . . . 1811. C. RHombifolia Gdgr mss

181 Fol. obovato-oblonga basi attenuata; discus planus - Rhône, Charbonnières (Chabert). . . 1812. C. PHLEBoleia Gdgr mss Fol. obovata basi attenuata; discus conicus - Hte-Savoie, Pringy (Puget) . . . . 1813. C. stictophora Gdgr mss

182 ( Ped. villosi . . . . . . . . . . . . . . . . . . . . . . Ped. glabri . . . . . . . . . . . . 184 
Discus conicus; fol. basi attenuata - Hte-Savoie, Argonnex 183 (Puget). ........1814. C. Acrodonta Gdgr mss Discus vix conicus; fol. basi rotundata - Tyrol, Hall (Gremblich). . . . . 1815. C. gracilicaulis Gidgr mss Fol. basi attenuata; pet. aculeati. . . . . . . 185

Is' Fol. basi rotundata; pet. inermes - Itte-Savoie, Argonnex (Puget). . . . . 1816. C. Alpestris Gdgr mss Pet. glandulosi ; fr. apice depressus. . . . . . . . 186 Pet. eglandulosi; $\mathrm{f}$. superne valde attenuatus - Rhône, Dardilly (Chabert) . . . 1817. C. semitanum Gdgr mss Ped. $12-16$ m. 1. ; pet. aculeati - Lot-et-Gar., Brax (Debeaux)

1 s6 $\ldots \ldots . . . . .1818$. C. Apostigma Gdgr mss Ped. 7-10 m. 1.; pet. subinermes - Lot-et-Gar., Moirax (Debeaux). . . . . 1819. C. obrusispind Gdgr mss

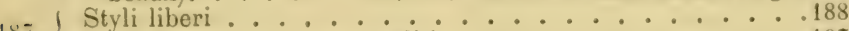

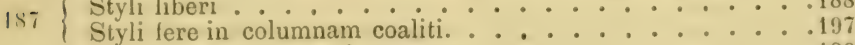

Iss Fol. basi attenuata vel cuneata . . . . . . 189 Fol. rotundata vel cordata . . . . . . . . 193

Rami flor aculeati. . . . . . . . . 190

18. Rami flor, inermes - Gallia, Annecy (Puget).1820. C. AvisoDiscus conicus . . . . . . . . . . 191

1910 Discus planus - Anglia, Cheshire : Raby (Harbord).1821.

Diseus planus - Parvifolia Gdgr mss Fol. obovata . . . . . . . . 19? Fol. oblonga; ped. $17-21 \mathrm{~m} .1$. ; discus vix conicus - IIte-

191 Savoie, St-Martin (Puget). 1822. C. Eccremopillata Gdgr mss Fol. elliptica; ped. $8-13 \mathrm{~m} .1$; discus conicus - Phine, Arnas (Gdgr herb. ros. n. 38).1823. C. Rhodantha Gdgr mss Pet. sparse pilosi; ped. 10-15 m. 1. - Sabaudia, Helv.1824.

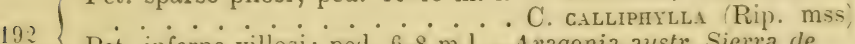
Pet. inferne villosi; pell. 6-8 m.1.-Aragonia austr. Sierra de Mosqueruela (Martin). . . 1825. C. Willkommina Gdgr mss Rami flor, aculeati ............... 194

193 Rami flor. inermes - Gallia, Oise, etc.1826. C. RECognita . . . . . . . . . Rouy) Pet. aculeati ................ 195 $194\{$ Pet. inermes - Hte-Savoie, Pringy (Puget). 1827. C. LaEteFol. obovata apice acuta. . . . . . . . . 196

195 $\{$ Fol. ovato-elliptica apice rotundata - Hte-Savoie, Argonnex (Puget) . . . 1828. C. Acutispisa Gdgr mss Ped. 7-9 m. l. ; fr. basi paulo attenuatus - Wurtemb., Donns-

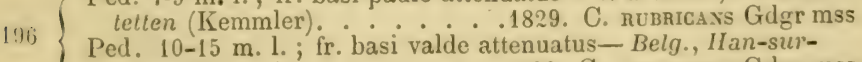
Lesse (Crépin). . . . . . . 1830. C. Loxgissima Gdgr mss Rami flor. inermes; pet. aculeati . . . . . . 198 Rami flor, et pet. toto aculeati - Tyrol, irolveno (Gelmi)

197 . . . . 1831. C. RHyscropirsa Gdgr mss Rami flor. aculeati; pet. inermes - Hte-Savoie, Pringy (Puget). .......... 1832. C. rlebera Gdgr mss Fol. ovata basi rotundata. ............ . . . 99

198 Fol. obovata hasi attenuata - IIte-saroie, Pringy (Puget) .1833. C. stignatotes Gdgr mss 


\section{$-144-$}

(Costa vix glandulosa ; pet. unifariam villosi - Sabaudia. 1834

99 Costa toto glandulosa; pet. undique villosi - Hte-Savoie,

Pringy (Puget). . . . . . 1835. C. Leiophlaea Gdgr mss

200 Fr. rotundatus vel ovato-rotundatus. . . . . . . 201

Fr. ovatus ovoideus vel obovato-suboblongus. . . . . . .204

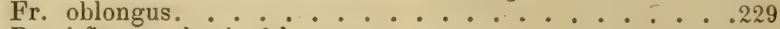

Rami flor. aculeati ; fol. ovata. . . . . . . . . 202

Rami flor aculeati; fol. oblonga - Anglia, Cheshire Har-

201 bord). . . . . . 1836. C. PERglobosa Gdgr mss Rami flor. inermes; fol. obovato-oblonga-Gallia, Clermont (F. Héribaud). . . . . 1837. C. claromontana Gdgr mss

20 ( Fol hasi late cordata . Fol. basi attenuata ; rami flor farinosi - Rhône. Gleizé(Gdgr herb. ros. n. 78 et 78 bis). . . . 1838. C. PApposa Gdgr Pet. aculeati ; costa eglandulosa; discus subconicus - Hte-

Savoie, Arenthon (Puget) . . 1839. C. HYPocycLA Gdgr mss

203 Pet. aculeati; costa gland.; discus planus - Rhône, Charbonnières (Chabert) . . . . 1840. C. MESOGYCLA Gdgr mss Pet. subinermes; costa gland.; discus conicus-Belg., Eich (Crépin) . . . . 1841. C. Luxemburgiana (Crép. mss)

?01 $\{$ Rami flor inermes. . . . . . . . . . . . 205

204 Rami flor aculeati. $\therefore . .2212$

205 Fol. obovato-oblonga vel oblonga. . . . . . . 206

205 Fol. ovata vel obovata . . . . . . . . . . 209

206 Pet. inermes. . . . . . . . . . 207

Pet. aculeati. . . . . . . . . . . 208

Ped. 10-12 m. 1.; fol. basi rotundata - Italia mer., Albe

207 (Gibelli). . 1842. C. Albanonum Gdgr mss Ped. 7-9 m. 1.; fol. basi attenuata-Tyrol, Hall (Gremblich) . . . . . . . 1843. C. Tenuicalyx Gdgr mss

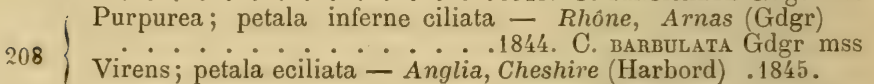
Virens; petala eciliata - Anglia, Cheshire (Harbord) . 1845. .......... C. Banksiana Gdgr mss

209 Discus planus. . . . . . . . . . . . 209

Discus conicus . . . . . . . . . . . 210

(Pet. subaculeati ; fol. basi rotundata - Pyrenaei, La Mala-

$210\left\{\begin{array}{l}\text { detta (Gdgr herb. ros. n. 216)..1846. C. PHALAcrophlata } \\ \text { Pet. inermés; fol. attenuata - Rhône, Pommiers (Gidgr herb. }\end{array}\right.$ ros. n. 314). . . . . . 1847. C. saxivaga Gdgr mss

$211\left\{\begin{array}{l}\text { Costa paulo glandulosa; pet. basi villosi - Rhône, Gleizé } \\ \text { (Gdgr herb. n. 542). . . - . 1848. C. curtipes Gdgr mss } \\ \text { Cos'a eglandulosa; pet. sparse pilosi - Silesia, Falkenberg }\end{array}\right.$ Costa eglandulosa; pet. sparse pilosi - Silesia, Falkenberg

212 Fol. basi rotundata vel subrotundata. . . . . . . . . . . . . 213

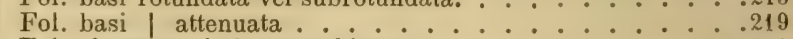

$213\left\{\begin{array}{l}\text { Fol. obovata vel obovato-oblonga } \\ \text { Fol. oblonga } \\ \text { Fol ovata }\end{array}\right.$

Fol. ovata; pet. inermes - Rhône, Montmelas (Gdgr). 1850. ................... Pinivaga Gdgr mss 
Fol. obovata; discus / conicus; pet. aculeati

Fol. obovato-oblonga ; discus planus; pet. subinermes Suecia, Vestrogothia : Mariestad (Lundquist).1851. C. EMAn............. . . . ginata Gidgr mss

Pet. sparse et paulo villosi ........... . . 216

215 Pet. dense pubescentes - Hie-Savoie, Habère-Poche (Puget) . . . . . . . 1852. C. Fabaefolia Gdgr mss Costa glandulosa; fol. acuta - Rhône, Pommiers (Gdgr

216 herb. ros. n. 169). . . 1853. C. Hintistyla Gdgr mss Costa eglandulosa; fol. obtusa - Austr. inf., Kalksburg (Wiesbaur). . . . . . 1854. C. Aggregata Gdgr mss

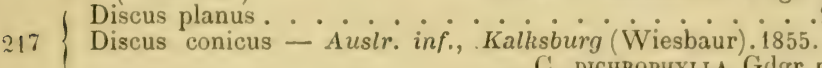

. . . . . . C. Dichrophyla Gdgr mss

Cor ciliata; fr. ovoideus - Rhône, Montmelas (Gdgr herb.

218 ros. n. 195) ...... 1856. C. oculus-Junonis Gidgr Cor. eciliata ; fr. ovatus - Rhône, Arnas (Gdgr).1857. C.

Fr. obovato-oblongus. . . . . . . . . . . 225

Fol. obovata. . . . . . . . . . . 221

220 Fol. obovato-oblonga. . . . . . . . . 222

Fol. oblonga. . . . . . . . . . . . . . 223

Bracteae amplissimae; cor. ciliata - Puy-de-Dôme, Mont-

221 baubier (Dubosclard) . . . 1858. C. vaginata Gdgr mss Bracteae minores; cor. eciliata - Rhöne, Pommiers (Gdgr herb. ros. n. 117). . . . . 1859. C. EpIPActis Gdgr

Discus planus ; ped. glabri - Thuringia, Oesterfeld (Haussknecht). . . . . 1860. C. FISSA Gidgr mss

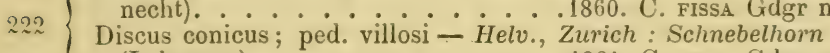
(Lehmann). . . . . . . 1861. C. Hurta Gdgr mss Ped. 10-12 m. 1. ..................224

223 Ped. 5-8 m. 1. - Gallia, Thonon (Puget).1862. C. GLaucina

.............. (Rip. mss

Fr. utrinque rotundatus; discus planus - Rhône, Charbonnières (Chabert). . . . 1863. C. Capnoniasa Gdgr mss

224 Fr. utrinque depressus; discus paulo conicus - Rhône, Villefranche (Gdgr herb. ros. n. 231; Gdgr Dec. plant. nov. III, p. 19). . . . . . 1864. C. Actinodroma Gdgr

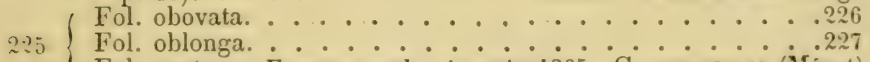

Fol. ovata - Europa med. et austr.1865. C. Bisennata (Mérat) Discus conicus; fol. apice attenuata - Rhône, firancheville

2. (Chabert) ....... 1866. C. illecta Gdgr mss Discus planus; fol. apice subrotundata - Gallia, Clermont (F. Héribaud). . . . . 1867. C. Rubrovotata Gdgr mss Pet. omnes inermes; fol. acuta . . . . . . . . .228

2.2 Pet. sup aculeati; fol acuminata - Rhóne, Villefranche (Gdgr herb. ros. n. 81) . . . 1868. C. podostrla Gdgr mss I'ed. 1't-16 m. 1.; discus paulo conicus - Isère, Les Abrets

228 (Gdgr) . 1869. C. Mesuclad Gdgr mss Ped. 11-12 m. 1.; discus planus - Anglia, Monmouth : Llanthony (Ley). . . . . . 1870. C. Smithiana Gdgr mss

229 Fol. ovata, obovato-elliptica. . . . . . . . 230 Fol. obovato-oblonga vel oblonga . . . . . . . . .237 
Rami flor inermes. . . . . . . . . 231

Rami flor aculeati. . . . . . . . . . 233

231 Petioli aculeati . . . . . . . . . . . . . . . . . .

Pet. inermes - Gallia, Thonon (Dés. herb. ros. n. 48). 1871.

............. c. cervina Gdgr mss Styli liberi ; fol. amplissime obovato-elliptica - Hte-Savoie,

$232\left\{\begin{array}{l}\text { Veyrier (Puget). - . 1872. C. CHamaEmespilus Gdgr mss } \\ \text { Styli subcoaliti ; fol. ovato-elliptica - Hte-Savoie, Arenthon }\end{array}\right.$ (Puget). . . . . . 1873. C. StYLARIS Gdgr mss

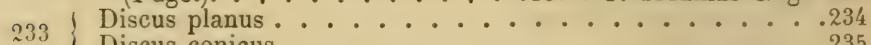

233 Discus conicus. . . . . . . . . . . . . . 235

Ped. 6-7 m. 1.; fol, obovata - Tyrol, Hall (Gremblich). 1874.

$23 \pm\left\{\begin{array}{l}\text { Ped.10-15 m. i.; fol. elliptica - Anglia, Germ.1875. C. Sar- } \\ \text { Parts }\end{array}\right.$

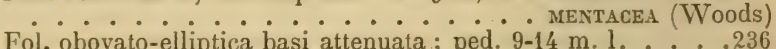
Fol. ovato-elliptica basi rotundata; ped. 5-8 m. 1. - Rhône, Montmelas (Gdgr). . . . 1876. C. stenoclada Gdgr mss

$236\left\{\begin{array}{l}\text { Costa glandulosa; stip. apice ovatae - Hte-Savoie, Argonnex } \\ \text { (Puget) } .1877 \text {. C Chinacantua Gdgr mss }\end{array}\right.$ Costa eglandulosa; stip. apice lanceolatae - Hte-Savoie, Pringy (Puget). . . . 1878. C. oligosticta Gdgr mss

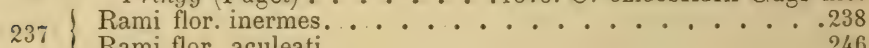

238 Fami flor aculeati. . . . . . . . . . . . . 246

238 Fol basi rotundata. $\quad \ldots 239$

Pet. aculeati; discus conicus - Pyrenaei, Gèdre (Bordère)

239 . . . 1879. C. oblongigema Gdgr mss Pet. inermes; discus subconicus - Gallia, Clermont (F. Héribaud). . . . . 1880. C. PHALACROCLADA Gdgr msS

240 ( Pet. inermes. . . . . . . . . . 241

Pet aculeati. . . . . . . . . . 243

| Discus planus. . . . . . . . . . . . 42

241 Discus conicus - Hibernia, Belfast (Stewart) 1881. C. HIBER.......................... Gdgr mss

$242\left\{\begin{array}{l}\text { Styli subcoaliti; fol. parva - Austria, Vienne (Wiesbaur) } \\ \text { Styli liberi; fol. magna - Puy-de-Dôme, Montbaubier (Du- }\end{array}\right.$ Styli liberi ; fol. magna - Puy-de-Dôme, Montbaubier (Dubosclard). . . . . 1883. C. Latisepala Gdgr mss

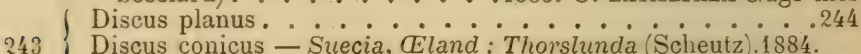
Discus conicus - Suecia, Eland: Thorslunda (Scheutz). 1884.

. . . . . . . Baltica Gdgr mss

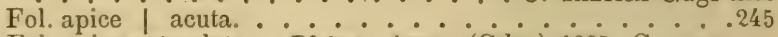

24 Fol, apice rotundata - Rhône, Amas (Gdgr).1885. C. Avoe. . . . . . NIFLORA Gdgr mss

$245\left\{\begin{array}{l}\text { Sep. apice angustata; ped. } 9-13 \mathrm{~m} .1 .- \text { Sabaudia, etc. (Gdgr } \\ \text { herb. ros. n. } 419 \text { et } 592 \text { var.). - - 1886. C. Insignis (Rip.) } \\ \text { Sep. apice foliacea ; ped. } 6-9 \text { m. 1. Austr. inf., Kalksburg }\end{array}\right.$ Sep. apice foliacea; ped. 6-9 m. 1. - Austr. inf., Kalksburg (Wiesbaur). . . . . 1887. C. Fraxinoides Gdgr mss

$2\{6$ Fol. oblonga. .............. 247

246 Fol obovato-oblonga. . . . . . . . . . 249

247 Pet. aculeati. . . . . . 248 Pet. inermes - Isère, Paladru (Gdgr).1888. C. Conoacantha 
Discus conicus ; ped. 9-13 m. 1. - Itte-Sacoie, I'ringy (P'uget)

Discus subonic. 1889. C. consociata Gdgr mss (Discus subconicus; ped. 15-23 m. 1. - Ite-Sawoie, Irinu!y (Puget). . . . . 1890. C. supravirens Gdgr mss

249 Pet. aculeati; discus | conicus . . . . . . . 250

Pet. inermes; discus planus - Rhone, Villefranche Gidgr herb. ros. n. 99) . . . . 1891, C. styloidea Gdgr

Fol. basi attenuata ; aculei conformes . . . . . . .251

$\because 50$ Fol. basi subrotundata; aculei dimorphi- IIibernia, Belfast Colin Gilen (Stewart) . . 1892. C. Heteracastia Gidgr mss

(Ped. 4-6 m. 1.; discus valde conicus - Aragonia austr., Sierra de IIosqueruela (Martin y Cercos). 1893. C. Cenco-

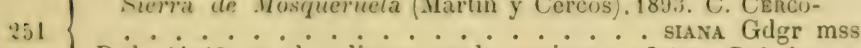

Ped. $14-18$ m. l.; discus paulo conicus - Isère, Paladru

(Gdgr). . . . . . 1894. C. FRuticetonum Gdgr mss

252 f Fr. rotundatus ovatus vel ovoideus. . . . . . . . 253

252 Fr. obovato-oblongus vel oblongus. . . . . . . . 265

253 Pet. glabri vel sparse pilosi. . . . . . . . . 254

Pet. saltem basi undique hirsuti. . . . . . . . .26?

(Fr. globosus vel rotundatus. . . . . . . . . . 255

254 Fr. ovatus. . . . . . . . . . . 257

Frovoideus. . . . 258

Fol. basi rotundatus; discus conicus . . . . . . . 256

255 Fol. basi attenuata; discus planus - Podolia (Indrz.) is95.

............... Ponolica (Tralt.)

Pet. aculeati; fol. ovata - Paris. .1896. C. MacrocanPa (Mérat)

256 Pet. inermes; fol. obovata - Itte-Savoie, Pringy (Puget)

. . . . . . 1897. C. oligophylla Gdgr mss

Fol. ovata basi rotundata; ped. 16-20 m. 1. - Hte-Savoie,

IIabère-Lullin (Puget). . . . . 1898. C. amoenifolia Gdgr mss

Fol. oblonga basi rotundata; ped. 7-12 m. 1. - Paris (Iort.

Paris 1869) . . . 1899. C. Actinochlamys Gdgr mss

Fol. obovato-oblonga basi attenuata; ped. 18-22 m. 1. - Silesia, Buchberg (Straehler) . 1900. C. Calostephana Gdgr mss

258 | Fol. basi rotundata. . . . . . . . . . 259

Fol. basi attenuata. . . . . . . 260

Ped. 4-7 m. 1.; fol. ovata - Rhône, Arnas (Gdgr).1901. C.

$2.94 \quad \cdots \cdots \cdots$

Ped. 11-14 m. 1.; fol. obovato-elliptica-Pyrenaei, Gèdre (Bordere). . . . . 1902. C. PSEUdo-Fentilis Gdgr mss Fol. obovato-oblonga; discus planus. . . . . . . .261

$210\{$ Fol. oborata; discus paulo conicus - Hte-Savoie, IIabèreLullin (Puget). . . . . . 1903. C. FentiLIs Gdgr-mss

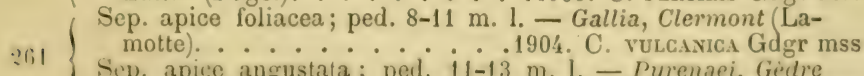
Sep. apice angustata; ped. 11-13 m. l. - Pyrenaei, Gédre

(Bordere). . . . 1905. C. srxstictonos Gidgr mss

Fr. rotundatus vel globosus. . . . . . . . . 263

$262\{$ Fr. ovoideus. . . . . . . . . . . . . . . 64

Ir. ovatus - Ilte-Savoie, Reyvroz (Puget) igon. C. PAcri. . . . . . . . . . canpa Gdgr mss

Ped. 10-17 m. 1.; fol. basi attenuata - Hte-Savoie, Habere-

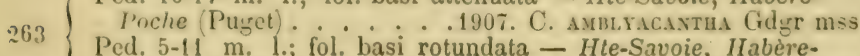
Ped. 5-11 m. 1.; fol. basi rotundata - Hte-Savoie, HabereLullin (Puget). . . . . 1908. C. sclenophlaea Gdgr mss 
( Discus conicus ; fol. ovato-elliptica - Hte-Savoie, St-Martin

264 (Puget). . . . . 1909. C. odontosepala Gdgr mss Discus planus; fol. obovato-oblonga - Hte-Savoie, HabèreLullin (Puget). . . . 1910. C. oligolePIS Gdgr mss

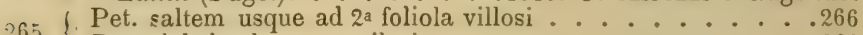

265 Pet. glabri vel sparse pilosi. . . . . . . . . 268

266 Ped. glabri • . . . . . . 267

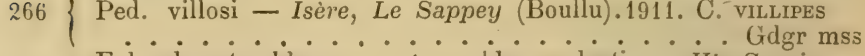
Fol. obovato-oblonga; pet. valde aculeati - Hte-Savoie,

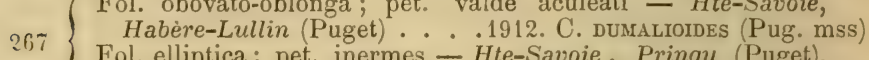
Fol. elliptica; pet. inermes - Hte-Savoie, Pringy (Puget).

Rami flor inermes. $\therefore$. 1913. C. Innocua (Rip. mss)

268 Rami flor aculeati.

$269\{$ Fol. basi attenuata. 0.270

269 Fol. basi rotundata - Hte-Savoie, Argonnex (Puget). 1914.

2 ( Discus planus. . . . . . Mcrotuba Gdgr mss

$270\left\{\begin{array}{l}\text { Discus planus. } \\ \text { Discus conicus. }\end{array}\right.$

( Fol. obovata ; fr. apice attenuatus - Hte-Savoie, Pringy

271 (Puget). . . . 1915. C. GeophILA Gdgr mss Fol. oblonga ; fr. apice longe constrictus - Anglia, Hereford: Aymestry (Ley) . . . . 1916. C. Hookeriana Gdgr mss

( Stip. rubentes; fol. oblonga - Hisp. bor., Sierra de Cameros $272\left\{\begin{array}{l}\text { (Zubia). } \cdot \dot{*} \cdot 1917 . \text { C. odontostephana Gdgr mss } \\ \text { Stip. virentes; fol. obovata - Dalmatia, Val di Ombla }\end{array}\right.$ (Studniczka) . . . . . . 1918. C. DINARICA Gdgr mss

773 Fr. obovato-oblongus . . . . . . . . . . 274

273 Fr. oblongus.

Discus conicus; fol. apice sensim attenuata - Hie-Savoie,

274 Habère-Lullin (Puget). - . 1919. C. Fraterna Gdgr mss

Discus subplanus; fol. apice rotundata - Hte-Savoie, Mt Sion (Puget). . . . . 1920. C. Polrsticta Gdgr mss

275 Fol. obovata vel obovato-oblonga, saltem apice | attenuata.276

275 Fol. ovato-elliptica utrinque rotundata - Hte-Savoie, Argonnex (Puget) . . . . . 1921. C. PARVA Gdgr mss

$276\left\{\begin{array}{l}\text { Ped. 10-16 m. l.; fr. utrinque depressus - Hte-Savoie, St- } \\ \text { Martin (Puget). . . . 1922. C. TRichostigra Gdgr mss }\end{array}\right.$ Ped. 4-9 m. 1.; fr. utrinque longe attenuatus - Hte-Savoie, St-Martin (Puget). . . . 1923. C. Elisocalyx Gdgr mss

\section{B. - Stictopodae Gdgr Essai p. 28. Canineae hispidae Crép. Prim. p. 18.}

Foliola undique glaberrima, simpliciter vel argute biserrata, virentia, subtus vero glaucescentia et ad costanı mediam 
plerumque glandulosa; Sepala deflexa interdum patula vel suberecta sero decidua, exfus saepius dorso glandulosa, pinnatipartita. Styli liberi, glabri ant villosi, ultra discum capitati. Corolla rosea, mediocris rel ampla. Fructus pulposus. Peduneuli semper glandulosi vel hispidi. Aculei plus minus falcato-dilatati. nommumquam dimorphi ac in setis glanduliferis (ad ramos praesertim floriferos) abeuntibus. Radix quandoque reptans.

A. $\left\{\begin{array}{l}\text { Fol. omnino simpliciter serrata } \text { Fol. sat persistentia alia simpliciter alia biserrata b. Marcidae } \\ \text { bol. }\end{array}\right.$ Fol. omnia argute biserrata. ........ Osmophyllae

a. - Euandegavenses Gdgr Essai, p. 28.

Foliolis simpliciter serratis ; sepalis post anthesim deflexis moxque deciduis; aculeis conformibus; radix non reptans.

Styli glabri vel vix hirtelli ............ 2

Styli hirsuti vel lanati ............ . . 18

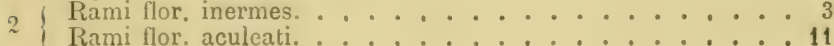

Fol. ovata. . . . . . . . . . . . 4

3 Fol, obovata. ............... 5

Fol oblonga. . . . . . . . . 8

Discus planus; pet. villosi. - Lot-et-Gar., Mourrens (de

4 Punmaret. . . . . . 1924. C. hirtella (Rip.)

I)iscus conicus; pet. glabri - Gallia, Montpellier (Loret).

. . . . . . . 1025. C. litigiosa (Crép. mss)

Pet. inermes. . . . . . . . . . . 6

Pet. aculeati. . . . . . . . . . . . 7

Costa eglandulosa; fol. basi suhtruncata - Catalauniae.

6 Falgas (Vayreda). . . 1926. C. Salvatoris Gdgr mss

Costa glandulosa; fol basi attenuata - Rhône, Charbonnières, etc. (Chabert) . . . 1927. C. Mrnopmylla Gdgr mss

Fol. basi attenuata; discus subconicus - Rhône, Francheville

- 1 (Chabert). . . . . 1928. C. edurescess Gdgr mss

Fol. basi subtruncata; discus conicus - Gallia, Perpignan

(Debeaux). . . . . 1929. C. Pexchisati Gdgr et Debx 
Fr. obovato-oblongus. . . . . . . . . . 9 9

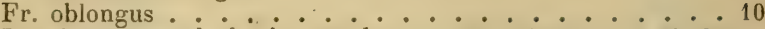

Pet. inermes; fol. basi rotundata - Deux-Sèvres, La Mothe

S. Héray (Sauzé et M.). . . 1930. C. Pictaviensis Gdgr mas

Pet. aculeati ; fol. basi attenuata - Lot-et-Gar., St Christien (de Pommaret). . . . . 1931. C. Glabristyla Gdgr mss

Discus paulo conicus; fr. basi decurrens - Doubs, Rosemont

10 (Billot exs. n. 3722). . . . 1932. C. Bavouxir Gdgr mss Discus planus; fr. basi attenuatus - Rhône, Poleymieux

(Chabert) . . . . . 1933. C. TuBrCalyx Gdgr mss

11 Sep. dorso eglandulosa. . . . . . . . . 12

Sep. dorso glandulosa . . . . . . . . . . . 13

Discus planus; fol. acuminata - Cher, Celle-Bruère (Déségl.).

12 Discus conicus; fol. subacuta - Hispania centr., El Escorial

(Laguna). . . . . . . 1935. C. Castellana Gdgr mss

13 Fol. basi rotundata ............. 14

Fol. basi attenuata. . . . . . . . . . . 15

Fr. ovoideus; discus planus - Rhône, Arnas (Gdgr) 1936.

14 . . . . . . C. Characias Gdgr

Fr. globosus; discus conicus - Persia, Schiras (Kotschy exs.

n. 5/47)............. 1937. C. ABstenta (Dés.)

15 Fr. ovoideus. ................... 16

Fr. obovato-oblongus vel oblongus . . . . . 17

Fr. laevis basi rolundatus - Rhône, Pommiers (Gdgr).1938.

$16\{$ Fr. toto glandulosus basi attenuatus - Cher, Turly (Ripart).

Ped. basi laeves ; fr. elongatus - Gard, Aulas (Tuezkiewicz).

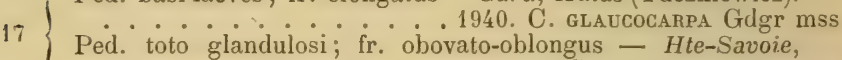

Habère-Lullin (Puget). . . . 1941. C. TRICHELlA Gdgr msS

Fr. oblongus . . . . . . . . . . 19

18 Fr. rotundatus vel globosus. . . . . . . . 25

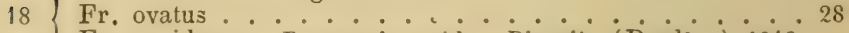

Fr. ovoideus - Pyrenaei occid., Biarritz (Bordère) 1942.

. . . . . . . . C. Extranea Gdgr mss

19 Fol ovata . . . . . . . . . 2 20

Fol. oblongata. $\ldots 24$

Virens; ped. dense glandulosi - Europa media, Oriens (Gdgr

$20\left\{\begin{array}{c}\text { herb. ros. n. } 439 \text { et } 509 \text { var). 1943. C. ANdEgAvensis (Bast.) } \\ \text { Rubens; ped. parce glandulosi-Sabaudia, etc.1944. C. PUR- }\end{array}\right.$

. . . . . . . . . . . purascens (Rip.)

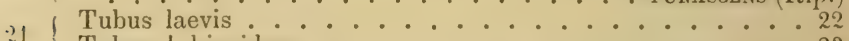

Tubus | hispidus ............... 23

$22\left\{\begin{array}{l}\text { Rami flor. inermes; discus conicus - Rhône, Dardilly (Cha- } \\ \text { bert) flor. aculeati ; discus planus - Rhône, Montmelas } \\ \text { Rami flor. }\end{array}\right.$

Rami flor. aculeati; discus planus - Rhône, Montmelas

(Gdgr) . . . . 1946. C. Gracilispina Gdgr mss $2.3\left\{\begin{array}{l}\text { Pet. omnes inermes; fol. basi attenuata - Hte-Savoie, Veyrier } \\ \text { (Puget) }\end{array}\right.$

Pet. inf. inermes; fol. basi attenuata - Hte-Savoie, Veyrier

(Puget). ...... 1948. C. Longa B. AfFinis Gdgr mss 
Pet. omnes aeuleati; fol. basi attenuata - IIte-Savoie, Veyrier

23 (Puget) . . . 1949. C' longa C. Glaydulosa Gdgr mss

Pet. omnes aculeati; fol. basi rotundata - Rhône, Ecully

(Morel) . . . . . . 1950. C. Monelin Gdgr mss

Sep. dorso glandulosa ; tubus toto hispidus - Persia, Laris-

tan, elc. (Kíotschy exs. n. 263 et $653-$ C. laristanica Gdgr;

24 Sep. dorso paulo glandulosa; tubus basi hispidus - Gallia,

Arles (Autheman). . . 1952. C. Arelatensis Gdgr mss

Sep. eglandulosa; tubus laevis - Hte-Savoie, Habère-Lullin

Pligit . . . . . . 1953. C. Pterti Gilgr mss

25 Sep. dorso eglandulosa; tubus laevis. . . . . . . 26

S. . . Iorso glandulosa; tubus glandulosus - Bararia 195i. . . . . . . . C. Raun (Tratt.)

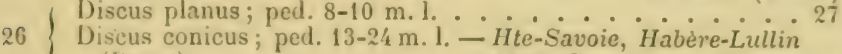
(Puget). . . . . . 1955. C. HeBegra Gdgr mss

Fol. apice subrotundata; pet. paree glandulosi - Aragonia,

27 Boucharo (Gdgr herb. ros. n. 322). .1956. C. cyclomonPHA

Fol. acuta ; pet. glandulosi - Pyrenaei centr., Ossau Gdgr mss dère). 1957. C ABRUPT Gor-

(Sep. dorso glandulosa ... . . . . . . 29

28 Sep. dorso eglandulosa - Gallia, Angers (Boreau).1958.

(Fol. elliptica vel rotundata; discus | conicus. . . . . . 30

29 Fol. oblonga ; discus planus - Tyrol, Anton (Wiesbaur) 1959.

Rami glauci; discus conicus - Anglia. .1960. C. surculosa

30 . . . . . . . . . . . . . . . . . . . . .

) Rami purpurei; disque vix conicus - Anglia. 1961 . C. surculosa

- B. MiNor Gdgr mss

\section{b. - Marcidae Gdgr Essai p. 29.}

Foliolis aliis simpliciter aliis crebre biserratis, glaucescentibus, serius quam in speciebus sectionis praecedentis deciduis; sepalis post anthesim mox deciduis; aculeis plerumque conformibus; radice haud reptante.

1 Sepala dorso eglandulosa. .............. 2

Sep. dorso glandulosa ............ $2 \tilde{3}$

2 Styli grabri vel subhirtelli ............. . 3

2 Styli hirsuto-lanati. . . . . . . . . . . . . $1^{3}$

3 / lroblongus. . . . . . . . . . . . 4

I Fr. ovoideus vel obovato-oblongus. . . . . . . . . 8 
Tuhus omnino laevis. . . . . . . . . . 5

Tubus in $1 / 3$ inf. hispidus . . . . . . . . . 6

Fol. ovato-elliptica plerumque biserrata - Hisp. bor., Sierra de Cameros (Zubia) . . . 1962. C. LePTodes Gdgr mss

Fol. obovato-oblonga plerumque simpliciter serrata - Pyrenaei centr.; Hisp., Barcelone (Gdgr herb. ros. n. 30) . . . . . . . 1963. C. gymnophlaea Gdgr mss

Rami flor. aculeati; fol. obovata vel obovato-oblonga. . . . 7

Rami flor. inermes ; fol. amplissime oblonga - Rhône, Chaponost (Chabert). . . 1964. C. Castaneaefolia Gdgr mss Yed. 10-16 m. l.; fol. basi attenuata - Rhône, Limonest (Chabert). . . . . . 1965. C. AdscEnsionis Gdgr mss Ped. 19-20 m. l.; fol. basi rotundata - Hisp. mer., Sierra de Segura (Canada). . . . . 1966. C. BAETICA Gdgr mss Aculei conformes ................. 9 Aculei dimorphi - Cher, Trouy (Ripart)..1967. C. INTER. . . . . . . . . veniens (Dés.)

Fol basi cordata

Fol. basi | attenuata .............. 11

Fol. ovata; discus planus - Anglia, Gilmaton (Ley). 1968.

........... C. CordFolis Gdgr mss Fol. ovata; discus paulo conicus - Pyrenaei, Gavarnie (Bordère). . . . . . . . 1969. C. INcisa Gdgr mss

Fol. obovato-oblonga; discus planus - Rhône, Arnas (Gdgr herb. ros. n. 90). . . 1970. C. oligoblephara Gdgr mss Fol obovata; discus valde conicus - Pyrenaei centr., Benqué (Bordère). . . . . . . 1971. C. Gaudens Gdgr mss Rami flor. aculeati; fol. obovata vel oblonga . . . . 12 Rami flor. inermes; fol. ovata - Cher, les Aix (Ripart) T. • • . 1972. C. enYTHRELA (Rip.) Tubus laevis; pet. aculeati. .......... 13 Tubus hispidus ; pet. inermes - Rhône, Mt-Ceindre (Chabert). . . . . . 1973. C. Calamaria Gdgr mss Discus subconicus; fol. obovata basi subattenuata - Pyrenaei, Gavarnie (Bordère). . . . 1974. C. Ambigens Gdgr mss Discus conicus; fol oblonga basi attenuata - Hispania, Logrono (Zabia). . . . . 1975. C. umbraticola Gdgr mss Pet. sparse hirtelli; fol. basi attenuata ad costam vix glandulosa . . . . . 1

Pet. minute hirsuti; fol. basi rotundata ad costam semper glandulosa ................. 20 Fr. ovoideus . . . . . . . . . . 16 Fr. oblongus . . . . . . 17 Discus planus; fol. oblonga basi attenuata - Rhône, Montmelas (Gdgr herb. ros. n. 207).1976. C. PHALACROSTEMa Gdgr mss Discus sat conicus; fol. elliptico-cordata - Rhône, Gleizé (Gdgr herb. ros. n. 537). 1977. C. BRAGHYstema Gdgr mss Fol. olovata vel oblonga. .......... 18

Fol. ovata - Cher, Trouy, etc . . 1978. C. viNealis (Rip.)

18 Rami flor. inermes; discus conicus - Hte-Savoie, St-Gervais (Puget). . . . . . 1979. C. PHLEBophora Gdgr mss 
Costa eglandulosa; fol. basi attenuata - Pyrenaei, Gèdre

19 (Bordère). . . . . 1980. C. Deliciosa Gidgr mss Costa glandulosa; fol. basi subrotundata - Pyrenaei, Lourdes (Bordère). . . . . 1981. C. Educts Gdgr. mss

Ped. omnes hispidi .............. . 21 Ped. plerique laeves - Rhone, Charbonnieres (Billot exs. n. 3586) . . . . . . . 1982. C. NuDIPEs Gidgr mas Fr. oblongus ................ . . 22 Ir. ovatus ; fol ovato-rotundata - Rhône, Franchevile (Billot. exs. n. 3723 ; Gdgr herb. ros. n. 381).1983. C. Au-

. . . . NienI (Cariot) Fr. ovoideus; fol. obovata - Hungaria, Presburg (Schneller) ................. C. opacifolia Gdgr mss Fol. ovato-elliptica - Rhône, Dardilly (Gdgr herb. ros. n. 1985. C. MYRIOCARPA Gdgr ms Fol. obovata - Rhône, Charbonnieres (Gdgr herb. ros. n. 323) ................ Potens Gdgr mss Styli glabri vel glabrescentes. . . . . . . . . . 24 Styli villosi vel hirsuti. ............. . . 48

2 ( Pet. glabri vel sparse pilosi . . . . . . . . . . 25 Pet. llaxe et undique pubescentes. . . . . . . . . . . . . . 42

.5 Tubus saltem usque ad 1/2 infer partem glandulosus. . . . 26

Tubus laevis vel basi infima tantum glandulosus . . . . 39

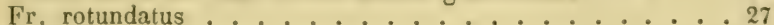

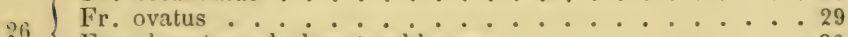

Fr. obovatus vel obovato-oblongus . . . . . . . 30

Fr. oblongus . . . . . . . . . . . . . 34

I Rami flor aculeati. . . . . . . . . . 28

27 Rami flor inermes - Rhône, Beaunant (Chabert). .1987.

C. BRachycocca Gdgr mss

Pet. 10-14 m. 1.; tubus toto aculeolatus - Rhône, Montmelas Ped. 5-8 m. 1.; tubus ad 2/3 sup. hispidus - Rhône, Ville s. J. (Gdgr herb. ros. n. 163).1989. C. Mesostema Gdgr mss Tubus aculeolatus; rami flor. aculeati - Caucasus, Dido

29 (Ruprecht)......... 1990. C. Didoessis (Boiss.) Tubus glandulosus ; rami flor, inermes - Rhone, Grézieux (Chabert). . . . . 1991. C. pimonPHocycla Gdgr mss Pet. omnes aculeati. ............. 31

30 Pet. omnes inermes. . . . . . . . . . . 32

Pet. sup aculeati. ............. . . 33 Pinnulae integerrimae; ped. 14-16 m. 1. - Helv., Genève

31 (Sandoz).............. G92. Geverexsis Gdgr mss Pinnulae dentatae; ped. 10-11 m. 1. - Pyrenaei, Gedre (Bordère). . . . . . 1993. C. Fastidita Gdgr mss Fol. basi cordata; discus planus - Rhône, Francheville

i2 (Chabert) ............... C. crvis Gidgr mss Fol. attenuata ; discus paulo conicus - Rhóne, Grézieux (Chabert) ......... 1995. C. perturbata Gdgr mss

Styli exserti; tubus in 1/2 inf. glandulosus-Rhône, Vaugneray Chabert). . . . . 1996. C. Nenatostipula Gdgr mss bier (Gdgr herb. ros. n. 337).1997. C. CiswamomcoloR 
34 Discus planus ........................ 35

34 Discus conicus. $\ldots 37$

35 Rami flor aculeati . . . . . . . 36

35 Rami flor. inermes - Rhône, Arnas (Gdgr herb ros. n. 285) . . . . 1998. C. Dolabrifolia Gdgr mss

( Cor. subalba; fol. ovata - Rhône, Montmelas (Gdgr herb. ros. n. 77). . . . . 1999. C. ActinopHL:EA Gdgr Cor. amoene rosea; fol: obovato-oblonga - Rhône, Montmelas (Gdgr herb. ros. n. 113). 2000. C. MARcescens Gdgr

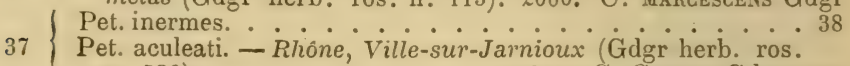
n. 520). . . . . . 2001. C. Carrae Gdgr mss Sep. apice angustata et integra; fr. oblongus - Doubs, Rosemont (Paillot Flora Sequaniensis exsicc. n. 369). .2002.

. . . . . . C. Ludibunda (Gren. et Paill.) Sep. apice foliaceo-dentata ; fr. elongatus - Rhône, Francheville (Cariot). . . . . 2003. C. EXPAllens Gdgr mss

$39\left\{\begin{array}{l}\text { Fr. ovoideus } \\ \text { Fr. oblongus } \\ \text { Fr. }\end{array}\right.$ Fr. rotundatus - Hérault, Ganges (Loret ; C. agraria Rip. in. Dés. Cat. n. 204). . . . . 2004. C. Agrestina (Crép.) Cor. ciliata; fol. elliptica - Rhône, Pommiers (Gdyr herb.

40 ros. n. 307) . . . 2005. C. Cladocampta Gdgr Cor. glabra; fol. ovato-acuta - Gallia, etc: Gdgr herb. ros. n. 530 ; Dés. herb. ros. n. 18?) . .2006. C. Lemaitrei (Rip.) Ped. 11-15 m. 1.; fol. basi valde attenuata - Gallia, etc. 2007. 41 Ped. $7-9 \mathrm{~m} . \dot{1}$; fol basi rotundata - Valais, St-Maurice (Favrat). . . . . 2008. C. Macrodon Gdgr mss

42 Fr. ovoideus vel obovato-oblongus . . . . . 43 Fr. oblongus........ 44 Tubus laevis ; fol ovato-elliptica - Rhône, Limonest (Cha-

43 bert). . . 2009. C. PEtiolaris Gdgr mss Tubus toto glandulosus; fol. obovato-oblonga - Vaucluse, Vallon de la Bonne (Autheman).2010. C. Ptrlodes Gdgr mss

44 Fol. obovata vel obovato-oblonga. ......... 45 Fol. ovata . . . . . 46 Rami flor gracillimi inermes; ped. $12-13 \mathrm{~m}$. 1 - Rhône,

45 Arnas (Gdgr herb. ros. n. 196). . 2011. C. Ischnodendron Rami flor robusti aculeati; ped. $4=6$ m. i. - Gard, GtAmbroix (Tuezkiewicz). . 2012. C. Nemausensis Gdgr mss Fol. lata apice / acuta; fr. parum attenuatus. . . . 47 46 Fol. parva apice rotundata; fr. utrinque valde attenuatus Hte-Savoie, Pringy (Puget) . . 2013. C. Ecdona Gdgr mss Sep. apice angustata; fol. acuta - Gallia, Annecy (Puget) 47 Sep. apice dilatata; fol. subacuta - Gallia, Annecy (Puget) Sep. apice dilatata; fol. subacuta - Gallia, Annecy (Puget)

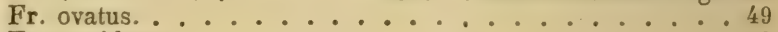

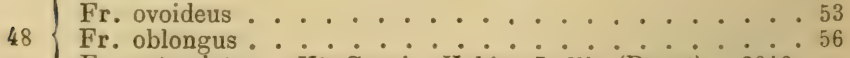
Fr. rotundatus - Hte-Savoie, Habère-Lullin (Puget). .2016. 


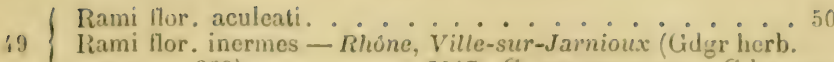
ros. n. 369). . . . . 2017. C. streptomonpina Gidgr mss

. I Costa eglandulosa. . . . . . . . . . 5!

I Custa glandulusa. . . . . . . . . . 5.

( Fol. et corolla mediocria - Helvetia occid. . . . . 2018.

Foi . . - . C. Dolosa (Godet)

Fr. basi rotundatus; fol. parva - Rhone, Amas (Gdgr herb.

.2 ros. n. 227)...... 2020. C. Clypelaria Gdgr

Fr. basi attenuatus; fol. ampla - Rhône, Charbonnières (Cariot). . . . . 2021. C. pseudo-Chaberti Gdgr mss

(3) I Rami flor inermes. . . . . . . . . 54

53 Rami flor aculeati. . . . . . . . 55 Discus planus, ped. 16-18 m. 1. - Anglia, Stafford Penn

$\therefore$ (Fraser). . . . . 2022. C. Hudsoxiasa Gdgr mss

Discus conicus; ped. 11-14 m. 1.; - Rhône, Châtoux (Gdgr herb. ros. n. 17). . . . 2023. C. enemocianis Gdgr

(Virens; rami flor inermes .......... 56

$\therefore$ Purpurea; rami flor aculeati - Isere, Le Sappey (Boullu)

Ped. $7-9$ m. i.; discus valde conicus - Serbia bor., Slanci

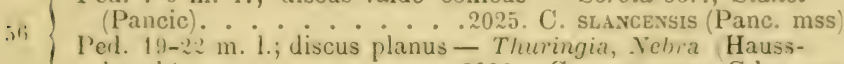
knecht . . . . . 2026. C. CAlycifera Gdgr mss

\section{c. - Osmophyllae Gdgr Essai p. 29.}

Foliolis omnino et argute biserratis, odoratis, ad costam mediam glandulosis, subcoriaceis nervosis; sepalis post anthesim interdum erectis, usque ad colorationem fructus persistentibus, dein caducis; aculeis saepe confornibus, interdum in setas glanduliferas prodeuntibus. - Radix nonnumquam reptans, tunc frutices vel suffrutices humiles graciles ramisque tenuibus praediti.

1 Aeulei conformes . . . . . . . . . . . . . : Aculei dimorphi alii setaceo-glanduliferi alii adunci. . . . 72

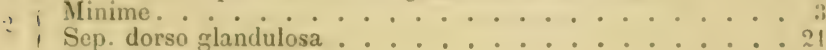

: 1 Styli glabri................. 4

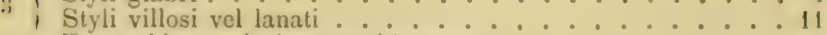

4 Fr. ovoideus vel obovato-oblongus. . . . . . . . 5

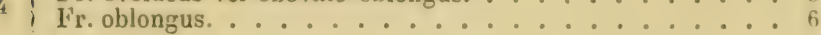




\section{$-156-$}

Rami flor. inermes ; fol. ample obovalo-elliptica - Pyrenaei centr., Campvieil (Bordère). .2027. C. Macromegas Gdgr mss Rami aculeati ; fol. minute elliptica - Hisp. bor., Sierra de Cameros (Zubia). . . . . 2028. C. Apposita Gdgr mss

Discus planus. .............. 7

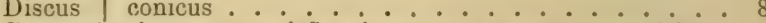

Sep. apice integra; rami flor. inermes - Gard, Bréau (T'uezkiewicz). . . . . . . 2029. C. Majcscula Gdgr mss Sep. apice laciniata ; rami aculeati - Catalaunia, Olot (Vayreda)........ 2030. C. AmeLYotis Gdgr mss

Rami flor.inermes............ . 9

Rami flor aculeati. . . . . . . . . 10

Sep. apice dilatato-laciniata; fol. rotundato-obtusa - Gard, Le Vigan (Tuezk.). . . . 2031. C. orbicularifolia Gdgr mss Sep. apice angustata, integra; fol. obovato-acuta - Gard, Bréau (Tuezkiewicz). . . . . .2032. C. unicolor Gdgr mss

Discus conicus; fol. apice acuta - Pyrenaei, Gèdre (Gdgr herb. ros. n. 214) . . . 2033. C. STEPHANOPHORA Gdgr msS Discus vix conicus; fol. apice subobtusa - Catalaunia, Falgas (Vayreda). . . . . 2034. C. subgLaúcA Gdgr mss

11 Fr. ovatus. . . . . . . . . . . 12

11 Fr, ovoideus vel obovato-oblongus. . . . . . . 13 Fr. oblongus. . . . . . . . . . . . 19 Aculei tenues; discus planus - Htes-Alpes, Chaudun (Burle) Aculei validi; discus sat conicus - Rhône, Charbonnières (Boullu). . . . . . 2036. C. Chabertioides Gdgr mss Tubus laevis. . . . . . . . . . . . . 14 Tubus | hispidus. . . . . . . . . 16 Discus I conicus; fol. basi cordata. . . . . . 15

14 Discus planus; fol. basi subattenuata - Isére, Charancieu (Gdgr herb. ros. n. 192) • . 2037. ('. Adexodoxtos Gdgr mss Ped. 16-18 m. l.; discus conicus - Gard, Aulas (Tuezkiewicz) Ped. 10-12 m. l.; discus paulo conicus - Catalaunia, Falgas (Vayreda). . . . . . . . 2039. C. Lentula Gdgr mss Fol. basi attenuata. . . . . . . . . . 17

16 Fol. basi rotundata - Gallia centr. (Déségl.).2040. C. INcons. . . . . . . . . . . . PICUA (Dés.)

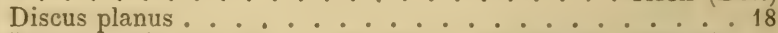

17 Discus paulo conicus - Thuringia, Wallendorf (Haussknecht) . . . . . 2041. C. oxyonon Gdgr mss Fr. ovoideus toto hispidus - Belg., Verviers, etc.2042. C.

Fr. obovato-oblongus inferne hispidus - Anglia, Monmouth Llanthony (Ley). . . . . . 2043. C. AdMIXTA Gdgr mss

Rami flor. aculeati; discus conicus . . . . . . . 20

19 Rami flor. inermes; discus planus - Gironde, St-irédard (Motelay). . . . . . 2044. C. sisxrodites Gdgr mss Ped. hispidi; fol. basi attenuata - Hte-Savoie, Pringy (Puget) Ped. sublaeves; fol basi rotundata - Hte-Savoie, Draillant (Puget). . . . . . 2046. C. LAEvIPES Gdgr mss

Styli glabri vel subhirsuti. . . . . . . . . . 22

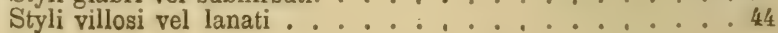


Tubus | hispidus. . . . . . . . . . . . 23

T'ubus lacvissimus. . . . . . . . . . . . . . . . . 27

Discus conicus. . . . . . . . . . . . 24

Discus planus - IIisp. bor., Sierra de Cameros, Logrono (Zubia) . . . . . 2047. C. Reuterrava Gidgr mss

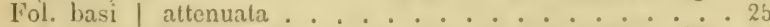

Fol. basi cordata - IIispania, Logrono (Zubia) 2048 . C. DEu-

Fr. basi attenuatus; fol. parva obovata vel obovato-oblonga. 26

Fr. basi rotundatus; fol. ample oblonga - Itte-Savoie,

Pringy (Pugret). . . . 2049. C. Maematodes Gdgr mss

Fol. obtusiuscula, ad costam eglandulosa - Hisp., Murcia Pliego (Codorniu). . . . . 2050. C. Codonnir Gdgr mss

Fol. acuta, ad costam glandulosa - Gallia, Toulouse 'Timbal; C. aspernata. Dés.). . . . 2051. C. Aspratilis (Crép.)

Fr. ovoideus vel obovato-oblongus. . . . . . . . 28

$\mathrm{Fr}_{\mathrm{r}}$ oblongus ........................ 35

Fol. basi rotundata (18 seq. species ad gregem C. Pouzini

Tratt. spectant) . . . . . . . . . 29

Fol. basi attenuata. . . . . . . . . . 31

Discus planus . . . . . . . . . 30

99 Discus conicus - Gard, Le Vigan (Billot exs. n. 3850).2052.

. . . . . . . . . C. Ilomenis Gren.)

Sep. toto glandulosa ; fol. ovata - Rhône, Ville. s. J. (Gdgr

30 herb. ros. n. 561) Sep. in $1 / 4$ inf. laevia; fol. oblonga - Pyrenaei Orient., St Paul de Fenouillet (Debx) . .2054. C. Corbarrexsis (Debeaux)

Discus planus; rami flor. aculeati. . . . . . . . . . 32

Discus conicus; rami flor. saepe inermes - Pyı enaci Or., Amélie-les-Bains (Debeaux). . . .2055. C. Caudata Gdgr mss

Fol. elliptica. . . . . . . . . . . . . 33

Foloblonga. $\ldots 31$

(Ped. 14-17 m. l.; fr. obovato-oblongus apice attenuato-conicus - I'yrenaei Orient., Castelnau Debeaux; Grtwr in Delsx

Mater. Prren. I, p. 19.) . . . . 205f. C. Drmarxir Gdgr Ped. 8-9 m. 1.; fr. oroideo-sublepressus - Pyrenaei Orient., Amélic-les-Bains (Deheaux) .2057. C. cer.tupurlua Gilgr mss Fr. hasi attenuatus olovato-oblongus; perl. 6-9 m. l.-Europa
mer. . . . . 2058. C. Pouzini (Tratt.) Fr. basi rotundatus; ped. $12-14 \mathrm{~m} .1 .-V a r$, Roussargues (Roux) . . . . . . 2059. C. provinglalis Gdgr mss

Fol. basi | attenuata ........... . . 36

Fol. basi rotundata vel cordata . . . . . . . . . 38

(Ped. 12-21 m. l.; fr. basi attenuatus. . . . . . . 37

Ped. 10-11 m. 1.; fr. basi depressus - Pyrenaei Or., Amélieles-Bains (Debeaux). . . 2060. C. Castaveicola Gdgr mss Pet. subglaberrimi ; ped. $12-21 \mathrm{~m}$. 1. - R'ıône, Ville. s. J. (Gdgr herb. ros. n. 189) . . . 2061. C. DiAcaylos Gdgr Pet. sparse hirtelli ; ped. 15-16 m. 1. - Hisp., Murcia, Sierra de Espuna (Canada) . .2062. C. Gumaosna Gdgr mss Fol. parva obovata; rami flor aculeati. . . . . . . 39 Fol. ample oblonga; rami flor. inermes . . . . . . . 43 
Fol: ovato-elliptica vel elliptica; discus | conicus . . . 40

Fol. obovata ; discus planus. . . . . . . . . . 41

Fol. obovata; discus conicus - Hisp. austr., Sierra de Segura (Canada). . . . . 2063. C. orocharis Gdgr ms s

Ped. 11-14 m. l.; fr. basi longe attenuatus - Isère, Verna

(Billot exs. n. 3721 bis). . . . . 2064. C. Manu Gdgr mss

Ped. 14-16 m. l.; fr. basi subattenuatus - Hisp. centr., El Escorial 'Laguna). . . . 2065̃. C. escurialensis Gdgr mss

4 Rami flor. aculeati; fol. apice obtusa. . . . . . 42

Rami saepius inermes; fol apice attenuata - Rhône, Villes.-J. (Gdgr herb. ros. n. 88). . 2066. C. uUsCARIA Gdgr mss

Fr. basi rotundatus apice attenuatus; ped. 7-8 m.1. - Hisp. bor., Sierra de Cameros (Zubia). .2067. C. Durifolia Gdgr mss

42 Fr basi utrinque depressus; ped. 10-12 m. 1. - Gard, Le Vigan (Billot exs. n. 3721 parte).2068. C. TENUISGAPA

............ Gdgr mss

Discus planus; fr. utrinque attenuatus; costa sublaevis Hisp. bor., Sierra de Cameros (Zubia).2069. C. Duriakana

..... Gdgr mss

Discus paulo conicus; fr. basi depressus apice longissime attenuatus; costa valde aculeolata - Hisp., Valence : St-

Felipe de Xativa (Hegelmaier). .2070. C. valentrina Gdgr mss

Fr. rotundatus vel ovatus. .......... 45

Fr. ovoideus. . . . . . . . . 50

Fr. obovato-oblongus vel oblongus. . . . . . . 58

Fr. glaber. . . . . . . . . . 46

45 Fr. hispidus - $\dot{P}_{\text {aris }}$ (Mérat).2071. C. verticillacantra

6 Discus planus. $\ldots \ldots 47$

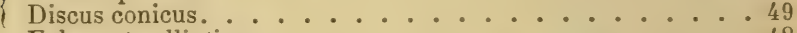

Fol. ovato-elliptica. . . . . . . . 48

47 Fol. oblonga - Pyrenaei, Gèdre (Bordère).2072. C. obJur-

............................ Gdgr mss

Fr. rotundatus; fol. lucida - Podolia, hrzemenie (Besser).

Fr. ovatus; fol. paulo glauca - Hérault, Le Larzac (Loret)

. . . . . . 2074. C. HIstricosa (Crép. mss)

Fr. ovatus; fol. basi rotundata apice subobtusa - Sabaudia

(Dés. herb. ros.n. 20). . . . 2075. C. Haberiana (Pug.)

Fr. ovato-rotundatus; fol. utrinque attenuata - Hte-Savoie,

Habère-Lullin (Puget) . . . . 2076. C. cuneata Gdgr mss

Fr. subglobosus; fol. basi abrupte attenuata apice vix constricta - Sabaudia . . . . 2077. C. FIRMa (Pug.)

Tubus saltem usque ad $1 / 3$ inf. hispidus. . . . . . . 51

Tubus glaber vel basi infima glandulosus . . . . . . 56

51 Fol. basi rotundata vel cordata . . . . . . . . 52

Fol. basi | attenuata .............. 53

Rami flor. aculeati; fol. ovata vel rotundata - Isère, Villard

52 de Lans (Boullu). . . . 2078. C. stictocarpa Gdgr mss Rami inermes; fol. obovato-oblonga - Borussia, Spremberg

(Riess). . . . . . 2079. C. Borussica Gdgr mss

53 , Rami flor inermes. . . . . . . . . 54

53 Rami flor aculeati. . . . . . . . . . 55 
Fol. elliptica - Podolia (Besser); Silesia (W. et G. fl. sil. II Isere, Le Sappey (Boullu).2081. C. Diseus planus; fol. ovata basi attenuata - Belg., Luxembourg 55 (Dum.). . . . . . 2082. C. Dumontieni Gdgr Discus conicus; fol. obovata basi subattenuata - Finistère, Daoulas (Thiébaut) . . . . 2083. C. Legallir Gdgr mss

56 Fol. ample obovato-oblonga vel oblonga. ...... 57 Fol. parva ovato-obtusa - Cher, Turly (Ripart).208\%. C.

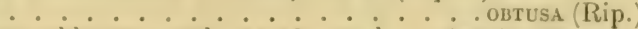
Fol. obovato-oblonga; ped. 16-18 m. 1. - Anglia, York (IVebster). . . . . . . .2085.C. DIDYMopoda Gdgr mss Fol. obovato-oblonga; ped. 9-11 m. 1. - Tyrol, IIall (Gremblich). . . . . . 2086. C. laxistrua Gidgr mss Fol. obovato-oblonga ; ped. 5-6 m. 1. - IIte-Savoie, Pringy (Puget). . . . . . 2087. C. Insolata Gdgr mss Fol. suboblonga; ped. 19-21 m. 1. - Anglia, Cheshire (Harbord) . . . . . 2088. C. vlamoldes Gdgr mss Fol. oblonga; ped. 7-12 m. 1. - Hte-Savoie, IIabère-Poche (Puget). . . . . . . . 2089. C. somnians Gdgr mss

ss , T'ubus glaber .................... 59

Tubus hispidus

.1 , Ped. alii laeves alii glandulosi . . . . . . 660 1 Ped. omnes I glandulosi. . . . . . . . . 62

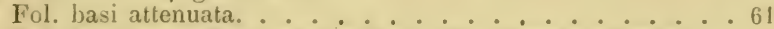
(Fol. basi rotundata; pet. villosi ; fr. obovato-ololongus -

60 Bel!y., Rochefort (Crepin). 2090. C. Psilophrluondes (Crip. mss) Fol. basi rotundata; pet. sparse pilosi; fr. oblongrus - IIteSaroie, Pringy (Puget) . . 2051. C. polyphlaea Gilgr ms: Rami flor. inermes; fol. oblongn-acuminata - rallia, Annecy

61 (Puget). . . . 2092. C. Lancaefonmis Gdgr mss Rami paulo aculeati; fol. acute sbovato-oblonga - Hisp. bor., Sierra de Cameros (Zubia). .2093. C. PRETIOSA Gdgr mss

6.) (Fol hasi attenuala. . . . . . . . . . . . . . 68

Fol. basi rotundata .............. 64 Discus planus; rami flor. aculeati - Anglia med. 209 '.

Discus planus; rami flor. inermes - Pyrenaei centr., Mousca (Bordère). . . . . . 2095. (\% enectisepala Gdgr mas Discus conicus ; rami inermes - Angl., Westmoreland Grasmere (Fisher) . . . . . 2096. C. sancobasis Gdgr mss

64 , Discus paulo conicus. . . . . . . . . . 65

, Discus planus . . . . . . . . . . . . 66 Rami flor. inermes; fr. oblongus - Ite-Savoie, Pringy (Pu-

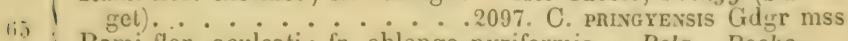
Rami flor. aculeati; fr. oblongo-pyriformis - Belg., Rochefort (Crépin). . . . . 2098. C. macteosa (Crép. mss)

(66 Fr. obovato-oblongus vel suboblongus; sep. caduca. . . . . 67 Fr. oblongus; sep. erecto-persistentia - Suecia; Gall. mer. (Gdgr herb. ros. n. 385) . . . . 2099. C. Achiaril (Billberg) Rami validi; fol obovata. . . . . . . . . 68

67 Rami graciles; fol. ovata - Hisp.; Lusit.; Sicilia (C. hispanica B. et R. non Mill.; C. anticaria Gdgr Essai 1876 et in herb. 1871)..... 2100. C. INconsiderata (Dés. 1873) 
Costa glandulosa; ped. 13-17 m. 1. - Rhône, Gleirẻ (Gdgr herb. ros. n. 177) . . . . 2101. C. Micradena Gdgr Minime ; ped. 9-10 m. 1. - Basses-Alpes, Bouzolières (Proal).

Fo. . . 2102. C. opportuna Gdgr mss

69 Fol. oblongo-lanceolata.

Fol. obovato-acuta - Vaucluse, Mont-Ventoux (Autheman).

Fol. minutissima; ped. $8-9$ m. 1 ; fr. C. ruberrima Gdgr ms

Sierra Nevada: St-Géronimo (Hegelmaier).2104. C. Nevs-

70 . . . . DENsis Gdgr mss

Fol. lata; ped. 14-16 m. 1; fr. ovoideus - Austria, Znaim (Oborny). . . . . 2105. C. Moravica Gdgr mss

Rami et pet. aculeati; fr, sparse aculeati - Isère, Le Sappey

$71\left\{\begin{array}{l}\text { (Verlot) } \\ \text { Rami et pet. inermes; fr. valde aculealus - Hte-Sanoie, }\end{array}\right.$

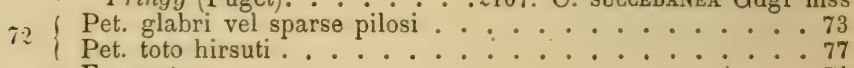

(Fr. ovatus. . . . . . . . . . 74

73 Fr. ovoideus. . . . . . . . . . . . 75

Fr oblongus . . . . . . . . . 76

it Styli lanati - Rhône, Dardilly (Gdgr herb. ros. n. 385) 2108.

t

Styli parce villosi - Anglia. . 2109. C. HaILstoni (Baker)

Fol. late ovato-elliptica apice rotundata - Hte-Savoie. St-

i5 $\left\{\begin{array}{l}\text { Martin (Puget). - - - - 2110. C. LIMITANEA (Crép. mss) } \\ \text { Fol. parva obovato-acuta - Belg., Namur (C. latebrosa Dés.) }\end{array}\right.$

Sep. dorso glandulosa; fr. basi depressus - Rhône, Dardilly

(Chabert; Gdgr herb. ros. n. 391). .2112. C. Chaberti (Dés.)

Minime; fr. basi rotundatus - Hisp. austr., Sierra de Segura

(Canada) . . . . . 2113. C. DIversispina Gdgr mss

Minime; fr. basi longe attenuato-clavatus - Anglia, Wolver-

hampton (Fraser). . . 2114. C. DIMORPHACAytha Gdgr mss

$77\left\{\begin{array}{l}\text { Fr. ovoideus. } \\ \text { Fr. ovatus } \\ \text { Lyon, etc. (Gdgr herb - . } 179 ; C \text {. } .78\end{array}\right.$ Fr. ovatus - Lyon, etc. (Gdgr herb. ros. n. 179; C. lugdunensis Timeroy herb. 1849!). - 2115. C. TIMeroyi (Chabert)

$78\left\{\begin{array}{l}\text { Fol. ovato-rotundata vel subrotundata } \\ \text { Fol. obovato-acuta }- \text { Cher, Trouy (Déségl.).2116. } \dot{\mathrm{C}} \text {. odonto- } 79\end{array}\right.$

(Rami recti; discus subplanus. $\therefore$. 80

79 Rami decumbentes; discus conicus - Cher, Grange-St-Jean (Ripart). . . . . . 2117. C. Decumbens Gdgr mss

( Fr. glaber - Helv. occid. (Billot exs. n. 3377).2118.

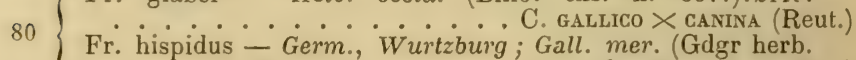
ros. n. 384 et 405$)$. . . . 2119. C. Psilophylla (Rau) 


\section{C. - Trichophiylae Gdgr Essai p. 30. Canineae} pubescentes, collinae et tomentellae Crép. Prim. p. 18.

Foliola plus minus (subtus saltem ad costam mediam) hirsuta, villosa vel tomentosa, saepius simpliciter serrata. Sepala post anthesim reflexa dein caduca (exceptis in quibusdam sect. Solstitiales, extus laevia vel glandulosa, pinnatipartitı. Styii liberi, ultra discum plus minus capitati. Corolla alba rel rarius intense rosea, mediocris, interdum parva tunc albo-ochroleuca. Fructus pulposus. Pedunculi glabri villosi glandulosi vel villoso-pubescentes. Aculei conformes, e basi late dilatata adunci validi. Radix haud reptans.

$\int$ Ped. et sepala eglandulosa; aculei conformes. I. Eupubescentes a. Ped. et sepala | glandulosa; aculei nonnunquam dimorphi.

1. - Eupubescentes Gdgr Essai p. 30. Canineae pubescentes Crép. Prim. p. 18.

Foliola plus minus villosa, ad costam mediam ordinarie eglandulosa saepiusque eglandulosa. Sepala extus plerumque eglandulosa. Pedunculi glabri vel rarius paululum glandulosi. Corolla alba mediocris, vel pallide rosea. Fructus glaber; aculei validi, conformes.

Fol. subtus ad costam mediam tantum paulo villosa. 1. Decalvatae Fol. subtus ad costam normaliter villosa cum pilis raris ad nervos secundarios obducta. ......2. Semi-glabrae Fol. subtus ad nervos tantum villosa . . . 3. Pilosiusculae

1. Fol. subtus ad nervos villosa cum pilis raris hinc inde adhuc obsita. . . . . . . . . 4. Urbicae Fol. subtus toto villosa, supra subhirsuta dein glabra. 5. Pilosae Fol. adulta undique molliter pubescentia fere tomentosa. . . . . . . . 6olstitiales 


\section{1. - Decalvatae Gdgr Essai p. 30.}

Foliolis mediis ramorum fioriferorum subtus ad costam mediam tantum plus minusve villosis, serraturis eorum simplicibus vel compositis.

Fol. omnino biserrata ............ 2

Fol. alia simpliciter alia biserrata. . . . . . . . . 6

Fol. omnino simpliciter serrata.......... 17

Fr. ovoideus vel obovato-oblongus. . . . . . . . . . 3

Fr. oblongus - Rhoune, Amas (Gdgr herb. ros. n. 300).

....... 2120. C. Cosmophylla Gdgr

Fr. ovatus - Rhône, Tassin (Billot exs. n. 3849 parte).2121.

. . . . . . . . C. odontosticta Gdgr mss

Fr. globosus - Liguria or., Mte Ariona (Gennari pl. ligust.

Cent. III, n. 50). . . . . 2122. C. Gennari (Huet)

Pet. molliter pubescentes. . . . . . . . . 4

Pet. sparse pilosi . . . . . . . . . 5

Discus paulo conicus; fr. utrinque valde attenuatus - Anglia,

4 York Boltby (Baker) . . . . 2123. C. Glandluosa Gidgr mss

Discus valde conicus; fr. utrinque vix depressus - Pyrenaei, Perpignan (Debeaux) . . . .2124. C. Oliveri (Debx mss)

( Rami flor. inermes; ped. 4-6 m. 1. - Anglia, York Thirsk (Baker). . . . . . . 2125. C. stylosA Gdgr mss

Rami flor. parce aculeati; ped. 7-8 m. 1. - Anglia, Warwich Shresby (Fraser) . . . . 2126. C. CONIFERA Gdgr mss Ped. villosi .............. 7

Ped. glabri . . . . . . . . 8

Styli sparse hirsuti; discus conicus; fol. ovata - Sabaudia, Salins (Puget). . . . .2127. C. sclerodonta Gdgr mss Styli paulo hirsuti; discus subplanus; fol. oblonga - Belg., Rochefort (Crépin). • . . .2128. C. DECALvata (Crép. mss) Styli villosi; discus planus; fol. obovata - Sabaudia, Salins (Puget) . . . . . . 2129. C. Trichopus Gdgr

Fr. globosus. . . . . . . . . . . 9

Fr. ovoideus. . . . . . . . . . . 12

Fr oblongus . . . . . . . . . 14

○ Fol. ovata vel elliptica basi cordata. . . . . . . 10

Fol. obovata vel oblonga. . . . . . . . . . 11

Pami flor. inermes; pet. aculeati-Pyrenaei centr., Trémouse

10 (Bordère). . . . . 2130. C. Cordata Gdgr mss Rami flor. aculeati; pet. inermes - Pyrenaei centr., Montné (Bordère) . . . . . . 2131. C. spalnsa Gdgr mss

( Styli glabri; pet. aculeati - Scotia, Perth (Drummond).2132.

11 . . . . . . . . . Trichodonta Gdgr mss Styli pubescentes; pet. inermes - Rhône, Francheville (Chabert). . . . . . . 2133. C. Latiflora Gdgr mss 
Styli subglaberrimi; rami flor. saepius inermes. . . . 13 Styli paulo hirsuti; rami aculeati - Pyrenaei, Gèdre (Bordère). . . . . 2134. C. villinaeva Gdgr mss Fr. 8-111 m. latus; costa toto villosa-Pyrenaei, Gedre (Bordère) . . . . . . . 2135. C. stiblaEvis Gder mss Fr. 14-15 m. latus; costa basi tantum villosa - Pyrenaei, Gèdre (Bordère), .2136. C. stblaevis B macnocanpa Gidgr mss

Fol basi rotunda . . . . . . . . . . . 15

Fol. basi attenuata. . . . . . . . . . 16

(Styli villosi; rami flor. aculeati - Hte-Savoie, Pringy (Puget)

Styli glabri; rami flor inermes-Pyrenaei, Arran (Bordere). . . . . . . . 2138. C. pogonata Gdgr mss Ped. 7-8 m. 1.; discus planus - Thuringia, Ettersberg (Haussknecht) . . . 2139. C. inaequeserrata Gdgr mss Ped. 12 m. 1.; diseus planus - Anglia, Cheshire (Harbord). . . . . . . . 2140. C. efFERTA Gdgr mss Ped. 14-15 m. 1.; discus sat conicus. - IIisp., Navarre Larvion (Ruiz) . . . . . 2141. C. Navarnensis Gdgr mss Ped. glabri.. . . . . . . . . . 18 Ped. villosi.. . . . . . . . . . . . 31 Fr. ovato-rotundatus, rotundatus vel globosus. . . . 19 Fr. ovoideus vel obovato-oblongus. . . . . . . 21 Fr. oblongus . . . . . . . . 29 Fr. ovato-ellipticus - Pyrenaei, Gèdre (Bordère) . . 2142. ... . . . . . C. FAlciFera Gdgr mss Styli villosi; fr. rotundatus vel globosus. . . . . . 20 Styli glabri ; fr. ovato-rotundatus - Pyrenaei, Lourdes (Bordère). . . . . . . 2143. C. Acora Gdgr mss Discus planus; rami flor. inermes - Isere, Recoin (Gdgr) Discus conicus; rami flor, aculeati - Ite-Savoie, HabèreLullin (Puget). . . . . 2145. C. LAxula Gdgr mss

21 Styli glabri. . . . . . . . . . . . 22 Styli hirsuti vel lanati.............. 24

$22\left\{\begin{array}{l}\text { Discus planus vel paulo conicus; pet. aculeati. } \\ \text { Discus conicus; pet. inermes - Italia, Modène (Gibelli) }\end{array}\right.$ . . . . . . . 2146. C. Gibellil Gdgr mss Rami flor. aculeati; ped. 10-12 m. 1. - Isère, Charancieu

28 (Gdgr) . . . 2147. C. Muscescexs Gdgr mss Rami for. inermes; ped. 5-6 m. 1. - Var, Aix (Autheman)

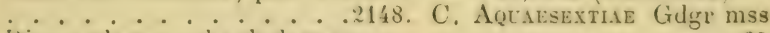

?f f liseus planus vel subplanus ........... . . . Discus conicus, . . . . . . . . . . . 27 Pet. aculeati ; fol. oblonga. . . . . . . . . 26 Pet. aculeati ; fol. parva obovata - Oise, Noyon (Magnier)

$\therefore \quad$. . . 2149. C. Godnoniava Gdgr mss Pet. inermes; fol. ovata - Hte-Savoie, etc. . . . .2150 ............ Hispiuela Rip.) Discus paulo conicus; rami gracillimi - Rhône, Arnas (Gdgr 21 herb. ros. n. 98) validi - Hungaria, Pesth (L. Richter)

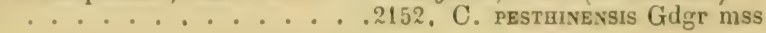


Fol. ovata vel obovato-oblonga; pet. inermes. . . . . . 28 Fol. oblongo-lanceolata; pet. aculeati - Bouches-du-Rhône, Martigues (Autheman). . . . 2153. C. вомвAx Gdgr mss Rami flor. inermes - Podolia austr. (Besser). . . . .2154

28 - : - C. Frondosa (Stev.) Rami flor. aculeati - Hte-Savoie, Thorens (Puget). . . 2155.

C. platystigma Gdgr mss Styli hirsuti ; rami flor. | aculeati . . . . . . . 30

Styli sparse pilosi ; rami flor. aculeati - Hisp., austr. Sierra de Segura (Canada). . . . . 2156. C. Segurae Gdgr mss Styli glabri; rami flor. inermes - Hanovre, Gertenbach (Zabel). . . . . . 2157. C. Acutifolia Gdgr mss

$30\left\{\begin{array}{l}\text { Cor. eciliata; fol. basi subrotundata - Italia or., Faenza } \\ \text { (Caldesi). . . 2158. C. patentiramea Gdgr mss }\end{array}\right.$ Cor. ciliata; fol. basi acuta - Gallia, Riom (Gdgr herb. ros. n. 371). . . . . 2159. C. Crassinervia Gdgr mss

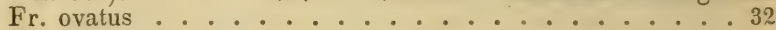

31 Fr. ovoideus vel obovato-oblongus. . . . . . . . 33 Fr. oblongus - Rhône, Arnas (Gdgr herb. ros n. 54).2160. ............ C. CarrieziI Gdgr mss Styli glabri ; ped. 5-6 m. i. - Rhône, Tassin (Cariot). 2161. Styli lantio $\cdot$ Rhône, Pommiers (Gdgr)

Styli glabri .................. 34

Styli hirsuti. . . . . . . . . . . . 36

34 Rami aculeati; fol. parva ovata - Pyrenaei or., Amélie-lesBains (Debeaux) . . . . 2163. C. Nummulariordes Gdgr Ped. 5-6 m. 1. lanati; fol. acute oblonga-Hisp. Santiago de Galicia (Quet). . . . . 2164. C. Galloecica Gdgr mss

Ped. 9-10 m. 1. villosi; fol. obtuse obovata - Italia, Parme Staffora (Gibelli)). . . . . 2165. C. PAPIENSIS Gdgr mss

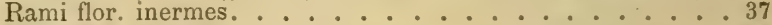

Rami flor aculeati. . . . . . . . 38 Fol. orato-elliptica; fr. basi attenuatus - Bouches-du-Rhóne, $37\left\{\begin{array}{l}\text { Martigues (Autheman). } \cdot \dot{2} \cdot 2166 \text {. C. Actheмani Gelgr mss } \\ \text { Fol. obovato-oblonga; fr. basi depressus - Rhône, Chaponost }\end{array}\right.$ (Chabert) . . . . . . 2167. C. Ficoldes Gdgr mss

( Discus valde conicus; fol. obovata vel oblonga. •. . . . 39

38 Discus subplanus; fol. ovata - Sabaudia, etc.2168. C. GLO................ rata (Dés.)

$39\left\{\begin{array}{l}\text { Styli subcoaliti ; pet. inermes - Sabaudia, Moutiers (Puget). } \\ \text { Styli liberi ; pet. aculeati - Bouches-du-Phone, Martigues }\end{array}\right.$ Styli liberi ; pet. aculeati - Bouches-du-Rhone, Martigues (Autheman). . . . . 2170. C. Gerardiana Gdgr mss

\section{2. - Semi-glabrae Gdgr Essai p. 30.}

Foliolis mediis ramol um floriferorum supra glabris, sub- 
tus vero ad costam mediam villosis, cum pilis raris ad nervos primarios; serraturis interdum compositis.

Fol. omnino simpliciter serrata . . . . . . . . 2

1 Fol. omnia biserrata. . . . . . . . . . 29

I Fol. alia simpliciter alia biserrata. . . . . . . . 32

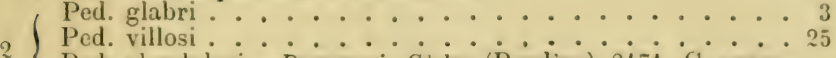

Ped. glandulosi - Pyrenaei, Gedre (Borderc).2171. C. Anexo-

Fr globosus vel rotundatus . . . PoDA Gdgr mss

Fr. ovatus. . . . . . . . . . . . 7

3

Fr. breviter ovoideus. ............ 10

Fr. ovoideus vel obovato-nblongus. . . . . . . 11

Fr. oblongus vel elongatus. . . . . . . . . 17

4 Fol. basi | attenuata. . . . . . . . . 5

( Fol. basi rotundata. . . . . . . . . . . . 6

( Styli villosi ; fol. elliptica ; fr. magnus - Sabaudia (Dés.

5 herb. ros. n. 68). . . 2172. C. spiraerocanpa (Puget)

Styli subglabri; fol. oblonga; fr. parvus - IIibernia, Moira Down (Stewart). . . . . 2173. C. Basaltica Gdgr mss

$6\left\{\begin{array}{l}\text { Ped. 8-9 m. l.; fol. lanceolata - Py/renaei or., Amélie-les- } \\ \text { Bains (Debeaux) . . . 2174. C. LEucustyla Gdgr mss }\end{array}\right.$ Ped. 12-15 m. 1.; fol. acuta - Anglia, York (Webster).2175.

To . C. microsphaena Gdgr mss

7 Fol basi attenuata; rami flor inermes. . . . . . . 8

I Fol. basi rotundata; rami flor. aculeati . . . . . . 9

(Petala eciliata albo-carnea; fr. basi rotundatus - Isère, Recoin (Gdgr) . . . . . 2176. C. conTonTa Gdgr mss

Petala ciliata amoene rosea; fr. basi depressus - Puy-deDôme, Savy (Gdgr herb. ros. n. 136). 2177. C. haemantia

..................... Gdgr mss

Styli Lanati; discus planus - Gallia, Gap (Burle).2178.

9 . . . . . . tomostigma Gdgr mss Styli giabri; discus paulo conicus - Hibernia, Belfast New Forge (Stewart). . . . 2179. C. Templetoniana Gdgr mss Styli lanati ; fol. basi attenuata - Isere, Charancieu (Gdgr herb. ros. n. 109). . . . 2180. C. PAppostrla Gdgr mss Styli paulo hirsuti; fol. basi rotundata - Anglia, York (Webster). . . . . 2181. C. pallidula Gdgr mss Styli glabri; fol. basi cuneata - Scotia, Perth Dunkeld (Drummond). . . . . 2182. S. S'tuantiana Gdgr mss

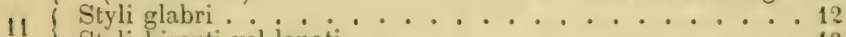
Styli hirsuti vel lanati ............ . . . 13 Ped. 8-10 m. I.; fol. obovato-elliptica - Gironde, Montfer-

12 rand (Motelay). . . . 2183. C. Fobrancm Gdgr mss Ped. 1'1-16 m. 1.; fol. oblonga - Isère, Charancieu (Gdgr herb. ros. n. 20) . . . . 2181. C. xudicatlis Gdgr mss

13 Rami flor inermes. . . . . . . . . 14

13 I Rami flor aculcati, ................. 15 

$\left\{\begin{array}{l}\text { Ped. 5-7 m. 1.; fol. obovata basi rotundata - Austr. inf., } \\ \text { Münchendorf (Wiesbaur). - . 2185. C. AUstriacA Gdgr mss }\end{array}\right.$ Ped. 12-14 m. 1.; fol oblonga basi attenuata - Italia or., Faenza (Caldesi) . . . 2186. C. Macrophylda Gdgr mss

(Fol. basi / attenuata; discus planus . . . . . 16 Fol. basi rotundata; discuś paulo conicus - Lyon (Gdgr herb. ros. n. 398) … 2187. C. Montsuiana (Chabert mss)

Ped. $11-13 \mathrm{~m}$. l.; fol obovato-oblonga - Pyrenaei or., Oletteles-Bains (Debeaux). . . . 2188. C. crispatula Gdgr mss Ped. 7-11 m. 1.; fol. obovato-elliptica - Armenia, Elisabethpol (Hohenacker Un. itin. 1835 sub nom. C. leucanthae). . . . 2189. C. elisabethopolensis Gdgr mss

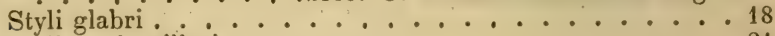

17 Styli paulo villosi ............. 21 Styli dense hirsuti. . . . . . . . . . . . 23

18 Fol. basi rotundata. . . . . . . . 19 Fol. basi l attenuata . . . . . . . . 20

$19\left\{\begin{array}{l}\text { Fr. utrinque valde constrictus; fol. obovato-oblonga-Rhône, } \\ \text { Ecully (Chabert). . C }\end{array}\right.$ Fr. fusiformis; fol. ovato-elliptica - Rhône, Charbonnières (Chabert). . . . . 2191. C. Fusiformis Gdgr mss Discus planus; fol. apice sensim attenuata - Anglia, York

20 Scanton (Baker). • . . 2192. C. Dumetonordes (Crép. mss) Discus conicus; fol. apice acuminata - Rossia, Riga (Bienert). . . . . . 2193. C. Livonica Gdgr mss

( Ped.12-13 m. 1.; fr. utrinque attenuatus. . . . . 22 Ped. 5-10 m. l.; utrinque depressus - Hte-Savoie, HabèreLullin (Puget). . . . . 2194. C. Leucoxoma Gdgr mss Rami flor. inermes; fol. basi rotundata - Oise, Noyon (Ma-

22 gnier). . 2195. C. AMPHoricarpa Gdgr mss Rami flor aculeati; fol. basi cuneata - Anglia, York (Webster). . . . . 2196. C. Davisiana Gdgr mss Fr. apice valde strangulatus; ped. $5-7 \mathrm{~m}$. 1. . . . . 24 Fr. apice valde strangulatus; ped. 17-20 m. 1. - Thuringia,

23 Haardorf (Haussknecht). - 2197. C. RHYNCHOPHORA Gdgr mss Fr. apice attenuatus; ped. 8-11 m. l. Hte-Savoie, Mont-Sion (Puget) . . . . 2198. C. sp.rsipila Gdgr mss Fol. et fr. basi rotundata - Oise, Noyon (Magnier). 2199. $24\left\{\begin{array}{l}\text { Fol. et fr. basi attenuata - Aisne, St-Quentin (Magnier).2200. } \\ \text { St }\end{array}\right.$

Fr o. C. veromanduorum Gdgr mss

(Fr. ovoideus vel obovato-oblongus. . . . . . 26

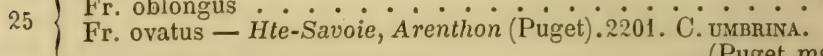
.................... (Puget mss)

Styli villosi; discus conicus. . . . . 27

26 Styli subglabri; discus planus - Rhône, Arnas (Gdgr).2202.

$27\left\{\begin{array}{l}\text { Pet. tomentosi subinermes - Sabaudia, etc. 2203. C. SEmi- } \\ \text { Pet. sparse pilosi aculeati - Bouches-du-Rhóne, Martigues }\end{array}\right.$ (Autheman). . . . . 220t. C. Havryasa Gdgr mss 
Ped. 14-16 m. 1.; fol. obovato-elliptica apice subattenuata -

28 Rhone, Dardilly (Chabert). . .2205. C. IhLigata Gdgr mss Ped. $10-11$ m. 1.; fol. oblongo-lanceolata - Rossia, Molilew (Laupmann) . . . . . 2206. C. nossica Gidgr mss

Fr. rotundatus vel globosus. . . . . . . . . . 30

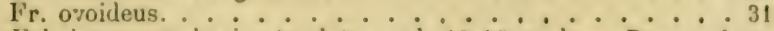
Fol. late ovata basi rotundata; ped. $13-17 \mathrm{~m}$. 1. - Pyrenaei, Perpignan (Debeaux) . . . . 2207. C. Anthopmona Gdgr mss

Fol. anguste oblonga basi attenuata; ped. 9-12 m. 1. Rhöne, Tassin (Billot exs. n. 3849 parle). 2208. C. ningu........... Donta Gdgr mss

Discus planus; rami flor. aculeati - Rhone, Oullins (Boullu).

....... 2209. C. AfFinita (Puget mss) Discus conicus; rami flor. inermes - Gard, Le Vigan (Tuezkiewicz). . . . . 2210. C. Teezkiewiczir Gdgr mss

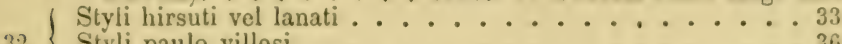

32 Styli paulo villosi....................... 36

Styli glabri............... . . 38

33 i Discus planus. . . . . . . . . . . . . . 34

Discus conicus. $\ldots 35$

Fr. oblongus; rami flor. inermes - Podolia mer. (Besser).

Fr. globosus; rami flor. aculeati — Rhône, Francheville (Chabert) . . . . 2212. C. spHaERocalyx Gdgr mss

Tami flor. aculeati; ped. 4-10 m. I villosissimi - Rhóne,

35 Chaponost (Chabert). . . . 2213. C. nobustion Gdgr mss Rami flor. inermes; ped. 13-15 m. 1. glabri - Hung., Ofen

(L. Richter). . . . . . 2214. C. Riceterir Gdgr mss

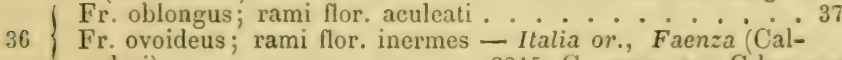
desi). . . . . . 2215. C. Mrsocarpa Gdgr mss Discus planus; ped. glabri $13-15$ m. l. Italia or., Faenza

(Caldesi) . . . 2216. C. GL.1Brescexs Gdgr mss

37 Discus valde conicus; ped. villosi 7-9 m. 1. - Bouchesclu-Rhöre, Le Réaltor (Autheman).2217. C. Derbesiani

. . . . . . . . . . . . Gdgr mss

Fr. ovoideus vel oblongus; rami flor aculeati $\ldots 39$

Fr. obovato-oblongus; rami flor. inermes - Tyrol, Trente

(Gelmi). . . . . 2218. C. inaequalis Gdgr miss

Fr. rotundatus; rami flor. inermes - Anglia, York (Vebster).

. . . . . 2219. C. pellita Gdgr mss

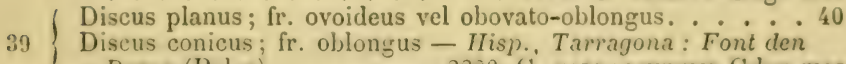
Dorca (Bolos).... . 2220. C. Tannacusexsis Gidgr mss Serraturae plerumque compositae; pel. 10-11 m. 1. - Rhine, Arnas (Gdgr). . . . . . 2221. C. subcomposita Gdgr mss Serraturae aefue compositae et simplices; perl. 1'í-17 m. 1. - Pihöne, Armas (Gdyr herb. ros. n, 16j).2222. C. TRaxi-

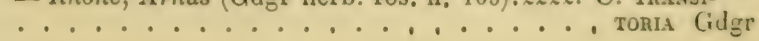




\section{3. - Pilosiusculae Gdgr Essai, p. 30.}

Foliolis medianis ramorum floriferorum supra glabris subtus tantum ad nervos primarios normaliter pubescentibus, caeterum glaberrimis; serraturis plerumque omnino simplicibus.

Styli glabri vel vix adsperse pilosi

Styli paulo hirsuti.............. 19

Styli villosi vel lanati . . . . . . . . . 51

Fr. ovatus. . . . . . . . . . . . 3

Fr. obovato-oblongus vel oblongus . . . . . . . 4

Fr. ovoideus; sep. valde dentato-glandulosa - Pedem.,

2 Vallées Vaudoises (Rostan).2223. C. Adenostephana Gdgr mss

Fr. ample ovoideus; sep. eglandulosa - Pyrenaei centr.,

Mourère (Bordère) . . . . .2224. C. latipetala Gdgr mss

Fr. rotundatus - Anglia, Hereford Clom-y-on (Ley).2225.

................ Anglorum Gdgr mss

Fol. utrinque sensim rotundata; pet. inf. inermes - Isère,

3 Charancieu (Gdgr herb. n. 155).2226. C. Pteroidea Gdgr mss

Fol. utrinque attenuata; pet. omnes aculeati - Puy-de-

Dôme, Montbaubier (Dubosclard).2227. C. Foligera Gdgr mss

4 Ped. villosi ............... 5

Ped. glabri............... 6

Discus valde conicus; fol. ovato-elliptica - Rhône, Charbon-

5 nières (Chabert). . . . 2228. C. PILOSELla Gdgr mss

Discus planus; fol. obovato-oblonga - Helv., Zurich Scline-

belhom (Lehmann). . . . 2229. C. orodexdnon Gdgr mss

6 Fr. obovato-oblongus vel suboblongus ........7

6 Fr. oblongus ..................... 14

7 Discus conicus. . . . . . . . . . . . 8

7 Discus planus vel subconicus. . . . . . 10

(Fol. obovato-oblonga vel oblonga basi attenuata . . . 9 9

8 Fol. obovata basi rotundata - Catalaunia, Maza (Vayreda). . . . . . . . . 2230. C. Costae Gdgr mss ( Ped. 9-13 m. l.; pet. omnes aculeati - Hte-Savoie, Mt-Sion

9 (Puget)....... 2231. C. RACEMulosa Gdgr mss Perl. 14-16 m. 1.; pet. sup. inermes - Basses-Alpes, Bouzolières (Proal). . . . . . . 2232. C. Proalin Gdgr mss

10 Fol. basi | attenuata. $\therefore 11$ Fol. basi rotundata - Rhône, Amas (Gdgr).2233. C. самpтo-

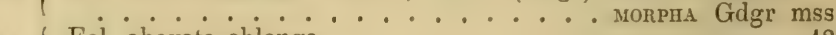

11 Fol. obovato-oblonga. $\ldots \ldots \ldots 12$

Fol. oblonga ................... 13 
Dentes plerumque compositae; pet. aculeati-Loire, Mt-Pilat

(Gdgr). . . . . . . 2234. C. scmisennata Grlgr mss

Dentes simplices; pet. inermes - Ilte-saroie, Habere-I'oche (Puget). . . . 2235. C. Bnevepubess Gdgr mss

Rami flor. incrmes; ped. 11-16 m. 1. - Sabaudia, etc. (Gdgr herb. ros. n. 503) . . 2236. C. PLATYPHYLlordes (Chabert)

Ram flor. aculeati; ped. 15-20 m. 1. - Isère, Charancieu (Gdgr) . . . . . . 2237. C. FIlopes Gdgr mss

14 Discus planus . . . . . . . . . . 14

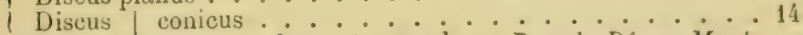

Fol. basi acuta ; ped. $13-16$ m. 1. - Puy-de-Dôme, Mont-

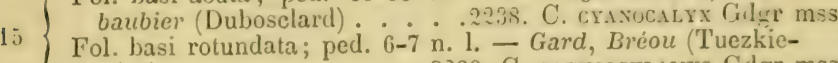
wicz) . 2239. C. CALYCOCHLAMYs Gdgr mss Fol. oblonga ................. 17 Fol. obovato-oblonga basi attenuata biserrata - Italia or., Faenza (Caldesi). . . 2210. C. Glaxdulosella Gdgr mss

Fol. elliptica basi rotundata-simpliciter serrata - Hisp. bor., Sierra de Cameros (Zubia). . . .2241. C. elliptica Gdgr mss

Serraturae omnes simplices. 18

Serraturae inf. compositae - Italia or., Brisighella (Caldesi).

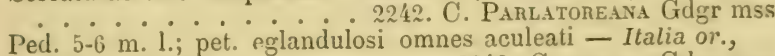
mnes aculeati - Italia or., Faenza (Caldesi). . . . . 2243. C. Mrvuta Gdgr mss

Ped. 6-9 m. 1.; pet. paulo glandulosi, sup. inermes - Anglia, York Ijoltby (Baker). . .2244. C. Decurrens Gdgr mss

Ped. 13-15 m. 1.; pet. eglandulosi subinermes - Cantal, Le Lioran (F. IIériljaud). . . . .2215. C. c.1rtalica Gilgr mss

Fr, globosus. . . . . . . . . . 20

19 Fr. ovatus vel ovato-rotundatus ........22

Fr. ovoideus vel obovato-suboblongus. . . . . . 26

Fr, oblongus. . . . . . . . . . . 41

Rami flor. aculeati; fol basi rotundata . . . . . . 21 $20\left\{\begin{array}{l}\text { Rami flor. inermes; fol. basi truncata - Hte-Savoie, Leschaux } \\ \text { (Bouvier). . . . . 2246. C. obesa Gdgr mss }\end{array}\right.$ Pet. inermes; fol. oblonga - Anglia, Hereford Aymestry (Ley). . . . . . 2247. C. Heniglaucos Gdgr mss

21 Pet. aculeati; fol. oblonga - Anglia, Cheshire (Harbord). Pet. aculeati; fol. obovata - Anglia, Plymoulh (Briggs.)

Fol basi attenuata ............... 23

Fol. basi rotundata . . . . . . . . . 24

Ped. glabri; dentes subbiserratae - Gallia, Thonon (Puget).

Ped. paulo villosi; fol simpliciter serrata - Isère, Charancieu (Gdgr herb. ros. n. 221) . . 2251. C. Illecebrosa Gdgr mss Styli elongati ; fol. apice attenuata; fr. ovatus . . . . . 25

21 Styli breves; fol. obtusa ; fr. subrotundatus - Hisp., Burgos : Soncillo Estebanez). . . . 2252. C. Bungontm Gdgr mss Ped. 12-15 m. 1.; fol. obovata-elliptica - Isère, Charancieu - Isère, Charancieu (Gdgr herb. ros. n. 48). . . . .2254. C. Uwania Gdgr mss 
Fol. ovata vel elliptica. . . . . . . 27

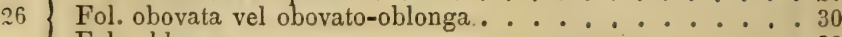

Fol. oblonga ................ . . 39

27 ( Ped. villosi. . . . . . . . . 28

Ped. glabri . . . . . . . . . . . . . 29

28 Discus planus; fol. paulo biserrata- Rhône, Tassin (Boullu).

28 Discus conicus; fol. simpliciter serrata - Rhône, Ecully (Chabert) . . . . . 2256. C. BLEPHAROPODA Gdgr mss Ped.15-17 m. I.; pet. omnes aculeati - Italia, Modène (Gibelli). . . . . . . 2257. C. Mutinensis Gdgr mss

Ped.11-12 m. 1. ; pet. sup. inermes - Tyrol, Hall (Gremblich) . . . . . . 2258. C. Leyboldiana Gdgr mss Ped. 8-12 m. 1.; pet. omnes inermes - Hte-Savoie, Pringy (Puget). . . . . . 2259. C. Leptophlaea Gdgr mss Fol. inaeque serrata; ped. glabri . . . . . . . 31

30 Fol, omnino simpliciter serrata . . . . . . . 32 Fol. omnia biserrata; ped. villosi - Tyrol, Trier (Ilse).

. . . . 2260. C. REMOta Gdgr mss Pet. aculeati ; fol. basi cordata - Gard, Le Vigan (Tuezkie-

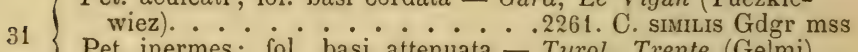
Pet. inermes; fol. basi attenuata - Tyrol, Trente (Gelmi)

32 Fol basi rotundata $\ldots$ 2262. C. DIVERsidens Gdgr mss

Fol. basi attenuata. . . . . . . 34

Pet. omnes aculeati; ped. 11-15 m. i. - Rhône, Arnas (Gdgr

33 herb. ros. n. 154). . . . 2263. C. SAPINI Gdgr mss Pet. inf. inermes; ped. 15-20 m. 1. - Isere Charancieu (Gdgr herb. ros. n. 199). . 2264. C. PEduncularis Gdgr mss

( Rami flor aculeati. . . . . . . . . 35

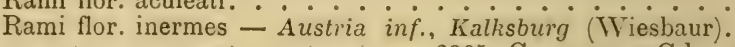
. . . . . . 2265. C. ARcUata Gdgr mss

35 Discus subplanus . . . . . . . 36 Discus conicus - Rhône, Ecully (Chabert). 2266.C. LAxepilosa

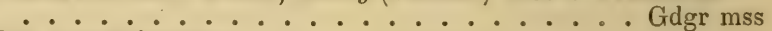
Pet. omnes inermes . . . . . . . 37

36 Pet. omnes aculeati . . . . 38 Pet. inf. inermes - Rhône, Dardilly (Chabert).2267. C. Lovata . . . . . . . . . . . Gdgr mss Cor. subciliata; ped. 10-13 m. 1. Rhône, Villefranche (Gdgr

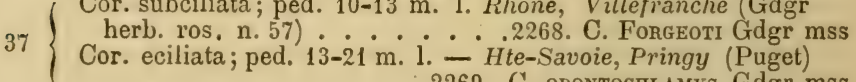

* . 2269. C. odontochlamys Gdgr mss Ped. 8-11 m. i.; fol. subobtusa - Helv., Fribourg: Cases

38 d'Allières (Favrat). . . . 2270. C. Friburgensis Gdgr mss Ped. 14-16 m. 1, fol. apice acuta - Austria inf., Kalksburg (Wiesbaur). . . 2271. C. Neilreichiana Gdgr mss

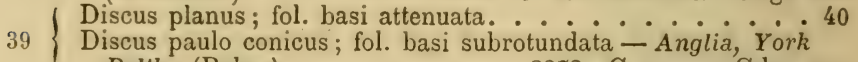
Boltby (Baker). ...... 2272. C. ANGLIGAGdgr mss 


\section{$-171-$}

Pet. omnes aculeati; ped. 12-15 m. 1. - Hisp., Logrono (Zubia) ....... 2273. C. menessis Gdgr mss Pet. inf. inermes; ped. 10-15 m. 1. - Rhöe, Arnas (Gdgr herb. ros. n. 183). . . - . 2274. C. TozzA Gdgr Pet. omnes inermes; ped. 9-10 m. 1. - Rossia mer., Manytsch (Laupmann) . . . 2275. C. cuensonensis Gdgr mss Serraturae aliae simplices aliae compositae. . . . . 42

Serraturae omnes simplices. . . . . . . . . . 44

Fol. basi rotundata. . . . . . . . . . 43

4. Fol. basi attenuata - Gard, Le Vigan (Tuezkiewicz). 2276. C.

. . schisticola Gdgr mss

Pet. glandulosi ; dentes plerumque simplices - Italia or., $43\left\{\begin{array}{l}\text { Pesaro (Caldesi) d - - . . 2277. C. Apriatica Gdgr mss } \\ \text { Pet. eglandulosi ; dentes plerumque compositae - Dania, }\end{array}\right.$ IIornalet Marh'(Zahrtmann) . . . 2278. C. DANica Gdgr mss

$44\left\{\begin{array}{l}\text { Fol. oblonga vel obovato-oblonga } \ldots \ldots \\ \text { Fol. obovata vel } \quad \ldots 46\end{array}\right.$

Pet. eglandulosi ; ped. 20-22 m.1. - Italia or, Faenza (Cal-

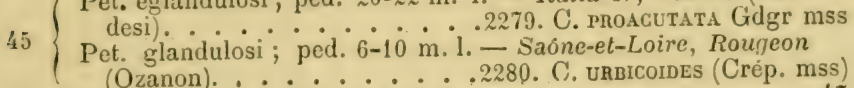
(Ozanon). ...... 2280. C. urbicoides (Crép. mss) Ped. villosi ; fol. basi rotundata. ........ 47

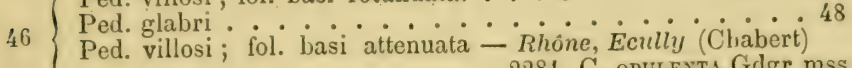

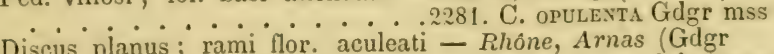

Discus planus ; rami flor. aculeati - Rhône, Arnas (Gdgr

47 herb. ros. n. 130) . . . 2282. C. venix Gdgr mss Discus conicus; rami flor. inermes - Gall., Thonon (Dés. herb. ros. n. 66) . . . 2283. C. ramealis (Puget mss)

48 Fol. basi rotundata. . . . . . . . 49

Fol. basi attenuata. . . . . . . . . . 50

Rami flor. inermes; discus planus - Istria, MI-Maggiore

49 (Freyn). . . . 2284. C. FreynII (Tommasini mss) Rami aculeati; discus conicus - Austria inf., Kulksburg (Viesbaur). . . . 2285. C. ovatipetala Gdgr mss Rami flor. et pet. inermes - Hisp. bor., Sierra de Cameros

50 (Zubia). . . . 2286. C. Anoenipetala Gdgr mss Rami flor. et pet. aculeati - Aragonia austr., Sierra de Mosqueruela (Martin) . . . . .2287. C. IBEnonUm Gdgr mss

Fr. globosus ............. . . 52

51 Fr. ovato-rotundatus vel ovatus......... 5 . . 5 i

Fr. subovoideus. - vel oblongus . . . . . . . . 58

Fr. obovato-oblongus vel oblongus

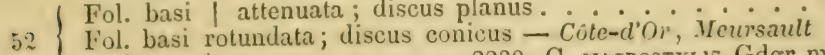

(Ozanon) ......2288. C. MAcrostruls Gdgr mss Pet. aculeati; fol. ovata - Austria, Zypsen (Mauksch).2289.

53 Pet. subinermes; fol o. - . . C. Mckscrm (Kit.) herb. ros. n. 271). . . . . . .2290. C. spicant Gdgr Fol. simpliciter serrata ; ped. glabri. . . . . . . 55 Fol. simpliciter serrata; ped. villosi - Belg., Rochefort

if (Crépin). . . . . 2291. C. Rupefontiana (Crép. mss) Fol. subbiserrata - Hte-Savoie, Leschaux (Bouvier).2292. C. 
Discus planus; fol obovata. . . . . . . . 56

55 Discus conicus; fol. ovata - Rhône, Arnas (Gdgr herb. ros. n. 226). . . . . 2293. C. Acrocomata Gdgr

(Sep. persistentia foliacea; pet. inf. inermes - Tyrol, Valle di Fiemme (Gelmi) . . . 2294. C. AMoexiconora Gdgr mss Sep. caduca angustata; pet. omnes aculeati - Hte-Savoie, Habère-Lullin (Puget). . . 2295. C. Tonertulosa Gdgr mss

Fol. omnia simpliciter serrata - Hte-Savoie, Habère-Poche (Puget). . . . . 2296. C. Compacta Gdgr mss Fol. alia simpliciter serrata alia necne - Basses-Alpes, Bouzolières (Proal). . . . . . .2297. C. LENIS Gdgr mss

Fol. omnia biserrata - Rhône, Francheville (Chabert). 2298. . . . . . . . . . C. odontophora Gdgr mss Ped. villosi vel paulo glandulosi. . . . . . . . 59

58 Ped. glabri . . . . . . . . . . 64

Fol. simpliciter serrata. . . . . . . . 60

59. Fol. inf. biserrata - Pyrenaei, Gèdre (Bordère).2299. C. . . . . . . . . . supranuda Gdgr mss Rami flor aculeati. . . . . . . . . 61

60 Rami flor. inermes - Pyrenaei cent., Arrens (Bordère). 2300. ......... C. miriranea Gdgr mss

61 ( Discus planus. . . . . . . . . 62

Discus / conicus . . . . . . . . . . 63

Tubus basi glaber; ped. 10-13 m. 1. - Rhône, Arnas (Gdgr

62 herb. ros. n. 240) . . 2301. C. cINEREosula Gdgr mss

Tubus basi hirsutus; ped. 16-20 m. 1. - Hte-Savoie, HabèrePoche (Puget). . . . . 2302. C. convergens Gdgr mss

Rami virentes; ped. 11-13 m. 1. - Suecia, Scania Sillarod (Tullberg) . . . . . . . 2303. C. OPERTA Gdgr msS

Rami violaceo-glauci; ped. 5-10 m. 1. - Hte-Savoie, HabèrePoche (Puget). . . . . 2304. C. violascens (Puget mss)

Fol. alia simpliciter serrata alia necne. . . . . . . 65

64 Fol. omnia biserrata. . . . . . . . 66 66

Fol. omnia simpliciter serrata. . . . . . . . . 69

Rami flor. inermes; fol. basi rotundata - Austria inf.,

65 Kalksburg (Wiesbaur). . . 2305. C. DichtLII Gdgr mss

Rami flor. aculeati ; fol. basi attenuata - Dania, Helleboek (Lundquist). . . . . 2306. C. Langeana Gdgr mss

Fol. basi rotundata. . . . . . 67

Fol. basi attenuata. . . . . . . . . . 68

Ped. 8-10 m. 1.; fol. ovata - Bohemia, Kuchelbad (Opiz).

Ped

Ped. 17-19 m. 1.; fol. oblonga - Italia or., Faenza (Caldesi)

.......... C. Calundescens Gdgr mss

Rami flor. inermes; pet. villosi eglandulosi - Pyrenaei or., St Paul de Fenouillet (Debeaux).2309. C. INFragladca Sicilia, Mirtô (Tod. fl. sicula exs. n. 1082 ex parte).2310. . . . . . . . . . . C. Todaror Gdgr mss

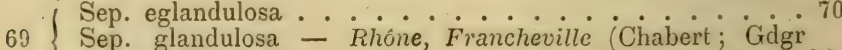
Dec. pl. nov. III, p. 20). . . . 2311. C. CARDIopHora Gdgr 
Fol. ovata elliptica vel obovato-elliptica. . . . . . . 71

Fol. oblonga. . . . . . . . . . 73

Fol. obovato-ohlonga - Anglia, Surrey Barnes (Groves 2312

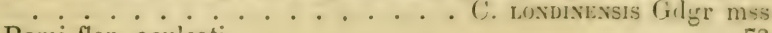

( Rami flor. aculeati. . . . . . . . . . . 72

Rami flor. inermes - Hte-Savoie, Pringy (Puget).2313. C. . . . . . . . . . . . LEucotricha Gdgr mss

72 (Puget). $\begin{aligned} & \text { Discus planus; fol. basi attenuata - Ite-Savoie, Pringy } \\ & \text { (Pu }\end{aligned}$

) Discus conicus; fol. basi rotundata - IIte-Savoie, Pringy

(Puget). . . . . . . 2315. C. Rigididenta Gdgr mss

(Pet. haud aut vix glandulosi; fol. basi rotundata. . . . . 74

73 Pet. glandulosissimi; fol. basi paulo altenuata - Pedem., Pinerolo (Rostan). . . . . 2316. C. Rostani Gdgr mss

(Fr. basi depressus ; pet. subinermes - Rhöne, Arnas (Gdgr)

(Gdgr herb. ros. n. 366). . 2318. C. sceistocarra Gdgr mss

\section{4. - Urbicae Gdgr Essai p. 31.}

Foliolis medüs ramorum floriferorum supra glabris, subtus ad nervos pubescentibus, cum pilis raris inter nerros hine inde sparsis ; serraturis simplicibus.

1 Styli glabri vel sparse pilosuli . . . . . . . . . 2

Styli hirsuti vel lanati . . . . . . . . . 33

f Ped. villosi . . . . . . . . . . . . 3

2 Ped glabri ................ 7

3 Fr. ovoideus. . . . . . . . . . . 4

Fr. oblongus ...................... 5

Fol. ovata basi rolundata ; pet. inermes - Pyrenaei (Bordère); Rhone, Alix (Gdgr herb). ros. n. 336).2319. C. Bon-

4 . . . . . . . . . DERI Gdgr

Fol. ohovato-oblonga basi attenuata; pet. aculeati - Rhöne,

Chaponost (Boullu). . . . 2320. C. Degurtata Gdgr mss

( Ped. 6-12 m. 1.; rami flor inermes. . . . . . . . . 6

5 Ped. 14-18 m. l.; rami flor. aculeati - Rhône, Arnas (Gdgr herb. ros. n. 7) . . . . 2321. C. ciscixisata Gdgr mss

Discus planus; pet. inermes - Rhône, Arnas (Gdgr herb.

(i) ros. n. 148). . . . . 2322. C. Eunyale Gdgr mss

Diseus conicus; pet. aculeati-IIte-Saroie, Annecy (Bouvier)

. . . . . . . . 2323. C. Latifolia Gdgr mss

7 Fol. basi rotundata. ........... 8

Fol basi attenuata. . . . . . . . 18 
Fr. ovoideus vel obovato-oblongus. . . . . . . . 9

Froblongus,

Fr. ovatus - Hte-Savoie, Pringy (Puget).2324. C. SILvu-

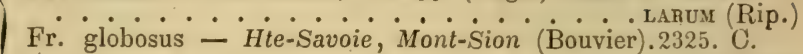

re-Savoie, Mont-Sion (Bouvier).2325. C.

$9\left\{\begin{array}{l}\text { Rami flor aculeati } \\ \text { Rami flor inermes - Rhône, Arnas (Gdgr), 2326. C. DEnTI- }\end{array}\right.$

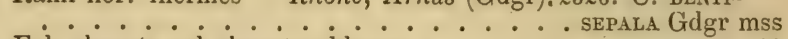

Fol. obovata vel obovato-oblonga . . . . 11

$10\{$ Fol. oblonga - Pyrenaei, Gèdre (Bordère).2327. C. Alligata

Fol. ovata - Anglia. $\therefore \therefore$ 2328. C. Forsteri (Sm.)

11 Pet. inf. inermes. $\therefore \ldots \ldots$. . . . .

11 Pet omnes aculeati . . . . . . . . . 13

Ped. 7-10 m. 1.; discus paulo conicus - Rhône, Montmelas

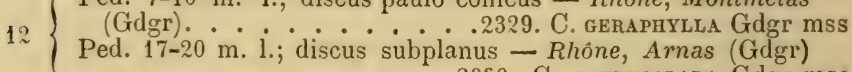

. . . . 2330. C. вотryoclada Gdgr mss

$13\{$ Discus planus vel paulo conicus; fol obovata. -14 Discus conicus; fol. obovato-oblonga - Hte-Savoie, Pringy (Puget). . . . . . 2331. C. sconodonia Gdgr mss Ped. 8-11 m. i.; discus paulo conicus - Hte-Savoie, St-

14 Martin (Puget) ...... 2332. C. PAupera Gdgr mss Ped. 15-18 m. l.; discus planus - Rhône, Arnas (Gdgr).2333

Fol. obovato-oblonga..... . . Pallidipetala Gdgr mss

15 Fol oblonga. . . . . . . . . 17

Fr. utrinque depressus ; ped. 9-10 m. 1. - Italia or., Faenza

16 (Caldesi) . . . 2334. C. Notarisiana Gdgr mss

Fr. utrinque attenuatus; ped. 14-16 m. 1. - Italia, Selva Pisana (Savi) . . . . . 2335. C. Errusca Gdgr mss

$17\left\{\begin{array}{l}\text { Ped. 7-8 m. 1.; fr. parvus utrinque attenuatus - Gard, Bréau } \\ \text { (Tuezkiewicz). . . . . 2336. C. Elevata Gdgr ms }\end{array}\right.$

17 Ped. 8-10 m. l.; fr. magnus basi longe decurrens - Helv., La Sarraz (Favrai). ....2337. C. RIGIDIFolia Gdgr mss

$18\left\{\begin{array}{l}\text { Fr. rotundatns vel globosus } \\ \text { Fr. ovoideus vel obovato-oblongus } \ldots \ldots \\ \text { Fr. oblongus }\end{array}\right.$

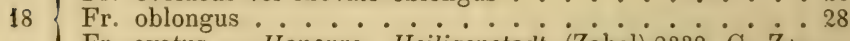

Fr. ovatus - Hanovre, Heiligenstadt (Zabel).2338. C. $\dot{\mathrm{Z}}_{\mathrm{A}-}$

. . . . . . BELII Gdgr mss Ped. 5-7 m. l.; fol. obovata - Pyrenaei, Biarritz (Bordère) $19\left\{\begin{array}{l}\text { Ped. } 10-15 \text { m. 1.; fol. oblonga - Isère, Paladru (Gdgr) } \\ \text { Pares }\end{array}\right.$ . . . 2340. C. TERRoRIS Gdgr mss

20 Fol inf. biserrata . . . . . . . 21

Fol. omnia simpliciter serrata . . . . * 22

Discus sat conicus; fol. obovata - Hungaria, Bude (L. Rich.

21 ter). . . . 2341. C. Sadleriana Gdgr mss Discus planus; fol. oblonga - Austria inf., Kalksburg (Wiesbaur) ........ 2342. C. Flexiramea Gdgr mss

22 Pet. inermes .................... 23

Pet. aculeati. . . . . . . . . . . 26 
Discus I conicus; fol. olovata vel obovato-oblonga. . 24 Discus planus; fol. oblonga - Pyrenaei, Gèdre (Bordère). Fol. obovato-oblonga - . . 2343. C. Mnutula údgr mss Fol. obovata ; sep. apice dentata; ped. $12-15 \mathrm{~m}$. 1. - Austria inf., Kalhsburg (Wiesbaur). . .2344. C. SEPIUM Gdgr mss

Fol. obovato-elliptica; sep. apice interra; ped. $9-12 \mathrm{~m} .1$. - Hte-Savoie, St Martin (Puget). .2315. C. PAllidevinexy

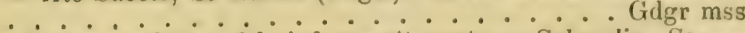
Fr. basi rotundatus; fol. inferne attenuata - Sabaudia, Sa- Ilelv., Vaud Tartegnins (Favrat).

Discus planus; fol. obovato-elliptica vel obovato-oblonga . . 27

21 Discus conicus; fol. oblonga - Itte-Savoie, Habère-Lullin (Puget) . . . . 2348. C. penscrutata Gdgr mss Ped. 8-10 m. l.; fol. glaucescentia - Suecia, Eland Tveta $27\left\{\begin{array}{l}\text { (Scheutz). } 1 \text {; fol. amoene viridia - Hte-Savoie, Pringy } \\ \text { Ped. 9-16 m. 1.; }\end{array}\right.$ (Puget) . . . . . 2350. C. BRevifolih Gdgr msS

28 Rami flor inermes . . . . . . . . 29

28 I Rami flor aculeati . . . . . . . . . 32

Pet. aculeati; discus / conicus. . . . . . . . 30 $29\left\{\begin{array}{c}\text { Pet. inermes; discus planus - Helv., Valais Bramois }(\mathrm{Fa}- \\ \text { vrat). . . . . . 2351. }\end{array}\right.$

$30\left\{\begin{array}{l}\text { Fol. oblonga; discus conicus. } \\ \text { Fol. elliptica; discus vix conicus - Rhone, Limonest (Cha- }\end{array}\right.$ bert) . . . 2352. C. AMphinhyncha Gdgr mss Pet. eglandulosi; fr. 16-18 m. diam. latus - Rhône, Arnas (Gdgr herb. ros . n. 76). . . . 2353. C. clavordes Gdgr

31 Pet. paulo glandulosi ; fr. $10-11 \mathrm{~m}$. diam. latus - Rhone, Arnas (Gdgr herb. ros. n. 540).2354. C. Clavoides B steno-

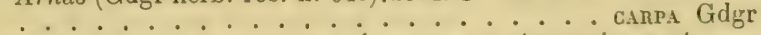

Discus planus ; pet. omnes inermes - Aragonia austr., Sierra de Mosqueruela (Martin).2355. C. CELTiberica

Discus subconicus; pet. sup. aculeati - Itte-Savoie, Pringy (Puget) . . . . 2356. C. erythropetala Gdgr mss

33 ～Ped. villosi. . . . . . . . . . 34

Ped. glabri. . . . . . . . . . . . 43

Fr. rotundatus vel globosus. . . . . . . . . 35

Fr. ovoideus vel obovato-oblongus. . . . . . . . 36

34 Fr. oblongus. . . . . . . . . . . 38

Fr. ovatus - Rhône, Arnas (Gdgr. herb. ros. n. 151)

. . . . . . . 2377. C. mracaxtua Gdgr mss

3.) $\left\{\begin{array}{l}\text { Dentes lanceolatae; fr. conicus apice attenuatus - IIelv., } \\ \text { Taud Chexbres (Favrat). - . 2358. C. DIMidexs Gidgr miss }\end{array}\right.$ Dentes obtusae; fr. apice subrotundatus - Bouches-duRhône, Martigues (Autheman).2359. C. BRachYodox Gdgr mss Pet. aculeati; discus | conicus. ........ 87

36 Pet. inermes; discus planus - Irie-Savoic, Habère-Poche (Puget). ...... 2360. C. Fonnicata Gdgr mss 
(Styli villosi; ped. 9-12 m. 1. - Sabaudia, Salins (Puget)

37 Styli lanati ; ped. 12-15 m.1. - Rhône, Chaponost (Chabert). . . . . . 2362. C. spHoenoclada Gdgr mss

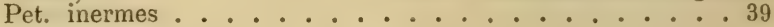

38 Pet. aculeati. . . . . . . . . . . 41

39 Rami flor. aculeati ; fol. basi attenuata . . . . . 40

Rami flor. inermes; fol. basi rotundata - Rhône, Limonest (Chabert).........2363. C. Podotricira Gdgr mss

Discus conicus ; ped. 5-8 m. l. - Hte-Savoie, Habère-Poche

40 (Billot exs. n. 3588 - C. pyriformis Dés. mss non Sw.)

. . . . . . . 2364. C. Pyrus Gdgr mss

Discus planus ; ped. 11-13 m. 1. - Gard, Le Vigan (Tuez-

kiewicz). . . . . . . 2365. C. PYrrHA Gdgr mss

41 Fol. basi attenuata; rami flor. inermes. . . . . . 42

Fol. basi rotundata; rami flor. aculeati - Rhône, Ecully

(Chabert). . . . . 2366. C. ILICETORum Gdgr mss

Discus planus; ped. $7-10 \mathrm{~m} . \mathrm{i}$. - Ardeche, Annonay (Boullu)

42 Discus conicus; ped. $10-13$ m. 1. - Austria inf., Kalksburg

(Wiesbaur). . . . . 2368. C. vindobonensis Gdgr mss

Fr. rotundatus vel globosus. . . . . . . . . 44

43 Fr. ovatus vel ovato-rotundatus. . . . . . . 50

Fr. ovoideus vel obova to-oblongus. . . . . . . 57

Fr. oblongus. ...................... 72

$44 \mid$ Fol. inaeque vel biserrata. . . . . . . . . 45

Fol. omnino simpliciter serrata. ......... 47

Fol. basi attenuata. . . . . . . . . . 46

Fol. basi rotundata ovata; pet. inf. inermes - Gallia, Belg. etc.

Fol. basi rotundata obovata; pet. omnes aculeati - Bouchesdu-Rhône, Martigues (Autheman).2370. C. CALOSPHAERA

............................. mss

Ped. 5-6 m. l.; pet. glandulosi - Thuringia, Oesterfeld

46 (Haussknecht) . . . . 2371. C. Loxgispina Gdgr mss

Ped. 1:-13 m. 1.; pet. eglandulosi - Anglia, Codsall (Fraser). . . . . . . 2372. C. CunEIfolia Gdgr mss

47 ( Pet. inermes . . . . . . . . . 48

Pet. aculeati $\ldots 49$

Discus conicus ; rami flor. inermes - Pyrenaei, Gèdre (Gdgr

$48\left\{\begin{array}{l}\text { herb. ros. n., 308). Discus planus; rami flor. aculeati - Tyrol, Hall (Grem- } \\ \text { Discus }\end{array}\right.$ blich). . . . . . . 2374. C. oRogenes Gdgr mss

Fol. ovato-rotundata; discus planus - Jurasso (C. opaca Gren.

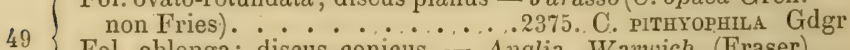
Fol. oblonga; discus conicus - Anglia, Warwich (Fraser)

. . . . . . 2376. C. Wartwichiana Gdgr mss

( Pet. omnes inermes ............. 51

50 Pet. inf. inermes. . . . . . . . . . 52

Pet. omnes aculeati............. 53

Fol. ovata basi rotundata; rami flor. aculeati - Rhône, Ar-

51 nas (Gdgr herb. ros. n. 250).2377. C. a.uphisbaexa Gdgr mss Fol. obovata basi subtruncata; rami flor. valde aculeati Tyrol, Hall (Gremblich) . . .2378. C. Steiniana Gdgr mss 
il Fol. obovato-oblonga, basi attenuata; rami flor. subinermes - Pyrenaei, Gédre (Bordère).2379. C. sYngenoldes Gdgr mss Rami flor. inermes; ped. 9-13 m. 1. - Isère, Paladru (Gdgr

52 herb. ros. n. 35I). - 2380. C. cironomelanos Gdgr mss Rami flor aculeati; ped. 13-15 m. 1. - Rhone, Arnas (Gdgr herb. ros. n. 132). . . 2381. C. labnellata Gdgr

Fol. basi rotundata ........... . 54 Fol. basi attenuata - Rhóne, Arnas (Ġdgr herb. ros. n. 346$)$

1. . . . . . . 2382. C. Colluen Gdgr Fol. omnino simpliciter serrata . . . . . . 55

51 Fol. inaeque subbiserrata - Sabaudia (Dés. herb. ros.n. 67) . . . . . . . 2383. C. uncinelloides (Puget mss)

( Discus conicus; fol. obovato-oblonga. ....... 56

55 Discus planus; fol. ovato-elliptica - Valais, Louéche (Lorenti). . . . . . 238'. C. ontuoculamys Gdgr mss Rami Hor. subinermes; fr. rotundatus ovatus et simul ovoideus - Rhone, Mont-Ceindre (Chabert).2385. C. METERo-

Rami flor, aculeati fr. omnes ovati - Rhóne, Montmelas (Gdgr herb. ros. n. 289). . . 2386. C. SYNGENEs Gdgr mss

5 i Fol. basi attenuata vel cuneata. . . . . . . 58

57 Fol. basi rotundata ............. . 62

is $\{$ Pet. omnes aculeati. . . . . . . . . . 50

Pet. saltem inferiores inermes. . . . . . . . 60

Rami flor aculeati; fol. oblonga - Pyrenaei, Gèdre (Gdgr

59 herb.ros. n. 265). - . 2387. C. ACANTHoclada Gdgr mss Rami flor. inermes; fol. obovato-elliptica - Rhône, Ecully (Chabert). . . . . . 2388. C. PLACIDA Gdgr mss Discus planus; rami flor, aculeati. . . . . . . 61

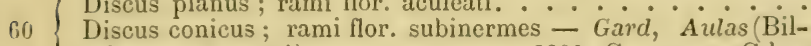
lot exs. n. 3724) . . . . 2389. C. Olearia Gdgrmss

$61\left\{\begin{array}{l}\text { Dentes subconvergentes; pet. omnes inermes - Rhône, Dar- } \\ \text { dilly (Chabert). . . 2390. C. PLATYPETALA Gdgr mss }\end{array}\right.$ Dentes apertae; pet. sup. aculeati - Hte-Savoie, HabereLullin (Puget). . . . . 2391. C. Pluniceps Gdgr mss

I Pet. omnès inermes. . . . . . . . . . 63

Pet. inf. inermes . . . . . 64

Pet. omnes aculeati. . . . . . . . . 66 66

Virens; discus planus - Rhône, Arnas (Ġdgr herb. ros.

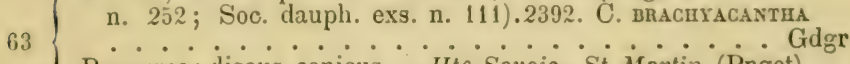
Purpurea; discus conicus - Itte-Savoie, St Martin (Pnget). ............ C. Rubristipula Gdgr mss

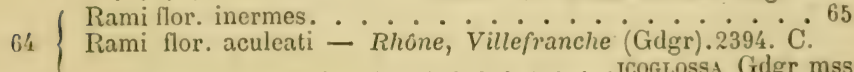

1................ ICoglossa Gdgr mss

$65\left\{\begin{array}{l}\text { Styli lanati ; ped. } 13-16 \mathrm{~m} .1 . \text { - Ain, Trévoux (Fray). 2395. C. } \\ \text { Styli villosi ; ped. } 15-20 \text { m. i. - Rhône, Arnas (Gdgr herb. }\end{array}\right.$ Styli villosi ; ped, $15-20$ m. 1. - Rhône, Armas (Gdgr herb. ros. n. 343 ). . . . . . .2396. C. stenopetala Gdgr

$66\left\{\begin{array}{l}\text { Fol. simpliciter serrata } \\ \text { Fol inf. biserrata - Ardèche, Annonay (Puget).2397. C. } 67\end{array}\right.$ (................................. (Puget mss) 
67 Discus planus ...................... 68

Discus | conicus. . . . . . . . . 699

Fol. obovata ; ped. 12-14 m. 1. - Croatia, Prekrize (Vuko-

68 tinovic). . . • . 2398. C. Vukotinovici Gdgr mss Fol. oblonga; ped. 9-11 m. 1. - Suecia, Scania Torup (Tullberg). . . . . . 2399. C. vireticola Gdgr mss

69 Rami flor. inermes vel subinermes........ 70

\{ Rami flor. aculeati. . . . . . . . . . . . 71

Sep. apice foliaceo-laciniata; fol oblonga - Pyrenaei, Gè-

70 dre (Bordère). . . . 2400. C. Nisus Gdgr mss

Sep. apice angustata integra ; fol. obovato-oblonga - Rhône, Chaponost (Chabert). . . 2401. C. BELoxidimorPHA Gdgr mss

Fol. ovato-elliptica; discus conicus - Rhône, Gleizé (Gdgr herb. ros. n. 65) . . . 2402. C. Gracilenta Gdgr mss

Fol. obovato-subobtusa; discus conicus - Bouches-du-Rhône, Martigues (Autheman). . . . 2403. C. Tinctilis Gdgr mss

Fol. obovato-acuta; discus vix conicus - Cher, Turly etc.

72 Fol. inaeque vel biserrata. $\ldots 2404$. TRICHONEURA (Rip.)

72 Fol. omnino simpliciter serrata. . . . . 74

Rami flor. inermes; fol. obovata basi rotundata - Austria inf., Kalksburg (Wiesbaur) .2405. C. BRAcHYstYLis Gdgr mss

73 Rami flor. paulo aculeati; fol. ovato-elliptica basi attenuata - Thuringia, Neuhaldensleben (Baenitz Herb. eur. n. 2234)

Foi . . 2406. C. MaAsI Gdgr msS

74 Fol basi attenuata. $\ldots \ldots 78$

Pet. omnes aculeati . . . . . . . . . 76

Pet. omnes inermes; fol. obovato-elliptica - Savoie, etc.

Pet. inf. inermes; fol. oblonga - Pyrenaei, Gavarnie (Gdgr herb. ros. n. 10). . . 2408. C. PlatYsTEPHANA Gdgr mss

Pet. sup. inermes ; fol. obovata - Austria inf., Kalksburg

(Wiesbaur). . . . . 2409. C. oxypetala Gdgr mss

\} Discus planus; fol. ovata vel elliptica. . . . . . 77

Discus conicus; fol. obovata - Helv., Zurich (Lehman). 2410.

. . . . . . . . C. Gaudiniana Gdgr mss

Fr. maximus utrinque altenuatus; fol. apice rotundata -

Ardèche, Annonay (Boullu). • .2411. C. Nervigera Gdgr mss Fr. angustissimus basi subdepressus apiceque ut et fol. attenuatus - Rhône, Arnas (Gdgr).2412. C. AMPHICARPA .................... Gdgr mss

Fol. oblonga ................... 79

78 Fol. ovata - Europa media (Gdgr herb. ros. n. 59,501 et 502 var.) . . . . . 2413. C. unbica (Lem.)

7 Pet. omnes I aculeati. . . . . . . 80 Pet. omnes inermes - Austria inf., Kalksburg (Wiesbaur). . . . . . . 2414. C. Approximata Gdgr mss

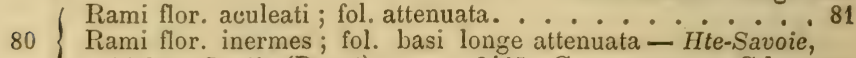
Habère-Lullin (Puget) . . .2415. C. ElisophyLLA Gdgr mss

( Ped. 6-10 m. 1.; discus sat conicus - Hte-Savoie, Fessy.

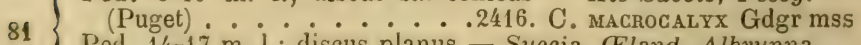
Ped. 14-17 m. 1.; discus planus - Suecia, Eland, Albrunna (Scheutz) ....... 2417. C. RUBRovirens Gdgr mss 


\section{5. - Pilosae Gdgr Essai p. 31.}

Foliolis medianis ramorum floriferorum supra pilosis dein glabris, subtus vero, etiam adultis, toto pubescentibus simpliciterque serratis.

(Ped. glaberrimi . . . . . . . . . . . . 2

1 Ped. glandulosi et paulo hirsuti. . . . . . . . 64

Ped. tantum villosi............. 66

() Styli glabri vel sparse pilosuli ... . . . . . . 3

2 Styli villosi vel lanati. . . . . . . . . . . 23

3 Fol. basi rotundata ............. 4

Fol. basi | attenuata . . . . . . . . . . . 14

4 Ir. ovoideus vel obovato-oblongus ......... 5

Fr. oblongus .................... 11

Rami flor inermes. . . . . . . . . . . . 6

I Rami flor aculeati. . . . . . . . . . . . 7

(Styli glaberrimi ; ped. $8 \cdot 10$ m. 1.- Rhône, Arnas (Gdgr).

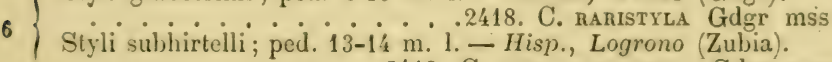

*...... 2419. C. Caesaraugustana Gdgr mss Pet. | aculeati.............. 8

7 Pet. omnes inermes - Pyrenaei Or., Le Boulon (Pellét).

Discus planus................ . . 9

Discus conicus. . . . . . . . . . . . . 10

(Fol. oblonga; pet. omnes aculeati - Isère, Paladru Gdgr

$9\{$ herb. ros. n. 373) . . .2421. C. crataeginaria Gdgr mss Fol. ovato-rotundata; pet. subinermes - Suecia (Fr.); Dania (Meyer); Germ. (Rchb.). . . . .2422. C. Columnifera (Fries) $10\left\{\begin{array}{l}\text { Ped. 6-11 m. 1.; fr. basi depressus - Hte-Savoie, Habere- } \\ \text { Poche (Pugret). . . . . 2123. C. Inementigna Gigr mss }\end{array}\right.$ I'ed. 14-16 m. 1.; fr. basi rotundatus - Bouches-du-Rhône, Berre (Autheman) . . . . 2424. C. australis Gdgr mss

11 Rami llor inermes............... 12

Rami flor aculeati. . . . . . . . . . 13

Discus planus; pet. inermes - IIte-Savoie, st-Martin (Puget). $12\left\{\begin{array}{l}\text { Discus conicus ; pet. aculeati - Iisp. bor., Logrono (Zubia). } \\ \text { - 2425. C. Paucrsprsa Gdgr mss }\end{array}\right.$

. . . . . . . . 2426. C. staunoclada Gdgr mss Fol. obovata; pet. inf. inermes - Rhöne, Gleize (Gdgr herb. ros. n. (66) . . . . 24:27. C. Axdnosices Gder mss Fol. olovata; jet. omnes inernes - Tyrol, Trente (Crelmi).

F * - . 2428. C. Infracondata Gdgr mss fol. ovato-elliptica ; pet. omnes aculeati - IIsp. bor., Sierra de Cameros (Zubia) . . . . 2429. C. probara Gdgr mss 
14 Fr. ovoideus vel obovato-oblongus. . . . . . . 15

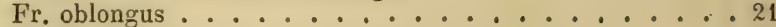

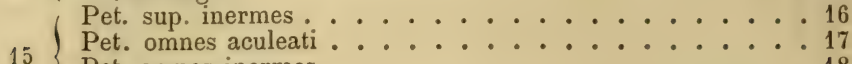

Pet. omnes inermes . . . . . . . . . 18

Pet. inf. inermes. . . . . . . . . . 19

Rami flor. inermes; fr. basi longe attenuatus - Tyrol, Hall

16 (Gremblich) . . . . 2430. C. IMprovida Gdgr mss Rami flor. aculeati; fr. basi paulo attenuatus - Tyrol, Trente (Gelmi) . . . . . . 2431. C. stilbe Gdgr mss Pami flor. aculeati; discus conicus - Rhône, Arnas (Gdgr herb. ros. n. 258). . . . . 2432. C. Arcturus Gdgr mss Rami flor. inermes; discus planus - Anglia, Cournouailles Trevol (Briggs). . . . . 2433. C. BriggsI Gdgr mss

( Sep. glabra; fol. obovato-oblonga - Rhône, Arnas (Gdgr).

18 Sep. villosa; fol. elliptico-obovata C. Rhône, Villef

(Rami flor aculeati.............. 20 Rami flor. inermes - Rhône, Arnas (Gdgr).2436. C. тітнY-

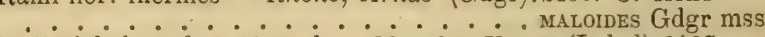

(Styli glabri; ped. $7-10$ m. i. - ianche, Yvetôt (Lebel). 2437. 20 Styli paulo hirsuti ; ped. $10-14$ m. 1. - Isère, Charancieu

21 ( Pet. et rami flor. inermes. . . . . . . . 22

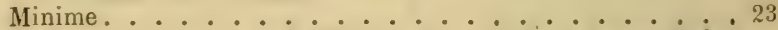
Styli paulo hirsuti ; ped. 16-20 m. 1. - Rhône, Arnas (Gdgr

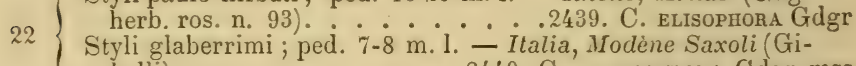
belli). . . . . . . 2440. C. LEUcochlora Gdgr msS

(Fr. basi rotundatus apice attenuatus; fol. obovato-oblonga

23 - Gallia, Annecy (Bouvier) - 2441. C. stenocanpa Gdgr mss Fr. basi depressus apice strangulatus; fol. oblonga - Rhóne, Soucieu (Chabert). . . 2442. C. strangulituba Gdgr mss

( Fr. globosus rotundatus vel ovatus . . . . . . . 25

24 Fr. breviter ovoideus. . . . . . . . . . . 38

Fr. obovato-oblongus vel oblongus. . . . . . . . 446

25 Fol. inaeque vel biserrata. . . . . . . 26

Fol. simpliciter serrata. . . . . . . . . . 29

26 Fr. rotundatus vel globosus . . . . . . . . 27

Fr. ovatus. . . . . . . . . . 28 Aculei adunci ; pet. glandulosi - Podolia, Krzemeniec (Bes-

27 ser) .......... 2443. C. Frutetorum (Bess.) Aculei inclinati ; pet. eglandulosi - Pyrenaei or., Mt-Louis (Pellet). . . . . .2444. C. DIDrMoxis Gdgr et Debeaux Fol. basi attenuata ; pet. aculeati - Scotia, Edimbourg (Bor$28\left\{\begin{array}{l}\text { rer). } \text { Fol. basi rotundata; pet. subinermes - Rhone, Gleizé (Gdgr). } \\ \text { - } 2445 \text {. C. Borreri (Woods) }\end{array}\right.$

Fr glo

Fr. globosus vel rotundatus. ............ 30

Fr. ovatus. ......................... 
Pet. omnes inermes ............... . 31

Pet. omnes aculeati . . . . . . . . . 32

I'et. inf. inermes - Pyrenaei, Gèdre (Gdgr herb. ros. n. 37\%),

. . . . . 2447. C. platysepala Gdgr mss

( Styli lanati; ped. 6-7 m. 1. - Anglia, York (Webster).2448.

31 . . . . . C. Webstent Gdgr mss

Styli hirsuti ; ped. 12-15 m. 1. - Rhöne, Francheville (Cha-

bert)........ 2449. C. DAsYcanPa Gdgr mss

32 f Fol. obovato-oblonga. . . . . . . . . 33

Fol. obovata. . . . . . . . . . . . 34

Sep. reflexa caduca; fr. apice haud attenuatus - Belg., St-

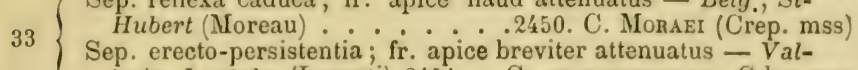
lesia, Loueche (Lorenti).2451 . .C. sclerostephasa Gdgr mss

Fol. acuta; fr. non decurrens. . . . . . . . 35

Fol. obtusa; fr. centralis decurrens - Vallesia, Mayens de Sion (Favrat). . . . 2452. C. \#ELvetica Gdgr mss

( Ped. 10-12 m. 1. . . . . . . . . 36

Ped 5-6 m. 1.; fr. parvus - Anglia, Hereford Wormbridge

(Ley). . . . . . . 2453. C. Cerasoldes Gdgr mss

Fol. oborato-spathulata - Pyrenaei Or., Mont-Louis (Pellel). 36 Fol. obovato-attenuata - Pyrenaei Or., Mont-Louis (Pellet).

. . . . . . . 2455. C. Accipitrina Debeaux

Styli breves; rami flor. aculeati - Suecia, Mt-Kinnekulle

37 (Zetterstedt) . . . . 2456. C. LEucANTHA Gdgr mss

Styli subcoaliti; rami flor. inermes - Rhoone, Chaponost

(Chabert). . . . . 2457. C. LONGISTYLA Gdgr mss

38 Fol. inaeque vel biserrata. . . . . . . 39

Fol. omnino simpliciter serrata . . . . . . . 40

Pet. aculeati; fol. inodora - Helv. occid. (C. Friesii Lagg. et $39\left\{\begin{array}{l}\text { Puget non Scheutz) - - - - 2458. C. Eglandulosa Gdgr } \\ \text { Pet. subinermes; fol. odorata - Anglia.2459. C. Canescens }\end{array}\right.$

................. (Baker)

Pet. omnes aculeati ............... 41

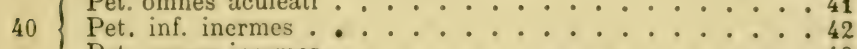

Pet. omnes inermes . . . . . . . . . 43

Cor. amoene rosea; fol. ovata - Gallia centr. (Gdgr herb. $41\left\{\begin{array}{l}\text { ros. n. } 557 \text { var.) oblonga - Pyrenaei, Bayonne (Bordere). } \\ \text { Cor. subalba; fol. oblor. }\end{array}\right.$

. . . . . 2461. C. BayonNENsis Gdgr mss

Discus planus; styli lanati - Rhône, Arnas (Gdgr herb. ros.

$42\{\mathrm{n} .144) \ldots . . . . .2462$. C. Macrostigma Gdgr

Discus conicus; styli parum viliosi - IIte-Savoie, Mt-Sion (Puget). ... .2463. (. onophila (Rip.! mss, an Gren. ?)

43 Fol. oblonga. ................. 44

Fol. obovata ................... 45

Rami flor inermes; fol basi rotundata-ilelv., Sion (Wolf). Rami flor. aculeati; fol. hasi attenuata - Suecia, Blek.

Lyckeby (Lundquist). . . . .2465. C. A.jgustifolia Gdgr mss 

$\therefore$ Discus planus; fol. basi subtruncata - Suecia, Mt-Kinnekulle Discus conicus; fol. basi rotundata - Hte-Savoie, Pringy (Puget) . . . . 2467. C. EPIMEDIUM Gdgr mss

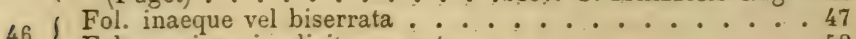

46 Fol. omnino simpliciter serrata......... . 52

47 ( Rami flor. inermes. . . . . . . . . . . 48

Rami flor aculeati. . . . . . . . . 49

Pet. inermes; fol oblonga basi attenuata - Norvegia, Sogn

48 (C. pubescens Blytt non al.). . 2468. C. Blytrir Gdgr Pet. aculeati; fol. obovata basi rotundata - Dalmatia, Giovanni (Studniczka). . . . 2469. C. Studniczkae Gdgr mss

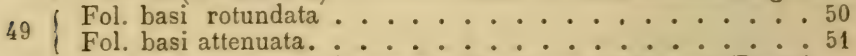
Discus conicus; pet. inermes - Hte-Savoie, St-Gervais (Puget) $50\left\{\begin{array}{l}\text { Discus planus; pet. aculeati - Bohemia, Kuchelbad (Opiz) } \\ \text { - } 2470 \text {. C. Sapida Gdgr mss }\end{array}\right.$

Fol caesia elliptica discus planus-Anglia 241 . C. Pilosa (Opiz) Fol. virentia oblongo-lanceolata; discus planus - Anglia,

51 Cheshire (Harbord) . . . 2473. C. Exerrans Gdgr mss

I Fol. virentia obovata ; discus subconicus - Suecia, Blekinge Nattraby (Lundquist). . . . 2474. C. DENTIFERA Gdgr mss

52 ( Pet. inf. inermes. . . . . . . . . . 53

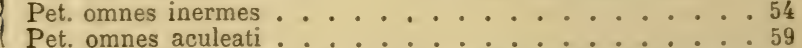

Styli lanati; ped. $8-10$ m. 1. - Rhône, Pommiers (Gdgr). 53 Styli villosi; ped. 9 -14 m. 1. - Rhône, Charbonnières (Chabert). . . . . . 2476. C. sYNCARPa Gdgr mss

54 Fol. basi rotundata vel subrotundata. . . . . . . 55

54 Fol. basi | attenuata ............... 57

Discus conicus. . . . . . . . . . . . 56

55 Discus planus - Helv., Jura Couvet (Baenitz herb. europ.

n. 1866). . . 2477. C. Leontonyx Gdgr

Fr. basi decurrens; fol. ovato-elliptica - Hte-Savoie, Pringy-

$56\left\{\begin{array}{l}\text { Lullin (Puget). } ; \text { fol. obovata - Italia or., Faenza (Cal- } \\ \text { Fr. basi rotundatus; fol }\end{array}\right.$ desi) . . . . 2479. C. paupercula Gdgr mss

$57\left\{\begin{array}{l}\text { Rami flor. aculeati; fr. ovoideus vel obovato-oblongus } \therefore \text { Rami flor subinermes; fr. oblongus - Hte-Savoie, Habère- } \\ \text { Ras }\end{array}\right.$ Lullin (Puget). . . . . 2480. C. stenotuba Gdgr mss

Ped. 9-11 m. l.; fr. apice attenuatus - Suecia, Blekinge Nattraby (Lundquist). . . : 2481. C. Rubripetala Gdgr mss

58 Ped. 4-6 m. 1; fr. apice longissime strangulatus - Suecia, Vestrog. Mt-Kinnekulle (Zetterstedt). 2482. C. LAGENARIA

Fol basi rotundata $\therefore \ldots \ldots$ Gdgr mss

59 Fol basi | attenuata. . . . . . . . . 62

60 Rami flor. aculeati. - Jura etc 2483 C 61

Rami flor inermes - Jura, etc. - 2483. C. suburis (Gren.)

( Pet. glandalosi; ped. 15-18 m. l. - Ain, St-Didier-sur-Cha-

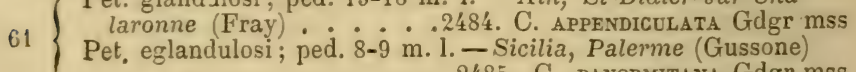
........2485. C. Pañormtana Gdgr mss 
Fr. oblongus; fol. oblonga. . . . . . . . 63

62 Fr. ovoideus; fol. elliptica - Rhône, Arnas (Gdgr herb. ros. n. 52)................ Confinis Gdgr mss Discus planus ; costa inermis - Pyrenaei, Gèdre (Bordère) 2487. C. Acidula Gdgr ms Discus conicus; costa basi aculeata - Pyrenaei, Biarritz (Bordère). . . . 2488. C. aGanthophylda Gdgr mss Styli glabri; fr. oblongus. .......... 65

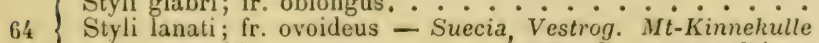
(Zetterstedt). . . . . . 2489. C. ElEgans Gdgr mss Fr. basi attenuatus; ped. 10-16 m. 1. - Rhône, Chaponost (Chabert); Arnas (Gdgr her. ros. n. 558 var.).2490. C. CURFr. basi decurrens; ped. i5-17 m. 1. - Gard, Arphy (Tuezkiewicz) ......2491. C. Integrata Gdgr mss

$66\left\{\begin{array}{l}\text { Styli glabri vel adsperse pilosi } . . . \\ \text { Styli villosi vel lanati . . . . . . . . } 67\end{array}\right.$

67 Fol. elliptica vel obovata. . . . . . . . . 68 Fol, oblonga . . . . . . . . . . . . . 75 Fr. ovoideus vel obovato-oblongus . . . . . . . 69

68 Fr. oblongus .................... 71 Fr. ovatus - Manche, Yvetôt (Lebel).2492. C. INcoxcessa ................. Gdgr mss

\{ Rami flor inermes .............. 70 Rami flor. aculeati - Rhône, Arnas (Gdgrr herb. ros. n. 244)

$69\{$. . . 2493. C. Ramusculosa Gdgr Ped. 7-8 m.1.; fr. basi depressus - Catalaunia, Valdemiras $70\left\{\begin{array}{l}\text { (Vayreda). } \\ \text { Ped. 10-14 m. i. fr. basi valde attenuatus - Rhône, Denicé }\end{array}\right.$ (Gdgr herb. ros. n. 143).2495 . . C. glauciramea Gdgr mss

71 Rami flor aculeati .................. 72 Rami flor inermes . . . . . . . 7 73 Pet. omnes inermes; fol. basi attenuata - Rhône, Chaponost

72 (Chabert). . . 2496. C. Mitoclada Gidgr mss Pet. inf. aculeati; fol. basi rotundata - Rhône, Ville-surJarnioux (Gdgr) . . . . 2497. C. ANaCanpseros Gdgr mss

Pet. omnes aculeati ............ 74 Pet. omnes inermes; fol. basi attenuata - Rhône, Arnas (Gdgr). . . . . . 2498. C. Lonchitis Gdgr mss

73 Pet. sup. inermes; fol. basi truncato-rotundata - Hisp. centr., Escorial (Laguna). .2499. C. Guadarramae Gdgr mss

Pet. inf. inermes; fol. basi subrotundata - Hte-Savoie,

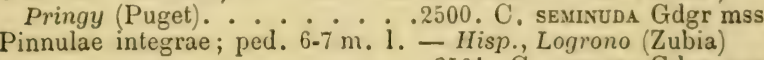
$74\left\{\begin{array}{l}\text { Pinnulae integrae; ped. 6-7 m. 1. - Hisp., Logrono (Zubia) } \\ \text { Pinnulae dentatae; ped. 10-12 m. 1. - Hisp., Barcelone }\end{array}\right.$ Vallvidrera (Vayreda) . . 2502. C. BARcINONENSIS Gdgr mss

75 | Fr. ovoideus .................. 76

75 Fr. oblongus ........................ 77

$76\left\{\begin{array}{l}\text { Ped. } 7-8 \mathrm{~m} \text {. 1.; pet. et rami flor. inermes - Hungaria, } \\ \text { Podhrad (Holuby) } \\ \text { Ped }\end{array}\right.$ Ped. 13-20 m. 1.; pet, et rami flor. aculeati - Hte-Savoie, Arenthon (Puget)......2504. C. FAtula Gdgr mss 
Rami flor aculeati. . . . . . . . 78

Ramí flor. inermes. . . . . . . . . . 80

Fol. basi attenuata . . . . . . . 79

78 Fol. basi rotundata - Rhône, Denicé (Gdgr herb. ros. n. 333)

( Pet. aculcati ; fr. basi depressus - Rhône, Arnas (Gdgr).

Pet. inermes; fr. basi valde attenuatus - Rhône, Arnas (Gdgr herb. ros. n. 376) . .2507. C. PLAGIopHylla Gdgr mss

Ped. $7-13 \mathrm{~m} .1 . \ldots 81$

Ped. $15-18$ m.1. - Rhône, Arnas (Gdgr herh. ros. n. 14)

......... 2508. C. ANDROPOGON Gdgr

Pinnulae integrae; styli subhirsuti - Rhône, Ville-sur-Jarnioux (Gdgr herb. ros. n. 275).2509. C. HYpochiosaea Gdgr Pinnulae dentatae; styli subglabri - Rhone, Arnas (Gdgr)

. . . . . . . 2510. C. Agricola Gdgr mss

Fr. globosus ovatus vel obovato-oblongus . . . . . . 83

Fr. oblongus . . . . . . . . . . 94

83 Fol. inaeque vel biserrata . . . . . . . . 84

Fol. omnia simpliciter serrata . . . . . . . 85

Fr. ovato-subrotundatus; ped. 4-5 m. 1.-Bouches-du-Rhône

84 Martigues (Autheman) . . 2hl1. C. cyclodonta Gdgr mss Fr. ovoideus; ped. 7-9 m. 1. - Helv., Valais Bramois ( $\mathrm{Fa}-$ vrat) . . . . . 2512. C. PARviceps Gdgr mss Fr. omnes ovati. . . . . . . . . . 86

Fr. omnes ovoidei. . . . . . . . . . . 89

Fr. omnes rotundati - Cher, Couet (Déségl.).2513. C. con-

Fr. alii rotundati vel ovati; alii ovoidei - Hte-Savoie, Arenthon (Puget) . . . . 2514. C. DIMORPHOCARPA Gdgr mss

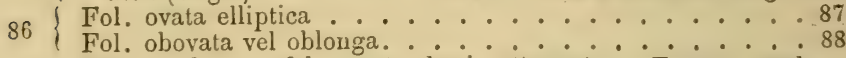

Discus planus; fol. ovata basi attenuata - Europa med.

(Gdgr. herb. ros. n. 578).. .2515. C. ConYMBifera (Borkh.)

87 Discus subconicus; fol. elliptica basi rotundata - Ain, StDidier-sur-Chalaronne (Fray).2516. C. STEPHANOCALYX

................ Gdgr mss

Pet. inermes; fol. obovata - Rhône, Charbonnières (Boullu)

Pet - . 2517. C. virgosa Gdgr mss (Lundquist). . . . . 2518. C. LundQuistil Gdgr mss

89 Fol basi rotundata ........... . 90

Fol. basi attenuata . . . . . . . . . . 91

Rami flor. aculeati; fol. ovata - Germ., Wurtzburg (Rau)

$90\{$ Gallia (Gdgr herb. ros. n. 506).2519. (. PLATyphylla (Rau) Rami flor. inermes; fol, obovata-Catalaunia, Olot (Vayreda)

. . . . . . . 2520. C. subpyrenaica Gdgr mss

91 Discus conicus. . . . . . . . . . 92

91 Discus planus ....... .993 Pet. omnes aculeati; fol. pallide riridia - Phône, Arnas

$92\{$ (Gdgr) . . 2521. C'. DIFFICILIS Gdgr mss Pet. inf. inermes; fol. atrovirentia - Sabaudia, Salins (Puget)

.2522. C. Pachyphylla Gdgr mss 
Fol. elliptica; pet. omnes inermes - Rhöne, Arnas (Gdgr herb, ros. n. 70). . . . 2523. C. VAriLlosil Gdgr Fol. obovato-oblonga; pet. omnes aculeati - Basses-Alpes Condamine (Proal). . . . 2524. C. Villarsiana Gdgr msg Fol. oblonga; pet. inf. incrmes - Rhône, Ecully (Chabert) D. . . . 2525. C. LAMPococca Gdgr mss

Discus planus. ................. 95

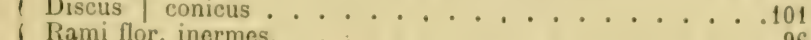

Rami flor inermes. . . . . . . . . . . . . 96

Rami flor aculeati. . . . . . . . 97

Pet. aculeati; fol. obovata - Rhône, Arnas (Gdgr herb, ros. n. 21). . . 2526. mnuxcaxula Gdgr mss Pet. inermes; fol. oblonga-Dalmatia (Schalch).2527. C. DAL-

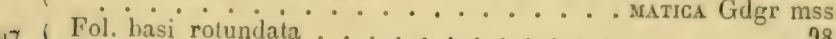

Fol. basi attenuata. . . . . . . . 98

Fr. ample turbinato-oblongus; fol. ohovata - Rihone, Denicé 38 Fr. anguste oblningus; fol. elliptica - Rhine, Montmelas (Gdigr herb. ms. 11. 330). . . 2529. C. mifonmis Gdgr mss

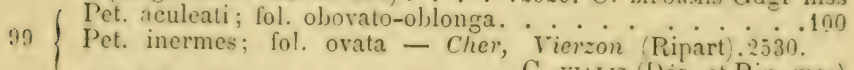

Ped. corrminsi $8-13$ i. C. Vhatis (Dés. et Rip. mss) $100\left\{\begin{array}{l}\text { Ped. corrmlosi } 8-13 \mathrm{~m} \text {. i.; fol. acuminata - Deux-Sévres, La } \\ \text { Mothe-St-IIéray (Sauzé); }-2531 \text {. C. AMoenıpes Gdgr mss }\end{array}\right.$ Ped. subsolitarii 6-8 m. 1.; fol. subacuta - Pyrenaei or., Mont-Louis (Pellet). . . .2532. C. Leucoacavtra (Debeaux)

101 Rami flor inermes. . . . . . . . . 102

Rami flor aculeati.

102 ( Fol. basi rotundata - Hte-Savoie, Arenthon (Puget).2533. 102 Fol. basi attenuata - Rhône, Tassin (Billot exs. n. 3589 bis parte, ut videtur). . . 2534. C. Leptostepirasa Gdgr mas

Fol. hast rotumdata oborata. . . . . . . . 101

103 Fol. basi attenuata. .20105

Fol. basi rotundata obovato-oblonga - Rhóne, Arnas (Gdgr herb. ros. n. 184) . . . 2535. C. vepREcularum Gdgr miss

Fr. hasi longe attenuatus - Isere, La Cute Saint-André $104\left\{\begin{array}{l}\text { (Boullu). } \text { Fr. basi subdepressus - Hte-Savoie, Arenthon (Billot exs. } \\ \text {. 2536. C Loxgruscl Gdgr mss }\end{array}\right.$

n. 3589 . . . . . 2537. (. HoLopond Gidgr mss

105 Fol. olnvaln-oblonga. . . . . . . . . 106

Fol. oblonga. . . . . . . . . . . . . 107

10. ( Fr. hasi attenuatus - Rhone, Alix (Gdgr) 25ss. C Arnita

Fr. basi longe decurrens - Helv., Bale (Christ).2539 Gdgr ms

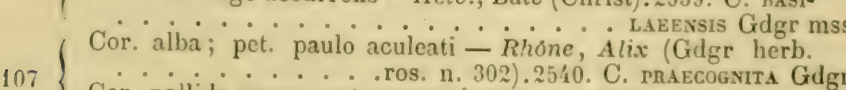

Cor. pallide rosea; pet. omnes incrmes - Rhóne, Iontmelas

(Gdgr herb. ros. n. 287). . . . 2541. C. persimilis Gdgr mss 


\section{6. - Solstitiales Gdgr Essai, p. 32.}

Foliolis, etiam adultis, utrinque dense et molliter hirsutis vel subtomentosis simpliciter serratis. - Facies fere Pugetiarum (Tomentoseae Auct.), sed pedunculi et sepala laevia nec normaliter glandulosa vel hispida.

1 Pedunculi glabri. . . . . . . . 2 Pedunculi villosi vel parcissime glandulosi. . . . . . 86

Fr. globosus. . . . . . . . . . . . 3

Fr. rotundatus. . . . . . . . . . . 8

Fr. ovato-rotundatus ................. 13

Fr. ovatus. . . . . . . . . . . 19

Fr. ovoideus vel obovato-oblongus. 25

Fr. oblongus. . . . . . . . . . . 5 57

Fol. obovata obovato-oblonga vel oblonga . . . . . 4

Fol. elliptica; pet. aculeati ; rami flor. inermes - Silesia

3 (Krocker) . . . . . 2542. C. Krockeri (Tratt.) Fol. ovata; pet. inermes; rami flor. aculeati - Scandinavia

4 Fol basi attenuata. $\therefore \ldots$

Fol basi rotundata. . . . . . . 6

Pet. inermes - Dania. . . 2544. C. Crassifolia (Wallm.)

5 Pet. aculeati - Suecia, Vestrog., Mt-Kinnekulle (Zetterstedt)

. . . . . . . . 2545. C. DEPRESSA Gdgr msS

Pet. subinermes . . . . . . . . . . 7

Pet. omnes inermes; ped, 15-16 m. 1. - Helv., Sion (Wolf;

6 Christ Rosen p. 191). . . 2546. C. Campicola Gdgr

Pet. omnes aculeati ; ped. 7-10 m. 1. - Helv., Valais Herbriggen (Wolf). . . . . 2547. C. Cononata Gdgr mss

Pinnulae eglandulosae angustatae; fol. obovata - Anglia

7 Pinnulae églandulosae foliaceae; fol. obovato-oblonga -

Tyrol, Trente (Gelmi) . . 2549. C. PRuinovinens Gdgr mss

Sep. dorso glandulosa; fol. oblonga - Suecia, Mt-Kinnekulle (Zetterstedt) . . . . . 2550. C. Platycarpa Gdgr mss

8 Fol. biserrata. ................. 9

Fol. simpliciter serrata. . . 10

Costa eglandulosa; pet. inermes. - Silesia, Goerbersdorf

9 (Straehler).......2551. (\%. SAmbucifolia Gdgr mss Costa glandulosa; pet. aculeati - Pyrenaei Or., Mont-Louis (Debeaux). . . . . 2552. C. cribrosa Gdgr mss

Pet. omnes inermes ............. 11

$10\left\{\begin{array}{l}\text { Pet. omnes aculeati fol ovato-rotundata - Hispania (Miller) } \\ .\end{array}\right.$

Pet. sup. aculeati ; fol obovato-oblonga - Borussia rhenana, Hecken (Braeniker). . . . .2554. C. RHENANA Gdgr mss 
Rami flor. aculeati. ............. 12

11 Rami flor inermes - Pyjenaci, Gidie (Bordère).255. C. ............... pyrenaeicola Gdgr mss Discus planus; fol. obovato-oblonga - Vallesia, Champex (Delasoic). . . . . . 2556. C. globulosa Gdgr mss Discus conicus; fol ovato-rotundata - Asia occ., Afr. bor., Europa (Gdgr herb. ros. n. 352, 585 et 586).2557. C. DU-

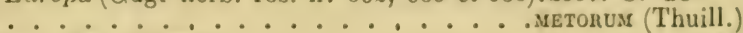
Rami flor. inermes............ 14 Rami flor aculeati. ............... 15 Discus conicus; ped. 7-9 m. 1. - Hisp. bor., Sierra de Cameros (Zubia). . 2558. C. secundaria Gdgr mss Discus planus; ped, 5-6 m. 1. - Suecia, Blekinge (Svanlund) . 2559. C. Andersoniana Gdgr mss Fol. basi attenuata .................... Fol. basi rotundata - Suecia, Scania Ortofta (Tullberg). ........ 2560. C. onthosepala Gdgr mss Pet. sup. aculeati ; fol. oblonga. . . . . . . . . 17 Pet. omnes inermes; fol. obovato-oblonga. . . . . . 18

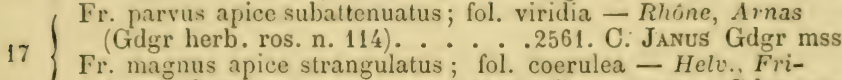
Fr. magnus apice strangulatus; fol. coerulea - Helv.. Fribourg (Cottet) . . . . 2562. C. Iodoprivlta Gdgr mss Perl. 12-15 m.1. - Rhöne, Craponne (Cariot).2563. C. CYAPed. 6-7 m. 1. - Suecia, Vestrog. Sattra (Lundquist).2564.

Fol. omnia simpliciter serrata. . Fol. infima biserrata - Austria inf., Kalksburg (Wiesbaur).

. . . 2565. C. avormalis Gdgr mss

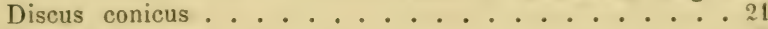

Discus planus. . . . . . . . . . . . 22 Rami flor. aculeati; pet. sup. aculeati - Pyrenaei centr.,

21 Luz (Bordère) . . . . . 2566. C. ApERTIDENS Gdgr msS Rami flor. et pel. omnes inermes - Rhône, Arnas (Gdgr herb. ros. n. 238) . . . . 2567. C. subdiscolon Gdgr mss

Pet. omnes inermes. . . . . . . . . . 23

22 Pet. omnes aculeati . . . . . . . 24 Pet. inf. inermes - Scotia, Perth (Drummond). 2568. C. ME-

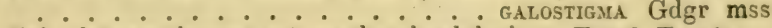
Fol. obovato-obtusa; pet. paulo glandulosi - Tyrol, Trente

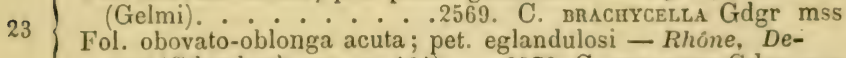
nicé Gdgr herb. ros. n. 161). . 2570. C. Petasites Gdgr mss

Rami flor. inermes; fol. minute ovata - Loire, Chalmazelle (Peyron $-C$. cinerosa Dés. Cat., 1876).2571. C. Pexrovir

| Rami flor aculeati ; fol ample oblonga - Rhóne, Montmelas (Gdgr herb. ros. 1. 12). . . . . 2572. C. Luvata Gdgr Fol. basi rotundata. ..............26 Fol. basi | attenuata . . . . . . . . 42

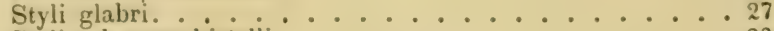

Styli adsperse hirtelli............ . . 28

Styli pubescentes. . . . . . . . . 30 Styli lanati ..................... 33 
( Discus conicus; rami flor. aculeati - Rossia, Astrakhan SaDiscus planus; rami flor. inermes - Rhône, Tassin (Cariot). . . . . . . . . 2574. C. Coxcolor Gdgr mss

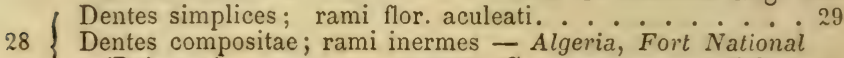
(Debeaux). . . . 2575. C. ADExodortordes Gdgr mss Pet. inermes; discus planus - Rhône, Arnas (Gdgr herb. ros. n. 89). . . . . 2576. C. GNaPHALOdES Gdgr Pet. aculeati; discus conicus - Tyrol, Hall (Gremblich) . . . . . . . 2577. C. hallensis Gdgr mss

Fol obovata . . . . . . . . . . . . . 31

Fol oblonga . . 32

Pet. eglandulosi, inf. inermes - Rhône, Arnas (Gdgr). 2578.

Pet. paulo ..... C. GRISESCENS Gdgr mss Pet. paulo glandulosi omnes aculeati - Gironde, Bassens Discus planus; fr. obovatus - Vallesia, Bovernier (Lagger)

32 . . . 2580. C. Holophylda Gdgr mss Discus conicus; fr. obovato-oblongus - Rhône, Arnas (Gdgr) ............. C. Apaloxylon Gdgr msS

33 Rami flor inermes ........... . . 34

Rami flor, aculeati. . . . . . . . . . . 35

Fol, acute obovato-oblonga; ped. 8-9 m. l. - Suecia, Smol.

34 Dref (Hyltén) . . . . . 2582. C. vaerendica Gdgr mss Fol. obtuse obovata ; ped. 12-13 m. 1. - Helv., Sion (Wolf ; Christ Ros. Schw. p. 189) . . . 2583. C. Pachystema Gdgr

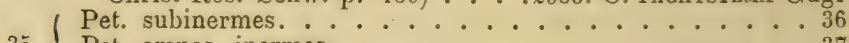

35 Pet. omnes inermes................ 37

Pet. omnes aculeati............ 40

Fol. ovata; fr. ovoideo-subattenuatus - Rhône, Tassin ( $\mathrm{Pu}-$

Fol

Fol oblonga; fr. oroideo-conicus - Vallesia, Vercorin (Favrat) . . . . . 2585. C. TENUICOLLIS Gdgr mss

Fol. ovata vel elliptica. . . . . . . . . . 38

Fol. obovata. . . . . . . . . . . . . . 39

Fol. obovato-oblonga; ped. $6-7 \mathrm{~m}$. 1. - Suecia, Mt-Kinnekulle (Zetferstedt). . . . . 2586. C. CALOpetala Gdgr mss

Fol. oblonga; ped. 15-17 m. 1. - Suecia, Mt-Kinnekulle (Zetterstedt) . . . . . 2587. C. BorEalis Gdgr mss Fol. acuminata - Galicia, Cracovie (Besser).2588. C. solsti-

Fol. 'subattenuata; ' fr. 'basi depressus - Rhône, Arnas (Gdgr herb. ros. n. 198). . . 2589. C. theratophila Gdgr

Fol. obtusata; fr. basi longe decurrens - Suecia, Vestrog. Sattra (Lundquist). . . 2590. C. phyllostephana Gdgr mss Sep. apice angustata ; ped. 6-7 m. 1. - Suecia, Mt-Kinnekulle (Zetterstedt) . . . 2591. C. ACANTHOPHORA Gdgr mss Sep. apice foliacea; ped. 11-13 m. 1. - Suecia, Smol. Barkeryd (Scheutz). . . . 2592. C. Nymaniana Gdgr mss

40 (Fol. obovata .................. 41 Fol. obovato-oblonga ; fr. basi valde attenuatus - Vallesia, Heremence (Wolf) . . 2593. C. scleroacantha Gdgr msS 

- decurrens - Suecia, Mt-Kinnekulle (Lundquist) . . . . . 2595. C. municata Gdgr mss Sep. apire foliacea; fol. inf. hiserrata - Suecia, TEland

41 Tveta (Scheutz). . . 2596. C. spinaenostigma Gdgr mss Sep. apice angustata; fol. simpliciter serrata - Pyrenaei Or., Le Capsir (Gautier).2597. C. Lapeynouslana Gidgr mss

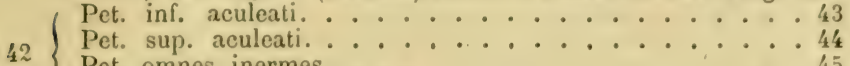

Pet. omnes inermes. . . . . . . . . . . 45

Pet. omnes aculeati. . . . . . . . . . . 52

Ped. 6-7 m. 1. ; fol minute oblonga - IIungaria, Csepel (Feich-

43 tinger). . . . 2598. C. nanubialis Gdgr mss Ped. 12-14 m. 1. ; fol. late obovato-oblonga - Basses-Alpes, Bouzolieres (Proal). . . . . 2599. C. Alpriva Gdgr mss Fr. utrinque rotundatus - Helv., Alpes rhacticae (Seringe) $44\left\{\begin{array}{l}\text { Fr. utrinque valde attenuatus - Vallesia, Champex (Delasoie). }\end{array}\right.$ . . . . . 2601. C. Jugularis Gdgr mss

$45 \mid$ Sep. dorso eglandulosa. . . . . . . . . 46 Sep. dorso normaliter glandulosa - Anglia, Cheshire (Harbord). . . . . . 2602. C. Glandulifera Gdgr mss Styli sparse pilosi ............ . 47 Styli lanati ................... 48 Styli glabri ; discus conicus - Rossia, Astrakhan Sarepta

46 Secker). . . . . 2603. C. SAreptana Gdgr ms Styli villosi ; discus planus - Suecia, Mt-Kinnehulle (Zetterstedt) ........ 2604. C. scandicA Gdgr mss

$47\left\{\begin{array}{l}\text { Rami flor. inermes; ped. 11-14 m. l. - Rhône, Francheville } \\ \text { (Chabert). C. BLxpranopylad Gdgr mss }\end{array}\right.$ Rami flor aculeati; ped. 7-11 m. 1. - Pyrenaei, Gèdre (Bordère) . . . . . 2606. C. tepinopHylla Gdgr mss

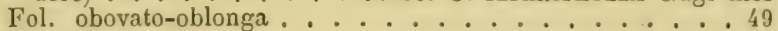

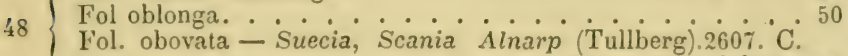
............. polyacastha Gdgr mss Stip. et pet. purpurea; ped. 3-5 mill. 1. - Hte-Savoie,

49 Mit-Sion (Puget). . 2608. C. LEucophona Gdgr mss Stip. et pet. virentia; perl. $8-10 \mathrm{~m}$. 1. - Suecia, Eland Tveta (Scheutz). . . . 2609. C. Mcropmylla Gdgr mss

$50\left\{\begin{array}{l}\text { Fol. apice subattenuata; fr. basi rotundatus vel depressus. . } 51 \\ \text { Fol. lanceolata; fr. basi attenuatus - Suecia, Scania Pine- }\end{array}\right.$ lierna (Tullberg). . . . . 2610. C. nIssinexs Gdgr mss

$51\left\{\begin{array}{l}\text { Ped. 5-10 m. 1. ; fr. ovoideus - Rhône, Craponne (Cariot). } \\ \text { a 2611. C. CEnrs Gdgr mss }\end{array}\right.$ Ped. 11-12 m. 1.; fr. obovato-oblongus - Finlandia, Abo (Hollmen). . . . . . .2612. C. ABoensis Gdgr mss

$52\left\{\begin{array}{l}\text { Styli villosi vel lanati. } \\ \text { Styli glabri - Hte-Savoie, La iandallaz (Puget).26i3. C. }\end{array}\right.$

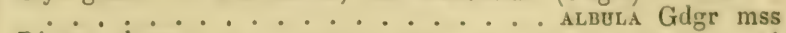

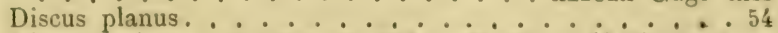

53 Discus conicus - Bouches-du-Rhone, Vartigues (Autheman). ................ C. olivetorum Gdgr mss 
Fol. oblonga ........................ 55

Fol. obovato-oblonga; ped. $10-12 \mathrm{~m}$. i. - Vallesia, Les $\dot{V}$ il-

lettes (Lagger). . . . . . 2615. C. supracana Gdgr mss

Fol. obovata ; ped. 6-8 m. 1. - Hung., Korenic (Kit.).2616.

. . . . . . . . C. intermedia (Kit.)

55 Ped. 10-15 m. l.; fol. mediocria. . . . . . . 56

Ped. $20-27 \mathrm{~m}$. l.; fol. $40-45 \mathrm{~m}$. longa - Rhône, Arnas

(Gdgr herb. ros. n. 256). . 2617. C. Melanophylla Gdgr mss

( Styli villosi ; sep. apice subdilatata - Isère, Charancieu (Gdgr.herb. ros. n. 91). • - . 2618. C. TRIstis Gdgr mss

Styli lanati; sep. apice foliacea - Suecia, Mt-Kinnekulle (Lundquist). . . . . 2619. C. Macrosepala Gdgr mss

Styli glabri vel subsparse hirtelli. . ....... 58

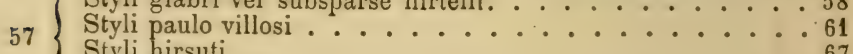

Styli hirsuti. . . . . . . . . . . . 67

Styli lanati . . . . . . . . . 72

58 Discus planus; pet. omnes inermes. . . . . . . 59

Discus conicus ; pet. | aculeati. . . 60

Fol. simpliciter serrata; rami flor inermes - Rhône, Dardilly

$59\left\{\begin{array}{l}\text { (Chabert) - . } \text { Fol. inf. biserrata; rami flor. aculeati - Serbia, Belgrade }\end{array}\right.$

(Pancic). . . . . 2621. C. BeLGRAdiensis Gdgr mss

Pet. omnes aculeati; fol. obovata - Algeria, Fort National

(Debeaux) . . . . . 2622. C. Kabylica Gdgr mss

Pet. sup. inermes; fol. oblonga - Gard, Cauvalat (Tuezkiewicz)...... 2623. C. DEnticalycina Gdgr mss

Pet. mediani tantum aculeati; fol. obovato-oblonga - Tyrol,

Pine (Gelmi) . . . . . 2624. C. Nigritella Gdgr mss

61

Fol. basi rotundata. . . . . . . . . . 62

Fol. basi attenuata. . . . . . . 64

62 Rami flor aculeati; fol obovato-oblonga vel oblonga. . 63

Rami flor. inermes; fol. obovata - Austria, Znaim (Oborny).

... . . . 2625. C. Moesta Gdgr mss

Pet. omnes aculeati ; fol. oblonga - Rhône, Montmelas (Gdgr).

Pet. omnes inermes; fol oblonga - Pyrenaei, Gèdre (Bordère) . . . . . 2627. C. Xatartiana Gdgr mss

Pet. inf. inermes; fol. obovato-oblonga - Rhône, Arnas (Gdgr herb. ros. n. 42) . . . . 2628. C. Onites Gdgr mss

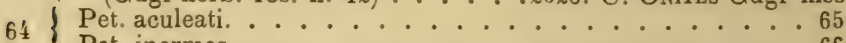

Pet. inermes.

Discus conicus; fol. oblonga - Gallia, Gde-Chartreuse (Gdgr).

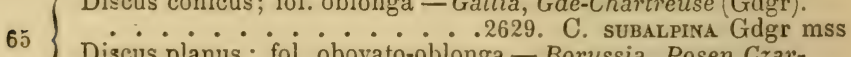

Discus planus ; fol. obovato-oblonga-Borussia, Posen Czarnikau (Hülsen). . . . . 2630. C. parvula Gdgr mss

Ped. 5-9 m. 1.; fr. utrinque attenuatus - Hte-Savoie, Brenthonne (Puget). . . . . . 2631. C. PRAsina Gdgr mss

Ped. 12-15 m. l.; fr. basi depressus apice longissime constrictus - Bouches-du-Rhône, Martigues (Autheman). 2632.

. . . . . . . RHYNchantha Gdgr mss

Ped. 17-20 m. l.; fr. utrinque attenuatus - Pyrenaei, Gèdre

(Bordère) . . . . . . 2633, C. obruta Gdgr mss

Fol. basi attenuata. . . . . . . . . 668

Fol. basi rotundata .................. 69 
Pet. incrmes ; fol. obovata - Isère, la Cüte-St-Andrè (Boullu).

Pet aculeati; fol oblonga - Rossia 2634. C. Oxycarpa Gdgr mss mann). . . . . . 2635. C Azovic Gaup-

Discus planus. .....................

Discus valde conicus. .............. 71

Fol. elliptica; fr. utrinque valde attenuatus - Rhone, Dar-

Fol. obovata; ir. utrinque parum attenuatus - Italia or. Faenza (Caldesi). . . . 2637. C. Capnotricha Gdgr mss

Rami flor. et pet. toto aculeati - Bouches-du-Rhône, Rognac

71 (Autheman) . * * : 2638. C. Reticulata Gdgr mss Vitrolles (Autheman)... 2639. C. Fusirera Gdgr mss

Fol. omnia biserrata. .............73

Fol. inaeque serrata ............ . 74

Fol. omnia simpliciter serrata. . . . . . . . 76

Stip. subtus glandulosø; ped. 5-6 m. 1. - Scolia, Braemar

Minime; ped. 12-14 m. 1. - Italia, Modène (Gibelli).2641.

. . . C. Bertoloniana Gdgr mss

$74\left\{\begin{array}{l}\text { Fol. basi attenuata; ped. } 7-9 \mathrm{~m} .1 . \\ \text { Fol. basi rotundata; ped. } 3-4 \mathrm{~m} .1 \text { - Suecia, Mt-Kinnekulle }\end{array}\right.$ (Zetterstedt). . . . . 2642. C. stexobasis Gdgr mss

Sep. apice angustata; dentes plerumque simplices - Suecia,

$75\left\{\begin{array}{l}\text { Smol., Moheda (Hyltén). 2643. C. Wahlenbergraxa Gdgr mss } \\ \text { Sep apice foliacea; ientes plerumque compositae - S }\end{array}\right.$ (Lundquist) 2644 C Cavptoct - Suecia,

TOCLADA Gdgr mss

76 Rami flor. inermes; discus conicus . . . . . . . . 77

Rami flor. aculeati; discus planus. . . . . . . 78

Pet. aculeati ; fol. ovato-elliptica - Hung., Albe (Tauscher).

-2645. C. TAuscheri Gdgr mss (Freyn)......2646. C. Tommasiviana Gdgr mss

Pet omnes aculeati. . . . . . . . . 79

Pet. omnes inermes . . . . . . . . . . 80

Aculei ad ramos flor. setacei; fol. acuta - Suecia, Mt-Kinne$79\left\{\begin{array}{l}\text { kulle (Lundquist) } \cdot \text { Minime; fol. lanceolata - Suecia, Eland Tveta (Scheutz). } \\ \text { Mr }\end{array}\right.$

Fol basi rotundata ....... 81 Fol. basi attenuata. . . . . . . . . 83

Fol. oblonga ................... 82 Fol. obovato-oblonga; ped. 7-9 m. 1. - Suecia, Mt-Kinnekulle (Lundquist) . . . . . . 2649. C. BICoLlis Gdgr mss Fol. obovata; ped. 5-7 m. 1. - Suecia, Wexio (Scheutz). .......... 2650. C. incrassans Gdgr mss Sep. apice foliacea; fol. apice rotundata - Suecia, Blek. Elleholm (Scheutz)......2651. C. Maxima Gdgr mss Sep. apice angustata; fol. acuminata - Helv., Outrerhóne (Favrat)........2652. C. Axceps Gdgr mss 
Fol. oblonga. ................. 84

Fol. obovalo-oblonga; fr. apice longissime attenuatus - Silesia, Breslau (Uechtritz). . . . .2653. C. crNerea Gdgr mss

Fol. obovata; Ir. apice strangulatus - Vallesia, Bornier (Wolf) . . . . . . 265'. C. Hirsutissima Gdgr Fol. omnia apice | acuta .......... 85

84 Fol. inf. obtusa, super, vero acutissima - Finlandia, Abo (Hollmén). . . . . . 2655. C. Finlandica Gdgr mss

$85\left\{\begin{array}{l}\text { Stip. rubentes; fr. basi subrotundatus - Rhône, Craponne } \\ \text { (Boullu) }\end{array}\right.$ Stip. virentes; fr. longe decurrens - Vallesia, Vollèges (Delasoie). . . . . . . 2657. C. Proxina Gdgr mss

Fr. globosus vel subglobosus. . . . . . . . . . 87

86 Fr. ovato-rotundatus vel ovatus. . . . . . . 88

Fr. ovoideus vel obovato-oblongus. . . . . . . . 92

Fr. oblongus . . . . . . . . . . 101

Fol. obovato-oblonga basi attenuata - Hte-Savoie, Belle-

87 vaux(Puget) ... . 2658. C. тивостсlA Gdgr mss Fol. elliptica basi rotundata - Anglia, Lancashire (Woods). ........2659. C. BRACTESGENS (Woods) Styli lanati ....................... 89 Styli hirsuti; pet. omnes inermes - Helv., Vaud Leysin (Favrat). . . . . . 2660. C. AlpINA Gdgr mss Styli subglabri ; pet. sup. aculeati - Pyrenaei, Gèdre (Gdgr herb. ros. n. 116) . . 2661. C. PORPHYRocarpa Gdgr mss

89 Rami flor. inermes; discus conicus . . . . . . . 90 Minime. . . . . . . . . . . . 91 Fr. apice valde strangulatus; fol acuta - Sabaudia, Salins

90 (Puget). . . . . . 2662. C. JoNides Gdgr mss Minime; fol. subobtusa - Pyrenaei, Perpignan (Debeaux). . . . . . . . . 2663. C. Reboudiana (Debeaux) Pet. inermes; fol. ovato-elliptica - Rhône, Arnas (Gdgr herb.

91 ros. n. 372) . . . 2664. C. PHYllochlora Gdgr mss Pet. aculeati; fol. oblonga - Germ. (C. silvestris Rchb exc. n. 3997). . . . . . . 2665. C. Altera Gdgr Fol. simpliciter serrata. . . . . . . 9 93

92 Fol. inf. valde biserrata - Algeria, Boghar (Debeaux). 2666. . . . . . . . . . . C. atlantica Gdgr mss Styli sparse pilosi. . . . . . . . . . . 94 Styli villosi . . . . . . . . . . . . . 996

93 Styli lanati .................. 97 Styli glaberrimi - Borussia, Posen Staykowo (Hülsen). 2667.

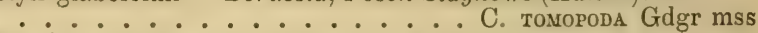

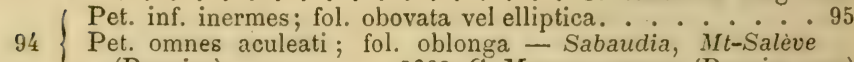
(Bouvier)... . . 2668. C. Monnetierensis (Bouvier mss)

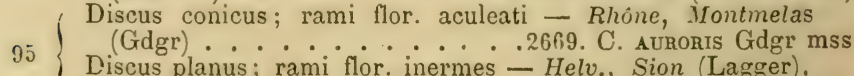
Discus planus; rami flor. inermes - Helv., Sion (Lagger). . . . . . . . 2670. C. constricta Gdgr mss

$96\left\{\begin{array}{l}\text { Pet. inermes; fol oblonga basi attenuata - Suecia, Scania } \\ \text { Nobbelof (Lundquist) } \\ \text { - . 2671. C. GLABrion Gdgr mss }\end{array}\right.$ Pet. aculeati ; fol. obovata basi rotundata - Pedem., Vallées Vaudoises (Rostan). . . . 2672. C. LANuginosa Gidgr mss 
97 Fol basi rotundata . . . . . . . . 98

Fol. basi attenuata. . . . . . . . 99

(Pet. inermes; fol. obovata - Basses-Alpes, Condamine

98 (Proal) . . . . 2673. C. machotnicha Gdgr mss Pet. aculeati ; fol. oblonga - Suecia, Mont-Kinnekulle (Zetterstedt). . . . . . 2674. C. ADENotricisa Gdgr mss

( Discus planus; rami flor. aculeati . . . . . . . . 100

Discus conicus; rami flor. inermes - Sabaudia, Salins (Puget) . . . . . . 2675. C. Semipilosa Gdgr mss (Pet. aculeati ; ped. 7-8 m. 1. - Suecia, Mt-Kinnekulle (Zet-

100 terstedt). . . . 2676. C. Kinsekullensis Gdgr mss Pet. inermes; ped. 3-5 m. 1. - Vallesia, Champex (Delasole).

Styli . . 2677. C. томоpHyLla Gdgr mss

Styli glaberrimi vel glabrescentes. ........ 102

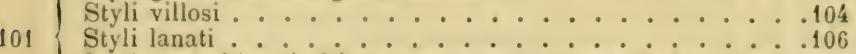

Styli paulo hirsuti; fol. supra aspero-punctata - Bouches-duRhóne, Rognac (Autheman). .2678. C. punctillata Gdgr mss

102 Dentes simplices; pet. aculeati . . . . . . . . 103

Dentes subbiserratae; pet. inermes - Pedem., St-Second (Rostan). . . . . 2679. C. stictochlamys Gdgr mss

( Rami flor. aculeati ; fol. oljovata - Belg., Dixmude (Crépin).

103 . . . . . 2680. C. poldemana (Crép. mss)

Rami flor. inermes; fol. oblonga - Borussia, Czarnikau (Hülsen). . . . . . . 2681. C. Hulsenir Gdgr mss

( Pet. inermes; fol. basi attenuata .........105

104 Pet. aculeati ; fol. rotundata - Pedem., St-Second (Rostan).

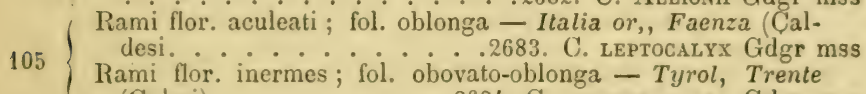
(Gelmi). . . . . 2684. C. CYLINDRocarPa Gdgr mss

(Pet. omnes inermes ; ped. 9-11 m. 1. - Suecia, Mt-Kinnekulle (Zetterstedt)..... 2685. C. suEcica Gdgr mss

106 Pet. omnes aculeati; ped. 7-8 m. 1. - Suecia, Mt-Kinnekulle (Zetterstedt).... 2686. C. Zetrenstedril Gdgr mss

Pet. inf. inermes; ped. 8-9 m.1. - Helv., Schaffouse (Lehmann). ...... 2687. C. scaphusiensis (Gremli mss)

\section{II. - Adexophorae Gdgr Essai p. 32. Canineae collinae et tomentellae Crép. Prim. p. 18.}

Foliola villosa, ad costam mediam saepius glandulosa, simpliciter vel biserrata. Sepala extus nune laevia nune normaliter hispida vel rubiginosa. Pedunculi (saltem in quibusdam individuis) semper villosi hispidi vel villoso-glandu- 
losi. Corolla rosea tunc ampla petalisque cordatis donata, aut albida vel ochroleuca parva, ergo petalis cordatocuneatis. Fructus glaber glandulosusve. Aculei robusti conformes, rarissime in setis prodeuntes. - Sectio sat abnormis speciesque inter se subdissimiles continens, et forsan ulterius aliter ordinaturae ; sed characteres firmiores hucusque non novi.

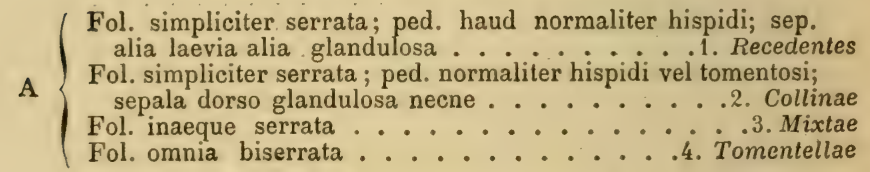

1. - Recedentes Gdgr mss.

Foliolis undique pubescentibus simpliciter serratis; pedunculis numquam normaliter hispidis; sepalis nunc laevibus nunc dorso plus minusve glandulosis; petalis albis vel carneis mediocribus cordatis, odorem moschatam ordinaire evidentius redolentibus. - Species subsectionis hujus eas Solstitialium (Trichophyll.) fere omnino referunt.

1 Fol. undique subtomentosa; sep. saepius glandulosa; cor.

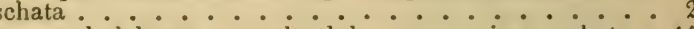

Fol. supra subglabra; sep. eglandulosa; cor. vix moschata . 14

Fr. globosus vel rotundatus. ........... 3

2 Fr. ovatus vel ovato-rotundatus ........... 4

Fr. oblongus . . . . . . . . . . . 6

Fr. ovoideus vel obovato-oblongus. . . . . . . 7

Styli glabrescentes; fol. obovata basi rotundata - Rhône, Montmelas (Gdgr herb. ros. n. 145).2688. C. LEUCOGRAPHA

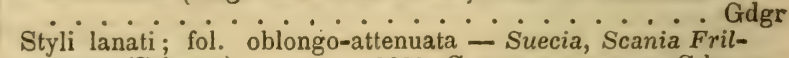
leslad (Scheutz). . . . 2689. C. Acanthothannos Gdgr mss

Tubus laevis ; pet. aculeati. . . . . . . . 5

4 Tubus apice hispidus; pet. inermes - Caucasus, Kutais (Radde). . . . 2690. C. transcaucasica Gdgr mss 
Fr. ovatus; sep. valde foliacea - Suecia, Mt-Kinnekulle

5 Fr (Zelterstedt) • - . . 2691. C. stephanomorpHa Gdgr mss r. ovato-rotundatus ; sep. angustata - Suecia, Mt-Kinnekulle (Lundquist). . . . . 2692. C, FLABELLATA Gdgr mss

$\left\{\begin{array}{l}\text { Styli hirsuti; fol. obovato-oblonga hasi attenuata - Rhóne, } \\ \text { Arnas (Gdgr). }\end{array}\right.$ $\left\{\begin{array}{l}\text { Arnas (Gdgr). } \text { Styli lanati; fol. oblonga basi rotundala - Suecia, Scania } \\ \text { Pinelierna }\end{array}\right.$ Pinelierna (Tullberg) . . . 2694. C. Cylindnica Gdgr mss

7 Pet. inf. inermes . . . . . . . . 8

Pet. omnes inermes . . . . . . . . . . 9 9

. . . . . . 11 (Gdgr herb. ros.n. 34 et 517 var.).2695. C. BAnBd-Jovis

Fol. elliptica basi rotundata Rhóne, ist-Ceindre (Chabert).

Fol. oblonga basi attenuata.

Fol. obovata basi rotundata - Suecia, Eland Thorsiund 10

(Scheutz) Horslunda

Ped. 4-5 m. 1. ; pet sup. glandulosi - Suecia Mt-Kinnehg mss (Zetterstedt) ; pet sup. glandulosi - Suecia, Mt-Kinnekulle

10 Ped. 8-9 m. l; pet. om 2698. C. Pulcma Gdgr mas kulle (Zetterstedt)

Fol. basi rotundata . . . 2699. C. DRYADEA Gdgr mss

11 Fol. basi rotundata . . . . . . . . . 12

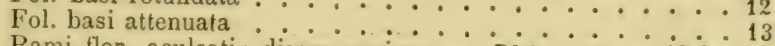

Rami flor aculeati; discus conicus - Rhone, Arnas (Gdgr

$12\left\{\begin{array}{l}\text { herb. ros. n. 233) discus planus - Rhone, Francheville } \\ \text { Rami flor. inermes; } \\ \text { (Chabert) }\end{array}\right.$

Iiscus conicus; rami flor. aculeati - Rhone, Chaponost

13 (Chabert).

Discus planus; rami flor. inermes - Suecia, Eland Tveta (Scheutz). . . . . . 2703, C. INTERTEXTa Gdgr mss

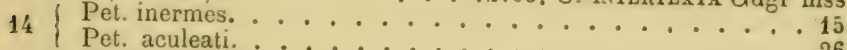

Pet. aculeati. . . . . . . . . . 26

15 Styli glabri

Styli villosi vel lanati . . . . . . 18

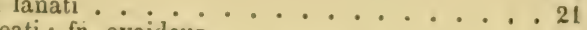

16 Rami flor inermes; fr. ovoideus . . . . . 17

Rami fr. oblongus - Rhóne, Arnas (Gdgr).

Discus cunicus; fol. elliptica basi rotu. C. GossYPINa Gdgr mss dilly (Chabert) $17\left\{\begin{array}{l}\text { dilly (Chabert) } \\ \text { Discus planus; fol. obovata basi attenuata - Rhône, A rnas } \\ \text { (Gdgr herb. ros. n. 316). . . 2706. C. DEFiciess Gdgr mss }\end{array}\right.$

Fr. oblongus

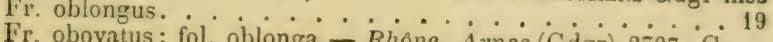

18

F. . . . e errabunda Gdgr mss

Fr. globosus; fol obovata - Rhone, Chaponost (Gdgr herb. ros. n. 504) basi attenuata . . .2708. C. variabilis Gdgr mss

$19\left\{\begin{array}{l}\text { Fol. obovala basi rotundata - Rhóne, Limonest (Chabert); } \\ \text { Arnas (Gdgr herb ros. } 20\end{array}\right.$ Arnas (Gdgr herb. ros. n. 532 var.).2709. C. Pachybasis Gdgr mss 
Fr. apice subattenuatus; fol. lata - Isère, St-Andié-le-Gaz

20 (Gdgr herb. ros. n. 167). . . 2710. C. DysGaulon Gdgr mss Fr. longe attenuatus; fol. angustata - Rhône, Arnas (Gdgr herb. ros. n. 361) . . . . 2711. C. podosticta Gdgr mss

21 Fol. basi rotundata. . . . . . . . . 22

Fol. basi attenuata. $: \ldots$

22 Fr. oblongus . . . . . . . . . . 23

Fr. ovoideus - Isère, Paladru (Gdgr).2712. C. vexata Gdgr mss

Discus paulo conicus; fol. ovato-elliptica - Rhône, Arnas

$23\{$ (Gdgr herb. ros. n. 85). . . . 27i3. C. stictopoda Gdgr

Discus planus; fol. obovato-oblonga - Suecia, Halland Halmstad (Scheutz) . . . 2714. C. Axgustispina Gdgr mss

Fol. oblonga basi attenuata. . . . . . . 25

24 Fol. obovata basi truncata - Rhône, Francheville (Chabert).

$\left\{\begin{array}{l}\text { Fr. et fol. apice subrotundata }- \text { Rhône, Arnas (Gdgr herb. } \\ \text { ros. n. } 223 \text { et } 223 \text { bis var.).2716. C. Tholeyroniana Gdgr mss }\end{array}\right.$

25 Fr. et fol. apice longe attenuata - Rhône, La Tour-de-Salvagny (Chabert). . . . . 2717. C. PILIFERA Gdgr mss

I Styli hirsuti vel lanati . . . . . . . 27 Styli glabrescentes - Rhône, Arnas (Gdgr herb. ros. n. 335 ).

$27\left\{\begin{array}{l}\text { Fol. subtus toto hirsuta. } \\ \text { Fol. subtus ad costam villosa }- \text { Asia occ., Laristan } \\ \text { C. macro- }\end{array}\right.$ Fol. subtus ad costam villosa carpa Boiss, non Mér.) . . . . .2719. C. stupenda (Crép.)

28 Minime; fol ovata. : * - - * - 29 Aculei sup. setacei ; fol. obovata - Rhône, Francheville (Chabert) . . . . . 2720. C. Adulterina Gdgr mss

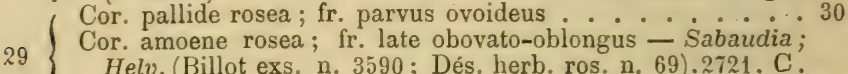
Helv. (Billot exs. n. 3590 ; Dés. herb. ros. n. 69).2721. C.

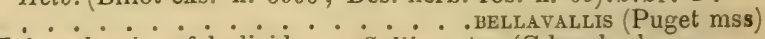

$30\left\{\begin{array}{l}\text { Tubus laevis; fol. livida - Gallia, etc. (Gdgr herb. ros. } \\ \text { n. } 507 \text { ). } \\ \text { Tubus basi hispidus ; fol. saturate viridia - Creuse, Grand- }\end{array}\right.$ Bourg (De Cessac). . . . . 2723. C. CEssacir Gdgr

2. - Collinae Crép. Prim. p. 18; ex parte ; Gdgr mss.

Foliolis pubescentibus, saepuis magnis, simpliciter serratis; pedunculi normaliter glandulosi hispidi vel tomentosi; sepalis dorso glandulosis necne; petalis amplis cordatis plerumque amoene roseis haud aut vix olentibus. 
$2 \int$ Styli hirsuti vel lanati ................. 3 Styli glabri - Isère, I'ortes (Boullu).272\%. C. Iseriexsis

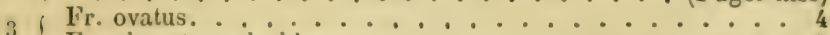

Fr. obnvatus vel oblongus. .......... . . 5 Aculei compresso-falcati ; fol. ovata - Austria.2725. C. Cor-

Aculei cylindrico-inclinati ; fol. rotundata - Cauc. LiNa (Jacq subspont. (Gidgr herb. ros. sine num. an. 1870).2726. C . . . . . . . ALBA (L.)

Pet. inermes. ............. . . 6

Pet aculeati. . . . . . . . 7

Uiscus planus; fol. ovata - Sabaudia, Moutiers (Puget).

Discus . . 2727. C. TRicholdea (Rip.) fol. obovato-elliptica - Gallia, Annecy (Puget). . . . . . 2728. C. PIIAlacnochlamys Gidgr mss Fr. et fol. obovata - Rhone, Craponne, etc. (Gdgr herb. ros.

7 n. 251 et 516) .......2729. C. oxcopiryla Gdgr Fr. et fol. oblonga - syria, Antiliban (Kotschy exs. n. 150),

Fr ovatus - . . 2730. C. Schergina (Boiss.) Fr. ovoideus vel obovato-oblongus. . . . . 13

Fr, oblongus .............. 16 Fr. globosus - Pyrenaei, Vénasque (Timbal).2731. C. CERA-

Styli hir . . SIfera (Timb.)

Styli huli vel lanati; aculei conformes. . . . . . . 10

Styli hirsuti; aculei dimorphi - Loire-Inf., St-Sébastien

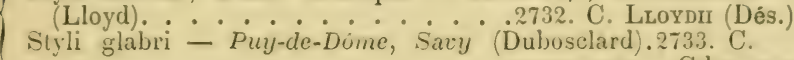

proteaefolia Gdgr mss

Tubus toto hispidus ............ 11

Tubus basi hispidus; fol. basi rotundata - Dania, Helleboek

(Lundquist). . . . 2734. C. tentaculata Gdgr mss

Tubus toto glaber; fol. attenuati - Austria; Galicia.2735.

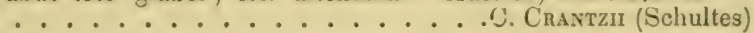

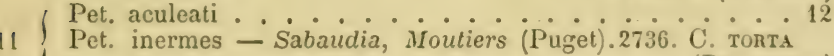

Pet. inermes - Sabaudia, Moutiers (Puget).2736. C. ToRTA

(................ (Puget mss)

Cor. purpurea; rami flor. inermes - Transs., Kronstadt, etc.

12 Cor. rosea ; rami flor. aculeati - Suecia med. et mer., etc.

2738. C. Clivonum (Scheutz)

Tubus toto hispidus .............. 14

Tubus basi hispidus; pet. inf. inermes - Arriège, Mijanès

13 (Gautier). . . . . . . 2739. C. Gautien Gler mss

Tubus glaber ; pet. omnes aculeati - Pyrenzei, Gèdre Bordère) ......... 2740. C. Leiocarpa Gdgr mss

Pet. omnes aculeati; fol. basi attenuata - Suecia or.; Germ. 15

14

(Rehb exc. n. 3996) . . . . . 2741. C. CAMpestris (Sw.)

Pet. inf, inermes; fol. basi rotundata - Dania, Sgalt Mark

(Zahrtmann)........2742. C. Echidne Gdgr mss 


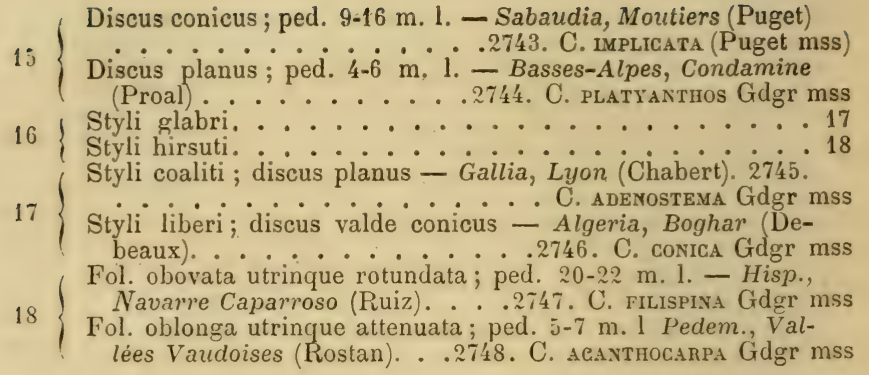

\section{3. - Mixtae Gdgr mss.}

Foliolis pubescentibus magnitudine mediocribus, infimis biserratis, superioribus vero simpliciter serratis; pedunculis normaliter glandulosis vel hispidis; sepalis dorso glandılosis vel non; petalis sat parvis albis vel pallidis.

1 Minime. . . . . . . . . .

Sep. dorso glandulosa . . . . . . . . . . . 8

Fr. globosus .............. 3

2 Fr. ovatus. $\ldots \ldots \ldots \ldots$

Fr. ovoideus - Rhône, Francheville, etc. (Gdgr herb. ros

n. 122 et 588)............ 2749. C. ERIogyna Gdgr

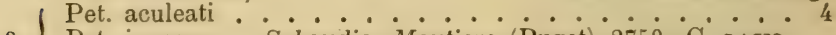

3 Pet. inermes - Sabaudia, Moutiers (Puget).2750. C. LAsio-

Fol. rotundata ; ped. sublaeves - Gallia centr. .2751.C. OB$4\left\{\begin{array}{l}\text { Fol. obovata; ped. hispidi - Anglia, Yorkshire.2752. } \\ \text { C. Watsoni (Baker) }\end{array}\right.$

(Aculei conformes; styli hirsuti . . . C. WATson (Baker)

5 Aculei dimorphi; styli glabrescentes - Gallia, Angers (Boreau) . . . . . . 2753. C. Fallaciosa (Dés.)

( Pet. omnes aculeati; fol. elliptica - Gallia centr. et mer.

6 . . . . . . . 2754. C. Nova Gdgr

Pet. inf. inermes ; fol. obovata - Rhône, Charbonnières (Boullu) ... 2755. C. obtusifolio-tomentella (Puget mss)

( Rami flor. inermes; ped. 16-18 m. 1. - Helv., Zurich

7 (Lehmann) . . . . 2756. C. Calochlona Gdgr mss Rami flor. aculeati ; ped. 6-8 m. 1. - Rhône, Charbonnières (Boullu) . . . . . 2757. C. DASYcalyx Gdgr mss 


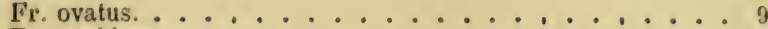

Fr. ovoideus ............................

Fr. oblongus .................. 14

Fr. rotundatus - Delph. etc. . . . 2758. C. arcana (Dés.)

Fr. magnus basi hispidus . . . . . . . . 10

9 Fr. parvus glaber - Caboul, Erak (Griffiths).2759. C. CAnu-

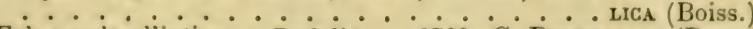

10 Fol. ample elliptica - Podolia. . 2760. C. Boneykiana (Bess.) Fol. mediocriter ovata - Podolia.2761. C. Ratomsciana (Bess.)

11 Aculei dimorphi. ................. 12 Aculei conformes . . . . . . . . . . . . 13

Tubus glaber; discus planus - Podolia.276?. C. nusmus (Bess.)

12 Tubus hispidus; discus conicus - Austria, Znaim (Baenitz herb. eur. an. 1878). . . . .2763. C. OBunxy Gdgr mss Pet. omnes aculeati; fol. obovata - Suecia, Blek. Lyckeby $13\left\{\begin{array}{l}\text { (Svanlund). } ; ~ \\ \text { Pet. inf. inermes; ovato-elliptica - Anglia, Yorkshire }\end{array}\right.$ (Baker). . . . . . . . 2765. C. Incerta (Dés.)

Pet. omnes inermes . . . . . . . 15

Pet. omnes aculeati ; fol. ovato-rotundata - Affghania, Kalou

14 (Griffiths).......2766. C. Latispina (Boiss.) Pet. inf. inermes; fol. obovata - Basses-Alpes, Condamine (Proal). . . . . . . 2767. C. Chaixiana Gdgr mss (Ped. 10-15 m. l.; ped. et tubus glandulosi - Sabaudia, Mou$15\left\{\begin{array}{l}\text { tiers (Puget) } \text { Ped. et tubus aculeati - Ilelv., Uri (Gisler) } \\ \text { Ped. } 5-10 \text { m. 1.; ped }\end{array}\right.$ .2769. C. Rigidula (Lagger et Puget mss)

\section{4. - Tomentellae Crép. Prim. p. 20 ex parte; Gdgr mss.}

Foliolis pubescentibus, coriaceis nervosis plerumque parvis omnino argute et crebre biserratis; pedunculis, in $\mathbf{1 4}$ specibus sequentibus, hispido-glandulosis, in sequentibus vero plus minus villosis; sepalis plerumque laevibus; petalis parvis ex albo ochroleucis.

Ped. normaliter et dense glandulosi. . . . . . . 2 Ped. glabri villosi vel $1-1-$ glandulosi (grex C. tomentellae

Lem.) . . . . . . . . . . . 13

Fr. globosus vel rotundatus ........... 3

Fr. ovatus ................. 5

Fr. ovoideus vel obovato-oblongus . . . . . . . . 8

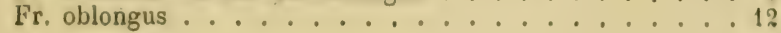


Styli glabri vel glabrescentes; tubus hispidus ....... Styli lanati; tubus laevis - Rossia, Podolia.2770. C. FrIEDFol ovato-elliptica; styli glabri - C . Landeriana (Bess.) Fol. elliptica ; styli paulo hirsuti - ${ }^{\circ}$ Guta (Mus. Pursch.) - Tauria.2772. C. Biebers.... teiniana (Tratt.)

5 ( Tubus hispidus ............. Tubus glaber ................ . . 7 ( Discus conicus; fol. obovata - Gallia, Chambéry (Songeon)

6 . . . . . 2773. C. Songeana (Puget mss) Discus planus; fol. ovata - Tauria.2774. C. SAXatilis (Stev.) Aculei dimorphi ; fol. ovata - Sarthe, Le Mans.2775. C. MA-

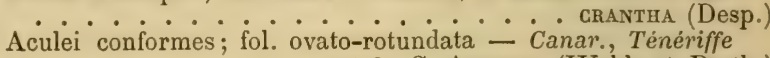
fol. ovato-rotundata - Canar., Ténériffe

Aculei dimorphi. . . . . . . . . . . . . . Aculei conformes . . . . . . . . . 10 ( Tubus glaber; fol. basi attenuata - Rossia, Podolia mer.

9 T $\because \cdots \cdots$ 2777. C. oвodotvskiensis Gdgr Tubus hispidus; fol. basi rotundata - Silesia, Breslau (Ansorge, etc.) . . . . 2778. C. КаватH (Ansorge mss)

$10\left\{\begin{array}{l}\text { Fol. obovato-oblonga basi attenuata. } \text { Fol. oblonga basi rotundata }- \text { Breslau (Krause) } \\ { }^{11}\end{array}\right.$ . . . . . . . . 2779. C. KrausiI Gdgr mss Cor. ciliata ; discus planus - Scotia, Perthshire (Drummond) $11\left\{\begin{array}{l}\text { Cor. eciliata ; discus conicus - Hte-Savoie, St-Martin (Puget) } \\ -2781 \text {. C. chlamyosticta Gdgr mss }\end{array}\right.$ Styli glabri; discus conicus - Hisp., Murcia (Cordoniu) $12\{$ Styli hirsuti; discus planus - Rhône, Francheville (Gdgr herb. ros. n. 423). . . . 2783. C. Roffavieri (Chabert) Fol. ovato-rotundata vel ovata .......... 14

13 Fol. elliptica vel elliptico-ovata ........... 23

Fol. obovata obovato-oblonga vel oblonga. . . . . . 35

Fr. globosus ............... . . 15

Fr. ovatus ..................... 17

14 Fr. ovoidéus ................. 19

Fr. ovato-rotundatus - Rhône, Tassin (Chabert). 2784.

. . . . . . . . C. obtusiuscula Gdgr mss

Pet. omnes aculeati ............. 16

Pet. et rami flor. omnes inermes - Rhône, Montmelas (Gdgr

15 herb. ros. n. 204). . . . 2785. C. Litterata Gdgr mss Pet. inf. inermes; rami flor. aculeati - Helv., Fribourg (Cottet). . . . 2786. C. Schneideri (Cottet et Pug. mss) Styli glabri; discus planus - Gallia, Helv. ete.2787. C. AMStyli hirsuti; discus paulo conicus - Europa media.2788.

Fol. apice obtusata vel subrotundata $\because$ C. Torentella (Lem.)

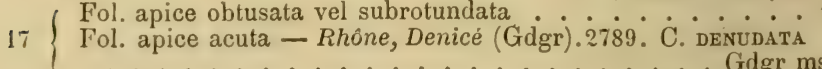
Gdgr mss 
Discus conicus; fr. basi depressus - Rhône, Arnas (Gdgr herb. ros. n. 87) . . . 2790. C. ARAchNordea Gidgr mss Discus subplanus; fr. basi attenuatus - Aude, Conques (Timbal) . . . . . 2791. C. Nervosa (Crép. mss)

Pet. inf inermes . . . . . . . . 2 20

19 Pet. omnes aculeati 21

Pet. et rami flor. omnes inermes - Rhône, Tassin (Cariot)

Styli *.... . 2792. C. Despicata Gdgr mss Styli glabrescentes; fol. apice rotundata - Rhône, Arnas

20 (Gdgr). . . . . . 2793. C. nololeuca Gdgr mss Siyli hirsuti ; fol. subacuta - Ilelv., Neuchtitel (Jacol) in soc. helv. exs. 1s74). . . . . . 2794. C. JACOBI Gdgr

Discus planus. . . . . . . . . . . . 22

Discus paulo conicus; fol apice subattenuata - Rhône, Alix

21 (Gdgr herb. ros. n. 157). .2795. C. ocmnolevcoides Gdgr mss Discus conicus; fol. obtusa - Rhône, Tassin (Cariot). 2796.

. . . . . . . . C. synantra Gdgr mss Cor. alba; fol. acuta - Rhóne, Gleizé (Gdgr herb. ros. Cor. pallide rosea ; fol. obtusa - Gallia, Autun; Angl. mer.

Fr. ovatus vel ovato-rotundatus - 2798. C. Lucanduna (Dés.

$23\left\{\begin{array}{l}\text { Fr. ovatus vel ovato-rotundatus. } \\ \text { Fr. ovoideus vel obovato-oblongus } \\ \text { Fr. }\end{array}\right.$ Fr. globosus vel rotundatus - Rhône, Gleizé (Gdgr herb. ros n. 72). . . . . 2799. C. INTERsita Gdgr

21 Styli hirsuti; discus planus. - A ros. n. 235). . . . . . 2800. C. stilnocirp. Gdgr mss

Rami flor, et pet. omnes aculeati . . . . . . 26

Rami flor. inermes; pet. aculeati - Rhône, Tassin (Cariot) Rami flor. aculeati ; pet. inermes - Bohemia, Prague (Opiz) . . . . . . 2802. C. IImbenRandtir (Weitenw.) Pet. glabrescentes; fol. ampla - (iallia, Angers (Boreau) $21 ;$ Pet. hirsuti; fol. mediocria - Bohemia, Mt-S.-Laurent Opiz)

Pet inf in . . . . . . . . 28

28 Fol basi|attenuata. . . . . . . 29

28 Fol basi rotundata $\ldots 30$ Styli glabri; ped. 15-18 m. 1. - Puy-de-Dóme, I'ionsat

$29 !$ (Gdgr herb. ros. n. 29) . 2805. C. PIAGAaloides Gidgr mss Styli villosi; ped. $7-12 \mathrm{~m}$. 1. - Itte-Savoie, Pring!l (Puget) . . . . . . . 2806. C. Nematella Gdgr mss Ped. 11-13 m. 1.; fr. apice longe constrictus - Anglia, York

(i1) (Webster) . . . 2807. C. Benthamina Gdgr mss Ped. 6-8 m. 1.; fr. apice subdepressus - Anglia, Survey (Groves). . . . . . 2808. C. sunutifolia Gdgr miss Diseus conicus. . . . . . . . . . . . . . . . Discus planus. . . . . . . . . . . . 33 Rami flor inermes; fol. apice subattenuata - Sabaudia, St$\therefore$ i Rami flor aculeati; fol. apice subrotundata - IIte-Savoie, Argonnex (Puget).. . . . 2810. C. мitoacantha Gidgr miss 
Styli villosi; fol. basi attenuata. . . . . . . . 34 Styli glabrescentes; fol. basi rotundata - Gironde, Barsac (Motelay). . . . . . . 2811. C. Bellula Gdgr mss

$34\left\{\begin{array}{l}\text { Fol. ampla supra glabra et lucida }- \text { Hung., Hradek (Fritze } \\ \text { et Ilse). }\end{array}\right.$ Fol. parva supra hirsuta et virentia - Rhône, Brignais (Chabert). . . . . . . 2813. C. pulvinaris Gdgr mss Fr. rotundatus vel globosus. . . . . . 36

35 Fr. ovato-rotundatus ovatus vel ellipticus. . . . . 39 Fr. ovoideus vel obovato-oblongus. . . . . . . . 44 Fr. oblongus. . . . . . . . . . . 5 53

36 Fol. obovato-oblonga; ped. villosi. . . . . . . 37 Fol. oblonga; ped. glabri - Anglia, Northumberland.2814

Fo. . . . C. celerata (Baker)

37 Fol. basi paulo attenuata - Rhône, Ecully (Chabert). 2815. . . . . . . . . . C. lobulata Gdgr mss Styli hirsuti ; ped. 13-15 m. 1. - Anglia, Whitace Junction

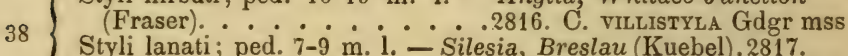
Styli lanati ; ped. $7-9$ m. 1. - Silesia, Breslau (Kuebel). 2817.

Fo

$39\{$ Fol basi rotundata ........... 40

Fol. basi attenuata. . . . . . . . . . 4 42

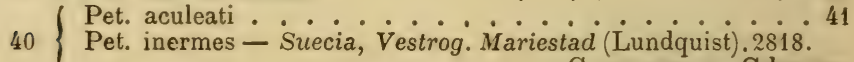

( . . . . . C. Mucronata Gdgr mss

$41\left\{\begin{array}{l}\text { Ped. villosi; fol. obovato-oblonga - Rhône, Arnas (Gdgr } \\ \text { herb. ros. n. 22).. 2819. C. PSEUDo-octroleucoIdes Gdgr mss }\end{array}\right.$ Ped. glabri ; fol. obovata - Caucasus.2820. C. CAuCASICA (II. B.)

$42\{$ Styli I villosi; discus planus. . . . . . . . 43 Styli glabri; discus coninus - Rhône, Fontaines (Chabert) . . . . . . 2821. C. cosmocarPa Gdgr mss

( Rami flor aculeati ; fr. ovato-ellipticus - Anglia, Warwich

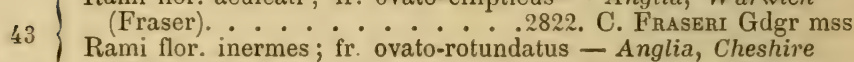
(Harbord). . . . . . . 2823. C. INDECORA Gdgr mss

( Styli glabri. . . . . . . . . . 45

44 Styli paulo villosi . . . . . . . . . 46

Styli hirsuti vel lanati . . . . . . . . 49 Rami flor. et pet. aculeati - Italia or., Faenza (Caldesi) $45\left\{\begin{array}{l}\text { Rami flor. et pet. aculeati - Italia or., Faenza (Caldesi) } \\ \text { Rami flor. inermes; pet. subinermes - Anglia, York }\end{array}\right.$ (Webster) ....... 2825. C. stenocoma Gdgr mss

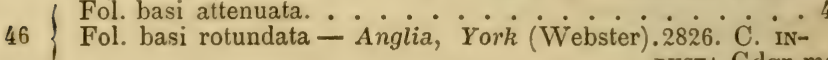

Rami flor inermes; pet. inf inermes . . DUCta Gdgr mss

47 Rami flor. et pet. omnes aculeati - Anglia, Cheshire (Harbord). . . . . . . 2827. C. Harbordir Gdgr mss

$48\left\{\begin{array}{l}\text { Fol. magna; ped. 8-10 m. 1. - Puy-de-Dôme, Savy (Gdgr } \\ \text { herb. ros. n. 94) }-2828 \text {. C. AvEllanaEfolia Gdgr mss }\end{array}\right.$ Fol. parva; ped. 4-6 m. I. - Loire, Sauvain (Gdgr).2829. 


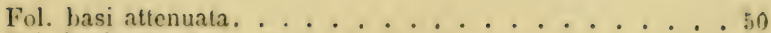

Fol. basi rotundata; discus planus. . . . . . . 51

Fol. basi rotundata; distus conicus - Rhöne, Arnas (Grigr)

. * . . 2830. C. MEgACANTHA Gdgr mss

Pet. aculeati; ped. 10-15 m. 1. - Rhône, Charbonnières (Cha-

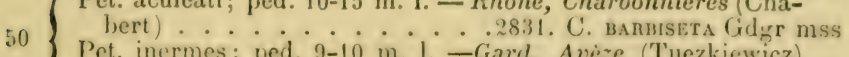

Pet. inermes; ped. $9-10 \mathrm{~m}$. 1. -Garel, Aveze (Tuezkiewicz) . . . . . . 2832. C. Lonetiana Gidgr mss

5 Discus planus vel subplanus; rami flor. aculeati . . . . 52

Discus conicus; rami flor. inermes - Isere, Charvieux (Boullu) . . . . . 2833. C. oxyBasis Gdgr mss

52 ( Ped. glabri; fol. mediocria - Anglia.2834. C. cilraris (Wilson) Ped. villosi; fol. ampla-IIanovre (Zabel).2835. C. Exsinfiess

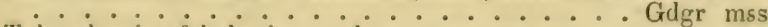

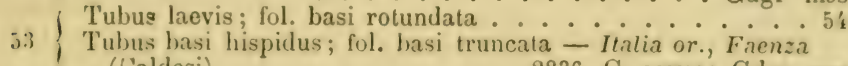
(Ualdesi). . . . . . . 2836. C. comosa Gdgr mss Discus conicus; styli hirsuti - Rhine, Amas (Gdgr herb.

34 ros. 11. 49) . . . . 2837. C. squannosuli Gidgr mss Discus planus; styli glabrescentes - Italia or., Faenza (Caldesi)........2838. C. Amabilis Gdgr mss

Subgenus IX. - Chavinia Gdgr Essai p. 34 (in sect.); Glandulosae Crép. Prim. mon. ros. p. 21.

Styli liberi, villosi lanati vel glabri, ultra diseum planım in capitulum sessile producti. Flores bracteati, rosei purpurei rarius albi, corymbosi, petalis amplis ad unguem glabris praediti. Fructus praecox, coriaceus, magnus, erectus, intense ruber, saepe glandulosus. Calycis laciniae (sepala) extus normaliter glandulosae, 2 integrae 3 late pinnatipartitae, post anthesim patulae dein reflexae tandem deciduae. Pedunculi erecti, hispido-glandulosi, interdum laeves. Folia imparipinnata, elongata, corum foliola ampla, coriacea, amoene virentia, subtus ad nervos saltem glandulosa, cum pube plus minus brev suffulta vel glabra, nervi prominule reticulati; serraturae late ef creherrime biserrato-glandulosae. Stipulae conformes dilatatae elongatae, ad margines renticulatae, dorso rubiginosae vel non. Aculei sparsi, nune. 
conformes, scilicet, falcato-compressi et dilatati, nunc vero dimorphi, hoc est in setas glanduliferas prodeuntes.

Frutices in sepibus Europae praesertim mediae et australis passim crescentes, erecti, sociales, parum elati, flexuosi, ramis ex virente paulo glaucescentibus saepius conformiter aculeatis, floribus corymbosis radiceque haud (an semper?) reptante praeditis; vel suffrutices nani graciles solitarii, vix 1-2 pedales, in nemoribus silvisque caeduis Europae mediae praesertim occidentalis et ad summum orientalis raro inhabitantes, ramis flexuosis gracilioribus, aculeis tenuibus saepe glanduliferis, floribus magnis subsolitariis radiceque longe reptante insignes. - Plantae attritu odorem gratam fructus Mali saepissime redolentes.

Genus hocce D. Chavin, helvetico, qui, vitam non solum Rosis sed plantis Helvetiae occidentalis inspiciendo, consecravit.

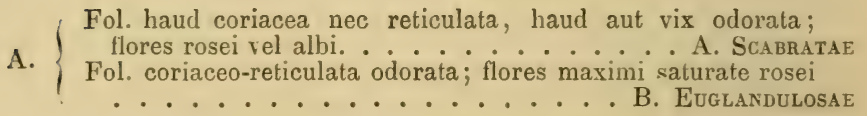

A. - Scabratae Gdgr Essai p. 34 (Canineae scabratue Crép. Prim. p. 21).

Foliola glabra villosave, haud aut vix reticulata, margine paulo crebre biserrato-glandulosa; sepala extus interdum eglandulosa; styli glabri aut villosi, ultra discum capitati vel fasciculati; corolla mediocris alba vel rosea; pedunculi glabri vel hispidi nonnunquam hirsuti; radix reptans in quibusdam. - Frutices plerumque sat elati, inodori, ramis 
rigidis aculeisque conformibus insignes, in tota Europa, hine inde sed ubique raro vel rarius crescentes.

Tribus, rhodologorum crux et scandalum, primo intuitu, in se valde abnormis, diagnosi generis fere nullo modo respondens et tamen huic certissime adnumeranda! Quamvis characterilus suis diversissimis singularis sit, ob glandas quae nervos inferiores obducunt, a variis Crepiniis Adenophoris (Canineae) speciebus quibus habitu, etc., sat accedere videtur, haec tamen apprime distinguenda. Nec cum Crepiniis (Canineae) adnunerari potest, ut existimavit (ne dicam somniavit) Cl. Crepin, propter folia subtus glandulosa, sed potius Chabertiis (Rubiginoseae) si, notis supra evolutis, tribus haec ab istis omnino distinctam prae se non feret.

1 Fol. plus minus hirsuta .............. 2

1 Fol. undique glaberrima ............ . 52

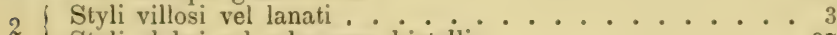

2 Styli glabri vel subsparse hirtelli. . . . . . . . . 37

3 Ped. hispidi vel glandulosi. . . . . . . . . . 4

3 Ped. laeves vel paulo villosi . . . . . . . . . 14

4 Tubus saltem inferne hispidus . . . . . . . . . 5

1 Tubus toto laevissimus. . . . . . . . . 10

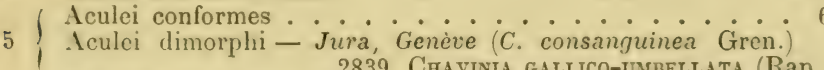

Fol. subtus ad costam mediam tantum villosa. . . . . 7

6 Fol. subtus ad nervos villosa ..........

\{ Fol. subtus toto villosa - Ilelv. Uri, Burglen (Gisler).2840.

........ Chavinia aceteserrata Gdgr mes

( Fr. ovoideus; fol. amplissima - Helv., Zurich (Lehmann).

$7\{$ Fr. oblongus; fol. angustata - Suecia media et austr.2842.

(Fr. ovoideus. CHAvinia sclenopHyLla (Scheutz)

Fr. oblongus ; pet. aculeati ; flos carneus - Gall, Bourges

s (Rip.) . . . . . . . 2843. C. vinetonum (Rip.)

Fr. oblongus ; pet. subinermes ; flos roseus - Gallia centr. etc. . . . . . 2844. C. Blondaledva (Dés. et Rip.)

| Rami flor inermes; fol. acuta - Alsatia, Soultzbach (Kirs-

9 chleger). . . . 2845. C. Wassermungensis (Kirschl.) Ninime; fol. subobtusa - IIelv. Fribourg, La Gottalaz (Cottet). . . . .2846. C. Dematranea (Lagger et Puget ms- 
Fol. supra glabra, subtus ad nervos vel toto hirsuta. . . . . 11

Fol. supra glabra, subtus ad costam hirsuta - Ins. Cyprus,

m. Troodos (Sintenis et Rigo plant. cypr. exs. n. 777).

ol. supra hirsuta, subtus giab - 2847. C. CYPRIA Gdgr mss Borgby (Scheutz).... 2848. C. sphaterica Gdgr mss

Fr. ovoideus vel obovato-oblongus. . . . . . . 12

Fr. oblongus ; rami flor. inermes - Naples, Ischia (Gussone).

Fr - - . * - . 2849. C. GussonII Gdgr mss

ovatus; rami flor. aculeati - Caucasus orient.2850. C.

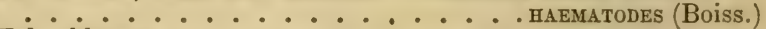

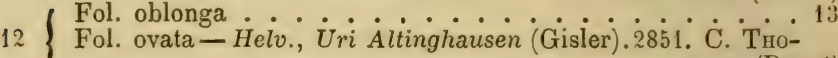

P. . . . . . . . . . PasI (Puget)

Ped. 9-12 m. l.; rami flor. inermes - Italia or., Faenza (Cal-

13 desi)..... 2852. C. PACHyPHylla Gdgr mss

Ped. 5-8 m. 1.; minime - Pedem., Vallées Vaudoises (Ros-

$\tan )$. . . . . 2853. C. valderia Gdgr mss

14 Fol. subtus ad costam vel ad nervos villosa. . . . . . 15

4 Fol. subtus toto vel undique villosa . . . . . . . . 24

15 Sep. dorso glandulosa . . . . . . . . 16

5 Minime. . . . . . . . . . . 19

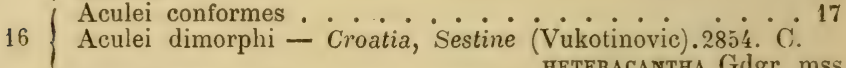

$17\left\{\begin{array}{l}\text { Fr. oblongus }- \text { Thuringia, Rockenholz (Wallroth) } 2855, C^{18} \\ \text { Fr. ovatus }\end{array}\right.$

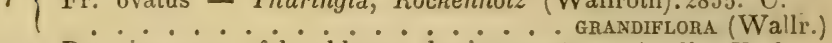

( Pet. inermes; fol. oblonga basi cuneata - Anglia, York

$18\left\{\begin{array}{l}\text { (Webster) } ; \text { Pet. aculeati ; fol. obovata basi rotundata - Helv., Vaud } \\ \text { Ps }\end{array}\right.$

Morcles (Favrat) . . . . . 2857. C. ALPESTRIs Gdgr mss

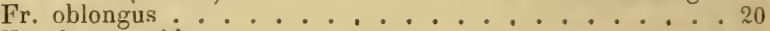

Fr. obovato-oblongus. . . . . . . . 22

Fr. ovatus - Suecia mer., ex hort. Lund (Tullberg).2858. C. Fr. globosus - Hung.; Hradek (Ilse).2859. C. Conjuncta

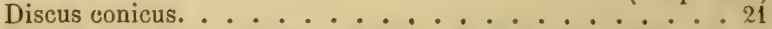

20 Discus planus - Croatia, Agram (Vukotinovic) 2860 . C.

. . . . . . . . . VuKotinovici Gdgr mss

$21\left\{\begin{array}{l}\text { Rami flor inermes; fr. angustatus - Suecia, Scania } m \text {. } \\ \text { Stenshufoud (Tullberg). }\end{array}\right.$

Minime; fr. latus - Anglia, York (Webster).2862. C.

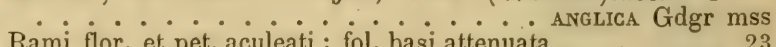

Rami flor. et pet. inermes; fol. basi rotundata - Tyrol, Lases in Pine (Gelmi). . . . 2863. C. Ambrosiana Gdgr mss

Sep. margine valde glandulosa; fol. angustata lanceolata -

Suecia, Scania Torup (Tullberg).2864. C. GLABREscers

Sep vix glandulosa fol lata subacuta - Anglia Gdgr mss (Webster) ........2865, C. Incerta Gdgr mss 
Aculei conformes; fr. ovatus . . . . . . . 25

Aculei conformes; fr. ovoideus vel obovato-oblongus . . . . 26

Aculei conformes; fr. oblongus. . . . . . . . 32 Aculei dimorphi - Naples, Ischia (Gussone).2866. C. INAnI-

Discus planus ; fol. oblonga basi attenuata - Anglia, York

(Webster). . . . . . 2867. C. ElEgans Gdgr mss

Discus conicus; fol. elliptica basi rotundata - Belg., Rochefort (Reliquiae Maill. n. 714) . . 2868. C. Crepisi Gdgr mss

Fol. basi rotundata. . . . 27

Fol. basi attenuata vel cuneata . . . . . . . 30

Rami flor. aculeati; fol, obovata vel obovato-oblonga . . . 28

Rami flor. inermes; fol. ovato-elliptica - Hte-Savoie, Pringy (Puget). . . . . 2869. C. Adenotrichia Gdgr mss

Dis'us subconicus; fol. obovata. . . . . . . . 29

.8 Discus conicus ; fol. obovato-oblonga - Rossia mer., Tscherkask (Laupmann) . . . . 2870. C. RutheNica Gdgr mss

Ped. 8-9 m. 1.; pet. aculeati - Angl., Cheshire Willaston

(Harbord L.) . . . . . . 2871. C. BRitannica Gdgr mss

Ped. 9-13 m. 1.; pet. subinermes - Hle-Savoie, Pringy (Puget). . . . . . 2872. C. sparsiglanda Gdgr mss

Discus planus ; fol oblonga. . . . . . . . 31

30 Discus conicus; fol. elliptica - Gall., Besançon (Billot exs. n. 3848). . . . . 2873. C. villosula (Paillot) Styli magni lanati ; ped. 10-13 m. 1. - Anglia, York (Webs31 ter). $\begin{aligned} & \text { Styli pubentes; ped. } 6-7 \mathrm{~m} .1 . \\ & \text { - Naples, Castallamare (Pas- }\end{aligned}$ quale) ........ 2875. C. stabiava Gdgr mss

32 Fol. basi rotundata. . . . . . . . . 33

Fol. basi attenuata. . . . . . . . . 34

Rami flor. aculeati ; pet. inf. inermes - Italia, Mantoue (GiRami flor. inermes; pet. omnes aculeati - Anglia, Surrey Barnes (Groves) . . . . . . 2877. C. MINon Gdgr mss

3 i Discus paulo conicus; rami flor. aculeati. . . . . . . 35

Discus planus; rami flor. inermes. . . . . . . . 36

Ped. 9-11 m. 1.; fol. ovato-elliptica - Italia or., Faenza Cal-

35 Petesi . . . . 2878. C. Coniacea Gidgr mss Ped. 14-15 m. 1.; fol. obovata - Italia or., Faenza (Caldesi). Styli lanati; pet, inermes - Suecia, Goteborg (Scheutz, Wins-

36 low)..........2880. C. Gotmica Gdgr mss Styli glabrescentes; pet. aculeati - Anglia, Yorks. Thirsk (Baker) . . . . . . 2881. C. oxycARPA Gdgr mss

\% 1 Ped. glabri . . . . . . . . . . . 38

37 Ped hispidi vel glandulosi. . . . . . . . . . 43

( Fol. suhtus ad costam tantum villosa . . . . . . . 39

38 Fol. subtus ad nervos plus minus villosa. . . . . . . 40

Fol. undique | hirsuta. ............. 42

Fr. rotundatus; fol. basi attenuata - Anglia, York (Webs-

39 ter). . . . . . 2882. C. Webstenir Gdgr mss

Fr. obovato-oblongus; fol. basi rotundata - Anglia, Leicestersh. Ashby (Ley) . . . . . 2883. C. GLaBnipes Gdgr mss 
Styli paulo villosi; fr. ovoideus vel obovato-oblongus. . . 41

Styli glaberrimi ; fr. oblongus - Tyrol, Hall (Gremblich). . . . . . . . . 2884. C. subalpina Gdgr mss Discus planus; fol. oblonga - Suecia, Vestrog. Branno

41 (Johanson)......2885. C. Macroacantha Gdgr mss Discus conicus; fol. obovata - Naples, Daunia Basilicata (Gussone) . . . . . . 2886. C. NEApolitana Gdgr mss

$42\left\{\begin{array}{l}\text { Fol. subbiserrata; sep. dorso laevia - Tyrol, Hall (Grem- } \\ \text { blich). . . . . . . 2887. C. HIRsuta Gdgr mss }\end{array}\right.$ Fol. biserrata ; sep. dorso paulo glandulosa - Istria, M.Maggiore (Freyn) . . . . 2888. C. Istriaca Gdgr mss Aculei conformes falcati ............ 4́

43 Aculei dimorphi recti setacei - Hisp. Murcia, Sierra de Espuna (Canada). . . . . 2889. C. Filispina Gdgr mss

6 Fol. supra glabra, subtus ad costam villosa...... 45

$\$ 4$ Fol. supra glabra, subtus ad nervos villosa . . . . . 47 Fol. supra glabra vel hirsuta, subtus toto villosa ..... 49

Sep. dorso glandulosa; fol. elliptica vel obovata . . . . 446

45 Minime; fol. ovato-elliptica - Aragonia austr., Sierra de Mosqueruela (Martin). . . . 2890. C. ARAGoNENsis Gdgr mss Fr. globosus; fol. basi attenuata - Croatia, Fiume (Kit.). . . . . . 2891. C. CROATICA (Kit.) Fr. oblongus; fol. basi rotundata - Gard, Arphy (Tuezkiewicz). . . . . . 2892. C. subintravs (Gren.)

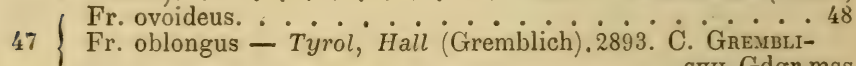
. . . . . . . . CHII Gdgr mss Rami flor. inermes; fol. basi rotundata - Austria, Znaim

48 (C. tomentella f. Obornyana Christ !).2894. C. Moravica

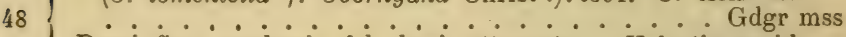
Rami flor. aculeati; fol. basi attenuata - Helvetia occid.

• . . . . . . 2895. C. concinna (Lagger et Pug.) Fr. oblongus ; fol basi attenuata. ....... 50

$49\left\{\begin{array}{l}\text { Fr. ohovato-oblongus; fol. basi rotundata - Hisp. mer., } \\ \text { Sierra de Orihuela (Canada) . .2896. C. Alicantra Gdgr mss }\end{array}\right.$ Fr. ovato-rotundatus ; fol. basi rotundata - Tyrol, Navis (Kerner). . . . . . 2897. C. TYRolensis (Kern.) Fol. oblonga

$50\{$ Fol. elliptica - Gallia, etc. (Dés. herb. ros. n. 24 ). 2898. C.

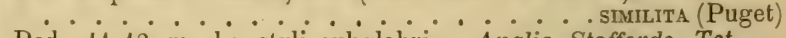
Ped. $11-12$ m. l.; styli subglabri - Anglia, Staffords. Tet-

51 tenhall (Fraser). . - 2899. C. RHYNCHOCARPA Gdgr mss Ped. 23-25 m. l.; styli glaberrimi - Anglia, Warwich Chesterton (Fraser) . . . 2900. C. Dichrophylla Gdgr mss

52 Ped. glabri.................. 53

52 Ped. hispidi.

53 Sep dorso paulo glandulosa . . . . . . . . . 54

Sep. dorso eglandulosa. . . . . . . . . . . 55

34 Discus conicus; fr. oblongus - Dalmatia, Val di Ombla Discus planus; fr. ovoideus - Suecia, Kalmar (Scheutz). . . . . . . 2902. C. suecica Gdgr mss Styli hirsuti jel i lanati. . . . . . . . . 56 55 Styli glaberrimi - Naples, Basilicata Potenza (Tenore!). 
56 , Fol. ovata basi rotundata ............ . . 57

Fol. obovata vel obovato-oblonga basi attenuata . . . . 58

Ped. 12-14 m. I.; fol. acuta - Bouches-du-Rhone, Roquefavour (Autheman). . ... . 2904. C. Mediternanea Gdgr mss

Ped. 1-6 m. 1.; fol. obtusa - Aragonia austr., Sierra de Mosqueruela (Martin). . . . . . 2!95. C. Hispanica Gidgr mss

( Discus conicus; fr. oblongus - Basses-Alpes, La Condamine (Proal). . . . . 2906. C. DELPHINENSIS Gdgr mss $\{$ Discus planus; fr. ovatus - Belg., Rochefort (Crépin).2907.

9 Sepala dorso eglandulo....... C. scabrata (Ćrép.) glandulosa. . . . . . . 60 60 Minimo ............... . 63 Fr. ovoideus vel obovato-oblongus; discus planus. . . . 661

60 Fr. oblongus; discus conicus. . . . . . . 62 Fr. ovoideus; discus conicus - Gall., etc. (C. depressa Gremli) . . . . . . . 2907 bis. C. Insidiosa (Rip.)

$61\left\{\begin{array}{l}\text { Pet. basi villosi ; ped. 11-13 m. 1. - Suecia, Blek. Nattraby } \\ \text { (Svanlund). . . . .2908. C. Scandisavica Gdgr mss }\end{array}\right.$ Pet. toto villosi; ped. 8-9 m. I. - Rossia mer., Tscherkask (Laupmann). . . . . 2909. C. CHersonensis Gdgr mss Fr. basi subdepressus; fol. lanceolata - Serbia, Belgrade

6. I (Pancic) . . . 2910. C. Belgnanexsis (Panc. mss) f Fr. basi attenuatus; fol. apice acuta - Suecia mer., Lund cult. (Tullberg). . . . . 2911. C. validil Gilgr mss

63 i Aculei dimorphi. .................... 64

Aculei conformes.

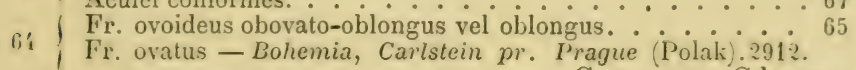

( Pet. et rami flor. aculeati; fol obovata. C. BoHemica Gdgr mss

65 Pet. et rami flor. inermes; fol.oblonga - Ilelv., Bále Sissach (Lehmann). . . . . 2913. C. Dimorphacaytua Gdgr mas Fr. obovatus; fol. basi attenuata - Ite-Savoie, Arenthon

661 (Puget) . . . . 2914. C. Microsticta Gdgr mss ( Fr. oblongus; fol. basi rotundata - Cher, Trouy (Pipart). ........... 2915. C. PROTEA (Rip.)

67 ( Fr. rotundatus. . . . . . . . 68

Fr. ovoideus vel obovato-oblongus. $\quad \ldots \quad \therefore 69$

Fr oblongus . . . . 81

68 Styli villosı; rami flor. aculeati - Savoie, etc. (C. viscida Styli subglabri ; rami flor. inermes - Pyren., Perpignan (Debeaux) . . . . . 2917. C. ocrmodora Gdgr mss

69 ) Styli glabri. . . . . . . . . 70 Styli villosi $\therefore 74$

Styli lanati $: \cdots 79$

70 Tubus laevis vel basi tantum hispidus . . . . . 71 Tubus toto hispidus . . . . . . . . 72 Discus planus; fol obovato-oblonga - Iisp. Murcia, Sierra

71 cle Carrascoy (Canada). . . 2918. C. Muncica Gdgr mss Discus conicus; fol. ovata - Helv., Sion, etc.2919. C. VALLE............... . SIAca (Lagger et Puget) $72\left\{\begin{array}{l}\text { Cor purpurea; suffrutex humilis - Alsatia, Ribeauville } \\ \text { (Süffert). }\end{array}\right.$

Cor. subalba; frutex elatus. . . . . . . . . 3 
Rami flor. inermes; fol. ovato-elliptica - Cher, Aude, etc. Minime; fol oblonga - Pyren., Perpignan (Debeaux).2922. ...................... PERdurans Gidgr

74 Tubus toto glandulosus.

74 Tubus laevis vel basi paulo glandulosus Fol. ovato-rotundata - Gallia, Bourges, etc.2923. C. DRYADEA

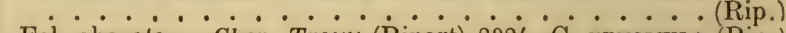
Fol. obovata - Cher, Trouy (Ripart).2924. C. NEMopHila (Rip.) Rami flor. aculeati; fol. basi rotundata . . . . . . 77

76 Minime; fol. basi cordata - Pyren. Perpignan (Debeaux)

Fol. acuta; fr. haud turbinatus . Perpignanensis Gdgr et Debx

77 Fol. obtusa; fr. turbinatus - Cher, Hautes-Aipes. $2926^{\circ}$

( Ped. alii glandulosi alii laeves; fr. apice attenuatus - Gallia

78 (Gdgr herb. ros. n. 424).2927. C. Titanophylla (Dés. et

Ped . . . . . . . . Rip. mss)

Ped. omnes parce hispidi; fr. nec attenuatus - Gallia,

Bourges, etc. . . . . . 2928. C. semiglandulosa (Rip.)

f Fol. basi attenuata. ......... 80

79 Fol. basi rotundata - Helv., Fribourg, etc. 2929. C. LAGGERI

(Rami flor aculeati; fol. obovata - Htes-Alpes, La Grave

80 (Reliq. Maill. n. 1082) . . . 2930. C. Monticola Gdgr mss Minime; fol. oblonga - Austria inf., Modling (Wiesbaur)

(Styli glabri. $\therefore \ldots . . . . . . .22$

81 Styli pubescentes ................... 83

Styli lanati .................. 84

$\left\{\begin{array}{l}\text { Rami flor. et pet. inermes; fol. oblonga - Cantal, Le Lioran } \\ \text { (Héribaud) }\end{array}\right.$

82 Rami flor. et pet. aculeati ; fol. obovata - Helv., Zermatt (C. sanguisorbifolia Delasoie in Christ Ros. der Schw. p. 181 non Donn). . . . 2933. C. Sanguisorbella Gdgr

$83\left\{\begin{array}{l}\text { Tubus in } 1 / 3 \text { inf. hispidus; fol. oblonga - Silesia, Breslau } \\ \text { (Kuebel) }\end{array}\right.$ Tubus laevis; fol. elliptica - Suecia, Blekingia Nattraby (Lundquist). . . . ......2935. C. LEPTOCLADA Gdgr mss

I Fol. lata; tubus | hispidus. .......... 85 Fol. minutissima; tubus laevis - Hispania austr., Sierra Nevada (Hegelmaier) . . . .2936. C. NEvadEnsis Gdgr mss

( Rami flor. et pet. inermes; fol. oblonga - Rossia austr.,

85 Tscherkask (Laupmann). . . 2937. C. Rossica Gdgr mss Minime; fol. ovato-elliptica - Pedem., Vallées Vaudoises (Rostan) . . . . 2938. C. PEdemontana Gdgr mss 


\section{B. - Euglandulosae Gigr Essai p. З̋̈ (Glandulosae Crép. Prim. p. 21).}

Foliola supra virentia lucida glabra rarissime villosa, sulstus toto sed prasertim ad nervos prominule reticulatos glandulosa, plus minusque hirsuta, copiose et creberrime marginato-biserrata, limbo saepius dilute purpureo; sepala magna, dorso semper hispido-glandulosa; styli villosi vel lanati, ultra discum planum sessiles conglobati; flos magnus eximie roseus vel purpureus, petalis obcordatis eciliatis ; radix saepe longe reptans. - Frutices vel suffrutices in ditione semper rari vel rarissimi, humiles, interdum soliIarii, attritu suaveolentes, ramis flexuosis subglaucescentilus, aculeisque aliis aduncis basi dilatatis, aliis vero in setas glanduliferas saepissime degenerantibus, insignes.

Fol. undique glaberrima ................ . 2

Fol. subtus ad costam mediam villosa . . . . . . . . 3

Fol. subtus ad nervos villosa.......... . . 10

Fol. subtus toto villosa. . . . . . . . . . . . 47

Ped. laeves; fol. ovata - Tyrol, Krems (Kerner; C. amoena

2 Kern. olim) . . . . . . 2939. C. DEcona (Kern.) Perl. hispidi; fol. oblonga - Gallia, Nancy (Millot). 2y'0

3) Fol. basi attenuata. . . . . . . . . . 4

Fol. basi rotundata vel subrotundata ......... 5

Fr. subglobosus; fol. oblongo-lanceolata - Rhône, Brindas $4\left\{\begin{array}{l}\text { (Chabert). } \text { Fr. obovatus; fol. subobtuse obovato-olionga - Rhóne, Dar- } \\ \text { Fr. }\end{array}\right.$ dilly (Chabert). . . 2942. C. Loxgepedixcllata Gdgrr mss Fr. ovoideus. . . . . . . . . . . . . 6

Fr. globosus vel subglobosus . . . . . . . . . . 7

Fr. ovotus - Rhône, Tassin (Boullu).2943. C. Flexiramea

Fr. oblongus - Tyrol, Krems, etc.2944. C. Reticulata (Kern.)

Tulus in 1/s inf. glandulosus; fol. obovato-elliptica -

6. Rhône, Denicé(Gdgr herb. ros. n. 324).2945. C. avgustata Gdgr Tubus toto glandulosus; fol. oblonga - Austria, Znaim (Oborny). ........2946. C. Obоnxy Gdgr mss 
Rami flor. aculeos glanduliferos gerentes. . . . . . 8

Minime .................... 9

Fol. oblonga; fr. basi glandulosus - Rhône, Tassin, etc.

(Cariot). . . . 2947. C. Cathaclines Gdgr mss

Fol. obovato-oblonga; fr. basi aculeolatus - Rhône, Beaunant (Chabert) . . . . 2948. C. RIGIDISETA Gdgr mss Fol. amplissime ovata - Alsatia, Thuringia, etc.2949

Fol - C. Marginata (Wallr.) (Fray) . . . . . . 2950. C. PRAETermissa Gdgr mss

10 Suffrutex plerumque humilis ( $5-8$ dec. altus); fol. amplissime Frutex 13-20 decim. altus ; fol. elliptica vel lanceolata $\cdot 20$

(Fr. ovatus. . . . . . . . . . . 12

11 Fr. ovoideus. . . . . . . . . . . 17 Fr. subglobosus - Thuringia . 2951. C. THURisgiacA (Wallr.)

12 Aculei dimorphi................. 13 1 Aculei conformes. . . . . . . . . . . 14 Ped. $7-8$ cent. longi; tubus laevis - Rhône, Tassin (Boullu)

13 P . * 2952. C. stupens Gdgr Ped. 1-2 cent. longi; tubus in $1 / 3$ inf. hispidus - Rhône, Francheville (Chabert). . . . . .2953. C. sotha Gdgr mss

14 Rami flor inermes. Rami flor aculeati. . . . . . . . . . 15 Pet. inf. inermes; fol. apice rotundata - Rhone, Charbon-

15 niéres, etc. (Chabert). . . 2954. C. PuMiLio Gdgr mss Pet. omnes aculeati; fol. subacuta - Rhône, Arnas (Gdgr herb. ros. 12. 5). . . . . 2955. C. ALxortir Gdgr Tubus glaber; pet. inf. inermes - Ain, La Pape (Chabert) 16 Tulus basi glandulosus; pet. omnes aculeati - Europa centr. (Gdgr herb. ros. n. 422, 427, 428, 429, 449, 450 et 474 var.) . . . . . . 2957. C. Flexuosa (Rau) Aculei ad ramos sup. conformes. . . . . . . . 18

17 Aculei sup. dimorphi - Rhône, Charbonnières (Gdgr hérb. ros. n. 463) . . . 2958. C. PSELdo-Flextosa (Ozanon)

Rami flor. inermes; tubus basi glandulosus. . . . . . 19

18 Pami flor. aculeati; tubus toto glandulosus - Gallia, Moselle (C. glandulosa Hol. fl. Mos. I, p. 232 non Bell.).2959

. . . . . . . . . . . Holandrei Gdgr Fr. suboblongus; pl. 3-5 dec. alta - Phóne, Arnas (Gdgr

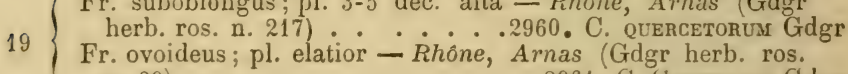
n. 39). . . . . . . . . G961. C. Gttardi Gdgr

20 Tubus globosus ovatus vel obovatus. . . . . . 21

20 Tubus oblongus . . . . . . . . . . . 39

21 Rami flor. haud (vel non normaliter) aculeato-setiferi. . . 22

21 Rami flor plerumque aculeato-setiferi. . . . . 30

( Tubus ovatus ....................... 23

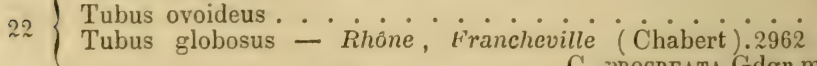
. . . . . . . C. PRocreata Gdgr mss

23 ( Rami flor inermes. . . . . . . . . 24

23 Rami flor aculeati. . . . . . . 25 
Fol. ovata; tubus basi infima glandulosus - Austria, Schonbrunn (Skofitz) . . . . 2963. C. enYtunoxyLoN Gdgr mss Fol. oblonga; tubus in $1 / 2$ inf. glandulosus - Rhone, Brindas (Chabert) . . . . . 2964. C. Laevicaulis Gdgr mss Tubus in $1 / 3$ inf. glandulosus; fol. ovata - Alsatia, Andelau (Billot exs. n. $206 t$ ter). . . . 2965. C. Matmae Gidgr mss Foliola elliptica; - Gallia bor-o"ient., etc. (Billot exs. n. 2061). . . . . 2966. C. Goderi (Gren.)

Tubus basi infina glandulosus; fol. oblonga - Rhóne, Craponne (Cariot) . . . . 2967. C. ontinacantila Gidgr mss

26 Rami flor inermes. . . . . . . . . 27

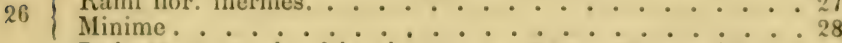
Ped. 17-20 m. 1.; fol. obovata - Rhone, Tassin (Cariot)

Ped. 13-17 m. l.; fol. elliptica - Rhone, Charbonnières (Chabert). . . . . 2969. C. CERAsifonmis Gdgr miss

Tubus basi glandulosus . . . . . . . . . 29

28 Tubus toto hispidus - Rhône, Frontenas (Gdgr).2970. . . . . . . . . C. calcareicola Gdgr mas Fol, ovato-clliptica - Germ., Wurtzburg (Rau).2971. C. TRA-

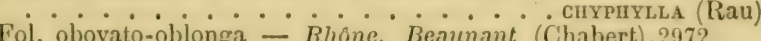
Fol. obovato-oblonga - Rhône, Beaunant (Chabert).2972. Aculei falcati ad ramos floriferos plerumque nulli . . . 31 Aculei falcati ad ramos floriferos |copiosi. . . . . . 34 Fr. ovoideus ............... . . 32

31 Fr. ovatus; fol. obovato-oblonga . . . 33 Fr. rotundatus; fol. obovata - Rhône, Charbonnières (Chabert). . . . . . . 2973. C. Adenophlaea Gdgr mss Discus paulo conicus; fol. ovato-elliptica - Cher, Fontmoreau, etc. (C. setulifera Timb.). 2974. C. NEmonrvaga (Dés.)

32 Discus planus; fol. ovato-rotundata - Cher, Fontmoreau, (Dés. herb. ros. n. 26).2975. C. Nemontraga B. AppropiN-

. . . . . . . . . . . QUATA Gdgr msS Sep. extus subglandulosa; pet. minute et parce villosi

33 Rhône, Tassin (Chabert) . . 2976. C. virudescens Gdgr mss Sep. valde glandulosa ; pet. tomentosi - Rhone, Tassin, etc. (Cariot) . . . . . 2977. C. determinata Gdgr mss

31 Fol basi rotundata vel cordata . . . . . . . 35 Fol. basi attenuata . . . . . . . 37

$35\left\{\begin{array}{l}\text { Fr. rotundatus } \\ \text { Fr. ovoideus }- \text { Austria, Vienne (Wiesbaur). 2978. C. Aus- }\end{array}\right.$ . . . . . . . . . . . . traca Gdgr mss Pet. tomentosi; ped. 20-25 m. 1. - Rhone, Francheville (Chaljert) . . . . . . 2979. C. LAticscela Gdgr mss

$36\left\{\begin{array}{l}\text { Pet. tomentosi; ped. } 13-15 \mathrm{~m} .1 .- \text { Rhone, Chartonnieres, } \\ \text { etc. (Cariot) }\end{array}\right.$ Pet. parce villosi ; ped. 2(1)-25 m. 1. - Rhüne, Tassin (C. speciosa Cariot Etude des fl. jo eil. II, P. 195 non Déségl.) . . . . . 2981. C. membranifolia Gdgr mss Fol. oblonga ; fr. ovatus vel globosus . . . . . . . . 38

37 Fol. obovata; fr. rotundatus - Rhône, Charbonnières (Chabert). . . . . . . 2982. C. IInTINaEva Gdgr mss 
Tubus plerumque totus glandulosus; fr. ovatus - Rhône, Francheville (Chabert) . . . 2983. C. RETiculosa Gdgr mss

Tubus basi infima aculeolatus; fr. globosus - Rhône, Charbonnières (Chabert). . . . 2984. C. triacantha Gdgr mss

39 Styli hirsuti vel lanati .............. 40

Styli glabri vel paulo pubescentes. . . . . 46

Aculei conformes . . . . . . . . . 4 41

40 Aculei alii majores alii setacei - Rhône, Tassin (Chabert) . . . . . . 2985. C. BREvicaulis Gdgr mss

41 Rami flor aculeati. ............. 42

Rami flor inermes .............. 43

Tubus in $1 / 4$ inf. hispidus; fol oblonga - Rhône, Tassin

$42\left\{\begin{array}{l}\text { (Billot exs. n. 3592) - .2986. C. PHylliriagfolia Gdgr mss } \\ \text { Tubus laevis ; fol. ovato-elliptica - Rhône, Arnas Gdgr }\end{array}\right.$ herb. ros. n. 563) . . . . 2987. C. ANceps Gdgr mss

$43 \int$ Fol. oblonga ; tubus toto vel in $1 / 3$ inf. hispidus.$\cdot 44$ Fol. elliptica; tubus in $1 / 4$ inf. hispidus - Hte-Savoie, Pringy (Puget) . . . . . .2988. C. ARIADNE Gdgr mss

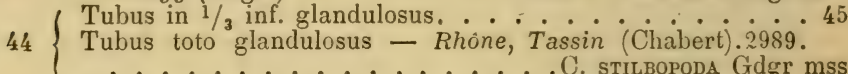

Fr. maximus $\cdot \dot{0}$ i.; fol . . . strllopoda Gdgr mss $45\left\{\begin{array}{l}\text { Fr. maximus } 16-19 \text { m. } \\ \text { Rhóne, Pollionay (Chabert). } 2990 . \text { C. CARYotoides Gdgr mss } \\ \text { Fr. minor 7-9 m. 1.; fol. basi rotundata - Ain, La Pape }\end{array}\right.$ (Chabert) . . . . . 2991. C. rostrata Gdgr mss

Fol. ovato-elliptica; tubus basi hispidus - Rhône, Arnas

(Gdgr herb. ros. n. 291) • . . 2992. C. leptoriphaeA Gdgr

Fol. obovato-rhomboidea ; tubus in $1 / 2$ inf. hispidus - HteSavoie, Pringy (Puget). . .2993. C. MEgalontodon Gdgr mss

Fol. ample oblonga; tubus laevis - Rhône, Arnas (Gdgr herb. ros. n. 359). . . . . . 2994. C. SATYRus Gdgr

47 Fol. undique hirsuta vel subtomentosa parceque glandulosa. 48

Fol. supra glabra vel glabrescentia . . . . . . . 53

48 Tubus laevis vel in $1 / 3$ inf. tantum glandulosus . . . . 49

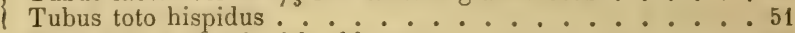

Rami flor. aculeati ; fol. oblonga . . . . . . . 50

49 Rami flor. inermes; fol. obovata - Rhône, Charbonnières (Chabert) . . . . . 2995. (') suprahinta Gdgr mss

Rami parce aculeati ; tubus laevis - Rhône, Charbonnières

$50\{$ (Chabert) . . 2996. C. PAucipinnata Gdgr mss Rami eopiose aculeati; tubus in $1 / 3$ inf. hispidus - Rhone, Francheville (Chabert). . . 2997. C. Dictamoides Gdgr mss

51 Fr. globosus : fol oblongn ... . . . . . 52

Fr. ovatus; fol. obovato-oblonga - Rhône, Charbonnières (Chabert). . . . . . . 2998. C. Gaespitosa Gdgr mss

$52\left\{\begin{array}{l}\text { Fol. apice lanceolata - Gallia, Lyon, etc. (Gdgr herb. ros. } \\ \text { n. 413, 420, 421, 462 et 473).2999. C. HIspIDocarpa (Chabert) }\end{array}\right.$

Fol. apice sensim attenuata - Rhône, Charbonnières (Chabert). . . . . . . 3000. C. HIRTIFolia Gdgr mss

53 Tubus globosus ovatus vel obovatus. . . . . . 54

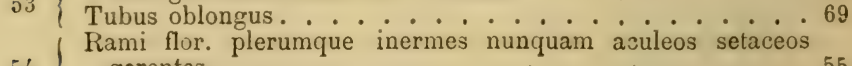

5 ' gerentes .................... 55

Rami flor. aculeos glanduliferos semper gerentes. . . . 65 
Fol. obovato-elliptica.

Fol. oblonga vel lanceolata. . . . . . . . . . 60

Fr. rotundatus .............. . . 57

56

Fr. ovatus . . . . . . . . . 59

Fr. obovatus - Rhône, Arnas (Gdgr herb. ros. n. 13). 3001.

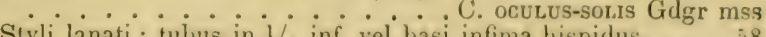
Styli lanati; tubus in $1 / 3$ inf. vel hasi infima hispidus . . 58 Styli villosi ; tubus in $1 / 2$ inf. hispidus - Rhône, Denicé (Gdgr herb. ros. n. 125) . .3002. C. Banmondenei Gdgr mss

Pet. aculeati; tubus basi infima hispidus - Podolia; Gall. or. (Gdgr herb. ros. n. 500 et 593).3003. C. Jundzilliaxa

Pot - . d buss. dus - Rhone, Poleymieux (Chabert) ...... . 3004. C. zYGorHyLLA Gdgr mss Styli pubentes; tubus in $1 / 3$ inf. glandulosus - Ain, Crépieux (Chabert)..... . 3005. C. silvulivaga Gdgr mss Styli pubentes; tubus toto hispidus - Podolia (C. glandulosa Bess. non Bell.) . . . . . . 3006. C. Polovica Gdgr Styli hirsuti ; tubus glaber - Itte-Savoie, Pringy (Puget)

. . . . 3007. C. Megasterhaxa Gidgr mss Styli lanati; tubus in $1 / 2$ inf. hispidus - Gallia, Lyon (Gdgr herb. n. 19) . . . . . 3008. C. gompnocanPA Gdgr mss

Aculei caulium sterilium vel inferiorum adunci. . . . . 61

Aculei omnes recti ............ . 62 62

Fr. ovoideus, fere toto hispidus - Rhône, Arnas (Gdgr)

Fr. ovatus . . . . . . . . . . . 63

Fr. ovoideus - Rhône, Arnas (Gdgr).3011. C. DEcunsiva

Fr. rotundato-globo . . . . . Gdgr mss n. 124) . . . 3012. C. LANCAEFolia Gdgr

Styli lanati ; fol. oblonga. . . . . . . . . . 64

63 Styli hirsuti; fol. obovata - Rhone, Tassin, etc. Gdigr herb. ros. n. 487,488 et 613 ) . .3013. C. vinidifolia (Chabert mss)

Tulus toto glandulosus; pet. omnino aculeati - Rhóne,

64 Tassin (Cariot) - . . 3014. C. INAEQuepilosa Gdgr mss Tubus in $1 / 3$ inf. glandulosus; pet. inf. inermes - Rhone, Alix (Gdgr). . . . . 3015. C. Dicthyocaulon Gdgr mss

65 Fr. rotundatus globosus vel ovatus ........66 66

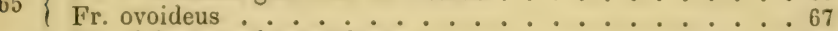

Fr. globosus vel rotundatus . . . . . . 68

Fr. ovatus - Rhône, St-Jean-d’Ádières (Aunier) $3016 .{ }^{\circ}$ C. $^{\circ}$

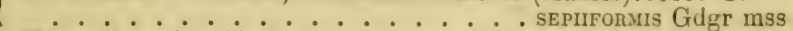
Tubus tolo hispidus; fol. oblonga - Rhône, Alix (Gưrr)

T. 3017 . C. crinita Gdgr mss Tubus in $1 / 3$ inf. hispidus; fol. obovato-olblonga - Rhone, Poleymieux (Chabert). . 3018. C. Inaequeanmata Gdgr mss Fol. oblonga ; tubus in $1 / 2$ inf. glandulosus - Ihöne, St-Genis, etc. (Cariot). . . . . . . 3019. C. setmamea Gder mss

Fol. olovata ; tubus in $1 / 3$ inf. glandulosus - Rhone, Chasselay (Chabert) . . . . 3020. C. majonaxa Gidgr mss

Fol. ovato-subrotundata ; tubus laevis - Paris; Rouen (Leturquier) . .....3021. C. texuiglasdulosa (Mérat) 


\section{$-216-$}

69

\section{exs. n. 3852 part.}

3034. C. ADENOCLADA Gdgr m

Fr. rotundatus; fol. obovato-oblonga - Rhône, Tassin (Billot exs. n. 3852 part.) . . . . . 3035. C. INCHOATA Gdgr mss Tubus in 1/, inf. glandulosus ; fol. oblonga - Rhône, Ecully

79 (Chabert) . 3036. C. ANGustisepala Gdgr mss

Tubus toto glandulosus; fol. ovato-elliptica - Cher; Rhône (C. speciosa Dés. non Andr.). . 3037. C. Formosissima Gdgr

Tubus toto vel fere toto hispidus . . . . . . . 81

Tubus in $1 / 3$ vel $1 / 2$ inf. hispidus. . . . . . . . . 82 Styli glabrescentes; fol. basi attenuata - Rhône, Arnas

81 (Gdgr herb. ros. n. 566). 3038. C. SAmbucifolia Gdgr mss

82 Sepalorum pinnula terminalis apice dilatata ..... 83

82 Sepalorum pinnula terminalis apice angustata . . . 86

83 Fr, ovoideus vel obovato-oblongus, ......... 84 
84 Fol. obovato-rhomboea basi rotundata - Rhóne, Arnas ol. oblonga basi paulo attenuata - Rhöne, Amas (Gidgr herb. ros. n. 342). . . . . 3041. C. POPLLNEA Gdgr mss Rami snbinermes; fol. ovato-elliptica - Rhone, Arnas

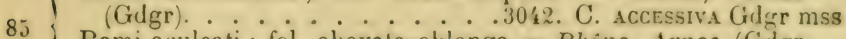
Rami aculeati ; fol. obovato-oblonga - Rhöne, Arnas (Gigr herb. ros. n. 128) . . . . 3043. C. INTECTa Gidgr mss

86 Fol. amplissime obovata vel oblonga ; ped. corymbosi. . . 87

86 Fol. mediocriter elliptica ; perl. 1-3-t. . . . . 88 Auriculae lanceolatae; styli hirsuti - Rhöne, Arnas (Gdgr

87 herb. ros. n. 315) .....3044. C. Cassiopes Gidgr mss A Ariculae truncatae; styli lanati - Rhône, Arnas (Gdgr herb. ros.॰n. 60). . . . . 3045. C. AMPLifolia Gdgr mss Fr, ovoideus vel obovato-oblongus. . . . . . 89

Fr. ovatus ; ? - Gallia orient. (Dés. herb. ros, n. 56).3046.

.......... Subolida (Dés.) Fr. ovatus; aculei dimorphi - Rhône, Arnas (Gdgr herb. ros. n. 280) . . . . . . 3047. C. texuicula Gdgr mss Fol. ovata vel obovata; rami flor. inermes - Rhóne, Arnas

$89\{$ (Gdgr herb. ros. n. 168) . . . 3048. C. Ligustri Gdgr mss Fol. oblonga ; rami flor. aculeati - Rhône, Arnas (Gdgr herb. ros. n. 309) . . . . 3049. C. prunicola Gdgr mss

Sul)genus X. - Chabertia Gdgr Essai p. 3ə̃ (in sect.); Rubiginoseae D. C. in Ser. Mus. helv. I (1818) p. 2; Crép. Prim. mon. ros. p. 23.

Styli liberi, ultra discum planum vel conicum in capitulum glabrum tunc subcoalitum, vel villosum ergo brevissime capitati vel sessiles. Flores bracteati, parvi, albi rosei vel purpurei, corymbosi, petalis cordatis margine glabris praediti. Fructus sat parvus, praecox vel serotinus, subcoriaceus vel semi-pulposus, erectus, glaber aut hispidus, ruber. Sepala pinnatipartita, ad marginem semper dentatoglandulosa, dorso nunc laevia nunc muricata vel rubiginosa, post anthesim deflexa tandem decidua, interdum vero erecta tune plus minus persistentia et serius decidua. Pedunculi erecti, glabri vel hispido-glandulosi, corymbosi lateque bracteati. Folia parva quandoque marcescentia, enrum 
foliolis subtus saltem toto rubiginoso-glandulosis, glabris vel villosis, coriaceis, prominule nervosis, acute et creberrime biserrato-glandulosis, tenuibus. Stipulae conformes, breviusculae, ad margines saltem glandulosae. Aculei sparsi, breves, interdum dimorphi, scilicet, alii e basi dilatata et compressa plus minus recurvati, alii vero setacei vel glanduliferi. Radix non reptans.

Frutices elati, sociales, glandulosi, sepes silvasque imprimis locorum petrosorum aridorumque frequentissime colentes, attritu suaveolentes (odor gratus Mali), atque plus minus glutinosi, ramosi, ramis tortuose articulatis, virentibus vel purpureis, rigidis aculeatisque, insignes.

Dicari in memoriam P. Chabert lugdunensis, de scientia rhodologica optime meriti. Ejus laborum fructus acquisivi hosque fateor ingenti auxilio mihi fuisse in meis indagationibus de Rosis quae Lugduni arva odoribus imbuunt.

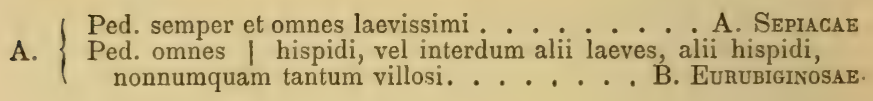

A. - Sepiacae Grép. Prim. mon. ros. p. 23 ; Gdgr Essai p. 36.

Styli liberi, nunc glabri vel glabriusculi tunc ultra discum plus minus planum subfasciculati, nunc vero villosi vel lanati ergo sessiles vel subcapitati; flores saepius albi vel dilute rosei, mediocris magnitudinis; fructus serotinus, subcoriaceus, glaberrimus, nitide sanguineus; sepala extus eglandulosa, pinnata, post anthesim decidua, rarius erecto-subpersistentia; pedunculi glaberrimi, corymbosi, graciles ; 
folia marcescentia diu sempervirentia vel sat decidua, subtus toto glandulosa parumque attritu olentia; aculei sparsi, conformes, falcato-dilatatı. - Frutices ubique vulgatissimi. a. Styli glaberrimi vel paululum hirsuti. . . . E 1. Eusepiacae

\section{1. - Eusepiacae Gdgr Essai p. 36.}

Styli liberi, glaberrimi vel paululum hirsuti nec villosi vel lanati, pauciores, ultra discum subfasciculati; flos albus mediocris, petalis cuneatis planiusculis patulisque praeditus; fructus angustior, serotinus, coriaceus ; sepala post anthesim deflexa, mox decidua; folia marcescentia basi saepissime longe cuneata, supra glabra saturateque virentia; aculei sparsi. - Frutices laxi, remote ramosi parciusque aculeati, subinodori.

1 Styli glaberrimi vel glabri ........... 2 Styli paululum hirtelli ............ . . . . 79

Fol. undique glaberrima ........... 3

2 Fol. subtus ad costam villosa. . . . . . . . . 5

Fol. subtus ad nervos villosa .......... 46

Fol. subtus toto villosa. . . . . . . . . 68

(Fr. oblongus basi I attenuatus . . . . . . . . 4

3 Fr. ovoideus basi rotundatus - Bouches-du-Rhone, Martigues (Autheman) ... 3050. Chabertia nobustion Gdgr mss

( Discus planus; ped. 5-6 m. 1. - Aragonia austr., Sierra de Mosqueruela (Martin). .3051. Chabertia mispanica Gdgr mss

Discus conicus ; ped. 12-14 m. 1. - B.-du-Rhône, Martigues

(Autheman)... . 3052. Chabertia glaberrima Gdgr mss

5 Pet. glabri vel sparse pilosi. .......... 6

5 Pet. toto minute pubescentes . . . . . . . . . . . . 20

6) $\left\{\begin{array}{l}\text { Fr. ovoideus } \ldots \\ \text { Fr oblongus }: \ldots\end{array}\right.$

( Pet. inermes; ped. 9-11 m. 1. - Dalmatia, La Trinita (Stud-

7 niczka). . . . . . 3053. CH. Dalmatica Gdgr mss Pet. aculeati ; ped. 11-14 m. 1. - Pyren. Or., IIaut-Ternel (Debeaux). .........3054. C. crclobasis G ! er mas 
Rami flor inermes, . . . . . . . . 9

Rami flor aculeati. . . . . . . . . . . 11

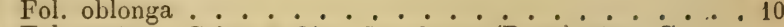

Fol. ovata - Seine-et-Oise, Les Loges (Rouy).3055. C. ELA-

Fol. obovata; pet inermes - Ain, Vancia (Chaber) (Rouy)

......... . . . supraglandulosa Gdgr mss

Fol. obovato-oblonga ; pet. aculeati - Tyrol, Hall (Kerner).

. . . . . . 3057. C. occultata üdgr mss Styli subpilosuli; pet. aculeati - Pyren. Or., Olette-les-

10 Bains (Debeaux) . . . . 3058. C. ANgusticeps Gdgr mss Styli glaberrimi; pet. subinermes - Gallia, Annecy (Puget). . . . . . . . . . . 3059. C. sENILIs Gdgr mss

Pet. inermes. . . . . . . . . . . 12

Pet. aculeati. . . . . . . . . . . 13 Discus conicus ; fol. apice subrotundata - Calvados, Bayeux

12 (Lebel)............. 3060. CunEIFolia Gdgr mss Discus planus ; fol. apice acuta - Italia, Moronico (Caldesi).

13 Fol. obovata vel obovato-oblonga .......... 14 Fol. oblonga. . . . . . . . . . . . 16 Discus planus. . . . . . . . . . 15

14 Discus paulo conicus - Rhône, Arnas (Gdgr herb. ros. n. 103). . . . . . 3062.C. ACAnthothamnos Gdgr Stipulae subtus glandulosae; pet. sparse hirtelli - Rhône,

15 Limas (Gdgr). . . . . 3063. C. Berthetiana Gdgr mss Minime; pet. glabri - Europa, etc. (Gdgr herb. ros. n. 607 et 608). . . . . . . 3064. C. sEpIum (Thuill.)

16 Discus paulo conicus. ............ 17

Discus planus. . . . . . . . . . . 18

Ped. 6-8 m. 1.; rami flor. subinermes - Pyren. Or., St-Antoine-de-Galamus (Debeaux). .3065. C. clavulata Gdgr mss

Ped. 10-12 m. l.; rami flor. copiose aculeati - Ain, Vancia (Chabert). . . . . . . . 3066. C. ornata Gdgr mss Ped. 16-18 m. 1. - Hisp. bor., Logrono San Felice (Zubia).

. . . . . . 3067. C. Navarrensis Gdgr mss Fol. subtus tantum glandulosa. . . . . . . . 19

18 Fol. inf. undique valde glandulosa - Catalaunia, Olot Font den Dorca (Vayreda). . . . . 3068. C. Vayredae Gdgr mss Ped. 15-17 m. 1.; pet. glabri - Gard, Bréau (Tuezkiewicz). $19\left\{\begin{array}{l}\text { Ped. } 5 \text { - } 6 \text { m. i. pet. sparse pilosi - Aragonia austr., Sierra } \\ \text { 3069. C. Cebennsis }\end{array}\right.$ de Mosqueruela (Martin) . . 3070. C. ARAgonensis Gdgr mss

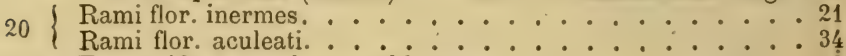

Fr: ovoideus vel obovato-oblongus. . . . . . 22 $21\left\{\begin{array}{l}\text { Fr. oblongus } \\ \text { Fr. globosus - Pyrenaei, Perpignan (Debeaux) } 3071 \text { C. }\end{array}\right.$ . . . . . . . . . PSEUDO-PISUM Gdgr mss

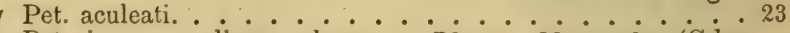
Pet. inermes; discus planus - Rhône, Montmelas (Gdgr

22 herb. ros. n. 180). . . 3072. C. RHIPIDOcLaDA Gdgr mss Pet. inermes ; discus conicus - Bouches-du-Rhône, Roquefavour (Autheman). . . . .3073. C. Moderata Gdgr mss 
Fol. ample oblonga - Rhóne, Ville-sur-Jarnioux (Gdgr) Fol. mediocriter obovato-oblonga - Tyrol, Hall Grembli ms ... 3075. C. editicola Gdgr mss Pet inermes. . . . . 25

Pet. I aculeati ............... . . 28 Discus paulo conicus. . . . . . . . . 26

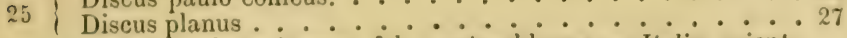
Stipulae subtus laeves; fol. ovato-oblonga - Italia orient., Faenza (Caldesi). . . . 3076. C. Faventrana Gdgr mss

Stip. subtus toto glandulosae; fol. oblonga - Bouches-duRhône, Pas des Lanciers (Autheman).3077. C. SUBUniflora

Ped. 9-12 m. i. ; fol. supra eglandulosa - Rhône Yzeron

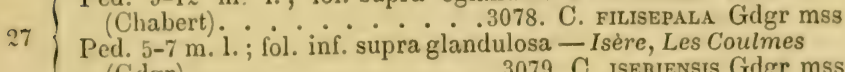
(Gdgr)............. C. Iseniensis Gdgr mss

28 Discus | conicus ............... 29

Discus planus . . . . . . . . . . . 31

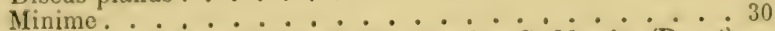

29 Stip. subtus glandulosae - Hte-Savoie, St-Martin (Puget)

. 3080. C. oligagantha Gdgr mss

Sep. apice integra; fol. basi cuneata - Bouches-du-Rhône, $30\left\{\begin{array}{l}\text { Gignac (Autheman). } \cdot \dot{0} \cdot \text { basi } \text { sensim attenuata }- \text { Tyrol, } \\ \text { Sep. apice dentata; fol. }\end{array}\right.$ Mulhaü (B. Stein). . . . . 3082. C. TYRolensis Gdgr msS

31 Fol. obovato-oblonga. . . . . . . . 32

Fol. oblonga ....................... 33

Pet. subinermes; styli 4-6 - Rhône, Villefranche (Gdgr.) $32\left\{\begin{array}{l}\text { Pet. aculeati ; styli numerosi - Gironde, Ste-Eulalie Mote- } \\ \text { - Mos3. C. oligostigra Gdgr mss }\end{array}\right.$ lay)............3084. C. epiglottis Gdgr mss Stip. rubentes; pet. subinermes - Rhône, Alix (Gdgr herb.

33 n. 230). . . . . . 3085. C. pinguissima Gdgr mss Stip. virentes; pet. aculeati - Gallia, Clermont (F. Héribaud)

Fr. ovatus ovoideus vel obovato-oblongus. AnvenNessis Gdgr mss

35 Fol, obovato-oblonga. 37 Ped. 10-12 m. l.; discus planus - Gallia, Lyon (Chabert)

. . . . 3087. C. Mrrtus Gdgr mss Ped. 15-17 m. i.; discus subconicus - Pyrenaei, Perpignan (Debeaux). . . . 3088. C. pYramdocanpa Gdgr mss Pet. I aculeati; fol. utrinque attenuata . . . . . 38

37 Pet. inermes; fol. utrinque rotundata - Savoie, Macôt (Puget)

.3089. C. IDIoNodonta Gdgr mss Aculei falcato-dilatati ; stip. dorso glandulosae - Ain, Van$38\left\{\begin{array}{l}\text { cia, etc. (Chabert) } \\ \text { Aculei inclinati nec dilatati; stip. subtus toto glandulosae - }\end{array}\right.$ Rhône, Dardilly, etc. (Chabert). .3091. C. Anvicola Gdgr mss Fol. apice rotundata vel obtusa........40 Fol. apice attenuata vel acuta. . . . . . . 42 
Fol. obovato-oblonga. . . . . . . . . . . 41

Fol. oblonga - Rhône, Ville-sur-Jarnioux (Gdgr).3092

............ G. gractlescens Gdgr mss Discus planus; pet. inf. inermes - Rhône, Arnas (Gdgr)

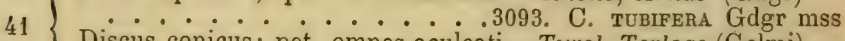
Discus conicus; pet. omnes aculeati-Tyrol, Terlago (Gelmi)

. . . . . . . 3094. C. murolita Gdgr mss

Discus conicus. . . . . . . . . . 43

Discus planus.

Pet. paulo aculeati ; fol. oblonga - Bouches-du-Rhone,

43 Martigues (Autheman). . . 3095. C. OLIVETonUm Gdgr mss Ped. inermes; fol. obovato-oblonga - Catalaunia, Gerone Vilademiras (Vayreda). . . 3096. C. Catalaunica Gdgr mss

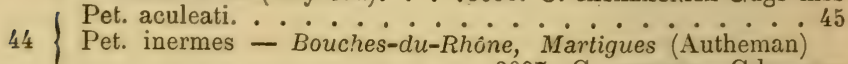

F - * * 3097. C. subtincta Gdgr mss Fol. minutissima; ped. $12-14$ m. l. - Bouches-du-Rhône, Velaux (Autheman) . . . .3098. C. ANGustissima Gdgr mss Fol. angustata; ped. 4-6 m. i. - Cher, etc. (Gdgr herb. ros.

n. 534) -

Fol. ampla; ped. 9-10 m. 1. - Rhône, Arnas (Gdgr). 3100

46 Pet. saltem super, sparse pilosi... BREviglanda Gdgr msS

Pet. omnes pubescentes. . . . . . . . . . . 58

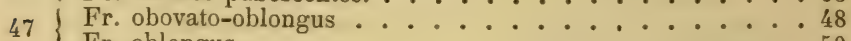

Fr. oblongus . . . . . . . . 50

Pet. omnes aculeati. . . . . . . . . . . . 49

Het. inf. inermes; ped. 11-12 m. l. Rhône, Ville-sur-Jarnioux

(Gdgr). . . . . . . 3101. C. vepreticola Gdgr mss

Pet. omnes inermes; ped. 8-11 m. l. - Ain, Sathonay, etc.

(Chabert) . . . . 3102. C. IGNorabilis Gdgr mss

Discus conicus; fol. oblonga - Bouches-du-Rhône, Martigues

49 (Autheman).......3103. C. Exuberans Gdgr mss Discus planus; fol. obovata - Gard, Le Vigan (Tuezkiewicz) ........... . . 3104. C. Dromedis Gdgr mss

$50\{$ Discus $\mid$ conicus ............. 51 Discus planus. . . . . . . . 52

Pet. subinermes; rami flor inermes - Etruria, Mte-Pisano

51 (C. myrtifolia Hall. f. Savi; Gdgr herb. ros. n. 572).3105

Pet. et rami flor - * - C. Agrestis (Savi)

Pet. et rami flor. aculeati - Italia, Modène (Gibelli).3106

| Rami flor inermes. . . . C. ApENnina Gdgr mss

52 Rami flor aculeati . . . . . . 53

(Styli minuti ; pet. subinermes - Vallesia, Champlan (Wolf) 53 Styli magni; pet. aculeati - Dania, Nyborg (Lange).3108

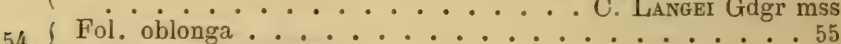

54 Fol obovato-oblonga. $\ldots . . .55$

( Ped. 10-11 m. 1.; fol. acuta - Pyren. Or., Vall del Bach

$55\{$ (Vayreda). Ped. 7 m. l.; fol. lanceolata - Hisp., Logrono (Zubia).3110 C. IBERENSIS Gdgr mss 
Ped. 9-13 m. 1. ; fol. apice attenuata, . . . . . 57

Ped. 5-7 m. 1.; fol. apice rotundata - Gironde, Vertheuil

(Motelay) . . . . . . 3111. C. Panviceps Gdgr mss

Fol. apice dilatata; nervi parce villosi - Rhóne, Pommiers

(Gdgr herb. ros, n. 3). . . . 3112. C. Jugonum Gdgr mss

Minime; nervi dense hirsuti - Catalaunia, Olot Montolivet

(Vayreda) . . . . . 3113. C. Costaeana Gdgr mss

(Fr. ovatus vel ovato-ellipticus. . . . . . . . 59

Fr. ovoideus vel obovato-oblongus. . . . . . . . 60

Fr. oblongus . . . . . 63

Fr. subglobosus; rami flor aculeati - Italia orient., Faenza

(Caldesi). . . . . . . 3114. C. subglobosa Gdgr mss

Fr. globosus; rami flor. inermes - Naples, Basilicata Po-

tenza (Gussone).....3115. C. NeApolitana Gdgr mss

Rami flor. et pet. omnes inermes - Sabaudia, etc.3116

59 . . . . . . . . . . . mentita (Dés.)

Rami flor. aculeati ; pet. sup. aculeati - Tyrol, Castellano Rovereto (Gelmi). . . . 3117. C. amblyrhyncha Gdgr mss

( Pet. inermes. ............ . . 61

Pet. aculeati.

Fol. basi cuneata; rami flor. inermes - Italia orient., Faenza

61 (Caldesi) . . . 3118. C. Parlatoreana Gdgr mss Fol. basi vix attenuata; rami flor. aculeati-Istria, $m$. Planik Vrana (Freyn). . . . . . 3119. C. Freynir Gdgr mss Rami flor. aculeati; fr. obovato-oblongus - Rhône, Arnas

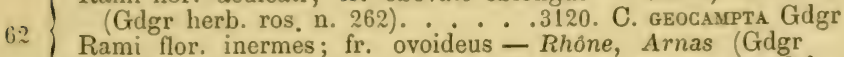
Rami flor. inermes; fr. ovoideus - Rhône, Arnas (Gdgr herb. ros. n. 546) . . . 3121. C. PSEudo-Campta Gdgr Pet. inf inermes. . . . . . . . . 64

Pet. omnes aculeati. . . . . . . . . . 65

Pet. sup. inermes; fol. obovato-oblonga - Serbia, Belgrade (Pancic). . . . . . . 3122. C. serbica Gdgr mss Pet. omnes incrmes; fol. oblonga - Bouches-du-Rhône, Berre (Autheman) . . . . 3123. C. Deniesiasa Gdgr mss Discus planus; fol. obovato-oblonga - Rhône, Montmelas (Gidgr herb. ros. n. 264) • . 3124. C. TExtiramea Gdgr mss Discus conicus; fol. oblonga - Tyrol, Molveno (Gelmi).3125

. C. RHYNCHOPHYSA Gdgr mss

Fol. basi rotundata; discus conicus - Istria, Canfanaro (Freyn). . . . . . 3126. C. Istriact Gdgr mss Fol. obovato-oblonga. . . . . . . . . 67 Fol. elliptico-obovata - Caucasus or., Daghestan (Becker)

F. . - * 3127. C. Catcasica Gdgr m

Fol. oblonga - Italia, Modène Roteglia (Gihelli).3128. C. ITA-

Ped. 4-6 m. 1.; fol. supra eglandulosa - Gallia, Marseille

(Bompart). ......3129. C. articulosa Gdgr mss Ped. 10-11 m. 1.; fol. supra glandulosa - Rhône, Arnas (Gdgr herb. ros. n. 193). . . . .3130. C. DAPHYoIdEA Gdgr Fr. rotundatus. .......... . . 69

Fr, ovatus vel ovato-rotundatus. . . . . . . . 70

Fr, ovoideus vel obovato-oblongus. . . . . . . . 72

Fr. oblongus .................... 76 
( Discus planus ; rami flor. inermes - Rhône Pommiers (Gdgr)

69 Discus conicus; rami flor. aculeati - Italia orient., Faenza (Caldesi). . . . . . 3132. C. Rugosula Gdgr mss

7 Pet. aculeati; fol. obovato-elliptica . . . . . . 71 $70\left\{\begin{array}{c}\text { Pet. inermes; fol. obovato-oblonga - Saône-et-Loire, Rou- } \\ \text { geon (Ozanon). . . . . 3133. C. seposita (Crép. mss) }\end{array}\right.$

$71\left\{\begin{array}{l}\text { Rami flor. aculeati; discus planus - Rhône, Arnas (Gdgr } \\ \text { herb. ros. n. 259). } \\ \text { Ram. flor. inermes; discus conicus - Tyrol, oto. } 3135 .\end{array}\right.$ Ram. flor. inermes; discus conicus - Tyrol, oto. 3135.

i2 Pet. omnes inermes ................. 73

i2 Pet. omnes aculeati ................ 74

Pet. inf. inermes. . . . . . . . . . . 75

(Fol. supra glabra; ped. 8 m. 1. - Italia orient., Faenza

73 (Caldesi). . . . 3136. C. Micheliana Gdgr mss Fol. supra hirta; ped. 12-13 m.1. - Tyrol, Seefeld (Gremblich). . . . . . . . 3137. C. HIRTA Gdgr mss

(Cor. alba; fol. supra glabra - Vaucluse, l'Isle (Autheman)

74 Cor. rosea; fol. supra hirta 3138 . C. VAltis-CLAUSAE Gdgr mss (Autheman). . . . 3139. C. Campylophylla Gdgr mss Fol. elliptica; fr. apice depressus - Rhône, Le Mont-Ceindre

$75\{$ (Chabert). . . . . 3140. Microsepala Gdgr mss Fol. oblonga; fr. apice attenuatus - Rhône, Arnas (Gdgr herb. ros. n. 45) .......3141. C. ARMigera Gdgr mss

76 ( Discus conicus. . . . . . . . . 77 Discus planus. Pet. sup aculeati; ped. $11-12$ m. 1. - Tyrol, valle di Cem-

77 bra (Gelmi).......... C. Cembra Gdgr mss Pet. omnes inermes; ped. 18-22 m. 1. - Italia or., Faenza (Caldesi)........3143. C. conophora Gdgr mss

Rami flor. inermes; ped. 12-14 m. 1. - Italia or., Faenza

78 (Caldesi). . . . . 3144. C. viridifolia Gdgr mss Rami flor. aculeati ; ped. 9-10 m. 1. - Italia, Abruzz. m. Mauro (Caldesi). . . . . . . 3145. C. BRUTIA Gdgr mss

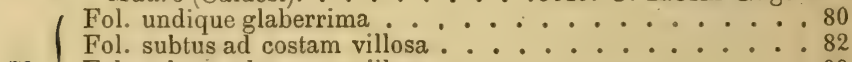

79 Fol. subtus ad nervos villosa . . . . . . . . 99

Fol. subtus praeter nervos hinc inde hirtula . . . . . 127

Fol. subtus omnino villosa . . . . . . . . . . 131

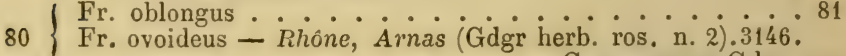

. . . . . . . . C. MYRSINiTES Gdgr mss Fol. obovato-oblonga supra paulo glandulosa - Rhone, Ville-sur-Jarnioux (Gdgr herb. ros. n. 562).3147. C. BUxI-

81 ............................. Gdgr mss Fol. oblonga supra eglandulosa - Rhône, Arnas (Gdgr herb. ros. n. 187) . . . . 3148. C. CAlocarpa Gdgr mss

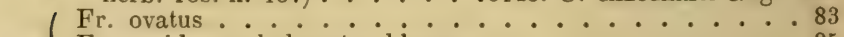

$82\left\{\begin{array}{l}\text { Fr. ovoideus vel obovato-oblongus. . . . . } 85 \\ \text { Fr. oblongus }\end{array}\right.$

Fr. globosus - Bouches-du-Rhône, Martigues (Autheman).

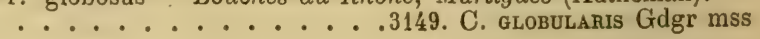


Fol. obovata; fr. apice haud aut vix attenuatus.

Fol, oblonga ; fr. apice valde strangulatus - Rhone, Limas

(Gdgr herb. ros. n. 222). . . .3150. C. stenonmyncha Gdgr Fr. et fol. minima - Iérault, Fontecouverte (Richter).3151.

Fr. et fol. majora - Rhöne, Tassin (Billot exs. n. 1871 ter).

. . . . 3152. C. gastrocarpa Gdgr mss

85 Rami flor aculeati. . . . . . . . 86

Rami flor, inermes. . . . . . . . . . 88

Ped. 6-9 m. l.; fol. supra eglandulosa . . . . . . 87

86 Ped. 4-6 m. 1.; fol. supra paulo glandulosa - Rhone, Villesur-Jarnioux (Gdgr herb. ros. n. 560).3153. C. CAfitel-

........ LATA Gidgr

Pet. omnes aculeati - Rhöne, Ville-sur-Jarnioux (Gdgr).

Pet. inf. inermes - Rhone, Denicé (Gdgr herb. ros. n. 147).

......3155. C. ANgulosa Gdgr mss

Pet. omnes aculeati; fol. oblonga . . . . . . 89

88 Pet. omnes inermes; fol. ovato-elliptica - Rhone, Montsuit

(Chabert). ......3156. C. enytmRophylla Gdgr mss

Pet. omnes villosi - Gironde, Bassens (Motelay).3157. C.

Pet. sup. sparse pilosi - Bouches-du-Rhône, Martigues (Autheman). . . . . . 3158. C. oxypHora Gidgr mss

I Pet. saltem sup. sparse pilosi. . . . . . . . . 91

Pet. omnes villosi. . . . . . . . . 93

Discus conicus; pet. inermes. . . . . . . . 92

91 Discus planus ; pet. aculeati - Rhône, Gleizé (Gdgr herb. ros. n. 43)............. C. CoRonaria Gdgr mss

Rami flor. aculeati ; fol. apice acuta - Bouches-du-Rhone,

92 Martigues (Autheman). . . 3160. C. Mrxta Gidgr mss Rami inermes; fol. apice rotundata - Bouches-du-Rhône, Rognac (Autheman). ....3161. C. Alphoxsi Gdgr mss

Pet. omnes inermes . . . . . . . . . . 94

93 Pet. omnes aculeati . . . . . . . . 9 96

Pet. inf. inermes - Rhône, Ouilly (Gdgr).3162. C. SAlits-

. cula Gdgr mss

Rami flor inermes. . . . . . . . . 95 Rami flor. aculeati - Cher, Graire (Billot exs. n. 1871 quater).

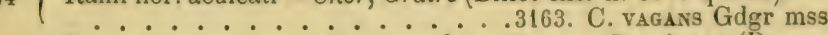
Discus planus; ped. 10-12 m.1. - Pyren., Perpignan (De-

95 beaux). . . . . 3164. C. Exsifolia Gdgr mss Discus paulo conicus ; ped. 9-10 m. 1. - Bouches-du-Rhóne, Le Réaltor (Autheman). . . 3165. C. RuYncroides Gdgr mss

Fol. apice rotundata. . . . . . . . . . 97

Fol. apice acuta. . . . . . . . . 98

Aculei conformes; discus planus - Gallia, etc.3166. C. Anra-

Aculei setace et adunci discus subconicus - Bouches-duRhône, Martigues (Autheman).3167. C. DIversispina Gdgr mss Rami flor. aculeati; discus planus - Rhöne, Alix (Gulgr herb. ros. n. 173) . . . 3168. C. LATERItia Gdgr mss Rami flor. inermes; discus subconicus - Pyren., Perpignan (Debeaux). . . . . . 3169. C. incubacea Gdgr mss 
Fr. globosus ovatus ovoideus vel obovatomoblongus . . . 100

Fr. oblongus . . . . . . . . . . . 116

100 Rami flor. inermes. . . . . . . . . . 101

Rami flor aculeati. . . . . . . . . . . 110

101 Fr. rotundatus ovatus vel ovato-ellipticus. . . . . . 102

Fr. obovatus vel obovato-oblongus. . . . . . 104

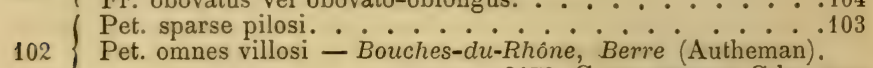

102 (............... C. Paupercula Gdgr mss

(Fr. rotundatus vel subglobosus - Rhône, St-Genis (Roffa-

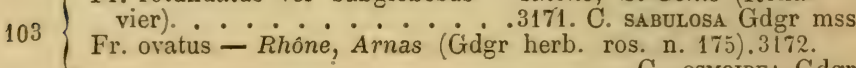

104 Pet. sup sparse hirsuti $\therefore \therefore \therefore \therefore . . . . . .205$

104 Pet. omnes sparse hirsuti. . . . . . . . 106

Pet. omnes villosi . . . . . . . . . . 108

Discus planus; pet. inermes - Rhône, Brindas (Chabert). 105 Discus conicus; pet. paulo aculeati - Bouches-du-Rhône, Martigues (Autheman). . . .3174. C. ovordalis Gdgr mss

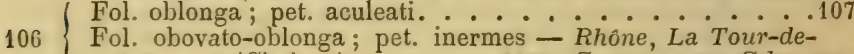
Salvagny (Chabert).....3175. C. oligantha Gdgr mss

$107\left\{\begin{array}{l}\text { Ped. 6-7 m. 1.; fol. apice acuta - Bouches-du-Rhône, Mar- } \\ \text { tigues (Autheman) . . . . . - C. Mrsutissima Gdgr mss }\end{array}\right.$ Ped. 12-13 m. l.; fol. obtusa - Catalaunia, Na Sa del Mont Gerone(Vayreda). . . .3177. C. Salvatoreana Gdgr mss Fol.oblonga. . . . . . . . . . . . 109 Fol. obovato-oblonga apice acuta - Rhône, Amas (Gdgr).

108 . . . . . 3178. C. MEsocalyx Gdgr mss Fol. ovato-elliptica apice rotundata - Rhône, Chaponost (Chabert) . . . . . . 3179. C. Nervigera Gdgr mss Discus planus; pet. sup. aculeati - Rhône, Arnas (Gdgr).

$109\{\ldots \ldots . \cdots 3180$. C. viridicans Gdgr mss Discus conicus; pet. inermes - Italia, Florence Impruneta (Areangeli). . . . . .3181. C. Florentina Gdgr mss

$110\left\{\begin{array}{l}\text { Fr. subglobosus. } \\ \text { Fr. ovoideus vel obovato-oblongus. } \ldots \ldots 111\end{array}\right.$ Fr. ovatus; pet. omnes aculeati - Pyren., Perpignan (Debeaux). . . . . . 3182. C. PODOPHYLLA Gdgr mss

$111\left\{\begin{array}{l}\text { Pet. inf. inermes; fol. oblonga }- \text { Rhône, Montsuit (Chabert). } \\ \text { Pet. omnes paulo aculeati fol. obovata - Saône-et-Loire, }\end{array}\right.$ Dract obovata - Saône-et-Loire, Dracy (Billot exs. n. 1871 bis).3184. C. SPHAerocephala ............................ Gdgr mss Pet. omnes pubescentes . . . . . . . . . . . 113

112 Pet. sup. sparse pilosi; fol. obovato-oblonga - Rhône, Theizé (Gdgr) . . . . 3185. C. Albocarnea Gdgr mss Pet. omnes sparse pilosi ; fol. oblonga - Pyrenaei, Perpignan (Debeaux). . . . .3186. C. Rufovirexs Gdgr mss

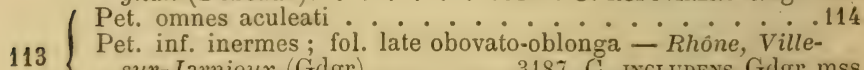
sur-Jarnioux (Gdgr) . . . . 3187. C. INCLudens Gdgr mss Pet. subinermes; fol. angustissime oblonga - Rhône, Liergues (Gugr herb. ros. n. 348).3188. C.serpyllifolia Gdgr mss 
Ped. 6-10 m. 1.; fol. supra eglandulosa. . . . . . . .115

Ped. 13-16 m. 1.; fol. supra glandulosa - Vaucluse, La Fontaine (Autheman) .... . 3189. C. Requexiasa Gilgr mss Fr. basi depressus; fol. lata - Rhone, Arnas (Gdgr herb.

115 ros. n. 301) ...... 3190. C. Nidulania Gdgr mss Fr. basi attenuatus; fol. parva - RhGne, Ville-sur-Jarnioux (Gdgr). . . . . . . 3191. C. Pinguifolia Gdgr mas

116 Rami flor inermes. . . . . . . . . . 117 \{ Rami flor aculeati. . . . . . . . . . 121

Pet. omnes inermes . . . . . . . . . . 118

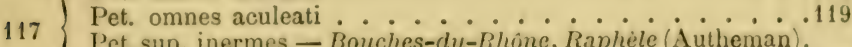
Pet. sup. inermes - Bouches-du-Rhóne, Raphèle (Autheman). ........3192. C. ARElatensis Gdgr mss Ped. 4-5 m. l.; fr. basi valde decurrens - Italia or., Faenza

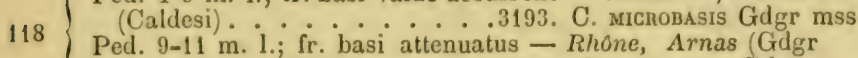
herb. ros. n. 102) . . . .3194. C. Filicaulis Gdgr mss

( Pet. omnes villosi . . . . . . . . . . . . . Pet. sparse pilosuli - Gallia, Lyon (Gdgr herb. ros. n. 79). .......... 3195. C. volubilis Gdgr mss

$120\left\{\begin{array}{l}\text { Discus planus; ped. } 8-9 \text { m. 1. - Suecia mer. (C. inodora } \\ \text { Hort. Lund. goth. non Fr.) - - 3196. C. INEnsis Gdgr mss }\end{array}\right.$ Discus conicus; ped. $14-15 \mathrm{~m}$. I. - Bouches-du-Rhône, Rognac (Autheman) . . . 3197. C. Rarispina Gdgr mss

121 Fol. obovata. . . . . . . . . . . . . . . . 22

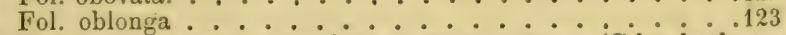

Rubens; fol apice rotundata - Rhône, Arnas (Gdgr herb. $122\left\{\begin{array}{l}\text { ros. n. 164) } \\ \text { Virens; fol. apice attenuata - Pedem., Vallées Vaudoises }\end{array}\right.$ (Rostan). . . . . 3199. C. valderia Gdgr mss

123 Pet. sup. sparse pilosi . . . . . . . . 124 Pet. omnes hirsuti. . . . . . . . . . 125 Fol. acuta; ped. 12-14 m. 1. - Italia, Modène Roteglia (Gi124 Folli). obtusa; ped. $9-10$ m. i. - Bouches-du-Rhône, Martigues (Autheman) . . . . . 3201. C. Gracilion Gdgr mss

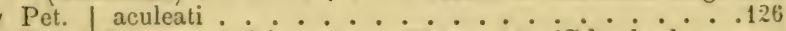
Pet. inf. inermes; fol. acuta - Rhone, Alix (Gdgr herb. ros.

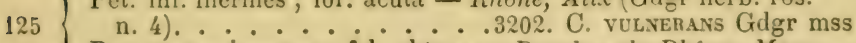
Pet. omnes inermes; fol. obtusa - Bouches-du-Rhône, Martigues (Autheman). . . . . 3203. C. Leptoclada Gdgr mss Stip. subtus glandulosae; fr. major - Valais, Bovernier (De$126\left\{\begin{array}{l}\text { lasoie). Pinime; fr. parvus - Saóne-et-Loire, Macon (Lacroix).3205. } \\ \text { Mas }\end{array}\right.$

Fr ovatus . . . . . . . . . . .

Fr. ovoideus . . . . . . . . 130

Fr. globosus - Helv., Fribourg Montboron (Coltet).3206. C.

.................. RIPIAEA Gdgr mss Fr. ollongus - Italia or., Faenza Brisigliella (Caldesi).3207.

• . C. Malpigulana Gdgr mss

$128\left\{\begin{array}{l}\text { Rami flor. et pet. inermes - Bouches-du-Rhone, iartigues } \\ \text { Rame }\end{array}\right.$ (Autheman) . . . . . 3208. C. Bnevicalyx Gdgr mss 
$129\left\{\begin{array}{l}\text { Fol. elliptica; pet. subinermes - Rhône, Montmelas (Gdgr } \\ \text { herb ros. n. 119). - - - HoAd Gdgr } \\ \text { Fol. oblonga; pet. aculeati - Hte-Savoie, Pringy (Puget). }\end{array}\right.$

Pet. sup. sparse hirsuti ; fol. magna - Suecia, Halland Bjor-

. . 3211. C. Lindebengir Gdgr mss

Pet. omnes hirsuti ; fol. parva - Rhône, Limas (Gdgr).3212.

Fr. globosus vel subglobosus .... C. Halleriana Gdgr mss

$131\left\{\begin{array}{l}\text { Fr. ovatus vel ovato-rotundatus. } \\ \text { Fr. }\end{array}\right.$

Fr. obovato-oblongus. . . . . . . . . . . 134

. . . . . . . . . . . . .

Rami flor. aculeati; fol. ovata - Angl.; Scand. (Fries herb.

$132\left\{\begin{array}{l}\text { norm. X, n. 51). } \text { fol. obovata - Basses-Alpes, Condamine } \\ \text { Rami flor. inermes; fol }\end{array}\right.$ (Proal) . . . . . . . 3214. C. spHaERICA Gdgr mss $133\left\{\begin{array}{l}\text { Virens; ped. 4-5 m. 1. - Rhône, Arnas (Gdgr).3215. C. EX- } \\ \text { Rubens; ped. 8-9 m. 1. - Rhône, Villefranche (Gdgr).3216. }\end{array}\right.$

( Rami flor. inermes; pet. omnes hirsuti - Rhône, Arnas

134 (Gdgr herb. ros. n. 281) . . . 3217. C. Sanlavillei Gdgr Rami flor. aculeati; pet. sup. sparse hirsuti - Rhône, Arnas (Gdgr). . . . . . . 3218. C. BReviramea Gdgr mss

Pet. omnes dense hirsuti. . . . . . . 136

135 Pet. sparse hirsuti - Rhône, Arras (Gdgr).3219. C. LYGium

136 Discus paulo conicus. $\therefore \ldots$ Gdgr ms

136 Discus planus. . . . . . . . . . . . 138

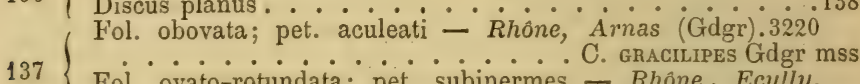
Fol. ovato-rotundata; pet. subinermes - Rhône, Ecully, (Chabert). . . . . . . 3221. C. SAUcia Gdgr mss

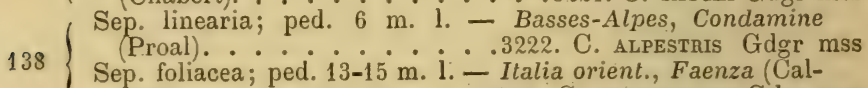
desi)............. C. chavinioides Gdgr mss

\section{2. - Hebegynae Gdgr Essai, p. 37.}

Styli liberi, semper villoso-hirsuti vel lanati, ultra discum planum sessiles; flores minores quam in Eusepiaceis, plerumque dilute rosei, petalis ovatis concavis suberectostellatis, intus margine superiori ad commissuram subplicatis, praediti; fructus sat major, subpraecox vel semi- 
pulposus; sepala post anthesim saepe erecta, usque ad maturitatem persistentia et tunc subemortua vel semivivida; folia eis sectionis praecedentis praecocius decidua, hirsutiora mollia pallide virentia et ordinarie minus longa; aculei sparsi ex areola basilari elongata acutissime falcati. Frutices praesertim regionis calcareae calidioris dense ramosi et aculeati, eorum ramis gracilibus brevioribusque.

Fol. undique glaberrima (grex C. Seraphini Viv. et spec.

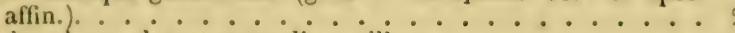
Fol. subtus ad costam mediam villosa . . . . . . $9_{9}$ Fol. subtus ad nervos villosa. ........... 14 Fol. subtus praeter nervos hinc inde villosa . . . . 30 Fol. subtus toto villosa. . . . . . . . . 4 ?

Sep. dorso eglandulosa. . . . . . . . . 3

2 Sep. dorso glandulosa - Sicilia (Gussone syn. fl sic. I, p. 564). C. Cupaniaxa Gdgr

(Aculei recti; fol. obovata vel oblonga . . . . . . . 4

\{ Aculei valde adunci; fol. ovata vel orbiculata . . . . . 5

| Fol. oblonga - Algeria, Tizi-Ouzou (Gdgr).3225. C. KärFol. obovata - Gallia, Gap. - 3226. C. vapensis (Gren. mss)

$5 \quad$ Fol. supra eglandulosa - Algeria, $\dot{m}$. Djurdjura Lella Kredidja (Debeaux) . . . .3227. C. DJunduUrensis (Debeaux)

6 Pet. tenuiter hirsuti ........... 7 Pet. glabri ${ }^{\text {Fr. globosus; tol. ovata - Sicilia, iradonia (Jan).3228 }}{ }^{8}$

$\mathrm{Fr}$ et fol rotundata - Italia a penn a C. viscosa (Jan) (Gibelli). . . . . . 3229. C. purpurea Gdgr ms

Fol. rotundata; fr, ovoideus - Corsica, etc.3230. C. SERA-

Fr. ovatus ; fr. globosus - Algeria, Djurdjura m. Tamgouth (Debeaux)................ C. CEDrorum Gdgr

I Fr. globosus ................ 10

9 Fr. ovoideus .................... 11

Fr. oblongus . . . . . . . . . . . . 12

$10\left\{\begin{array}{l}\text { Rami flor et pet. omnes inermes - Bouches-du-Rhône, Mar- } \\ \text { tigues (Autheman). . - . - . 3232. C. IMprobata Gdgr mss } \\ \text { Rami flor inermes }\end{array}\right.$ Rami flor. inermes; pet. sup. aculeati - Rhone, Liergues (Gdgr herb), ros.n. 345). . 3233. C. Divrmocycla Gdgr mss Pet. aculeati ; rami flor. inermes - Bouches-du-Rhone, Mar-

11 tigues (Autheman).....3234. C. Castagneana Gdgr mss Pet. et rami flor. inermes - Isère, Dessine (Chabert).3235

(Rami flor aculeati; pel. omnes sparse hirsuti. . . Gdgr mss Rami flor, inermes; pet, inf, hirsuti - Pyrenaei Or., HautTernet (Debeaux). . . 3236. C. nicthophlated Gidgr mis 
Discus conicus; fol. supra valde glandulosa - Suecia merid.

13 (Tullberg). Discus planus; fol. supra eglandulosa-Naples, m. S. Angelo di Stabia (Pasquale), . . .3238. C. stabiana Gdgr mss

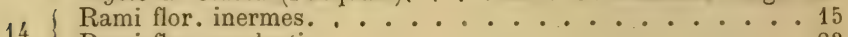

14 Rami flor aculeati. . . . . . . . . . 23

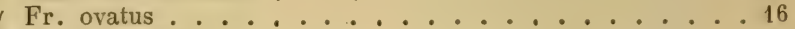

Fr. ovoideus . . . . . . . . . . . 17

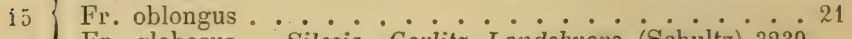

Fr. globosus - Silesia, Gorlitz Landskvore (Schultz). 3239

Pet. sup. aculeati ; fol. obovato-oblonga - Rhône, Pommiers

$16\left\{\begin{array}{l}\text { Pet. sup. aculeati ; fol. obovato-oblonga - Rhone, Pommiers } \\ \text { (Gdgr herb. ros. n. 242). . .3240. C. LEIocaulon Gdgr mss }\end{array}\right.$

6 Pet. omnes inermes; fol. oblonga - Gallia, Thonon (Puget)

$17\left\{\begin{array}{l}\text { Pet. omnes hirsuti. } \\ \text { Pet. sup. sparse hirsuti }- \text { Sabaudia, Thonon (Puget) } 3242\end{array}\right.$

17 Pet. sup. sparse hirsuti - Sabaudia, Thonon (Puget).3242

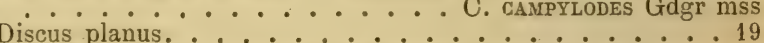

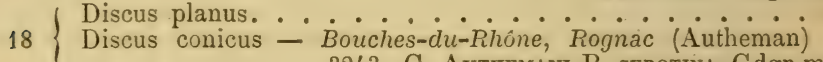

- . . . 3243. C. Authemani B. serotina Gdgr mss

19 Pet. omnes aculeati. . . . 20

19 Pet. inf. inermes - Rhône, Mont-Ceindre (Chabert).3244

. . . . . . . C. eustaninea Gdgr mss Pet. $8-10$ m. i. ; fr. ellipsoideus - Gallia, etc.3245. C. CHE-

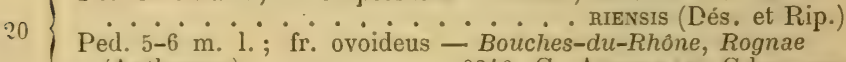

(Autheman). ....3246. C. Authenani Gdgr mss

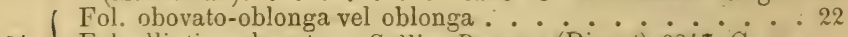

21 Fol. elliptico-obovata - Gallia, Bourges (Ripart).3247. C. PE-

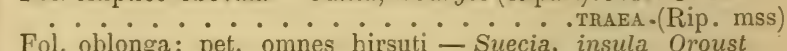

- Suecia, insula Oroust

(Forsell, etc.) . . . 3248. C. BAHUsiexsis Gdgr mss

Fol. supra eglandulosa ; pet. omnes sparse hirsuti - Silesia;

22 Posen, Czarnikau (Hülsen). . . 3249. C. silesiaca Gdgr mss

Fol. supra glandulosa; pet. sup. tantum sparse hirsuti Basses-Alpes, Bouzolieres (Proal).3250. C. MEGAstepHaxa

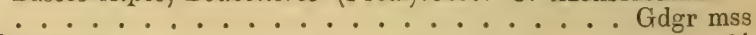

$23 \mathrm{Fr}$ ovatus $\mathrm{Fr}$. . . . . 24

23 Fr. globosus vel subglobosus . . . . . . . . . 25

Fr. ovoideus. . . . . . . . . . . . 26

Fol. ovata; pet. subinermes - Anglia.3251. C. CRYPTOPODA

24 Fol. obovata; pet. aculeati - Ardennes, Longué (Ć) (Baker)

........ 3252. C. PSEudo-Sepium (Callay mss)

( Pet. villosi; fol ovata - Sicilia, Madonia.3253. C. NEBRo$25\{$ Pet. sparse villosi; fol. obovata - Gallia, etc.3254. C. vir-

26 Fol. supra glandulosa . . . . . . . 27

Minime. . . . . . . . . . 28

Discus planus; pet. hirsuti - Gallia, Annecy (Bouvier). 3255

27 . . . . . . . . . Bouvieri (Crép. mss)

Discus conicus; pet. sup. sparse hirsuti - Algeria, Fort

National (Debeaux) .... 3256. C. AtLantica Gdgr mss 
Pet. omnes pubescentes . . . . . . . . 29

Pet. omnes sparse hirsuti, inf. vero inermes - Ain, Reyrieux

(l'ray) . . . . . . 3257. C. producta Gdgr mss

Pet. sup. sparse hirsuti omnesque aculeati - Rhone, Denicé

(Gdgr herb. ros. n. 292). - .3258. C. stid,BOANTnג Gdgr mss

Fr. conicus; fol oblonga - Rhone, Montmelas (Gdgr herb. ros. n. 299; Société dauph. exs. n. 110).3259. C. Boul-

Minim . . . . . . . Lun (Gdgr) Minime; fol. obovato-oblonga - Gallia.3260. C. Jondani (Dés.)

Fr, oblongus . . . . . . . . . . . . 31

30 Fr. globosus vel rotundatus . . . . . . . . . 32

Fr. ovatus ovato-rotundatus vel ellipticus . . . . . . 35

Fr. ovoideus vel obovato-oblongus. . . . . . . . . . 37

Fr. hasi decurrens; pet. aculeati-Basses-Alpes, Bonzolières

31 (Proal).........3261. C. macronasis Gdgr ms Ir. basi rotundatus; pet. inermes - Italia orient., Faenza (Caldesi)....... 3262. C. grandiconosa Gdgr mss Pet. et rami flor. aculeati . . . . . . . . . . . . 33

3? Minime - Ardèche, La Louvesc (Billot exs. n. 3853). 3263 . . . . . . . . C. actinostepinana Gdgr mss Discus planus; pet. pubescentes. . . . . . . . 34

33 Discus conicus; pet. sup. sparse hirsuti - Thuringia, Weimar (Baenitz herb. europ. n. 1274).3264. C. crmboldes . . . . . . . . . . . Gdgr mss Fol. supra glandulosa; fr. parvus - Vaucluse, Mt-Ventoux

34 (Autheman) - . 3265. C. Gallopnovincialis Gdgr mss Fol. supra eglandulosa; fr. major - Rhone, Couzon (Billot exs. n. 4028) . . . . . 3266. C. Calcarea Gdgr mss

35) Pet. hirsuti; fr. ovatus vel ovato-ellipticus. $-\dot{\text { Helv. }} \dot{\text { Bex }}{ }^{36}$ (Schleicher). . . . . 3267. C. Aspera (Schleich.) Fol. late oblonga; fr. ovato-ellipticus - Austria, Znaim

36 (Oborny) $\ldots . .3268$. C. Monavica Gdgr mss Fol. minutissime olovato-oblonga; fr. ovatus - Isêre, Crémieux, (Boullu)...... 3269. C. mixima (Puget mss)

Pet. omnes inermes. . . . . . . . . . 38

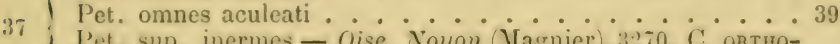
Pet. sup. inermes - Oise, Noyon Magnier .3270. C. ontuo. . . . . . . . . . . culamys Gdgr mss Rami flor. aculeati ; fol. obovata - Sabaudia, Salins (Puget)

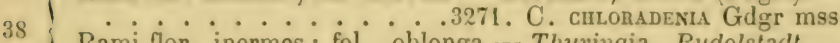
Rami flor. inermes; fol. oblonga - Thuringia, Rudolstadt (Dufft). . . . . . . 3272. C. Thunisgraca Gdgr mss Fol. obovata vel obovato-oblonga ........ . 40 Fol. ovato-elliptica; sep. dorso paulo glandulosa - Aragonia austr., Sierra de Mosqueruela (Martin). 3273 Fol. ovato-rotundata; sep. dorso e. C. Loscosuna Gdgr mss Salins (Puget). . . . 3274. C. Tanextasiensis (Pugret mss) Rami flor, aculeati ; fol obovato-oblonga. .... 41

40 Rami flor. subinermes; fol. obovata - Côte-d'Or, Meursault (Ozanon)........3275. C. comosordes (Ozan. mss) 
Sep. caduca; fol, apice subrotundata - Basses-Alpes, BouSep. persistentia; fol. subacuta - Basses-Alpes, Bouzolières (Proal). . . . . 3277. C. STEPHANOPHORA Gdgr mss

42 Tubus (fr.) ovatus rotundatus vel globosus. . . . . . 43

42 T Tubus (fr.) ovoideus vel oblongus. . . . . . 53

43 I Rami flor. inermes ............ . . 44

Rami flor. aculeati. . . . . . . . . . . 48

(Fr. globosus .............. 45

44 Fr. rotundatus. . . . . . . . . . 46

Fr. ovatus . . . . . . . . . . . 47

Cor. amoene rosea; fol oblonga - Gallia, Rouen (Rouy)

$45\left\{\begin{array}{l}\text { Cor. pallida vel alba; fol. obovato-oblonga - Tyrol, Trente } \\ \text { (Rouy) }\end{array}\right.$

(Gelmi) . . . . . . 3279. C. Cerasifera Gidgr mss

Fol. parva supra glabra - Isère, St-Nizier (J. B. Verlot)

Fol. lata supra hirta - Thuringia, Weimar (Haussknecht)

Pet. aculeati; ${ }^{\circ}-\cdots 3281$. C. Tomentella Gdgr ms

(Cottet). . . . . 3282. C. HETERobasis Gdgr ms

Pet. subinermes; fol. obovato-elliptica - Gallia, Chambéry

(Songeon) . . . . . 3283. C. Rufinervis Gdgr mss

Pet. inermes; fol. oblonga - Bouches-du-Rhône, St-Mitre

(Autheman).......3284. C. Macrostyla Gdgr mss

Fr. ovatus. . . . . . . . . . . . 49

Fr. subglobosus . . . . . . . . . . 50

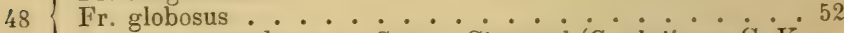

Fr. ovato-rotundatus - Germ., Stargard (C. glutinosa C. K.

Schultz prodr. fl. starg.). . . 3285. C. STARgardiensis Gdgr

Pet. hirsuti; fol. elliptico-obovata - Rhône, Montmelas

(Gdgr herb. ros. n. 357) …3286. C. Didymacantha Gdgr

Pet. hirsuti ; fol. obovato-oblonga - Bouches-du-Rhóne,

Istres (Autheman) . . . 3287. C. SERPYlloIDEs Gdgr mss

Pet. sparse hirsuti; fol. oblonga - Dania, Horneboeks (Lundquist) . . . . . . 3288. C. DANICA Gdgr mss

Fol. oblonga .............. . 51

Fol. obovato-oblonga - Sabaudia, Chambéry, Moûtiers

(Songeon) . . . . . 3289. C. vincta Gdgr mss

Fol. oborato-elliptica - Helv., Fribourg Montbovon (Cottet)

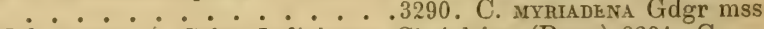

Fol: ovata - Seine-Inférieure, St-Adrien (Rouy).3291. C.

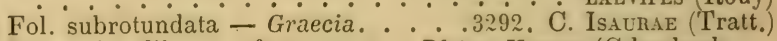

Fol. apice dilatata; fr. magnus - Rhône, Yzeron (Gdgr herb.

51 ros. n. 397).............. C. Zygrs Gdgr mss

Minime; fr. mediocris - Rhône, Dardilly (Chabert). 3294.

. . . . C. Fragrans Gdgr mss

Fr. parvus; fol. elliptica - Austria, Vienne (Skofitz).3295.

. . . . . . . C. Hinsutula Gdgr mss

Fr. parvus ; fol obovata - Basses-Alpes, Condamine (Proal)

52

Fr. minimus; fol. ovato-elliptica - Gallia, Lyon, etc. (Gdgr

herb. ros. n. 451 et 452).3297. C. LugDuNENSIS (Chabert; Dés.)

Fr. major; fol. rotundata - Podolia . .3298, C. KuukII (Bess.) 
Pet. toto villosi vel tomentosi. . . . . . . . 54

Pet. glabri, vel nune villosi nune sparse hirsuti . . . . . 70

Aculei magni adunco-dilatati; frutices validi. . . . . . 55

Aculei tenues inclinati parum dilatati ; frutices graciles. . 66

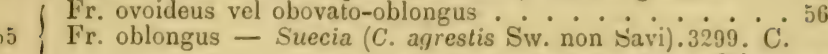

. .......... suecica Gidgr mss

( Pet. inf inermes ............. . . . . . .

Pet. omnes aculeati . . . . . . . . . 58

Pet. omnes inermes - Bouches-du-Rhöne, Berre (Autheman)

3300. C tortuosa Gdgr mss

Fol. elliptica basi rotundata - Rhone, Montmelis (Grler herb. ros. n. 212). . . . . 3301. C. cladophora Gdgr

Fol. obovata basi cuneata - Sabaudia, Salins (Puget).3302.

. . . . . . . C. zygoantra Gdgr mss Fol. obovato-oblonga basi cuneata - Thlone, Dardilly (Chabert).........3303. C. nypoacantira Gdgr mss

58 Fol. apice rotundata. . . . . . . . 59

Fol apice attenuata $\ldots 61$

5) Fol supra glandulosa - Podolia, hremeniec.330\%. C. Ca- 60 . . . . . . . . nyophylacea (Bess.)

( Ped. 8-10 m. 1.; fr. obovato-oblongus - Rhône, Montmelas (Gdgr herb. ros. n. 172). . . . .3305. U. PERAfFinis Gdgr

Ped. 4-5 m. 1.; fr. breviter ovoideus - Pedem., Vallées Vaudoises (Rostan). . . . . 3306. C. opAcA Gdgr mss

Fol. obovato-oblonga .............. 62

61 Fol. oblonga . . . . . . . 63 63

61 Fol. obovato-elliptica vel elliptica. isere, La Salette (Boullu).3307. C. AMACANTHA 65

( Ped. 12-14 m. l.; fol. supra hirta - Bouches-di-Iihone, Maitigues (Autheman) . . . 3:30s. C. Theratopuma Giler mss Ped. 5 -8 m. 1.; fol. supra glabra - Lin, Les Echeyx (Chabert) . . . . . . 3309. C. Astenoidea Gdgr mss

$63\left\{\begin{array}{l}\text { Fol. basi attenuata. } \\ \text { Fol. basi rotundata subtus flavescentia - Bouches-du-Rhône, }\end{array}\right.$ Martigues (Autheman) . . 3310. C. Flavescens Gdgr mss

$64\left\{\begin{array}{l}\text { Rami flor. aculeati ; fol. lanceolata - Bohemia, Kuchelbad } \\ \text { (C. albiftora Opiz non Stev.) - . 3311. C. Bomemica Gdgr }\end{array}\right.$ Rami flor. inermes; fol. subacuta - Germania occ., Nordhausen (Vocke) . . . . 3312. C. HencYNicA Gdgr mss Fol. elliptica subbiserrata - Bohemia (Tausch).3313. C.

65 . . . . . . Elliptica (Tausch) Fol. obovato-elliptica argute biserrata - Rhône, Montmelas (Gdgr herb. ros. n. 513) . . . . .3314. C. Arolu) Gidgr Fr. ovoideus vel obovato-oblongus ....... 6i

$66\left\{\right.$ Fr. ovatus - Helv, Fribourg Montbovon (Cottet). $3315^{\circ} \dot{C}^{\circ}$. Fr. globosus - Caucasus, etc. - 3316. C. AREmania (II. Bieb.)

Minime. . . . . . . . . . . 68

67 Sep. dorso glandulosa - Gallia, Chambery (Songeon). 33 i7.

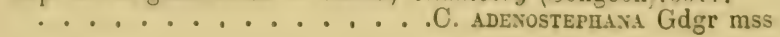




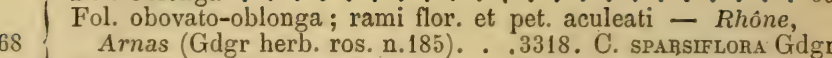

Fol. elliptico-obovata; pet. et rami flor. inermes - Gallia, Chambéry (Songeon) . . .3319. C. Camberiacensis Gdgr mss Rami flor. aculeati ; pet. omnes inermes - Thuringia, Wei-

69 mar (Haussknecht). . . . . 3320. C. BREvion Gdgr mss Rami flor. inermes ; pet. inf. aculeati - Bohemia, Kralupy (Polảk)............ C. PRAGENSIs Gdgr mss

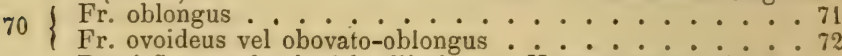

(Rami flor. aculeati; fol. elliptica - Naples, Vésuve (C. canina v. fr. ellipsoideo Pasq. fl. vesuv. p. 42).3322. C. PAS-

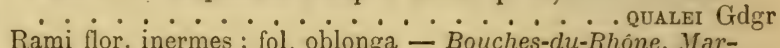
Rhône, Martigues (Autheman) . . . .3323. C. subaurata Gdgr mss

72 Fol. basi | attenuata . . - * * * 73 Fol. basi rotundata - Valais, irartigny (Delasoie).3324. ${ }^{\circ}$. . . . . . . . . . zygloIdes Gdgr mss

73 Pet. | aculeati . Pet. omnes inermes - Bouches-du-Rhône, Martigues (Autheman). . . . . 3325. C. nediterranea Gdgr mss

74 ( Rami flor inermes ............ . 75

it Rami flor aculeati . . . . . . . . 77

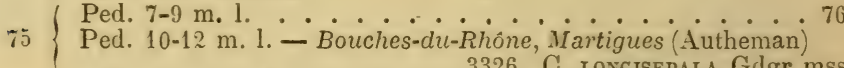
. . . . . . 3326. C. Longisepala Gdgr mss ( Sep. foliacea ; fol. amplissima - Dania, Hornboek (Jonsson)

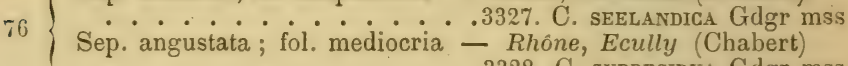

. . . . . . 3328. C. subdecidua Grdgr mss

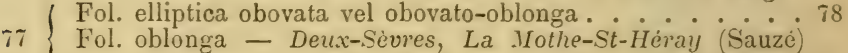

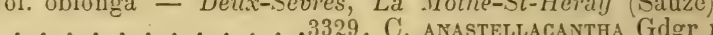

- 5 Fol. apice atlenuata. . . . . . . . . . 79

78 Fol, apice rotundata. $\quad . \quad 80$

Fr. subovoideus; fol. mediocria - Sabaudia, Salins (Puget) $79\left\{\begin{array}{l}\text { Fr. obovatus; fol. lata - Rhône, Limas (Gdgr herb. ros. }\end{array}\right.$ n. 16). . . . . . . 3331. C. ARgus Gdgr $80\left\{\begin{array}{l}\text { Stip. subtus eglandulosae; fol. obovato-oblonga - Basses- } \\ \text { Alpes, Condamine (Proal). 3332. C. Viluarsiana Gdgr mss } \\ \text { Stip. subtus glandulosae; fol. elliptico-obovata - Cher, }\end{array}\right.$ Chapelle-St-Ursin (Ripart). . .3333. C. FALLACINA (Kip. mss) 
B. - Echubiginosae Gdlgr Essai p. 38 (Rubiginoseae micranthae et suavifoliae Crép. Prim. mon. ros. p. 23).

Styli liberi, nune glabri vel glabriusculi tune subeoaliti vel fascieulati, nume vero villosi vel lanati igitur sessiles vel brevissime eapitati; flores parvi, intense purpurei cum ungue albo vel flavescente, rarius toto albi; fructus sat praecox, intense ruber, minor, glaber aut echinatus glandulosusve, subpulposus; sepala plerumque normaliter corso glandulosa, pinnatipartita, post anthesim defexa tandem decidua, hic et nune erecta sed modo emortuo fructum maturum coronantia ; pedunculi semper hispidi rel glandulosi, interdum alii laeves, alii glandulosi, alii tandem villosi vel villoso-glandulosi, breviores sat crassi; folia praecocius caduca, supra nitide virentia, infra toto rubiginoso-glandılosa, attritu amoene olentia ; aculei e basi dilatata compressaque falcati, inclinati vel rectiusculi, in setas glanduliferas praesertim ad ramos annotinos vel biennios interdum prodeuntes. - Frutices in locis lapidosis et praesertim calidis ubique rulgati, ramos sacpe fiexuose articulatos, jlus minusve aculeatos confertosque habentes.

\footnotetext{
( Ped. omnes normaliter glandulosi. . . . . . . . I

A. Ped. villosi, vel nune glabri nune paulo glandulosi 1. Iletero-

Styli glaberrimi, evidenter subcoàliti. . injpostyloidae

B. Styli glabrescentes tunc omnino liberi, vel villosi ergo ad basin | subcoaliti......... 3. Mesostyloidae Styli lanati liberi et sessiles. ....... 4. Apostyloidae
} 


\section{1. - Heteropodae Gdgr Essai p. 38.}

Pedunculi alii glabri, alii glandulosi, alii tandum villosi, vel omnibus sive villosi sive villoso-glandulosi (semper in eodem individuo vel ramo florifero); flos albus vel roseus ; caetera ut in Eurubiginosis.

Ped. alii glabri, alii villosi, alii paulo glandulosi (in eodem ramo). . . . . . . . . . . . 2 Ped. omnes villosi. . . . . . . . . . 4 Ped. alii glabri, alii paulo glandulosi . . . . . . . 10

( Styli hirsuti; fol. ovata vel obovata, supra glabra. . . . . 3

2 Styli glabrescentes; fol. supra hirta - Italia orient., Faenza (Caldesi) . . . . . . 3334. C. Acridentata Gdgr mss Fr. obovato-oblongus; fol. ovata ad nervos subhirta - Styria,

3 Gratz (Skofitz). . . . 3335. C. HETERopes Gdgr mss Fr. oblongus; fol. obovata subtus toto villosa - Pyren. Or., Mont-Louis (Debeaux) . . . .3336. C. OROCHARIS Gdgr mss

$\mid$ Rami flor. glabri et eglandulosi. . . . . . . 5

4 Rami flor. villoso-glandulosi - Italia, Modène (Gibelli). $3337^{\circ}$.

5) Fol. subtus ad costam tantum hirsuta. . ERIoclada Gdgr ms Fol. undique hirsuta. . . . . . . . . . 7 Rami flor. inermes; fol. elliptita-Pedem., Vallées Vaudoises

6 (Rostan) . . . . 3338. C. pedemontana Gidgr mss Rami flor. aculeati; fol. obovata - Aragonia austr., Sierra de Mosqueruela (Martin). . . . .3339. C. Pardoana Gdgr mss

7 Styli glabri vel glabrescentes. . . . . . . . . 8 Styli hirsuti vel lanati ............. 9

Fr. et fol. oblonga - Italia orient, Faenza (Caldesi). 3340

8 . . . . . . . . . ERIOPODA Gdgr mss Fol. ovato-elliptica; fr. obovato-oblongus - Italia orient., Faenza (Caldesi). . . . .3341. C. HeteromorpHa Gdgr mss Fr. globosus; fol. oblonga - Bouches-du-Rhône, St-Mitre (Autheman) ........3342. C. Robertiana Gdgr mss

9 Fr. ovatus; fol. ovato-rotundata - Persia, m. Elbrus (Kotschy exs. n. 276). . . . . . . .3343. C. Aucheri (Crép.) Fr. ovoideus; fol. obovato-elliptica - Sabaudia, etc. (Billot exs. n. 3594) .......3344. C. Billetil (Puget)

10 Sep. dorso eglandulosa. . . . . . . . . 11 Sep. dorso I dense glandulosa $\ldots$

11 Fol. supra eglandulosa ; fr. globosus ovoideus vel oblongus . 12

11 Fol. supra valde glandulosa; fr. ovatus - Hisp., Sierra de Yunquera, Malaga (Hegelmaier).3345. C. Gravatensis Gdgr mss 
12 Fr. globosus vel rotundatus.

Fr. ovoideus vel oblongus. . . . . . . 14

Fol. lanceolata; styli lanati - Rhơne, le Mont-Ceindre

13 (Gdgr herb. ros. n. 476). .3346. C. Montceindriana Gdgr mss Fol. obtusa; styli hirsuti - Rhône, Mont-Thou (Chabert) ........ 3347. C. HYlebia Gdgr msS

14 Styli glabri ; fr. ovoideus . . . . . . 15 Styli villosi; fr. oblongus-Basses-Alpes, Condamine (Proal) . . . . . . . 3348. C. Montigena Gdgr mss

$15\left\{\begin{array}{l}\text { Rami flor. aculeati ; nervi villosi - Austria, Znaim (Oborny) } \\ \text { Rami flor. inermes; costa fol. villosa - Valais, Branson }\end{array}\right.$ Rami flor. inermes; costa fol. villosa - Valais, Branson (Wuitel) . . . . . 3350. C. Inconstans Gdgr mss Fr. ovatus vel ovoideus. . . . . . . . . 17

Fr. elongatus; styli glabri - Ital. or., Faenza Brisighella

16 (Caldesi). . . . 3351. C. Lagenifonmis Gdgr mss Fr. globosus; styli lanati-IIisp. austr., Sierra de Yunquera (Hegelmaier). . . . . 3352. C. malacitana Gdgr mss

17 Aculei late adunci; fol. | acuta .......... 18 1 Aculei recti; fol obtusia . . . . . . . . . 19 Styli glabrescentes; costa villosa - Italia, Apenn. Modène

18 l'abetone (Gibelli)......3353. C. Latispina Gdyr mss Styli lanati ; fol. undique villosa - Aragon. austr., Sierra de Mosqueruela (Martin) . . 3354. C. Willkommina Gdgr mss Fol. rotundata; sep. toto glandulosa-Basses-Alpes, Con-

19 damine (Proal) . . . . 3355. C. pimpinelloides Gdgr mss Fol. obovata; sep. in $1 / 3$ inf. extus laevia - Basses-Alpes, Jausières (Proal) . . . . 3356. C. onopHILA Gdgr mss

\section{2. - Hypostyloidae Gdgr Essai p. 38 (Rubig. micran-} thae Crép. loc. cit. parte).

P'edunculi semper plus minus copiose glandulosi vel hispidi ; styli glaberrimi rarius subsparse hirtuli, post anthesim vel ad fructus maturitatem basi in columnam gracilem satque exsertam breviter subcoaliti; foliola glabra vel glabrescentia ; frutices laxi conspicue plerumque ramoso-articulati.

$1\left\{\begin{array}{l}\text { Ramorum floriferorum aculei conformes dilatati alii setaceo- } \\ \text { Ramorum floriferorum aculei alii falcato-d } \\ \text { glanduliferi }\end{array}\left\{\begin{array}{l}\text { Pet. omnes hirsuti vel tomentosi } \\ \text { Pet. alii sparse villosi alii vero glabri. } \\ \text { Pet. omnes sparse villosi. } \ldots\end{array}\right.\right.$ 
3 Tubus toto vel saltem usque in $1 / 3$ vel $1 / 2$ inf. glandulosus. . 4

3 Tubus glaber vel basi infima tantum glandulosus. . . . . 31

Fol. subtus toto hirsuta ............ 5

4 Fol. subtus ad costam vel nervos hirsuta. . . . . . 20

| Fr. ovato-rotundatus vel ovatus. . . . . 6

๖ Fr. ovoideus. ....................... 8

Fr. oblongus ...................... 13

( Discus conicus. . . . . . . 7

6 Discus planus - Europa med., etc. (Gdgr herb. ros, n. 492) . . . . . . . . 3357. C. mitrantha (Sm.) ( Rami flor. et pet. aculeati - Sardinia, Cagliari (Thomas)

7 Rami flor. inermes ; pet. subiner .3358. C. oligocarpa Gdgr mss exs. n. 3725). Rhône, Tassin (Billot

8 Rami flor. inermes. . . . . . . . . . 9

8 Rami flor. aculeati. . . . . . . . . . 11

Fol. ovata vel elliptica basi i attenuata. . . . . 10

9 Fol. obovata basi rotundata - Serbia bor., Klokocevac (Pancic) . . . . . . 3360. C. Pancicrr Gdgr mss Discus subconicus; fol. ovato-elliptica - Belg., Han-surDiscus planus; fol. ovata - Belg., etc. (Gdgr herb. ros. n. 475) . . . . . . . . . . N362. C. Norosa (Lib.)

Pet. aculeati; fol. ovata vel rotundata . . . . . . 12

Pet. aculeati ; fol. obovata - Isère, Vienne etc., (Gdgr herb.

11 ros. n. 389)......3363. C. DELPHINENSIS (Chabert) Pet. inermes; fol. oblonga - Oise, Noyon (Magnier). 3364

12 Fol. rotundata - Gallia, etc. $\therefore$. . . N365. C. DImInta (Bor.) Fol. ovata - Helvetia, etc. $33660^{\circ}$ C. Micrantho-sepium (Christ) f Fol. elliptica obovata vel oblonga. . . . . . 14

13 Fol. rotundata - Carinthia, Lienz (Pichler).3367. C. Cacosma ................. Gdgr mss

14 Discus conicus. . . . . . . . . . 15

14 Discus planus. . . . . . . . 17

(Fol. elliptica vol obovata. . . . . . . . 16

15 Fol. oblonga basi attenuata - Valais, Bovernier (Delasoie) . . . . . . 3368. C. prenoclada Gdgr mss Ped. 2i-24 m. i.; rami flor. inermes - Hisp., Biscaye Ur-

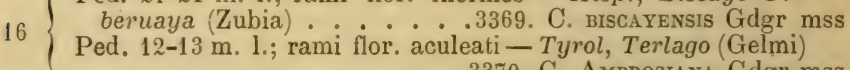

17 Fol. oblonga basi attenuata. 3370. C. Ambrosiana Gdgr mss

17 Fol. obovata vel obovato-oblonga basi rotundata $\ldots 19$ (Sep. apice angustata; rami flor. aculeati - Manche, Yvetôt

18 (Lebel). ......3371. C. cRyptostigMa Gdgr mss Sep. apice dilatata; rami inermes - Manche, Yvetôt (Lebel) . . . 3372. C. cryptostigna B. Lebeli Gdgr mss Ped. $1 \dot{7}-19$ m. l.; pet. aculeati - Hisp. centr., El Escorial

19 (Laguna). . . . . . 3373. C. Lagunae Gdgr mss Ped. 10-12 m. l.; pet. sup. inermes - Hisp. centr., El Escorial (Laguna). . . . 3374́, C. Cutandaeana Gdgr mss 
Fr. ovatus vel ovato-rotundatus . . . . . . . . 21

Fr. ovoideus vel obovato-oblongus. . . . . . . . . 23

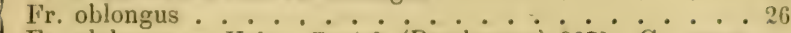

Fr. globosus - Helv., Zurich (Bamberger).3375. C. Ionos-

. . . . . . . . MANTII Gdgr mss

Rami flor. aculeati; fol. basi attenuata. . . . . . 22

21 Rami flor. subinermes; fol. basi rotundata - Rhone, Arnas (Gdgr). . . . . . 3376. C. paciryseta Gdgr mss Fol. lanceolata elliptica - Ain, St-Didier-de-Formans (Fray)

22 . . . . . 3377. C. Fray Gdgr mss Fol. acuta oljovato-oblonga - Cher, etc.3378. C. spinaro-

I Rami flor inermes. $\therefore \ldots \ldots$ phona (Rip.)

23 Rami flor aculeati. 2.25

Discus planus; fol. elliptica - Puy-de-Dome, Pionsat (Girlgr

21 lerb. ros. n. 225). . . . 3379. C. Intricans Gdgr mss Discus conicus; fol. obovato-oblonga - Anglia, Leicestersh. Ashby Parva (Ley) . . . . .3380. C. Anglica Gdgr mss

Pet. subinermes; discus conicus - Pyrenaci, Perpignan (1)e-

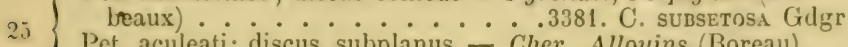
Pet. aculeati; discus subplanus - Cher, Allouins (Boreau).

......... 3382. C. Ducens Gdgr mss

$26\{$ Fol. ad nervos hirsuta. . . . . . . . . . 27

Fol. ad costam villosa - Pyren. Or., St-Paul-de-Fenouillet

(Debeaux). . . . . 3383. C. mutabilis (Debeaux)

27 Rami flor aculeati. . . . . . . . . 28

Rami tlor inermes. . . . . . . 29

Fol. lata; discus subconicus - Austria, Vienne (Skofitz)

Fol. minuta; discus conicus - Istria, Lupoglava (Ireyn)

$29\left\{\begin{array}{l}\text { Pet. inermes; fol. obovato-oblonga } \text { Pet. aculeati ; fol. elliptica - Tyrol, Lases in Pine (Gelmi) } \\ \text { Po }\end{array}\right.$

Pet. aculeati; fol. elliptica - Tyrol, Lases in Pine (Gelmi)

. . . . . . 3386. C. cylindrocarra Gdgr mss

Fr. basi depressus; fol. apice rotundata - Isère, Charancieu

30 (Gdgr herb. ros. n. 33). ‥3387. C. Cladocuanis Gdgr mss

Fr. basi attenuatus; fol. apice attenuata - Pyren. Or.., Caslelnau (Debeaux) . . . . 3388. C. strommifolia Gdgr mss

31 Fol. subtus ad nervos vel toto aut undique hirsuta . . . . 32

31 Fol. subtus ad costam villosa............. 54

Fol. subtus toto villosa. . . . . . . . . 58

$32\{$ Fol. ovata vel ovato-rotundata . . . . . . . 33

Fol. elliptica obovato-oblonga vel oblonga. . . . . . 36

Fr. rotundatus vel ovato-rotundatus . . . . . . . . 34

33 Fr. ovoideus. . . . . . . . . . . . 35

Fr. oblongus - Hte-Savoie, Annecy (Puget).3389. C. Palles-

........................ Gens Gdgr mss

(Pet. subinermes ; fr. rotundatus - Rhône, Brignais (Chabert)

34 . . . . . 3390. C. Quassa Gdgr mss

Pet. aculeati ; fr. ovato-rotundatus - Landes, Dax (Maillard)

. : . . . . 3391. C. blepharidea Gdgr mss

( Rami decorticati ; fol. recta - Hte-Savoie, Annecy (Puget) (Ite-Savoie, Pringy (Puget).3393. 
36 Sep. dorso eglandulosa. . . . . . . . . . 37

Minime . . . . . . . 40

37 Rami flor et pet. inermes . . . . . . . . . 38

37 Rami flor et pet. aculeati $\ldots 39$ Fr. obovato-oblongus; fol. basi rotundata - Hisp. centr.,

38 El Escorial (Laguna) . . . . 3394. C. Castellana Gdgr mss Fr. oblongus; fol. basi attenuata - Hisp. centr., El Escorial (Laguna). . . . . . . 3395. C. CARPETANA Gdgr mss Cor. purpurea; fol. supra eglandulosa - Hisp. centr., El

39 Escorial (Laguna) . . . . 3396. C. Madpritensis Gdgr mss Cor. alba ; fol. inf. supra glandulosa - Hisp. centr., El Escorial (Laguna) . . . .3397. C. Guadarramae Gdgr mss

$40\left\{\begin{array}{l}\text { Fr. oblongus } \\ \text { Fr. ovoideus vel obovato-oblongus. }\end{array}\right.$ Fr. ovatus - Gallia, etc. (C. salvanensis Delasoie). 3398.

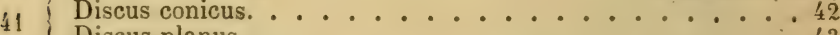

Biscus planus .

Fr. apice attenuatus; fol amplissima - Valais, Salvan (De-

42 lasoie) . . . . . 3399. C. Coryphaea Gdgr mss Fr. apice longe strangulatus; fol. mediocria - Istria, $m$. Maggiore (Freyn). . . . 3400. C. Tommasiniana Gdgr mss

( Fol. elliptica basi rotundata - Pyrenaei, Gèdre (Bordère).

43 Fol oblonga bi - * . . C. Assimuls Gdgr mss bert).......3402. C. RHynchantha Gdgr mss

Pet. sup. inermes . . . . . . 45

Pet. omnes aculeati. . . . . . . . . . 46

Pet. inf. inermes - Isère, Balmes Viennoises (Chabert). 3403.

P........ C. Blepharosepala Gdgr mss Pet. omnes inermes - Hisp. austr., Sierra de Alfacar (Hegelmaier)........3404. C. BaEtíca Gdgr mss

Discus subplanus ; ped. $13-16$ m. i. - Pyrenaei, Gèdre (Bor-

45 dère). . . . . . 3405. C. LAxistyla Gdgr mss I Discus conicus ; ped. 11-12 m.1.-Pyren. centr., Benqué (Bordère). . . . . . 3406. C. Borderi Gdgr mss

46 Fol. oblonga . . . . . . . . . 47

46 Fol. elliptico-obovata vel obovato-oblonga . . . . 48 Rami flor. et pet. aculeati - Oise, Compiègne (Maire).3407.

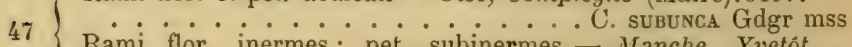
Rami flor. inermes; pet. subinermes - Manche, Yvetôt (Lebel). . . . . . . 3408. C. subattenuata Gdgr mss

( Discus planus. .................. 49

48 Discus conicus - Austria, Vienne (Wiesbaur).3409. C.

(Rami flor inermes. . . . . ConICA Gdgr mss

49 Rami flor aculeati. . . . . . . . . 52

50 (Pet. aculeati. . . . . . . . . 51

Pet. subinermes - Saône-et-Loire, Rougeon (Ozanon).3410.

. . . . . . . . C. septicoloides (Crép. mss)

$51\left\{\begin{array}{l}\text { Ped. 11-12 m. i, fol. basi rotundata - Belg., Rochefort } \\ \text { (Reliq. Maill. n. 1081) }\end{array}\right.$

Ped. 12-14 m. l.; fol. basi attenuata - Anglia, Warwich Chesterton (Fraser). . . . 3412. C. Fraseri Gdgr mss 
Fol. elliptica basi rotundata - Rhône, Villefranche (Gdgr). . . . . . . . . 3413. C. scrutata Gdgr mss (Ped. 12-15 m. 1.; fol. amplissima - Pyrenaei, Gedre (Bor-

53 dère) . . . . . 3414. C. viridipes Gdgr mss Ped. 7-9 m. l.; fol. mediocria - Finistere, Crozon (Thiébaut). ............... C. Peculiaris Gdgr mss Sep. dorso glandulosa . . . . . . . . . . 55

54 Sep. dorso eglandulosa - Italia, Selva Pisana (Savi) 3416. . . . . . . . . C. pisana Gdgr msa Fr. ovoideus vel obovato-oblongus ....... 56 55 Fr. ovatus; discus planus - Ain, Trévoux (Fray.).3417. C. Fr. oblongus; discus conicus - Savoie ${ }^{\circ}$ DIssidens Gdgr mss . Puget).3118. . . . . . . . C. graciliramea Gdgr mss

Rami flor inermes; fol elliptica . . . . . . . 57

56 Rami flor. aculeati; fol. obovata - Aragonia austr., Sierma de Mosqueruela (Martin) . . 3419. C. Langeava Gdgr mss Tubus laevis; pet. subinermes - Rhône, Liergues (Gdgr

57 herb. ros. n. 293). $\begin{aligned} & \text { Tubus in } 1 / 2 \text { inf. subsetosus; pet. aculeati - Rhône, Liergues } \\ & \text { Ths }\end{aligned}$ (Gdgr herb. ros. n. 321).3421. C. Desmocarpa B. Parvifolia . . . . . . . . . . Gdgr mss

is Rami flor inermes. . . . . . . . . . 59

is 1 Rami flor aculeati. . . . . . . 64

Fr. ovoideus vel obovato-oblongus. . . . . . . 60

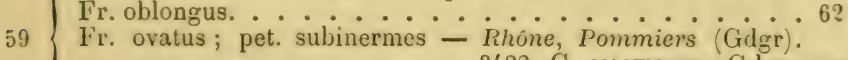
Fr. subglobosus ; pet. aculeati - Gallia C. Masticnina Gdgr mss velicula (Dés.)

60 Fol. obovata vel obovato-elliptica.. . . . . . 61 Fol. ovata - Helvetia occid. .3424. C. Lusseri (Lagg. et Pug.)

$61\left\{\begin{array}{l}\text { Fr. magnus; fr. basi rotundatus - Ain, St-Didier-de-For- } \\ \text { mans (Fray) } \\ \text { Fr. mediocris } ; \text { fol. basi depressus - Ain St-Didier-sur- }\end{array}\right.$ Fr. mediocris; fol. basi depressus - Ain, St-Didier-surChalaronne (Fray). . . 342fi. C. PSECDO-SEPTICol.a Gdgr mss Discus conicus; fr. basi rotundalus . . . . . . . 63

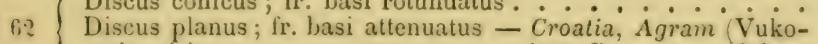
tinovic). . . . . . 3427. C. croatica Gdgr mss Pet. subinermes; fol. obovata - Ilelvetia, Altorf (Gisler).

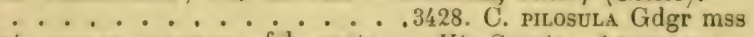
Pet. omnes mermes; fol. ovata - IIte-Savoie, Argonnex (Puget). . - . 3429. C. pHalacroclada Gdgr mss Pet. omnes aculeati; fol. ovata - Hte-Savoie, St-Martin (Puget). . . 3430. C. Phalacroclada B. Pugeti Gdgr mss Fr. ovoideus vel obovato-oblongus. . . . . . . . 65

Fr. oblongus .............. . . 68

65 Fol. basi attenuata................ 66 Fol. basi rotundata . . . . . . . . 67 Pet. subinermes; fol. obovata - Gard, Le Vigan (Billot exs.

66 n. 3.998 ex parte) . . . 3431. C. Stexoriylla Gdgr mss Pet. aculeati ; fol. obovato-elliptica - Rhone, Amas (Gdgr herb. ros. n. 202). ..... 3432. C. Heteroclita Gdgr 
Fol. obovata ; pet. subinermes - Rhône, Arnas (Gdgr herb.

67 ros. n. 232). . . . . . 3433. C. DISCEDENS Gdgr mss Fol. ovato-elliptica; pet. aculeati - Rhône, Arnas (Gdgr herb. ros. n. 55). . . . 3434. C. PATER-Familias Gdgr mss

68 Discus planus; fol. basi attenuata. . . . . . . 69

68 Discus conicus; fol. basi rotundata - Rhône, Arnas (Gdgr herb. ros. n. 565). . . . . 3435. C. Mesto Gdgr Pet. inf. inermes ; fol. ovato-elliptica - Rhône, Gleizé (Gdgr

69 herb. ros, n. 270). • . . 3436. C. Dichroopetala Gdgr Pet. omnes aculeati; fol. elliptico-obovata - Pyrenaei, Gèdre (Bordère). . . . 3437. C. TePHróacantha Gdgr mss

70 Sep. dorso eglandulosa. . . . . . . . . 71

70 Sep. dorso 1 dense glandulosa. . . . . . . . . 73

Pet. aculeati ; fol. basi rotundata . . . . . . . 72

71 Pet. inermes; fol. basi attenuata - Hisp. austr., Sierra de Segura, Campo de S. Juan (Canada).3438. C. Muncica

. . . . . . . . Gdgr mss Aculei adunci; ped. 19-22 m. l. - Hisp. centr., El Escorial Aculei recti ; ped. 12-13 m. 1. - Basses-Alpes, Condamine (Proal). . . . . 3440. C. calacantha Gdgr mss Fr. ovoideus vel obovato-oblongus. . . . . . . . 74

73 Fr, oblongus . . . . . . . . 78 Fr. ovatus - Hisp., Badajoz Olivenza (Torrepando).3441. . . . . . . . C. transtagana Gdgr mss

74 Fol. subtus ad costam villosa. .......... 75

Fol. subtus ad nervos vel toto villosa . . . . . . . . 76

Tubus in $1 / 2$ inf. glandulosus; fol. basi attenuata - Gard,

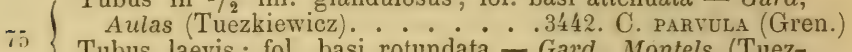
Tubus laevis; fol. basi rotundata - Gard, Montels (Tuezkiewicz) . . . . 3443. C. orthacantha Gdgr mss

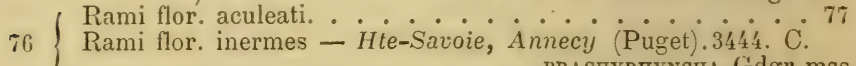

......... BRACHYRHYNCHA Gdgr msS Pet. inf. inermes; fol. ovata - Rhóne, Villefranche (Gdgr).

Pet omnes aculeati; fol obovato-oblong. C. EFFata Gdgr ms (Gelmi). ........3446. C. Ternispina Gdgr mss

Fol. subtus ad nervos vel toto hirsuta. . . . . . . 79

Fol. subtus ad costam mediam hirta. . . . . . . . 82

Tubus glaber vel basi glandulosus. . . . . . 80

79 Tubus toto glandulosus - Pyren. Or., Amélie-les-Bains (Debeaux). . . . . . 3447. C. Castanearum Gdgr mss

$80\left\{\begin{array}{l}\text { Fol. ovata vel obovata basi rotundata } \text { Fol. oblonga basi attenuata }- \text { Hisp. mer., Sierra de Segura, } \\ \text { de }\end{array}\right.$ las Mruelas (Canada) . . . . . 3448. C. Segurae Gdgr mss

Discus conicus ; pet. et rami flor. aculeati - Hte-Savoie, Ar-

81 gonnex (Puget). . . : 3449. C. ARGonnexensis Gdgr mss Discus planus; pet. et rami flor. inermes - Catalaunia, Sagaro (Vayreda). . . . 3450. C. celtiberica Gdgr mss Pet. omnes aculeati. . . . . . . . . . 83

$82\{$ Pet. inf. inermes - Rhône, Tassin (Chabert).3451. C. ELIsoPet. omnes inermes - Ṕyrenaei Or., Camélas (Debeaux) 
83 / Rami flor inermes. . . . . . . . . . 84

Rami flor aculeati, . . . . . . . . 85

Tubus in $1 / 3$ inf. glandulosus; sep. dorso subeglandulosa Catalaunia, Sagaro (Vayreda). .3453. C. Gerundensis Gdgr mss

Tubus toto laevis; sep. dorso dense glandulosa - Aragonia austr., Sierra de Mosqueruela (Martin).3454. C. Queruaxa . . . . . . . . . . Gdgr mss Tubus basi glandulosus; fol. obovato-oblonga - Rhone, Vérigny (Chabert). . . . . . 3455. C. Domita Gdgr mss Tubus toto glandulosus; fol. obovata - Rhône, Frontenas (Gdgr). . . . . . 3456. C. setulifera Gdgr mss Tubus oblongus. ............. . . . 87

Tubus rotundatus ovatus vel ovoideus . . . . . 104

Tubus toto vel saltem usque ad $1 / 3$ inf. glandulosus. . . . .88

Tubus laevis vel tantum basi infima glandulosus. . . . 95

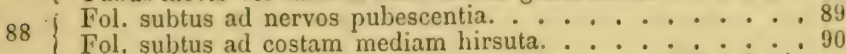
Rami flor, et pet. inermes - Rhône, Pommiers (Gdgr herb.

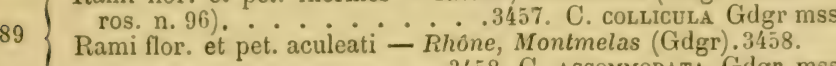
.3458. C. Accommodata Gdgr mss

90 Fol. elliptica obovata vel obovato-oblonga . . . . . . 91 Fol. oblonga - Lot-et-Garonne, Agen (Debeaux).3459. C. Fol basi rotundata $\because \because \because \because \ldots 2$ Fol. basi attenuata. .................. 93 Ped. 25-27 m. l.; discus subplanus - Catalaunia, Maza (Vay- $92\left\{\begin{array}{l}\text { reda) } \cdot 19 \cdot 1 . ; \text { discus conicus - Gard, Le Vigan (Tuez- } \\ \text { Ped. 16-18 }\end{array}\right.$ kiewicz). . . . . . . 3461. C. Lovgd Gdgr mss

( Discus I conicus .............. 94 Discus planus - Pyren. Or., Perpignan, Camélas (Soc. dauph. exs. n. 1197). . . . 3462. C. obloxgicalyx Gdgr (Fol. obovato-oblonga; discus paulo conicus - Hte-Savoie, Argonnex (Puget). . . . 3463. C. Minutifolia Gdgr mss

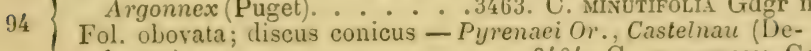

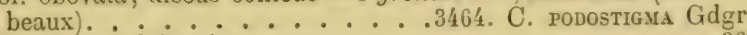

Fol. subtus glaberrima. ............ . . 96

95 Fol. subtus ad costam villosa. . . . . . . . 97

Fol. subtus ad nervos vel toto villosa . . . . . . 102

Rami flor. aculeati; discus planus - Pyrenaei Or., Camélas

96 (Debeaux).......3465. C. Asperula Gdgr mss Rami flor. inermes; discus conicus - Lot-et-Garonne, Moirax (Debeaux) . . . . . 3466. C. LAGENOIDES Gdgr ms3

97 Fol. basi rotundata. . . . . . . . 98

97 Fol. basi attenuata. . . . . . . . 100

$98\left\{\begin{array}{l}\text { Pet. et rami flor. aculeati; fol. elliptica. - Pot-et-Garonne, } \\ \text { Pet. et rami flor. inermes; fol. obovata - } 99\end{array}\right.$ Moirax (Debeaux). . . 3467. C. valdedilatata Gdgr mss

$99\left\{\begin{array}{l}\text { Sep. dorso eglandulosa; discus subconicus - Rhône, Mt- } \\ \text { Ceindre (Chabert). - . - 3468. C. SpHAERostruis Gdgr mss }\end{array}\right.$ Sep. dorso glandulosa; discus planus - Pyrenaei Or., Castelnau (Debeaux). ...3469. C. PSEUdo-RENOTIOR Gdgr mss 
Rami flor. aculeati ; fol. elliptica vel obovata. : . . . 101

Rami flor. inermes; fol. oblonga - Pyren. Or., Amélie-lesBains (Debeaux). . . . 3470. C. Longispina Gdgr mss Pet. aculeati; fol. elliptica - Gallia, etc (Gdgr herb. ros. n. 496 et 594)........ 3471. C. Lemanir (Bor.) beaux). . . . 3472. C. chalicophila Gdgr mss

102 Sep. dorso glandulosa; rami flor aculeati. . . . . 103

102 Minime - Lot-et-Garonne, La Gravette (de Pommaret).3473

• • • • • • • . C. Pommaretil (Puget) Fr. apice valde attenuatus; fol. subacuta - Rhône, Arnas

103 (Gdgr herb. ros. n. 149). .3474. C. ATtendatissima Gdgr mss Fr. apice subattenuatus; fol. acuta - Rhône, Dardilly (Chabert)..........3475. C. REMOTIOR Gdgr mss

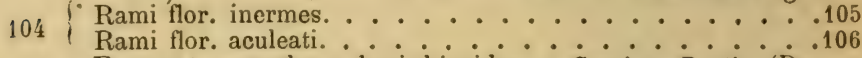
Fr. ovato-rotundatus basi hispidus - Corsica, Bastia (De-

105 beaux). . . . 3476. C. Mrcrococcos Gdgr mss Fr. oblongus laevis - Hte-Savoie, Thonon (Puget).3477. C.

$106\{$ Fol basi attenuata. $\therefore \therefore \therefore \therefore$ OPERTA (Puget)

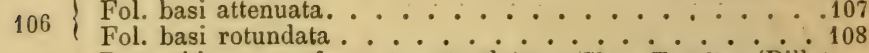

$107\left\{\begin{array}{l}\text { Pet. subinermes; fr. ovato-rotundatus-Cher, Fontiley (Billot } \\ \text { exs. n. } 3598 \text { bis) . . . . . 3478. C. JulcienI Gdgr mss }\end{array}\right.$

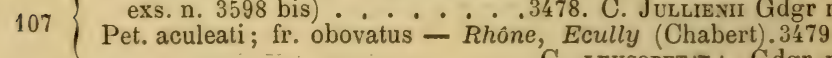
.............. LeUCOPFTATA Gdgr mss

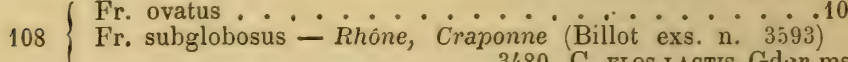

108 C. FLOS-LACTIS Gdgr mss Pet. aculeati ; fol. ovato-elliptica vel elliptica. . . . . 110

109 Pet. inermes; fol. ovata - Rhône; Chaponost (Gdgr herb. ros. n. 425). . . 3481. C. LACTiflora Gdgr mss, an Dés.?

$110\left\{\begin{array}{l}\text { Tubus basi hispidus; aculei juniores flavescentes - Rhône } \\ \text { (Gdgr herb. ros. n. 414) } 0 . .3482 \text {. C. Vallcantiana (Bor.) }\end{array}\right.$ Tubus saltem usque ad $1 / 2$ inf. glandulosus; aculei rubelliRhône, Brignais (Chabert) . .3483. C. PYracantha Gdgr mss

$111\left\{\begin{array}{l}\text { Rami flor. alii aculeos setaceos vel glanduliferos gerentes, alii } \\ \text { necne. . . . . . . . . . } 112\end{array}\right.$ Rami flor. omnes aculeos setaceos vel glanduliferos gerentes.115 Fol. ovata elliptica vel obovato-oblonga . . . . . 113

112 Fol. oblonga undique hirsuta, supra glandulosa - Hisp. austr., Sierra de Segura (Canada).3484. C. Guiraoana

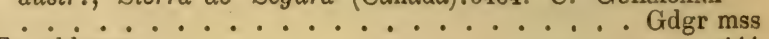
Fr. oblongus ..................... Fr. ovoideus; discus planus - Hte-Savoie, Pringy (Puget)

. . . . . . . 3485. C. REDUX Gdgr mss Fr. ovato-rotundatus; discus conicus - Hte-Savoie, Pringy (Puget). . . . . 3486. C. PERneglecta Gdgr mss (Pet. paulo aculeati; fol basi attenuata, ad costam hirsuta Rhône, Montmelas (Gdgr herb. ros. n. 208).3487. C. STE-

Pet. inf a... bo basi rotundata, ad nocalyx Gdgr mss Rhône, Arnas (Gdgr). . . 3488: C. GRateolens Gdgr mss 
Pet. glabri vel sparse hirsuti . . . . . . . . 116

Pet. villosi vel tomentosi. . . . . . . . . . 125

Tubus toto et copiose aculeolatus. . . . . . . 117

T'ubus laevis vel in $1 / 2$ inf. glandulosus . . . . . . . 122

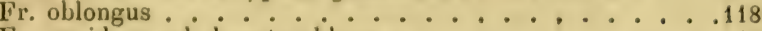

Fr. ovoideus vel obovato-oblongus. . . . . . 119

Rami flor. falcato-aculeati; fol. lanceolata - Lapponia,

118 Tornea (Lindberg). . . . 3489. C. Glaciarum Gdgr mss Rami flor. inermes; fol. subacuta - Gard, Le Vigan (Billot exs. n. 3598 parte). . . . 3490. C. Tuezkiewiczir Gdgr mss

119 Fol. subtus ad costam hirsuta. . . . . . . . 120

Fol. subtus toto hirsuta . . . . . . . 121

Fr. apice attenuatus; fol. obovato-acuta - Ain, St-Didier-de-

120 Formans (Fray).....3491. C. Hebetuba Gdgr mss

Fr. apice longe attenuatus; fol. elliptico-rotundata - Cala-

launia, Sagaro (Vayreda) . . 3492. C. Hispidela Gdgr mss

Ped. 12-15 m. 1.; fol. basi attenuata - Ain, Trévoux (Fray) 121 Ped. $9-10$ m. 1.; fol. basi rotundata - Rhône, Francheville (Chabert) . . . . 3494. C. Discolorifolia Gdgr mss

Tubus in $1 / 2$ inf. glandulosus. . . . . . . . . 123

122 Tubus basi glandulosus $\ldots . . .124$

Tubus glaber - Rhone, Charbonnières (Chabert).3495. C. Ix-

* . . . . . . FLEXA Gdgr msS

Fol. glaberrima basi attenuata - Ardèche, Tournon (Cha-

123 bert) .......3496. C. Atractiformis Gdgr mss Fol. ad costam villosa basi cordata - Silesia, Breslau (Kuebel). . . . . 3497. C. vratislaviensis Gdgr mss

Pet. aculeati ; fol. ovato-elliptica - Cher, etc.3498. C. sitvi-

124 . . . . . . . cola (Dés. et Rip.)

Pet. subinermes; fol. elliptica - Graecia, Athènes (de Ileldreich). . . . . . . 3499. C. INcLYTA Gdgr mss

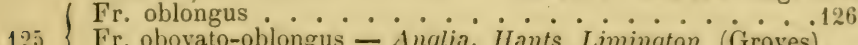
Fr. obovato-oblongus - Anglia, Hants Limington (Groves) ............... C. LONDINENSIS Gdgr mss

126 ( Discus conicus . . . . . . . . . 127

Discus conicus
Discusus. $\cdots \cdots \cdots$

Tubus toto glandulosus; pet. inermes - Pyrenaei, Perpignan

127 (Debeaux). : . . . . 3501. C. Elevata Gdgr mss Tubus in $1 / 2$ inf. glandulosus ; pet. aculeati - Italia, A penn. Modène Boscolungo (Gibelli). . .3502. C. GibeLLII Gdgr mss

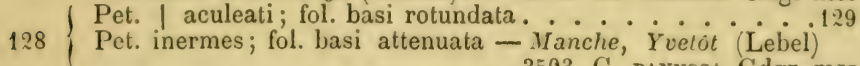
. . . . . . . 3503. C. panxosa Gdgr mss

$129\left\{\begin{array}{l}\text { Fol. subtus ad nervos hirsuta. } \\ \text { Fol. subtus toto hirsuta }- \text { Savoie, Brides (Puget).3504. C. }\end{array}\right.$ ....... . ACANTHOCALYX B. villosion Gdgr mss

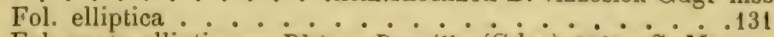

$130\{$ Fol. ovato-elliptica - Rhône, Brouilly $(\dot{G} \dot{d g r}) .3505 . \dot{C}$ MA................ DONAE Gdgr mss Styli paulo hirsuti; fr. apice valde attenuatus - Islandia,

131 Mt-Iléckla (Liebmann). . . .3506. C. Islaxdica Gdgr mss Styli glabri; fr. apice vix attenuatus - Saroie, Brides (Puget) .3507 . C. Acasthocalyx Gdgr mss 
3. - Mesostyloidae Gdgr Essai p. 39 (Rubiginoseae micranthae Crép. Prim. p. 24).

Pedunculi omnes hispidi aut glandulosi; styli nunc glabrescentes tunc haud aut vix basi subcoaliti, nunc plus minusve villosi ergo fere coaliti ; foliola villosula; flos parvus, purpureus, basi pallidus; frutices laxi, eorum ramis plus minus articulato-flexuosis. - Grex studio sat difficilis inter Hypostylosas et Apostylosas optime intermedius.

Styli glabrescentes haud coaliti . . . . . . . . . 2

Styli / hirsuti tunc basi paulo pedicellato-subcoaliti ... . 48

2 Rami flor. aculeos setaceos et glanduliferos haud gerentes.. ?

Secus. .................... 36

3 Tubus toto laevis . . . . . . . . . . . 4

Tubus basi vel toto glandulosus. . . . . . . . . 19

Fr. ovatus vel ellipticus . . . . . . . . 5

Fr. ovoideus. .................

Fr. obovato-oblongus. . . . . . . . . . . . 9

Fr. oblongus . . . . . . . . . 12

f Fol. ovata vel obovata .............. 6

Fol. oblonga - Rhône, Yzeron (Chabert).3508. C. NEvRosA

( . . . . . . . . . . . Gdgr mss

Pet. subinermes; fol. obovata subtus toto hirsuta - Savoie,

6 Salins (Puget). . . . . 3509. C. Amphismallum Gdgr mss

Pet. aculeati; fol. ovata subtus ad nervos hirsuta - Gallia, Chambéry (Songeon). . . . .3510. C. Parallela Gdgr mss

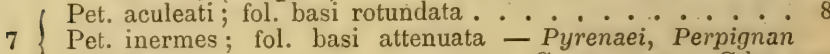
(Debeaux). . . . . . . .3511. C. MEGaPETALA Gdgr mss Rami flor. inermes; fol. ad nervos hirsuta - Hibernia, Antrim Cramlin (Stewart) . . . 3512. C. HiberNica Gdgr mss Rami flor. aculeati; fol. undique hirsuta - Silesia, Landeshut (Hoger) . . . . . . . 3513. C. Krauseana Gidgr mss

9 ( Ped. saltem sup. sparse hirsuti . . . . . . . . 10 Pet. omnes hirsuti vel tomentosi ........... 11 Fol. elliptica, subtus ad costam hirsuta - Pyren., Gèdre (Gdgr

10 herb. ros. $\left.n_{s} 245\right) \ldots \ldots \ldots$. . . . . S . SopHIA udgr mss Fol. obovata, subtus ad nervos hirsuta - Rhône, Montmelas (Gdgr herb. ros. n. 215). . . . 3515. C. RHIPIDOPHORA Gdgr

( Rami flor. aculeati ; fol. basi attenuata - Isère, Balmes-Tien-

11 noises (Chabert) Rami flor. inermes; fol. basi rotundata - Rhône, Cherviagr mass (Gdgr) . . . . . . . . 3517. C. chenocaULis Gdgr mss 
12 Pet. omnes hirsuti.

2 Pet. saltem sup. sparse hirsuti ......... 16

13 Sep. dorso eglandulosa. . . . . . . . . 14

Minime. . . . . . . . . . 15

Discus subconicus; pet. aculeati - Finistere, Crozon (Thie-

14 baut) . . . . . . 3518. C. Leptoceras Gdgr msS | Discus planus ; pet. inermes - Hisp., Sierra Nevada St-Geronimo (Hegelmaier). . . . .3519. C. NEvadexsis Gdgr mss

Fr. basi longissime attenuatus; nervi villosi - Isère, Villard

15 de Lans (Boullu). . . . . 3520. C. Clavifonmis Gdgr mss

Fr. basi subattenuatus; costa villosa - Rhóne, Arnas (Gdgr herb. ros. n. 539) ..... 3521. C. Collaris Gdgr mss

16 Sepala dorso glandulosa ............ 17

Minime.................... 18

Ped. 8-10 m. l.; fol. ovato-elliptica - Pyrenaei, Gédre (Bor-

17 dère). . . . . . . . . Compta Gdgr mss

Ped. 12-15 m. l.; fol. obovata - Gard, Le Vigan ('Tuezkiewicz). . . . . . . 3523. C. Delileana Gdgr mss

Discus conicus; rami flor. aculeati - Hisp., Sierra Nevada

18 S. Geronimo (Hegelmaier) . . .3524. C. Axdalusica Gdgr mss

Discus planus ; rami flor. inermes - Basses-Alpes, Jausières (Proal). . . . . . . 3525. C. LeIosepala Gdgr mss

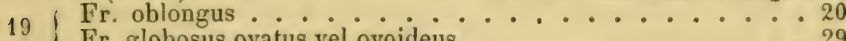

| Fr. globosus ovatus vel ovoideus. . . . . . . . . 29

20 Fol. subtus toto hirsuta . . . . . . . . . 21

20 Fol. subtus ad costam vel nervos hirsuta. . . . . . 25

21 Rami flor inermes. . . . . . . 22

Rami flor aculeati. . . . . . . . . . . 23

Fol. ovata supra glabra - Valais, Bovernier (Delasoie). 3526.

22 ( . . . . . . Acanthobasis Gelgr mss Fol. elliptica supra hirta - Rhône, Chaponost (Billot exs. n. 4029) .............. En27. EnIosa Gdgr mss

Tubus toto glandulosus. .......... 24

Tubus basi glandulosus; fol. ovata basi rotundata - Rhône,

23 Brignais (Chabert) . . . 3528. C. streptocallis Gdgr mss

Tubus in $1 /{ }_{3}$ inf. glandulosus; fol. elliptica basi attenuata Pedem., Fénestrelle (Rostan). . 3529. C. Rostani Gdgr mss

Pet. sulbinermes ; fol. rotundata - Rossia, Nijni-.Yowogorod

21 (Reimbole) . . . . 3530. C. NigRescens Gdgr mss Pet. aculeati ; fol. ovata - Gallia, Rouen (Malbranche).3331.

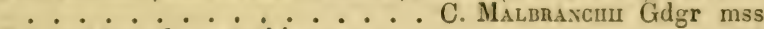

Fol. subtus ad nervos hirsuta......... 26

25 Fol. subtus ad costam hirsuta - Graecia, it-Parnasse Orphanides). . . . . . 3532. C. Microriphaea Gdgr mss

Pet. saltem sup. sparse pilosi. . . . . . . . . 27

Pet. omnes dense pilosi . . . . . . . . . . 28

Tubus toto glandulosus; rami flor. aculeati - Sabaudia, Méry (Puget). . . . . . 3533. C. idionacantea Gdgr mss Tubus in $1 / 3$ inf. glandulosus; rami flor. subinermes Rhône, Villefranche (Gdgr) . .3534. C. AMPHICALYX Gdgr mss

Tubus in $1 / 2$ inf. glandulosus; fol. ovata - Rhône, MtCeindre (Chabert). . . . 3535. C. solesordea Gdgr mss 
Tubus in $1 / 2$ inf. glandulosus; fol. rotundata - Rhône, Le Mt-Dore (Chabert).3536. C. SolenoIdeA B. MACrosepala

Tubus basi glandulosus; fol obovata - Italia, Florence Impruneta (Arcangeli) . . . 3537. C. ITAlica Gdgr mss

Pet. omnes villosi vel tomentosi . . . . . . . . 30

29 Pet. saltem sup. sparse hirsuti. . . . . . . . . 35

Tubus saltem basi glandulosus; pet. aculeati. . . . . . 31

30 Tubus glaber; pet. subinermes - Hisp., Saragosse (Bachès); Ain (Gdgr herb. ros. n. 579) .3538. C. conymbulosa Gdgr mss

Discus planus . . . . . . . . . . . 32

31 Discus conicus - Rhône, Alix (Gdgr herb. ros. n. 44).3539.

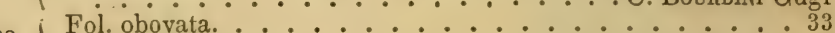

32 Fol ovata $\ldots 34$

Rami flor. aculeati; fr. ovatus - Rhône, Arnas (Gdgr herb.

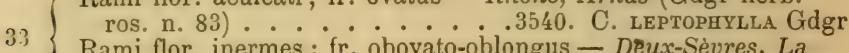
Rami flor. inermes; fr. obovato-oblongus - Deux-Sèvres, $L a$ Mothe (Maillard). . . . . 3541. C. Maillardi Gdgr mss

Tubus basi glandulosus; fr. ovato-rotundatus - Hte-Savoie, Pringy (Puget). . . . . .3542. C. promiscua Gdgr mss

Tubus basi glandulosus; fr. globosus - Hte-Savoie, Pringy (Puget). . . . 3543. C. Promiscua B. Glabrescens Gdgr mss

Tulsus in $1 / 3$ inf. glandulosus; fr. oblongus - Hte-Savoie, Pringy (Puget) . .3544. C. Promiscua C. Megacalyx Gdgr mss

Fr. ovato-rotundatus basi hispidus - Rhône, Soucieux (Cha-

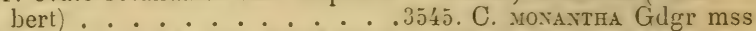
Fr. ovatus in $1 / 3$ inf. glandulosus - Helv., Fribourg Montbovon (Cottet). . . . . 3546. C. Exocisita Gdgr mss

Fr. obovatus glaber - Phóne, Pommiers (Gdorr lierb. rós. n. 284). . . . . . 3547. C. Calophylla Gdgr Rami flor. alii aculeos glanduliferos gerentes alii necne. . . 37

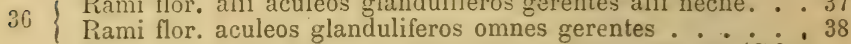

Fr. ovatus; fol. utrinque attenuata - Rhône, Ecully (Gdgr herb. ros. n. 197). . . .3548. C. MELaNOAdENA Gdgr mss

Fr. oblongus; fol. utrinque rotundata - Rhône, Arnas (Gdgr herb. ros. n. 150 ; Billot exs. n. 4031).3549. C. PAU-

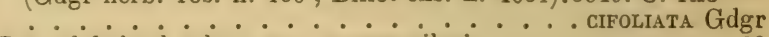

Pet. glabri vel saltem sup. sparse pilosi ...... 39

38 Pet. omnes dense pilosi. . . . . . . 43

Fr. oblongus. . . . . . . . . . . 40

39 Fr. ovato-rotundatus - Rhône, Brindas (Chabert) $35500^{\circ} \mathrm{C}$.

. . . . . . . . BRUNEOGLANDA Gdgr msS

Fol. subtus ad costam mediam hirsuta. . . . . . . 41

Fol. subtus glaberrima, oblonga - Rhône, Tassin (Cha-

40 bert) . . . . . 3551. C. xylomitis Gdgr mss

Fol. subtus ad nervos hirsuta, obovata - Cher, La Servanterie, etc. (C. anisopoda Christ 1875 ; C. subdola Dés. 1877; C. Klukii Auct. non Bess.!) .3552. C. Anisacantha Gdgr 1872

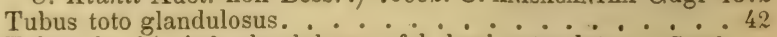

Tubus in $1 / 3$ inf. glandulosus; fol. basi rotundata - Gard, Pommiers (Tuezkiewicz). • - 3553. C. Telacantha Gdgr mss

Tubus basi glandulosus; fol. basi attenuata - Rhône, Charbonnières (Billot exs. n. 4034).3554. C. RHYNCHOCARPA

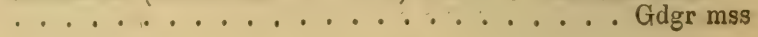


Discus conicus ; fol. elliptica - Baléares, Majorque (Bachès).

Discus subplanus; fol. oblonga 3555. C. Laguncula Gdgr mss beaux)...... 3556. C. Aspericalyx Ge-

( Tubus toto glandulosus. . . -

Tubus glaber vel basi tantum glandulosus

Fr. oblongus; pet. inermes - Ardèche, La Lombardière

4 't (Billot exs. n. 3854 partim). . .3557. C. Helviessis Gdgr mss (Frebovato-oblongus; pet. aculeati - Istria, Lupoglava (Freyn) . . . . . 3558. C. acantiocanpa Gdgr mss

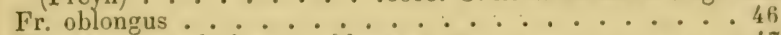

Fr. obovatus vel obovato-oblongus. . . . . . . . . 47

Fr. breviter ovoideus; discus conicus - Rhone, Vaugneray; Heln., etc. . . . . . . 3559. C. PEIraga Gilgr Fr. rotundatus ; discus planus - Rhone, Dardilly (Chalsert). 3560. C. trsticelata Gider mss Pet. inermes; fol. lanceolata - Rhône, Arnas (Gdgr herb.

46 ros. n. 310) . . 3561. C. Nesonoso-tonentosa Gdgr mss Pet. aculeati ; fol. apice rotundata - Pyrenaei, Gèdre (Bordère) ........ 3562. C. spivigera Gdgr mss

Pol. ovata; pet. inermes - Ardèche, La Lombardière (Billot exs. n. 3854 part.). . . . . .3563. C. Adversaria Gdgr mss Fol. elliptica; pet. aculeati - Isère, La Villette d'Anthon (Chabert) . . . . . 3564. C. cladosparsa Gdgr mss

18 Rami flor. aculeos setaceos vel glanduliferos gerentes. . . 49

48 Ramorum floriferorum aculèi falcati. . . . . . . . 55

Sep. dorso toto glandulosa; fol. basi rotundata. . . . . 50

49 Sep. dorso apice tantum glandulosa; fol. basi attenuata Hisp., Logrono (Zubia) . . .3565. C. AdENocLADA Gdgr mss

50 I Fr. oblongus . . . . . . . . . . 51

Fr. ovoideus. . . . . . . 52

Pet. sparse pilosi ; nervi hirsuti - Silesia, Goerbersdorf

51 (Straehler) . . . . 3566. C. Sancostephava Gdgr mss Pet. toto hirsuti ; costa villosa - Naples (Gussone).35fi. C.

Tubus toto glandulosus. . . . . . . . . 53

-2 Tubus toto laevis ............. 54

Tubus in $1 / 3$ inf. glandulosus - Valais, Lnterbach (Wolf).

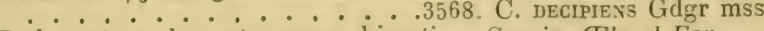

Ped. 9-11 m. l.; pet. sparse hirsuti - Suecia, Eland Fal-

$53\left\{\begin{array}{l}\text { jestaden (Scheutz) to hirsuti - Basses-Alpes, Condamine } \\ \text { Ped. 6-7 m. 1.; pet. toto }\end{array}\right.$ (Proal). . . . . 3570. C. Echinulata Gidgr mss

Pet. sparse hirsuti ; ped. corymbosi - Rhône, Gleizé (Gdgr

Pet. toto hirsuti ped i-2- - C 1-2-ni - Silesia, Breslau (Uechtritz).

Pet. glabrescentes, vel sup. saltem sparse hirsuti. . . . . 65

, Fr. ovatus vel ovato-rotundatus. . . . . . . . 57

Fr. ovoideus vel obovato-oblongus. . . . . . . . . 58

Fr. oblongus . . . . . . . . 61 Fr. globosus - Isère, Meyzieu (Chabert).3573. C. oExoxylon 


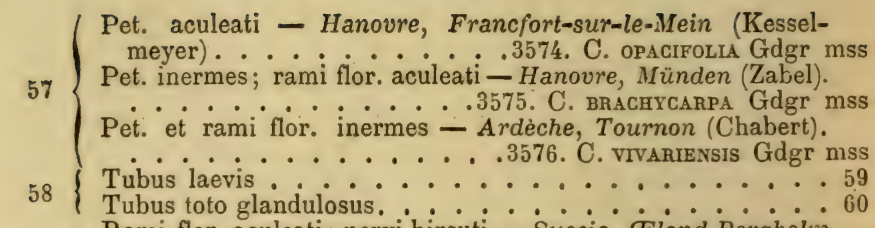

( Rami flor. aculeati; nervi hirsuti - Suecia, Eland Borgholm

$59\left\{\begin{array}{l}\text { (Scheutz) - - - } \dot{0} \text {. subtus toto hirsuta - Basses-Alpes, } \\ \text { Rami flor. inermes; fol. subs }\end{array}\right.$ Jausières (Proal). . . . . . .3578. C. ProAlII Gdgr mss

Fol. elliptica; pet. aculeati - Htes-Alpes, La Moucherolle

$60\{$ (Cariot) .........3579. C. orogenes Gdgr mss Fol. oblonga ; pet. subinermes - Italia, Apenn. Modène l'A betone (Gibelli) .... 3580. C. Bertoloxiana Gdgr mss

( Tubus basi vel in $1 / 4$ inf. glandulosus. . . . . . 62

61 Tubus toto glaber .63

Tubus toto glandulosus - Belg., Malmédy, Wagnez (C. resinosa Lej. fl. Spa non Sternb.). . . 3581. C. spadana Gdgr

( Rami flor. aculeati; fol. oblonga - Istria, Canfanaro (Freyn).

$62\left\{\begin{array}{cc}0 \\ 0\end{array} \cdot 3582\right.$. C. PAnNonica Gdgr mss

Rami flor. inermes; fol. obovata - Basses-Alpes, Jausières (Proal) . . . . . 3583. C. Florigera Gdgr mss

( Fol. subtus toto vel ad nervos hirsuta; rami flor. aculeati . . 64

63 Fol. ad costam hirsuta; rami flor. inermes - Suecia mer. (Tullberg). . . . 3584. C. MicrorHyNCHA Gdgr mss Ped. 10-12 m. 1.; fol. elliptica - Dania, ile Bornholm (Jorgensen)............ C. Vahliana Gdgr mss Ped. 10-12 m. 1.; fol. obovata - Rossia bor. occid., Kokenhusen (Fl. ross. occ. exsicc. n. 254).3586. C. Rossica

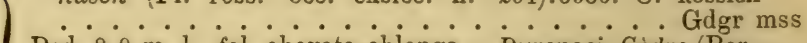
Ped. $8-9$ m. i.; fol obovato-oblonga - Pyrenaei, Gèdre (Bordère). . . . . . . 3587. (\%. Marchandiana Gdgr mss

( Fr. rotundatus vel subglobosus .......... 66

65 Fr. ovoideus vel obovato-oblongus. . . . . $\therefore 67$

Fr. oblongus . . . . 71

( Rami flor. et pet. aculeati - Deux-Sèvres, La Mothe (Sauzé). $66\left\{\begin{array}{l}\text { Rami flor. et pet. inermes - Gallia, Gde-Chartreuse (Gdgr). } \\ \text { - } 1358 \text {. C. Polychados Gdgr mss }\end{array}\right.$

3589. C. viridissima Gdgr mss

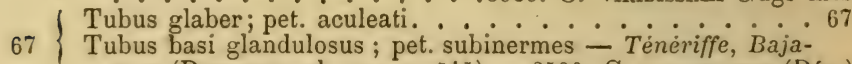
mar (Bourgeau pl. can. n. 545) . .3590. C. Cayariensis (Dés.)

68 Rami flor. inermes. . . . . . . 69

68 Rami flor aculeati. . . . . . . . . . . 70 (Fol. elliptica sußtus toto hirsuta - Gallia, Narbonne (Loret). $69\left\{\begin{array}{l}\text { Fol. obovato-oblonga subtus ad nervos hirsuta - Pyrenaei, } \\ \text { - Pars }\end{array}\right.$ Gèdre (Bordère). . . . 3592. C. Lapeyrouslana Gdgr mss

70 Ped. omnes hispidi $\therefore 71$

70 Ped. alii glandulosi alii sublaeves - Rhône, Charbonnières

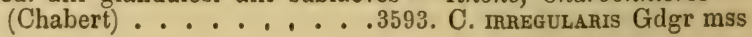


Ped. 7-8 m. 1.; fol. elliptico-acuta - Valais, Cauthey (Wolf) . . . . . . . 3594. C. stenoacantha Gdgr mss Ped. 7-9 m. 1.; fol. obtuse ovaí-elliptica - Silesia, Goerbersdorf (Straehler). . . . . . 3595. C. oxronos Gdgr mss Perl. 4-6 m. 1.; fol. obovata apice rotundata-Rhone, Pommiers (Gdgr herb. ros. n. 536). . . 3596. C. nupirnaga Gdgr mss

72 (Sep. dorso glandulosa........... 73

72 Minime - IIanovre (Zabel). . . 3597. C. collisa Gdgr mss

73 f Fr.oblongus. . . . . . . . 74

Fr. obovatus vel obovato-oblongus. . . . . . . . 75

(Tubus glaber; fol. ovata basi rotundata - Rhône, Brindas

(Chabert). . . . . 3598. C. DEsmophylla Gdgr mss

Tubus in $1 / 3$ inf. glandulosus; fol. obovato-oblonga basi attenuata - Gard, Le Vigan (D. Tuezkiewicz).3599. C. DECAL-

............................ Gatgr mss

Fol. ovata vel suborbiculata basi rotundata, subtus ad nervos

75 Fol. elliptica basi attenuata, subtus toto hirsuta - Rhone, 76 Brindas (Chabert) . . . . . .3600. C. Ixvisa Gdgr mss Sep. foliacea; fol. suborbiculata - Rhỏne, Ecully (Chabert)

.3602. C. stilbophylla Gdgr

\section{4. - Apostyloidae Gdgr Essai p. 39 (Rubiginoseae suavifolice Crép. loc. cit.)}

Pedunculi glandulosi vel hispidi; styli lanati vel villosissimi, ultra discum planum sessiles, capitati; foliola plus minus hirsuta, praecocius decidua, odorata; aculei validiores quam ut in sectionibus praecedentibus, flores etiam submajores.

\footnotetext{
- Aculei conformes. . . . . . . . . Isoacanthae

A. Aculei alii adunci, alii recti vel glanduliferi, b. Dimorpha-
} 


\section{a. - Isoacanthae Gdgr Essai p. 37.}

\section{Aculei validi, conformes, scilicet, omnes e basi compressa} dilatataque plus minus falcati vel adunci.

1 Pet. glabri vel saltem sup. sparse pilosi. . . . . . . 2

1 Pet. omnes dense villosi vel tomentosi. . . . 50

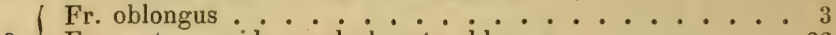

2 Fr. ovatus ovoideus vel obovato-oblongus . . . . 22

Fr. globosus vel rotundatus. . . . . . . . . 42

3 Pet. omnes sparse hirsuti. . . . . . . . . . 4

Pet. alii sparse alii dense hirsuti ......... 10

4 Rami flor aculeati. ............ 5

4 Rami flor. inermes. . . . . . . . . . 7

( Discus / conicus; fol. subtus toto vel ad nervos hirsuta. . . 6

5 Discus planus; fol. ad costam hirsuta - Aragonia austr., Sierra de Mosqueruela (Martin). .3603. C. Assoana Gdgr mss Fr. apice longe attenuatus; discus subplanus - Rhône, St-

6 Laurent-de-Vaux (Chabert). . .3604. C. Rosulenta Gdgr mss Fr. haud attenuatus; discus conicus - Isère, Vienne (Chabert). . . . . . . 3605. C. lepidula Gdgr mss

7 Fol. basi rotundata; ped. 10-15 m. 1. . . . . . 8

Fol. basi attenuata; ped. 4-6 m. 1. . . . . . . 9

( Pet. subinermes; fr. basi attenuatus - Pyrenaei, Gèdre

8 (Bordère) .......3606. C. oenopetala Gdgr mss Pet. aculeati ; fr. basi longe decurrens - Pyrenaei, Gèdre (Bordère) . . . . . 3607. C. crassibasis Gdgr mss Fol. subtus toto hirsuta; fr. basi decurrens - Rhône, MIont9 melas (Gdgr). . . . . 3608. C. BIoxycarpa Gdgr mss Fol. subtus ad costam hirsuta ; fr. basi attenuatus - Gallia, Clermont (F. Héribaud). . . . 3609. C. LEIoglada Gdgr mss

10 Fol. subtus ad costam mediam hirsuta. . . . . . . 11

10 Fol. subtus ad nervos vel toto hirsuta. . . . 15

11 Fol. basi rotundata ............. 12

Fol. basi attenuata. . . . . . . . 13 Pet. subinermes; fol. elliptica - Rhône, St-Didier-au-MtDore (Chabert). . . . 3610. C. Apogyna Gdgr mss Pet. inf. inermes; fol. ovato-elliptica - Isère, Balmes Viennoises (Chabert). . . . .3611. C. Rostrifera Gdgr mss Pet. omnes aculeati; fol. obovata - Rhône, Montmelas (Gdgr) . . . . . . 3612. C. ToRDYlioldes Gdgr mss Pet. et rami flor aculeati. . . . . . . . . 14

Pet. aculeati; rami flor. inermes - Rhône, Brindas (Chabert)

. . . . . . 3613. C. chlamydocarpa Gdgr msS Pet. inermes; rami flor, aculeati - Baléares, Majorque (Bachès). . . . . . . 3614. C. Atrofusca Gdgr mss 
Aculci falcati; ped. 6-8 m. 1. - Catalaunia, Olot (Vayreda).

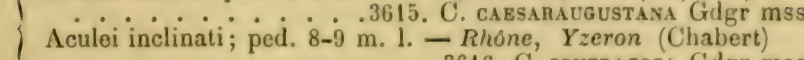
. . . . . . . . 3616. C. confragosa Gdgr mss

Pet. inermes .............. 16

Pet. aculeati. . . . . . . . . . 17

Fr. basi attenuatus; fol. elliptico-lanceolata - Rhone, La

Tour-de-Salvagny (Chabert).3617. C. heтEnodonra Gdgr mss

Fr. basi rotundatus; fol. subacute obovato-oblonga - Rhơne, Limonest (Chabert) . . . . 3618. C. HYaliva Gdgr mss

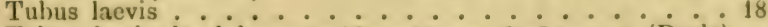
Tubus basi glandulosus - Htes-Alpes, St-Laurent (Burle) . . . . . . . . . 3619. C. Jtgicola Gidgr mss

Sep. dorso glandulosa . . . . . . . . . 19

18 Minime-Basses-1lpes, Condamine (Proal).3620. C. Chalxiana

| Fol basi | attenuata; fr. inferne valde decurrens . Gugr mss

Fol. basi rotundata; fr. inferne rotundatus vel depressus. 21

Ped. 2-3 m. I.; fol. ovato-elliptica - Ilelv., Fribourg, Mont-

3 bovon (Cottet). ......3621. C. sarcopoda Gdgr mss

$\because 0$ | Yed. 6-8 m. 1.; fol. obovato-oblonga - Rhône, Arnas (Gdgr) . . . . . . 3622. C. platycarpa Gdgr mss Fr, angustatus; ped. $7-9 \mathrm{~m} .1$. - Thuringe, Ettersberg

21 (Haussknecht) ..... 3623. C. spivosissima Gdgr mss Fr. magnus; ped. 9-12 m. 1. - Pyrenaei, Gèdre (Bordere) .......... 3624. C. caloacantha Gdgr mss

$2 . \quad$ Rami flor inermes ............. 23

Rami flor aculeati. . . . . . . . . . 27

Sep. extus glandulosa . . . . . . . . 24

23 Minime - Rhône, Vaugneray (Chabert).3625. C. Mitigata

Foi. basi attenuata. $\ldots \ldots$. Gdgr mss

24 Fol basi rotundata 25

$25\left\{\begin{array}{l}\text { Pet. omnes inermes; fol. obtuse ovato-rotundata - Loire, } \\ \text { Planfoy (Chabert) }\end{array}\right.$

Pet. paulo aculeati; fol. acute obovato-oblonga - Rhóne, StLaurent-de-Vaux (Chabert). . . . 3627. C. AMica Gdgr mss Fol. obovata apice rotundata; pet. omnes aculeati - HtesAlpes, Rabou (Burle). . . . .3628. C. пномвоеA Gdgr mss

Fol. obovato-obtusa; pet. subinermes - Pyrenaei Or., MtLouis (Debeaux).. . . . . 3629. C. RuFina Gdgr mss Fol. obovato-oblonga apice rotundata; pet. inf. inermes Isère, Dessine (Chabert). . . 3630. C. oenosepala Gdgr mss

( Rami graciles implexi ; sep. dorso hispidissima . . . 28 Rami validi nec implexi; sep. dorso tantum glandulosa vel laevia.

28 Tubus laevis; fol. ovata vel elliptica. . . . . . . . 29 Tubus toto glandulosus; fol. oblonga - Helv, Fribourg Montbovon (Cottet) . . . 3651. C. Adexosepala Gdgr mss

( Fol. subtus ad nervos villosa, basi rotundata. : . . . . 30 Fol. subtus ad costam hirsuta, basi attenuata - Helv., Carrouge (Chabert) ..... 3632. C. AsabiLion Gdgr mss

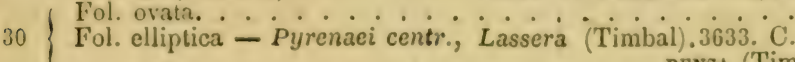
31

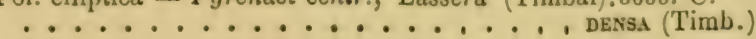


( Ped. 6-8 m. 1.; fol. obtusa - Ain, Néron (Chabert).3634 Ped. $10-14$ m. $\dot{1}$; fol. apice rotundata - Pyrenaei, Gedre (Bordère) . . . . . . 3635. C. DEBILIs Gdgr mss

32 Sep. dorso eglandulosa. . . . . . . . . 33

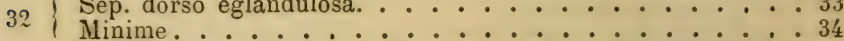
( Fol. elliptica subtus toto hirsuta - Rhône, Liergues (Gdgr)

33 . . . 3636. C. Lithophila Gdgr mss Fol. ovato-obtusa subtus ad costam hirsuta - Basses-Alpes, Condamine (Proal). . . . . 3637. C. BRevifolia Gdgr mss

34 Tubus glaberrimus. . . . . . . . . 35

34 Tubus sparse glandulosus - Loire, Chalmazelle (Peyron) . . . . . . . 3638. C. EBEnina Gdgr mss

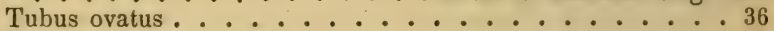

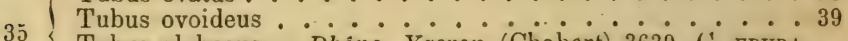
Tubus globosus - Rhône, Yzeron (Chabert).3639. C. EDURA

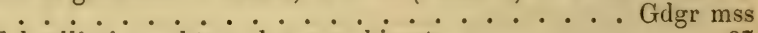
Fol. elliptica subtus ad nervos hirsuta. . . . . 37 Fol. obovata subtus ad nervos hirsuta - Côte-d'Or, Meursault (Ozanon) .......3640. C. minuscula (Crép. mss) Fol. ovata subtus toto hirsuta - Rhône, Theizé (Gdgr).3641

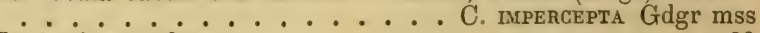

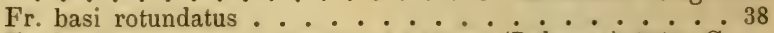

37 Fr. basi attenuatus - Corsica, Le Pigno (Debeaux).3642. C. ........... . . oxyponus Gdgr mss Stip. magnae subtus glandulosae; fol lata - Ain, Néron

38 (Chabert)............. C. validula Gdgr mss Minime - Rhône, Limas (Gdgr herb. ros. n. 282).3644. C.

...................... HYPONEMA Gdgr

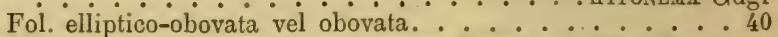
Fol. ovata basi attenuata, ad costam hirsuta - Htes-Alpes, La Grave (Reliq. Maill. n. 1086).3645. C. Mathonneti

Fol. ovato-elliptica basi rotundata, subtus toto hirsuta Thuringia, Ettersberg (Baenitz herb. europ. n. 1270).3646.

j Fol. subtus ad nervos hirsuta. . . C. DECIDENS Gdgr mss

40 Fol. subtus toto hirsuta - Saône-et-Loire, etc. (C. anomala Rip. olim). . . . . . . . 3647. C. Aeduensis (Dés.)

Ped. 8-10 m. 1.; fr. apice valde attenuatus - Pyrenaei, Gèdre

41 (Bordère). . . . . 3648. C. subcoronata Gdgr mss Ped. 6-8 m. l.; fr. apice strangulatus - Ain, Vancia (Chabert).............. C. SEnescens Gdgr mss

42 Fol subtus ad nervos vel toto hirsuta. . . . . . 43

42 Fol. subtus ad costam hirsuta. . . . . . . . 45

(Tubus laevis; pet. aculeati . . . . . . . . . 44

43 Tubus basi glandulosus; pet. subinermes - Rhône, Francheville (Chabert). . . . . .3650. C. heterochroa Gdgr mss Aculei falcati; ped. elongati - Rhône, Vaugneray (Chabert);

44 Helv., etc. : Aculei recti vel inclinati; ped. breves - Ain, Sathonay (Chabert) ......3652, C. ACAnthoclina Gdgr mss 
Discus conicus - Rhone, Mont-Ceindre (Chabert).3653. C.

46 Tubus basi giandulosus. . . . . . . . . . 47

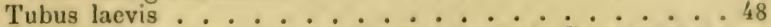

Fol. obovata apice obtusa - Gallia, ete.3654. C. Graveolens

47 Fol. oblongo-attenuata - Rhone, Francheville (Chabert).

48 Fol. minuta elliptico-rotundata - Rhône, Montmelas (Gdgr).

. . . . . . 3656. C. PEtnopula Gdgr mss

Sep. dorso glandulosa ; fr. $61 / 2-8 \mathrm{~m}$. diam. latus - Isere,

Balmes Viennoises (Chabert).3657. C. пAEMatocrnoa

49 Sep. dorso subeglandulosa; fr. $11 \dot{1} / 2-13 \mathrm{~m}$. diam. latus -

Lyon, Dessine (Chabert) . . . 3658. C. AENEA Gdgr mss

Fr. globosus vel rotundatus. . . . . . . . 51

50 Fr. ovatus vel subovoideus. . . . . . . . 61

Fr. ovoideus vel obovato-oblongus. . . . . . . . 73

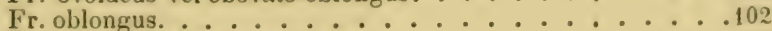

51 ( Fr. apice haud strangulatus ........... . 52

51 Fr. apice valde attenuatus . . . . . . . . . . 58

Tubus laevis . . . . . . . . . . . . 53

Tubus basi glandulosus; fol. ovato-rotundata - Ain, Tra-

moy (Chabert) . . . . 3659. C. pulchna Gdgr mss

52 Tubus in $1 / 3$ inf. glandulosus; fol. elliptica - Ain, Les Echeyx (Chabert). . . . .3660. C. DEsmopoda Gidgr mss

Tubus glandulosus; fol. rotundata - Graecia (Tratt.); Creta (Sieber), etc. . . . . . . 3661. C. cRETIGA (Tourn.)

I Rami flor inermes. . . . . . . . . . 54

Rami for aculeati.

Fol. ovata basi attenuata, subtus toto hirsuta - Gallia, Tou-

54 louse........3662. C. пeterophyla (Timb.)

Fol. oblonga basi rotundata, ad costam hirsuta - Catalaunia, Olot (Vayreda).....3663. C. Lagascana Gdgr mss

Fol. supra eglandulosa. . . . . . . . 56

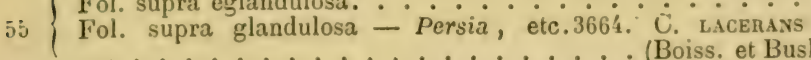

Pet. aculeati ; fol. ovata vel elliptica utrinque rotundata. . . 57

56 Pet. subinermes; fol. obovato-oblonga utrinque attenuata Pyren. Or., St-Paul-de-Fenouillet (Deheaux).3665. C. cr-

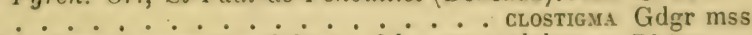

Sep. dorso parce glandulosa ; fol. supra glabra - Rhóne, Sep. dorso dense glandulosa; fol. supra hirsuta - Rhone, Denice Grlgr herb. ros. n. 82) .3667. C. Bl.scastua Gdgr mss

58 Rami flor. aculeati. . . . . . . . 59 Rami flor. inermes - Pyrenaei, Gèdre (Bordère).3668. C.

Unguis flavescens; fol, obovata vel obovato-oblonga

59 Unguis albus; fol. ovato-elliptica - Pyrenaei, Gèdre (Gdgr herb. ros. n. 356) .....3669. C. cosocarpa Gdgr mss 
Fol. supra glandulosa basi rotundata - Rhône, Montmelas (Gdgr herb. ros. n. 205) …3670. C. BENEARMATA Gdgr mss Fol. supra eglandulosa basi attenuata - Rhône, Ouilly (Gdgr herb. ros. n. 304) . . . . 3671. C. ScLEROXYLON Gdgr

61 Sep. dorso eglandulosa. . . . . . . . . . 62 Minime. . . . . . . . . . . . . 663 Pet. inermes ; rami flor. aculeati - Rhône, Chaponost; Isère, etc......... 3672. C. Comatula Gdgr mss Pet. aculeati; rami flor. inermes - Rhône, Montmelas (Gdgr herb. ros. n. 159) . . . . . 3673. C. Vesper Gdgr mss

Tubus basi glandulosus . . . . . . . . . 64 Tubus glaberrimus. . . . . . . . . . 66 Tubus in $1 / 2$ inf. glandulosus; fol. elliptico-obovata-Doubs, Mt-Brégille (Billot exs. n. 3596).3674. C. Pailloti Gdgr mss Tubus toto glandulosus; fol. ovata - Valais, Tourtmayne (Wolf). . . . . . . . . 3675. C. crinigera Gdgr mss Rami flor. aculeati ; fol apice attenuata. . . . . . . 65

64 Rami flor. inermes; fol. apice rotundata - Cher, Graire (Billot exs. n. 3596 bis). . . . .3676. C. Grainensis Gdgr mss Elata; cor. rosea - Tauria. . . . 3677. C. FLoRibundA (Stev.)

65 Nana; cor. alba - Tauria, Bachtschisarai (C. floribunda var. M. B. fl. t. c. III, p. 344).3678. C. FLORIBUNDA . . . . . . . . . . B. HuMilion Gdgr mss

Rami flor. aculeati. ............ . 67

66 Rami flor. inermes - Valais, Bovernier (Delasoie).3679. C.

| Fol. apice attenuata ......... . PURA Gdgr mss

67 Fol. apice rotundata vel obtusa. . . . . . . 71

$68 \mid$ Pet. subinermes. . . . . . . . . . . 699

Pet. aculeati. . . . . . . . . . . 70

Ped. 7-8 m. l.; fol. obovata subtus toto hirsuta - Rhône, Gleizé (Gdgr herb. ros. n. 75).. .3680. C. ACANTHOPHORA Gdgr

69 Ped. 8-11 m. l.; fol. obovato-oblonga subtus ad nervos hirsuta - Cher, Allouins (Boreau).3681. C. unBelliformis

....... Gdgr mss Sep. foliacea; fol. suborbiculata - Ain, Les Echeyx (Cha-

$7_{0}$ bert)........... 3682. C. Dimidiens Gdgr mss Sep. angustata ; fol. ovata - Rhône, Francheville (Chabert).

................. C. xylorerubra Gdgr mss

Aculei falcati vel inclinati ; fol. elliptica subtus ad nervos hirsuta ................... 72

71 Aculei recti tenues; fol. obovata subtus toto hirsuta - Anglia, Worcester Wye Forest (Fraser).3684. C. BRtTannica

...................... Gdgr mss

Stip. inf. subtus glandulosae; sep. angustata - Isère, Des-

72 sine (Chabert)...... 3685. C. ARMATIRAMEA Gdgr mss Irinime; sep. foliacea - Tyrol, Trente (Gelmi).3686. C. TRI. . . . . . . . . . . DENTINA Gdgr mss

73 ( Rami flor. inermes. . . . . . . . . . . 74

Rami flor. aculeati. . . . . . . . . . . 79

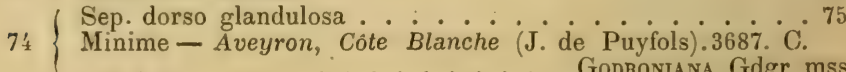

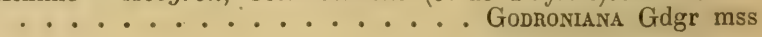


Tubus glaber ................ . . . . 6

Tuluus | glandulosus - IIte-Savoie, Mt-Sion (Puget).36 38 ........... C. Inamoena Gdgr mss

Fol. basi attenuata. . . . . . . . . 77

Fol basi rotundata. . . . . . 78

Pet. subinermes; fol. oblonga subtus toto hirsuta - Hte-Ga-

ronne, Laramette (Timbal) . . . .3689. C. olida (Crép. mss)

Pet. aculeati; fol. obovato-oblonga ad nervos hirsuta - Hungaria, Trencin Bosaca (Holuby). .3690. C. Holuby Gdgr mss Pet. subinermes; fol. apice attenuata ad nervos hirsuta Cóte-d'Or, Larray (Boullu). . .3691. C. Fnaudulosa Gidgr mss Pet. omnes inermes; fol. apice rotundata ad nervos hirsuta - Rhone, Poleymieux (Chabert).3692. C. oligopona Gdgr mss

Pet. inf. inermes; fol. apice attenuata subtus toto hirsuta - Tyrol, Nogare in Pine (Gelmi).3693. C. Hatssmansiasa

. . . . . . . . . . Gdgr mss Pet. omnes aculeati; fol. apice obtusa subtus toto hirsuta Basses-Alpes, Condamine (Proal).3694. C. Fastuosa Gdgr mss

79 Fol. latiora 9-10 mill. lata. . . . . . . . 80 Fol. latiora saepius $15-18 \mathrm{~m}$. lata. $\quad .94$

80 Aculei subulati recti (ut in $R$. spinosissima) . . . . . 81 Aculei falcato-dilatati. . . . . . . . . . . 84 Fol. supra glandulosa . . . . 82

S1 Minime - Hte-Savoie, Pringy (Puget).3695. C. tragacantea

Fol elliptica . . . . Gdgr mss

ptica subtus ad costam hirsuta. . . . . . . 83

Fol. obovato-oblonga ad nervos hirsuta - Aragonia, austr., Sierra de Mosqueruela (Martin).3696. C. Reuteriasa Gdgr mss

Tubus glaber; fol. basi rotundata - Aragonia austr., Sierra $83\left\{\begin{array}{l}\text { de Mosqueruela Martin). - } 93697 \text {. C. Filispisi Gdgr mss } \\ \text { Tubus in } \% / 3 \text { inf. glandulosus; fol. basi attenuata - Caucasus, }\end{array}\right.$ Daghestan (Becker). . . 3698. C. DAgnestayica Gdgr mss

8 \& Fol. supra | glandulosa . . . . . . . . . 85

Fol. supra eglandulosa. . . . . . . . . . . 87

Pet. omnes aculeati ............ . 86

Pet. omnes inermes; fol. obovata - Aisne, St-Quentin (Magnier). . . . . . . 3699. C. Mlagxieni Gdgr mas Pet. inf. inermes; fol. ovato-elliptica - Rhöne, Pommiers (Gdgr herb. ros. n. 153). . . 3700. C. Rufescens Gdgr mss Ped. j-6 m. 1.; fol. basi rotundata - Aisne, St-Quentin Ia-

86 gnier) ........3701. C. Texuispiva Gdgr mss Ped. 9-11 m. 1.; fol. basi attenuata - Pedem., Vallées Vau• doises (Rostan). . . . . 3702. C. myriosticta Gdgr ms3 Tubus laevis. .................. 88 Tubus in $1 / 3$ inf. glandulosus; pet. inf. inermes - Ain, Les Echeyx (Chabert) . . . . . 3703. C. Holocalyx Gdgr mss Tubus toto glandulosus; pet. omnes aculeati - Europa, etc. (Gdgr herb. ros. n. 430, 602, 603, 604, 605 et 606 var.). ................... C. rubigrnosa (L.) Fol. basi attenuata. . . . . . . . . . 89

88 Fol. basi rotundata $\ldots 91$

89 Pet. aculeati; fol. apice rotundata. . . . . . . 90 Pet. subinermes; fol. apice attenuata - Rhone, Pommiers (Gdgr herb. ros. n. 112). . . 3705. C. BRacirstylis Gdgr 
Fol. obovata acute dentata - Hanovre, Münden (Zabel).3706

. . . . . . . C. HaNoverana Gdgr mss Fol. oblonga - Arabia, Mt-Horeb (Schimper exs. n. 723;

Bové exs. n. 180). . . . . .3706 bis. C. ArAbica (Crép.)

Fol. ovato-elliptica obtuse dentata - Loire, Chatelneuf (Gdgr)

. . . . . . . 3707. C. Monticola Gdgr mss

Fol. subtus ad nervos hirsuta. ......... 92

Fol. subtus toto hirsuta . . . . . 93

Fr. basi attenuatus; fol. suborbiculata - Gallia, Cher, etc.

Fr. basi rotundatus; fol. obovata - Rhône, Montmelas (Gdgr

herb. ros. n. 312) . . . . 3709. C. sclenoacantha Gdgr

Aculei 2-ti; fr. basi rotundatus - Rhone, Arnas (Gdgr).3710

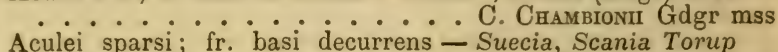
Tullberg). . . . . 3711. C. stepHanocarPa Gdgr mss Fol. supra | glandulosa . . . . . . . . . 95 Minime. . . . . . . . . . . . 99 Fol. utrinque rotundata; pet. inermes vel subinermes. . . 996 Fol. utrinque attenuata; pet. aculeati ........ 97 Pet. inf. inermes; fol. subtus ad nervos hirsuta - Hte-Savoie, Pringy (Puget). . . . . .3712. C. supramollis Gdgr mss Pet. subinermes; fol. subtus toto hirsuta - Rhône, Gleizé (Gdgr herb. ros. n. 203). . . . 3713. C. ostryaefolia Gdgr

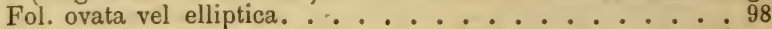
Fol. obovato-oblonga - Rhône, Alix (Gdgr herb. ros. n. 318) . . . . . . . . . 3714. C. Dentata Gdgr mss Ped. 7-9 m. l.; fr. ovoideus basi rotundatus - Rhône, Ouilly (Gdgr herb. ros. n. 101).3715. C. GLandulosissima Gdgr inss Ped. 9-11 m.l.; fr. obovato-oblongus basi attenuatus - Rhône, Gleizé (Gdgr herb. ros. n. 255).3716. C. RHIPIDothamnos Gdgr Fol. supra glaberrima basi rotundata . . . . . . 1 no Fol. supra villosa vel tomentosa basi rotundata. . . . . 101 Fol. supra adsperse hirsuta basi attenuata - Rhône, Yzeron (Chabert). . . . . . . 3717. C. Fugax Gdgr mss Tubus basi glandulosus; sep. dorso dense muricata - Tyrol, Hall (Gremblich). . . . . . 3718. C. HALLENSIs Gdgr mss Tubus laevis; sep. dorso sublaevia - Vaucluse, Mt-Ventoux (Autheman) . . . . 3719. (.. ventosana Gdgr mss Fol. amplissima, acuta, utrinque tomentosa - Podolia.3720. 101 Fol. mediocria, obtusa, utrinque tenuiter hirsuta - Oise, Noyon (Magnier) . . . .3721. C. erectisepala Gdgr mss Fr. apice strangulatus . . . . . . . . 103

Fr. apice attenuatus necne, nunquam strangulatus . . . 114

103 | Pet. aculeati ................. 104

1 Pet. inermes d. . . . . . . . . 109

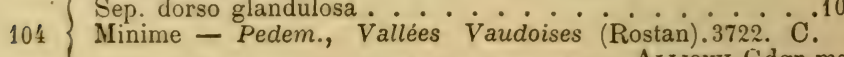
....................... Gdgr mss Tubus laevis; fol. subtus toto hirsuta ........106

105 Tubus laevis; fol. subtus ad nervos hirsuta.. .107 Tubus basi glandulosus; fol. ad costam hirsuta - Iles Canaries, Orotava (Sagot). . . .3723. C. onotavensis Gdgr mss 
Fol. ovata basi attenuata apice rotundata - Helv., Fribourg Montbovon (Cottet). . . . 3724. C. Longicalycina Gdgr mss

Fol. oblonga basi rotundata apice attenuata - Rhone, Ecully, Sathonay, etc. .......3725. C. PORRECTa Gdgr mss

107 Fol. obovata utrinque attenuata - Pyrenaei, Gèdre (Bordère) . . . . 3726. C. Griseispina Gdgr mss Ped. 10-13 m. 1.; stip. subtus eglandulosae - Isère, Balmes

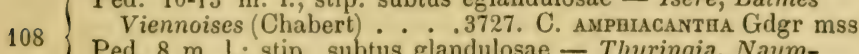
Ped. 8 m. l.; stip. subtus glandulosae - Thuringia, Naumburg (Staritz) . . . . 3728. C. Reichenbachlana Gdgr mss

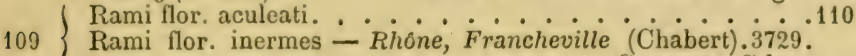

110 Fol. apice lanceolata.......... AfFIXA Gdgr mss

Fol apice lanceolata. . . . . . . . . . 111

110 Fol. apice rotundata . . . . . . . . . . 112 $111\left\{\begin{array}{l}\text { Fol. apice rotundata } \\ \text { Fol. apice lanceolata -Gironde, La Teste-de-Buch (Maillard). }\end{array}\right.$

112 Fol. elliptica basi rotundata - Isère, Balmes Viennoises (Chabert) . . . . 3731. C. biglandulosa Gdgr mss Virens ; fr. basi valde decurrens - Basses-Alpes, Jausières $113\left\{\begin{array}{l}\text { (Proal). f basi attenuatus - Rhône, Messimy (Chabert). } \\ \text { Rubens; fr. brass }\end{array}\right.$ . . . . . . . 3733. C. pulcherrna Gdgr mss

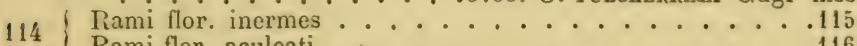

(Rami flor aculeati. . . . . . . 116

(Fol. elliptica apice breviter attenuata - Italia, Pise (Savi). $115\left\{\begin{array}{l}\text { Fol. oblongo-lanceolata - Rhône, Beaunant (Chabert).3735. } \\ \text { - B }\end{array}\right.$

. . . . . C. Pruniformis Gdgr mas

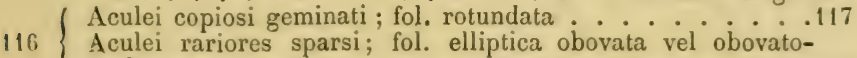
oblonga. . . . . . . . . 118

Stıp. subtus glandulosae; ped. $6-8$ m. $\dot{1}^{\circ}-$ - Rhône, Alix

117 (Gdgr herb. ros. n. 317). - . 3736. C. Alixensis Gdgr Minime ; ped. 2-5 m. 1.-Rhône, Pommiers (Gdgr herb. ros. n. 362). . . . 3737. C. synacantra Gdgr mss Fol. subtus ad costam hirsuta, apice rotundata vel obtusa. .119

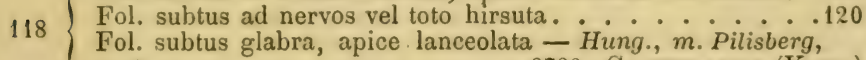
ete. . . . . . . . 3738. C. huvgarica (Kern.)

Pet. aculeati ; fr. apice breviter attenuatus - Rhone, Mont-

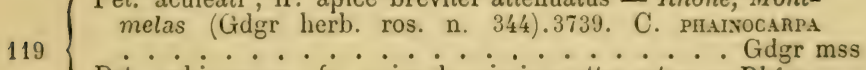

Pet. subinermes; fr. apice longissime attenuatus - Rhône, Chessy (Gdgr) . . . . . 3740. C. collifera Gdgr mss

$120\left\{\begin{array}{l}\text { Sep. dorso I glandulosa. Minime - Puy-de-D6me (Malarbet).374i. C. MaLARBETI } \\ \text { Mina } 12\end{array}\right.$ Fol. subtus ad costam aculeolata - Pyrenaei, Gèdre (Bordère).......3742. C. CalostepHaNa Gdgr ms\$ 
( Discus planus. . . . . . . . 123 Discus conicus - Rhóne, Montmelas (Gdgr herb. ros. n. 218).

$12.3\left\{\begin{array}{l}\text { Tubus glaber } 1 / 4 \text { inf. glandulosus - Isère, Dessine (Chabert). } \\ \text { Tubus in } 124\end{array}\right.$ .......3744. C. ANISOCARPA Gdgr mss Pet. omnes aculeati . . . . . . . . . 125 Pet. sup. inermes; fol. utrinque attenuata - Pyrenaei,

124 Gèdre (Bordère) . . . . 3745. C. Ramondiana Gdgr mss Pet. omnes inermes; fol. utrinque rotundata - Pyren. Or., Amélie-les-Bains (Debeaux) . . . 3746. C. Rufula Gdgr mss

125 Fol. basi rotundata. . . . . . . . . . . . . . . . .

Fol. basi attenuata. $\ldots 127$

Ped. 11-13 m. l.; fol. supra glabra - Pyrenaei, Gèdre (Gdgr $126\left\{\begin{array}{l}\text { herb. ros. n. 249). } \\ \text { Ped. 7-9 m. 1.; fol. undique hirsuta-Pyrenaei, Gèdre (Bor- }\end{array}\right.$ dère) . . . . . . 3748. C. hypoxantha Gdgr mss Fr. mediocris; fol. obovata - Pyrenaei, Gèdre (Bordère). 127 Fr. magnus; fol. elliptica - Gallia, Auxerre (R. d'Auxerre Ravin fl. de l'Yonne $2^{\circ}$ éd., II, p. 118).3750. C. Altissiodu-

b. - Dimorphacanthae Gdgr Essai, p. 40.

Aculei dimorphi, nempe vero, alii praesertim ramorum annotinorum vel biennium in setas glanduliferas abeuntes, alii autem e basi dilatata compressaque plus minus falcatoadunci.

1 Pet. saltem sup. sparse hirsurti . . . . . . . 2

\{ Pet. omnes hirsuti vel tomentosi .......... 17

Fr. globosus vel rotundatus ........... 3

2 Fr. ovatus ...................... 6

Fr, ovoideus vel obovato-oblongus . . . . . . . 9

Fr. oblongus . . . . . . . . . . 12

3 Tubus toto glandulosus; fol. obovato-oblonga . . . . . 4

\{ Tubus laevis; fol. ovata vel elliptica ........ 5

( Ped. 3-5 m. 1.; stip. subtus glandulosae - Rhône, Francheville (Chabert) . . . . . 3751. C. BREvipes Gdgr mss $4\left\{\begin{array}{l}\text { Ped. } 9-10 \text { m. } 1 \text {; ; stip. subtus eglandulosae - Anglia, York } \\ \text { (Wester). }\end{array}\right.$ Bracteae fructum aequantes; discus subconicus - Italia, Suse

5 (Chavanis). . . . . 3753. C. xyLOECHINA Gdgr mss Bracteae fructu multo breviores; discus planus - Rhône, Mont Ceindre (Chabert). . . . 3754. C. SEMIPEdunculata Gdgr msS 
'Tubus laevis. . . . . . . . . . . . . . . 7

Tubus basi glandulosus; fol. obovato-oblonga - Rhone, Tassin (Billot exs. n. 3595). .3755. C. PARcepunens Gdgr mss Tubus in $1 / 3$ inf. glandulosus ; fol. obovata - Hung., Trencin Bosaca (Ioluby). . . . . 3756. C. submitis Gdgr mss

Fol. elliptica subtus ad nervos hirsuta ; pet. subinermes. . . 8 Fol. obovata subtus toto hirsuta; pet. aculeati-Scotia, Perth Aberargie (Drummond). . . . .3757. C. scotica Gigr mss Aculei falcati ad ramos florif, sat copiosi - Rhone, St-Romain (Chabert). .......3758. ' J. unicolon Gdgr mss Aculei falcati ad ramos floriferos nulli - Gallia, Marseille (Roux). . . . . . 3759. C. Massiliaca Gdgr mss

Fol. supra glandulosa . . . . . . . . . . . 10

Minime. .............. 11

Fol. rotundata subtus toto hirsuta - Graecia, etc. (Heldr. herb. norm. n. 734, etc.). . . . 3760. C. glutinosa (S. S.) Fol. ovata subtus glabra - Persia, $m$. Kuh Nur (Haussknecht). . .............. C. Aspernima (Godet) Rami flor aculeati; fol. ovata ad costam hirsuta - Gallia,

11 Gap (Burle) ......... 3762. C. Bunler (Puget mss) Rami flor. inermes; fol. oblonga ad nervos hirsuta - HteGaronne, Balma ......3763. C. LAdANifera (T'imb.)

Tubus toto glandulosus. .............. 13

$12 \int$ Tubus toto laevis ........................ 14 Tubus basi glandulosus - Gard, Goulson (Tuezkiewicz). 3764 ........... C. lovgrteba Gigr mss Discus subconicus; fol. basi attenuata - Rhône, Brignais

$13 \int$ (Chabert). . . . . . 3765. C. pungers Gdgr mss Discus conicus; fol. basi rotundata - Austr. inf., Kalksburg (Wiesbaur). . . . . . .3766. C. ERroneA Gdgr mss

$14\left\{\begin{array}{l}\text { Fol. basi attenuata subtus ad costam hirsuta. . . . . . } 15 \\ \text { Fol basi rotundata subtus toto hirsuta. } 16\end{array}\right.$ Rami flor. et pet. aculeati - Rhône, Yzeron (Chabert). 3767.

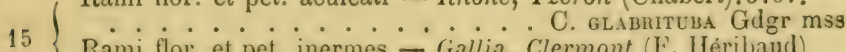
Rami flor. et pet. inermes - Gallia, Clermont (F. Héribaud) . . . . . . . . 3768. C. claromontana Gdgr mss Ped. 10-15 m. 1.; fol. supra glandulosa - Pyrenaei, Gedre

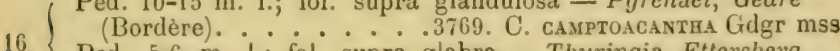
6 Ped. 5-6 m. 1.; fol. supra glabra - Thuringia, Ettersberg (Haussknecht) ......3770. C. Macrocarpa Gdgr mss

17 Tubus toto hispidus . . . . . . . . . 18

17 Tubus laevis vel basi aut in $1 / 2$ inf glandulosus . . . . 27 Fol. supra glandulosa . . . . . . . . . . 19

$18\{$ Ninime.

Aculei falcati ad ramos floriferos sat copiosi. . . . . 20

19 Aculei falcati ad ramos flor. nulli - Belg., Rochefort (Reliq. Maill. n. 603) . . . . . . . 3771. C. Crepini Gdgr mss Pet. inf. inermes; fol. obovata apice rotundata - Ain, Rey-

$20 \int$ rieux (Chevrolat, Fray). . . 3772. C. Cnevrolati Gdgr mss Pet. omnes aculeati; fol. ovato-obtusa - Gallia, etc. (C. spinourceolata Crép. mss). . . . .3773. C. Echinocarpa (Rip.) Aculei falcati ad ramos floriferos nulli. . . . . . . 22 
Fol. elliptica vel obovata basi rotundata. . . . . . 23 Fol. oblonga basi attenuata - Rhone, Brignais (Chabert). ............... C. ST74ENOGYNA Gdgr mss Discus planus; fol, obovata apice rotundata. . . . 24

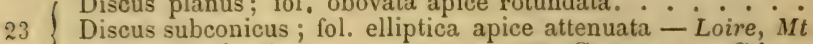
Pilat (Chabert). . . . . 3775. C. DEARMata Gdgr mss

$24\left\{\begin{array}{l}\text { Ped. 9-11 m. l.; sep. foliacea - Anglia, Kew (J.-G. Baker). } \\ -\ldots .3776 \text {. C. MacrostepHana Gdgr mss }\end{array}\right.$ Ped. 7-8 m. 1.; sep. angustata - Hollande, Zélande Domburg (Ankersmit). . . . . 3777. C. Batavica Gdgr mss Fol. subtus toto hirsuta $\ldots \ldots 26$

25 Fol. subtus ad costam hirsuta - Suecia mer.; Dania. 3778.

Ped 5-6 m. l; fol elliptica - Tyrol, Hall Gremblich) 3779 .

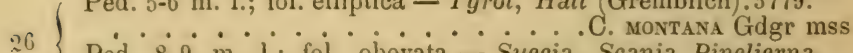
Ped. 8-9 m. l.; fol. obovata - Suecia, Scania Pinelierna (Tullberg). . . . . . . 3780. C. DIMORPHA Gdgr mss

(Fr. oblongus . . . . . . . . . . . 28

27 Fr. ovoideus vel obovato-oblongus. $\therefore 40$

Fr. rotundatus ovatus vel rarius subovoideus. . . . . . 45

Sep. dorso glandulosa . . . . . . . . . 29

Sep. subeglandulosa ; aculei falcati ad ramos flor. copiosi Rhône, Gleizé (Gdgr herb. ros. n. 15).3781. C. BRAchyste-

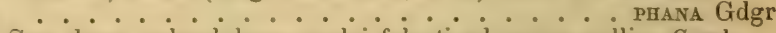
Sep. dorsn eglandulosa; aculei falcati ad ramos nulli-Gard, Aulas (Tuezkiewicz). . . . .3782. C. Lonetiana Gdgr mss Tubus basi glandulosus . . . . . . . . . 30 Tubus glabur . . . . . . . . . . . . 35 Tubus in $1 / 3$ inf. glandulosus; fol. elliptico-obovata-Pyren. centr., Mourère (Bordère). . . . . 3783. C. Nova Gdgr mss Tubus in $1 / 2$ inf. glandulosus; fol. obovato-oblonga - Tyrol, Hall Gremblich). . . . . . 3784. C. ELEGans Gdgr mss

30 Aculei falcati ad ramos floriferos nulli. . . . . . 31

30 Ninime. . . . . . 32 Ped. 4-5 m. 1., valde glandulosi - Gallia, Clermont (Lamotte;

31 F. Héribaud). . . 3785. C. LanotTi Gdgr mss 1 Ped. 7-8 m. l, parcius glandulosi - Rhône, Yzeron (Chabert). . . . . . 3786. C. scrupulosa Gdgr mss

$22\left\{\begin{array}{l}\text { Fol. subtus ad costam vel nervos hirsuta. } \\ \text { Fol. subtus toto hirsuta. } \ldots . .334\end{array}\right.$

Fol. sulstus ad costam hirsuta, basi rotundata - Baléares,

33 Iviça (Bachès). . . . 3787. C. AdelpHICA Gdgr mss Fol. subtus ad nervos hirsuta, basi paulo attenuata - Rhone, Yzeron (Chabert). . . . 3788. C. FAsciculata Gdgr mss

( Pet. aculeati ; fol.. acute obovato-oblonga - Borussia, Lyck

24 (Sanio) $\cdots$. . Prossica Gdgr mss

24 Pet. subinermes; fol. obtuse elliptica - Cantal, Le Lioran

(F. Héribaud). . . . . 3790. C. cantalica Gdgr mss

35 Aculei falcati ad ramos floriferos nulli........ 36

35 | Minime. . . . . . . . . . . . 39

(Minime.................... 37

(3) Fol. supra glandulosa - Italia orient., Faenza (Caldesi). ................ C. Tenoreana Gdgr ms 
37 ( Pet. I aculeati ; fol apice attenuata . . . . . . 38

Pet. sup. inermes; fol. apice rotundata - Pyrenaei, Gédre

(Gdgr herb. ros, n. 279). . . 3792. C. lampocarpa Gidgr mas Fol. elliptica subtus toto hirsuta - lihone, Ecully (Chabert.

Fol obovato-oblonga subtus ad nervos hirsuta - Itte-Savoie, Fessy (Puget). . . . . . 3794. C. Loxgicollis Gidgr mss

I'et. subinernes ; fol. obovata basi rotundata - Rhone, Chá-

$39 \int$ toux (Gidgr herb. ros. n. 294).3795. C. cBlamyophora

Pet. aculeati ; fol, oblonga basi attenuata - Rhone, Durette

$(\mathrm{Gdgr})$. . . . . . . 3796. C. Dumetonum Gidgr mss

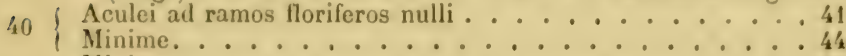

4 ) Mlinime. . . . . . . . 42

41 Fol. supra glandulosa - Saxonia, Komigstein (Kórieger, .3797.

Tubus laevis $\ldots \ldots$

42 Tubus basi glandulosus - Belg., IIan-sur-Lesse (Reliq Maill.

n. 603 a) .........3798. C. Dodonaei Gidgr mss

Fol. utrinque attenuata - Rhone, Beaunant (Chabert).3799.

$43\{$ Fol. utrinque rotundata - Loire, iri Pilat (Malarbet). 3800 .

Fol. oblonga utrinque attenuata ; tubus basi glandulosus mss Rhone, Soucieux (Chabert). . .3801. C. JUCUNDA Gdgr mss

Fol. ohovala utrinique rotunilata; tubus glaber - Anjlia,

44 Worcester Wye Forest (Fraser).3802. C. Ifudsoniana

Fol. obovata utrinque atienuata; tubus glaber - Rdgre, Charbonnieres (Chabert). ...3803. C. Comgena Gidgr mss

45 Aculei falcati ad ramos floriferos nulli. . . . . . . 46

i Minime. . . . . . . . . . . . 48

(Fr. ovatus ..................... 47

Fr. rotundatus; fol. elliptica subtus ad nervos hirsuta - IIte-

46 Savoie, Pringy (Puget). . . . . 3804. C. obesa Gdgr mss

Fr. subovoideus; fol. obovata subtus toto hirsuta - Rhóne, Villefranche (Gdgr) . . . . 3805. C. Fonmosula Gdgr mss

( Tubus basi glaber; fol. ad costam hirsuta - Rhone, La Roche $47\left\{\begin{array}{l}\text { d'A joux (Gdgr) } \\ \text { Tubus basi glandulosus; fol. ad nervos hirsuta - Austria, }\end{array}\right.$ Znaim (Oborny) . . . . . 3807. C. Austniaca Gdgr mss

( Fr. submoideus. . . . . . . . . . 44

48 Frovatus . . . . . . . . . . 50

Fr. globosus vel rotundatus. ........... 52

( 'Tubus laevis; fol, basi attenuata - Europa media, etc. 3808.

19 Tubus in 1 inf glandulosus Tubus in $1 / 2$ inf. glandulosus; fol. basi rotundata - Suecia, Scanie Hoor (Tullberg) . . . .3809. C. INCRassaxs Gdgr mss

$50\left\{\begin{array}{l}\text { Fol. ovata vel elliptica, } \text { Fol. obovato-oblonga; tubus in } 1 / 2 \text { inf. glandulosus-Suecia, } \\ 51\end{array}\right.$ Kalmar (Scheutz) . . . . 3810. C. Scuetrzir Lidgr mas 
( Pet. subinermes; fol. subtus virentia - Rhône, Beaunant (Chabert). . . . 3811. C. ceratoacantha Gdgr mss Pet. aculeati ; fol. subtus cinereo-glauca - Rhône, Pommiers (Gdgr herb. ros n. 329). • .3812. C. dicranodendron Gdgr

52 | Fol. ovata. . . . . . . . . 53

Fol. rotundata vel oblonga. Tubus glaber - Gallia, etc. (Gdgr herb. ros n. 464, 484, 485

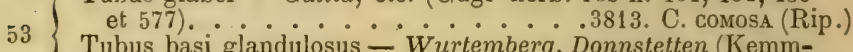
Tubus basi glandulosus - Wurtemberg, Donnstetten (Kemmler). . . . . . 3814, C. elegantissima Gdgr mss Fol. orbiculata utrinque rotundata - Gallia, etc (Gdgr herb. ros. n. 433 et 601). . . . 3815. C. Rotundifolia (Rau)

Fol. oblonga utrinque attenuata - Loire, Rive-de-Gier (Chabert). . . . . . 3816. C. Multiculars Gdgr mss Fol. rotundata ; ? - Gallia, Gap (Grenier). 3816 bis. C. PuY-

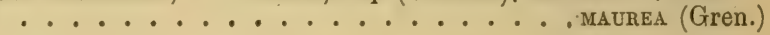

Subgenus XI. - Pugetia Gdgr Essai p. 40 (in sect.) Tomentoseae Dés.; Crép. Prim. mon. ros., et Villoseae D. C. in Ser. Mus. helv. I (1818), p. 2.

Styli liberi, glabri villosi aut lanati, ultra discum planum vel rarius conicum capitati, sessiles. Flores bracteati, c0rymbosi, rarissime solitarii, mediocris magnitudinis, albocarnei rosei vel intense purpurei, petalis ovato-cordatis attritu subglutinosis ad marginesque saepe ciliatis praediti. Fructus magnus vel maximus, saepissime coriaceus, subserotinus, glaber glandulosus vel echinatus, intense ruber, sepala pinnatifida vel subintegerrima, breviora, extus plerumque glandulosa aut muricata, post anthesim patula et decidua, vel erecta et tunc fructum maturum coronantia. Pedunculi erecti, glandulosi hispidi vel (rarius) laeves aut villosi, late bracteati. Folia majora, decidua, attritu digitorum saepe maleolentia, eorum foliola plerumque et undique molliter tomentosa, subtus nonnumquam plus minus glandulosa, nunc glabra vel glabrescentia tunc glandulosa, saepius biserrata. Stipulae conformes, angustatae vel dilatatae, 
margine dentato-glandulosae, subtus ordinarie villosae vel tomentosae. Aculei sparsi, tunc ex areola basilari anguste oblonga plus minus adunci aut rectiusculi, vel stipulares, ergo recti basique ex areola ovata vel subrotundata parum dilatati, interdum (sed abnormaliter, ut videtur) in setas glanduliferas abeuntes. Radix reptans vel non.

Frutices sericei, in sepibus et silvaticis totius Europae (in australioribus alpestres vel alpini) hine inde crescentes, sociales, erecti, plus minus elati, ad ramos floriferos sat frequenter hirsuti aut tomentosi, cum aculeis aduncis dilatatis sepalisque deciduis; vel suffrutices humiliores, praesertim regionis montanae vel alpestris (ubi semper rari), tunc saepe solitarii, ad ramos floriferos rarius hirsuti, aculeos stipulares rectos sepalaque erecto-persistentia habentes.

In honorem D. abb. Puget e Sabaudia, genus hocce dicavi, cui gratias agere sanctum mihi officium est.

A Aculei recti ; sep. fructum maturum coronantia. . . . . . B Aculei saltem inclinati; sep. decidua, vel fructum modo mortuo coronantia . . . . . . . A. Eutomentosae

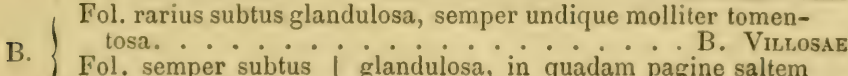
Fol, semper subtus I glandulosa, in quadam pagine saltem glabra vel glabrescentia....... C. Pualacroidae

\section{A. - Entomentosae Gdgr Essai p. 41}

(Tomentoseae Auct. recent.)

Rami laxi apice arcuati; aculei e basi plus minus dilatata saltem subincurvati faleati rarissime setacei rel aciculares; foliola utrinque molliter tomentosa, subtus ruhiginosi, necue, 1-3-serrato-glandulosa; sepala post anthesim plus minus scro decidua; styli glabri villosi aut lanati; llos sacpius 
pallide roseus, ad unguem ciliatus; fructus maturus glaber vel glandulosus, coccineus, calyce vivido haud coronatus. - Frutices per campos collesque passim crescentes, in australioribus Europae rarissimi vel alpestres.

Fol. omnino simpliciter serrata. ......... 2

Fol. alia simpliciter alii biserrata. . . . . . . 45

Fol. omnino biserrata............. 81

$\quad$ Ped. glabri villosi vel villoso-glandulosi. . . . . . 3

2 Ped. omnes glandulosi nec villosi. . . . . . . 18

3 Rami flor. glabri .............. 4

3 Rami flor hirsuti. . . . . . . . 7

4 Ped. glaberrimi. . . . . . . . . . . 5

4 Ped. hirsuti vel tomentosi... . . . . . . . . 6

(Sep. dorso eglandulosa; fol. apice rotundata - Helv., Genève

(Thomas)......3817. Pugetia glabripes Gdgr mss

Sep. dorso paulo glandulosa; fol. attenuata-Pedem., Vallées Vaudoises (Rostan). . 3818. Pugetia valdensium Gdgr mss (Fr. ovatus; ped. tomentosi - Vosges, Romont (Boulay).

$6\left\{\begin{array}{l}\text { Fr. oblongus; ped. paulo hirsuti - Htes-Alpes, Les Lau- } \\ \text { - } 3819 \text {. Pugetia trichopoda Gdgr mss }\end{array}\right.$ zières (Reliq. Maill. n. 170). 3820. Pugetia Mathonneti ................... Gdgr mss

- Fr. rotundatus vel ovatus ........... 8

7 I Fr. ovoideus vel oblongus. . . . . . . . . 13

s Tubus toto glandulosus ............... 9

Tubus laevis . . . . . 10

(Discus conicus; fol. obtusa - Armenia (Kotschy exs. n. 369

9 et 789). . . . . . . 3821. P. Vanheurckiana (Crép.) Discus planus; fol. lanceolata - Rhône, Arnas (Gdgr herb. ros. n. 325). . . . 3822. P. candrcans Gdgr mss

$10\left\{\begin{array}{l}\text { Fr. ovatus vel ovato-rotundatus; discus planus. } * \text { Fr. rotundatus; discus conicus }- \text { Volhynia, Jitomir (Golde). } 11 \\ \text {. }\end{array}\right.$ . . . . . . . 3823. P. Besseriana Gdgr mss

11 Styli glabrescentes; fr. ovatus hirsuti ; fr. ovato-rotundatus - Rhône, Tassin (Cha- 12 bert). . . . . . . 3824. P. ARgentea Gdgr mss Pet. inermes; fol. mediocria - Lyon, etc. (Gdgr herb. ros.

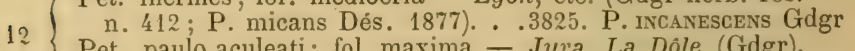
Pet. paulo aculeati; fol. maxima - Jura, La Dôle (Gdgr). . . . . . 3826. P. Subsericea Gdgr msS Tubus glaber . . . . . . . . . . 14

13 Tubus toto glandulosus ...................... 15 Tubus in $1 / 3$ inf. glandulosus; fol. obovato-oblonga - Cantal, Le Lioran (F. Héribaud). .3827. P. villicaulis Gdgr mss $14\left\{\begin{array}{l}\text { Discus planus; pet. subinermes - Rhône, Tassin (Chabert). } \\ \text { Discus conicus; pet. aculeati - Rhône, Charbonnières (Cha- }\end{array}\right.$ bert)... . . . . 3829. P. LANUginosa Gdgr msS

$15 \mid$ Fr. oblongus . . . . . . . . . . 16

15 Fr. obovatus $\ldots 17$ 
Styli paulo hirsuti ; fol. basi rotundata - Belg., Orchimont (Gravet). . .......3830. P. cinenacea (C'rép.) Styli lanati ; fol. hasi attenuata - Manovre, Münden (Zalel).

• • • • • • . . 3831. P. minsutissima Gidgr mss Fol. obovato-oblonga; styli subylabri - R'tine, Chartion-

17 nières (Chabert). . . . 3832. P. Leucopriylla Gdgr mss Fol. ovato-elliptica; styli hirsuti - Rhone, St-Genis-les-Ollières (Chabert). . . . 3833. P. subactrnacantia Gdgr mss

18 ( Fr. rotundatus vel globosus . . . . . . . 19 Fr. ovatus, obovatus, oblongus vel ovato-rotundatus (et tune

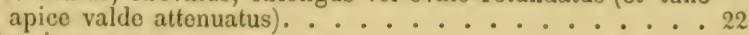

19 Pet. inermes . . . . . . . . . . 20

Pet. aculeati ............... . . 21

Styli lanati ; fol, obtusa - Belg., etc. $383^{\circ}$ P. Crnenascens

$20 \quad \ldots \ldots \ldots \ldots \ldots$. . . . . . Dum.) Styli pubescentes; fol. acuta - Rhone, Charbonnières (Gdgr herb. ros. n. 583). . . . .3835. P. nepilata Gidgr mss Styli pubescentes - Sarthe, Précigné (Chevallier).3836. P. 21 Styli glabri - Iite-Savoie, Brenthonne (Puget).3837. P. notex-

Tubus . . DATA Gdgr msS

?: Tubus toto glandulosus . . . . . . . . . 23

Tubus laevis vel basi glandulosus. . . . . . . . . 39

23 Pet. omnes inermes . . . . . . . . . . 24

Pet. aculeati . . . . . . . . . . 30

24 Rami flor apice villosi . . . . . . . . . 25

24 Rami flor glaberrimi. . . . . . . . . 27

25 Fr. ovoideus; fol. elliptica vel obovato-oblonga. . * . 26 Fr. ovato-rotundatus; fol. oblonga - Rhơne, Alix (Gdgr herb. ros. n. 182 bis). . . 3838. P. HEBECLADA Gdgr mss Rami flor. inermes; lol. ovato-elliptica - Ain, Divonne

96 (Gdgr herb. ros. n. 591). . . 3839. P. INCEnTA Gdgr mss Rami flor. I aculeati ; fol. obovato-oblonga - Rhône, Alix

(Gdgr). . . . . 3840. P. Roseipetala Gdgr mss

$27\left\{\begin{array}{l}\text { Fr. ovatus vel ovato-rotundatus. } \\ \text { Fr. subovoideus Rhón Tassin }\end{array}\right.$ Horovoldeus - Rhone, Tassin ( $P$ praecox Boullu non Hort. Angl.) . . . . . . 3841. P. properata Gdgr

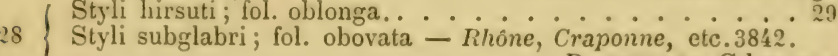

. . . . . . . P. SERICANs Gdgr mss

( Pet. paulo glandulosi ; rami flor. aculeati - Rhône, Alix

29 (Gdgr). . . . . 3843. P. chl.amyopetala Gdgr mss Pet. cylandulosi ; rami flor. inermes - Loire, soularges (Billot exs. n. 3855 ter). . . 3844. P. Henvieri Gdgr mss

30 Ramiflor villosi............... . 31

Rami flor glabri Styli hirsuti; ped. 12-14 m. 1. - Suecia, Scania Orup (Lund-

31 quist) . . . 3845. P. ABenn.sve Cidgr mss Styli glabri; ped. 14-15 m. 1. - Isere, Bourgoin (Gilgr). . . . . . . . . . 3846. P. Leptoclad Gdgr mss

32 Fr ovatus $\therefore \ldots \ldots$

82 Fr, ovoideus . . . . . . . 35 
iscus planus ; pet. eglandulosi - Ain, La Pape (Gdgr herb. ros. n. 283). . . . . 3847. P. LinguA Gdgr mss Discus subconicus; pet. paulo glandulosi - Saône-et-Loire, Cluny, etc. . . . 3848. P. Cluniacensis Gdgr mss

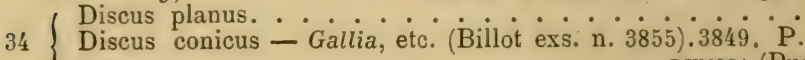

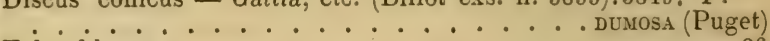
Fol. oblonga $\ldots \ldots \ldots$ Fol. obovato-oblonga; styli lanati - Basses-Alpes, Faucon

35 (Proal). . . . 3850. P. PAchyphylla Gdgr mss Fol. elliptica; styli hirsuti - Asia Minor, Lazistan (Balansa exs. n. 318) ......3851. P. Balansaea (Dés.)

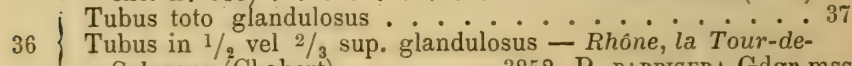
Salvagny (Chabert). . . . 3852. P. BARBIGERA Gdgr mss

Styli hirsuti; fol. basi rotundata . . . . . . . 38 Styli lanati; fol. basi attenuata - Pomerania, Czarnikau (Hülsen). . . . . 3853. P. AEQuidentata Gdgr mss

$38\left\{\begin{array}{l}\text { Ped. et tubus glandulosi - Savoie, Annecy, etc.3854. P. PEL- } \\ \text {. P }\end{array}\right.$

38 Ped. et tubus longe aculeolati - Rhône, Ecully (Chabert)

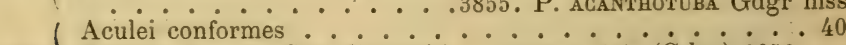

39 Aculei ad ramos flor. dimorphi - Rhône, Alix (Gdgr).3856. Rami flor apice l pubescentes $\therefore \ldots . . .41$

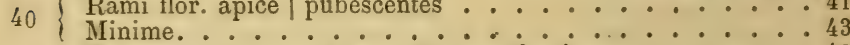

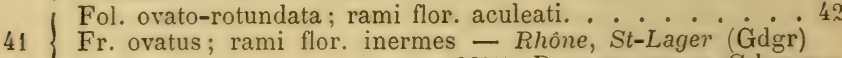
. . . . . . . 3857. P. CARPINIFOLIA Gdgr ms

$42\left\{\begin{array}{l}\text { Ped. 14-17 m. l.; sep. apice foliacea - Rhône, Alix (Gdgr } \\ \text { herb. ros. n. 182). . P P . P. LACTEscEns Gdgr }\end{array}\right.$ Ped. 25-32 m. 1.; sep. apice angustata - Rhône, Alix (Gdgr) . . . . . 3859. P. Lactescens B. subnuda Gdgr Fr. oblongus .................... 44 Fr. ovoideus ; fol. oblonga - Volhynia, Jitomir (Golde exs. 1873). . . . . . . 3860. P. Rossica Gdgr mss

43 Fr. ovatus; fol oblonga - Rhône, Beaunant (Billot exs. n. 3855 bis). . . . . 3861. P. Dumetivaga Gdgr mss Fr. ovato-rotundatus; fol. elliptica - Valais, Entremont (Delasoie). . . . . 3862. P. ANGusticollis Gdgr mss

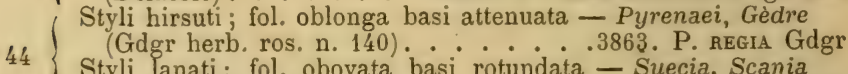
Styli lanati; fol. obovata basi rotundata - Suecia, Scania Kjells Nobbelof (Lundquist). . .3864. P. DENudata Gdgr mss

45 Fol. subtus ad costam plerumque eglandulosa . . . . 46 Fol. subtus ad costam semper glandulosa . . . . . . 62 Fr. ovoideus vel obovato-oblıngus . . . . . . . . 47

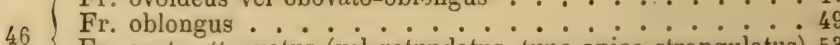
Fr. ovato-attenuatus (vel rotundatus, tune apice strangulatus) 53 Fr, rotundatus vel globosus . . . . . . . . 58 
Aculei conformes; ped hispidi. . . . . . . . 48

Aculei conformes; ped. lanati - Belg., Louette-St-Pierre

(Gravet).

Aculei dimorphi ; ped. hispidi - Pedem., Vallées Vaudoises (Rostan). . . . . . 3866. P. Rostan Gdgr mss Styli villosi ; fol. obovato-oblonga - Ilelv., Fribourg Allieres

(Cottet). . . . . . 3867. P. analoga Gdgr mss Styli subglabri; fol. oblonga - Ilelv., Solothurn (Alioth)

Rami flor. aculeati; ped. toto glandulosi ..... 50

49 Rami flor. inermes; ped. inferne hirsuti - IIarz, Sinlberg (Vocke). . . . . 3869. P. Hencysici Gdgr mss

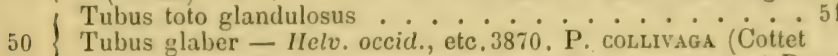
. . . . . . . . . . . . . et Pug.)

$51\left\{\begin{array}{l}\text { Rami flor. glabri } \text { Rami flor. hirsuti - Wurtemb., Donnstetten (Kemmler) } \\ \text { 52 }\end{array}\right.$

. . . . . . . . . 3871. P. Lovglfolia Gder mss

Discus planus; fol. obovata - Cúte-d'Or, Meursault (Ora-

non) . . . . . . . 3872. P. pulposa (Crép. mss)

52 Discus subconicus; fol, ovata - Hte-Savoie, Mt-Sion (Puget)

Discus valde conicus; fol. oblonga - Isère, La Gde Chartreuse (Gdgr). . . . . 3874. P. canthusiana Gdgr mss

53 ( Ped. glandulosi . . . . . . 54

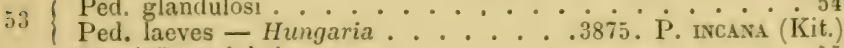

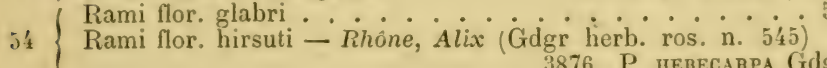

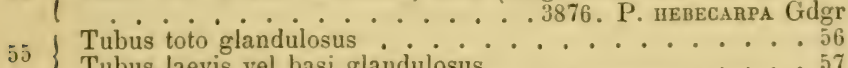

Tubus laevis vel basi glandulosus . . . . . 57

Rami flor. et pet. aculeati - Helv., Bovernier (Delasoie)

$56\{$. . . . 3877. P. Bovernierana (Crép.)

Rami flor. et pet. inermes - Gironde, Le Verdon (Notelay)

. . . . . . . 3878. P. Emargivata Gdgr mss

(Pet. aculeati ; fol. oblonga - Sabaudia, etc.3879. P. PERMU-

Pet. aculeati ; fol. obovata - Rhóne, Armas (Gdgr herb. ros.

n. 58). . . 3880. P. nosella Gdgr

Pet. inermes; fol. elliptica - Belg., Rochefort (Crepin). 3881.

-. P. intromissa (Crép.)

58 Acule dimorphi - Rhóne, Tassin, etc. 3ss?. P' MAncrava

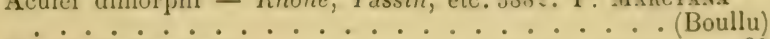

Pet. omnes aculeati . . . . . . . . 60 60

Pet. sup. inermes; rami flor. glabri - Rhône, Francheville

59 (Chabert) . . 3883. P. velutinafoli Gdgr mss Pet. inf. inermes; rami flor. hirsuti - Rhune, Alix (Gulgr)

. . . . 3884. P. PHLEwornuara Gilgr mss

Tubus basi glandulosus ........... 61

Tubus in $1 / 3$ inf. glandulosus; fol. obovata - Ilte-Savoie,

60 Habère-Lullin (Puget). . . . .3885. P. viness Gdgr mss

Tuhus toto glandulosus; fol. oblonga - IIte-saroie, Bellevaux (Puget) .....3886. P. imframolis Gdgr mss 
( Stip. subtus glandulosae; fr. rotundato-globosus - Hte-Sa-

61 voie, Habère-Lullin (Puget).3887. P. PHYLlostephana Gdgr mss Minime; fr. globosus - Delph.; Sabaudia.3888. P. Otiтes .................. Gdgr mss

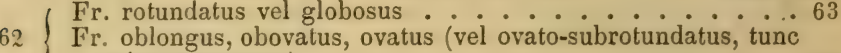
Fr. oblongus, obovatus, ovatus (vel ovato-subrotundatus, tunc apice attenuatus) ............. 65

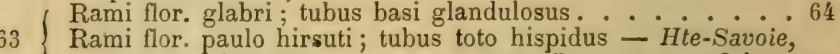
Argonnex (Puget). . . . .3889. P. Pallidula Gdgr mss Fol. obovata; ped. basi paulo hirsuti - Hle-Savoie, Habère-

64 Lullin (Puget) . . . . . 3890. P. AcropHILA Gdgr mss Fol. oblonga ; ped. tantum glandulosi - Hte-Savoie, HabèrePoche (Puget). . . . . 3891. P. tentaculata Gdgr mss

65 Tubus laevis; sep. dorso laevia vel fere eglandulosa . . . 66

| Tubus | glandulosus; sep. dorso glandulosa . . . . . . 67

Ped. glandulosi ; fol. elliptico-ovata - Htes-Alpes, St-Lau-

66 rent(Burle). 3. - 3892. P. LEIocALYX Gdgr mss Ped. laeves; fol. oblonga - Palatinat, Waldmoor (Fries)

......... . 3893. P. LAEVIPES Gdgr mss

Fr. oblongus ................ 68

67 Fr. ovatus vel ovato-rotundatus. . . . . . 70

Fr. ovoideus vel obovato-oblongus . . . . . . . 74

Rami flor. hirsuti ; pet. aculeatĩ . . . . . . . 69

68 Rami flor. glabri ; pet. inermes - Thuringia, Rudolstadt (Dufft). . . . . . . 3894. P. saxonica Gdgr mss Styli hirsuti; fol. inf. subtus toto glandulosa - Tyrol, Hall

69 (Gremblich)........3895. P. TENERA Gdgr mss Styli lanati; fol. subtus eglandulosa - Suecia, Scania Alnarp (Tullberg). . . . . . 3896. P. Tullbergir Gdgr mss

$70\left\{\begin{array}{l}\text { Rami flor. et pet. aculeati - Cantal, Le Lioran (F. Héribaud) } \\ \text { Rami flor. et pet. inermes }\end{array}\right.$

71 Styli lanati; fol obovato-oblonga vel oblonga . . . . . 72 Styli hirsuti; fol. obovata . . . . . . . . . . 73 Fol. amplissime obovato-oblonga subacuta - Hte-Savoie,

72 Argonnex (Puget) .....3898. P. Latifolia Gdgr mss Fol. sat anguste oblongo-acuminata - Hte-Savoie, Argonnex (Puget). . . . . . 3899. P. HaEsitans Gdgr mss Discus subconicus; rami flor. glabri - Hte-Savoie, Draillant

73 (Puget). . . . 3900. P. conomea Gdgr mss Discus planus; rami flor. hirsuti - Tyrol, Hall (Gremblich) . . 3901. P. Hallensis Gdgr mss

if Tubus basi glandulosus . . . . . . . . 75

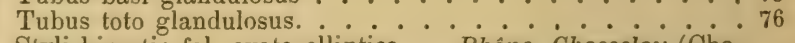

$75\left\{\begin{array}{l}\text { Styli hirsuti; fol. ovato-elliptica }- \text { Rhône, Chasselay (Cha- } \\ \text { bert). }\end{array}\right.$ Styli subglabri fol obovato-oblonga - Italia, Apenn. Modène Boscolungo (Gibelli). . . .3903. P. APENNINA Gdgr mss

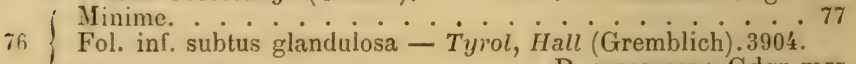
................ P. ColıIncola Gdgr mss 
Rami flor. glabri

Iiami flor. hirsuti et aculeati; fol olovalo-elliptica - Rhione,

Rami flor, tomentosi et inermes; fol. oblonga - Tyrol, MtVasone (Gelmi). . . . . . 3906. P. Tnidentina Gdgr mss

Fol. oblonga ..................... 79

Fol. obovala - inglia, York (IVebster).3907. P. Hudsoniana

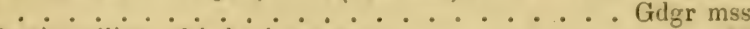

Petala eciliata; fol. basi attenuata. ...... . 80

Petala hasi ciliata; fol. inferne rotundata - Rhone, Alix (Gdgr) . . . . . . . . 3908. P. Ecurops Gdgr mss

Albicans; ped. 12-13 m. 1. - Pomerania, Czarnikau (Hülsen) Flavescens ; ped. 8-10 m. 1. - Itte-Savoie, Bellevaux (Puget)

3910. P. poliocnlona Gdgr mss Fol. subtus eglandulosa, vel ad costam tantum glandulosa. . 82

Fol. inf. subtus toto glandulosa. . . . . . . . 144

Fol. omnia subtus toto glandulosa. . . . . . . 166

Fr. ovato-rotundatus. . . . . . . . . 83

Fr. globosus vel subglobosus. . . . . . . . 84

Fr. ovatus, obovatus (vel oblongus tunc apice haud aut vix altenuatus) ................. 91

Fr. obovato-oblongus, oblongus (vel ovatus tunc apice valde

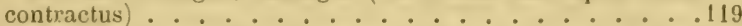

Rami flor. inermes; fol. obovata - Suecia, Scania Alnary

(Tullberg) . . . . . 3911. P. crassiuscula Gdgr mss Rami flor. aculeati; fol. obovato-oblonga - Hibernia, Belfast (Stewart). . . . . . 3912. P. Mlooreaxa Gdgr mss Pet. inermes .......... . . 85

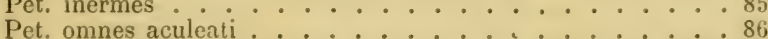

Pet. sup. inermes - Rhône, Limonest, etc. (Chahert). 3913.

...P. Melanocerasus Gidgr mss Fol. obrvato-elliptica apice rotundata - Itte-Savoie, St-Nicolas (Puget). . . . . . .3914. P. sericorhylla Gdgr miss Fol. oblongo-acuta - Anglia, Staffordsh. Beechouse Tywell (Fraser). . . . . 3915. P. Hookeriana Gdgr mss Tubus toto laevis ............. . . 87 Tubus toto glandulosus; styli hirsuti vel lanati. . . . . 89 Tubus in $1 / 3$ inf. glandulosus; styli sulglabri - IIelv., Bale (Christ). . . . . 3916. P. CalostepHana Gdgr mss Fol. apice attenuata ........ 88

87 Fol. apice obtusa - Suecia, Śmol. Bachaby (Ślicutz).39i7. . . . . . . . . P. AMOENA Gdgr mss Fol. oblonga - Isère, St-André-le-Gaz (Gdgr herb. ros. n. 290) . . . . . . 3918. P. Hiriciva Gdgr mss Fol. obovato-oblonga - Hte-Savoie, Habère-Lullin (Puget)

. . . . . 3919. P. sphaerotuma Gdgr mss Fol. ovata - Germ, in agro Werthem. (Wibel).3920. P. Du-

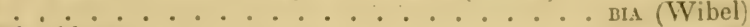

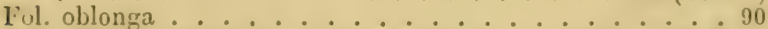
Fol. obovato-oblonga apice rotundata - Anglia, York (Webster) . . . . . . .3921. P. globulanis Gdgr mss Fol. ovato-acuta - Europa, elc. (Gdgr herb. ros. n. 410 , 431 et 454 . . . . . . . . 3922. P. subglobosa (Sm.) 

$\left\{\begin{array}{l}\text { Ped. 10-12 m. 1.; costa glandulosa - Anglia, Warwich } \\ \text { Harbwo (Bloxam). . . . . . 3923. P. Bloxami Gdgr mss }\end{array}\right.$ Ped. 22-25 m. l.; costa eglandulosa - Tyrol, Valle di Cembra (Gelmi) . . . . . . 3924. P. Cembra Gdgr mss

91 Styliglabri. . . . . . . . . . . . . 92

I Styli hirsuti vel lanati . . . . . . . . . . 996

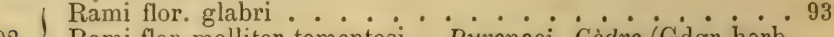
Rami flor. molliter tomentosi - Pyrenaei, Gèdre (Gdgr herb. ros. n. 95). . . . . 3925. P. ERIOPHLAEA Gdgr msS

93 ( Pet. aculeati ; fr. ovoideus . . . . . . . . 94 Pet. inermes ; fr. oblongus . . . . . . . . . . . 95 Tubus glaber; fol. obovata - Pyrenaei Or., Vernet-les-

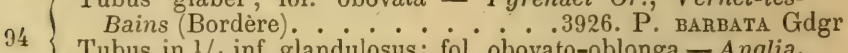
Tubus in $1 / 4$ inf. glandulosus; fol obovato-oblonga - Anglia, Cornwallis Trescastle (Briggs).3927. P. ConnubIENsIs Gdgr mss Pet. glandulosi ; fr. basi attenuatus - Pyrenaei, Gèdre (Gdgr

95 herb. ros. n. 375). . . 3928. P. PHalacrostigna Gdgr mss Pet. eglandulosi ; fr. basi rotundatus - Catalaunia; Olot (Vayreda). . . . . 3929. P. catalaunica Gidgr mss

96 Tubus glaber vel in $1 / 3$ aut $1 / 2$ inf. glandulosus . . . . 97 Tubus toto glandulosus. ............... . . .

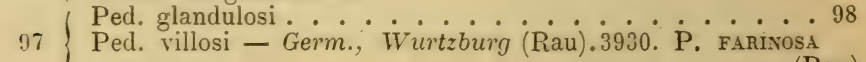

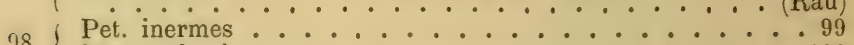

Pet aculeati 100

Rami flor. aculeati ; fol. obovato-oblonga - Manche, Yvetôt

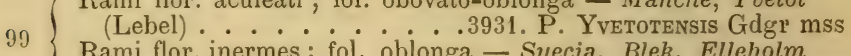
Rami flor. inermes; fol. oblonga - Suecia, Blek. Elleholm (Scheutz). . . . . . . .3932. P. Mrtis Gdgr mss Tubus toto laevis $\ldots \ldots . . . . . .101$ Tubus basi glandulosus; fol. parva - Suecia, Ystad (P. um-

100 belliflora var. minor Scheutz). . .3933. P. MINoR Gdgr mss T'ubus in $1 / 2$ inf. glandulosus; fol ampla - Vosges, SteMarie-aux-Mines (Christ). . . .3934. P. vogesIaCA Gdgr mss $101\left\{\begin{array}{l}\text { Fol. apice acuta. } \\ \text { Fol. obtusa - Anglia, York (Webster).3935. P. WEBSTERII }\end{array}\right.$ . . . Gdgr mss

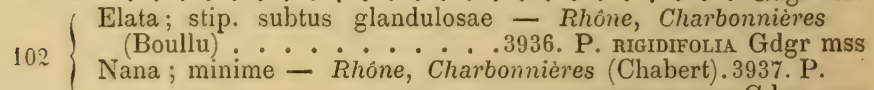

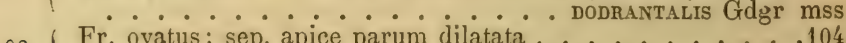

$103\left\{\begin{array}{l}\text { Fr. ovatus; sep. apice parum dilatata . Fr. obovatus vel oblongus; sep. apice saepius foliacea } .104 \\ \text {. }\end{array}\right.$

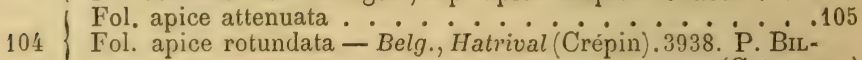

104 Fol. apice rotundata - Belg., Hatrival (Crépin).3938. P. BiL

105 Pet. dense aculeati. .................. 106

Fol. ovata - Germ; Silesia. . 3939. P. BonkHAusenir (Tratt.)

106 Fol. oblonga - Anglia, Warwich Chesterton (Fraser).3940. 
Styli parce hirsuti; discus subconicus - Belg., Orclimont 107 Styli lanali; discus planus-Suecia, ete. (I'. venusla sicheut\% non Waitz) . . . . . . . 3942. P. ScreutzIr Gdgr

108 / Pet. valde aculeati. . . . . . . . . . 109

Pet. inermes ............... . . . . . . . .

109 / Rami flor. villosi ; ped. hirsuto-glandulosi. . . . . . . 110

Rami flor. glabri ; ped. tantum glandulosi . . . . . . 112

110 Fol. obovata vel obovato-oblonga; fr. ovoideus. . . . . .111

Fol. et fr. oblonga - Tyrol, Hall (Gremblich).3943. P. TYno-

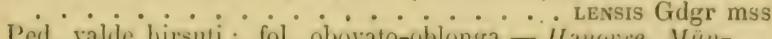

Ped. valde hirsuti ; fol. obovato-oblonga - Hanovre, Münden (Zabel). . . . . . . 3944. P. EnIOPODA Gdgr mss

111 Ped. subhirsuti; fol. olovata - Thuringia, schwarzburg ( $P$. thuringiaca Crép. non Wallr.).3945. P. trunisgensis

....................... Gder

Fr, ovoideus vel obovatus ..............

112 Fr. oblongus - Sabaudia (Dés. herb. ros. n. 74 ) $3946 .{ }^{\circ}{ }^{\circ}$.

. . . . . . . . . annesiensis (Dés.)

Discus planus. ......................

113 Discus conicus - IIte-Saroie, Choisy (Puget). 39í. P'. Nemo.

. . . . . . . Ricola Gdgr mss

Fol. elliptica ....................... . . . . .

Fol. obovato-oblonga - Sabaudia, etc. (Dés. herb. ros. n. $31 ;$

114 . . . . . . . 3948. P. tunoniensis (Dés.)

Fol. oblonga - Itibernia, Belfast (Stewart).3949. P. munn-

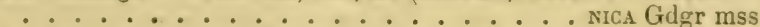

Cor. amoene rosea; dentes patulae - Europa, etc. (Gdgr

herb. ros. n. $499,514,610,611$ et 612$) .3950$. P. TOMEx-

115 . . . . Tosa (Sm.)

Cor. pallida; dentes fol. convergentes - Ain, Le Reculet ( $P$. foetida Cariot Etud. des fl. $2 \circ$ édit. II, 1854, non Bast.)

. . . . . . . . . . . . P. IDANENSIS Gdgr

Fol. apice attenuata ................ . .

116 Fol. apice rolundata - Calvados, Bernesq Lebel $3952 . \mathrm{P}$.

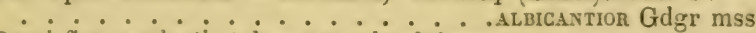

Rami flor. aculeati; tubus toto glandulosus. .....48

117 Rami flor. inermes; tubus in $1 \%$ inf. glandulosus - Caucas., Kaischaur. . . . . . . 3953. P. Ledebouri (Spr.)

Ped. 12-14 m. 1.; fol. oblonga tomentosa - Aisne, St-Quen-

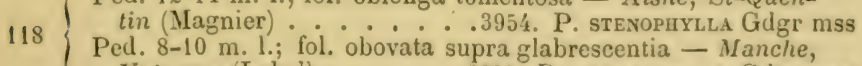
Valognes (Lebel) . . . . 3953. P. gLabricsclla Gdegr mss

119 Tubus glaber vel basi tantum glandulosus. . . . . 120

Tubus toto vel saltem in $1 / 2$ inf. glandulosus. . . . . . 126

Fr. oblongus . . . . . . . . . 121

120 Fr. ovoideus vel obovato-oblongus. $\quad \ldots 122$

Fr. ovatus - Rossia bor., ile Oesel (Schmidt llor. ross. hor.occ. exs. n. 255). . . . . . 3956. P. Livonica Gdgr mss

Ped. villoso-glandulosi; styli hirsuti - Rhone, Charbonniéres (Gdgr herb. ros. n. 599).3957. P. PSEUdo-Aldida

121 Ped. tantum glandulosi ; styli lanati - Suecia, CEland Treta

(Scheutz). . . . . . . 3958. P. TExuIs Gdgr mss 
1.2 (Discus planus ; rami flor. glabri . . . . . . . . . .

Discus conicus; rami flor. hirsuti - Hisp., Sierra Nevada;

Barcelone. . . . . . . 3959. P. BAETica Gdgr mss

123 Styli glabrescentes. . . . . . . . . 124

Styli hirsuti. . . . . . . . . . . . . .

Ped. tantum glandulosi - Isére, Crémieux (Gdgr).3960. P.

$124\left\{\begin{array}{c}0 \\ 0\end{array}\right.$

Ped. villoso-glandulosi - Deux-Sèvres, La Mothe (Sauzé).

. . . . 3961. P. Sauzeana Gdgr mss

(Tubus basi glandulosus ; fol oblonga - A in, Trévoux (Fray)

125 Tubus laevis; fol. elliptica - 3962. P. Cochlearis Gdgr mss . . . . . . . . . FriesII (Scheutz)

$126\{$ Styli glabri vel subsparse hirtelli. . . . . . . . . . . 27

126 Styli paulo villosi . . . . . . . . . . . . . . . . . . . . . . .

Styli hirsuti vel lanati . . . . . . . . . . . 138

127 Rami flor. glabri et aculeati - * - $\cdot 128$

127 Rami flor. hirsuti et inermes - Pyrenaei, Gèdre (Bordère).

Fr. oblongus; discus paulo conicus . . . . . . . . 129

128 Fr. ovoideus; discus planus - Ain, St-Didier-sur-Chalaronne (Fray). . . . . . 3965. P. strumosa Gdgr mss

$129\left\{\begin{array}{l}\text { Fol. supra sparse hirsuta, atrovirentia - Helv., Altorf (Gis- } \\ \text { ler). } \\ \text { Fol. undique albo-tomentosa - Hte-Savoie, Mt Sion (Puget). }\end{array}\right.$ . . . . . . . 3967. P. AlbifoliA Gdgr mss

130 Stip. subtus tomentosae . . . . . . . . 131

130 Stip. subtus subglabrae nunquam tomentosae. . . . . . 135

$131\left\{\begin{array}{l}\text { Fr. ovoideus } \\ \text { Fr. oblongus - Rhône, Arnas (Gdgr herb. ros. n. 26) } 3968 .\end{array}\right.$ . . . . P. HEBephora Gdgr mss

(Tubus toto glandulosus. . . . . . . . 133

$132\{$ Tubus in $2 / 3$ sup. glandulosus - Rhóne, Tassin (Chabert).

Rami flor glabri.

133 Rami flor. hirsuti - Helv., Berne (Séringe).3970. P. BER-

$131\left\{\begin{array}{l}\text { Pet. subinermes fol. elliptica - Rossia mer.occid.3971. } \\ \text { Pet. aculeati ; fol. oblonga - Wurtemberg, Donnstetten }\end{array}\right.$ (Kemmler). . . . . . . 3972. P. AfFinis Gdgr mss

(Fr. ovoideus vel obovato-oblongus; fol. oblonga $\cdot 136$

135 Fr. oblongus; fol. obovato-oblonga - Hte-Savoie, HabèreLullin (Puget) . . . . . 3973. P. confusa (Puget)

$136\left\{\begin{array}{l}\text { Ped. villoso-glandulosi. } \\ \text { Ped. tantum glandulosi }- \text { Hte-Savoie, Arenthon (Puget). }\end{array}\right.$

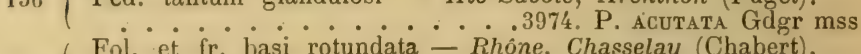
Fol. et fr. basi rotundata - Rhône, Chasselay (Chabert).

137 Fol. et fr. basi altenuata - Rhône, Dardilly (Chabert). 3976.

138 Rami flor hirsuti $\therefore \ldots$ P. PSEdo-Acutata Gdgr mss

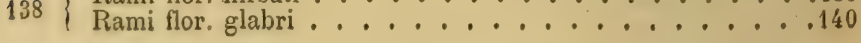


( Fr. oblongus; rami flor. inermes - Isère, Gide-Chartiruse

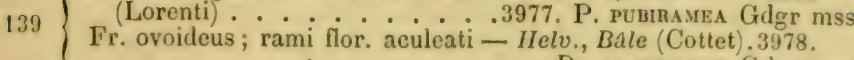
r. ovoideus; rami flor. aculeati - IIelv., Bale (Cottet).3978.

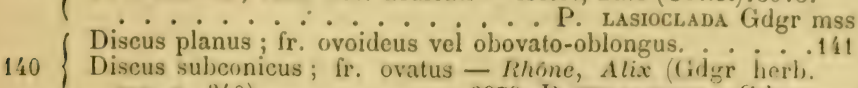

Diseus subeonicus; fr. ovatus - Rhone, Alix (idgr herb). ros. n. 340). . . . . . 3979. I’. tinamsurina Gidgr mss

141 Fol. oblonga .................. 14?

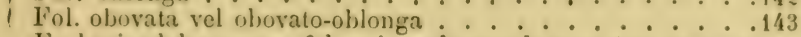

Fr. basi subdepressus; fol. apice subrotundata - Ain, St-Jean-

14:2 de-Thurignieux (Fray) . - . 3980. P. wealbesiens Gidgr mss Fr. basi attenuatus; fol. apice acuta - Rhöne, Arnas (Gilgr herl, ros. n. 237) d i basi attenuata - IIte-Savoie. Aren-
Rami flor. aculeati ; fol. brestica Gidgr

143 thon (Puget). . . . 3982. P. nigidispisa Gidgr mss Rami flor. subinermes; fol. basi rotundata - Manche, Lieusaint (Lebel) . . . . . 3983. P. normanniana Gdgr mas

144 I Styli villosi vel lanati. . . . . . . . . 145

Styli glabri vel subglaberrimi.

(Fr. globosus vel rotundatus. . . . . . . . . . 146

Fr. oblongus . . . . . . . . . 150

145 Fr. ovoideus vel obovato-oblongus. .154

Fr. ovatus - Bohemia, Carlstein (Polak).3984. P. Pragensis

................ Gdgr mss

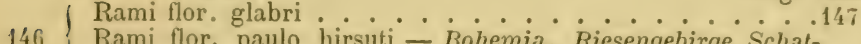

Rami flor. paulo hirsuti - Bohemia, Riesengebirge Schatzelar (Patze) . . . . . . 3985. P. BOHEMICA Gdgr mss

147 | Tubus glaber . . . . . . . . . . . . . . . . . . . . . .

Tubus toto glandulosus. $\ldots 149$

Ped. $13-15 \mathrm{~m}$. l.; fol apice acuta - Thuringia, Weimar

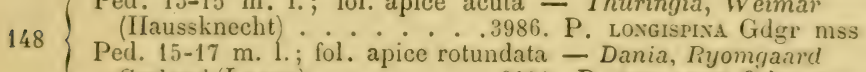
Seeland (Lange). . . . . 3987. P. sublaevis Gdgr mss

$149\left\{\begin{array}{l}\text { Fol. ol,longo-obtusiuscula, basi attenuata - Hanovre, Linz } \\ \text { (Zabel). } \\ \text { Fol. acuminata basi rotundata - Isère, Crémieux (Billot exs. }\end{array}\right.$ Fol. acuminata basi rotundata - Isère, Crémieux (Billot exs. n. 4035). . . . . 3989. P. macrostephasa Gidgr mss

150 ) Rami flor hirsuti ........................

Rami flor. glabri. . . . . . . . 152

Discus planus ; fol. basi attenuata - Dania, Jonstmup (Mor-

$151\left\{\begin{array}{l}\text { tensen). } \\ \text { Discus coni. }\end{array}\right.$

Discus conicus; fol. basi rotundata - Tyrol, Hall (Gremblich). . . . . . . 3991. P. Gremblicuir Gdgr mss

Tubus toto glandulosus . . . . . . . . . 153

$152\left\{\begin{array}{l}\text { Tubus basi glandulosus; ped. 19-22 m. 1. - Anglia, War- } \\ \text { wich Shrewly Pool (Fraser). } 2.3992 . \text { P. Fraser Gdgr mss }\end{array}\right.$

Tubus laevis; ped. 7-8 m. 1. - Dania, Bornholm Bodrlshes

(J. Lange). . . . . . 3993. P. Baltica Gdgr mss

(Pet. aculeati; fol. oblonga - Rhone, Charbonnières (Cariot).

153 Pet. subinermes; fol. obovato-oblonga - Helv, occid., Couvet (Baenitz herb, eur. exs. an. 1877).3995. P. HELvetica .................... Gdgr mss 
Rami flor. hirsuti. . . . . . . . . . . . 155

154 Rami flor. glabri . $\ldots .157$

Rami flor. apice glanduliferi - Suecia, Scania Flyinge (Lundquist). . . . . . . . . 3996. P. patula Gdgr mss

Discus subplanus; rami flor. aculeati . . . .....156

155 Discus conicus; rami flor. inermes - Hte-Savoie, Pringy etc. (Puget). . . . . . .3997. P. glandulosella (Rip. mss) Stip. subtus eglandulosae; fr. basi rotundatus - Gallia, Be-

156 sançon (Billot exs. n. 3726 parte). 3998. P. INsidiosa (Gren.) Stip. subtus glandulosae; fr. basi abrupte decurrens - Silesia, Goerbersdorf (Straehler). .3999. P. AcUTIFolia Gdgr mss

Tubus toto glandulosus . . . . . . . . . . 158

Tubus basi glandulosus; fol. acuta - Gallia, Chambéry

157 (Songeon). . . . . . .4000. P. inornata Gdgr mss

Tubus glaber; fol. obtusata - Suecia, Smol., Alsheda (Scheutz). . . . . . . 4001. P. PURPUREA Gdgr mss

158 Fol. basi altenuata .............. 159

Fol. basi rotundata . . . . . . . . . . 160

Ped. 15-17 m. l. ; pet. omnes paulo aculeati - Dania,

159 Aamup (J. Lange) . . . . . .4002. P. FIONICA Gdgr" mss Ped. 12-14 m. 1.; pet. sup. inermes - Gallia, Brest (Thiébaut). . . . . . . 4003. P. Thiebautn Gdgr mss

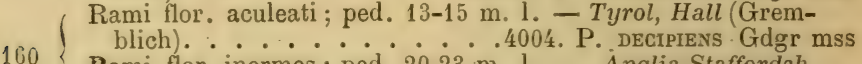
Rami flor. inermes; ped. 20-23 m. 1. - Anglia,Staffordsh. Baggeridge (Fraser). . . . 4005. P. BRitannica Gdgr mss

Rami flor. glabri. . . . . . . 162 161 Rami flor. paulo hirsuti - Rhône, Alix (Gdgr).4006. P. LUPet. | aculeati. ..................... Pet omnes inermes; fr. oblongus - Doubs, les St-Martin

162 (Billot exs. n. 3727).4007. P. Pseudo-Seringeana Gdgr mss Pet. sup. inermes; fr. ovoideus - Helv., Bâle (Christ). ........ . . 4008. P. FLEXILIS Gdgr msS Tubus toto glandulosus. . . . . . . . . . 164 Tubus in $1 / 2$ inf. glandulosus; fol. obovata - Hte-Savoie;

163 Pringy (Puget). . . . . . . .4009. P. errosa (Rip. mss) Tubus basi glandulosus; fol. obovato-oblonga - Anglia, Cheshive Grange (Harbord L.).4010. P. HARBORDII Gdgr mss

Fol. obovata vel oblonga basi attenuata. ........165

164 Fol. ovata basi rotundata - Gallia, Nancy, etc.4011. 1 .

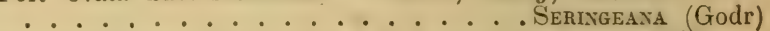

$165\left\{\begin{array}{l}\text { Pet. subinermes; fol. oblonga - Doubs, Mt-Brégille (Billot } \\ \text { exs. n. } 3726 \text { parte). . . 4012. P. vEsorertaca Gdgr mss } \\ \text { Pet. aculeati; fol elliptico-obovata - Gallia, Chambémy }\end{array}\right.$

165 Pet. aculeati; fol. elliptico-obovata - Gallia, Chamberry (Songeon). . . . . . . . 4013. P. cana Gdgr mss

166 \{ Rami flor. | hirsuti . . . . . . . . 167

166 Rami flor. glabri. . . . . . . . . . . . . 174

167 $\{$ Fr. globosus vel rotundatus ..........

Fr. ovatus . . . . . . . . . . 169

Fr. ovoideus .......................

Discus subconicus ; fol. acuta - Rhône, Alix (Gdgr).4014. $168\left\{\begin{array}{l}\text { Discus planus ; fol. subobtusa - Harz, Kunstein (Vocke). } \\ \text { Ders }\end{array}\right.$ .4015. P. Vockir Gdgr mss 
169 Rami flor. inermes; styli hirsuti.

Rami flor. aculeati; styli glahrescentes - Ain, Trevoux (Fray). . . 401fi. P. cemtostaga Grler mas

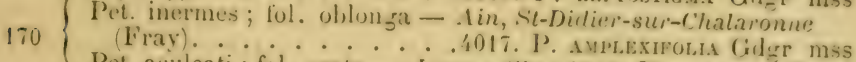
Pet. aculeati ; fol. ovata - Lyon; Cher.úls. P. FLocciba (Dés.)

171 Fol. basi attenuata, obovato-oblonga vel oblonga. . . . 172 Fol basi rotundata ovata vel obovata. . . . . . . . . . Styli hirsuti; rami flor. paree hirsuti - Iielv., Bale Cottet: $172\left\{\begin{array}{l}\text { Styli glabri ; rami flor. tomentosi - IIte-Savoie, Argonnex } \\ \text { (Puget). }\end{array}\right.$

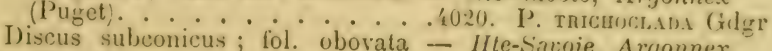

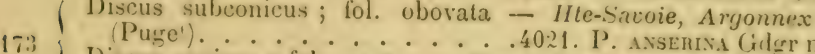
Discus conicus ; fol. ovata - Helv, Onex, etc. (Rapin; $P$.
fimbriata Gremli). fimbriata Gremli) ${ }^{\prime}$ tyli glabri vel paulo hirteli. . .4022. P. GENEvensis (Puget)

174 Styli glabri vel paulo hirtelli. ......... . . . . . . .

Styli hirsuti vel lanati .................... 179

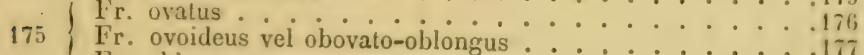

Fr, oblongus ol obovalo-oblongus . . . . . . 17

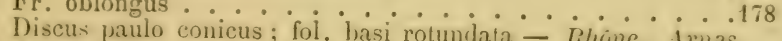
(Glione, Arnas 176 Discus planus; fol. basi attenuata - Jura, Pontarlier (Grenier)........ . . 4024. P. Abietina (Gren.) ....... Jura (Grenier). 4025.

Rami fior. aculeati, . . . . P. onopula (Gren.)

( Rami flor. aculeati, pet. omnes paulo aculeati - Rhóne.

177 Ramias (Gdgr herb. ros. n. 266). . 4026. P. Deccalion Gidgr Thames Dilton (Ges. sup. inermes - Anglia, Surrey

Thames Dilton (Groves). . . 4027. P. Londinensis Gdgr mss

Discus planus; pet. inermes - Helv., Fribourg (Puget).

178 Discus conicus; pet. aculeati; fol. oblonga - Angens Gdgr mss ford Aymestry (Ley). . . . 4029. P. Elovgata Gdgr msš

Discus conicus ; pet. aculeati ; fol. ovata - Gallia, etc. (Baker herb. ros. n. 11). . ...4029 bis. P. FoETIDA (Bast.)

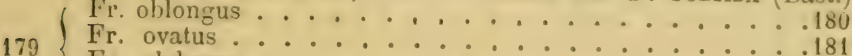

Fr. o. . . . . . . . . . . . . . . .

Fr. glolinsus ............. 184

Fr. ovoideus vel obovato-oblongus. . . 190

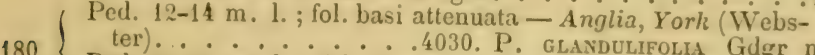
Ped. 20-2i m. i.; fol. basi roturdata Glandulifolia Gdgr mss (Magnier). . . . . Aisne, St-Quentin

Magnieri Gdgr mss

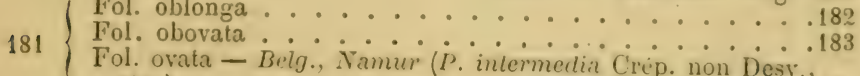

Fol. ovata - Belg., Namur (P. intermedia Crep. non Desv.

etc.) - . . . . . 4032. P. Tuelexsir Gilgr

Fol. basi longe cuneata - IIelvetia, ete.4033. P. tomentoso-

182 Fol. basi subrotundata - Rhône, Tassin (C... SEPIUn (Christ) $\ldots \ldots \ldots$ psanMophyLla Gdgr mss 


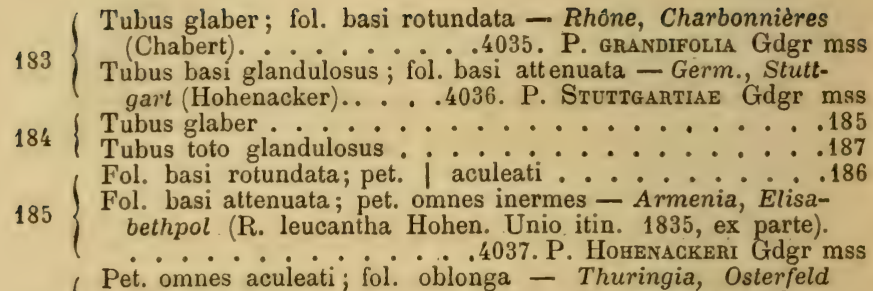

$186\{$ (Haussknecht). .....4038. P. condensata Gdgr mss

186 Pet. inermes; fol, elliptica - Aisne, St-Quentin (Magnier).

. . . . . . . . 4039. P. DePICTA Gdgr mss

Tubus dense glandulosus; fol lanceolata vel acuminata. . 188

$187\left\{\begin{array}{l}\text { Tubus vix glandulosus; fol. apice subacuta - Thuringia, } \\ \text { Weimar (Baenitz herb. europ. n. 1271):4040. P. AcINAcI- }\end{array}\right.$

. . . . . . FERA Gdgr mss

$188\left\{\begin{array}{l}\text { Ped. } 10-15 \mathrm{~m} \text {. l.; fol. magna } \text { Ped. 6-8 m.l.; fol. parva - Silesia, Bromberg (Koehler) } 4041 . \\ .189 .\end{array}\right.$

Corolla basi ciliata - Belg., Clausen (Ćrépin).4042. P. cus-

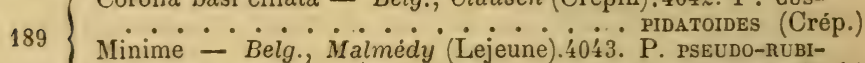

Minime - Belg., Malmédy (Lejeune).40ł3. P. pseudo-nubI-

Aculei conformes . . . . . . . . 191

$190\left\{\begin{array}{l}\text { Aculei ad ramos flor, alii inclinati alii glanduliferi }-D a n i a, \\ -\infty\end{array}\right.$ Helleboek Seeland (Scheutz). . .4044. P. DANicA Gdgr mss

Tubus glaber vel basi glandulosus. . . . . . . . . 192

191 Tubus toto glandulosus. $\ldots 193$

Tubus in 1\%, inf. glandulosus - Rhône, Chasselay (Gdgr herb. ros. n. 580). . . 4045. P. TRichosticta Gdgr mss

$192\left\{\begin{array}{l}\text { Rami flor. subaculeati ; tubus toto laevis - Suecia, Kalmar } \\ \text { (Lundquist) } . .4046 . \text { P. SAMBucifolia Gdgr mss }\end{array}\right.$ Rami flor, inermes; tubus basi glandulosus - Suecia, Eland Tveta (Scheutz). . . . 4047. P. FRaxinifolia Gdgr mss

193 | Rami flor inermes. . . . . . . . . . 194

Rami flor aculeati . . . . . . . 195

Pet. aculeati ; fol. basi attenuata - Suecia, Blek. Nattraby

$194\{$ (Lundquist)

Pet. inermes; fol. basi rotundata - Hanovre, Francfort-sur-

le-Mein (Kesselmeyer) . . . 4049. P. Asperifolia Gdgr mss

Pet. omnes aculeati. ....................

$195\left\{\begin{array}{l}\text { Pet. sup. inermes; fol. basi rotundata }- \text { Manche, Yvetot (Le- } \\ \text { bel) . }\end{array}\right.$

Pet. omnes inermes; fol basi attenuata - Suecia, Kalmar

(Lundquist) . . . . 4051. P. CRAssifolia Gdgr mss

$196\left\{\begin{array}{c}\text { Fol. late ovato-lanceolata - Europa orient., etc.4052. P. } \\ \text { (M. Bieb.) }\end{array}\right.$

Fol. mediocriter ovata - Tyrol, Hall, etc.4053. P. Capnoines 


\section{B. - Villosae Grdgr Essai p. 42 /Villoseae Auct. quoad sect. gen.)}

Rami rigidi compacti, apice haud arcuati; aculei saepissime graciles, rectiusculi vel subulati ; foliola utrinque molliter tomentosa (pube tamen breviore quam in Eutomentosis), subtus glandulosa vel non, biserrata; sepala post anthesin erecto-conniventia ac persistentia; styli plerumque lanati; flos sat parvus, intense roseus, odoratus; fructus maturus glaber vel setulosus, coccineus, saepe maximus, calyce vivido semper coronatus. - Frutices venusti, graciles, sat humiles, in Europa boreali vel australi (et tunc alpestres vel montani) hinc inde et non frequenter crescentes.

Fol. subtus eglandulosa vel tantum ad costam mediam glan-

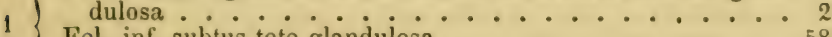
Fol. inf. subtus toto glandulosa . . . . . . . . . . 58 Fol. omnia subtus toto glandulosa. . . . . . . . . .98

Fr. sphaericus vel subglobosus, saepe aculeatissimus . . . . 3

2 Fr. ovatus vel subovoideus . . . . . . . . . 28 Fr. obovato-oblongus vel oblongus . . . . . . . . 44

3 Fol, simpliciter (vel inaeque) serrata. . . . . . . . . 4 4 I Fol. omnino biserrata ............. 7

(Ped. 6-10 m. 1.; rami flor glabri ........... 5

4 Ped. 16-17 m. I.; rami flor. hirsuti - Wurtemberg, Donnstetten (Kemmler) . . . . . . .4054. P. crinita Gidgr mss

(Pet. inermes; fol oblonga .............

5 Pet. aculeatissimi ; fol. obovato-oblonga - Valais, Louèche (Lorenti) . . . . . . . . 4055. P. conRrVaLIS Gdgr mss

Fol. amplissima ; rami flor. aculeati - Valais, Louèche (Lo-

6 renti) ........4056. P. cyclocepriala Gdgr mss Fol. mediocria; rami flor. inermes - Valais, Louéche (Lorenti). . . . . . . 4057. P. vaEvclifera Gidgr ms3

Stip. saepius angustatae, subtus glabrae vel pubentes nec

7 tomentosae ............... 8

Stip. I dilatatae, subtus semper tomentosae. . . . . . 18

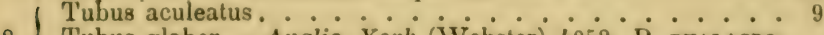

Tubus glaber - Anglia, York (Webster).4058. P. PHalacko............... c... carpa Gdgr mss 


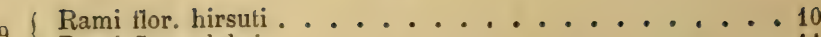

Rami flor. glabri ................. 11

(Fol. oblongo-attenuata - Bavaria, Schloss Zeil (Kugler).

... . . . . . 4059. P. BAvarica Gdgr mss Fol. obovato-obtusa - Finlandia, Abo Turku (Hollmen). . . . . . . . 4060. P. FEnNiGa Gdgr mss Fol. oblonga . . . . . . . . . . 12 Fol. obovato-oblonga, utrinque attenuata - Rossia, ad $\mathrm{fl}$. Wolchow (Meinshausen herb. fl. Ingricae n. 219).4061. P.

Foi obovata - Rossia bor ilniska PETERSBURgensis Gdgr ms non Wrede) . . . . . . . . 4062. P. IngRIGA Gdgr

Fol. elliptico-obovata, basi rotundata apice attenuata - HtesAlpes, La Grave (Billot exs. n. 3062 partim).4063. P. CINE-

. . . . . . . . . REIFOLIA Gdgr mss

12 Fol. basi rotundata ............. 13

Fol. basi attenuata. . . . . . . . . 14

Aculei grisei ; fol. apice subrotundata - Pyrenaei, Gèdre

13 (Bordère).......4064. P. Adoptata Gdgr mss Aculei flavi; fol. apice obtusa - Pyrenaei, Gèdre (Bordère).

........ 4065. P. Flavispina Gdgr mss

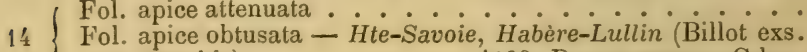

n. 3062 bis). . . . . 4066. P. oblongelLA Gdgr mss Corolla calycem superans .......... 16

15 Cor. calyce duplo brevior - Htes-Alpes, La Grave, etc.4067.

. . . . . . . . . P. MINUTA (Bor.)

Rami flor. aculeati ; fr. utrinque rotundatus . . . . . 17

16 Rami flor. subinermes; fr. utrinque breviter attenuatus Htes-Alpes, Le Lautaret. . . . 4068. P. oneites Gdgr mss Stip. subtus glandulosae ; sep. foliacea - Suecia, Mt Kinne-

17 kulle (Zetterstedt). . . . . 4069. P. Gothica Gdgr mss Minime - Caucasus. . . .4070. P. Ruprechti (Boiss.) Rami flor. glabri ........... 19

18 Rami flor. paulo hirsuti - Valais, Vercorin (Wolf). 4071. P. Pet. subinermes. . . . . 20 Pet. omnes aculeati . . . . . . . . . 22 Pet. omnes inermes. . . 4072. P. LoRENTII Gdgr mss Fol. basi rotundata . . . . . . . . 21

20 Fol. basi attenuata - Alpes Europae.4073. P. PoMrFenA Tubus hispidus; fol. apice obtusa - Anglia, York Helmsley

21 (Webster) . . . . 4074. P. Benthanrana Gdgr mss Tubus aculeatus; fol. apice attenuata - Anglia, York (Webster). . . . . 4075. P. EBoracensis Gdgr mss

22 Fol. apice rotundata vel obtusa . . . . . . . 23 Fol. apice attenuata . . . . . . . . 24 Tubus glandulosus; fol. elliptica basi rotundata - Valais, Loueche (Lorenti). . . . . 4076. P. cograta Gdgr mss

Tubus aculeatus; fol. ovato-rotundata basi rotundata - Podolia, etc. . . . . . 4077. P. ciliato-PETAla (Bess.) Tubus hispidus; fol. oblonga basi attenuata - Suecia media, Carlsko a (Reuterman). . . 4078. P. Reutermani Gdgr mss 
24 Fol basi attenuata. . . . . . . . . 25

Fol. basi rotundata. . . . . . . . . . 26

Ped. 6-8 m. l.; fol. ovato-elliptica - Scantinavia.4079. P.

25 . . . . . . . Mollissima (Fries)

Ped. 4-5 m. I.; fol. ohlonga - Italia, Mt Cenis, Lans-le-

Bourg (Morthier) . . . . . 4080. P. AlpiNa Gilgr mss

Fol. ovata - Pelem., Suse (Balbis).4081. P'. Penemontaxi

Scp. dorso aculeata; tulus inaeciue aculeatus - d . Gdgr mss

La Grave (Billot exs. n. 3602 ex parte).4082. P. subalpes-

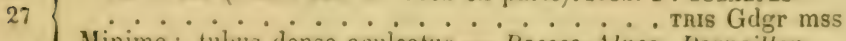

Minime; tulus dense aculeatus - Basses-Alpes, Parpaillon (Proal). . . . . . 4083. P. ulmordes Gdgr mss

28 ; Fr. subovoideus . . . . . . . . . 29

28 / Fr ovatus. . . . . . . . . . . . 32

? (Fol. biserrata. . . . . . . . . 30

Fol. simpliciter serrata - Asia Minor, Laristan (Balansa, pl. exs. 11. 314) . . . . . .4084. P. Bolssieri (Ćrép.)

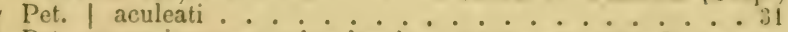

Pet. omnes inermes; tubus laevis - Valais, etc. 4085 . $\mathrm{P}$. LEN-

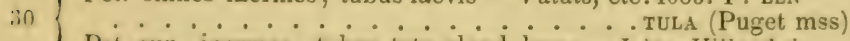

Pet. sup. inermes; tubus toto glandulosus - Isère, Villard de Lans (Ravaux) . . . . . . 4086. P. RavauxiI Gdgr mss

Rami flor. inermes; fol. ample oblonga - Anglia, Bre-

31 conshire Llanthony (Ley) . . 4087. P. CAMbrica Gdgr mss Rami flor. aculeati ; fol. parva elliptica - Isère, Les Coulmes (Gdgr) . . . . . . 4088. P. oligacastha Gdgr mss

(Fol. omnia simpliciter serrata. . . . . . . . 33

32 Fol. omnia biserrata. . . . . . . . . 34

Fol. inf. bi, sup. uniserrata - Scotia, Aberdeen Balmoral (Ley) . . . . . . . 4089. P. Lanata Gdgr mss Aculei adunci ; rami rigidi - Persia (Bunge pl. pers. exs. n. 7). . . . . 4090. P. albicans (Godet) Aculei arcuati ; rami flexuosi - Asia, Kurdistan (IIaussknecht). . . . . . 4091. P. rlexicaulis (Godet)

34 ( Pet. omnes tomentosi............ . . 35 Pet. sup. sparse pilosi - IIibernia, Antrim Larne (Stewart). . . . . . . . . 4092. P. Basaltica Gdgr mss

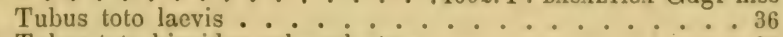
Tubus toto hispidus vel aculeatus . . . 37

Tubus basi glandulosus; fol. inferne attenuata - Italia, Pistoie Boscolungo (Gibelli) . . . 4093. P. Italica Grlgr mss

Tubus apice aculeatus; fol. basi rotundata - Italia, Pisloie Boscolungo (Levier). . . . .4094. P. PIstoniessis Gdgr mss

Fol. obovato-oblonga utrinque attenuata - Pyrenaei, Gedre (Bordère). . . . . . 4095. P. IodAcantina Gdgr mss

Fol. obovata ; basi attenuata apice obtusa-Suecia, Mt Kinnekulle (Zetlerstedt) . . . . .4096. P. nwmakiss Gilgr mss

Fol. oblonga, utrinque rotundala - Basses-Alpes, Condamine (Proal) . . . . . 4097. P. Hypoleuca Gdgr mss 
Pet. omnes aculeati. . . . 38

Pet. subinermes; ped. $12-14$ m. 1. - Hibernia, Antrim Cramlin (Stewart) . . . . 4098. P. Stewartil Gdgr mss

Pet. sup. inermes; ped. 10-11 m. 1. - Tyrol, Rovereto (Gelmi). . . . . . 4099. P. ventricosa Gdgr mss

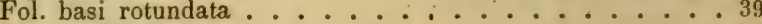

Fol. basi attenuata . . . . . . . . . . 41

Tubus hispidus; fol. apice attenuata . . . . . . 440

39 Tubus aculeatus; fol. apice rotundata - Pyrenaei, Gédre (Bordère) . . . . . 4100. P. Rhipidoacantha Gdgr mss

Fr. apice strangulatus; fol. obovato-oblonga - Anglia, Cheshire Little Neston (Harbord L.).4101. P. INSULARIS

Fr. apice rotundatus; fol. oblonga - Suecia, Stockholm

(Lofgren). . . . . . . . .4102. P. HolMIENSIS Gdgr mss

Fol. ovata vel obovato-oblonga . . . . . . . 42

Fol. oblonga . . . . . . . . . 43

Fol. obovato-oblonga; tubus aculeatus - Anglia, York

(Webster). . . . . .4103. P. BREvion Gdgr mss Fol. ovata; tubus hispidus - Podolia, etc.4104. P. ANDR-

Ped. 4-6 m. l.; fol. angustata - Delph., etc. (P. Perusiana Timb.; Dés. herb. ros. n. 38 et 38 bis).4105. P. Grenieri

Ped $8 \ldots \ldots \ldots$. . . (Dés. ed. 8-10 m. l.; fol. ampla - Italia, Apenn. Modène l'Abetone (Gibelli). . . . . .4106. P. GibeLLII Gdgr mss

44 Rami flor. glabri ; styli semper lanati. . . . . . 45

Rami flor. I hirsuti ; styli saepius glabri . . . . . . . 56

Fol. omnino biserrata ............ 46

Fol. omnino simpliciter serrata; pet. inermes - Italia, Mt

45 Cenis, Lans-le-Bourg (Morthier) . .4107. P. CENISIA Gdgr mss Fol. sup. simpliciter serrata; pet. aculeati - Anglia, York (Webster). . . . . . 4108. P. SARCocarpa Gdgr msS

\{ Ped. hispidi vel aculeati .......... 47 Ped. laeves - Anglia, York (Webster).4109. P. BAKERIOIDES

Tubus toto hispidus

Tubus glaber ; pet. sup. inermes - Anglia, Monmouth, Llanthony (Ley). . . . . . . : .4110. P. obesa Gdgr mss Tubus glaber; pet. omnes aculeati - Anglia, York (Webster). . . . 4111. P. Murrayana Gdgr mss

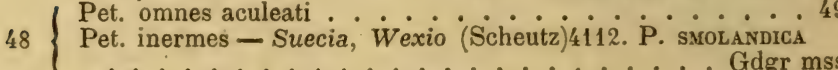

Fr. ovoideus vel obovato-oblongus. . . . . . . 50 Fr. suboblongus; rami flor. aculeati - Isère, villard de

49 Lans (Boullu) . . . . . 4113. P. colligena Gdgr mss Fr. oblongus; rami flor. inermes - Hungaria.4114. P. BAL-

....................... (Willd.)

$50\{$ Fol. basi attenuata. ........... 51

50 Fol basi rotundata. $\ldots 53$

51 Fol. oblonga; fr. ovoideus . . . . 52 51 Fol. et fr. obovato-oblonga - Pyrenaei, Gèdre (Bordère) 4115 . 
Styli lanati ; fr. utrinque depressus - Basses-Alpes, Condamine (Proal) . . . . . .4116. P. TEnuifolia Gidgr mss Styli sparse hirsuti ; fr. utrinque attenuatus - Pyrenaei, Gèdre (Bordère) . . . . 4117. P. DEalbata Gdgr mss

53 Fol. apice obtusata vel rotundata . . . . . . . 54

Fol. apice I attenuata................. 55 Aculei albi ; fr. basi rotundatus - Pyrenaei, Gedre (Bordère) 54 Aculei grisei; fr. basi attenuatus - Isère, Villard de Lans (Soc. dauph. exs. n. 113). . 4119. P. Delpurnensis Gdgr mss Ped. 11-13 m. 1.; sep. apice dentata - Suecia, Scania Fogel-

55 sang (Lundquist). . . . . 4120. P. scanica Gdgr mss Ped. 7-10 m. l.; sep. apice integra - Anglia, Thirsk (Baker) . . . . 4121. P. Bakert Gdgr mss

Fr. elongatus; fol acuta. ......... 57 Fr. ovoideus; fol. obtusa - Suecia, Mt-Kinnekulle (Zetterstedt) . . . . . . 4122. P. Flexuosa Gdgr mss Rami flor. aculeati ; tubus hispidus - Pyrenaei, Gèdre (Bor-

57 dère)......4123. P. odontostephana Gdgr mss Rami flor. inermes; tubus laevis - Pyrenaei centr., Bué (Bordère) . . . . . . 4124. P. LEIOGYNA Gdgr mss

58 Fr. globosus vel rotundatus . . . . . 59 Fr. ovatus, ovoideus, obovato-oblongus vel oblongus . $\cdots 75$

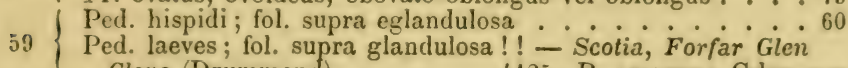
Clova (Drummond). . . . 4125. P. LeIAstria Gdgr mss

$60\left\{\begin{array}{l}\text { Rami flor. glabri - } \text { Rami flor. villosi - Alsatia, Haguenau (Reliq. Maill. n. 1084 }\end{array}\right.$ Rami flor. villosi - Alsatia, Haguenau (Reliq. Maill. n, 1084)

$61\left\{\begin{array}{l}\text { Tubus toto laevissimus. } \\ \text { Tubus basi laevis apice glandulosus. } \\ \text { Tubus toto }\end{array}\right.$

61 Tubus toto et dense glandulosus vel hispidus $:-64$ Tubus toto et sparse glandulosus - Savoie, Pringy (Billot exs. n. 3600). . . . . . 4127. P. omissa (Dés.)

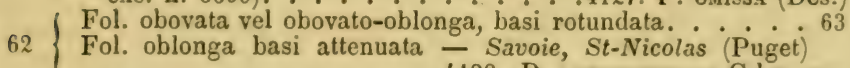

62 Fol. Stip. inf. tantum subtus glandulosae; fol. attenuata - Suecia, Mt-Kinnekulle Westerplana (Zetterstedt). 4129. P. cossoStip. omnes subtus glandulosae; fol. obtusa - Brisa Gdgr mss trog. Hallebergs (Zetterstedt) . .4130. P. Leiocarpa Gdgr mss

$64\left\{\begin{array}{l}\text { Pet. omnes aculeati; fol. obovata - Suecia, Mt-Kinnekulle } \\ \text { Windsater (Zetterstedt). - . P131. P. suEcrca Gdgr mss } \\ \text { Pet. sup. inermes; fol. oblonga-Pedem., Vallées Vaudoises }\end{array}\right.$ Pet. sup. inermes; fol. oblonga - Pedem., Vallées Vaudoises

(Rostan). . . . . . . 4132. P. Allionir Gdgr mss

65 Pet. sup. inermes . . . . . . . . 66

Pet. omnes aculeati . . . . . . . . . . . . . 67

$66\left\{\begin{array}{l}\text { Fol. mediocria; ped. 9-11 m. 1. - Pedem., Vallees Vaudoises } \\ \text { (Rostan). } \text { Fol. amplissima; ped. 11-14 m. 1. - Tyrol, m. Vasone Gel. }\end{array}\right.$ Fol. amplissima ; ped. 11-14 m. 1. - Tyrol, m. Vasone (Gel. mi) .......4134. P. DICtAMNoIdes Gdgr mss

67 Rami flor inermes ............ 68

\{ Rami flor aculeati ............... 69 

. . . . . . . 4136. P. sudetica Gdgr mss

69 Fol. basi attenuata. ............ 70

Fol. basi rotundata . . . . . . . . . 72

Fol. obovato-oblonga . . . . . . . . . 71

Fol. obovata; ped. 9-10 m. 1. - Suecia, Kinnekulle Wester-

70 plana (Zetterstedt) ....4137. P. Microcarpa Gdgr mss

Fol. oblonga; ped. 6-7 m. 1. - Valais, Bourg St-Pierre

(Guinet). . . . . . 4138. P. vallesiaca Gidgr mss

Ped. 6-8 m. 1.; fr. mediocris - Isère, La Salette (Puget)

71 . . . . . 4139. P. Amrcta Gdgr mss

Ped. 11-13 m. 1.; fr. major - Suecia, Kinnekulle Blomberg (Zetterstedt)....... . 4140. P. similis Gdgr mss

(Fr. globosus . . . . . . . . 73

i2 Fr. ovato-rotundatus - Suecia, Mt-Kinnekulle Osterplana (Zetterstedt). . . . . 4141. P. ZetterstedtI Gdgr mss

Fol. obovata . . . . . . . . . . . 74

Fol. ovato-elliptica; ped. $4-6$ m. 1 - Pedem., Mt-Cenis Lans-

73 le-Bourg (Mathonnet). . . 4142. P. echixophora Gdgr mss

Fol. obovato-oblonga; ped. 7-8 m. 1. - Suecia, Vestrog. Sattra (Lundquist). . . . . 4143. P. ELEgans Gdgr mss Stip. dorso tantum tomentoso-glandulosae - Pyren. Or.,

i4 Bach Zisern (Vayreda) - . .4144. P. Xatarti Gdgr mss Stip. subtus toto hirto-glandulosae - Suecia, Kinnekulle Morkeklefv (Zetterstedt). . ... 4145: P. Jucunda Gdgr mss

75 \{r. saepius laevissimus raro adsperse hispidus. . . . . 76

75 Fr. semper dense hispido-aculeatissimus. . . . 81

I Fol. undique tomentosa; pet. omnes aculeati. . . . . . 77

if Fol. supra glabra; pet. sup. inermes - Pedem., Vallées Vaudoises (Rostan). . . . 4146. P. Discolon Gdgr mss

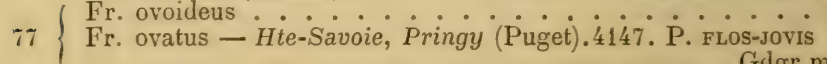

................. Gdgr mss

i8 Fol obovata ........................ 79

Fol. oblonga . . . . . . . . . . . 80

Sep. apice angustata; ped. 3-6 m. 1. - Hte-Savoie, St-Martin

79 (Puget) . . . . . . . 4148. P. PANvuceA Gdgr mss Sep. apice foliacea; ped. 7-9 m. 1. - Suecia, Mt-Kinnekulle (Lundquist). . . . . 4149. P. Lundeuistir Gdgr mss

( Ped. 3-4 m. l.; fr. conicus basi rotundatus - Valais, Vercorin $80\left\{\begin{array}{l}\text { (Favrat). } \text { Ped. 8-9 m. 1.; fr. basi decurrens - Isère, St-Christophe } \\ \text { - } 150 \text {. P. TenuisPna Gdgr mss }\end{array}\right.$ (Soc. dauph. exs. n. 114) . . .4151. P. PARvifolia Gdgr mss

81 (Fr. oblongus . . . . . . . . . . 82

81 Fr, ovatus . . . . . . . . 85

Fr. ovoideus vel obovato-oblongus . . . . . . . 89

82 Rami flor inermes; fol. oblonga ........ . . 83

Pami flor. aculeati; fol. obovato-oblonga - Dania, Helleboek Seeland (Lundquist). . . .4152. P. crassinervia Gdgr mss Pet. omnes inermes; fr. basi attenuatus . . . . . . 84

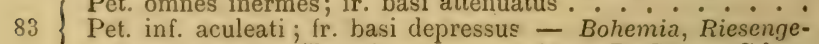
birge Schatzlar (Patze) . . . .4153. P. Patzll Gdgr mss 
Aculei subadunci; ped. 12-15 m. 1. - Silesia, Waldenburg (Firle) . . . . . 4154, P. ozavonioldes Gdgr mss Aculei recti ; ped. $16-20$ m. 1. - Silesia, Goerbersdorf $(P$. vestita f. Straehleri Uechtr. in Verh. Bot. Ver. Brand. 1875) . . . . . . \$155. P. Stnaeuleri Gdgr mss

(Rami flor glabri ; pet aculeati. . . . . . . 86

8. Rami flor. hirsuti; pet. inermes - Suecia, Kinnekulle $\dot{R}^{\circ}$ backstorp (Zettetstedt) . . . . 415f, P. ovita Gder mss

Fol. basi attenuata ....... 87

Fol. basi rotundata . . . . . . 88

Ped. 8-10 m. 1.; fol basi attenuata - Sabaudia, etc. $\{157$

87 . . . . . P. nesinosordes (Ćrép. mss)

Ped. 10-15 m. i.; fol. basi rotundata - Itte-Savoie, HaberePoche (Billot exs. n. 3601).4158. P. semi-incrassata Gdgr mss

Fol. obovata utrinque rotundata - Ite-Savoie, Habère-Poche (Puget) . . . . . . . 4159. P. CAdvca Gdgr mss Fol. oblonga utrinque attenuata - Suecia, Scania Pinelierna (Tullberg). . . . . . 4160. P. Didxмaмтия Gdgr mss

Aculei conformes . . . . . . . . . 90 Aculei dimorphi - Valais, etc. . 4161. P. Gaudisi (Puget)

9 I Rami flor hirsuti . . . . . . . . . . . 91

Rami flor glabri . . . . . . . . 92

Pet. inermes; fol. utrinque attenuata - Valais, Vercorin

91 (Favrat). . . . . 4162. P. Alpestris Gdgr mss Pet. aculeati; fol. utrinque rotundata - Suecia, Scania Nobbelof (Coster). . . . . \$163. P. macropiyla Gider mss

Pet. inermes ............... . . 93

Pet. | aculeati . . . . . . . . 94

Rami flor. inermes; fol. basi rotundata - Scotia, Perth Scone

93 (Drummond) . . 4 . 164. P. Lightfootiana Gdgr miss Rami flor. aculeati; fol. basi attenuala - Tyrol, Regnana (Gelmi). . . . . 4165. P. Ambrosiaxa Gdgr mss

94 Fol. basi attenuata ................ 95

Fol basi rotundata $: \cdots 9$.

Ped. fructifer carnosus, $6-10$ m. 1. - Ilelv., Fribourg, Mont-

95 bovon (Cottet). . . . 4166. P. Inflatipes Gdgr mss Ped. fructifer haud carnosus, 12-14 m. 1. - Suecia, Vestrog. Mariestad (Lundquist). . . .4167. P. BonEalis Gdgr mss

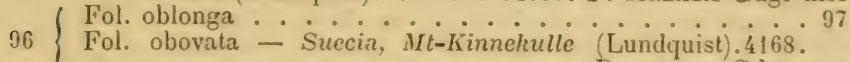

• . . . . . . . . Candida Gdgr mss

(Fr. basi depressus; fol. obtusa - Helv., Zurich Albishamm

97 (Lehmann) . . . . 4169. P. Turicensis Gdgr mss Fr. basi attenuatus; fol. acuta - suecia, simol. Moheda (Hyltén). . . . . . 4170. P. Axdensovisa Gdgr mss

98 Fr. rotundatus . . . . . . . . . . 99

98 Fr. sphaericus . . . . . . . 102 Fr. ovatus, ovoideus vel oblongus. . . . 120

99 Tubus toto hispidus . 100 Tubus laevissimus - Cantal, Le Lioran (F. Héribaud). 4171 . . . . . . . . . P. Delanbnsava Gdgr mss $100\left\{\begin{array}{l}\text { Rami flor. et pet. aculeati - Rami flor. et pet. incrmes - Suecia, Smol. Carlskrona } 101 \\ \text { Ro }\end{array}\right.$ (Scheutz).........4172, P. spLsescens (Christ) 
Tubus dense hispidus; fol. obtuse obovata - Suecia, Kinnekulle Westerplana (Zetterstedt). 4173. P. Kinnekullensis

Tubus sparse aculeatus; fol acute obovato-oblonga - Gdgr m-

tal, Le Plomb (F. Héribaud). 4174. P. ARvernensis Gdgr mss

Rami flor. I hirsuti ..................

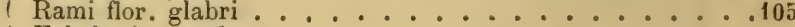

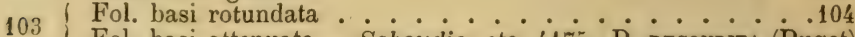
Fol. basi attenuata - Sabaudia, etc. 4175. P. RECondita (Puget) Ped. 10-12 m. l.; pet. inermes - Valais, Münster (Favrat)

Ped. $7-8$ m. l.; pet. inermes - Borussia, Dantzig (Baenitz herb. europ. n. 2236). . . . 4177. P. Baenitzir Gdgr mss Ped. 8 m. l.; pet. aculeati - Suecia, Smol. Moheda (Hyltén) . . . . . . 4178. P. PAllidifolia Gdgr mss Tubus laevissimus. . . . . . . . . . 106

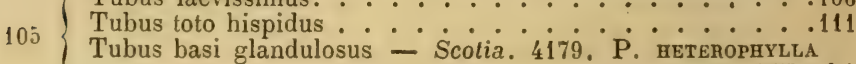

$106\left\{\begin{array}{l}\text { Pet. aculeati } \\ \text { Pet. inermes - Anglia, York (Webster) } 4180^{\circ} \text { P. LEPTo- }\end{array}\right.$ .................. pHYLLA Gdgr msS

107 Rami flor inermes . . . . . . . . 108

Rami flor aculeati. . . . . . . . . . 109 Fr. basi depressus; fol. oblonga basi rotundata - Silesia,

108 Carlowitz (Ansorge). - 4181. P. vratislaviensis Gdgr mss Fr. basi rotundatus; fol. elliptica basi attenuata - Tyrol, Ritten (Kerner) . . . . . 4182. P. Australis (Kern.)

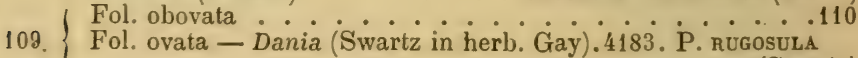

Aculei falcati; fol. obtusa - Hte-Savoie, St-Martin (Puget) $110\left\{\begin{array}{l}\text { Aculei recti; fol. acuta - Suecia, Kinnekulle Westerplana } \\ \text { - Sur }\end{array}\right.$ (Zetterstedt) . . . . .4185. P. GLABRicalyx Gdgr msS Pet. omnes aculeati .................. Pet. omnes inermes; fol. utrinque attenuata - Suecia, Scania Esperod (Tullberg). . . . .4186. P. CladopHora Gdgr mss

111 Pet. sup. inermes; fol. utrinque attenuata - Valais, Mayens de Sion (Wolf). . . . . 4187. P. SPINULEscens Gdgr mss Pet. sup. inermes; fol. basi rotundata apice attenuata - Tyrol, m. Vasone (Gelmi). . . . . 4188. P. GeLMII Gdgr mss

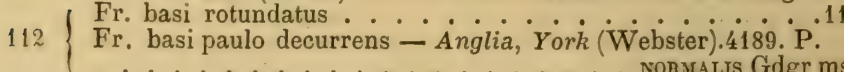

113 Fol basi i attenuata . . . Nonmalis Gdgr mss Fol. basi rotundata $\ldots 114$ Fol. ovata - Pyrenaei (Gay). $\because 4190$. P. GAYANA (Wallr.)

114 Fol. obovato-oblonga - Helv., Fribourg (Lagger).4191. P. 14 Fol. oblonga - Gallia, Annecy (Bouvier).4192. P. Clusiana

115 Fol. apice obtusa vel rotundata. $\therefore \ldots$ Bouvier)

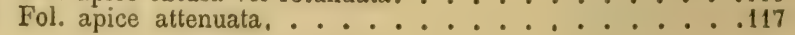


(Tubus aculeatus; fol. obovato-elliptica - Loire, Mt-Pilat (Gdgr herb. ros. n. 390). . .4193. P. THURIfera Gdgr mss Tubus hispidus; fol. obovata - Suecia, Hallebergs Storgarden (Zetterstedt).. . . . . 4194. P. RIGescriss Gidir mss Fol. elliptica ..................... 118

117 Fol. oblonga ...................... . . . . . . Fol. obovato-oblonga - Helv., Fribourg (Lagger).4195. P'.

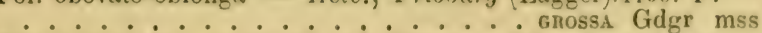
Tubus aculeatus - Styria, m. Buchberg.4196. P. nesrivosa $118\{$ Tubus hispidus - Tyrol, Krems (Kerner). G197. P. Cnemser-

119 (Billot exs. n. 3602 partim).4198. P. osmonendnon Gdgr mss Ped. 6-7 m. 1.; fol. 20-25 m. 1. - Suecia, Smol. Dref (Hyltén). . . . . . . 4199. P. scaninavica Gdgr mss

120 Fr. laevissimus . . . . . . . . . 121

f Fr. hispidus vel aculeatus. . . . . . . . 126

Fr. ovoideus . . . . . . . . 122 Fr. oblongus - Suecia, Eiand Borgholm (Scheutz) $4200 . \mathrm{P}$. Fr. ovato-ellipticus - Italia, Modène (Gibelli).4:01. P. P'An-

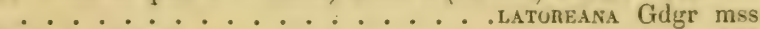

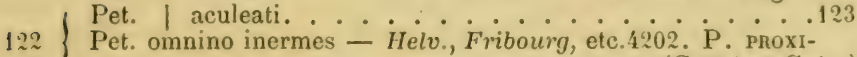
Pet. omnino inermes - Helv., Fribourg, etc.4202. P. proxi-

( . . . . . . . . . . Cottet et Crép.)

123 | Fol. basi attenuata ............. . 124

Fol basi rotundata. . . . . . . . 125

Ped. 7-9 m. l. ; fr. basi attenuatus - Dania, Kioge Seeland

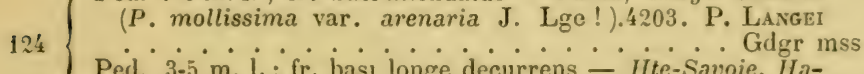

Ped. $3-5$ m. i. ; fr. bası longe decurrens - Itte-Savoie, Habère-Lullin (Puget). . . . 4204. P. Decunness Gilgr mss

$125\left\{\begin{array}{l}\text { Fr. apice longissime attenuatus; fol. petiolulata emarginata } \\ \text { - Valais, Biel (Favrat) .4205. P. nurwchocanpa Gdgr mis }\end{array}\right.$ Fr. apice strangulatus; fol, sessilia basi rotundata - Tyrol, m. Vasone (Gelmi). . . . 4206. P. tunbixata Gdgr mss Rami flor. tantum hirsuti. . . . . . . . . . 127 $126\left\{\begin{array}{l}\text { Rami flor glabri } \\ \text { Rami flor. villoso-glardulosi - Liban, Les Cédres (Kintschy) }\end{array}\right.$

. . . . . . . 4207. P. libanotica (Boiss.)

Fr. ovoideus vel obovato-oblongus . . . . . 128

Fr. oblongus - Helv., Fribourg Montbovon (Conttet). 4208.

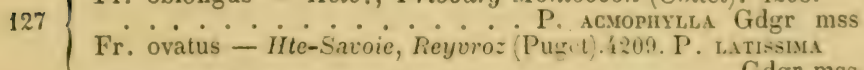

. . . . . . . . Gdgr mss

$128\left\{\begin{array}{l}\text { Pet. aculeati; fol. oblonga. } \\ \text { Pet. inermes; fol. ovato-elliptica - IIelv.. Iri iospenn }\end{array}\right.$ (Gisler). . . . . 4210. 1'. resisiftera Gider mss Rami flor. aculeati; fol. et fr. basi attenuata . . . . .130

129 Rami flor. inermes; fol. et fr. hasi rotundata - Ifelv., Fribourg Charmey (Thomas). . 4211. P. clanotricha Gdgr mss 
( Petala glabra ; pet. et tubus longe aculeati - Belg., Namur

$130\{$ (Crépin).......4212. P. Echinocalyx Gdgr mss Petala basi hirsuta ; pet. et tubus minute glandulosi - Silesia, Carlowitz (Ansorge). . . .4213. P. Krauseana Gdgr mss

131 Fr. oblongus . . . . . . . . . . . . . . . . . . . . . . .

Fr. ovatus . . . . . . . . . . . 134

Fr. ovoideus vel obovato-oblongus . . . . . . . . 137

Rami flor, aculeati ; fol. basi attenuata. . . . . . . .133

132 Rami flor. inermes; fol. basi rotundata - Silesia, Goerbersdorf (P. spinulifolia f. Uechtritziana Straehler in litt.!).4214. .............. P. JUGULARIS Gdgr mss Fol. ovata - Hibernia. . . 4215. P. Dicksonı (Lindl.)

133 Fol. oblonga - Anglia media et mer.4216. P. scabriuscula .......................... (Winch.)

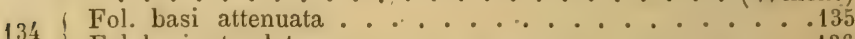

Fol. basi rotundata. . . . . . . . 136

Pet. aculeati; fr. apice valde attenuatus - Helv., Fribourg

135 la Gotallaz (Puget). . . . . 4217. P. scabida (Puget mss)

Pet. inermes; fr. apice parum attenuatus - Hanovre, Lüddenhagen (Zabel). . . . .4218. P. ZabeLII (Crép. mss)

Fr. basi rotundatus; fol. oblongo-acuta - Pyrenaei Or.,

136 Mt-Louis (Debeaux). - . 4219. P. MEspiliformis (Debeaux)

Fr. basi decurrens; fol. obtuse obovato-oblonga - Suecia,

Scania Albrunna (Scheutz). . . 4220. P. oelandica Gdgr mss

( Pet. omnes tomentosi; fol. apice | acuta vel rotundata. .138

137 Pet. sup. sparse hirsuti ; fol. longe acuminata - Anglia, York Strensal (Webster). . . 4221. P. JONIDEs Gdgr mss

138 ) Pet. inermes . . . . . . . . . 139

Pet. aculeati ................. . . 140

Fol. ovata basi attenuata - Suecia, etc. $(P$. scanica et $P$.

139 Scheutzii Gdgr olim) ....4222. P. SCANorum Gdgr mss Fol. obovato-oblonga basi rotundatá - Helv., Fribourg

Mühlibach (Lagger). . .4223. P. echinostephana Gdgr mss

140 | Fol. apice obtusata ......................

Fol. apice | attenuata . . . . . . . . . . 143

Fol. basi rotundata . . . . . . . . . . 142

141 Fol. basi attenuata - Suecia, Vestrog. Sattra (Lundquist).

. . . . . . . 4224. P. opobalsanum Gdgr mss

Ped. 9-11 m. l.; fr. utrinque longe attenuatus - Suecia

$142\{$ centr., Carlskoga (Reuterman).4225. P. setuliflora Gdgr mss Ped. 16-17 m. 1.; fr. utrinque depressus - Thuringia, Rudolstadt (Dufft). . . . 4226. P. Germaxica Gdgr mss Fol. obovata basi rotundata .................

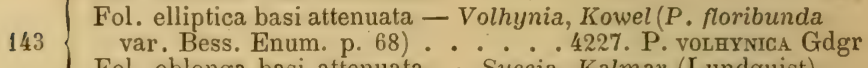
Fol. oblonga basi attenuata - Suecia, Kalmar (Lundquist).

. . . . . . 4228. P. PYRIFERA Gdgr mss Ped. $6-8$ m. i.; fr. utrinque depressus - Cantal, Le Plomb (F. Héribaud). . . . . . 4229. P. Leconuil Gdgr mss

144 Ped. 10-12 m. 1.; fr. utrinque valde attenuatus - Anglia, Carnarvonsh. Llamberis (Harbord L.).4230. P. oxronon ..................... Gdgr mss 
C. - Phalacroidae Gdgr Essai p. 43 (Villosene Auct. ex parte; Sabineae Crép. Prim., ex parte; Elymaiticue Boiss. fl. Orient. II, p. 669, ex parte)

Rami erecti, rigidi apice hatud arcuati; aculei recti subulati vel setacei, conformes, sat rari ; toliola argute biserrata, subtus (saltem inferiora) ad nervos semper glandulosia, utrincque glaberrima, vel supra parcissime hirtella, aut subtus plus minus villosa, nee tomentosa; pedunculı saepius hispidi, sepala glandulosa, plus minusve pinnatipartita, post anthesim erecto-conniventia et persistentia; styli lanati; flos ruber vel intense roseus; fructus coriaceus, calyce vivido semper coronatus. - Frutices alpestres, imprimis helvetici et orientales.

Grex ille parvulus praesertim a Villosis recedit foliolis utrinque haud semper tomentosis, aculeis (ut in Ozanoniis - Alpince) interdum eranidis, facie altera, ete. Sectio pulcherrimia atque valde singularis ab omnibus cognitis longe recelens, cum formas primo intuitu fere hybridas ideoque curiosissimas exhibeat; etenim, notae listinctivae quae tribui solent Rosae alpinae, montance, pimpinellifoliae, psilophyllae. tomentellae, pomiferce, ete., in Phalacroidis evidenter inveniuntur atque manifestantur! sed, certo certius,'eas hybridas esse non antumno (quidquid dicat $\mathrm{cl}$. Dr. Christ, de hybriditate nimis amator', cum, ex copia speciminorum ditissima, fructus maturos fertiles semper vidi. 
Fol. undique glandulosa. ............. 3

Fol. subtus tantum glandulosa . . . . . . 6

Aculei dimorphi et glanduliferi. .

Aculei conformes - Iberia, Krzchnival (Steven). 4231 , P. IBE-

4

Fr. globosus hispidus - Thessalia, etc. $4232 . \dot{\mathrm{P}}$. HELDrEIcHI

Fr. ovoideus glaber - Italia; Sicilia, etc.4233. Poiss. et Reut.)

(Acule plerumque falcati; fol. lata - Tauria, etc. ${ }^{2} 34$ (Bert.)

............ pulverulenta (M. Bieb.)

Aculei plerumque recti ; fol. parva - Caucasus, Kasbeck ( $P$. pulverulenta f. microphylla C. A. Meyer enum. plant. Kolen. p. 46). . . . . 4235. P. Kolenatiana Gdgr mss

Ped. laeves . . . . . . . . . . . . 7

6 Ped. glandulosi ................. 8

Fr. globosus; fol. elliptico-obtusa - Bohemia, Prague m. Zizkow (P. mollis Presl fl. cech. p. 102, non Led.).4236.

- P. CECHICA Gdgr

Fr. suboblongus; fol. oblongo-attenuata - Valais, etc. $(P$. pomifero-cinnamomea Christ).4237. P. GoMbensis (Lagger

Fr. globosus . . .

Fr, oblongus ........................ 11

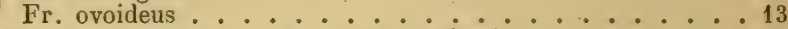

S Tubus glandulosus; pet. omnes aculeati. . . . . . . 10

Tubus laevis; pet. inf. inermes - Pyrenaei centr., Héas

(Bordère). . . . . . 4238. P. platystema Gdgr mss

Aculeata ; fol. attenuata - Belg., Luxembourg, ets.4239.P.

- * * * ARduenensis (Crép.)

Inermis; fol obtusa - Delph., Huez, etc. (P. pomifero-alpina Christ) . . . . . 4240. P. AlPICOLA (Rouy)

Pet. aculeati f fol obovato-oblonga . . . . . 12

11 Pet inermes; fol oblonga - Valais, Les Combes (Puget).

. . . . . . 4241. P. galbanifera Gdgr mss

Fol. basi rotundata; pet. hirsuti - Hte-Savoie, Mt-Salève

$12 \mathrm{~F}$ (Sandoz) .......... . . P242: Palaevensis Gdgr mss Fol. basi attenuata; pet. sparse hirsuti - Ht-Savoie, Mt-Salève, etc. (P. Semproniana (Favrat).4243. P. Berneti(Schmi............... dely mss)

Rami flor. glabri, aculeati ............ 14

13 Rami flor. villosi, inermes - Hte-Savoie, Mi Salève (Schmidely) . . . . . . . 4244. P. hiRtella Gdgr mss

14 Fol. obovata vel ovato-elliptica . . . . . . . 15

Fol. oblonga vel obovato-oblonga . . . . 16

Fr. utrinque subdepressus; fol. obovata - Helv., Uri (P. ste-

15 nosepala ('hrist) . . 4245. P. UnIENSIs (Lagger et Pug.) Fr. utrinque attenuatus; fol. ovato-elliptica - Helv. mer., Locarno (Franzoni). . . . .4246. P. HORRIDULA Gdgr mss Sep. apice angustata; fol. subtus toto hirsuta - Anglia, Yorksh. Scarbro (Webster).4247. P. Trachynella Gdgr mss Sep. apice foliacea; fol. subtus ad nervos hirsuta - Helv., Fribourg, Cases d'Allières (Cottet).4248. P. Cotreti 
17 Fr. ovoideus. . . . . . . . . . . 18

Fr, oblongus .................... 19

Rami flor. et pet. inermes; fol. obtusa - Valais, Conches, etc. (Lagger). . . . . . . 4249. P. resprixea (Puget mss)

Rami flor. et pet. aculeati ; fol. acuta - Belg., Malmédy ( $P$. spinulifolia var. Foxiana Red. Roses I, 94).4250. P. Foxiana

Pet. aculeati

Pet. aculeati. . . . . . . . . . . 20

19 Pet. inermes. . . . . . . . 22

$20\left\{\begin{array}{l}\text { Rami flor. aculeati ; fol. basi attenuata }- \text { Hede-Savoie, }_{\text {Mit }}{ }^{21} \\ \text { Rami flor. inermes; fol. basi rotundata }\end{array}\right.$ Salève (Guinet). . . . . .4251. P. AMpHonicanpa Gdgr mss

$21\left\{\begin{array}{l}\text { Tubus valde aculeatus; fol. oblonga }- \text { Helv., Neuchatel } \\ \text { Chaumont (Sire). } \\ \text { Tubus sparse hirsutus ; fol, obovato-elliptica - Echrose Gdgr }\end{array}\right.$

Tulus sparse hirsucus ; fol. obovato-elliptica - Isère, Prémol (Foudras) . . . . . . . 4253. P. Foudnasir Gdgr mss

Fol. rotundata vel obovato-oblonga ; rami flor. aculeati . . . 23

Fol. elliptica; rami flor. subinernes - Itte-Savoie, Brenthonne (Puget) . . . . . . 4254. P. piralacroidea Gdgr Fol. parva suborbiculata ; ped. 5-8 m. 1. - Persia austr. (IIaussknecht). . . . . . . . .4255. P. elyMartica (Boiss.)

Fol. ample obovato-oblonga; ped. 25-30 m. 1. - Helv. occid., etc. (P. glabrescens, multivaga, camberiacensis, propinqua et jurana Dés. Cat. n. 362, omnes pessime descriptae ideoque dubiae) . . . 4256. I'. spisclifolia (Dematra)

| Ped. hispidi.............. . 25 Ped laeves - Hte-Savoie, Habère-Poche(Puget).4257. P. GLO............. Bifera (Puget mss) Fr. subglobosus. . . . . . . . . . 26

Fr. ovoideus. . . . . . . . . . . 27

Fr. ovatus; pet. glabri - Helv., Selkingen, etc.4258. P.

Fr, oblongus; pet. hirsuti - Helv., Geneve Pommier (Thomas) . . . . . . . 4259. P. Lexana Gdgr mss

$26\left\{\begin{array}{l}\text { Pet. hirsuti ; fol. obovato-elliptica - Helv., Fribourg La } \\ \text { Comballaz (Cottet). . . . 4260. P. ougocepraLA Gdgr mss }\end{array}\right.$ Pet. glabri ; fol. ovata - Scand. austr.4261. P. commutata

Thus hispidus ............. (Scheutz)

27 Tubus laevis - Itte-Savoie, it Saléve (Ǵainet) $4262 .{ }^{\circ}$. dic-

$\ldots \ldots \ldots \ldots$ Tamifolia Gdgr ms6

Fol. basi rotundata apice attenuata . . . . . . 29

Fol. basi attenuata apice lanceolata; pet. subglahri - IIelv., Fribourg Montbovon (Cottet).4263. P. FRiBungeNsis (Lagger $\ldots \ldots \ldots \ldots$. . . . . . . . . Puget)

Ped. 9-10 m. 1.; sep. ample foliacea - Hte-Savoie, $M t$ Sa-

29 lève (Schmidely)...... .4264. P. Sabauda Gdgr mss Ped. 5-9 m. 1.; sep. parum dilatata - Helv., Fribourg Cases d'Allières (Cottet). . . . 4265. P. acasthochlamys Gugr mss 
Sulgenus XII. - Hulthemia Dumort. diss. 1824; Boiss. fl. Orient. II, p. 668 ; Lourea Lindl. Bot. Reg. tab. 1261 ; Rhodopsis Bge in Ledeb. fl. Alt. II, p. 224; ejus Ic. Ross. tab. 370; Rosa berberifolia Pall. in Nov. Act. Petrop. X, p. 397, tab. 10, fig. 5.

Stỵli liberi, plerumque lanatı; sepala dorso aculeolata, lirta, acute oblongo-lanceolata, integerrima, post anthesim patentia, persistentia ; fiores solitarii, terminales, in genere minuti, petalis quinque, basi nectario maculaeformi olsitis, aureis inferne atropurpureis; fructus avellana minor, globosus vel ovatus toto densissime aculeolato-hirsutus (variat etiam glaber, sed non vidi) ; calycis tubus fauce constrictus, limbo quinquepartito ; carpella glalerrima ; folia simplicia, orata, oborato-cuneata rèl lineari-oblonga, glabra rel breviter tomentella, obtusa, dentata, parallele nerrosa; aculei omnes stipulares, fere oppositi, patenti-sulincurvi, grisei vel alloi; stipulae nullae. - Fruticulus vix pedalis, Persiam et Desertum Songaro-Khirghisicum incolens, ramosissimus, glaber vel cinereo-tomentellus, ejus rami angulosi, breves, torluosi.

Characteres omnes generis - Persia bor. et occid. (Kotschy exs.

n. 698 , ete. . . . . 4266. H. BERBERIFolia (Pall.) 


\title{
INDEX ALPHABETICUS NOMINUM
}

\author{
NotA. - Ubi nomen auctoris speciei deest, pone : GDGR.
}

Bakeria adenophlaea, no 705 anglica, 694

armaena (Boiss.), 682

biturigensis (Bor.), 711

britannica, 700

coronata (Crép.), 701

crassibasis, 702 et 703

Doniana (Woods), 685

dubia, 693

echinantha, 697

gentilis (Sternb.), 717

rlaberrima, 713

gracilis (Woods), 692

helvetica (Hall. f.), 710

hibernica $(\mathrm{Sm}$.), 709

hispanica, 684

involuta $(\mathrm{Sm}$.) 704

Lehmanniana (Bge), 698

leiocarpa, 690

longisepala , 689

Malyi (Kern.), 707

megalochlamys, 708

normalis, 695

Ederiana (Tratt.), 712

pilosa, 686

pomponia (DC), 706

Reuteriana, 699

rhipidophlaea, 691

Robertsonii (Bak.), 687

rubella (Sm.), 716

sabauda (Rap.), 715

Sabini (Woods), 696

suavis (Willd.), 714

tephrosa, 688

tomentosa, 683

Wilsoni (Borrer), 681

Chabertia acanthobasis, 3526 acanthocalsx, 3501-3507

aeanthocarpa, 3558

a: anthoclina, 3652

acanthophora, 3680

acanthothamnos, 3c62

acanthotuba, 3493
Chabertia accommodata, 3458

acridentata, 3334 .

actinostephana, 3263

adelphica, 3787

- adenoclada, 3565

adenophylla, 3237

adenosepala, 3631

aderiostephana, 3317

admixta, 3360

adriatica, 3385

advena, 3204

adveısaria, 3563

aeduensis (Dés.), 3647

aena, 3658

aflinis, 3107

aftixa, 3729

agrestis (Sw.), 3299

agrestis (Savi), 3105

albillora (Opiz), 3311

albocarnea, 3185

alixensis, 3736

Allionii, 3722

alpestris, 3222

Alphonsi, 3161

alsogenes, 3743

alsophila, 3384

altissiodurensis, 3750

amabilior, 3632

amacantha, 3307

amblyrhyucha, 3117

Ambrosiana, 3370

amica, 3627

amphiacantha, 3727

amphicalyx, 3534

amphismallum, 3509

.anastellacantha, 3329

andalusica, 3524

anglica, 3380

angulosa, 3155

angusticeps, 3058

angustissima, 3098

anisacantha, 3552

anisocarpa, 3744

anisopoda (Christ), 3552
Chabertia anomala (Rip.), 3647

apennina, 3106

a pocarpa, 3655

apogyna, 3610

Apollo, 3314

apricorum (Rip.), 3708

arabica (Crep.), 3706 bis

aragonensis, 3070

arelatensis, 3192

arenaria (M.B.), 3316

argonnexensis, 3449

Argus, 3331

armatiramea, 3685

armigera, 3141

articulosa, 3129

arvatica (Puget), 3166

arvernensis, 3086

arvicola, 3091

aspera (Schleich.), 3267

aspericalyx, 3556

asperrima (Godet), 3761

asperula, 3165

assimilis, 3400

Assoana, 3603

asteroidea, 3309

atlantica, 3256

atractiformis, 3196

atrofusca, 3614

attenuatissima, 3474

Aucheri (Crép.), 3313

Augusti, 3653

austriaca, 3807

Autheman, $3246 \mathrm{et} 3243$

(var.)

baetica. $3: 04$

bahusiensis, 32.18

baltica, 3577

barcinonensis, 3160

batavica, 3777

belgica, 3411

benearmata, 3670

Berthetiana, 3063

Bertoloniana, 3580 
Chabertia biacantha, 3667

biglandulosa, 3731

Billietii (Puget), 3314

bioxycarpa, 3608

biscayensis, 3369

bispina, 3666

blepharidea, 3391

blepharosepala, 3403

bohemica, 3311

Borderi, 3406

Boulluii, 3259

Bourdini, 3539

Bouvieri (Crép.), 3255

brachyearpa, 3575

brachyrhyncha, 3144

brachystephana, 3781

brachystylis, 3705

brevicalyx, 3208

brevifolia, 3637

breviglanda, 3100

brevior, 3320

brevipes, 3751

breviramea, 3218

britannica, 3684

bruneoglanda, 3550

brutia, 3145

Burlei (Puget), 3762

buxifolia, 3147

cacosma, 3367

caesaraugustana, 3615

calcarea, 3266

Caldesii, 3061

calacantha, 3440

caloacantha, 3624

calocarpa, 3148

calophylla, 3547

calostephana, 3742

camberiacensis, 3319

camptoacantha, 3769

campylodes, 3242

campyllophylla, 3139

canariensis (Dés.), 3590

cantalica, 3790

capitata, 3601

capitellata, 3153

cariophyllacea (Bess.), 3304

carpetana, 3395

Castagneana, 3234

castellana. 3394

castanearum, 3347

catalaunica, 3096

caucasica, 3127

cerasifera, 3279

ceratoacantha, 3811

cebennensis, 3069

cedrorum, 3231

celtiberica, 3450

Cembra, 3142

Chaixiana, 3620

chalicophila, 3472

Chambionii, 3710

chavinioides, 3223
Chabertia chenoraulis, 3517 cheriensis (D. et Rip.), 3245

Chevrolatii, 3772

chlamydocarpa, 3613

chlamydophora, 3795

chloradenia, 3271

cladocharis, 3387

cladophora, 3301

cladosparsa, 3564

claromontana, 3768

claviformis, 3520

clavulata, 3065

collaris, 3521

collieula, 3457

collifera, 3740

collina, 3597

comatula, 3672

comigera, 3803

comosa (Kip.), 3813

comosoides(Ozan.),3275

compta, 3522

confragosa, 3616

conica, 3409

conocarpa, 3669

conophora, 3143

coronaria, 3159

corylifolia, 3425

corymbulosa, 3538

coryphaea, 3399

Costaeana, 3113

crassibasis, 3607

crassipes, 3732

Crepini, 3771

cretica (Tourn.), 3661

crinigera, 3675

croatica, 3427

cryptopoda (Bak.), 3251

cryptostigma, 3371,3372

cuneifolia, 3060

Cupaniana, 3224

curvula, 3393

Cutandaeana, 3374

cyanescens, 3459

cyclobasis, 3054

cyclostigma, 3665

cylindrocarpa, 3386

cymboides, 3264

daghestanica, 3698

dalmatica, 3053

danica, 3288

daphnoidea, 3130

dearmata, 3775

debilis, 3635

decalvata, 3599

decidens, 3646

decipiens, 3568

decorticans, 3392

Delileana, 3523

delphinensis (Chab.), 3363

densa (Timb.), 3633

dentata, 3714
Chabertia Derbesiana, 3123

desmocarpa, 3420,3421

desizophyila, 3598

desm poda, 3660

dichroopetala, 3436

dichrophylla, ined.

dicranodendron, 3812

dictamnoides, ined.

dicthyophlaea, 3236

didymacantha, 3286

didymocycla, 3233

dimidiens, 3682

diminuta (Bor.), 3365

dimorpha, 3780

dimorphacantha (Martinis), 3813

Diomedis, 3104

discedens, 3433

discolorifolia, 3494

dissidens, 3417

diversispina, 3167

djurdjurensis (Debx), 3227

Dodonaei, 3798

domita, 3455

ducens, 3382

dumetorum, 3796

ebenina, 3638

eboracensis, 3752

echinocarpa (Rip.), 3773

echinulata, 3570

editicola, 3075

edura, 3639

effata, 3445

elatior (Rouy), 3055

elegans, 3784

elegantissima, 3814

elevata, 3501

elisocalyx, 3451

elliptica (Tausch), 3313

elongatula, 3571

elongella, 3749

ensifolia, 3164

epiglottis, 3084

erectisepala, 3721

erioclada, 3337

eriopoda, 3340

eriosa, 3527

erronea, 3766

erythrochlamys, 3134

erythrophylla, 3156

escurıalensis, 3439

eustaminea, 3244

eusticta, 3090

exquisita, 3546

exsurgens; 3215

exuberans, 3103

fallacina (Rip.), 3333

fasciculata, 3788

fastuosa, 3694

faventina, 3076

filicaulis, 8194

filisepala, 3078 
Chabertia filispina, 3697

flavescens, 3310

flexuosella, 3747

florentina, 3181

floribunda (Stev.), 3677, 3678

florigera, 3583

flos lactis, 3480

formosula, 3805

fragrans, 3294

Fraseri, 3412

fraudulosa, 3691

Frayi, 3377

Freynii, 3119

fugax, 3717

galbanum, 3152

galloprovincialis, 3265

gastrocarpa, 3152

geocampta, 3120

gerundensis, 3453

Gibellii, 3502

glaberrima, 3052

glabrituba, 3767

glaciarum, 3489

glandulosissima, 3715

globosa, ined.

globularis, 3149

glutinosa (S. S.), 3760

Godroniana, 3687

gracilescens, 3092

gracilior, 3201

gracilipes, 3220

graciliramea, 3418

grairensis, 3676

granatensis, 3345

grandicorona, 3262

grateolens, 3488

graveolens (G. G.), 3654

griseispina, 3726

Guadarramae, 3397

Guiraoana, 3481

Gussonii, 3567

haematochroa, 3657

hallensis, 3718

Halleriana, 3212

hanoverana, 3706

Haussmanniana, 3693

hebetuba, $3-191$

helviensis, 3557

hercvnica, 3312

lieterobasis, 3282

heterochroa, 3650

heteroclita, 3.132

heterodonta, 3617

heteromorpha, 3341

heterophylla (Timb.), 3662

heteropes, 3335

hibernica, 3512

hirsutula, 3295

hirta, 3137

hispanica, 3051

hispidula, 3192
Chabertia histricula, 3516

holocalyx, 3703

Holubri, 3690

Hudsoniana, 3812

hungarica (Kern.), 3738

hyalina, 3618

hylebia, 3347

hypoacantha, 3303

hypoxantha, 3748

hyponaema, 3644

iberensis, 3110

idionacantha, 3533

idionodonta, 3089

ignorabilis, 3102

impercepta, 3641

impolita, 3094

improbata, 3232

inamoena, 3688

includens, 3187

inclyta 3499

inconstans, 3350

inconspicua, 3730

incrassans, 3809

incubacea, 3169

inermis, 3196

inflexa, 3495

inodora (Fries), 3213

intermixta, 3330

intricans, 3379

invisa, 3600

ionosmantha, 3375

irregularis, 3593

Isaurae (Tratt.), 3292

iseriensis, 3079

islandica, 3506

istriaca, 3126

italica, 3537

italicicola, 3128

Joadi, 3209

Jordani (Dés.), 3260

Jordanoides(Crép.),3280

jucunda, 3801

jugicola, 3619

jugorum, 3112

Jullienii, 3478

Kabylica, 32:2.5

Klukii (Bess.), 3298

Krauseana, 3513

lacerans (B. et Bushe), 3664

Lacroixii, 3205

lacticolor (Crúp.), 3315

lactillora (Dís., Gdgr) 3.181

ladanifera (Timb.), 3763

laevipes (Rouy), 3291

Lagascana, 3663

lageniformis, 3351

lagenoides, 3166

Lagunae, 3373

laguncula, 3555

Lamotti, 3785

lampocarpa, 3792
Chabertia Langeana, 3119

Langei, 3108

Lapevrousiana, 3592

lateritia, 3168

latispina, 3353

laxistyla, 3405

leiocaulon, 3240

leioclada, 3609

leiosepala; 3525

leiostephana, 3210

Lemanii (Bor.), 3471

lepidula, 3605

leporina, 3241

leptoceras, 3518

leptoclada, 3203

leptophylla, 3540

leptopoda, 3651

leucopetala, 3479

Lindebergii, 3211

lithophila, 3636

londinensis, 3500

longa, 3461

longicalycina, 3724

longicollis, 3794

longisepala, 3326

longispina, 3470

longituba, 3764

Loretiana, 3782

Loscosiana, 3273

lugdunensis (Ch.), 3297

Lusseri (Lagg.et Puget), 3424

lycium, 3219

macrobasis, 3261

macrocarpa, 3770

macrostephana, 3776

macrostyla, 3281

Madonae, 3505

madritensis, 3396

Magnieri, 3699

Maillardi, 3511

malacitana, 3352

Malarbeti, 3741

Malbranchii, 3531

Malpighiana, 3207

Marchandiana, 3587

massiliaca, 3759

mastichina, 3422

mastoidea, ined.

Mathonnetl, 36-15

mediterranea, 3325

megapelala, 3511

megastephana, 3250

melanoadena, 3518

mentita (Dés.), 3116

mesocalyx, 3178

mesoceras, 3157

Mesto, 3435

Micheliana, 3136

micrantha (Sm.), 3357

micrantho-sepium(Chr ), 3366

microbasis, 3193 
Chabertia microeoccos, 3476 microriphaea, 3532 microrhyncha, 3584 microsepala, 3140 minima (Puget), 3269 minuscula (Crep.), 3640 minutifolia, 3463

minutissima, 3176

mitigata, 3625

mixta, 3160

moderata, 3073

modesta, 3572

monantha, 3545

montana, 3779

Montceindriana, 3346

monticola, 3707

montigena, 3348

montivaga, 3276

moravica, 3268

multiculmis, 3816

murcica, 3438

mutabilis (Debx), 3383

mutinensis, 3200

myophylla, 3131

myriadena, 3290

myrioclada, 3173

myriosticta, 370\%

myrsinites, 3146

myrtifo!ia (Hall. f.), 3105

Myrtus, 3087

narbonensis, 3591

navarriensis, 3067

neapolitana, 3115

nebrodensis (Guss.), 3253

nemorosa (Lib.), 3362

nemoroso - tomentosa 3561

nervigera, $\mathbf{3 1 7 9}$

nevrosa, 3508

nevadensis, 3519

nidularia, 3190

nigrescens, 3530

nova, 3783

noviodunensis, 3364

obesa, 3804

oblongicalyx, 3462

Obornyi, 3349

occultata, 3057

oelandica, 3569

oenacantha, 3198

oenopetala, 3606

oenosepala, 3630

oenoxylon, 3573

olida (Crép.), 3689

oligantha, 3175

oligacantba, 3080

oligocarpa, 3358

oligopoda, 3692

oligostigma, 3083

olivetorum, 3095

opaca, 3306

opacifolıa, 3574
Chabertia operta (Puget) 3477

ornata, 3066

orucharis, 3336

orogenes, 3579

orophila, 3356

orotavensis, 3723

orthacantha, 3443

orthochlamys, 3270

osmoidea, 3172

ostryaefolia, 3713

ovoidalis, 3174

oxyodon, 3595

oxyphora, 3158

oxyporus, 3642

pachyseta, 3376

Pailloti, 3674

pallescens, 3389

Pancicii, 3360

pannonica, 3582

pannosa, 3503

parallela, 3510

parcepubens, 3755

parcespinosa, 3668

Pardoana, 3339

Parlatoreana, 3118

parviceps, 3111

parvula (Gren.), 3442

Pasqualei, 3332

pater-familias, 3434

paucifoliata, 3549

paupercula, 3170

peculiaris, 3415

pedemontana, 3338

peraffinis, 3305

permixta (Dés.), 3398

perneglecta, 3486

pervaga, 3559

petraea (Rip.), 3247

petrophila, 3656

phainocarpa, 3739

phalacroclada, 3429 et 3430

pilosula, 3428

pimpinelloides, 3355

pinguifolia, 3191

ringuissima, $308{ }^{\circ}$

pisana, 3416

platycarpa, 3622

platychlamys, 3183

podophylla, 3182

podostigma, 3464

polyclada, 3588

Pommaretii (Puget)3473

porrecta, 3725

pragensis, 3331

prenoclada, 3368

Proalii, 3578

producta, 3257

promiscua, 3542, 3543 et 3544

pruniformis, 3735

prussica, 3789
Chabertia pseudo-campta, 3121

pseudo-pisum. 3071

pseudo-remotior, 3469

pseudo-sepium (Callay)

3252.

pseudo-septicola, 3426

pulcherrima, 3733

pulchra, 3659

pungens, 3765

pura, 3679

purpurea, 3229

Puymaurea (Gren.),3816

bis

pyracantha, 3483

pyramidocarpa, 3088

pyrenaica, 3109

quassa, 3390

Queriana, 3454

quieta, $362 \theta$

Ramondiana, 3745

rarispina, 3197

redux, 3485

Reichenbachiana, 3728

remotior, 3475

Requieniana, 3189

Reuteriana, 3696

rhipidoclada, 3072

rhipidophora, 3515

rhipidothamnos, 3716

rhombaea, 3628

rhynchantha, 3402

rhynchocarpa, 3554

rhynchoides, 3165

rhynchophysa, 3125

Richterii (Crép.), 3151

riphaea, 3206

Robertiana, 3342

robustior, 3050

rossica, 3586

Rostani, 3529

rostrifera, 3611

rosulenta, 3604

rothomagensis (Rouy), 3278

rotundifolia ( $\mathrm{Rau}$ ) 3816

rubelliformis, 3216

rubiginosa (L.),3704

rufescens, 3700

rufina, 3629

rufinervis, 3283

rufovirens, 3186

rufula, 3746

rugosula, 3132

rupifraga, 3596

sabulosa, 3171

saliuscula, 3162

salvanensis (Delasoie), 3398

Salvatoreana, 3177

Sanlavillei, 3217

sarcopoda, 3621 
Chabertia sarcostephana, 3566

saucia, 3221

saxetorum, 3806

saxonica, 3797

scaberrima, 3778

Scheutzii, 3810

scleroacantha, 3709

scleroxylon, 3671

seotica, 3757

serupulosa, 3786

scrutata, 3413

seclandica, 3327

Segurae, 3.48

semi-armata, 3154

semi-pedunculata, 3751

senescens, $36-19$

senilis, 3059

sepium (Thuill.), 3064

seposita (Críp.), 3133

septicola (Dés.), 3423

septicoloides (Crép.) 3410

Seraphini (Viv.) 3230

serbica, 3122

serpyllifolia, 3188

serpylloides, 328

setulifera, 3456

silesiaca, 3249

silvicola (Dés. et Rip.), 3.198

solenoidea, 3535 et 3536

Sophia, 3514

spadana, 3581

sparsillora, 3318

sphaerocephata, 3184

sphaerophora (Rip.) 3328

sphaerostylis, 3468

sphaerica, 3214

spinigera, 3562

spinosissima, 3623

spinourceolata (Crép.) 3773

stabiana, 3238

stenoacantha, 3594

stenuealy, 3-187

stenocarpa (Rip.), 3099

stenogyna, 3774

stenophylla, 3.131

stenorhyncha, 3150

stephanocarpa, 3711

stephanophora, 3277

stilboantha, 3258

stilbophylla, 3602

stargardiensis, 3285

streptocaulis, 3528

strombifolia, 3388

suavefragrans, 3631

subalpina, 3296

subattenuata, 3108

subaurata, 332:

subcoronata, $36-18$

subdecidua, 3328

subdola (Dés.), 3552
Chabertia subglobosa, 3114

sublinearis, 3235

submitis, 3756

subsetosa, 3381

subspinosa, 3074

subtincta, 3097

subunca, 3407

subunillora, 3077

suecica, 3299

supraglandulosa, 3056

supramollis, 3712

synacantha, 3737 .

tarentasiensis, (Puget), 3274

tassinensis, 3359

telacantha, 3553

Tenoreana, 3791

tenuispina, 370

tephroacantha, 3437

ternispina, 3446

terebinthinacea (Bess.), 3720

testiculata, 3560

textiramea, 3124

theratophila, 3308

thuringiaca, 3272

tomentella, 3281

Tommasiniana, 3400

tordylioides, 3612

tortuosa, 3300

tragacantha, 3695

transtagana, 3445

tridentina, 3686

tubifera, 3093

Tuezkiewiczii, 3490

tuscanica, 3734

tyrolensis, 3082

uberta, 3799

umbellata (Leers), 3808

umbelliformis, 3681

ulmicola, 3800

unicolor, 3758

vagans, 3163

Vahliana, 3585

Vaillantiana (Bor.) 3482

valdedilatata, 3467

valderia, 3199

validu'a, 3643

vallis-clausae, 3138

vapensis (Gren.), 3226

Vayredae, 3068

ventosana, 3719

vepreticola, 3101

versicolor ('Timb.) 3383

Vesper, 3673

Villarsiana, 3332

vincta, 3289

vinodora (Kern.) 3135

virgultorum (Rip.). 3251

viridicans, 3180

viridifolia, 3144

viridissima, 3588

viridipes, 3414
Chabertia viscosa (Jan), 3228

vivariensis, 3576

volubilis, 3195

vratislaviensis, 3497

vulnerans, 3202

Willkommiana, 3354

Wimmeriana, 3239

xyloechina, 3753

xylomitis, 3551

xylorubra, 3683

xylotes, 3081

zephirophila, 3793

zygioides, 3324

Zygis, 3293

zygoantha, 3302

Chavinia accessiva, 3042

acuteserrata, 2810

adenoclada, 303.4

adenophlaca, 2973

adenotrichia, 2869

alicantina, 2896

alnorum, 2955

alpestris, 2857

Ambrosiana, 2863

amoena (Kern.), 2939

amplexifolia, $30-15$

anceps, 2987

anglica, 2862

angustata, 2915

angustisepala, 3036

aragonensis, 2890

ariadne, 2988

arvermensis, 2932

asinina, 3040

aspreticola(Gremli),284]

austriaca, 2978

Barmondierei, 3002

belgradensis (Pancic), 2910

Bertoloniana, 2876

Blondaeana (Rip.), 2814

bohemica, 2912

brachystigma, 3025

brevicaulis, 2985

britannica, 2871

caespitosa, 2998

calcareicola, 2970

Caryotoides, 2990

Cassiopes, 3044

cathaclines, 2947

cerasiformis, 2969

chersonensis, 2909

chrmopetala, 3026

cohaeres, 3028

concinna (Lags. et Pug.) 2895

conjuncta (Críp.). 2859

consanguinea (Gren.), 2839

controversa (Rip.), 2921

coriacea, 2878

coronata, 2874 
Charinia Crepini, 2868 crinita, 3017 croatica (Kit.), 2891 cuneifolia, 2856 crpria, 2847

dalmatica, 2901 decora (Kern.), 2939 decursiva, 3011 delphinensis, 2906 Dematranea(Lagg.), 2846 depressa (Gremli), 2907 bis

determinata, 2977

dictamnoides, 2997

dichrophylla, 2900

dicthyocaulon, 3015

dilatata, 2972

dimorphacantha, 2913

drvadea (Rip.), 2923

elatior, 3010

elegans, 2867

ervthroxylon, 2963

falcispina, 3009

filispina, 2889

llexiramea, 2943

flexuosa (Rau), 2957

formosissima, 3037

gallico-umbellata(Rap.), 2839

glabrescens, 2864

glabripes, 2883

Godeti (Gren.), 2966

gomphocarpa, 3008

gothica, 2880

Gouttardi, 2961

grandiflora(Wallr.), 2855

Gremblichii, 2893

Gussonii, 2849

haematodes(Boiss.), 2850

heteracantha, 2854

hirsuta, 2887

hirtifolia, 3000

hirtinaeva, 2982

hispanica, 2905

hispidocarpa: (Chabert), 2999

Holandrei, 2959

inaequearmata, 3018

inaequepilosa, 3014

inarimensis, 2866

incerta, 2865

inchoata, 3035

insidiosa (Rip.), 2907 bis

intecta, 3043

istriaca, 2888

italica, 2879

Jundzilliana(Bess.),3003

laevicaulis, 2964

Laggeri (Puget), 2929

lancaefolia, 3012

latiuscula, 2979

leptacantha, 3027

leptoclada, 2935
Chavinia leptoriphaea, 2992

ligustri, 3048

longeappendiculata, 3030

longepedunculata, 2942

longipes, 3032

macroacantha, 2885

majorana, 3020

marginata (Wallr.), 2949

Mathiae, 2965

mediterranea, 2904

megalontodon, 2993

megastephana, 3007

membranifolia, 2981

microsticta, 2914

minor, 2877

monticola, 2930

moravica, 2894

murcica, 2918

neapolitana, 2886

nemophila (Rip.), 2924

nemorivaga (Dés.), 2974-

75 (var.)

nevadensis, 2936

- notha, 2953

obliqua, 3029

oblonga, 2861

Obornyi, 2946

oculus-solis, 3001

ocymiodora, 2917

orthacantha, 2967

oxvcarpa, 2881

pachyphylla, 2852

parvifolia, 2858

paucipinnata, 2996

paupercula, 3031

pedemontana, 2938

perdurans, 2922

perpignanensis, 2925

phylliriaefolia, 2986

polita, 3039

polonica, 3006

populnea, 3041

praeterita (Dés. et Rip.), 2926

praetermissa, 2950

pi ocreata, 2962

protea (Rip.), 2915

prunicola, 3049

pseudo-flexuosa (Ozan.), 2958

pseudo-gallica, 2968

Pugeti (Bor.), 3024

pumilio, 2954

purpurascens, 2931

Pyriha, 3022

quercetorum, 2960

reticulata (Kern.), 2944

reticulosa, 2983

rhynchocarpa, 2899

rigidiseta, 2948

rostrata, 2991

rossica, 2937

rubrinaeva, 3033
Chavinia ruthenica, $28 \%$

salicina, 2941

sambucifolia, 3038

sanguisorbelia, 2933

sanguisorbifolia (Delasoie), 2933

Satyrus, 2994

scabrata (Crép.), 2907

scabricaulis, 2980

scandinavica, 2908

sclerophylla (Scheutz), 2842

semi-glandulosa (Rip.), 2928

sepiiformis, 3016

setiramea, 3019

setulifera (Timb.), 2974

silesiaca, 2934

silvulivaga, 3005

similita (Puget), 2898

speciosa (Dés.), 3037

sphaerica, 2818

sparsiglanda, 2872

spectabilis, 2940

stabiana, 2875

stilbopoda, 2989

stupens, 2952

subalpina. 2884

subolida (Dés.), 3046

subintrans (Gren.), 2892

suecica, 2902

Süffertii (Kirschl.), 2920

suprahirta, 2995

temula, 3023

Tenori, 2903

tenuicula, 3047

tenuiglandulosa (Mér.). 3021

Thomasii (Puget), 2851 thuringiaca (Wallr.), 2951

titanophylla (D. et Rip.), 2927

trachyphylla (Rau), 2971

triacantha, 2984

turneraefolia, 2956

tyrolensis (Kern.), 2897

valderia, 2853

valida, 2911

vallesiaca (Lagg.), 2919

villosula (Paillot), 2873

vinetorum (Kip.), 2843

viridescens, 2976

viridifolia, 3013

viscida (Puget), 2916

viscidula, 2916

Vukotinovicii, 2860

wasserburgensis(Kirs.), 2845

Websterii, 2882

zygophylla, 3004

Cottetia abscondita, 573 
Cottetia acanthocaulon, 633 acanthodesma, $60-4$ acanthopoda, 577 aciculosa, 600 adenophora (Kit.), 626 adenostephana ined. atlinis (Sternb.), 665 alpestris, 620 amoena, 587 angliea, 578 apposita, 553 aprica, 670

aragonensis, 623 a renastrum, 556 arenosa, 630 Assoana, 656 autaretica, 643

Balloniana (Mous.), 555

Besseri (Tratt.), 628

bohemica, 557

borealis (Tratt.), 560

britamnica, 598

caltanisettensis, 617

Caroli, 561

catalaunica, 559

conjuncta, 655

consimilis (Dés.), 606

contingens (Dús.), 586

croatica, 572

danubialis, 558

delphinensis, 619

dichroa (Lerch), 674

dichroearpa, 575

dinarica. 659

disparata, 597

exasperita, 654

fissifolia, 642

glaberrima, 56

glabrata (Vest), 658

glabrinaeva, 639

glandulosissima, 666

glaucescens, 652

grammicolepis, 671

granatensis(Willk.), 675

hibernica, 65

hirtella, 624

hispanica, 644

horrefacta, 661

immixta, 610

inaequearmata ined.

insueta, 602

interjecta, 595

isoacantha, 622

juratensis, 603

Kemmleri, 650

laciniosa, 667

laevissima, 562

Lansaeensis, 593

latebrans, 589

legionensis, 601

Leibnitzensis, 627

Lenormandit, 647

lepidophora, 668

\section{Cottetia Leyi, 618}

Loscosiana, 680

macroacantha, 584

macropoda (Rip.), 662

macropodoides, 591

maculans, 55-

Maitleana, 631

maracandica (Bge), 664

mariaeburgensis, 607

maritima, 580

Martini, 646

Mathonneti (Crêp.), 640

melanocarpa (Link), 611

melanopoda, 592

microcarpa (Bess.), 628

mitissima (Gm.), 588

mitissimoides, 590

montenegrina, 614

montigena, 645

Montis Aurei, 637

myriacantha (D. C.) , 672

myriadena, 673

nevadensis, 677

Neyraiana, 585

Noeana, 568

nubigena, 621

oblongifolia, 599

oenocarpa, 629

oreades, 566

oxyacantha (M. B.), 608

Ozanonii (Dés.), 618

pachypoda, 565

palatina, 613

Pantocsekii, 574

pedemontana, 616

pellucina (Arvet T.), 606

petricola, 594

petrogenes (Ozan.), 657

pimpinellifolia (L.), 555

pimpinellifolio-rubiginosa (Rchb), 679

platyacantha, 636

platvcalyx, 641

poteriffolia (Bess.), 562

bis?

pubens, 649

Pugeti, 582

pycnacautha, 632

Reimbolei, 609

reversa (IV. K.), 679

Ripartii (Dés.), 663

rothomagensis, 567

rubiginoidea, 669

Scopoliana (Tratt.), 562 bis

scotica (Tratt.), 635

septentrionalis, 63

siphonocarpa, 60:

Songaeana (Puget), 596

spinosissima (L.), 571

spinosissimo - coronala

(Crép.), 653

spreta (Dés.), 563
Cottetia Sternbergii, 665

Stuartiana, 581

subinermis, 612

suboblonga, 638

taraconensis, 676

Tauscheri, 583

telonensis, 678

tuschetica (Boiss.), 660

Utzka, 570

valdensium, 615

velutina (Clairv.), 625

Ventenatiana (Th.), 570

viréscens, 569

vogesiaca, 570

Crepinia aboensis, 2612 abrupta, 1957

abstenta (Dís.), 1937

acanthocarpa, 27.18

acanthoclada, 2387

acanthophylla, 2488

acanthophora, 259)

acanthothamnos, 2689

accedens (Ri).), 2169

accipitrina (Debx), 2455

acerina, 1219

Acharii (Billb.), 2099

acicarpa, 1460

aciculatis, 1672

aciculifera, 2081

aciculifolia, 1428

acidula, 2487

aciodonta, 1814

aciphylla ( $\mathrm{Rau}$ ), 148-1

aciphylloides (Crép.), 1249

acmodonta, 1336

acmophrila, 1526

acora, 2143

acridentata, 1645

acrocomata, 2293

actinochlamys, 1899

actinoclada, 1677

actinophlaea, 1999

actinodroma, 1864

aculeata, 1754

acuta (Crép.). 1951

acuta Gdgr, 1472

acutifolia, 2157

acutispina, 1828

acutiuscula, 1208 bis

addita (Dés.), 1951

adenochlamyda, 1423

adenodontoides, 2575

adenodontos, 2037

adenophora, 1717

adenosepala, 1573

adenostema, 2745

adenostephana, 22:23

adenotricha, 2674

admissa (Crép.), 1580

admixta, 2043

adornata, 1353 
Crepinia adriatica, 2277 adscensionis, 1965 adscita (Dés.), 1577 adulterina, 2720 aequalis, 2202 affinis (Rau), 1723 affinita (Puget), 2209 aggerivaga, 1220 aggrezata, 1854 aginensis (Rip.), 1164 agraria (Rip.), 2004 agrestina (Crép.), 2004 agricola, 2510 ailanthoides, 1585

alba (L.), 2726 albanorum, 1842

albistyla, 1539 albogilva, 2137 albo-lutescens (Rip.), 1331

albula, 2613

aliena, 2636 alligata, 2327

Allionii, 2682 alpestris, 1816 alpicola, 1149 alpina, 2660 alpivaga, 2599 altera, 2665 amabilis, 2838

Amansii (Dés.), 1164 ambigens, 1974 ambigua (Lej.), 2042 amblyacantha, 1907 amblyotis, 2030 amblyphylla (Rip.), 2787 Ambrosiana, 1295 amoena, 1509 amoenevirens, 1415 amoenicorona, 2294 amoeniflora, 1885 amoenifolia, 1898 amoenipetala, 2286 amoenipes, 2531 amphicarpa, 2412 amphiloga, 1298 amphirhyncha, 2352 amphisbaena, 2377 amphoricarpa, 2195 amplifolia, 1184 ampliuscula, 1212 anacampseros, 2497 analoga (D is.), 1289 anceps, 2652 andegavensis (Bast.), 1943

Andersoniana, 2559

andropogon, 2508

androsacea, 2427

anglica, 2272

anglorum, 2225

angustifolia, 2465

angustior, 1385
Crepinia angustispina, 2714 angustula, 1261 anisodonta, 1820 anisophlaea, 1766 Annoniana (Puget), 2397 anor'malis, 2565 anthophora, 2207 antiqua, 1223 anticaria, 2100 anxia, 1315

apaloxylon, 2581 apennina, 1736 apertidens, 2566 apiceacuta, 1365 apiculata, 1704 apostigma, 1818 appendiculosa, 1183 apposita, 2028 appropinquata, 1392 approximata, 2414 Aquaesextiae, 2148 arachnoidea, 2790 aragonensis, 1321 arbutifolia, 1338 arcana (Dés.), 2758 Arcturus, 2432 arcuata, 2265 arelatensis, 1952 argutiserrata, 140 7 arguta (Muss.), 2771 armata (Stev.), 1537 armatissima (Dés.), 1783 Armidae (Webb), 2776 armoricana, 1632 asclepiadea, 1756 aspera, 1740 aspernata (Dés.), 2051 aspratilis Crép.), 2051 astrachanica, 2573 astreata, 1396 asturica, 1231 atlantica, 2666 atrovirens, 1348 altenuata (Rip.). 1627 attenuatifolia, 2349

Aunieri (Cariot), 1983 aurita, 2538 auroris, 2669 australis, 2424

austriaca, 2185

Authemani, 2166

avellana, 1786 avellanaefolia, 2828 azovica, 2635

baetic2, 1966

Bakeri, 1448

b altica, 1884

Banksiana, 1845

barba-jovis, 2695

barbulata, 1844

barbiseta, 2831

barcinonensis, 2502

basaltica, 2173
Crepinia basilaeensis, 2539

batavica, 1463

bavarica, 1408

Bavouxii, 1932

bayonnensis, 2461

belgradiensis, 2621

bella, 1545

Bellavallis (Puget), 2721

bellojocensis, 1229 bellula, 2811

belonidimorpha, 2401

Benthamiana, 2807

Berheri, 1474

Bertoloniana, 2641

biacuta, 1252

bicollis, 2649

Bienerti, 1258

Biebersteiniana (Tratt.), 2772

biformis, 2529

Billotiana, 1595

biserrata (Mérat), 1865

blekingensis, 2764

blepharella, 1490

blepharopetala, 1681

blepharophilla, 2605

blepharopoda, 2256

Bluffiana, 1882

Blyttii, 2468

bohemica, 1549

Boitrayana, 1621

bombax, 2153

bombycella, 1763

bombycina, 1217

Borderi, 2319

borealis, 2587

Boreychiana 2760

Borreri (Woods), 2445

borussica, 2079

botryoclada, 2330

brachiata (Dés.), 2751

brachyacantha, 2392

brachycalyx, 1327

brachrcarpa, 2339

brachycella, 2569

brachycocca, 1987

brachyodon, 2359

brachypoda (Dés. et R.) 1730

brachyphylla, 2797

biachystem\&, 1977

brachystylis, 2405

bracteosa (Crép.), 2098

bractescens (Woods), 2659

Braunii, 1296

bregillensis, 1796

brevepubens, 2235

brevicalyx, 1571

brevicollis, 1410

brevifolia, 2350

brevior, 1857 
Crepinia brevipila, 2656 brevispina, 1785 brevituba, 2248 breviuscula, 2824 Briggsii, 2133 britamica, 1508 burgorum, 2252 buxigena, 1244 cabulica (Boiss.), 2759

caesaraugustana, 2419 caesia (Sm.), 2172 calamaria, 1973 calcarea, 1335 Caldesii, 1200 caledoniorum, 1747 callicanthos, 173: callichroa, 2014 calliphylla (Rip.), 1824 calochlamys, 2466 calochlora, 2756 calodonta, 1326 calopetala, 2586 calophyton, 1669 calorhyncha, 1323 calostephana, 1900 calosphaera, 2370 ealycifera, 2026 calycina (M. Bieb.), 1454

calycochlamys, 2239

calycogloba, 1728

calyptocalyx, 1733

calva, 1166

calvescens, 2308

camerina, 1163

campestris (Sw.), 2741

campicola, 2546

camporum, 1660

camptoclada, 2644

camptodes, 1182

camptomorpha, 2233

campylophylla, 1273

canescens (Bak.), 2459

canina (L.), 1297

cantabrica, 1146

cantalica, 2245

capnotricha, 2637

caprifica, 1314

caproniana, 1863

cardiophora, 2311

Carestiae, 1328

Carionii (Dés.), 2369

Carioti (Chabert), 1591

carneillora, 1388

carneipetala, 2175

carpetana, 17ซ1

Carrae, 2001

Carriezii, 2160

Castagneana, 1452

castaneicola, 2060

castaneaefolia, $196-1$

castellana, 1935

catalaunica, 1194
Crepinia calteasica (M. B.), 2820

caudata, 2055

caulifolia, 1770

cebennensis, 16.t]

relerata (Bak.), 2814

celtiberica, 2355

cerasifera ('Timb.), 2731

cerasiformis, 1502

cerasoides, 2453

ceratophora, 1235

ceratophyila, 2057

Cercosiana, 1893

cerina, 2611

cerino-alba, 2693

ina, 187

Cessacii, 2723

Chaberti (Dis.), 2112

Chabertioides, $2036^{\circ}$

Chaixiana, 2767

chamaemespilus, 1872

Chapusii (Gren.), 2519

characias, 1936

chersonensis, 2275

chlamydoadena, 1430

chlamydocalyx, 1619

chlamydosticta, 2781

chloromelanos, 2380

ciliaris (Wilsoni), 2834

cincinnata, 232]

cinerascens, 2591

cinerea, 2653

cinereosula, 2301

cinerosa (Dés.), 2571

cinnamomicolor, 1997

civis, 1994

cladobotrys, 1607

cladocampta, $200 \%$

cladoleia (Rip.), 1624

cladophora, 1424

claromontana, 1836

clavata, 1485

clavigera, 2190

clavoides, 2353 et 2354

clinacantha, 1877

clinella, 1510

clinophlaea, 1259

clivorum (Scheutz), 2738

clypeolaria, 2020

coadunata, 120

Codornii, 2050

coerulescens, 1473

collaris, 1154

Collieri, 2382

collina (Jacq.), 2725

collincola, 1426

colloeata, 1319

colorata, 1227

columnifera (Fr.), 2422

commiserata, 2.19-1

comosa, 2836

compacta, 2290
Crepinia Companyoii(Dehx.), 2151

Companyonis, 1362

compressispina, 1530

coneolor, 2574

condensata (Puret), 1215

conferta, 1792

confertillora, 1787

confertifolia, 1531

confertior, 1226

confidens, 1761

continis, 2486

confusa, 1611

conglobata, 2513

congruens, 1351

conica, 27.46

conifera, 2126

conimbricensis, 1608

conoacantha, 1888

conocarpa, 1178

conomorplaa, $16-12$

conopliora, 1683

consobrina, 1516

consociata, 1889

consolationis, $127 t$

constricta, 2670

contorta, 2176

convergens, 2302

conversa, 1665

convexula, 2367

conyzoides, 2700

corbariensis (Debeaux), 205

Corda, 1290

cordata, 2130

cordifolia, 1968

cordiger'a, 1368

coriacea (Crép.), 1951

coriacea (Opiz), 2801

coriascens (Boullu), 1518

coriifolia (Fries), 2513

cornubiensis, 1144

coronata, $25-17$

corrlicola, 1169

coryloides, 1376

corymbifera (Borkh.), 2515

corymbulosa, 1512

cosmocarpa, 2821

cosmophylla, 2120

Costac, 22:30

Crantzii (Schult.), 2735

crassifolia (Wallm:), 2.544

crassidens, 1303

crassinervia, 2159

crassior, 1159

crassiuscula, 1200

crataegina ria, 2121

cremorlada, 1142

Criacantha, 1.470

cribrosa, 2552

crinisepala, $165 \mathrm{~J}$ 


\section{2}

Crepinia crinita, 1726

crispatula, 2188

croatica, 1512

cruentata, 1435

cryptostylis, 2718

csepeliensis, 1401 cuneata, 2076

cuneifolia, 2372

cuneipetala, 1137

curticola (Puget), 1692

curtifolia, 1805

curtipes, 1848

Curtisiana, 1718

curtispina, 2490

curvidens, 1277

curvispina, 1405

curvula (Timb.), 2543

cynocalyx, 2238

cyanopoda, 2563

cyclocalyx, 2016

cyclocarpa, 1208

cyclodonta, 2511

cycloidea, 1628

cyclomorpha, 1956

cyclophylla, 1772

cylindrica, 2694

cylindrocarpa, 2684

cymigera, 1275

dalmatica, 2527

danica, 2278

danubialis, 2598

dasicalyx, 2757

dasycarpa, 2449

dasystyla, 1230

dasvphora, 2373

dasyphylla, 1256

Davisiana, 2196

Debeauxii, 2056

decalvata (Crép.), 2128

decipiens, 1205

decumbens, 2117

decuriens, 2244

decurtata, 2320

dedolata, 1312

deficiens. 2706

deliciosa, 1980

Delileana, 1674

delphinensis, 1409

densifolia, 1742

densiuscula, 1278

denticalycina, 2623

dentifera, 2474

dentisepala, 2326

dentosa, 1425

denudata, 2789

depressa, 2545

Derbesiana, 2217

Deseglisei (Bor.), 2722

desiderabijis, 1308

despicata, 2792

Desvauxii, 1325

detruncata, 1748

Deucalion, 2048
Crepinia deviata, 1243

devota, 1598

diachylon, 2061

dichrophylla, 1855

dicranoclada, 1560

dicranoides, 1752

Dichtlii, 2305

didoensis (Boiss.), 1990

didymocarpa, 1693

didymodonta, 2208

didymopoda, 2085

didymorhyncha, 1153

didymoxys, 244

differens, 1242

difficilis, 2521

dimidiens, 2358

diminuta, 1305

dimorphacantha, 2114

dimorphocarpa, 2514

dimorphocycla, 1991

dinarica, 1918

Diomedis (Gren.), 2052

diphtheritis, 1429

discolor, 1658

discors, 2801

discostyla, 1657

dissidens, 2610

dissimilis, 1701

divaricata, 1441

diversidens, 2262

diversispina, 2113

dolabrifolia, 1998

dolata, 1737

dolosa (Godet), 2018

Dommartini, 1745

dombensis, 1604

Donniana, 1517

dormiens, 1156

dryadea, 2699

drymophila, 1515

Dubosclardii, 1565

Duffiti, 1455

dumalis (Bechts.), 1789

dumalioides (Puget), 1912

dumetoides (Puget), 2584

dumetoroides (Crép.), 2192

dumetorum (Thuill.), 2557

Dumortieri, 2082

dumosa, 1311

dura, 1250

Duriaeana, 2069

durifolia, 2067

duriuscula, 1254

dysphoroidea, 1945

dyscaulon, 2710

dysclados, 1741

dysphorica, 1445

dysthamnos, 1562
Crepinia eboracensis; 1503

eccremoclada, 2620

eccremophlaea, 1822

Echeandiana, 1345

Echidne, 2742

echinopoda, 2697

edimburgensis, 1I51

edita (Dés.), 1934

edita Gdgr, 1699

edocenda, 1630

edocta, 1486

educta, 1981

eduensis (Dés.), 1711

edurescens, 1928

efferta, 2140

effusa, 1775

eglandulosa, 2458

elegans, 2489

elevata, 2336

elisabethopolensis, 2189

elisantha, 1359

elisocalyx, 1923

elisophylla, 2415

elisophora, 2439.

elliptica, 2241

elongata, 1586

emarginata, 1851

epimedium, 2467

epipactis, 1859

erectisepala, 2095

erectiuscula, 1493

eremocharis, 2023

ericetorum, 1417

eriogyna, 2749

errabunda, 2707

erronea, 1265

erythrantha (Bor.), 2460

erythrella (Rip.), 1972

erythrocarpa (Rip.),1458

erythropetala, 2356

escurialensis, 2065

etrusea, 2335

eucharis, 1757

eudicthyon, 1499

eudora, 2013

eunuchophylla, 1673

Euryale, 2322

eusynphylla, 1634

exaltata, 2626

exarmata (Crép.), 1207

exerrans, 2473

exilis (Crép. et Wirtg.), 1337

exoptata, 1357

expallens, 2003

exsertistyla, 1352

exsul, 1684

exsurgens, 2835

extranea, 1942

fabaefolia, 1852

fagifolia, 1495

falcatula, 1399

falcifera, 2142 
Crepinia falcinella, 169.

fallacina, 1199

fallaciosa (Dés.), 2753

fallax (Puget), 1299

fallens (Dís.), 1366

falsa, 1739

fastibilis, 1389

fastidita, 1993

fatula, 250.1

faventina, 1309

Favrati, 1170

fennica, 1228

ferox (Chabert), 1482

fertilis, 1903

ticoides, 2167

filamentosa, 2702

filiformis (Ozanon), 1196

tilispina, 27.47

tilopes, 2237

finitima, 1201

finlandica, 2655

firma (Puget), 2077

firmior, 1561

fissa, 1860

fissidens, 1301

fissifolia, 1216

fissispina (Wielzb.)1789

flabellata, 2692

flexibilis (Dés.), 1139

flexibilis Gdgr, 153.1

flexicaulis (Crép.), 1638

flexiramea, 23.12

fodinarum, 2183

foliosa, 1412

foliigera, 2227

foresiaca, 1287

Forgeoti, 2268

fornicata, 2360

Forsteri (Sm.), 2328

fragilis, 1666

Fraseri, 2822

fratema, 1919

fraxinoides, 1887

Freynii, 228:1

friburgensis, 2270

Friedlanderiana (Bess.), 2770

Friesii (Lagg: et Pug.), 2.458

frondosa (Ster.), 2154

- fruteticola, 1311

frutetorum (Bess.), 2413

fruticetorum, 1891

furcellaria, 1197

fusifera, 2639

fusiformis, 2191

gallico-canina (Reut.), 2118

galloecica, 2161

Gandogeri (Puget), 1162

gastı oidea, 1538

gaudens, 1971

Gaudiniana, 2410
Crepinia Gautieri, 2730

Gelmii, 1762

genevensis, 1992

genistaceola, 1506

Gemmarii (IInct), 2122

geophila, 1915

geracantla, 1575

geraplivlla, 2:3-20

Gerardiana, 2170

gerundensis, 1705

Gibellii, 21.16

glabella; 1161

glaberima (Dum.), 1592

glabrata (Kánitz), 1161

glabreseens, 2216

glabriot, 2671

glabristvla, 1931

glandulitera, 2002

glandulimaera, 1217

glandulisepala, 1390

glandulosa, 212:3

glandulosella, 2212

glaucescens( I) esv.),1325

glancitolia (Opiz), 1807

glaucina (Rip.), 1862

glauciramea, 2195

glaucocarpa, 1910

glaucorubra, 1809 ,

glaueovirens, 2015

globata (Dús.), 2168

globosa, 1501

globularis (Franchet), 1373

globulosa, 2556

gnaphalodes, 2576

Godroniana, 21.19

goniodenta, 2701

gonionlyylla, 2715

gossypina, 2704

-gothica, 1522

gracilenta, 2102

graculicaulis, 1815

gracilituba, 1332

gracilior, 1381

graciliramea, 1525

gracilis, 122:-2

gracilispina, 19.16

grandillora, 1550

grandispina, 1521

granitica, 1196

grata, 1801

gremaria 1511 et 1781

Gremblichii, 1871

grisescens, 2578

grossularioides, 1.198

Guadarramae, 2199

Guepini (Bast., Desv.), $280: 3$

Guilloti, 1300

Guira0ana, 2062

gymnophlaea, 1963

gymnostigma, 2137

gynoleia, 1138
Crepinia Fraberiana (Puget), $2(1) 75$

haemantha, $217 \pi$

haematodes, 2019

Hailstoni (Baker), 2109

latlensis, 2.57

hanoveraua, 1710

Haแrvana, 2201

Harliordii, 2527

Hanssmaniana, 1688

hebechlamrs, 1582

hebervina, 1955

helvotien, 2152

hemicvela, 1501

hemiglatteos, 2217

henitricha (Rip.), 1671

heteracantha, 189:

heterocarpa, 1214

heteromorpha, 2385

heterostisma, 2123

hiberuorum, 1881

Hildbrandtii (Opiz),2802

lirsutissima, 2054

hirta, 1861

hirtella (Rip.), 1921

hirtistrla, 1853

hirtipes, 2533

hispaniea (IIill.), 2553

hispanica (B. et R.), 2100

hispidula (Rip.), 2150

histricosa (Crép.), $207 t$

hololeuca, 2793

holopetala, 1732

holophaea, 1656

holophylla, 2580

holopoda, 2537

Holubri, 2503

Hookeriana, 1916

horridula (Dés.), 1482

Hurlsoniana, 2022

Hüisenii, 2681

humicampta, 1804

humilis (Bess.), 2762

hungarica, 16.19

hriochionaea, 2509

hypoevela, 1839

hipotricha, 2138

ibercensis, 2273

iberorum, 2287

iemacoplivila, 1489

icorlossa, 2394

Ildetomsi, 1720

ilicetorum, 2366

illecebrosa, 2251

illecta, 1806

illibata, 155.

illigata, 2205

illnstrata, 1475

imitata (Dés.), 2364

implexa (Gien.), 2236

implicata, 2743

impolita, 1381

improvida, 2430 
Crepinia inaequalis, 2218 inaequeserrata, 2139 inaequidens, 1788

inanis, 1307

incerta (Dés.), 2765

incisa, 1969

incompta, 1427

inconcessa, 2492

inconsiderata (Dés.), 2100

inconspicua (Dés.), 2040

incrassans, 2650

incurva, 1395

indecora, 2823

indifferens, 1743

inducta, 2826

inermis, 1543

infundubiformis, 2528

infracordata, 2428

infraglauca, 2309

innocua (Rip.), 1913

insignis (Rip.), 1886

insolata, 2087

insularis, 1712

insulsa, 1141

intacta, 1167

integrata, 2491

intenta, 2501

intermedia (Kit.), 2616

interobscura, 1584

intersita, 2799

intertexta, 2703

intervallata, 1459

interveniens (Dés.), 1967

intricata, 1524

involucrata, 1431

iodophylla, 2562

Irpex, 1988

ischnoclada, 2151

ischnodendron, 2011

iseriensis (Puget), 2724

istriaca, 1547

italica, 1355

Jacobi, 2794

Jacquiliana, 1333

jactata (Dés.), 2211

Janus, 2561

jonides, 2662

jugularis, 2601

juncta (Puget), 2250

juniperorum, 1224

Kabathi (Ansorge), 2778

kabylica, 2622

Keckii, 1668

Kerneriana, 1760

kinnekullensis, 2676 .

Kitaibeliana, 1188

Kosinsciana(Bess.),2019

Krausii, 2779

Krockeri (Tratt.), 2542

labrellata, 2381

kaburnifolia, 1550

laeteglaucescens, 1682
Crepinia laeterubens, 1827

laevipes, 2046

laeviramea, 1172

laevis, 1440

laeviuscula, 1294

lagenaria, 2482

laguncula, 1635

Lamotti, 1350

lampococca, 2525

lampophvlla, 2506

lancaefolia, 1557

lancaeformis, 2092

lanceolata (Opiz), 1803

lancifolia, 1528

Langeana, 2306

Lannesiana, 1271

lanuginosa, 26\%2

Lapeyrousiana, 2597

lapidicola, 1447

laristanica, 1951

lasiophylla, 2750

latebrans, 1279

latebrosa'(Dés.), 2111

latedentata, 1306

latepartita, 1583

latiflora; 2133

latifolia, 2323

latipetala, 2224

latisepala, 1883

latispina (Boiss.), 2766

latistipula, 1779

laxepilosa, 2268

laxifolia, 1659

laxipila, 2361

laxistyla, 2086

laxula, 2145

Legallii, 2083

legionensis, 1602

leiocarpa, 2740

leiochlamys, 1450

leioclada, 2809

leiophlaea, 1835

leiosepala, 1636

leiostigma, 2705

leiostvla (Rip.), 1597

Lejeunii (Crép.), 1782

Lemaîtrei (Rip.), 2006

lenis, 2297

lenta, 1397

lentula, 2039

Leontonyx, 2477

lepidiflora, 1613

lepidota, 2314

leptocalyx, 2683

leptocarpa, 1639

leptoclada, 1691

leptodes, 1962

leptophlaea, 2259

leptostephana, 2534

leptosticta, 1724

Lerchii, 1143

leucantha, 2456

leucochlora, 2440
Crepinia leuconoma, 2194

leucostigma, 2395

leucostyla, 2174

leucoacantha (Debx) 2532

leucographa, 2688

leucotricha, 2313

leucophora, 2608

Leyboldiana, 2258

Leyi, 1176

Lightfootiana, 2640

limitanea (Crép.), 2110

litigiosa (Crép.), 1925

litterata, 2785

livonica, 2193

Lloydii (Dés.), 2732

lobulata, 2815

Loeflingiana, 1687

lonata, 2267

lonchitis, 2498

londimensis, 2312

longa, 1947, 48, 49

longecoronata, 1574

longifolia, 2647

longior, 1238

longiramea, 1670

longiscapa, 1462

longispina, 2371

longissima, 1830

longistyla, 2457

Iongituba, 1546

longiuscula, 2536

Loretiana, 2832

Loscosiana, 1514

Lucandiana (Dés.), 2798

lucorum, 1236

lucroniensis, 1631

ludibunda (Gren.), 2002

lugdunensis (Timeroy), 2115

lunata, 2572

Lundquistii, 2518

lustrata, 1685

lutetiana (LEm.), 1195

lupulina, 1406

luxemburgiana (Crép.), 1841

Maasii, 2406

macrantha (Desp.), 2775

macroacantha (Rip.). 1647

macrocalyx, 2416

macrocarpa (Boiss.), 2719

macrocarpa (Mérat), 1896

macrochlamys, 1497

macrodiscu $\div, 1768$

macrodon, 2008

macromegas, 2027

macrophylla, 2186

macrosepala, 2619

macrosphaera, 1157

macrostigma, 2462 
Crepinia macrostylis, 2288 macrotricha, 2673

maculisparsa, 1267

magna, 1938

Magnieri, 1134

magnoliaefolia, 2141

majuscula, 2028.

Malmundariensis (Lej.), 1729

mammosa, 1443

Mandonii (Dés.) , 1810

marcescens, 2000

Marchantiana, 1793

Maru, 2064

Martini, 1643

massiliensis, 1360

Massotiana (Debx), 2420

mastoidea, 1260

Mathonneti, 1541

Maukschii (Schult.Kit.), 2289

maxima, 2651

medioxima (Dís.), 1808

mediteranea, 1708

megacantha, $28: 30$

megalocarva (Dés.),2719

megalocarpa Gdgr, 2317

megalophylla, 1281

megalosepala, 1690

megalostigma, 2568

megalostyla, 1959

melanochloros, 1322

melanoidea, 1477

melanophylla, 2617

melastoma, 1302

mentacea ('Puget), 1559

mesocarpa, 2215

mesoclada, 1869

mesocycla, 18.10

mesomorpha, 2164

mesopoda, 1225

mesostema, 1989

micradena, 2101

microacantha, 1652

microbasis, 2478

microcalyx, 1625

microcarpa, 1377

microclada, 1383

microcoidea, 1468

micrococcos, 2249

micromegas, 1379

micropappa, 1421

microphylla, 2609

microrhyncha, 2015

microstyla, 1147

microtuba, 1914

microsphaera, 2175

millefoliata, 1605

minor, 1505

minuscula, 1480

minuta, 2213

minutifolia, 2808

minutiramea, 1626
Crepinia minutispina, 1203

niuutula, 2343

mitiramea, 2300

mitis, 1442

mitoacantha, 2810

mitoclada, 2496

modesta, 1466

modica, 1174

moesta, 2625

Monnetierensis (Bouvier), 2668

mentana, 2325

monticola (Rapin), 1213

montivaga (Dis.), 1500

Montsuiana (Chabert), 2187

Moraei (Crêp.), 2450

moraviea, 2105

Morelii, 1950

mortaniensis, 1654

mosqueruelensis, 1469

Motelayi, 1558

mucronata, 2818

mucronulata (Dés.),1148

multiceps, 1569

multispina (Puget), 1561

murcica, 2782

muricata, 2595

musearia, 2066

muscescens, 2147

mutata (Rip.), 2108

mutimensis, 2257

myacantha, 2357

myriocarpa, 1985

myriophylla, 1444

myriosticta, 1722

myriotricha, 1798

myrophylla, 1927

myrtilloides, 1483

myrtoides, 1451

naevulifolia, 1280

Naias, 1616

narbonensis, 1777

navarrensis, 2141

Neilreichiana, 2271

nematella, 2806

nematostipula, 1996

nemausensis, 2012

nervifolia, 1548

nervigera, 2411

nervosa (Crép.), 2791

nervulosa, 1744

nevadensis, 2101

nldulans, 1563

nigrescens, 1478

nigritella, 2624

Nisus, 2100

nitens (Desv.), 1177

nitidula (Bess.), 2073

nivescens, 2696

normalis, 1513

Notarisiana, 2331

nova, 2754
Crepinia nuda (Woods), 1364

nudicaulis, 2184

nudipes, $198 \%$

nummularioides, 2163

Nymaniana, 2:592

obattenuata, 1190

obcordata, 1576

obodowshiensis, 2777

obesa, 2216

objurgata, 2072

oblecta, 1231

obliqua, 26.18

oblonga (Dés.), 1679

oblonga Gdgr, 1465

oblongicalyx, 1679

oblongifolia, 1316

oblongigemma, 1879

Obornyi, 2763

obruta, 2633

obscura (Puget), 2107

obtusa (Rip.), 208

obtusifolia (Desy.), 2751

obtusifolio - tomentella

(Puget), 2755

obtusiramea, 1750

obtusispina, 1819

obtusiuscula, 2784

occulta (Crép.), 2111

oculus-Junonis, 1856

ochroleucoides, 2795

odontochlamys, 2269

odontoceras, 2116

odontophora, 2298

odontophylla, 1721

odontosepala, 1909

odontostephana, 1917

odontosticta, 2121

odontoxys, 1414

oelandica, 1476

oenensis (Kern.), 1599

oenophlaea, 1179

oenophora, 1437

oenophylla, 1721

olearia, 2389

oleiformis, 1507

oligoblephara, 1970

oligolepis, 1910

oligophylla, 1897

oligopoda, 1152

oligosticta, 1878

Oliveri (D baux), 2124

olivetorum. 2614

ololeia (Rip.), 1330

oncophylla, 2729

Onites, 2628

opaca (Fries), 1725

opaca (Gren.), 2375

opacifolia, 1981

operta, 2303

oporina, 1232

opportuna, 2102

opulenta, 2281

orbicularif lia, 2031 
Crepinia orocharis, 2063

orodendron, 222 1

orogenes, 2374

orophila (Rip.; Gren. ?) 2463

orthacantha (Kern.) 1181

orthochlamys, 2384

orthosepala, 2560

orthostephana, 1434

orthodon, 1776

ovatifolia, 1339

ovatipetala, 2285

oviformis, 1375

ovoidalis, 1343

ovoidituba, 1158

oxybasis, 2833

oxycarpa, 2634

oxyodon, 2041

oxypetala, 2409

oxyphylla (Rip.), 1173

Ozanonii, 1535

pachybasis, 2709

pachyearpa, 1906

pachyphora, 1257

pachyphylla, 2522

pachistema, 2583

pallescens; 1319

pallidevirens, 2315

pallidillora, 1572

pallidipetala, 2333

pallidula, 2181

Palomet, 1553

pampinosa, 1664

Pancicii, 1461

panormitana, 2485

papiensis, 2165

papillosa, 1767

papposa, 1838

pappostyla, 2180

paradoxa, 2146

parcespinosa, 1661

Pardoana, 1363

parietina, 1422

Parlatoreana, 2242

parva, 1921

parviceps, 2512

parvifolia, 1820

parvispina, 11 1

parvula, 2630

paseua, 1596

pascuicola, 1210

Patatii, 1282

patens, 1581

patentiramea, 2158

patula, 1284

paucispina, 2425

paupera, 233:

paupercula, 2479

pectinata, 1398

pectinella, 1663

peculiaris, 1136

peduncularis, 2264
Crepinia pelligera, 2579

pellita, 2219

Penchinati, 1929

pentecostes, 1901

perglobosa, 1836

permiscibilis, 1519

permixta, 1286

permutata, 1347

peroblonga, 1386

peronata, 1622

perscrutata, 2348

persimilis, 2541

perturbata, 1995

pesthinensis, 2152

petásites, 2570

petersburgensis, 1494

petiolaris, 2009

petiolulata, 1187

petrivaga, 1240

Perronii, 2571

phoenicantha, 1186

phoenicella, 1180

phoenicocarpa, 1487

phagnaloides, 2805

phalacroclada, 1880

phalacrochlam ys, 2728

phalacrostema, 1976

phalacrophlaea, 1846

phleboleia, 1812

phlebophora, 1979

pliyllochlora, 2664

phyllostephana, 2590

picacea, 1165

pietaviensis, 1930

pilifera, $2 \pi 17$

pilinaera, 2199

pilosa (Opiz), 2471

pilosella, 2228 .

pilosiuscula

(Opiz), 2307

pinetorum, 1301

pinguis (Crép.), 2292

pinivaga, 1850

pithyophila, 2375

placida, 2388

plagiophylla, 2507

platyanthos, 2744

platicalsx, 2021

platycarpa, 2550

platvodon, 1667

platipetala, 2390

platyphrlla (Rau), 2519

platyphylloides (Chab.), 2256

platrsepala, 2447

platysphaera, 1533

platistephana, 2408

platistigma, 2155

plebeia, 1832

plicata, 1245

plicatilis, 1806

pluriceps, 2391

podantha, 1221

\section{Crepinia podolica (Bess.), 1895}

podosticta, 2711

podostyla, 1868

podotricha, 2363

poetica, 1351

pogonata, 2138

pogonobasis, 1251

pogonopetala, 1464

polderiana (Crép.), 2680

polyacantha, 2607

polycephala, 1361

polyclada, 1849

polyodon, 1610

polyphlaea, 2091

polypoda, 1570

polysticta, 1920

pomeranica, 1419

porphyrocarpa, 2661

potens, 1986

Pourretiana, 1155

Pouzini (Tratt.), 2058

praecognita, 2540

praelonga, 1675

praemicans, 1262

praemissa, 1380

praeruptorum, 2829

praestans, 1233

prasina, 2631

pressa, 1413

pretiosa, 2093

primaria, 1285

pringyensis, 2097 .

privata, 1370

Proalii, 2232

proacutata, 2279

probata, 2129

prostrata, 1555

proteaefolia, 2733

provincialis, 2059

provirens, 1253

proxima, 2657

pruinosa (Baker), 2548

pruinovirens, 2549

Pruna, 1356

prussica, 1578

prunoides, 1615

pseudo-cerasus, 1609

pseudo-Chaberti, 2021

pseudo-fallax, 1192

pseudo-fertilis, 1902

pseudo-ochrolencoides , 2819

psilophylla (Rain), 2119

psilophylloides (Crép.), 2090

pteroidea, 2226

ptyloides, 2010

pubens, 1374

pubescens (Blytt), 2468

puberula Gdgr, 1491

puberula (Desv.), 2751

Pugeti, 1953 
Crepinia pulchra, 2698

pu vimaris, 2813

punctillata, 2678

purpurascens Gdgr, 1532

purpurasceus (Rip.), 1551 et 1944

purpurata, 1551 et 1552

pycnophylla, 1433

pyramidata, 1680

pyramidula, 1614

pyrenaica, 1568

pyrenaeicola, 2555

pyriformis (Dés.), 2364

Pyrus, 2364

Pyrrha, 2365

Queriana, 1637

racemulosa, 2231

radians, 1707

rugusina, 1150

ramealis (Puget), 2283

ramigera, 1795

Ramondiana, 1342

ramosissima (Rau), 1164

ramulosa (Godr.), 1255

ramusculosa, 2493

rarillora, 1467

rarispina, 1759

raripes, 1702

raristyla. 2418

Ratomsciana (Bess.), 2761

Rauii (Tratt.), 1954

Reboudiana (Debx), 2663

recognita (Rouy), 1826

redolens, 1387

religiosa, 1317

remissa, 2505

remota 2260

resecta, 23.46

reticulata, 2638

Reuteriana, 2047

rhaetica, 2600

rhenana, 255

rhipidoclada, 2564

rhipidodendron, 1618

rhoddantha 1823

rhodella, 1755

rhombifolia, 1811

rbynchantha, 2632

rhynchocarpa (Rip.), 1633

rhynchophora, 2197

rhynchophy6a, 1831

Richterii, 2214

rigididenta, 2315

rigidifolia, 2337

rigidior, 1703

rigidiramea, 1145

rigidula (Lagg. et Pug.), 2769

rivalis, 1175

robusta, 1211
Crepinia robustior, 2213

R. ffivieri (Chabert), 2783

romana, 1457

rorida (Puget), 1048

rosalis, 1698

roseillora, 1.403

rossica, 2206

Rostani, 2316

rostellata, 1321

rougeonensis (Crêp.), 1727

Rousselii (Rip.), 1939

rubelliflora (Rip.), 1797

rubens, 1765

ruberrima, 2103

rubescens (Rip.), 1790

rubricans, 1829

rubricolor, 1218

rubrillora, 1479

rubrifolia, 1198

rubripetala, 2481

rubristipula, 2393

rubronotata, 1867

rubrovirens, 2417

rutidula, 2780

ruinarum, 1686

Rupefortiana (Crép.), 2291

Sadleriana, 2311

salicetorum, 2434

saltivaga, 1394

Salvatoris, 1926

sambucifolia, 2551

sapida, 2470

Sapini, 2263

sarcobasis, 2096

sareophylla, 1802

sareplana, 2603

sarmentacea (Woods) 1875

sarmentoides (Puget), 1738

Savii, 1544

Saxatilis (Ster.), 2774

saxivaga, 1847

saxonica, 1588

scabrata, 1716

scabrella, 1320

scandica, 2604

scanica, 1263

seaphusiensis (Gremli), 2687

scapigera, 1370

Schergiana (Boiss.), 2730

schisticola, 2276

schistocarpa, 2318

Schneideri (Puget), 2786

Schottiana, 2080

scita, 1697

scleroacantha, 2593

sclerodonta, 2127
Crepinia sclerophlaca, 1908

sclerophilla, 1731

silerosteplana, 2451

scorodonia, 2331

scotica, 1.196

scotophylla, 1678

scrupea, 1.102

Sebastianii, 1589

secundaria, 2558

Segurae, 2156

semiglabra (Rip.), 2203

semihirta, 1372

seminuda, 2500

semipilosa, 2675

semitarum, 1817

senticosa (Achar.), 1181

separabilis (Dés.), 1290

sepium, 2314

sepivaga, 1316

septica, 1358

sequaniensis, 1209

serapionis, 1696

serbica, 1140

serrulata (Dess.), 1616

Serum, 1758

sicula (Tod.), 1676

Sierrae, 1556

silesiaca, 1432

silvestris (Rchb), 2665

silvularum (Rip.), 2324

similis, 2261

singularis, 1794

siphonoides, 1310

sisyrodites, 2041

slancensis (Panc.), 2025

Smithiana, 1870

smolandica, 1288

solstitialis (Bess.), 2588

somnians, 2089

Songeana (Puget), 2773

sorbifolia, ined.

sorbiformis, 1270

sparsa, 2131

sparsiglanda, 1276

sparsipila, 2198

sphaerica (Gren.), 1209 his

sphaerocalyx, 2212

sphaerocarpa(Pug.),2172

sphaeroidea, 1778

sphaerophora, 1612

sphaerostigma, 2590

sphoenoclada, 2362

sphoenogvna, 2817

spieant, 2290

spilophylla, 1317

spinifolia, 2038

spininaeva, 1391

spissa, 1.181

Spuria (Puget), 1436

squarrosa (Rau), 1753

squarrosula, 2837

stabiana, 1603 
Crepinia stauroclada, 2426

Steiniana, 2378

stenoacautha, 1189

stenobasis, 2642

stenocarpa, 2441

stenocaulis, 1269

stenoclada, 1876

stenocoma, 2825

stenodiscus, 1640

stenopetala, 2396

stenorhyncha, 1506

stenotuba, 2480

stephanocalyx, 2516

stephanodon, 1293

stephanomorpha, 2691

stephanophora, 2033

stictocarpa, 2078

stictochlamys, 2679

stictophora, 1813

stictopoda, 2713

stictosepala, 2768

stigmatophora, 2253

stigmatotes, 1833

stilbe, 2431

stilbocarpa, 2800

stipulacantha (Bast.), 2019

stipu'aris (Mérat), 1781

strangulata, 1600

strangulituba, 2442

streptoclada, 1590

streptomerpha, 2017

strictidenta, 1400

Stuartiana, 2182

Studniczka, 2469

stupencla (Crép.), 2719

stylaris, 1873

styloidea, 1891

stylosa, 2125

subalpina, 2629

subbiserrata, 2234

subcomposita, 2221

subconferta, 1393

subcontorta, 1540

subcordata, 1620

subdiscolor, 2567

subefiusa, 1593

Suberti (Rip.), 2007

subglabrata, 2161

subglauca, 2034

subglobosa, 1492

subgracilis, 1601

subhirta, 1439

subinermis, 1329

sublaevis, 2135, 2136

sublustris, 1579

submitis (Gren.), 2183

subnitens, 1272

subpyrenaica, 2520

subrectisjina, 1746

subsecunda, 1623

subtorta, 1438

subtrillora, 1709
Crepinia succedanea, 2107

sudetica, 1529

suecica, 2685

suevica, 1719

supracana, 2615

supranuda, 2299

supravirens, 1890

surculosa (Woods), 1960,

1961

Swartzii (Fries), 1715

synantha, 2796

syncarpa, 2476

syngenes, 2386

syngenoides, 2379

synstictodon, 1905

synphylloclada, 1695

syntrichopoda, 2162

tarraconensis, 2220

Tauscheri, 2645

telocarpa, 1646

Templetoniana, 2179

teneripes, 1239

tentaculata, 2734

tenuicalyx, 1843

tenuicarpa (Dés.), 1639

tenuicaulis, 1160

tenuicollis, 2585

tenuifolia, 1420

tenuior, 1331 bis

tenuiramea, 1773

tenuiscapa, 2068

tenuispina, 2035

tephrophylla, 2606

terebinthifolia, 1237

teretispina, 1168

terroris, 2340

texta, 1135

theratophila, 2589

thermophila, 1606

Thevenetii, 1369

Tholeyroniama, 2716

thuringiaca, 1378

Timbaliana Gdgr et Debx, 1411

Timeroyi (Chabert), 2115

tincta, 1800

tinctilis, 2403

tithymaloides, 2436

Todaroi. 2310

tomentella (Lem.), 2788

tomentelloides(Ozanon), 1780

tomentulosa, 2295

Tommasiniana, 2646

tomophylla, 2677

tomopoda, 2667

tomostigma, 2178

torrentum, 1611

torta (Puget), 2736

tortiramea, 1617

tortuosa (Wierzb.), 1943

Touranginiana (Dés. R.), 1291
Crepinia Tozza, 2274

transcaucasica, 2690

transito ria, 2222

transmota (Crép.), 1958

transsilvanica (Schur), 2737

triangularis, 1418

trichella, 1941

trichodonta, 2132

trichoidea (Rip.), 2727

trichoneura (Rip.), 2404

trichoneuroides (Puget), 2255

trichopetala, 1769

trichophora, 1283

trichopus, 2129

trichosepala, 2435

trichostigma, 1922

tridentina, 1651

tristis, 2618

truncatula, 2526

tnbicalyx, 1933

tubiformis, 1313

tubocycla, 2658

tubulosa, 1594

Tuezkiewiczii, 2210

Tullbergii, 1456

tumulina, 1520

turgida, 1218

turicensis, 1764

tyrolensis, 1523

Uechtritzii, 1266

Uhlwormiana, 1653

ulicicola, 1488

ulmaria, 2254

ulmiformis, 1340

ulmoides, 2088

ulullans, 1700

umbilicata, 1382

umbraticola, 1975

umbrigena, 1268

umbrina (Puget), 2201

umbrosa, 1751

uncinella (Bess.), 2211

uncinelloides (Puget) 2383

uncinula, 1344

unicolor, 2032

urbica (Lem.). 2413

urbicoides (Crép.), 2280

Utzka, 1629

vaccinioides, 1334

vaerendica, 2582

vafra, 1706

vaga, 1713

Vagiana (Crép.), 2612

vaginata, 1858

valdefoliosa, 1185

valdepubens, 1791

valentina, $20 \% 0$

vallesiaca, 2351

vallicola, 1241

Vapillonii, 2523 
Crepinia variabilis, 2\%08

vaudensis, 2317

Vayredae, 1367

venix, 2282

venosa (Sw.), 1749

veprecularum, 2535

Verloti (Crép.), 2106

veromanduorum, 2200

ver'suta, 1416

verticillacantha (Mérat), 2071

vexata, 2712

vexionensis, 1527

vialis, 2530

viarum, 1246

Villarsiana, 2524

rilligera, 1799

villinaeva, 2134

villipes, 1911

villistyla, 2816

villosiuscula (Rip.),1671

vimiualis, 1191

vinacea (Baker), 2094

vindobonensis, 2368

vinealis (Rip.), 1978

vinosa, 1404

violascens (Puget), 2304

virens, 1471

vireticola, 2399

viretorum, 1449

virginea (Rip.), 1689

virgosa, 2517

viridicata (Puget), 1834

viridiglauea, 1714

viridis, 1567

Visianiana, 1202

vitivaga, 1660

volhynica, 1587

Vratislaviensis, 1536

Vukotinovici, 2398

vulcanica, 1904

Wahlenbergiana, 2643

Warwichiana, 2376

Watsoni (Baker), 2752

Websterii, 2418

Wiesbaurii, $17 \% 4$

Willkommiana, 1825

Wimmeriana, 1446

wolgensis, 1261

xanthoacantha, 2053

xanthochlora, 1735

Xatartians, 2627

Zabelii, 2338

Zetterstedtii, 2686

Zubiae, 1453

Hultemia berberifolia (Pall.), 4266

Laggeria argutidenta, 518

bicolor (Jaci.), 510

Bungeana (Boiss.), 513

caesarea (Buiss.), 5331
Lasgeria cerea (Roess.), 514

chlorophyfla (Ehrh.), 533

chrysocephala, 538

chersonensis, 539

campanulata (Ehrh.), 546

dichroopetala, 535

eglanteria (L.), 544

fallax, 552

flava (Wickstr.), 542

foetida (Herm.), 544

glaucophylla (Ehrh.),537

hemisphaerica (Herm.), 537

ignescens, 536

lasiopoda, 551

lutea (Mill.), 532

punicea (Mill.), 534

Rapini (Boiss.), 549

sulfurea (Ait.), 541

turbinata (Ait.), 517

Wuitelii, 550 .

vulpina (Wallr.), 532

Yellowiana, 545

Ozanonia abieticola, 830

acanthocarpa, 1093

acanthophlaea, 749

acanthophora, 742

acieularis (Lindl.), 900

aciphylla, 1116

aciphylloides, 957

actinosepala, 931

adenophora, 1013

adenopoda, 941

adjecta (Dis.), 724

admirabilis, 1100

adriatica, 806

adscita, 790

adversa, 991

Allionii, 1089

alpestricola, 839

alpestris (Dés.), 846

alpigena, 1067

alpina (L.), 724

alpino - pimpinellifolia

(Reut), 803

alpiphila (Arvet), 1051

alnivaga, 998

amoenevirens, 751

Amphora, 832

amplifolia, 1109

amygdaloides, 730

Andrewsii (Tratt.), 744

angustifolia, 813

angustituba, 770

anisopoda, 812

apennina, 820

applanata, 836

aragonensis, 1038

argutifolis, 975

ariaefolia, 807

aristata (La).), 89

arre:nensis, 1003
Ozanonia assurgens, 1125

atrovirens, 956

attenuatifolia, 825

aucuparioides, 715

auriculata, 85]

aurosica, 1110

austriaca, 877

bactiacensis, 811

Balfouriana, 1008

baltica, 1039

Bellardii. 921

Bertolonii, 779

beugesiaca, 815

biadena, 734

Bichetii, 936

binata, 973

biserrata, 1070

blepharostephana, 870

bombax, 950, 954 et 963

bombycifera, 991

Borbasii, 722

Borderi, 878

borealis, 964

Braunii, 1022

brevifolia, 881

brevitrons, 876

brevisepala, 754

brevituba, 800

brigantiaca, 1097

brutia. 781

caballicensis(Pug.), 1018

calcarea, 1015

caledoniae (Borrer), 987

calopetala ined.

calycina, 971

calyptopoda, 1080

camptopoda, 865

campylacantha ined.

cantalica, 101]

carelica (Fries), 740

Carlscronae, 1053

carniolica, 974

carthusianorum, 718

catalaunica, 788

caturigensis, 1073

cebemensis, 939

celsicola (Arvet T.), 908

cenisia, 746

cerasifera, 912

cerasoides, 929

Chaixiana, 1006

Chavini (Rap.), 1122

clavata, 948

clinocarpa, 816

coerulescens ined.

collecta, 817

colorata, 855

complicata (fren.), 1016

condensata, ys3

conglobata, 10-44

congregata, 733

conjurata, 906

conocalys, 915 
Ozanonia conostyla, 953

coronularia, 856

crassifolia, 781

crassispina, 1050

crataegina, 966

cremocephala, 808

Crepini (Mieg.), 1042

Crepiniana (Des.), 926

croatica, 789

cuneata, 873

cuspidata, 1066

cuspidens, 1031

cycla, 928

cyclophora, 1048

cylindrica, 859

cylindrocarpa, 841 et 719

dalmatica, 761

danica, 1004

dasycarpa, 1096

Debeauxii, 795

decipiens, 725

Delasoii (Lagg.et Pug.), 1060

delphinensis, 940

demissa, 818

desertorum, 898

detonsa, 728

dictamnifolia, 858

didymorhyncha, 1117

dimorpha, 958

dinarica (Vis.), 805

discreta (Rip.), 1106

dissimilis (Pug.), 1132

djimilensis (Boiss.), 802

dryophila, 976

Ducrosii, 925

eccremocarpa, 831

Echidne ined.

echinulata, 866

editicola, 1112

editorum (Rip.), 846

effugiens, 869

elabens, 943

elegantula, 741

elisocarpa, 753

eminens, 1056

Enwaldii, 890

eriostyla, 1009

ervthrocaulon ined.

fagetorum, 959

falcata (Pug.), 985

falcatula, 1082

fallacina, 989

farinosa, 909

fausta, 756

fennica, 885

ferruginea (Host), 908

ficoides, 1010

filispina (Debx), 797

fontinalis, 840

Frevnii, 1057

frigida, 739

fugax (Gren.), 1136
Ozanonia fusiformis, 1108

Gibellii, 935

glabrior, 861

glandulifolia, 827

glandulosa (Bell.), 1090

glauca (Vill.), 1051

glaucescens(Wulf.), 1040

glaucoalba, 951

glaucopurpurea, 799

globosa, 793

Gmelini (Bge), 896

gothica, 1078

gracilifolia, 826

gracillıma, 760

gracilis, 727

grandifolia, 1061

gratiosa, 1133

gra $i$ ensis, 736

Gravetii (Crép.), 937

gravida (Puget), 946

Grovesii (Baker), 920

guttensteinensis (Jacq. f.) 908

gymnopoda, 968

gymnostephana, 783

Hacquetii, 1035

Hampeana (Gris.), 1107

hastulata, 947

Haussknechtii, 1081 bis.

helvetica, 918

heterocarpa, 945

hirsuta, 889

hirtula, 880

hispanica, 1062

hispida (Kroch.), 792

hispidissima, 1105

horripila, 772

hyposticta, 833

icmadophylla, 1084

idionopoda, 822

Ilseana (Crép.), 919

inponens (Rip.), 1046

inclinata (Kern.), 1124

imcumbens, 771

incrassans, 1033

inermis (Mill.) 743

inflata, 853

inflaticalyx, 1111

intercalaris (Dés.), 846

interposita, 867

intricata (Dés.), 804

ircutiana, 897

istriaca, 905

italica, 961

jugorum, 842

juratensis, 757

Kerneri, 934

Kitaibelii, 891

laciniata, 768

lagenaria (Vill.), 766

lageniformis, 764

Lamotti, 911

Lapeyrousii, 895
Ozanonia lapponica, 884

latebracteata ined.

latibasis, 970

latidens, 810

laxa (Retz), 848

leiantha, 829

leioclada, 955

leiosepala, 838

leiostyla, 1059

Lereschii (Rap.), 1128

Levieri ined.

lissinensis, 875

livida (Host), 979

longepedunculata (Dela.

soie), 1085

longicalyx, 852

longicuspis, 823

longipes, 767

lindens, 980

Lundquistii, 988

lurida (Andr.), 981

macrocarpa, 996

macrosepala, 962

macroseta, 1095

macrosphaera, 999

macrostephana, 933

macrostigma, 1019

marginata, 997

Mariana, 729

Mathonneti, 910

megacarpa, 824

megaphylla, 1101

megathamnos, 723

micradena ined.

micronuegas, 1041

Miegevillei, 1042

mira, 844

misera, 752

monspeliaca (Gou.), 780

montana (Chaix), 1118

montenegrina, 879

montosicola, 1055

moravica, 1064

Morthieri, 984

multisecta, 1114

mutinensis, 972

myriodon, 1126

myrioseta, 1088

navarrensis, 847

Neilreichiana, 773

nervifolia, 1034

nervulosa, 786

nutans, 814

obesa, 1091

oblongifolia, 1081

obstinata, 863

obtruncta, 750

obtusata, 747

odontostephana, 787

opacifolia, 1069

oplisthes (Boiss.), 801

oppositispina, 1102

Orbanisiana, 874 
Ozanonia oreinosa (Rip.), 83.5 orthosepala (Schimp.), 982

ovata, 1002

ovoidalis, 785

oxyodon (Boiss.), 872

oxyodonta, 1092

pallidifolia, 922

pallidipes, 721

patentíramea (Debeaux), 1121

Parisii, 1072

parvillora (Lap.), 895

parvifolia, 1077

pedemontana, 94

vedemontii, 917

Pelletii (Debx), 952

pendulina (Ait.), 809

pennina (Delasoie), 987

permixta, 763

Perrieri (Songeon), 1120

personata, 1052

petersburgensis, 882

petiolulata, 1023

phoeniceoclada, 837

pilatensis, 942

pilosa, 1032

pilosula, 886

pimpinellifolio - alpina (Ray.), 868

pistoriensis, 8.13

platycarpa, 1079

platyphylla, 737

platystigma, 1021

plurillora ined.

podophylla, 1123

politula, 759

polyclada, 1017

Portenschlagiana(Tratt.), 776

praegracilis, 949

Preslii, 883

propria, 932

provincialis, 923

pseudo-cerasus, 990

pseudo-suberistata, 1043

pubescens, 893

pubipes, 735

pubinaeva, 821

pulcherrima, 930

purpurans, 969

pycnacantha, 798

pyrenaica (Gou.), 849

rarispina, 995

Ravaudi (Boullu), 1083

refracta, 992

repressa ined.

Requimiana, 828

retusa, 1099

Reuteri (Godet), 987

Reynicri (Hall. f.), 1118

rhaetica (Kern.), 804
Ozanonia rhynchocalyx, 1021

Rostani, 1087

ruberrima, 907

rubicunda (Hall. ..), 908

rubiginosa, 762

rubrifolia (Vill.), 908

rubronotata, 978

rupestris (Crantz), 748

Ruprechtiana, 899

salaevensis (Rap.), 1026. 1027 et 1036

Sambrancheriana (Delasoic), 1118

Sanctae-Balmae, 1058

sarcocarpa, 769

sarcostephana, 8.15

Savii (Puget), 794

saxea, 834

scandica, 967

Scheutzii, 1005

Schlosseriana, 913

Schultzii (Rip.), 927

sciacephala, 921

scotica, 726

semidecidua, 1030

septorum, 1130

sericonaeva, 977

serratidenta, 1103

sessilis, 1054

seticalyx, 791

setipes, 993

setisepala, 871

setulisepala, 1020

sfakiensis, 1012

Sieberi, 1131

silesiaca, 901

Sipho, 730

siphuncula, 864

sitiens, 1025

smolandica, 1037

sparsispina, 765

sphaerica, 778

spiniramea, 965

spinosa ined.

stenocalyx, 1098

stenocarpa ined.

stenoriphaea, 755

stephanocarpa (Dés. et Rip.), 1074-76

stictochlamys, 1000

stictophrlla, 758

subalpina, 862

subcrassa, 797

subcristata (Baker), 1045

subretusa, 1094

subrotunda, 782

subsessilis, 1016

sudetica, 1127

suprapilosa, 1113

Srmii, 1029

tentaculata, 1063

tenuipubens, 887

testudinaria, 1119
Ozanonia thuringiaca, 1065

tomentella, 904

tortidenta, 775

transiens, 986

trichocarpa, 860

trichopoda, 819

tridentina ined.

Tullbergii, 1068

Turrae, 774

tyrolensis, 914

uberta, 1001

Uechtritzii, 1007

Utzka, 731

vagans, 1014

valbonnaisiensis, 1115

valderia, 902

vallesiaca, 1086

vaudensis, 857

Vay redae, 720

ventricosa, 960

verticillaris, 1071

vestita (Sternb.), 1017

vesula, 738

Villarsii (Tratt.), 851

villosa, 903

vinosa, 916

Visianii, 850

volemus, 777

vogesiaca (Moug.), 1051

Vrabelyi, 888

Vukotinovicii, 892

vulcanorum, 1129

Wolfii, 1104

Wulfenii (Tratt.), 796

Zabelii, 1049

Zetterstedtii, 1028

Pugetia aberrans, 3815

abietina (Gren.), 4024

acanthochlamrs, 4265

acanthotuba, 3855

acinacifera, $\mathbf{4 0 1 0}$

acmophylla, 4208

acrophila, 3890

acutata, 3974

acutifolia, 3999

adoptata, 4064

aequidentata, 3853

affinis, 3972

albescens, 3905

albicans (Godet), 4090

albicantior, 3952

albida, 3969

albifolia, 3967

albispina, 4118

Allionii, 4132

alpestris, 4162

alpicola, 4210

alsatica, 4126

Ambrosiana, 4165

amicta, 4139

amoena, 3917

amphorica 117,4251 
Pugetia amplexifolia, 4017

analoga, 3867

ancyclacantha, 4181

Andersoniana, 41\%0

Andrzeiowil (Bess.), 4104

anglica, 3940

angusticollis, 3862

annesiensis (Dés.), 3946

anserina, 4021

apennina, 3903

arduenensis (Crép.), 4239

argentea, 3824

arvernensis, 4171

asperifolia, 4019

asphaltitica, 3981

Aublancii, 3856

australis (Kern.), 4182

Baenitzii, 4177

baetica, 3959

Bakeri, 4121

bakerioides, 4109

Balansaea (Dés.), 3851

balsamica (Bess.), 4114

baltica, 3993

barbata, 3926

barbigera, 3852

basaltica, 4092

bavarica, 4059

Bellardiana, 4133

Benthamiana, 4074

bernensis, 3970

Berneti (Schmidely), 4243

Besseriana, 3823

Billotiana (Crép.), 3938

Bloxamii, 3923

bohemica, 3985

Boissieri (Crép.), 4081

bombrcina, 3988

borealis, 4167

Borkhausenii (Tratt.), 3939

Bovernierana (Crép.), 3877

brevior, 4103

britannica, 4005

caduca, 4159

calostephana, 3916

camberiacensis (Dés.) 4256

cambrica, 408 -

cana, 4013

candicans, 3822

candida, 4168

cantalica, 3897

capnoides (Kem.), 4053

carpinifolia, 3857

carthusiana, 387

catalaunica, 3929

cechica, 4236

Cembra, 3924
Pugetia cenisia, 4107

cephalorhyncha, 3975

chlamydopetala, 3843

ciliato-petala (Bess.), 4077

cineracea (Crép.), 3830

cinerascens (Dum.), 3834

cinereifolia, 4063

cladophora, 4186

cladotricha, 4211

cluniacensis, 3848

Clusiana (Bouvier), 4192

coccinea (Rupr.), 4062

cochlearis, 3962

cognata, 4076

colligena, 4113

collineola, 3901

collivaga (Cottet), 3870

commutata (Scheutz), 4261

condensata, 4038

confusa (Puget), 3973

conoidea, 3900

consobrina, 4129

cormubiensis, 3927

corrivalis, 4055

Cotteti (Lagg. et Pug.), 4248

crassibasis ined.

crassifolia, 4051

crassinervia, 4152

crassiuscula, 3911

cremsensis (Kern.), 4197

crinita, 4054

cuspidata (II. B.), 4052 cuspidatoides (Crép.), 4012

cyclocephala, 4056

danica, 4044

dealbata, 4117

dealbescens, 3980

decipiens, 4004

decurrens, 4204

Delarbreana, 4171

delphinensis, 4119

denudata, 3861

depicta, 4039

depilata, 3835

Deucalion, 4026

Dicksoni (Lindl.), 4215

dictamnifolia, 4262

dictamoides, 4134

didymantha, 4160

dimorpha (Bess.), 3971

discolor, 4146

divergens, 4096

dodrantalis, 3937

dubia (Wibel), 3920

dumetivaga, 3861

dumosa (Puget), 3849

eboracensis, $40 \% 5$
Pugetia Echidne, 4252

echinocalyx, 4212

echinophora, 4142

Echinops, 3908

echinostephana, 4223

elegans, 4143

elongata, 4029

elymaitica (Boiss.), 4255

emarginata, 3878

erioclada, 3865

eriophlaea, 3925

eriopoda, 3944

eriosa (Rip.), 4009

espinea (Puget), 4249

farinosa (Rau), 3930

fennica, 4060

fimbriata (Gremli), 4022

fionica, 4002

flavispina, 4065

flexicaulis (Godet), 4091

flexilis, 4008

flexuosa, 4122

floccida (Dés.), 4018

flos-jovis, 4147

foetida (Bast.), 4029 bis

Foudrasi, 4253

Foxiana, 4250

Fraseri, 3992

fraxinifolia, 4047

friburgensis (Lagg. et Puget), 4263

Friesii, 3963

galbanifera, 4241

Gaudini (Puget), 4161

Garana (Walir.), 4190

Gelmii, 4188

Gisleri (Puget), 3966

generensis (Puget),4022

germanica, 4226

Gibellii, 4106

glabrescens (Dés.), 4256

glabricalyx, 4185

glabripes, 3817

glabriuscula, 3955

glandulifolia, 4030

glandulosella (Rip.), 3997

globifera (Puget), 4257

globularis, 3921

gombensis (Lagg. et Pug.), 4237

gothica, 4069

graeilescens, 3868

grandifolia, 4035

Gremblichii, 3991

Grenieri (Dés.), 4105

grossa, 4191 et 4195

haesitans, 3899

hallensis, 3901

Harbordii, 4010

hebecarpa, 3876

hebeclada, 3838

hebephora, 3968 
Pugetia Heldreichii (Boiss.), $42: 32$

helvetica, 3995

heterophylla (Woods), 4179

hereynica, 3869

helvetica, 3995

Hervieri, 3814

hibernica, 3949

hircina, 3918

hirtella, 4244

hirtiramea, 4119

hirsutissima, 3831

Hohenackeri, 1037

holmiensis, 4102

Hookeriana, 3915

horrida, 4176

horridula, 4216

Hudsoniana, 3907

hypoleuca, 4097

ianthinochilora, 4023

iberica (Stev), 4231

idanensis, 3951

incana (Kit.), 3875

incanescens, 3825

incerta, 3839

incumbens, 4028

indistincta, 3828

intlatipes, 4166

inframollis, 3886

ingrica, 4062

inornata, 4000

iusidiosa (Gren.), 3998

insula is, 4101

intromissa (Crép.), 3881

iodacantha, 4095

italica, 4093

jonides, 4225

jucunda, 4145

jugularis, 421

jurana (Dés.), 4256

Kinnekullensis, 4173

Kolenatiana, 4235

Krauseana, 1213

lactescens, 3858 et 3859

laevipes, 3893

lanata, 4089

Langei, 4203

lannginosa, 3829

lasioclada, 3978

latifolia, 3898

latissima, 4209

Lecoquii, 4229

Ledebourii (Spr.), 3953

leiantha, 4125

leiocalyx, 3892

leiocarpa, 4130

leiogyna, 4124

Lemana, 4259

lentula (Puget), 4085

leptoclada, $3816^{\circ}$

leptodendron, 4128

leptophylla, 4180
Pugetia leptostigma, 4016

leucophylla, 3832

libanotica (Boiss.), 4207

Lightfootiana, 4l6-

Lingua, 3817

livenica, 3956

longifolia, 3871

longispina, 3986

londinensis, 4027

Lorentii, 4072

Lundquistii, 1149

luteovirens, 4006

macrophylla, 4163

macrostephana, 3989

Magnieri, 4031

Marcyana (Boullu), 3882

Nathonneti, 3820

melanocerasus, 3913

mespiliformis (Debx) 4219

micans (Dés.), 3825

microcarpa, 4137

minor, 3933

minuta (Bor.), 4067

mitis, 3932

mollissima (Fr.), 4079

Mooreana, 3912

multivaga (Dés.), 4256

Murithii (Lagg. et Pug.), 4258

Murrayana, 4111

naevulifera, 4057

nemoricola, 3947

nervulosa, 3990

nitida, 4048

normalis, 4189

normanniana, 3983

obesa, 4110

oblengella, 4066

oblongifolia, 4014

odontostephana, 4123

oelandica, 4220

olens, 4050

oligacantha, 4088

oligocephala, 4260

omissa (Dés.), 4127

oophora, 4115

opobalsammm, 4221

oreites, 4068

orophila (Gren.), 4025

osmodendron, 1198

Otites, 3888

oxyodon, 4230

ovata, 4156

ozanonioides, 4154

pachyphylla, 3850

pallidifolia, 4178

pallidula, 3889

bannucea, 4148

Parlatoreana. 4201

parvifolia, 4151

patula, 3996

Patzii, 4153
Pugetia pedemontana, 4081

pellita (Rip.), 3854

pergracuis, 3902

permutata (Rip.), 3879

P'erusiana ('Timb.), 4105

petersburyensis, 406

phalacrocarpa, 4058

phalacroidea, 4254

phalacrostigma, 3928

phlebotricha, 3881

phyllostephana, 388 i

pistoriensis, 409.1

platystemma, 1238

poliochlora, 3910

pomifera (Herm, , 40\%3

pomifero-alpina (Christ), 4240

pomifero - cinnanomea

(Christ), 42:37

pragensis, 3984

properata, 384

propinqua (Dés.), 4256

proxima (Cottet), 4202

psammophylla, 4031

pseudo-acutata, 3976

pseado-albida, 3957

pseudo-rubıginosa (Lej.) 4043

pseudo-Seringeana, 4007

pubiramea, 3977

pulchra ined.

pulposa (Crép.), 3872

pulverulenta (M. B.), 4234

purpurea, 4001

pustulosa (Bert.), 4233

pyrenaica, 3964

pyrifera, 4228

Ravauxii, 4086

recondita (Puget), 4175

regria, 3863

resinifera, 4210

resinosa (Sternb.), 4196

resinosoides (Crép.) 4157

Reutermani, 4078

rhipidoacantha, $\$ 100$

rhynchocarpa, 4205

rhynchophora, 3960

rigescens, 4194

rigidifolia, 3936

rigidispina, $3982^{\circ}$

roseipetala, 3810

rosella, 3880

rossica, 3860

Rostani, 3866

rotundata, 3837

rugosula (Sw.), 4183

Ruprechti (Boiss), 4070

sabauda, 4261

salaevensis, 4212

sambucifolia, 4016

sarcocarpa, 4108 
Pugetia Sauzeana, 3961

sazonica, 3894

scabida (Puget), 4217 seabriuscula (Winch.), 4216

scandinavica, 4199

scanica, 4120

scanorum, 4222

Scheutzii, 3942

scotica

semi-incrassata, 4158

semproniana (Favrat), 4243

sericans, 3842

sericophylla, 3914

Seringeana (Godr.), 4011

setosa, 3909

setuliflora, 4225

silesiaca, 4041

similis, 4140

smolandica, 4112

sphaerica, 3836

sphaerotuba, 3919

spinescens (Christ), 4172

spinulescens, 4187

spinulifolia (Dematra), 4256

stenophylla, 3954

stenosepala (Christ), 4245

Stewartii, 4098

Straehleri, 4155

strumosa, 3965

Stuttgartiae, 4036

subactinacantha, 3833

subalbida, 3873

subalpestris, 4082

subglobosa (Sm.), 3922

subhirsuta, 3994

sublaevis, 3987

subsericea, 3826

sudetica, 4136

suecica, 4131

tenera, 3895

tentaculata. 3891

tenuifolia, 4116

tenuis, 3958

tenuispina, 4150

thamnophila, 3979

Thiebautii, 4003

Thielensii, 4032

thurifera, 4193

thuringensis, 3945

tomentosa (Sm.), 3950

tomentoso-sepium(Ch.), 4033

Trachynella, 4247

trichoclada, 4020

trichopoda, 3819

trichosticta, 4045

tridentina, 3906
Pugetia Tullbergii, 3896

tunoniensis (Dés.), 3948

turbinata, 4206

turicensis, 4169

tyrolensis, 3943

ulmoides, 4083

umbraticola, 3941

uriensis (Lagg. et Pug.), 4245

valdensium, 3818

vallesiaca, 4138

Vanheurckiana (Crép.), 3821

velutinaefolia, 3883

ventricosa, 4099

vesontiaca, 4012

venusta (Scheutz.), 3942

villicaulis, 3827

virens, 3885

viridifolia, 4200

Vockii, 4015

vogesiaca, 3934

volhynica, 4227

vratislaviensis, 4181

Websterii, 3935

Wimmeriana, 4135

Wolfii, 4071

Xatartii, 4144

Yretotensis, 3931

Zabelii (Crép.), 4218

Zetterstedtii, 4145

Ripartia abyssinica (Salt.),

aciculosa ined,

adenantha, 39

adenoclada, 118

adenophora, 92

admixta, 16

adriatica, 187

aestuans, 49

affinior, 8 .

africana, 51

albens, 23

- algeriensis, 34

alpestris, 166

amici, 90

anceps (Bouteiller), 290

anglica, 141

anisodonta, 280

apennina, 247

apta, 157

arborea (Oliv.),

armoricana, 195

arundana, 71

arvensis (L.), 150

asturica, 153

atrovirens (Viv.), 75

australis. 283 bis

austriaca, 181

Ayreshirea (Hort.),

baldensis (Kern.), 192

baleariea (Desf,), 88
Ripartia barbulata, 226

barcinonensis, 36

basaltica, 130

bavarica, 183

Beggeriana (Schrenk.)

belonoides, 138

belophora, 128

Bertolonii, 13

bibracteata (Bast.), 102

bichroa, 159

bichroophylla ined.

biscayensis, 53

Bompardii, 67

bonensis, 74

brachystylis, 22

brevidens, 38

brevistipulata, 219

brevistyla (D. C.), 251

Briggsii, 179

Broteri (Tratt.), 45 ,

Brownii (Spr.)

Brunonii (Lindl.),

burdigalensis, 4

calabrica, 82

Caldesii, 3

calopoda, 235

calostyla, 285

cantabrica, 20

carnatula, 275

carniolica, 190

catalaunica, 32

Caruelii, 63

cebennensis, 116

cerasoides ined.

Chaubardiana, 6.5

Chouletti, 85

ciliolata, 276

Clotildea (Timb.), 281

collinita, 243

colorata, 212

commisserata, 169

confertiramea, 173

congesta (Rip.), 278

congruens ined.

conifera, 140

conspersa (Puget.), 256

conspicua (Bor.), 100

contemnata, 227

controversa ined.

cordatıfolia, 12

coriacea, 10

corsica, 86

croatica, 191

cucurbitacea, 149

cuspidifera, 267

Cutandaeana ined.

crelocarpa, 113

dalmatica, 80

decalvata, 277

declinata, 272

deducta, 233

densifolia, 44

dichrosepala, 237 


\section{$-315$}

Ripartia Dierbachii, 202

dilatans, 11

dioxys, 142

discolor, 18-1

dumeticola, 19.1

dumetigena, 199

Dupontii (Dés.), 94

Durandoi, 87

durifolia, 127

elegantissima, 119

eriopoda, 98

erratica (Rip.), 263

erronea (Rip.), 151

erythroclada, 109

evolvens, 144

exigua, 279

fastigiata (Bast.), 218

faventina, 25

ficta, 68

limbriata, 14

firmata, 248

fissidens, 273

flaccida, 250

florida ined.

Fraseri, 120

Freynii, 40

fusca (Fl. Wett.), 150

gallicoides (Dés.), 121

Gandogeriana (Deb.), 62

Gariodiana, 25;

Garroutei (Pug.), 266

ga-umnae, 6

glabrescens, 174

glabrifolia, 107

glabripes, 245

glandulifera (Crép.), 117

glandulifera (Roxb.)

glaucocarpa, 26-1

globigemma, 30

globulifera (Rip.), 274

grandis, 46

gratianapolitana, 156

Gremlii. 257

guestphalica, 182

Gussonii, 186

Halleri (Revn.)

herporhodon (Ehrh.), 114

heterogyna, 281

heteropoda, 37

hibernica, 101

hirtella, 2

hispanica, 89

hispidobasis, 211

humifusa ('Tratt.), 111

ianthinacantha, 239

idanicola, 211

idionocarpa, 176

immitis (Dís), 270

impressa, 163

inaequevillosa, 136

inarimensis, 66

infrapubens, 188

insignita, 222
Ripartia intybacea, 228

ischnophylla, 152

istriaca, 69

jacens, 175

Kabylica, 33

Keckii, 1.18

Kenmmleri, 158

Kiibleri (Crép.), 283

laetevirens, 225

lamprotes, 236

latidens, 139

laxistigma, 250

Lebelii, 210

leiophylla, 101

leiosepala, 185

lentiscifolia, 253

leptodon, 168

leptostyla, 5

Leschnaultiana (Red. et Th.)

leucochroa (Desv.), 251

longicuspis (Bert.)

longipes, 7

Luciae (Franchet),

lugens, 201

lusitanica, 64

macrocarpa, 103

macrodon, 147

macrostrla, 58

maderensis, 271

madritensis ined,

marcescens, 165

mauritanica, 43

mediterranea, 48

meridionalis, 26

micans, 28.

microdon, 242

microphyila (D. C.), 27

minoricensis, 56

modesta (Rip.), 244

moschata (Yill.), 95

multiflora (Reyn.),

Munbyana, 50

murciea ined,

myriadena, 11

myriolepis, 223

neapolitana, 70

neglecta, 258

nigrovirens, 214

nivea (Dup.)

Neotria, 115

nudata, 162

numidica, 76

obesa, 217

oblongifolia, 215

obtusata, 105

oceanica, 238

odontophora, 177

oenocallos, 205

oenophylla, 198

olivetorum, 42

opsostema (Ehrh.), 96

ovata (Lej.), 150
Ripartia ovatifolia, 209

ovato-cordata, 208

oxvihylla, 178

pacliyacantha, 229

palmensis, 91 bis

panormitana (Tod.), 93

parrifolia, 59

parvula (Sauzé et M.) 287

patellaris, 286

peruotata, 2:9

pervirens (Gren.), 18

phatacropada, 161

phoenicia (Boiss.), 91

phrilomegas, 17

pinicola, 171,172

pinnatula, 19

pisiformis, 126

pleiantha, 210

plurillora, 35

podophylla, 289

pogronophylla, 232

Pollichii, 213

polysticta, 81

probata, 132

prostrata (D.C.), 9

proxima, 73

prunella, 108

puberula Gdgr, 211

puberula (Rip.), 23\%

pusilla (Rip.), 279

ragusina, 57

raripila, 180

recumbens, 137

reflexifolia, 221

repens (Scop.), 164

reptans (Crép.), 122

Requienii, 83

rhenana, 203

rhynchocarpa, 77

rigescens, 269

Rodriguezii, 52

romana, 79

Rouxii, 29

rubripes, 261

rubrisepala, 231

ruscimonensis (Gren.), 2

rusticana (Dés.), 268

scandens (Mill.), 60

Schimperiana (A.),

sclerothamnos, 1.43

semi-calva, 246

semi-nuda, 155

semi-pubens, 145

sempervirens (L.), 81

seperina (Dís, ?),

sepicola, 154

seposita (Dés.), 285

septicola. 133

servens (Fihrh.), 150

serpenti-canina, 251

setigemma, 5

silvulicola, 167 


\section{$-316$}

Ripartia singularis, 262

soli ia, 249

sphaerica, 123

spininaeva, 170

stabiana, 72

staminosa, 31

stenobasis, 216

strata, 193 et 197

stylosa (Desv.), 234

subbiserrata, 124

subcinerea, 135

subintegrisepala, 129

subovata, 255

subrecta, 110

subumbellata ( Schur.), 99

syntrichostyla (Rip.), 268

systyla (Bast.), 254

Talenceana, 207

tamnoides, 196

terracina, 78

thamnocharis, 230

Thumbergii (Tratt.),

Todaroi, 61

tomentelloidea, 288

Torrepandoi ined,

Tratinickii, 265

tridentina ined,

tu , furiorum(Willd), 150 ?

tyrolensis, ined.

umbellifera, 131

umbilicata, 112

umbrosa (Kip.), 146

vacillans, 260

variifolia, 55

Vayredae, 28

velutina, 189

Verrieti, 1

villosa, 106

vireticola, 15

virginea (Rip.), 281 ?

viridula, 200

Visianii, 47

viticula, 160

vogesiaca, 134

Wichurae (C. Koch.),

Zubiae, 206

Rosa (eu) abortiva, 309

actinacantha, 316

acutiformis, 321

adducta, 357

adpres sa, 346

adriatica, 441

aеqua, 389

aggrea, 313

agrestis (Gm.), 372

ailanthifolia, 393

albida, 345

ambigens (Gremli,), 343

amoenicolor, 364

apennina, 354
Rosa (eu' apiculata, 361

approximata (Dés.), 365

arenivaga (Jullien), 409

arnassensis, 385

arvensi-gallica (Gremli), 343

arvina (Schwenk.), 296

aspernata, 360

aipreticola (Boullu), 330

assimilis (Dés.), 430

atroviridis, 383

attenuatocalyx, 420

austriaca (Cr.), 376

Axmanni (Gm.), 291

badensis, 372

Balbisiana, 419

belgica (Mill.), 451

betulina, 423

bichroophylla, 382

bohemica, 448

Boraeana (Béraud), 348 canino-gellica (Gremli), 343

cantabrica, 428

cardiophyllos, 430

cartilaginosa, 300

cebennensis, 337

centifolia (L.), 435

Champagneuxii, 434

chlorella, 335

commixta, 350

concessa, 421

congestifolia, 429

conica (Chabert), 342

cordata (Cariot), 430

cordifolia (Host), 437

coryliformis, 318

cribrosa, 450

croatica, 347

curtistyla, 293

cuspididenta, 427

cyclotuba, 397

Czackiana (Bess.), 305

damascena (Mill.), 443

debilior, 329

deci iens (Bor.), 384

dendroidea, 341

densiramea, 405

dentifera, 332

desiderata, 297

dichroantha, 402

dilecta, 362

dimorphophylla, 459

diospyros, 407

disseminata, 317

ebracteata, 324

echinantha, 442

echinoclada, 302

echinophlaea, 449

echinophora, 424

elata, 367

eminens (Chabert), 370

erythrophora, 455
Rosa (eu) excogitata, 415

eximia, 356

festina, 452

flectidenta, 351

florentina, 394

Fourraei (Dés.), 341

fulgens (Adamow.), 387

fusiformis, 426

gallica (L.), 435

gallico-arvensis (Chr.), 310

gallico-biserrata (Chr.), 435

gallico-dumetorum(Chr.) 435

gallico-dumetoro-obtusifolia (Christ), 435

gallico-lutetiana (Chr.), 435

gallico-mollissima

(Rap.), 435

gallico-serpens

(Kirschl.), 310

gallico-stylosa (Timb.), 296

gallico-tomentosa

(Rap.), 435

geminata (Rau), 359

geminatoidea (Puget), 396

Gibellii, 333

glaberrima, 314

glycyphylla, 323

gynocrates, 369

Harpago, 436

hispida (Munchl.), 376

holosericea (Ser.), 432

humilis (Tausch.), 458

hybrida (Schleich.), 310

hypomelanocarpa, 399

inarimensis; 353

incarnata (Mill.), 349

incomparabilis (Cha bert), 299

indecora, 311

indita, 363

integriuscula, 398

intertexta, 366

intricans, 425

isodonta, 334

italica, 315

ladenburgensis

(Schimp.), 307

laevis (Boullu), 349

lateovata, 326

laticalyx, 444

laureola, 380

leiosepala, 331

leucophora, 294

likana (Vuk.), 376

livescens (Bess.), 392

locata, 406

longicalyx, 445 
Rosa (eu) lugdunensis, 327 macrosepala, 416 macrosphaera, 451 mirabilis (Dés.), 338 mixta (Chabert), 341 muscosa (Ait.), 413 nemocharis, 339 nemorum (Rip.), 325 notata, 390

nummularia, 314 odontotricha, 312 olbiensis, 453 opacifolia (Clabert), 322 orbicularifolia, 456 parvifolia (Ehrh.), 111 permissa, $3: 0$ phyllophora, 368 platysepala, 403 Polliniana (Spr.), 3.13 polyadena, 413 pomponia (D. C.), 443 praesensa, 303 prolixa, 439 propingua, 328 provincialis (Ait.), 457 pseudq-acutiformis, 319 pseudo-ruralis, 412 pubigera, 355 pulchella (Woods), 375 pumila (L. f.), 458 pustulifera, 438 pygmaea (M. Bieb.), 418 remensis (D. C.), 408 ramosula, 447 remota, 308

revoluta, 358

Rhodani (Chabert), 401

rhomboidalis, 414

rigescens, 395

rigidiuscula, 371

Rostani, 378

rubra (Lam.), 435

ruralis (Dés.), 391

sancta (Rich.), 413

silesiaca, 446

silvatica (Tausch), 373

slavica, 377

Spenneri, 295

spilantha, 431

stenodendron, 417

streptogyna, 301

stylophora, 292

subglabrescens, 388

subinermis (Chabert), 410

subleia, 374

subrhombeea, 304

transmutata, 386

trichopetala, 440

trillora, 430

valderia, 381
Rosa (eu)varia(Schimp.), 400 variata, 336 varians (Pohl.), 433 velutinaeflora (D. et Ozan.), 401

virescens (Dés.), 352

Vukotinovicii, 422

Waitziana (Waitz), 298

Willdenowii ('T'ratt.), 379

Wolfgangiana (Bess.), 300

Scheutzia adenosepala, 468 adenodontos, 483

alpina, 479

amblyotis (C. et M.), anserinaefolia (Boiss.), 46.4

arvernensis, 487

assyriaca, 517

astrachanica, 480

baltica (Roth.), 526

bataviea, 521

Beckeri, 482

Biebersteinii (Spr.), 528

biserrata, 510

blanda (Jac氏.), 507

borysthenica, 475

carolina (L.), 529

cinnamomea (L.), 462

cinerea (Sw.), 460

collincola (Ehrh.), 489

coruscans (Waitz.),

cremocephala, 476

cuneifolia, 478

daënensis' (Boiss.),

dahurica (Pall.)

dalecarlica, 473

desertorum, 463

dissimilis (Dés.),

domestica (Matth.), 462

echinata (Dupont),

elegans, 530

faecundissima (Moench), 465

fastuosa (Tratt.), 502

fennica, 486

Fischeriana (Bess.), 504

fluviatilis (Retz), 494

francofurtana (Münch.), 525

fraxinea (WV.), 507

fraxinifolia (Borkh.), 507

gallica, 467

Gebleriana (Schrenk),

gemella (Willd.), 493

Girgensohnii, 523

glabrifolia (C. A. M.), 505
Scheutzia Gmelini (Bge),

gorenkensis (Bess.), 491

nothica, 470

Guile'mi-WValdemarii

(Klotzsk).

Heckeliana (Tratt.), 518

hercynica, 17.4

Hofmeisterii (Klotzsk),

Hoppii, 498

hypoleuca, 490

ingriea, 511

ircutiana, 488

ivicensis, 499

Iwara (Sieb.)

Kamtschatica (Vent.), 501

lacerans (Boiss.), 520

Ledebouriana, 509

lucida (Elırh.), 521

macrophylla (Lindl.),

majalis (Retz), 492

mitis (Boiss. et Bushe), 516

mollifolia, 466

moravensis, 500

muricata (Waitz), mutica (Fl. dan.), 497

nervosa, 485

obcuneata, 484

orcidentalis, 527

orientalis (Dup.), 522

Orphanidis (Boiss.), 514

pendula, 515

peroblonga. 469

platyacantha (Schrenk),

pseudo-lucida (Hess.),

vyriformis (Siv.), 496

Regeliana (Linden),

Roxburghii (Tratt.)

rugosa (Thumb.),

sareptana, 513

sericea (Lindl.),

sibiriea (Tratt.), 512

sicula (Tratt.), 506

Silverhielmi (Schrenk),

simplex (Scop.), 462

Solandri (Tratt.), 503

Stocksiana, 520. a.

suecica, 177

taurica (M. Bieb.), 460

tomentella, 471

trillora, 472

turbinella (Sw.), 495

velutina,

vesuviana, 519

virginiana (Mill.),

Wallichii (Tratt.),

Willdenowii (Spr.),

Wolgensis, 481

Woodsii (Lindl.), 508 



\section{INDEX RERUM}

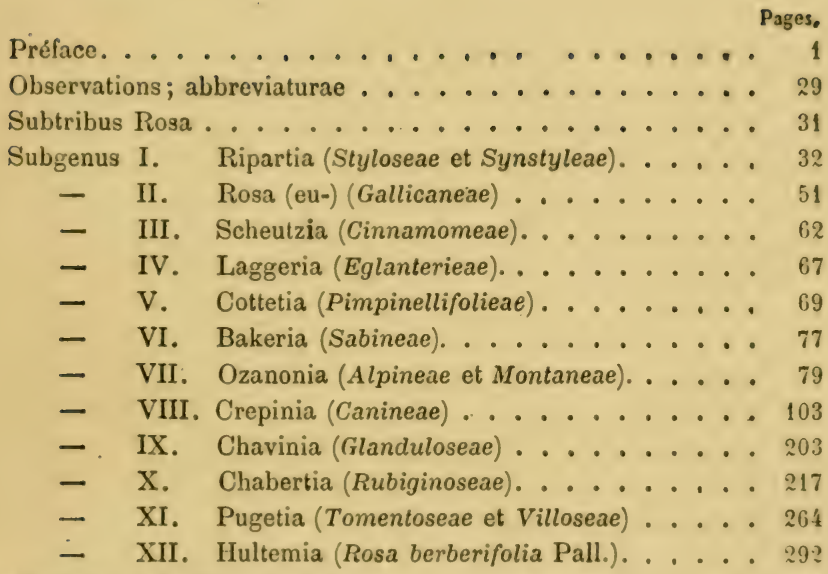

Index alphabeticus nominum ........... 293

Addenda et corrigenda. ................ 1

Rouen. - Léon DESHAYS, imprimeur de plusieurs Sociélés savantes, 





QL 145. A1 G35
Gandoger, Michel/Tabulae rhodologicae Eu

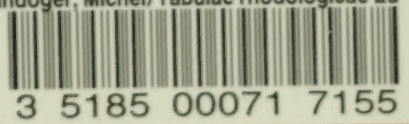


